LA W RENCE LIVERMORE N A TIONAL LABORATORY
Wide Bandgap Extrinsic Photoconductive Switches

J. S. Sullivan

July 3, 2013 
This document was prepared as an account of work sponsored by an agency of the United States government. Neither the United States government nor Lawrence Livermore National Security, LLC, nor any of their employees makes any warranty, expressed or implied, or assumes any legal liability or responsibility for the accuracy, completeness, or usefulness of any information, apparatus, product, or process disclosed, or represents that its use would not infringe privately owned rights. Reference herein to any specific commercial product, process, or service by trade name, trademark, manufacturer, or otherwise does not necessarily constitute or imply its endorsement, recommendation, or favoring by the United States government or Lawrence Livermore National Security, LLC. The views and opinions of authors expressed herein do not necessarily state or reflect those of the United States government or Lawrence Livermore National Security, LLC, and shall not be used for advertising or product endorsement purposes.

This work performed under the auspices of the U.S. Department of Energy by Lawrence Livermore National Laboratory under Contract DE-AC52-07NA27344. 


\title{
Wide Bandgap Extrinsic Photoconductive Switches
}

\author{
By \\ JAMES S. SULLIVAN \\ National Security Engineering Division \\ Lawrence Livermore National Laboratory
}

\begin{abstract}
Semi-insulating Gallium Nitride, $4 \mathrm{H}$ and $6 \mathrm{H}$ Silicon Carbide are attractive materials for compact, high voltage, extrinsic, photoconductive switches due to their wide bandgap, high dark resistance, high critical electric field strength and high electron saturation velocity. These wide bandgap semiconductors are made semi-insulating by the addition of vanadium $(4 \mathrm{H}$ and $6 \mathrm{H}-$ $\mathrm{SiC}$ ) and iron (2H-GaN) impurities that form deep acceptors. These deep acceptors trap electrons donated from shallow donor impurities. The electrons can be optically excited from these deep acceptor levels into the conduction band to transition the wide bandgap semiconductor materials from a semi-insulating to a conducting state. Extrinsic photoconductive switches with opposing electrodes have been constructed using vanadium compensated $6 \mathrm{H}-\mathrm{SiC}$ and iron compensated $2 \mathrm{H}-\mathrm{GaN}$. These extrinsic photoconductive switches were tested at high voltage and high power to determine if they could be successfully used as the closing switch in compact medical accelerators.
\end{abstract}

The successful development of a vanadium compensated, 6H-SiC extrinsic photoconductive switch for use as a closing switch for compact accelerator applications was realized by improvements made to the vanadium, nitrogen and boron impurity densities. The changes made to the impurity densities were based on the physical intuition outlined and simple rate equation models. The final $6 \mathrm{H}-\mathrm{SiC}$ impurity 'recipe' calls for vanadium, nitrogen and boron 
densities of $2.5 \mathrm{e} 17 \mathrm{~cm}^{-3}, 1.25 \mathrm{e} 17 \mathrm{~cm}^{-3}$ and $\leq 1 \mathrm{e} 16 \mathrm{~cm}^{-3}$, respectively. This recipe was originally developed to maximize the quantum efficiency of the vanadium compensated $6 \mathrm{H}-\mathrm{SiC}$, while maintaining a thermally stable semi-insulating material. The rate equation models indicate that, besides increasing the quantum efficiency, the impurity recipe should be expected to also increase the carrier recombination time.

Three generations of $6 \mathrm{H}-\mathrm{SiC}$ materials were tested. The third generation vanadium compensated $6 \mathrm{H}-\mathrm{SiC}$ has average impurity densities close to the recipe values. Extrinsic photoconductive switches constructed from the third generation vanadium compensated, $6 \mathrm{H}-\mathrm{SiC}$, $1 \mathrm{~mm}$ thick, $1 \mathrm{~cm}^{2}$, substrates have achieved high power operation at $16 \mathrm{kV}$ with pulsed currents exceeding 1400 Amperes and a minimum on resistance of $1 \mathrm{ohm}$. The extrinsic photoconductive switch performance of the third generation $6 \mathrm{H}-\mathrm{SiC}$ material was improved by a factor of up to 50 for excitation at the $532 \mathrm{~nm}$ wavelength compared to the initial $6 \mathrm{H}-\mathrm{SiC}$ material. Switches based on this material have been incorporated into a prototype compact proton medical accelerator being developed by the Compact Particle Acceleration Corporation (CPAC).

The vanadium compensated, 6H-SiC, extrinsic photoconductive switch operates differently when excited by 1064, or $532 \mathrm{~nm}$, wavelength light. The 6H-SiC extrinsic photoconductive switch is a unipolar device when excited with $1064 \mathrm{~nm}$ light. The carriers are electrons excited from filled vanadium acceptor levels and other electron traps located within $1.17 \mathrm{eV}$ of the conduction band. The switch is bipolar at $532 \mathrm{~nm}$ since the carriers consist of holes, as well as electrons. The holes are primarily generated by the excitation of valence band electrons into empty trap/acceptor levels and by two-photon absorption. Carrier generation by two-photon absorption becomes more important at high applied optical intensity at $532 \mathrm{~nm}$ and contributes to the supralinear behavior of switch conductance as a function of optical power. 
The $6 \mathrm{H}-\mathrm{SiC}$ switch material is trap dominated at low nitrogen to vanadium ratios. The trap dominated vanadium compensated $6 \mathrm{H}-\mathrm{SiC}$ exhibits low quantum efficiency when excited with 1064 and $532 \mathrm{~nm}$ light and has a carrier recombination time of $150-300$ ps. The vanadium compensated $6 \mathrm{H}-\mathrm{SiC}$ transitions to an impurity dominated material as the ratio of nitrogen to vanadium is increased to 0.5 . The increased nitrogen doping produces a material with much higher quantum efficiency and carrier recombination time of 0.9 to $1.0 \mathrm{~ns}$.

The iron compensated $2 \mathrm{H}-\mathrm{GaN}$ did not perform well as an extrinsic photoconductive switch. The density of carriers generated at $1064 \mathrm{~nm}$ was, low indicating that there were very few electrons trapped in the iron acceptor level located at $0.5-0.6 \mathrm{eV}$ below the conduction band. Carrier generation at $532 \mathrm{~nm}$ was dominated by two photon absorption resulting in the switch conductance increasing as the square of applied optical intensity. A minimum switch resistance of 0.8 ohms was calculated for the $400 \mu$ m thick, 1.2 by $1.2 \mathrm{~cm}, 2 \mathrm{H}-\mathrm{GaN}$ switch for an applied optical intensity of $41.25 \mathrm{MW} / \mathrm{cm}^{2}$. An optical intensity of $\sim 70 \mathrm{MW} / \mathrm{cm}^{2}$ at $532 \mathrm{~nm}$ would be required to achieve a $0.8 \mathrm{ohm}$ on resistance for a $1 \mathrm{~mm}$ thick, $1 \mathrm{~cm}^{2}, 2 \mathrm{H}-\mathrm{GaN}$ switch.

This work was performed under the auspices of the U.S. Department of Energy by Lawrence Livermore National Laboratory under Contract DE-AC52-07NA27344. 


\section{CONTENTS}

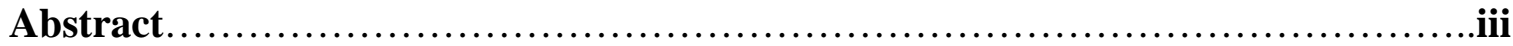

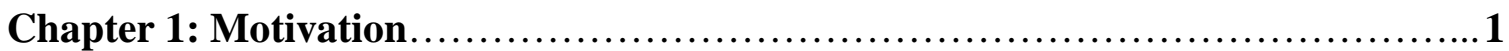

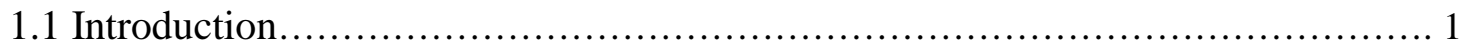

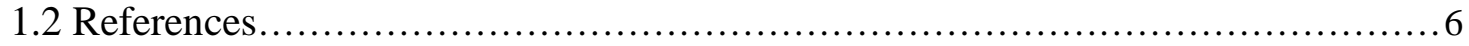

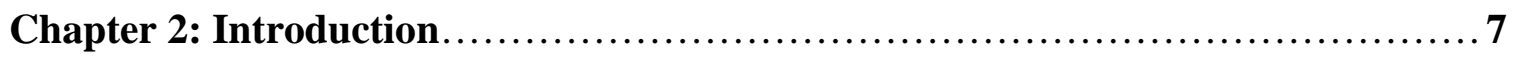

2.1 Photoconductive Semiconductor Switches (PCSS) ............................

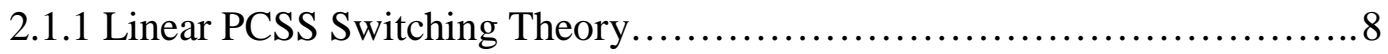

2.1.2 Intrinsic Optical Aborption.............................................11

2.1.3 Extrinsic Optical Absorption........................................... 15

2.1.4 Nonlinear PCSS Switching Results...................................16

2.1.5 Linear Intrinsic Switching Results..................................17

2.1.6 SiC PCSS Device Results........................................22

2.2 Wide Bandgap Semiconductors...........................................24

2.2.1 Silicon Carbide........................................................

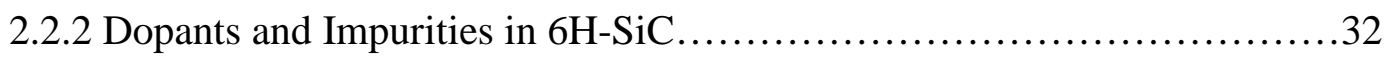

2.2.3 Gallium Nitride....................................................... 34

2.2.4 Impurities in Gallium Nitride........................................38

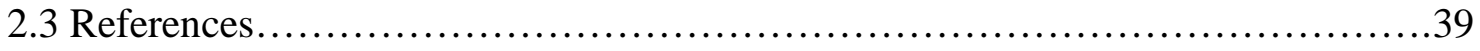

Chapter 3: Electronic Properties of 4H-SiC, 6H-SiC and 2H-GaN............... 42

$3.14 \mathrm{H}$ and $6 \mathrm{H}-\mathrm{SiC}$ Band Structure and Effective Mass.......................... 42

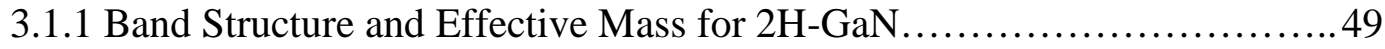


3.2 Intrinsic Carriers in 4H-SiC, $6 \mathrm{H}-\mathrm{SiC}$ and $2 \mathrm{H}-\mathrm{GaN}$.

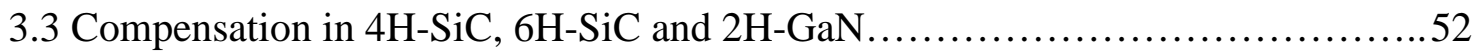

3.3.1 Incomplete Ionization........................................54

3.3.2 Semi-insulating 4H, 6H-SiC and 2H-GaN by Compensation with Deep Acceptors......................................................... 59

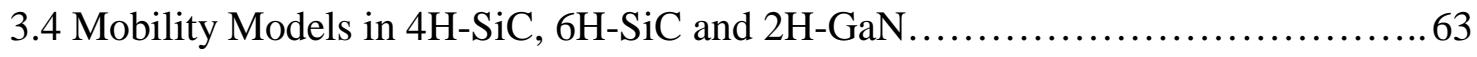

3.5 Recombination........................................................68

3.5.1 Radiative Recombination.....................................68

3.5.2 Auger and Shockley - Read - Hall (SRH) Recombination................70

3.5.3 Traps..................................................... 74

3.6 References........................................................ 79

Chapter 4: Below Bandgap Optical Properties of 4H-SiC, 6H-SiC and 2H-GaN.... 81

4.1 Introduction........................................................... 81

4.2 Above Bandgap Optical Absorption in 2H-GaN, 4H and 6H-SiC...............82

4.3 General Optical Properties of Uniaxial Crystals.............................. 86

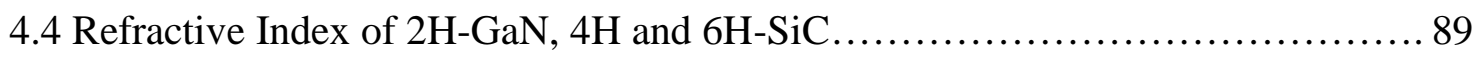

4.5 Optical Generation of Carriers in Extrinsic Photoconductive Switches............ 96

4.5.1 Radiative Absorption in Semiconductors........................... 97

4.5.2 Models for Optical Absorption at Deep Impurities in Semiconductors... 102

4.5.3 Lucovsky Model for Photoionization of Deep Impurities................ 104

4.5.4 Ridley Model for the Photoionization of Deep Impurities.............. 107

4.5.5 Franck - Condon Shift........................................... 112 
4.5.6 Two Step Optical Transitions.

4.6 Nonlinear Optical Properties of 4H-SiC, 6H-SiC and 2H-GaN.................119

4.6.1 Second Harmonic Generation.................................. 120

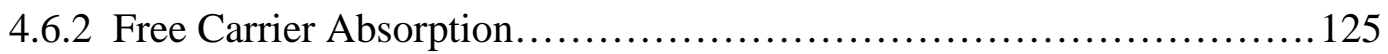

4.7 Conclusions......................................................... 130

4.8 References........................................................ 132

\section{Chapter 5: Initial Testing of Vanadium Compensated 6H-SiC and Iron}

Compensated 2H-GaN Extrinsic Photoconductive Switches..................................136

5.1 6H-SiC Test Results................................................ 136

5.1.1 6H-SiC Dark Resistance Measurements............................137

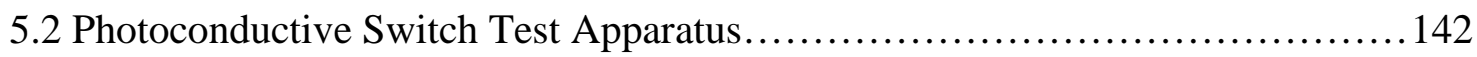

5.2.1 Electrical Components......................................... 142

5.2.2 Optical Components........................................... 148

5.3 Extrinsic Photoconductive Switch Tests of Semi-insulating, Vanadium

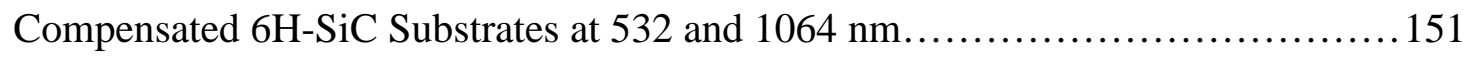

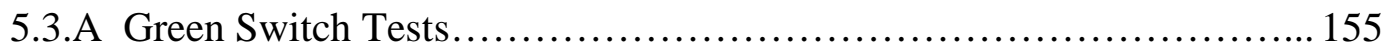

5.3.B Green Switch Photoconductive Switch Results at $532 \mathrm{~nm} . . . . . . . . . . . . .164$

5.3.C Black and Silver Switch Photoconductive Switch Tests................ 169

5.4 Summary of Initial Photoconductive Switching Results for $6 \mathrm{H}-\mathrm{SiC} . . . . . . . . . .190$

5.4.1 Supralinear Response of 6H-SiC Switches..........................195

5.5 2H-GaN Photoconductive Switching Test Results..........................199

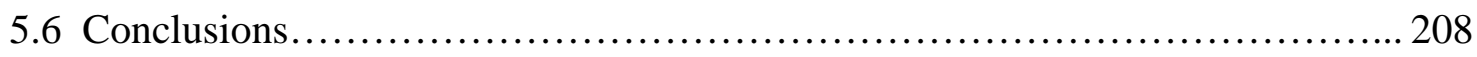


Section 5.7 References.

Chapter 6: Parameters Limiting Switch Performance.

6.1 Switch High Voltage Breakdown.

6.2 Improving the Quantum Efficiency of Vanadium Doped 6H-SiC 218

6.3 Optical Transmission Measurements in 6H-SiC and 2H-GaN. 224

6.4 Switch Triggering with Different Optical Profiles and at Multiple Facets

6.5 Switch Turn-on and Turn-off Times 235

6.6 Conclusions

6.7 References

Chapter 7: Analysis of Initial Test Results of Vanadium Compensated, Semiinsulating, 6H-SiC Extrinsic Photoconductive Switches.

7.1 Photoconductive Switch and Test Circuit Interaction. 248

7.2 Switch Analysis 258

7.3 Vanadium and Nitrogen Co-doped 6H-SiC in Thermal Equilibrium at Room Temperature 260

7.3.1 Point Defects. 268

7.4 Switch Model for Operation at $1064 \mathrm{~nm}$ 276

7.4.1 Switch Model at $1064 \mathrm{~nm}$ Including Only the Effects of Nitrogen, Vanadium and Boron.

7.4.2 Switch Model at $1064 \mathrm{~nm}$ Including the Effects of Impurities and a Deep Trap Located Close to the Vanadium Acceptor Level....

7.4.3 Switch Model at $1064 \mathrm{~nm}$ for Increased Nitrogen and Vanadium Doping 290 
7.5 Conclusions

7.6 References

298

Chapter 8: Comparison of Switches Fabricated with Improved Doping Profile

6H-SiC to Switches Fabricated with Initial 6H-SiC Material. 300

8.1 Introduction 300

8.2 Substrate Doping Profile, Physical Dimensions and Micropipe Density for Second Generation, Vanadium and Nitrogen Doped 6H-SiC.... 304

8.3 Dark Current in Improved Doping Profile Second Generation 6H-SiC Substrates .311

8.4 Photoconductive Switching Tests of Second Generation 6H-SiC Substrates......313

8.5 Third Generation 6H-SiC Material 329

8.5.1 Third Generation 6H-SiC Material Switch Test Results at $532 \mathrm{~nm}$.... 333

8.6 Conclusions.

8.7 References 340

\section{Chapter 9: High Voltage, High Power Testing of Third Generation 6H-SiC}

Extrinsic Photoconductive Switch.

9.1 High Voltage Tests.... 348

9.2 Conclusions 354

Chapter 10: Conclusions and Reccomendations. .356

10.1 Conclusions 356

10.2 Recommendations. 358 


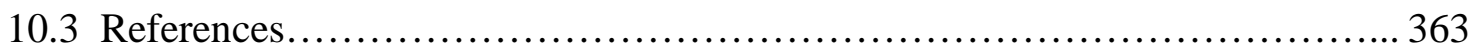

-ix- 


\section{Chapter 1: Motivation}

\section{1) Introduction}

There has been a recent increase in research to develop inexpensive, compact, charged particle accelerator systems intended for pulsed radiography and medical applications. This research activity has been motivated, in part, by the dielectric wall accelerator (DWA) concept [1.1] illustrated in Figure 1.1. The DWA can reduce the length of an accelerating structure by up to two orders of magnitude as compared to Linear Induction and standard RF Linear Accelerators of comparable energy. The advantage of the DWA approach is that the pulsed

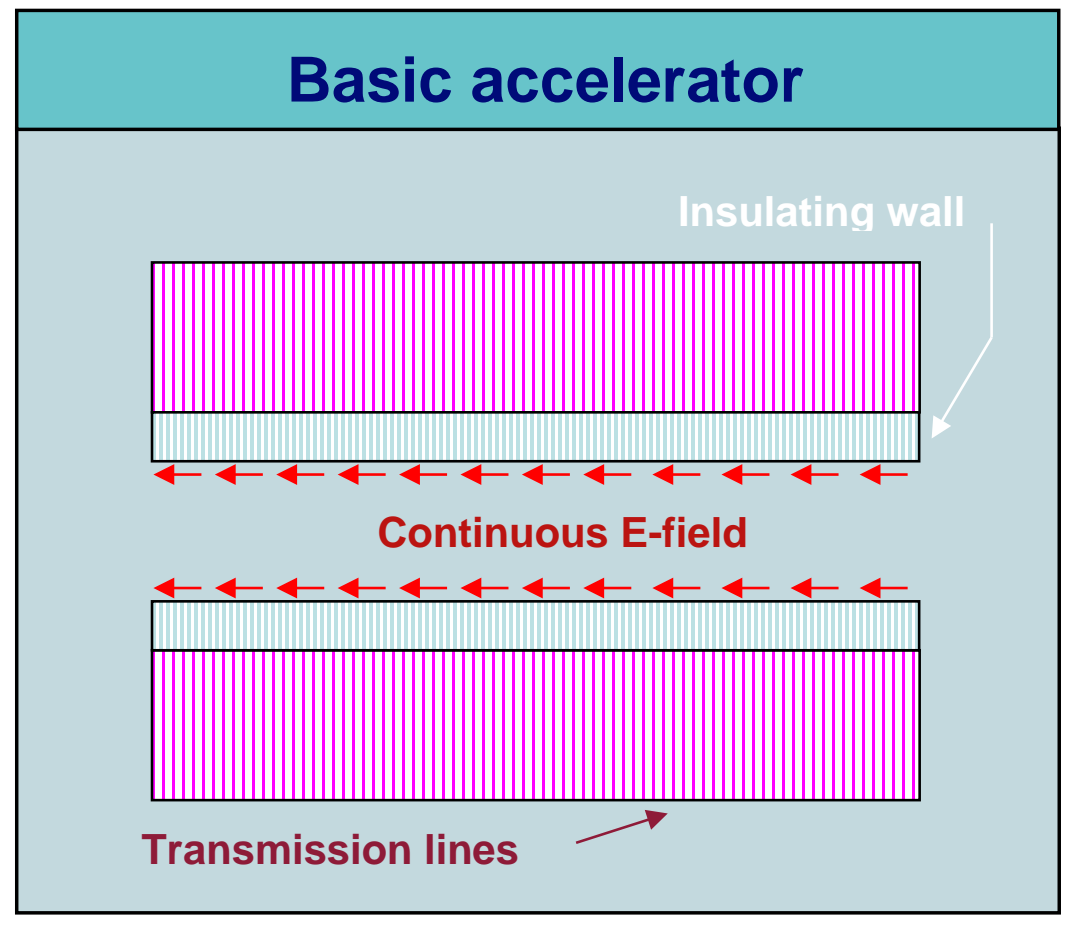

Figure 1.1 Dielectric Wall Accelerator concept

accelerating field can be applied along the entire length of the high gradient insulator (HGI) [1.2] vacuum wall. The magnitude of the applied field in the DWA is limited only by the dielectric strength of the HGI, which is believed be up to $100 \mathrm{MV} / \mathrm{m}$ for pulse widths of several nanoseconds duration. In comparison, Linear Induction and standard RF Linear Accelerators 
couple energy into the particle beam using accelerating gaps periodically spaced along the length of the accelerator. A fraction of the entire length of these accelerator structures is used to accelerate the beam. As a result, the average field gradients for Linear Induction and standard RF Linear Accelerators range from $1-8 \mathrm{MV} / \mathrm{m}$ [1.3]. The maximum DWA gradient of $100 \mathrm{MV} / \mathrm{m}$ also compares favorably to the gradients of $50-70 \mathrm{MV} / \mathrm{m}$ obtained with $[1.4,1.5] \mathrm{X}$-band RF linear accelerators.

Figure 1.1 shows a stack of pulse forming transmission lines that deliver the pulsed electric field that appears across the HGI of the dielectric wall accelerator. A Blumlein stack can be used for this purpose. Single Blumlein pulse forming lines can be stacked one upon another to form a Blumlein stack. A single Blumlein can consist of two parallel plate transmission lines stacked upon one another and an electrical switch mounted between the center and bottom conductor shown on the left of Figure 1.2. The center conductor of the Blumlein is charged to a voltage $\mathrm{V}$ with respect to the top and bottom conductors. A negative voltage wave is launched on the bottom transmission line of the Blumlein when the electrical switch is closed. A negative voltage $\mathrm{V}$ appears across the matched load when the negative voltage wave traveling on the bottom transmission line reaches the load. The negative voltage remains across the load for a time equal to the round trip transit time of the transmission lines forming the Blumlein. Any desired output voltage can be obtained by stacking single Blumleins as shown in Figure 1.3. The desired pulse width is obtained by proper selection of line length ( $\mathrm{L}$ ) and dielectric material. Identical Blumlein stacks can be connected electrically in parallel to drive high current loads. For example, if four Blumlein stacks like that shown in Figure 1.3 were connected in parallel, it would apply a voltage of $5 \mathrm{~V}_{\mathrm{ch}}$ across a load of $10 \mathrm{Z}_{\text {line. }}$. High voltage pulses can be applied to 


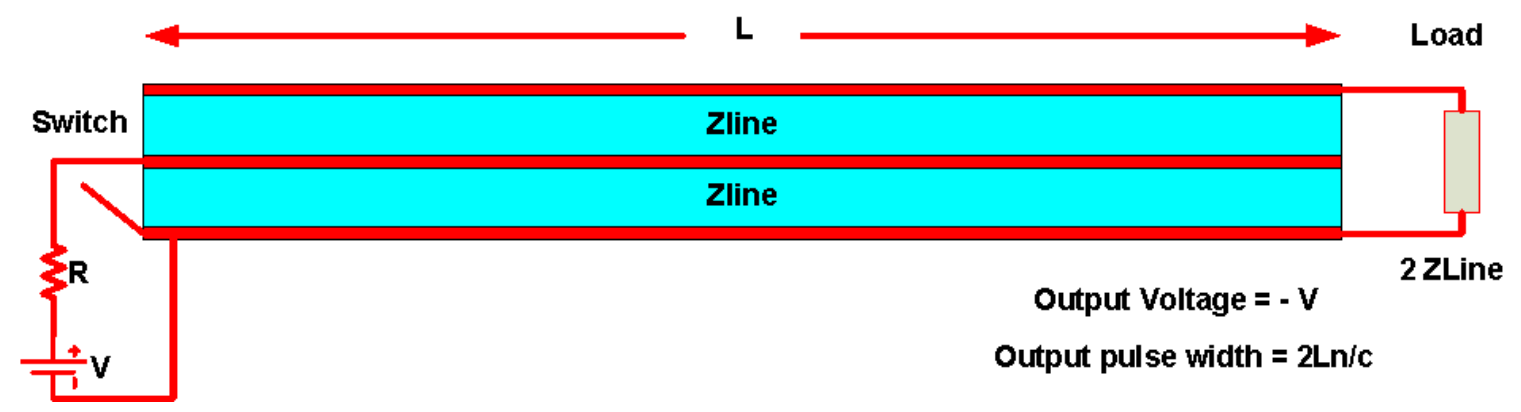

Figure 1.2. Matched single Blumlein pulse forming line.

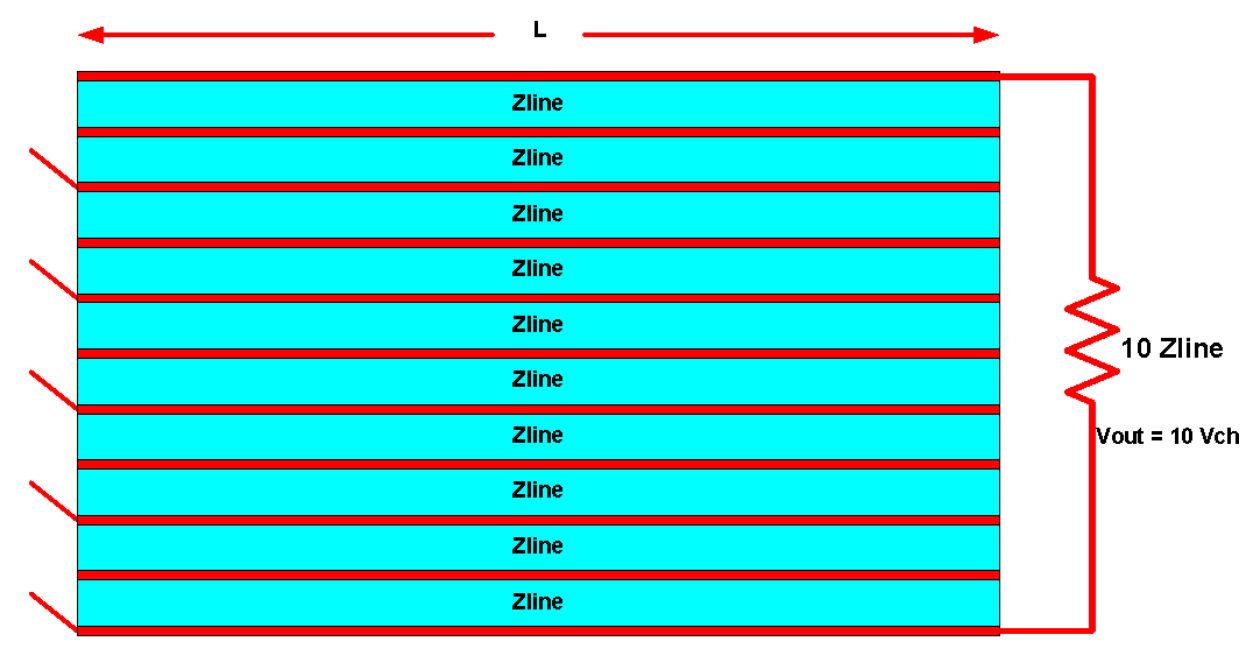

\section{5-high Stack of Single Blumleins}

\section{Figure 1.3. Blumlein stack}

arbitrary loads by constructing Blumlein stacks from single Blumleins and combining the stacks in parallel.

The only active component in the Blumlein is the switch. The Blumlein electrical switch has to withstand the charge voltage $\left(\mathrm{V}_{\mathrm{ch}}\right)$ and switch the line current $\left(\mathrm{V}_{\mathrm{ch}} / \mathrm{Z}_{\text {line }}\right)$. The DWA application places stringent requirements on the electrical switches incorporated into the single Blumlein assembly. The switches must be compact, withstand electric fields on the order of 50 $100 \mathrm{MV} / \mathrm{m}$, switch several kiloamperes of current with sub-nanosecond risetime, be precisely triggered and exhibit low jitter and high reliability. The DWA based compact medical accelerator 
application has the additional requirement that the electrical switch operate for extended periods of time at repetition rates of a few Hertz. This requires an electrical switch with lifetimes in the $10^{7}-10^{8}$ pulse range. A switch meeting the DWA requirements would also be useful in other applications. If the switch has a fast recovery time it can also be used in both hard tube and transmission line modulators applications. These would include high voltage "adder" and ultrawideband RF circuits. The need to meet the switching requirements for the DWA application lead us to consider high voltage, photoconductive semiconductor switches (PCSS). These are compact, optically triggered, high voltage devices.

Wide bandgap extrinsic photoconductive switches quickly became the focus of the research due to their wide bandgap, high critical electric field strength compared to silicon and gallium arsenide, high thermal conductivity $(4 \mathrm{H}$ and $6 \mathrm{H}-\mathrm{SiC})$ and recent availability in semiinsulating substrates. Operating the wide bandgap photoconductive switches in the extrinsic mode enabled the construction of switches with electrodes on the opposing faces on the semiinsulating substrates, so full advantage could be taken of the high critical electric field strength of the materials. However, there was no existing data for $4 \mathrm{H}$ and $6 \mathrm{H}-\mathrm{SiC}$ used as extrinsic photoconductive switches and no data for $2 \mathrm{H}-\mathrm{GaN}$ as a high power photoconductive switch of any kind. In addition, the candidate photoconductive switches would have to be capable of attaining an on resistance of $\sim 1 \mathrm{ohm}$, hold off a minimum of $15-20 \mathrm{kV}$ and switch greater than a kilo-amp of current. These switching requirements prompted an extensive review of the existing literature concerning the pertinent electrical and below bandgap optical properties of $4 \mathrm{H}-\mathrm{SiC}, 6 \mathrm{H}-\mathrm{SiC}$ and $2 \mathrm{H}-\mathrm{GaN}$. Models for the compensation mechanism in $4 \mathrm{H}, 6 \mathrm{H}-\mathrm{SiC}$ and $2 \mathrm{H}-$ $\mathrm{GaN}$ had to be developed based on the location of the nitrogen, boron and vanadium impurities in $4 \mathrm{H}$ and $6 \mathrm{H}-\mathrm{SiC}$, and iron and oxygen impurities in $2 \mathrm{H}-\mathrm{GaN}$. During the literature review, 
proof of principal, photoconductive switches were constructed and tested from available vanadium and nitrogen co-doped $6 \mathrm{H}-\mathrm{SiC}$ and iron compensated, $2 \mathrm{H}-\mathrm{GaN}$ semi-insulating semiconductor materials to establish the feasibility of the wide bandgap materials as high power, extrinsic, photoconductive switches. Additional switch testing identified device limitations with respect to operating voltage, optical absorption, switch rise-time and rate of rise of switch recovery voltage. These initial tests determined that both the iron compensated 2H-GaN and vanadium and nitrogen co-doped $6 \mathrm{H}-\mathrm{SiC}$ were both suitable materials for extrinsic photoconductive switch construction. However, the test results also indicated that significant improvement in quantum efficiency would be required to obtain a practical switch material. A detailed knowledge of the vanadium, nitrogen and boron dopant densities of the initial $6 \mathrm{H}-\mathrm{SiC}$ material and the physical understanding of how manipulate these dopant densities to improve quantum efficiency and photoconductive switch performance had to be established to develop a practical 6H-SiC extrinsic photoconductive switch.

Secondary Ion Mass Spectroscopy (SIMS) measurements of the vanadium, nitrogen, boron and aluminum impurity levels in the initial 6H-SiC material (first generation), initial switching results and a simple rate equation model for photo-generated carrier density formed the basis for specifying nitrogen, vanadium and boron impurity profiles for improved photoconductive switch performance. Photoconductive switches were constructed from two successive generations of $6 \mathrm{H}-\mathrm{SiC}$ material with improved vanadium, nitrogen and boron levels based on the specifications developed. Switch testing of the third generation material $6 \mathrm{H}-\mathrm{SiC}$ material showed an improvement in quantum efficiency by a factor of 20 - 30 times compared to the initial $6 \mathrm{H}-\mathrm{SiC}$ (first generation) substrate material. Third generation material $6 \mathrm{H}-\mathrm{SiC}$ was 
used to construct a $1 \mathrm{~cm}$ by $1 \mathrm{~cm}, 1 \mathrm{~mm}$ thick switch that successfully operated at a charge voltage of $17 \mathrm{kV}$ while switching 1500 Amps.

\section{2) References}

1.1) S.E.Sampayan, et al., ”Development of a compact radiography accelerator using dielectric wall accelerator technology”, presented at the $15^{\text {th }}$ International IEEE Pulsed Power Conference, Monterey, CA June 2005

1.2) S.E.Sampayan, et al., "High gradient multiplayer insulator technology”, Proceedings of the $26^{\text {th }}$ International IEEE Power Modulator Symposium and High Voltage Workshop 2004 IEEE Power Modulator Conference, May 2004, pp 46 - 49

1.3) S.Humphries, Jr., Principles of Charged Particle Acceleration, pages 288 and 440, Wiley - Interscience, 1986

1.4) C. Christou, "X-band linac technology for a high repetition rate light source", Nuclear Instruments and Methods in Physics Research A, Volume 627, pp. 13 - 21, 2011

1.5) F. Albert, F. Hartemann, S. Anderson, R. Cross, D. Gibson, et al., "Precision linac and laser technologies for nuclear photonics gamma-ray sources”, Physics of Plasmas, Volume 19, No. 5, 059902, 2012 


\section{Chapter 2: Introduction}

Photoconductive semiconductor switches (PCSS) have been investigated since the late 1970s [2.1, 2.2]. Some devices have been developed that withstand tens of kilovolts and others that switch hundreds of amperes [2.3]. However, no single device has been developed that can reliably withstand both high voltage and switch high current. Yet, photoconductive switches still hold the promise of reliable high voltage and high current operation with sub-nanosecond risetimes. Particularly since good quality, bulk, single crystal, wide bandgap semiconductor materials have recently become available. In this chapter we will review the basic operation of PCSS devices, status of PCSS devices and properties of the wide bandgap semiconductors $4 \mathrm{H}-$ SiC, $6 \mathrm{H}-\mathrm{SiC}$ and $2 \mathrm{H}-\mathrm{GaN}$.

\section{1) Photoconductive Semiconductor Switches (PCSS)}

A PCSS device is constructed by depositing metallic contacts on the same side (lateral geometry) or, opposing sides (vertical geometry) of a piece of semi-insulating semiconductor material. A lateral geometry PCSS is illustrated in Figure 2.1. The PCSS is switched on by applying optical energy to the interelectrode gap. PCSS devices can have linear and nonlinear operating modes. A linear PCSS device is gated 'on' by generating carriers in the bulk semiconductor material by applying photons of the proper wavelength. An electron-hole pair is generated for each absorbed photon in the ideal case. A nonlinear PCSS device is gated 'on' by optically generating a small number of carriers in the semiconductor bulk that are multiplied by an electric field avalanche mechanism termed 'lock-on'. 


\section{Simple Photoconductive Switch Geometry}

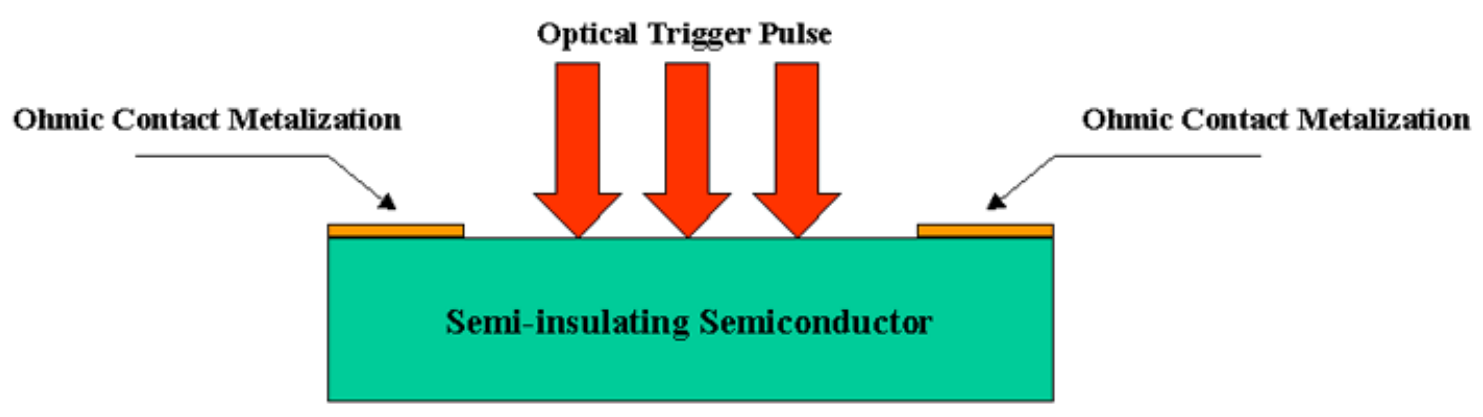

Figure 2.1. Lateral geometry photoconductive semiconductor switch (PCSS)

\subsection{1) Linear PCSS Switching Theory}

A vertical, linear PCSS geometry is shown in Figure 2.2. This is the primary geometry of PCSS devices constructed for this work. Figure 2.2 shows the side view of a rectangular semiconductor substrate of width $\mathrm{W}$, thickness $\mathrm{d}$ and length $\mathrm{L}$ (into page). The dark resistance of this device is given by:

$$
R_{\text {dark }}=\frac{\rho_{o} \cdot d}{W \cdot L}
$$

Where $\rho_{0}$ is the dark resistivity of the semiconductor material and is given by:

$$
\rho_{0}=\frac{1}{q\left(n_{0} \cdot \mu_{n 0}+p_{0} \cdot \mu_{p 0}\right)}
$$

Where $\mathrm{n}_{0}$ and $\mathrm{p}_{0}$ are the free electron and hole densities and $\mu_{\mathrm{n} 0}$ and $\mu_{\mathrm{p} 0}$ are the electron and hole mobilities. The resistivity of the PCSS device is reduced when a uniformly distributed pulse of light is applied on the left hand facet. The optical excitation generates carriers governed by [2.4]

$$
\frac{\partial n(t)}{\partial t}=G(t)-\frac{n(t)}{\tau_{r}}
$$


Where we have assumed only electrons are generated and $G$ and $\tau_{\mathrm{r}}$ are the electron generation rate and recombination time, respectively. In general, the total number of electrons present in the semiconductor is the sum of the free and photogenerated electrons.

$$
n=n_{0}+\Delta n
$$

Where $\Delta \mathrm{n}$ is the amount of optically generated electrons. The PCSS devices are usually constructed using semi-insulating semiconductor material. This justifies the following simplification.

$$
n=\Delta n
$$

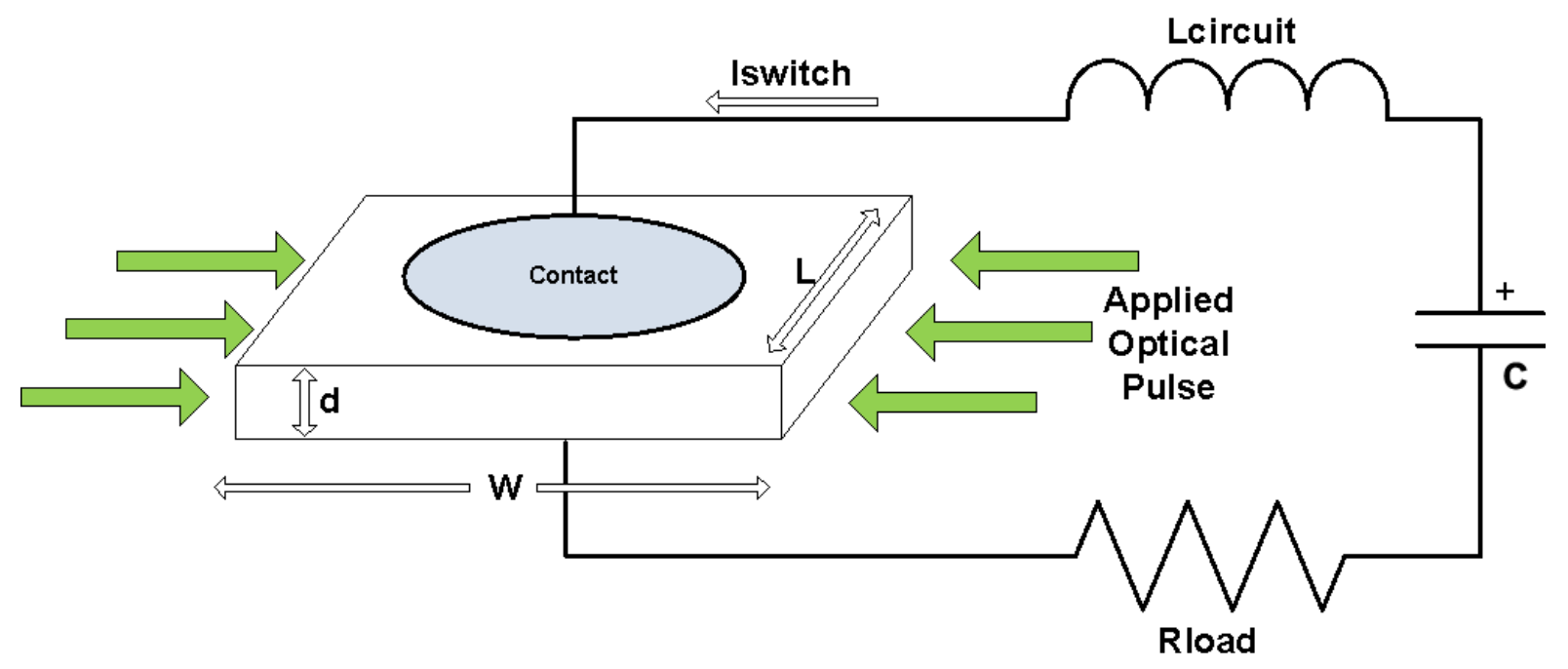

Figure 2.2. Vertical Geometry PCSS

The LaPlace transform is used to obtain a general solution for equation (2.3).

$$
n(t)=\int_{-\infty}^{t} G(s) e^{(s-t) / \tau_{r}} d s
$$


The carrier density is given by the convolution of the carrier generation rate and the exponential decay due to recombination. The optical generation rate of carriers for a linear, intrinsic semiconductor is given by [2.5]:

$$
G(t)=\frac{P(t) \cdot \eta}{W \cdot d \cdot L \cdot \hbar \omega}(1-r) \cdot\left(1-e^{-\alpha W}\right)
$$

Where $\mathrm{P}(\mathrm{t})$ is the applied optical power, $\alpha$ is the linear absorption, $\hbar \omega$ is the photon energy, $\eta$ is the quantum efficiency and $\mathrm{r}$ is the switch surface reflectance. Let us assume that the applied optical trigger has constant power $\left(\mathrm{P}_{0}\right)$ for pulse duration, $\mathrm{T}$, and the recombination time $\left(\tau_{\mathrm{r}}\right)$ is much larger than optical pulse width $\left(\mathrm{T}<<\tau_{\mathrm{r}}\right)$. Equation (2.6) reduces to the following solution for this limiting case.

$$
n(t)=\frac{(1-r)\left(1-e^{-\alpha W}\right) \cdot \eta \cdot e^{-t / \tau_{r}}}{h v \cdot W d L} \int_{-\infty}^{T} P(s) d s=(1-r)\left(1-e^{\alpha W}\right) \cdot \eta \cdot P_{0} \frac{T}{h v \cdot W d L} e^{-t / \tau_{r}}
$$

The carrier density and switch current are proportional to the total applied optical energy $\left(\mathrm{P}_{\mathrm{o}} \mathrm{T}\right)$ for the case when the recombination time is long compared to the optical pulse width. The dynamic PCSS resistance for this case is written:

$$
R(t)=e^{t / \tau_{r}} \cdot \frac{d^{2} \cdot h v}{P_{0} T \mu_{n}(1-r)\left(1-e^{-\alpha W}\right) q \eta}
$$

Where $\mu_{\mathrm{n}}$ is the electron mobility and $\mathrm{q}$ is the elementary unit of charge. It is important to note that the PCSS resistance is inversely proportional to the applied optical energy and proportional to the square of the switch length when the recombination time $\left(\tau_{r}\right)$ is long compared to the optical pulse width (T). A second solution for equation (2.3) exists for the case where the optical pulse width $(\mathrm{T})$ is much greater that the recombination time $\left(\tau_{\mathrm{r}}\right)$.

$$
n(t)=\frac{P(t)(1-r)\left(1-e^{-\alpha W}\right) \cdot \eta}{h v \cdot W d L} \cdot \tau_{r}
$$


The dynamic resistance for this case is

$$
R(t)=\frac{d^{2} \cdot h v}{P(t) \tau_{r} \mu_{n}(1-r)\left(1-e^{-\alpha W}\right) q \eta}
$$

The optically generated carrier density and switch current for a semiconductor material with short recombination time will have a temporal profile similar to the applied optical power. The PCSS resistance is proportional to the square of the switch length and inversely proportional to the applied optical power. The PCSS resistance expressions $(2.9,2.11)$ for the limiting cases show that an increase in quantum efficiency $(\eta)$ results in a reduction of switch resistance for constant applied optical energy, or power. An increase in quantum efficiency will also decrease the amount of optical energy, or power required to obtain a given switch resistance. There are several ways to express quantum efficiency in a photoconductive switch. We define the quantum efficiency as the ratio of the number of photon generated carriers created per second to the number of absorbed photons. This can be written using equation (2.10) as

$$
\eta=\frac{n(t) \cdot W L d}{(P(t) / h v) \cdot(1-r)\left(1-e^{-\alpha W}\right)}
$$

It must be kept in mind that the equations developed for photocarriers and dynamic resistance for the two extreme cases of optical excitation have been developed assuming that absorption coefficient is dominated by photogeneration, saturation of the absorption and nonlinear absorption effects are neglected. These effects need to be considered in actual devices.

\subsection{2) Intrinsic Optical Absorption}

Linear PCSS devices are based on photogenerated carriers created by intrinsic, or, extrinsic optical absorption in the semiconductor material. Intrinsic optical absorption [2.5] corresponds to the creation of an electron-hole pair in a semiconductor material by the absorption 
of a photon with energy greater than, or equal to, the bandgap energy. This process is called intrinsic optical absorption since it is due solely to the intrinsic properties of the semiconductor material. Intrinsic absorption results in the promotion of an electron from the valence band into the conduction band, leaving a hole in the valence band. The excitation of the electron from the valence to the conduction band is termed an optical transition. Energy and momentum must be conserved in all optical transitions. Optical transitions require a photon with a minimum energy greater than, or, equal to the semiconductor material bandgap. The excitation of an electron from the valence band to the conduction band is a direct, or vertical, intrinsic optical transition if the energy minimum of the conduction band occurs at the same point in $\mathbf{k}$ space as the energy maximum of the valence band. A direct optical transition is depicted in Figure 2.3. Energy and momentum are conserved in a direct transition since the absorbed photon energy (hv) equals the bandgap energy $\left(E_{g}\right)$, the conduction band minimum and valence band maximum are aligned in $\mathbf{k}$ space and the photon momentum is negligible. The absorption coefficient $(\alpha)$ for a direct transition has units of $\mathrm{cm}^{-1}$ and is proportional to the square root of the energy difference between the absorbed photon and material bandgap [2.4].

$$
\alpha(\hbar \omega) \propto\left(\hbar \omega-E_{G}\right)^{\frac{1}{2}} \quad \text { for } \quad \hbar \omega \geq E_{G}
$$

The absorption coefficient for a direct transition grows rapidly as the photon energy (hv) exceeds the bandgap. Table 2.1 lists the absorption coefficient $(\alpha)$ and penetration depth $(1 / \alpha)$ in the direct bandgap semiconductor Gallium Arsenide for photon energies of 1.5, 2.0 and $2.34 \mathrm{eV}$. The resulting optical penetration depth $(1 / \alpha)$ for photon energies above $2.0 \mathrm{eV}$ are fractions of a micron. So, as the photon energy increases above the bandgap energy, most of the photons are absorbed in a thin layer at the surface of the material. 


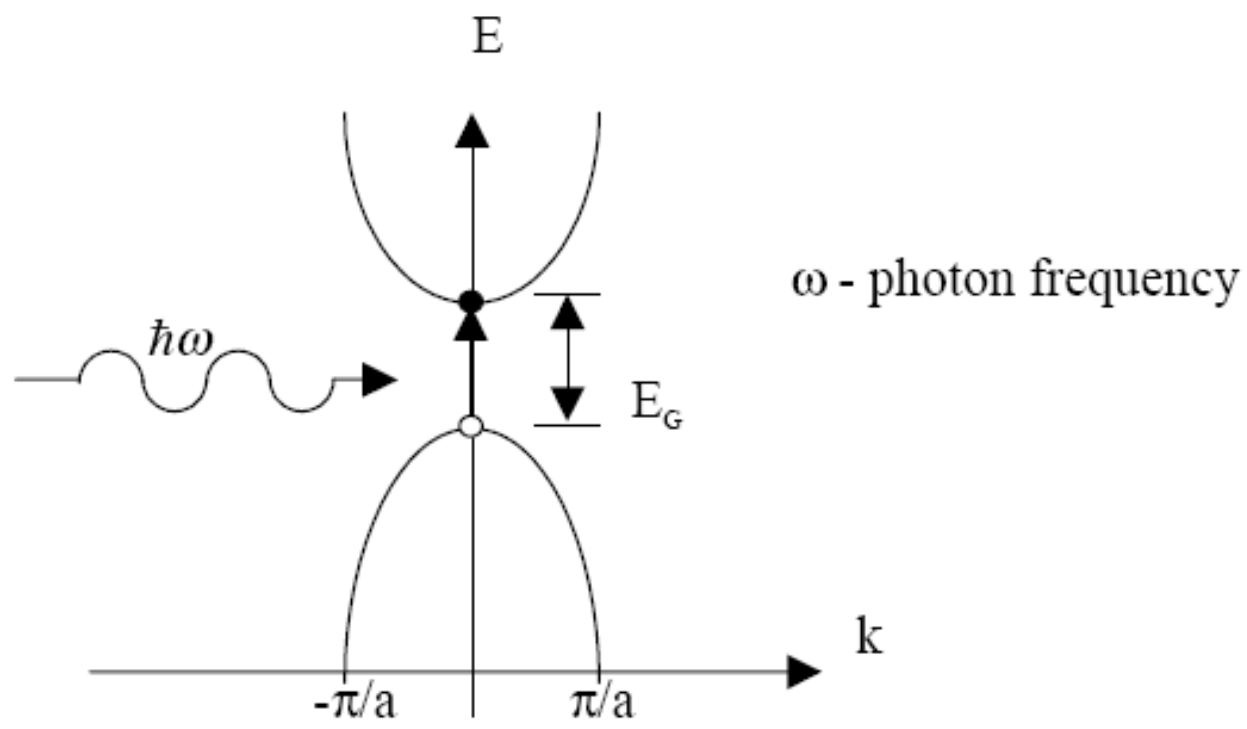

Figure 2.3. $\mathrm{E}$ - $\mathrm{k}$ Band diagram for a direct transition

\begin{tabular}{|c|l|l|l|}
\hline Photon Energy(eV) & 1.5 & 2.0 & 2.34 \\
\hline$\alpha\left(\mathrm{cm}^{-1}\right)$ & 100 & $3 \times 10^{4}$ & $9 \times 10^{4}$ \\
\hline $1 / \alpha(\mu \mathrm{m})$ & 100 & 0.33 & 0.11 \\
\hline
\end{tabular}

Table 2.1 Absorption Coefficient in GaAs at $300 \mathrm{~K}\left(\mathrm{E}_{\mathrm{G}}=1.42 \mathrm{eV}\right)$

An indirect intrinsic optical transition occurs when an electron is excited from the valence to the conduction band in a material where the minimum of the conduction band and maximum of the valence band are not aligned in $\mathbf{k}$ space. An indirect transition requires the absorption of a photon and the emission, or, absorption of a phonon to conserve both energy and momentum. As a result, an indirect transition has a lower probability of occurring compared to a direct transition. Figure 2.4 shows the $\mathbf{E}-\mathbf{k}$ band diagram for an indirect transition. In general, the absorption coefficient for an indirect bandgap material is smaller than the absorption 
coefficient for a direct bandgap material. The absorption coefficient for an indirect optical transition can be approximated as

$$
\alpha(\hbar \omega) \propto\left(\hbar \omega-E_{G}+\hbar \Omega\right)^{2}+\left(\hbar \omega-E_{G}-\hbar \Omega\right)^{2} \quad\left(\text { for } \hbar \omega \geq E_{G}\right)
$$

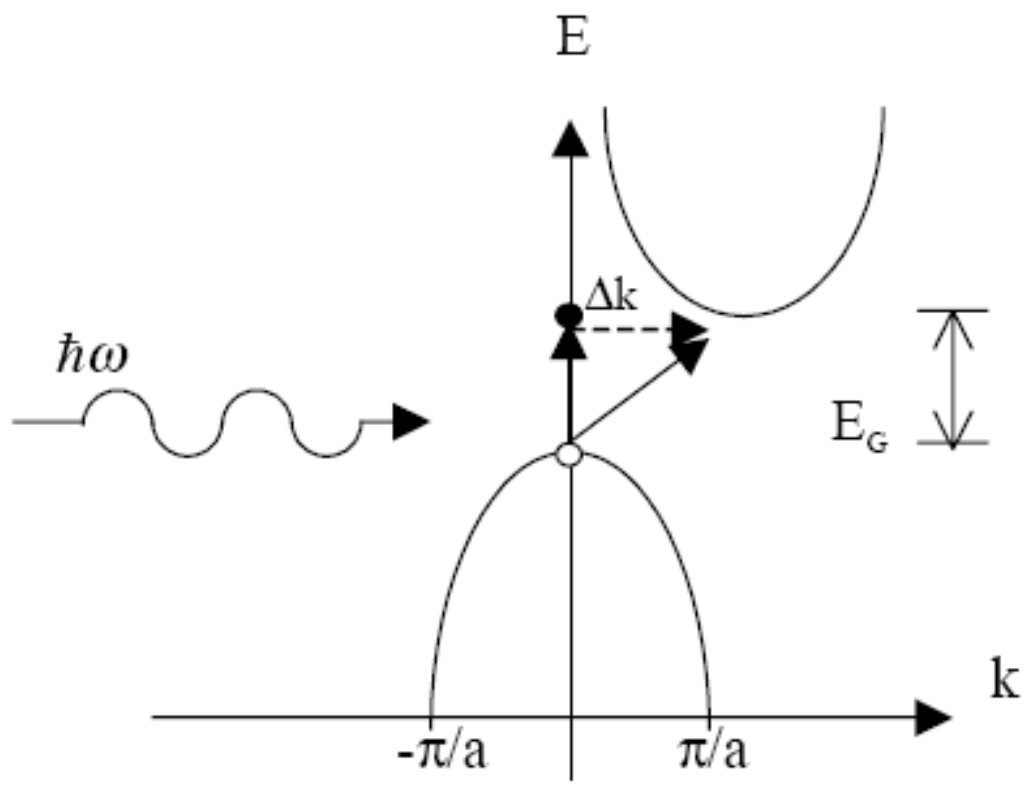

\section{Figure 2.4. E-k diagram for an indirect optical transition}

Where $\hbar \Omega$ is the phonon energy. The absorption and penetration depth at several photon energies is shown in Table 2.2 for the indirect bandgap material silicon. The absorption increases rapidly in silicon when the photon energy exceeds the bandgap energy. The stronger optical absorption in direct compared to indirect bandgap material becomes clear upon comparison of Tables 2.1 and 2.2, despite the significantly lower bandgap of silicon. However, both materials display a shallow penetration depth when the photon energy is sufficiently greater than the bandgap energy. The difference is that the penetration depth will be a few microns in a direct bandgap material compared to a few tens of microns for an indirect bandgap material. In either event, intrinsic optical absorption results in the generation of carriers in a shallow layer at the surface of the material. This leads to lateral PCSS devices similar to that shown in Figure 2.1. Lateral 
devices are limited in their operating voltage by surface flashover and carrier transport is limited by surface mobility.

\begin{tabular}{|c|r|r|r|r|}
\hline Photon Energy(eV) & 1.17 & 1.5 & 2.0 & 2.34 \\
\hline$\alpha\left(\mathrm{cm}^{-1}\right)$ & 15.2 & 486 & $3.2 \times 10^{3}$ & $6.8 \times 10^{3}$ \\
\hline $1 / \alpha(\mu \mathrm{m})$ & 658 & 20.6 & 3.1 & 1.48 \\
\hline
\end{tabular}

Table 2.2. Optical absorption in Silicon at $300 \mathrm{~K}\left(\mathrm{E}_{\mathrm{G}}=1.12 \mathrm{eV}\right)$

\subsection{3) Extrinsic Optical Absorption}

Extrinsic optical absorption occurs at impurity and defect levels located between the valence and conduction band. Electrons (holes) located at an impurity/defect level, can be optically excited into the conduction (valence) band, or, into another available impurity/defect level, leaving ionized impurity levels behind. We are particularly interested extrinsic absorption when it corresponds to the electrons captured by deep acceptor and trap levels. The extrinsic optical absorption is given by [2.4]

$$
\begin{gathered}
\alpha(\hbar \omega)=\sigma(\hbar \omega) N \\
\sigma(\hbar \omega)=\frac{\left(\hbar \omega-E_{D A}\right)^{\frac{3}{2}}}{(\hbar \omega)^{3}}
\end{gathered}
$$

Where $\mathrm{N}$ is number density of absorbing extrinsic levels, $\sigma$ is the optical absorption transition cross section for exciting an electron from the absorbing extrinsic level to the conduction band and $E_{D A}$ is the deep acceptor energy level. The energy required to optically excite carriers from the extrinsic levels depends upon the position of the extrinsic levels in the semiconductor 
bandgap. This energy can range from a few millielectron-volts for shallow levels to hundreds of millielectron-volts for extrinsic levels located deep in the bandgap. Extrinsic absorption presents the opportunity to choose the absorption wavelength by the proper selection of impurity atom. However, the absorption coefficient for extrinsic optical absorption is relatively low since impurities and defects are usually present in densities that are measured in parts per million. As a result, the penetration depth for extrinsic optical absorption is on the order of centimeters, a much deeper penetration than intrinsic absorption. Optically exciting extrinsic levels will allow for PCSS designs that take advantage of the bulk properties of the semiconductor. Extrinsic absorption is unlikely to be the dominant absorption mechanism. Optical loss due to extrinsic absorption will be or par with other loss terms like scattering. Also, saturation of absorption of extrinsic levels becomes a more important effect since there are a limited number of levels. We should expect low quantum efficiency for extrinsic devices.

\subsection{4) Nonlinear PCSS Switching Results}

Nonlinear PCSS devices (primarily GaAs and InP based) switch hundreds of amps of current with modest amounts of applied optical energy $\left(\mu \mathrm{J} / \mathrm{cm}^{2}\right)$. This is a result of electric field avalanche multiplication of the optically generated electron hole pairs termed "lock-on" [2.6, 2.7]. Current persists in nonlinear PCSS after the optical trigger pulse terminates. Current decays after the external circuit can no longer maintain the "lock-on" field $(6-10 \mathrm{kV} / \mathrm{cm})$ in the semiconductor material. Nonlinear PCSS currents flow in constricted filaments a few tens of microns in diameter. Current densities can exceed a few tens of $\mathrm{MA} / \mathrm{cm}^{2}$ and limit switch life. Nonlinear GaAs PCSS are the most highly developed of the high power, photoconductive switching devices. Research has been performed on both lateral and vertical nonlinear GaAs PCS devices for use in Pockels cell drivers, compact accelerators, high power microwave generation, 
high voltage laser diode drivers, ground penetrating radar and firing sets [2.8]. Lateral, nonlinear, GaAs PCSS switch arrays have been fabricated that hold off greater than $100 \mathrm{kV}$ (pulsed), switch kiloamps of current, have pulsewidths of a few ns and rise and fall times of hundreds of picoseconds. Device lifetimes have ranged from $10^{4}$ to $10^{6}$ pulses for high and low current GaAs PCSS devices, respectively. The longest lifetime devices are limited to a pulse width of a few ns and peak current of a few hundred amps. Device failure is caused by GaAs and metalization erosion at the GaAs-contact interface. Recent work [2.9] has concentrated on improving device lifetime by incorporating heavily doped, $2 \mu \mathrm{m}$ thick, $\mathrm{p}$ and $\mathrm{n}$-type layers below $\mathrm{p}^{+}$and $\mathrm{n}^{+}$contact metallizations, respectively. These changes led to a more diffuse current at the device contacts and increased lifetime by an order of magnitude. Nevertheless, GaAs nonlinear PCSS do not have sufficient lifetime for the DWA application.

\subsection{5) Linear Intrinsic PCSS Switching Results}

Linear, intrinsic PCSS are switched on by irradiating the interelectrode gap with photons of energy (hv) equal to, or exceeding, the semiconductor bandgap energy. Each absorbed photon generates an electron-hole pair within a few microns of the material surface due to the shallow absorption depth. Consequently, optical energy densities of a few $\mathrm{mJ} / \mathrm{cm}^{2}$ [2.3] are required to reduce PCSS resistance to the sub-ohm level.

Linear PCSS are usually constructed in the lateral geometry due to the limited depth of penetration of photons in the semiconductor material. Switch current is concentrated at the semiconductor surface and surface flashover limits the PCSS maximum operating voltage. Carriers are generated in the volume of the semiconductor determined by the optically irradiated area and the depth of penetration of photons. The depth of penetration of photons is determined by the optical absorption of the semiconductor material at the applied wavelength and can be 
several microns for direct and tens of microns for indirect bandgap semiconductors. Current distribution is determined by the spatial profile of the optical pulse and is of the order of MA/cm² and a few $100 \mathrm{kA} / \mathrm{cm}^{2}$ for direct and indirect semiconductor material, respectively. Current flows until optical excitation is removed and the switch recovers by carrier recombination, or carrier

\begin{tabular}{|c|c|c|c|c|c|}
\hline Properties & Si & GaAs & 6H-SiC & 4H-SiC & Diamond \\
\hline Bandgap (eV) & 1.12 & 1.43 & 3.0 & 3.2 & 5.5 \\
\hline Lattice Constant (A) & 5.43 & 5.65 & $\begin{array}{l}a=3.08 \\
c=15.12\end{array}$ & $\begin{array}{l}a=3.08 \\
c=10.08\end{array}$ & 3.567 \\
\hline Breakdown Field (MV/m) & 0.25 & 0.3 & 2.5 & 2.2 & 5.0 \\
\hline $\begin{array}{l}\text { Saturated Electron } \\
\text { Velocity }\left(\mathbf{1 0}^{7} \mathrm{~cm} / \mathrm{s}\right)\end{array}$ & 1.0 & 1.0 & 2.0 & 2.0 & 2.7 \\
\hline Hole Mobility $\left(\mathrm{cm}^{2} / \mathrm{V}\right.$-s) & 480 & 400 & 80 & 120 & 1600 \\
\hline Electron Mobility $\left(\mathrm{cm}^{2} / \mathrm{V}-\mathrm{s}\right)$ & 1300 & 8500 & $\begin{array}{l}415 \text { perp. c } \\
87 \text { parall. } c\end{array}$ & \begin{tabular}{|l|}
947 perp. c \\
1141 parall. c
\end{tabular} & 2200 \\
\hline Dielectric Constant $\left(\varepsilon_{\mathrm{r}}\right)$ & 11.9 & 13.0 & 10.0 & 10.0 & 5.0 \\
\hline Thermal Cond. (W/cm-K) & 1.5 & 0.5 & $3-5$ & 4.8 & 20.0 \\
\hline $\begin{array}{l}\text { Thermal Expansion } \\
\text { Coefficient }\left(10^{-6} / \mathrm{K}\right)\end{array}$ & 2.6 & 5.73 & 4.5 & 3.7 & 0.8 \\
\hline Density $\left(\mathrm{g} / \mathrm{cm}^{2}\right)$ & 2.3 & 5.3 & 3.2 & 3.2 & 3.5 \\
\hline Melting Point (C) & 1420 & 1240 & 2830 & 2830 & 4000 \\
\hline Direct/Indirect Bandgap & I & D & $\mathbf{I}$ & I & $\mathbf{I}$ \\
\hline
\end{tabular}

Table 2.3. Properties of some semiconductor materials used to fabricate linear PCS [2.20 2.14

sweep out. Linear PCSS devices have the potential of longer switch life due to reduced switch current density. Linear, intrinsic PCSS devices have been fabricated using Silicon, Gallium Arsenide, CVD and natural Diamond, Silicon Carbide and other materials. The physical properties of Silicon (Si), Gallium Arsenide (GaAs) and several wide bandgap semiconductor materials are listed in Table 2.3 [2.10 - 2.14]. The wide bandgap materials have obvious 
advantages over Si and GaAs in breakdown field, saturated electron velocity, bandgap and thermal conductivity. These advantages make the wide bandgap materials a better choice for high power, high voltage, high field and high temperature applications. However, bulk Diamond substrates cannot be obtained and good quality semi-insulating, single crystal $4 \mathrm{H}$ and $6 \mathrm{H}-\mathrm{SiC}$ have only recently became available. As a result, past work focused on linear PCSS devices fabricated from Silicon and Gallium Arsenide since these materials were available in wafer form.

Poly-crystalline, semi-insulating, silicon is available in resistivities up to $\sim 100-500 \mathrm{k} \Omega$ $\mathrm{cm}$. However, the higher resistivity is achieved at the expense of reduced carrier mobility. All silicon PCSS devices have been fabricated using bulk silicon wafers. Bulk silicon is available with resistivity up to $10-20 \mathrm{k} \Omega$-cm. This relatively low value of resistivity results in PCSS devices with dark (off) resistance on the order of tens of kilo-Ohms. If a number of these Si PCSS devices are connected in parallel, the effective dark resistance can become low enough to allow unacceptably high leakage currents from energy storage components in the circuit. Silicon also exhibits thermal runaway that limits the time that a Silicon PCSS can hold off high electric fields to a few microseconds. This requires that the Si PCSS be operated in the pulsed bias mode. Energy storage components in the electrical circuit (capacitors, pulse forming lines) must be pulse charged to permit use of a Si PCSS as the switching element. In addition, the long recombination time in Silicon (>10 $\mathrm{s}$ ) prevents rapid opening of the PCSS. Nevertheless, linear, intrinsic, lateral, Silicon PCSS devices have successfully operated at pulsed electric fields of $82 \mathrm{kV} / \mathrm{cm}$ and peak switch currents of 2 kiloamps (not simultaneously) [2.3].

GaAs is available in wafer form with resistivity greater than $10^{8} \Omega$-cm, which should limit leakage current through a GaAs PCSS device. High purity GaAs has a critical electric field strength of $350-400 \mathrm{kV} / \mathrm{cm}$. These physical properties suggest that linear, GaAs PCSS devices 
with very high voltage hold off should be realizable. This is unfortunately not the case. The dc dark breakdown field for GaAs is $8-20 \mathrm{kV} / \mathrm{cm}$ [2.3]. This low dc holdoff is attributed to carrier injection at deep traps in the bulk GaAs. The low dark dc breakdown field of GaAs PCSS requires that these devices be used in pulsed circuits where the high electric field appears across the PCSS for a few microseconds. In addition, GaAs PCSS can collapse into the nonlinear "lock-on" mode of operation if sufficient energy is left in the circuit when the optical pulse terminates. Control of the GaAs PCSS is lost during lock-on and current continues to flow until the external circuit can no longer maintain the "lock-on" field in the PCSS. Linear, GaAs PCSS have not achieved the high switch current and field operating parameters demonstrated by linear Si and nonlinear GaAs PCSS. The DWA switch application requires simultaneous operation at high electric field ( $250-500 \mathrm{kV} / \mathrm{cm})$ and high switch current (1-2 kA). Neither linear Si, nor GaAs, PCSS are capable of achieving the required DWA operating parameters.

\subsection{6) SiC PCSS Device Results}

Fabrication and testing of linear SiC PCSS began in the mid- nineteen nineties when $\mathrm{n}$ type, $6 \mathrm{H}-\mathrm{SiC}$ substrates became commercially available. In initial experiments [2.15], lateral, ohmic contacts were deposited on a 20 um thick, p-type (300 $\Omega$-cm), epitaxial layer grown on an n-type $6 \mathrm{H}-\mathrm{SiC}$ substrate. PCSS devices with electrode separations of 50 to $1000 \mu \mathrm{m}$ were tested at charge voltages of 40 Volts. The devices were optically triggered at $337 \mathrm{~nm}$ with energy densities of up $320 \mu \mathrm{J} / \mathrm{mm}^{2}$. The optical penetration depth $(1 / \alpha)$ into $6 \mathrm{H}-\mathrm{SiC}$ at $337 \mathrm{~nm}$ is 10 $\mu \mathrm{m}$. The devices achieved dynamic on resistances of 25 and 18 Ohms for optical energy densities of 160 and $320 \mu \mathrm{J} / \mathrm{mm}^{2}$. Devices with a $1 \mathrm{~mm}$ switch gap were tested at a charge voltage of $1 \mathrm{kV}$. These tests resulted in the measurement of high dark currents and unrepeatable switching results. The authors attributed these results to high impurities in the 6H-SiC. 
Sheng et al [2.16, 2.17] constructed low voltage PCSS, with interdigitated, lateral, ohmic contacts, on thick (600 $\mu \mathrm{m})$, CVD, polycrystalline, high resistivity (1 M $\Omega$-cm) 3C-SiC, and thin (5-20 $\mu \mathrm{m}$ ) boron doped and undoped CVD 3C-SiC. The PCSS were optically triggered using ArF (193 nm) and XeCl (308 nm) excimer lasers with 15 and 75 ns pulsewidths, respectively. Best results were obtained for the thick, polycrystalline, 3C-SiC PCSS with a $10 \mu \mathrm{m}$ gaps and $\mathrm{Ni}$-Au ohmic contacts triggered by the ArF laser. This device was tested to 250 volts ( $25 \mathrm{MV} / \mathrm{m}$ ) in air and 560 volts (56 MV/m) submerged in Fluorinert. Surface flashover was the reason for failure. These switches withstood electric fields five times greater than the maximum fields switched by either Si, or, GaAs lateral PCSS devices. The photocurrent pulsewidth ranged from 15 to 30 ns for 15 ns wide optical pulses for several devices constructed with 10 um gaps. The variation in photocurrent pulse width was attributed to differences in grain boundaries and surface states in the polycrystalline 3C-SiC. Peak photocurrent varied linearly with bias voltage up to 170 volts and saturated at higher bias voltages. The authors believed this was a result of saturation of the electron drift velocity. The highest peak photocurrent was $1.7 \mathrm{~A}$, which corresponds to a calculated current density of $10 \mathrm{kA} / \mathrm{cm}^{2}$. Photocurrent saturated at $1.4 \mathrm{MW} / \mathrm{cm}^{2}$ of applied optical energy at the $193 \mathrm{~nm}$ wavelength. Minimum on resistance was 45 Ohms and the dark resistance was 4.5 M $\Omega$. The authors believed that operation at 1000 volts bias could be achieved for switch gaps of $200 \mu \mathrm{m}$. In addition, lower on resistances could be obtained with longer wavelength optical triggers.

Experiments by Dogan et al $[2.18,2.19]$ were performed on lateral PCSS fabricated from semi-insulating 4H-SiC and conductive 6H-SiC. $\mathrm{H}_{2}$ annealing $(1650 \mathrm{C})$ and molten $\mathrm{KOH}$ etching (210 C) were used to remove surface damage due to polishing and defects. Ni/Ti/Au electrodes were deposited in a circular, bull's-eye pattern with the anode surrounded by the 
cathode separated by a $1 \mathrm{~mm}$ gap. A dye laser with a $307 \mathrm{~nm}$ wavelength and $8 \mathrm{~ns}$ (FWHM) pulse width was used for the optical trigger. Dark current and peak photocurrent were measured for bias voltages up to 1000 Volts. Dark current values of $0.37 \mathrm{nA}$ and $2.5 \mathrm{~mA}$ and peak photocurrent values of 49.4 and 15.4 A were measured at a bias voltage of 1000 Volts for the $4 \mathrm{H}-\mathrm{SiC}$ and the $6 \mathrm{H}-\mathrm{SiC}$ PCSS, respectively. The peak photocurrent showed no sign of saturation with bias voltage for either material. The average on-state resistance was 20 and 60 Ohms at optical pulse energy of $1.44 \mathrm{~mJ}$ for the $4 \mathrm{H}-\mathrm{SiC}$ and $6 \mathrm{H}-\mathrm{SiC}$, respectively.

Zhu et al [2.20] extended the work of Dogan $[2.18,2.19]$ by adding $100 \mathrm{~nm}$ thick $\mathrm{n}^{+}-\mathrm{GaN}$ sub-contact layers below the electrode metalizations for PCSS constructed from semi-insulating, vanadium compensated, and high purity, semi-insulating 4H-SiC. Lateral, bulls-eye type contacts, with and without the $\mathrm{n}^{+}-\mathrm{GaN}$ sub-layers, having electrode separations of 0.5, 0.75, 1.25 and $1.75 \mathrm{~mm}$ were tested. The ${ }^{+}{ }^{-} \mathrm{GaN}$ layer was located below Ti/Al/Ti/Au electrodes. Ni/Ti/Au was used for electrode metalization for PCSS without the $\mathrm{n}^{+}$-GaN sub-layers. The purpose of the $\mathrm{n}^{+}$-GaN sub-contact layer was to spread the switch current to decrease current density at the PCSS electrodes. The PCSS devices were triggered with a frequency tripled Nd:YAG laser (355 $\mathrm{nm}$ ) with a ten nanosecond pulsewidth. The PCSS devices constructed with the $\mathrm{n}^{+}$-GaN sublayers had an order of magnitude lower dark resistance, two orders of magnitude higher photocurrent efficiency $(01.454 \mathrm{~A} / \mu \mathrm{JV})$, two orders of magnitude lower on resistance (115 Ohms) and showed no signs of contact degradation at high switch current. The PCSS devices without contact sub-layers showed contact damage at the cathode after operation at high current (100 - 200 A). Contact damage manifested as blackening of the cathode metalization and reduced switch performance. Switch damage at the cathode was attributed to the low hole mobility in the material $\left(40 \mathrm{~cm}^{2} / \mathrm{V}\right.$-s), which tends to focus the PCSS photocurrent at the cathode 
metalization/semiconductor edge. Peak photocurrent versus bias voltage was plotted for PCSS with, and without, the $\mathrm{n}^{+}-\mathrm{GaN}$ contact sub-layers. The peak photocurrent for the two PCSS devices increased linearly to approximately 20 amps at a bias of 1000 volts. The photocurrent for PCSS without the contact sub-layers shows a much higher rate of rise for bias voltage greater than 1000 volts. The photocurrent for this device increases to approximately 180 amps for a bias voltage of 1500 volts. The photocurrent for the PCSS with the $n^{+}-\mathrm{GaN}$ sub-layers continues a gradual linear increase until it reaches a bias voltage of 1500 volts. This device exhibits a rapid increase in photocurrent for bias voltages greater than 1500 volts. The authors were more concerned that the PCSS without the $\mathrm{n}^{+}$-GaN sub-layer had significantly higher photocurrent than the layered device, rather than the fact that both devices exhibited a highly nonlinear I - V characteristic.

In summary, experiments on $4 \mathrm{H}$ and $6 \mathrm{H}-\mathrm{SiC}$ PCSS demonstrated high electric field operation (25 - $56 \mathrm{MV} / \mathrm{m})$ [2.16,2.17], fast recovery [2.20], and switched up to 200 amps. However, some of these results can be misleading. The high field operation was demonstrated for lateral electrodes with a $10 \mu \mathrm{m}$ separation holding off 250 volts. It is well known that surface flashover occurs at higher electric fields for smaller gaps [2.21]. The breakdown field will decrease as the electrode separation increases to allow operation at useful voltage levels ( $\sim 10$ $\mathrm{kV})$. I believe the operating field for SiC lateral PCSS devices will be close to that demonstrated by lateral Si and GaAs PCSS devices $(100 \mathrm{kV} / \mathrm{cm})$, since surface flashover is the common failure mechanism. Zhu et al demonstrated a lateral, 4H-SiC, PCSS with $100 \mathrm{~nm}$ thick $\mathrm{n}^{+}$-GaN electrode sub-layers that switched 200 amps for a bias of 1500 Volts. These operating conditions damaged the cathode electrode of PCSS devices without the $\mathrm{n}^{+}$-GaN sub-layers. The sub-layers serve the same purpose as the $2 \mu \mathrm{m}$ deep, $\mathrm{p}$ and n-type diffusion layers below the cathode and anode 
electrodes on lateral, nonlinear, GaAs PCSS. These layers have a higher conductivity than the bulk semiconductor material and cause the current density to decrease prior to being collected by the electrodes. However, the problem of high current density remains and electrode damage may re-emerge at higher switch current.

We believe that linear, intrinsic PCSS devices have several disadvantages. These devices are usually limited to operation in the lateral geometry due to the shallow penetration of the optical triggering pulse. The operating limits of lateral geometry PCSS are determined by surface flashover, surface carrier mobility and high current density. These limitations prevent linear, intrinsic PCSS from achieving the operating characteristics required for the DWA switch application. Wide bandgap semiconductors offer superior PCSS performance due to large values of critical field strength, electron saturation velocity, bandgap, and thermal conductivity. However, we can only take advantage of all these properties if we switch through the bulk of the material. We decided to pursue linear, extrinsic PCSS using wide bandgap semiconductor material for the DWA application.

\subsection{Wide Bandgap Semiconductors}

Wide bandgap semiconductors have advantages over Si and GaAs in electric field strength, saturated electron velocity, thermal conductivity and operating temperature. Good quality three to four inch diameter $4 \mathrm{H}$ and $6 \mathrm{H}$-SiC wafers are commercially available, while $2 \mathrm{H}$ GaN has recently become available in bulk substrates. We will review the general properties of $4 \mathrm{H}$ and $6 \mathrm{H}-\mathrm{SiC}$ and $2 \mathrm{H}-\mathrm{GaN}$. 


\subsection{1) Silicon Carbide}

Silicon Carbide ( $\mathrm{SiC}$ ) is composed of the group IV elements Silicon and Carbon, each with four valence electrons. Carbon (C) is tetrahedrally surrounded and covalently bonded to its four nearest neighbors, which are Silicon (Si) atoms [2.22]. The bonding structure is shown in Figure 2.6. The distance between the $\mathrm{C}$ atom and each of its four neighboring Si atoms is 1.89 Angstroms. This short bond distance is an indication of the strong bond between the $\mathrm{C}$ and $\mathrm{Si}$ atoms. As a result, $\mathrm{SiC}$ is second only to Diamond in hardness. This makes $\mathrm{SiC}$ difficult to polish and etch. The distance between same kind atom neighbors (C-C or Si-Si) is 3.08 Angstroms. This tetrahedral arrangement is the basic structural unit of all the SiC polytypes.

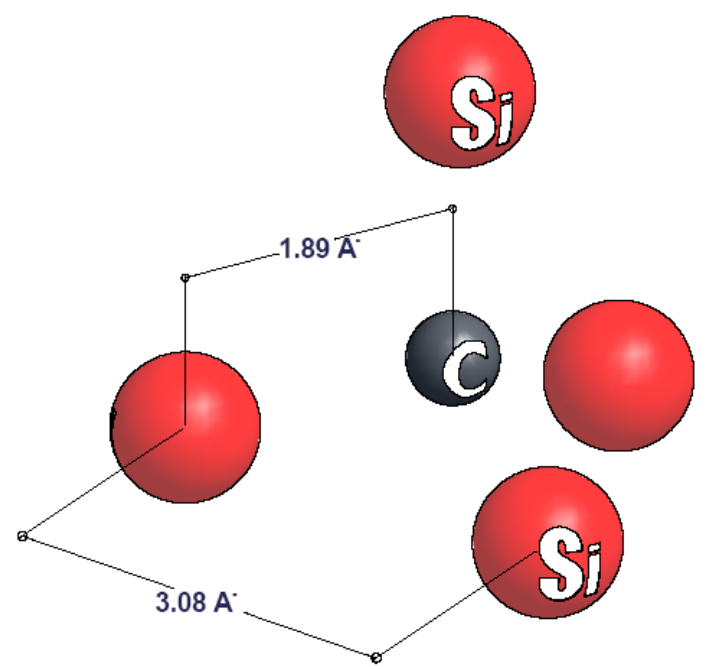

Figure 2.6. Basic SiC tetrahedral bond arrangement (similarly, each $\mathrm{Si}$ atom is surrounded by four $\mathrm{C}$ atoms) 


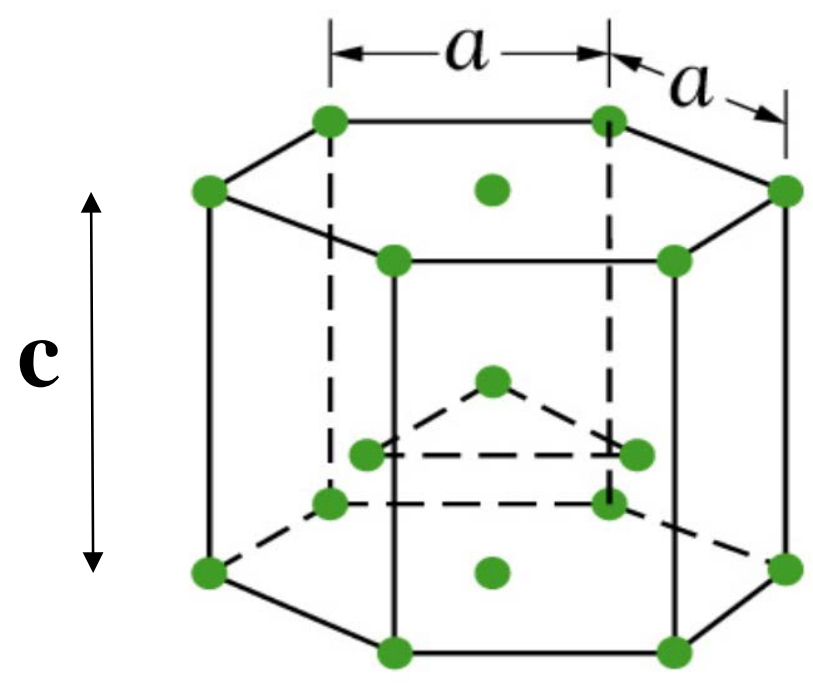

Figure 2.7. Hexagonal close packed structure

SiC occurs in over 150 , and as many as 250 , polytypes [2.23]. SiC polytypes occur in cubic, hexagonal and rhombohedral crystal structures, but all SiC polytype crystal structures can be described using the hexagonal system. The $6 \mathrm{H}$ and $4 \mathrm{H}-\mathrm{SiC}$ crystal structures can be described using the hexagonal close packed structure shown in Figure 2.7.

A hexagonal lattice structure is built by stacking layers of atoms in the arrangements shown in Figure 2.8. The hcp structure shown above could be described by the stacking sequence ACA. The $4 \mathrm{H}$ and $6 \mathrm{H}-\mathrm{SiC}$ hexagonal lattices are constructed using $\mathrm{C}-\mathrm{Si}$ bilayers of atoms

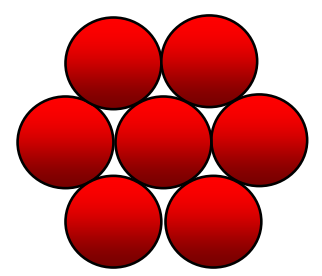

A

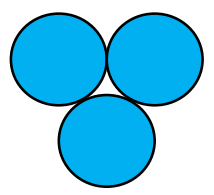

B

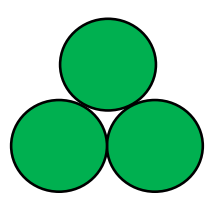

C

Figure 2.8. Atom positions in the layers used to construct a hexagonal lattice structures. 
in the A, B and C positions shown in Figure 2.8. The SiC tetrahedra are grouped so that all atoms lie on parallel planes on the node points of a close packed hexagonal lattice. The different SiC polytypes are determined by variations in the stacking sequence of the $\mathrm{Si}-\mathrm{C}$ bilayer sheets in the "c" direction of the hexagonal lattice. Figure 2.9 [2.24] shows the atom positions of Si - C bilayers and stacking sequence for 3C, $4 \mathrm{H}$, and $6 \mathrm{H}-\mathrm{SiC}$.

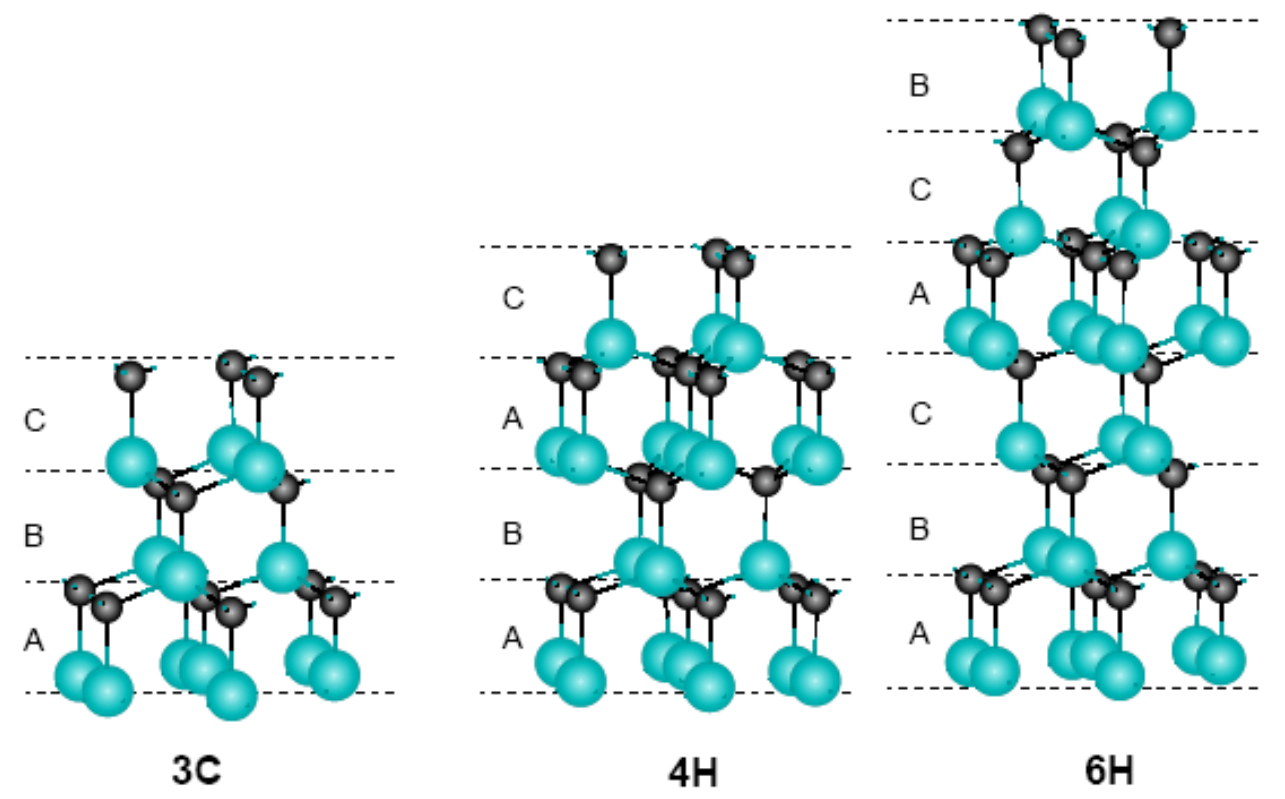

Figure 2.9. Stacking sequence of $3 \mathrm{C}, 4 \mathrm{H}$, and $6 \mathrm{H}$-SiC: Side views of $\mathrm{Si}-\mathrm{C}$ bilayers of $3 \mathrm{C}$ $\mathrm{SiC}, 4 \mathrm{H}$ and $6 \mathrm{H}$-SiC polytypes [2.24].

The polytypes are named using the Ramsdell nomenclature. $6 \mathrm{H}-\mathrm{SiC}$ is so named because there are six layers in its unit cell (ABCACB) and it has a hexagonal crystal structure. $6 \mathrm{H}$ and $4 \mathrm{H}-\mathrm{SiC}$ are the only polytypes that are currently available as bulk wafers and substrates. Our primary focus is on semi-insulating $4 \mathrm{H}$ and $6 \mathrm{H}-\mathrm{SiC}$ substrates. $6 \mathrm{H}-\mathrm{SiC}(4 \mathrm{H}-\mathrm{SiC})$ has 12 (8) atoms per unit cell, 3 (2) inequivalent sites, lattice constant "a” of 3.08 (3.08) Angstroms and a unit cell length "c" of 15.079 (10.050) Angstroms [2.25]. The inequivalent lattice sites result from the carbon, or associated silicon atom, in a quasihexagonal, or quasicubic, stacking 
environment with respect to its neighboring stacking planes. The inequivalent sites are shown in Figure 2.10 [2.12]. The cubic inequivalent lattice sites are shaded. Figure 2.10 shows the stacking sequence in the [1120] plane for both $4 \mathrm{H}$ and $6 \mathrm{H}-\mathrm{SiC}$ lattice. The inequivalent sites are labeled $\mathrm{h}$ for hexagonal and $\mathrm{k}$ for cubic. The $4 \mathrm{H}-\mathrm{SiC}(6 \mathrm{H}-\mathrm{SiC})$ polytype has one (two) cubic and
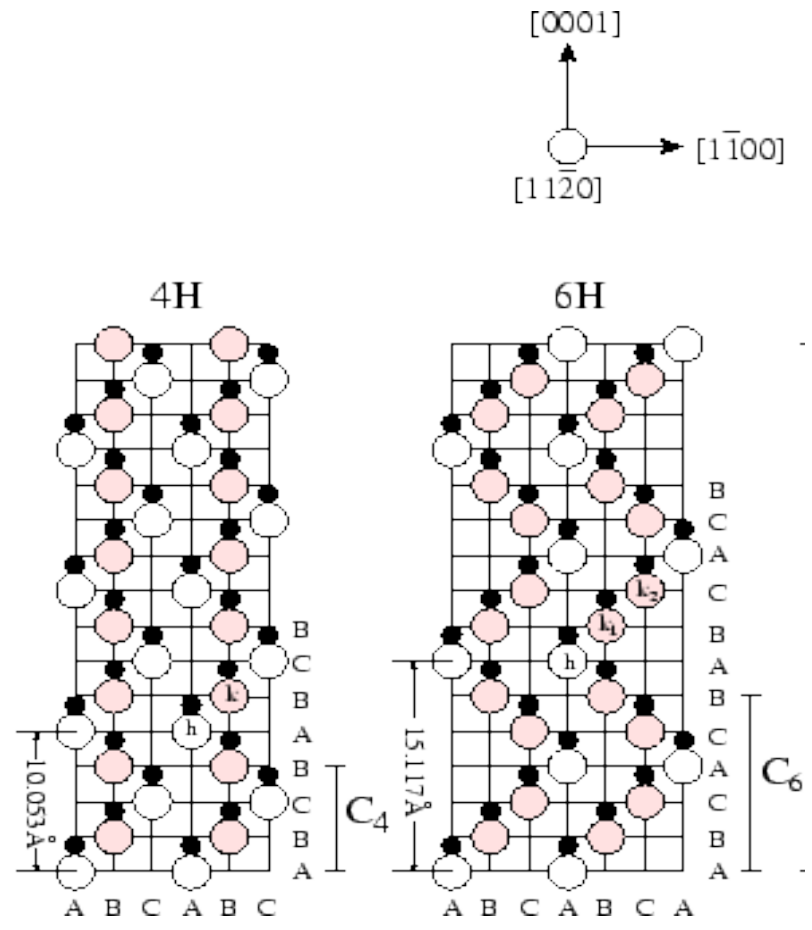

Figure 2.10. Cross section of $4 \mathrm{H}$ and $6 \mathrm{H}-\mathrm{SiC}$ stacking sequence [2.12]

one (one) hexagonal inequivalent sites. As a result, the $4 \mathrm{H}(6 \mathrm{H})$ polytype is referred to being 50 (33) percent hexagonal. The inequivalent sites have slightly different electronic properties. This point becomes important when dopants, or impurities substitute at these sites.

$\mathrm{SiC}$ does not melt at atmospheric pressure. Stoichiometric melting of SiC occurs for pressures and temperatures greater than $10^{5}$ atmospheres and $3200 \mathrm{C}$, respectively [2.25]. As a result, the Czochralski and Bridgeman techniques cannot be used to grow SiC crystals. High Temperature Chemical Vapor Deposition (HTCVD) and Liquid Phase Epitaxy (LPE) have been used to grow epitaxial SiC layers on SiC substrates. However, bulk SiC crystals are grown by Physical Vapor Transport (PVT) [2.25, 2.26]. PVT takes advantage of the sublimation of SiC 


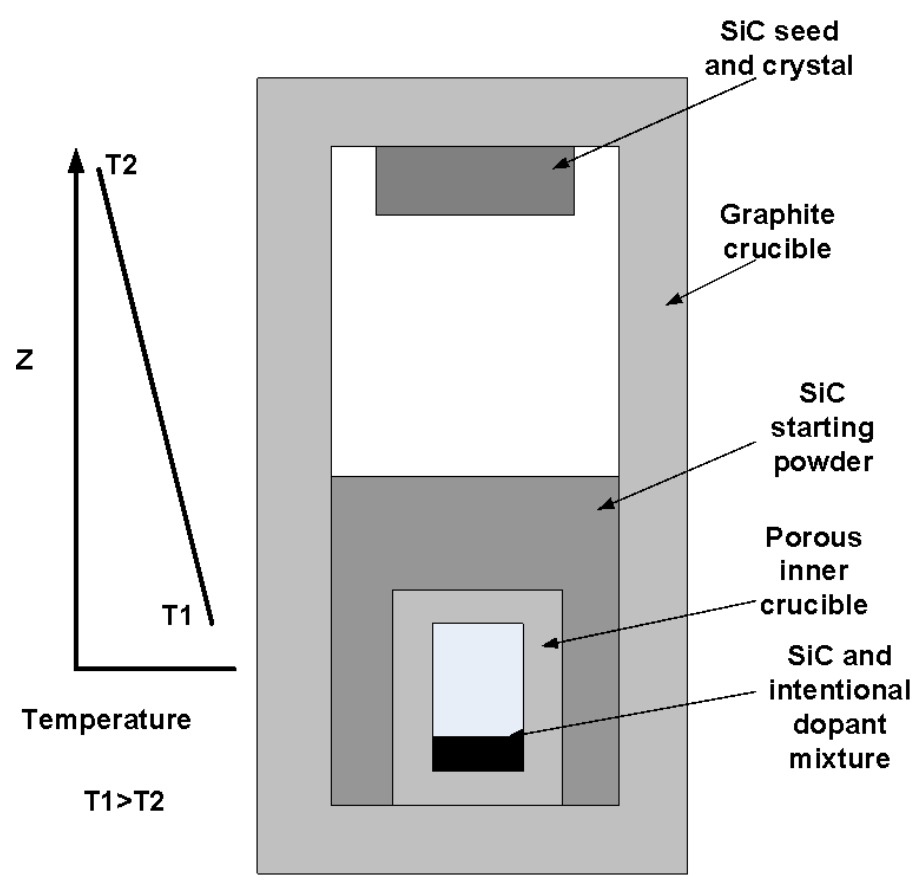

Physical Vapor Transport System

Schematic

\section{Figure 2.11. Simplified schematic of Physical Vapor Transport growth chamber}

above 1800 C. A simplified schematic of a PVT system is shown in Figure 2.11. The graphite crucible is enclosed in thermal insulation and heated with an induction coil. The crucible is maintained at a background pressure of 20 - 40 Torr of Argon during crystal growth. A temperature of $2200-2400 \mathrm{C}$ is maintained at the SiC starting powder source. A negative temperature gradient of $10-35 \mathrm{C} / \mathrm{cm}$ is established between the SiC starting powder source and the SiC seed crystal. Si and C species are driven from the hotter source zone and condense on the cooler seed crystal. The seed crystal is usually maintained at a temperature of $100 \mathrm{C}$ cooler than the source. Typical growth rates of $\mathrm{SiC}$ crystals are 0.5 to $5 \mathrm{~mm} / \mathrm{hr}$ using the PVT technique. Four inch diameter crystals have recently been grown by PVT. The PVT growth technique has the penalty of unintentional impurities and crystal defects. 
PVT grown SiC crystals exhibit several defects including stacking faults, screw dislocations, growth spirals, inclusions and polytypism [2.25, 2.26]. One particular defect, termed micropipes, limits the usable area of wafers cut from the $4 \mathrm{H}$ and $6 \mathrm{H}-\mathrm{SiC}$ crystal. Micropipes are hollow core screw dislocations that propagate along the growth direction (c axis) of the crystal [2.25 - 2.27]. The micropipes form cylindrical voids, ranging in diameter from 0.1 to $10 \mu \mathrm{m}$, through the entire length of the crystal. Improvements in crystal growth techniques have reduced micropipe densities to $15-30 / \mathrm{cm}^{2}$ for standard wafers and premium zero micropipe wafers [2.29]. Wafers are usually sliced from the c-plane of a SiC crystal. Micropipes manifest themselves as small holes running through the wafer from face to face. Micropipes can be revealed by etching the face of a $\mathrm{SiC}$ wafer with molten potassium hydroxide (KOH). Several micropipes are shown on the c-plane of a $6 \mathrm{H}-\mathrm{SiC}$ wafer in Figure 2.12. Figure 2.13 shows a microscopic image of a $400 \mu \mathrm{m}$ thick, $6 \mathrm{H}-\mathrm{SiC}$ substrate that was cut from the a-plane of the crystal. This image is of a 250 micron wide, 150 micron high substrate section. The micropipes and inclusions appear throughout the thickness of the substrate. These micropipes are a few microns in diameter.

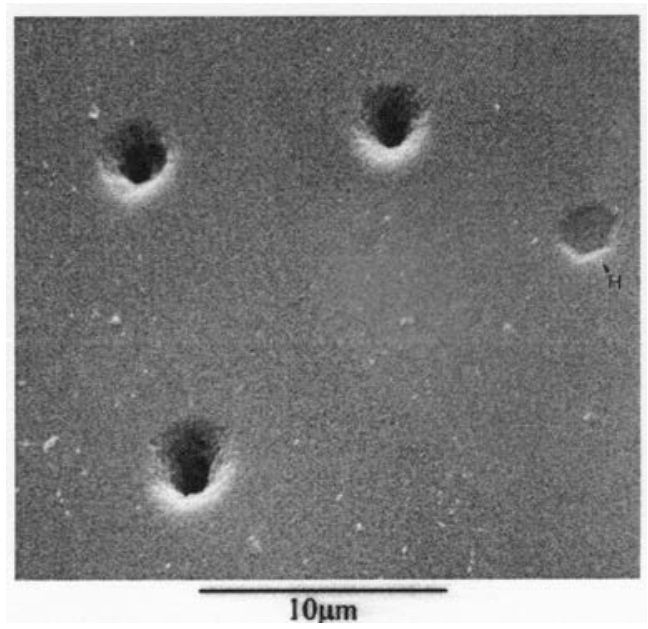

Figure 2.12. Micropipes on the etched face of a $6 \mathrm{H}-\mathrm{SiC}$ wafer [2.29] 


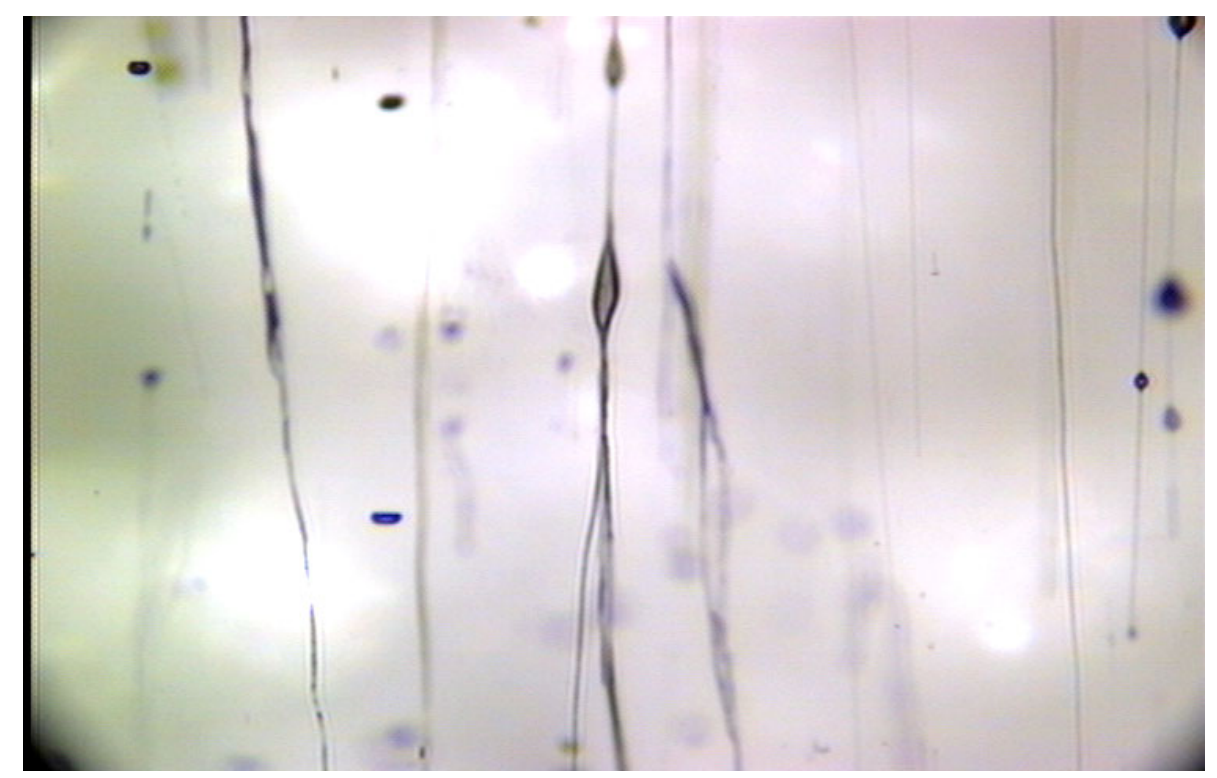

Figure 2.13. Micropipes and inclusions in a $400 \mu \mathrm{m}$ thick, a-plane $6 \mathrm{H}-\mathrm{SiC}$ substrate

Micropipes reduce the voltage holding capability through the wafer bulk. High voltage, broad area electronic devices require micropipe-free $4 \mathrm{H}$ and $6 \mathrm{H}-\mathrm{SiC}$ substrates. We propose to obtain micropipe-free $4 \mathrm{H}$ and $6 \mathrm{H}-\mathrm{SiC}$ substrates by requiring that the substrates be sliced from the a-plane of the $4 \mathrm{H}$ and $6 \mathrm{H}-\mathrm{SiC}$ crystals. The hexagonal geometry of a $6 \mathrm{H}-\mathrm{SiC}$ crystal, highlighting the a and m-planes, is illustrated in Figure 2.14 [2.28]. The micropipe-free substrates are obtained by identifying a region on the crystal face ( $\mathrm{Si}$ or $\mathrm{C}$ ) that is micropipe-free and slicing down through the length of the crystal. The light green shaded a-plane in Figure 2.14 represents a face of a substrate obtained by this method. There are two disadvantages in using the micropipe-free, a-plane substrates. It is more expensive, but obtaining micropipe-free substrates is worth the expense. The second disadvantage is that dopant and impurity (unintentional dopants) densities vary in the growth (c) direction. It is possible that the variation in dopant density along the c direction of the a-plane substrate can have a negative effect on device performance. 


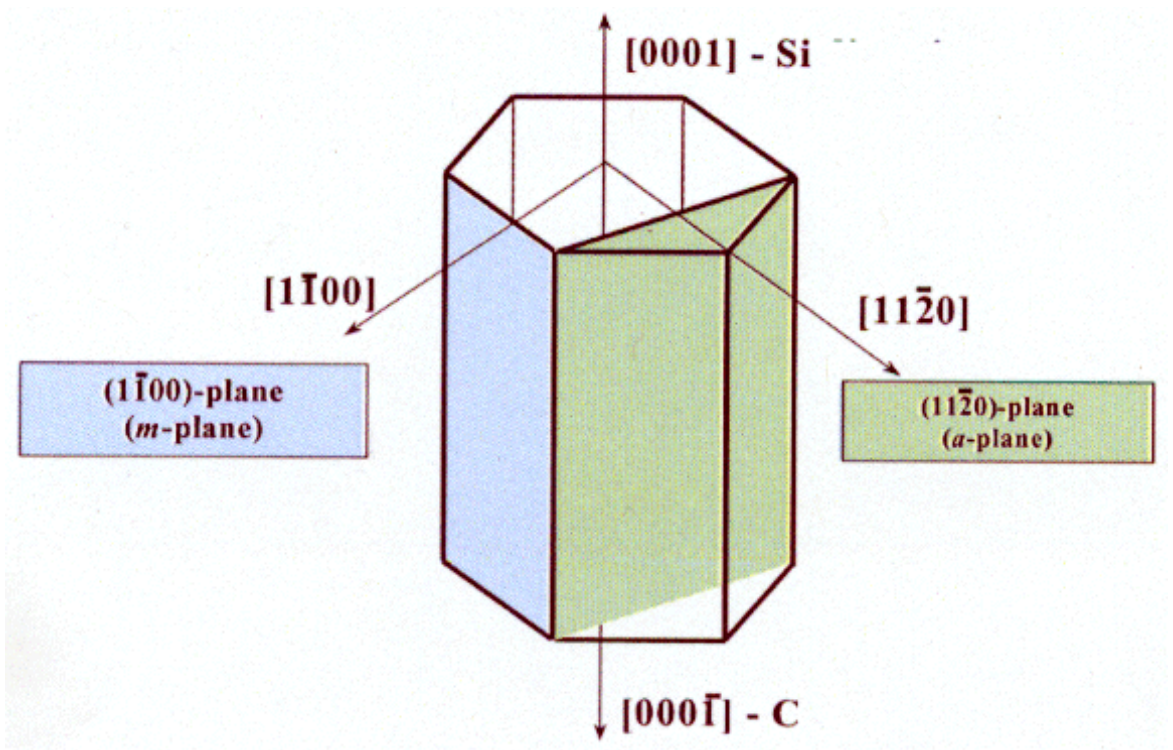

Figure 2.14. Hexagonal geometry illustrating the position of a and m-planes [2.28]

\subsection{2) Dopants and Impurities in $6 \mathrm{H}-\mathrm{SiC}$}

The PVT growth technique usually yields n-type $4 \mathrm{H}$ and $6 \mathrm{H}-\mathrm{SiC}$ crystals. This is a result of the presence of nitrogen as an unintentional n-type dopant in the PVT growth crucible. The nitrogen originates from the adsorption of air by graphite parts and the $\mathrm{SiC}$ powder source. Nitrogen $(\mathrm{N})$ is a shallow donor that substitutes on the three (two) inequivalent Carbon sites in the $6 \mathrm{H}(4 \mathrm{H})-\mathrm{SiC}$. The three nitrogen donor levels reside at $80(\mathrm{~h}), 137\left(\mathrm{k}_{1}\right)$ and $142\left(\mathrm{k}_{2}\right) \mathrm{meV}$ below the $6 \mathrm{H}-\mathrm{SiC}$ conduction band [2.30]. Here $\mathrm{h}$ and $\mathrm{k}$ correspond to the hexagonal and cubic inequivalent sites. In $4 \mathrm{H}-\mathrm{SiC}$, the nitrogen donor levels reside at $92(\mathrm{k})$ and $52 \mathrm{meV}$ below the conduction band [2.13]. The nitrogen impurity density depletes exponentially during crystal growth. Boron is another impurity present in the growth chamber due to graphite parts. Boron is a p-type dopant that substitutes primarily at silicon sites with energy levels of $300-330 \mathrm{meV}$ and $650 \mathrm{meV}$ above the valence band for the $6 \mathrm{H}$ and $4 \mathrm{H}$-SiC polytypes, respectively. The boron impurity depletes more slowly than nitrogen during crystal growth. The nitrogen and boron concentrations in undoped $6 \mathrm{H}-\mathrm{SiC}$ can be as high as $1 \times 10^{17} / \mathrm{cm}^{3}$. High purity $\mathrm{SiC}$ material has 
nitrogen and boron impurity densities in the mid $10^{15} / \mathrm{cm}^{3}$ range. The energy levels for impurities/dopants and intrinsic defects in the 6H-SiC polytype are shown in Figure 2.15 [2.31]. Intrinsic defects represent levels due to vacancies, antisites and double vacancies.

An interesting aspect of Figure 2.15 is the appearance of vanadium as both a donor and acceptor. Vanadium is an electrically amphoteric impurity due to interactions between its d-shell electrons and the $4 \mathrm{H}$ and $6 \mathrm{H}-\mathrm{SiC}$ lattice. Vanadium forms a deep donor $\mathrm{D}^{0} / \mathrm{D}^{+}\left(3 \mathrm{~d}^{1} / 3 \mathrm{~d}^{0}\right)$ and a deep acceptor $\mathrm{A}^{0} / \mathrm{A}^{-}\left(3 \mathrm{~d}^{2} / 3 \mathrm{~d}^{1}\right)$ level at $0.67-0.8$ and $1.6 \mathrm{eV}$ below the conduction band in $6 \mathrm{H}-$ $\mathrm{SiC}[2.31,2.32]$. The vanadium deep donor and acceptor levels reside at 1.7 and $0.9 \mathrm{eV}$ below the conduction band in $4 \mathrm{H}-\mathrm{SiC}$ [2.10, 2.32 ,2.33]. As a result, vanadium (V) doping is used to compensate $\mathrm{n}$ and p-type $4 \mathrm{H}$ and $6 \mathrm{H}-\mathrm{SiC}$ to obtain semi-insulating material. Let us assume that the only impurities in a substrate of $6 \mathrm{H}-\mathrm{SiC}$ are $\mathrm{V}$ and $\mathrm{N}$, with the $\mathrm{V}$ concentration the higher of the two impurities. The $\mathrm{V}$ acceptors capture the electrons from the $\mathrm{N}$ donors and compensation is achieved. The Fermi level is pinned to the $\mathrm{V}$ acceptor level and high resistivity $6 \mathrm{H}-\mathrm{SiC}$ is

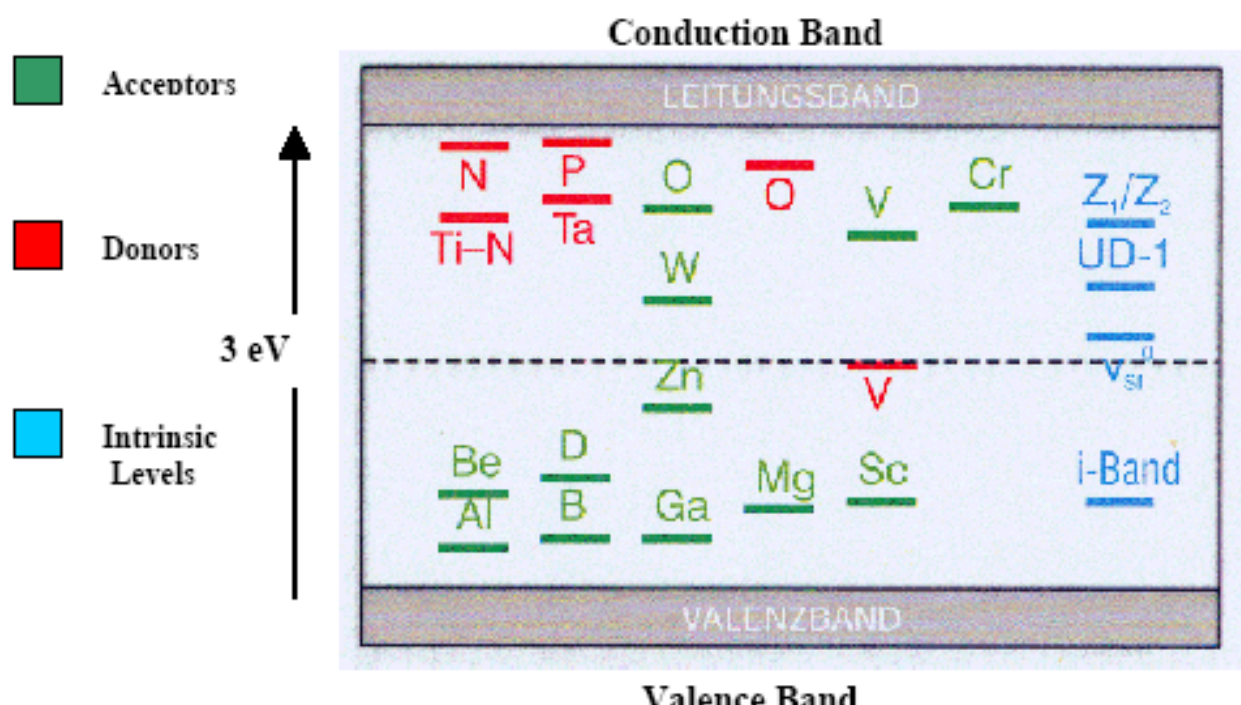

Figure 2.15. Energy levels of dopants and intrinsic defects in 6H-SiC [2.31] 
obtained. We now have a significant portion of Vanadium sites, located $0.67-0.8 \mathrm{eV}$ below the conduction band and distributed throughout the volume of the $6 \mathrm{H}-\mathrm{SiC}$ substrate, with an electron captured from an $\mathrm{N}$ donor. These captured electrons can be excited into the conduction band using below bandgap wavelengths (450 - $1550 \mathrm{~nm})$. The below bandgap wavelengths will penetrate several centimeters into the substrate because absorption is low. This enables electrodes to be placed on opposing faces of the substrate with optical excitation applied to the substrate facets. A photoconductive switch based on exciting the captured electrons with below bandgap light would have the advantages of switch current excited throughout the volume of the material and holdoff voltage determined by the dielectric strength of the bulk $6 \mathrm{H}-\mathrm{SiC}$. A similar device can be fabricated using vanadium compensated 4H-SiC.

\subsection{3) Gallium Nitride}

Gallium Nitride is another attractive wide bandgap semiconductor material for the high power switching application. Gallium Nitride $(\mathrm{GaN})$ is thermodynamically stable in both the wurtzite and zincblende crystalline structure. GaN is usually grown by epitaxy. The growth substrate material determines the crystalline structure of the GaN epitaxial layer. The GaN structure will be wurtzite if it is grown on a hexagonal substrates and zincblende if grown on a cubic GaAs substrate.

The wurtzite GaN structure is shown in Figure 2.16. The GaN wurtzite "a” and "c” lattice constants are 3.189 and 5.185 Angstroms, respectively. The GaN wurzite lattice [2.34] is comprised of two interpenetrating hexagonal closed packed (hcp) sublattices.. One hcp sublattice consists of nitrogen atoms. The second hcp sublattice consists of gallium atoms. The two sublattices are offset from one another by 3.24 angstroms along the c axis. Table 1.2 [2.10, 2.35] 


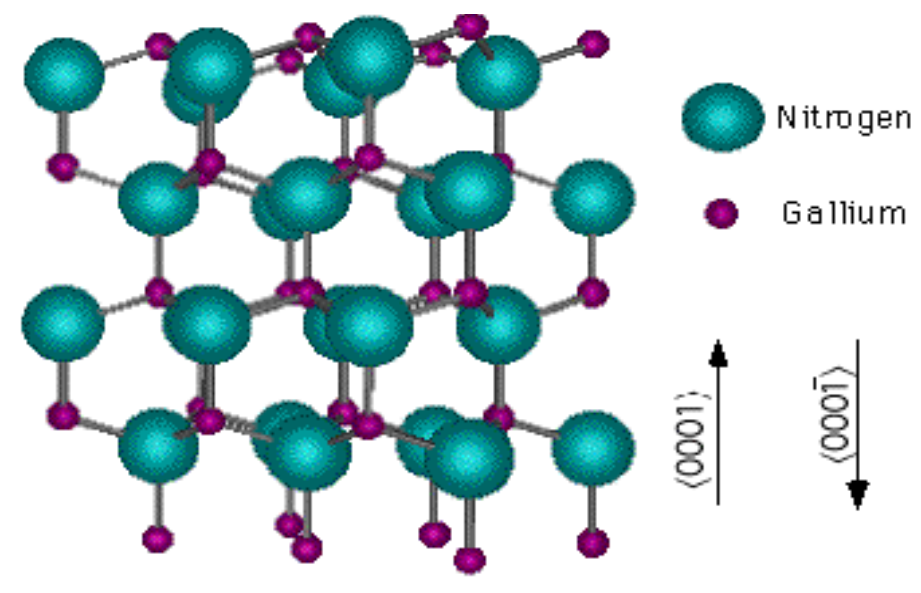

Figure 2.16. GaN wurzite crystalline structure [2.34].

lists the properties of $\mathrm{GaN}$ along with those of $6 \mathrm{H}-\mathrm{SiC}$ and $4 \mathrm{H}-\mathrm{SiC}$ for comparison. $\mathrm{GaN}$ offers higher breakdown field, saturated electron velocity and electron mobility compared to silicon carbide. In addition, $\mathrm{GaN}$ has a direct bandgap. $\mathrm{GaN}$ melts at high temperature $\left(2500{ }^{\circ} \mathrm{C}\right)$ under

\begin{tabular}{|c|c|c|c|}
\hline Properties & 6H-SiC & $\underline{4 H-S i C}$ & 2H-GaN \\
\hline Bandgap (eV) & 3.0 & 3.2 & 3.4 \\
\hline Lattice Constant (A) & $\begin{array}{l}a=3.08 \\
c=15.12\end{array}$ & $\begin{array}{l}\mathrm{a}=\mathbf{3 . 0 8} \\
\mathrm{c}=\mathbf{1 0 . 0 8}\end{array}$ & $\begin{array}{l}\mathrm{a}=3.189 \\
\mathrm{c}=\mathbf{5 . 1 8 5}\end{array}$ \\
\hline Breakdown Field (MV/m) & 2.5 & 2.2 & 3.0 \\
\hline $\begin{array}{l}\text { Saturated Electron } \\
\text { Velocity }\left(10^{7} \mathrm{~cm} / \mathrm{s}\right)\end{array}$ & 2.0 & 2.0 & 2.5 \\
\hline $\begin{array}{l}\text { Electron Mobility } \\
\left(\mathrm{cm}^{2} / \mathrm{V} \text {-s) }\right.\end{array}$ & $\begin{array}{l}415 \text { perp. } C \\
87 \text { parall. } C\end{array}$ & $\begin{array}{l}947 \text { perp. C } \\
1141 \text { parall. C }\end{array}$ & 400 \\
\hline Hole Mobility $\left(\mathrm{cm}^{2} / \mathrm{V}-\mathrm{s}\right)$ & 80 & 120 & 30 \\
\hline Dielectric Constant $\left(\varepsilon_{\mathrm{r}}\right)$ & 10.0 & 10.0 & 9.5 \\
\hline Thermal Cond. (W/cmK) & $3-5$ & 4.8 & 1.3 \\
\hline $\begin{array}{l}\text { Coefficient Thermal } \\
\text { Expansion }\left(1^{-6} / \mathrm{K}\right)\end{array}$ & 4.5 & & 5.6 \\
\hline Density $\left(\mathrm{g} / \mathrm{cm}^{3}\right)$ & 3.2 & 3.2 & 6.1 \\
\hline Melting Point ( C ) & 2830 & 2830 & 2500 \\
\hline Direct/Indirect Bandgap & I & I & D \\
\hline
\end{tabular}

Table 2.4. Comparison $\mathrm{GaN}$ and $4 \mathrm{H}$ and $6 \mathrm{H}-\mathrm{SiC}$ properties [2.10, 2.35] 
high nitrogen pressure (45 kbar) [2.36]. As a result, crystals of $\mathrm{GaN}$ cannot be grown by the standard Czochralski, or, Bridgeman methods used to grow Si and GaAs boules. A reliable method to grow large, single crystal boules of $\mathrm{GaN}$ has yet to be developed. GaN has been available in thin high quality films. Thin films of $\mathrm{GaN}$ are grown epitaxially, usually by MetalloOrganic Vapor Phase Deposition (MOCVD), on growth templates of sapphire, SiC and Aluminum Nitride. The MOCVD technique is limited to growth rates of $1-2 \mu \mathrm{m} / \mathrm{h}$ for GaN. As a result, most early experimental data on GaN has been obtained by tests performed on thin epitaxial films five to ten microns thick.

Bulk GaN substrates have only recently become available due to the development of faster growth rate methods that includes Hydride Vapor Phase Epitaxy (HVPE) [2.36]. Bulk GaN substrates are grown by HVPE on sapphire, silicon carbide and aluminum nitride (AlN) substrates. The GaN substrate is then removed from the sapphire, SiC, or AlN growth template. Figure 2.17 is a schematic of a horizontal GaN HVPE reactor [2.37]. The HVPE reactor is a quartz tube surrounded by graphite insulation enclosed in RF induction coils. The applied RF power is absorbed in the graphite insulation, heating the volume of the reactor but, preventing the penetration of the RF fields in to the quartz reactor volume. The reactor runs at atmospheric pressure and uses hydrogen $(\mathrm{H})$ and nitrogen $\left(\mathrm{N}_{2}\right)$ as the carrier gases. The reactor has two different temperature zones. The lower temperature zone is used to synthesize gaseous $\mathrm{GaCl}$ by flowing gaseous hydrochloric acid $(\mathrm{HCl})$ over molten Gallium $(\mathrm{Ga})$. This reaction occurs at $~$ $900{ }^{\circ} \mathrm{C}$ and is given by equation 2.16. The gaseous $\mathrm{GaCl}$ reacts with ammonia gas $\left(\mathrm{NH}_{3}\right)$ to deposit a GaN film on the surface of a rotating substrate in the hot zone $\left(\sim 1100{ }^{\circ} \mathrm{C}\right)$. The reaction in the hot zone is given by equation $2.17 . \mathrm{GaN}$ is deposited on the substrate and the $\mathrm{HCl}$ 


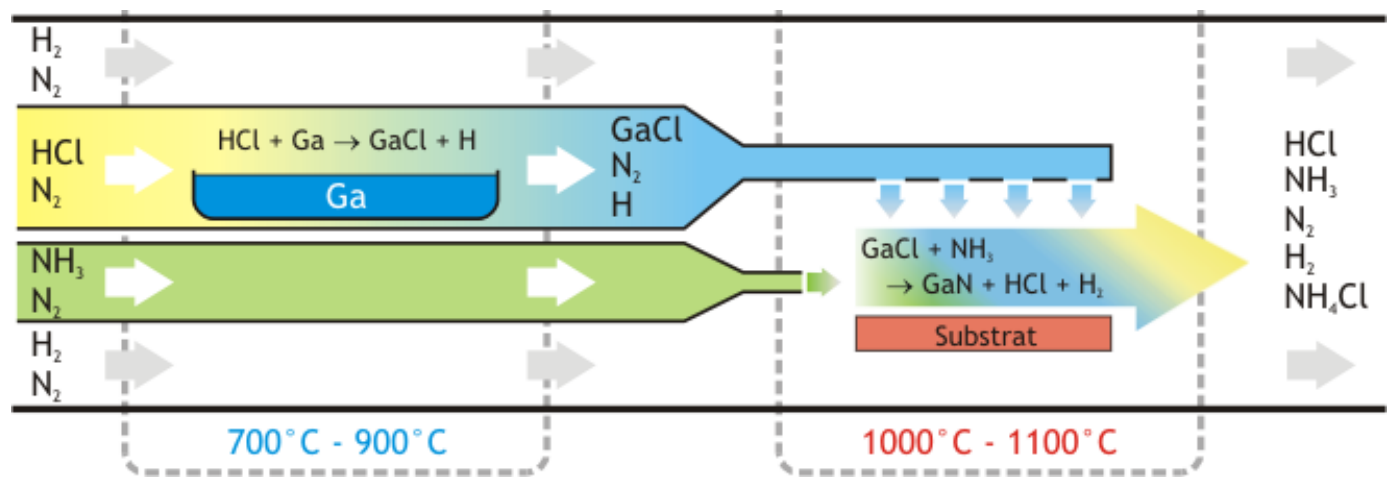

Figure 2.17. Horizontal HVPE reactor for GaN substrate production [2.37].

$$
\begin{gathered}
\mathrm{HCl}+\mathrm{Ga} \rightarrow \mathrm{GaCl}+\mathrm{H} \\
\mathrm{GaCl}+\mathrm{NH}_{3} \rightarrow \mathrm{GaN}+\mathrm{HCl}+\mathrm{H}_{2}
\end{gathered}
$$

and $\mathrm{H}_{2}$ gas are exhausted from the reactor. The temperature profile of the reactor must be maintained to prevent parasitic deposition of GaN on surfaces other than the target substrate. Growth rates of $100-200 \mu \mathrm{m} / \mathrm{h}$ [2.36] are standard for the HVPE growth method. Bulk GaN substrates are now commercially available with areas of $1-2 \mathrm{~cm}^{2}$ and thickness up to $1-2 \mathrm{~mm}$. Recent developments [2.38, 2.39] using a vertical HVPE reactor forecast the capability of growing up to $7 \mathrm{~cm}$ long, 2 inch diameter boules of $\mathrm{GaN}$ at growth rates of up to $400-500$ $\mu \mathrm{m} / \mathrm{h}$.

HVPE bulk GaN substrates exhibit dislocations, stacking faults and hexagonal pit defects. The dislocation density is high and ranges from $10^{4}-10^{6} / \mathrm{cm}^{2}[2.39,2.40]$. The dislocations and stacking faults are believed to be a result of the lattice mismatch between the $\mathrm{GaN}$ and the growth substrate $\left(\mathrm{Al}_{2} \mathrm{O}_{3}, \mathrm{SiC}\right.$, or $\left.\mathrm{AlN}\right)$. Pit defects are hexagonal pits that can measure up to 100 's of microns in diameter and contain a hollow core at its center, similar to a micropipe in SiC [2.41]. A microphotograph of a hexagonal pit in HVPE grown GaN is shown in 
Figure 2.18. Hexagonal pits in GaN present a problem in constructing a high voltage device. GaN substrates that are to be used in photoswitch construction should be free of hexagonal pits.

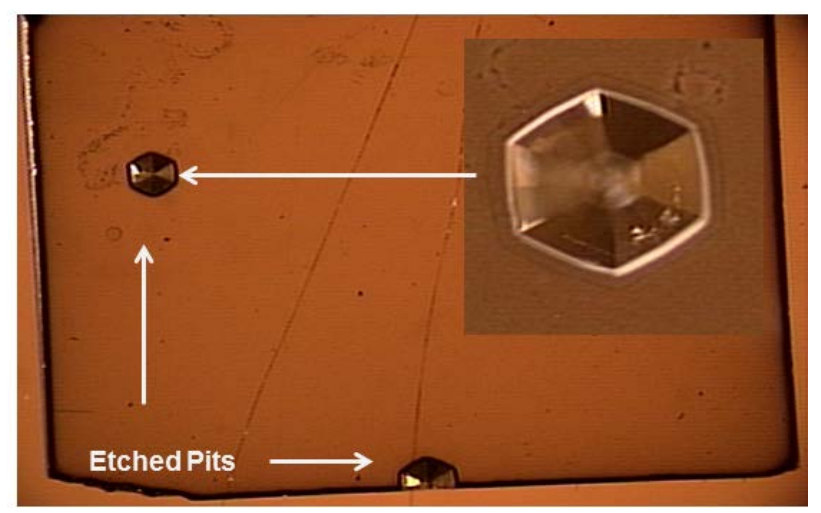

Figure 2.18. Two Large etched pits in a $9 \mathrm{~mm}$ by $9 \mathrm{~mm}, 400$ micron thick $2 \mathrm{H}-\mathrm{GaN}$ substrate, insert shows an etched pit magnified by a factor of 5

\subsection{4) Impurities in GaN}

GaN is n-type due to unintentional impurities. The primary unintentional n-type impurities in GaN are oxygen and silicon. Oxygen and silicon are shallow donors in GaN that reside $0.02-0.03 \mathrm{eV}$ below the conduction band [2.10, 2.42]. The nitrogen vacancy $\left(\mathrm{V}_{\mathrm{N}}\right)$ has been shown to be a shallow donor at $0.07 \mathrm{eV}$ below the conduction band [2.42]. The dominant shallow acceptor appears to be the gallium vacancy $\left(\mathrm{V}_{\mathrm{G}}\right)$. Iron is a deep acceptor $(0.5-0.6 \mathrm{eV}$ below conduction band) in GaN [2.43]. Semi-insulating GaN is obtained by compensating the shallow oxygen donors with deep iron acceptors. However, iron densities of $10^{17}-10^{18} / \mathrm{cm}^{3}$ are required to compensate for oxygen densities of $10^{15}-10^{16} / \mathrm{cm}^{3}$ indicating only a small fraction of the iron atoms are active [2.44, 2.45]. The situation in iron compensated, semi-insulating, GaN is similar to vanadium compensated, $4 \mathrm{H}$ and $6 \mathrm{H}-\mathrm{SiC}$. A deep acceptor captures electrons from a shallow donor. These electrons can be excited into the conduction band by below bandgap light. We have constructed extrinsic photoconductive switches from iron compensated GaN and 
vanadium compensated $6 \mathrm{H}-\mathrm{SiC}$. The following chapters will review the electronic and optical

properties of the $2 \mathrm{H}-\mathrm{GaN}, 4 \mathrm{H}$ and $6 \mathrm{H}-\mathrm{SiC}$ wide bandgap materials.

\subsection{References}

2.1) P.LeFur and D.H.Auston, “A kilovolt picosecond optoelectronic switch and Pockel's cell”, Applied Physics Letters, vol. 28, no. 1, January 1976, pp. 21 - 23

2.2) G.Mourou and W.Knox, " High-power switching with picosecond precision”, Applied Physics Letters, vol. 37, October 1979, no. 7, pp. 492 - 495

2.3) A.Rosen and F.Zutavern, High-Power Optically Activated Solid-State Switches, page 245 Artech House, 1994

2.4) R.H. Bube, Photoelectronic Properties of Semiconductors, Chapter 1 - Introductory Concepts, Cambridge University Press, 1992

2.5) S. L. Chuang, Physics of optoelectronic devices, page 586, Wiley - Interscience, 1995

2.6) G.M.Loubriel, et al., "Photoconductive semiconductor switches", IEEE Transactions on Plasma Science, vol. 25, no. 2, April 1997, pp 124 - 130

2.7) G.M.Loubriel, et al., "Longevity of optically activated, high gain GaAs photoconductive semiconductor switches”, IEEE Transactions on Plasma Science, vol. 26, no. 5, October 1998, pp1393 - 1402

2.8) F.J.Zutavern, et al.,” Photoconductive semiconductor switch technology for short pulse electromagnetics and lasers", Digest of Technical Papers - IEEE International Pulsed Power Conference, vol. 1, 1999, pp. 295 - 298

2.9) A. Mar, et al., "Doped contacts for high-longevity optically activated, high gain GaAs photoconductive semiconductor switches", IEEE Transactions on Plasma Science, vol. 28, no. 5, October 2000, pp 1507 - 1511

2.10) M.E.Levinshtein, S.L.Rumyantsev and M.S.Shur, Properties of Advanced Semiconductor Materials, pages 1 and 93, Wiley - Interscience, 2001

2.11) W.J.Choyke and G.Pensl, "Physical properties of SiC”, MRS Bulletin, March 1997, pp $25-29$

2.12) A. R. Powell and L. R. Rowland, "SiC materials - Progress, status and potential roadblocks", Proceedings of the IEEE, vol. 90, no. 6, June 2002

2.13) G. Pensl et al., "SiC material properties", International Journal of High Speed Electronics and Systems, vol. 15, no. 4, 2005, pp 705 - 745

2.14) C. -M. Zetterling, Process Technology for Silicon Carbide Devices, page 4, IEE INSPEC, 2002

2.15) P.S.Cho, J.Goldhar, C.H.Lee,S.E.Saddow and P.Neudeck, "Photoconductive and photovoltaic response of high-dark-resistivity 6H-SiC devices”, Journal of Applied Physics, vol. 77, no. 4, February 1995, pp 1591 - 1599

2.16) S.Sheng, et al., "An investigation of 3C-SiC photoconductive power switching devices", Materials Science and Engineering B, vol. 46, 1997, pp 147 - 151

2.17) S.Sheng, et al., "Polycrystalline cubic Silicon Carbide photoconductive switch", IEEE Electron Device Letters, vol. 18, no. 8, August 1997, pp 372 - 374 
2.18) S.Dogan, et al., "Large area $6 \mathrm{H}$ and $4 \mathrm{H}-\mathrm{SiC}$ photoconductive switches”, Materials Research Society Symposium Proceedings, vol 764, Materials Research Society 2003, pp $389-394$

2.19) S.Dogan, et al., " $4 \mathrm{H}-\mathrm{SiC}$ photoconductive switching devices for use in high-power applications”, Applied Physics Letters, vol. 82, no. 18, May 2003, pp 3107 - 3109

2.20) K.Zhu, et al., "Effect of $n^{+}-\mathrm{GaN}$ subcontact layer on $4 \mathrm{H}-\mathrm{SiC}$ high-power photoconductive switch”, Applied Physics Letters, vol. 86, no. 26, June 2005, pp 261108-1 - 261108-3

2.21) L.L.Alston, High-Voltage Technology, Oxford University Press, 1968

2.22) W.F.Knippenberg, “Growth phenomena in Silicon Carbide”, Philips Research Reports, vol. 18, no. 3, June 1963, p 168

2.23) M.Willander, M.Friesel, Q. Wahab and B. Straumal, "Silicon Carbide and Diamond for high temperature device applications”, Journal of Materials Science: Materials in Electronics, vol. 17, 2006, pp 1 - 25

2.24) S.M.Koo, Design and Process Issues of Junction- and Ferroelectric- Field Effect Transistors in Silicon Carbide, Ph.D. Thesis, KTH, Royal Institute of Technology, Department of Microelectronics and Information Technology, Stockholm, Sweden, 2003

2.25) R.C.Glass, D.Henshall, V.F.Tsvetkov and C.H.Carter, Jr., "SiC-seeded crystal growth”, Materials Research Society Bulletin, March 1997, pp 30 - 35

2.26) M.Yoganathan, et al., "Growth of large diameter semi-insulating 6H-SiC crystals by physical vapor transport”, Materials Research Society Proceedings, vol. 815, Materials Research Society, 2004, pp 21 - 26

2.27) H. P. Strunk, W. D. Dorsch and J. Heindl, "The nature of micropipes in 6H-SiC single crystals” Advanced Engineering Materials, vol. 2, no. 6, 2000

2.28) Intrinsic Semiconductor Website, www.intrinsicsemi.com

2.29) Cree Research Website, www.cree.com

2.30) M.Bickermann, D.Hofmann, T.L.Straubinger, R.Weingartner and A.Winnacker, "Preparation of semi-insulating Silicon Carbide by Vanadium doping during PVT bulk crystal growth", Conference Proceedings of the $4^{\text {th }}$ European Conference on Silicon Carbide and Related Materials, Linkoping, Sweden, September 2002

2.31) M.Bickermann, “Own publications about SiC and AlN”, http://serww6.ww.unierlangen.de/ bicki/e-publish.html

2.32) J. R. Jenny et al., "Deep level transient spectroscopic and Hall effect investigation of the position of the vanadium acceptor level in $4 \mathrm{H}$ and 6H-SiC”, Applied Physics Letters, vol. 68, no. 14, April 1996

2.33) W. C. Mitchel et al., "Vanadium donor and acceptor levels in semi-insulating $4 \mathrm{H}$ and $6 \mathrm{H}-$ SiC”, Journal of Applied Physics, vol. 101, 013707, 2007

2.34) M. Golio, Editor, RF and Microwave Device Handbook, Chapter 11, page 11 - 12, CRC Press, 2003

2.35) M. Shur and R. Davis, GaN-Based Materials and Devices, Chapter 1, World Scientific, 2004

2.36) M. Bockowski, "Review: Bulk growth of gallium nitride: challenges and difficulties”, Crystal Research Technology, Vol. 42, No. 12, pp. 1162 - 1175, 2007

2.37) wikipedia.org/wiki/File:Schema_HVPE-Reaktor_de.eng

2.38) Schineller, J. Kaeppeler and M. Heuken, "Vertical - HVPE as a production method for free - standing GaN - substrates”, CS MANTECH Conference Proceedings, pp. 123 126, May $14-17,2007$, Austin, Texas, USA 
2.39) B. Monemar, et al., "Growth of thick GaN layers with hydride vapour phase epitaxy”, Journal of Crystal Growth, Vol. 281, pp. 17 - 31, 2005

2.40) B. Lucznik, et al., "Deposition of thick GaN layers by HVPE on the pressure grown GaN substrates”, Journal of Crystal Growth, Vol. 281, pp. 38 - 46, 2005

2.41) T. B. Wei, et al., "Hillocks and hexagonal pits in a thick film grown by HVPE", Microelectronics Journal, Vol. 39, pp. 1556 - 1559, 2008

2.42) D. C. Look, Z. - Q. Fang and B. Clafin, "Identification of donors, acceptors and traps in bulk - like HVPE GaN”, Journal of Crystal Growth, Vol. 281, pp. 143 - 150, 2005

2.43) R. P. Vaudo et al., "Characteristics of semi-insulating, Fe-doped GaN substrates", Physica Status Solidi A, vol. 200, no. 1, pp. 18 - 21, 2003

2.44) A. Y. Polyakov et al., "Properties of Fe-doped, thick, freestanding GaN crystals grown by hydride vapor phase epitaxy”, Journal of Vacuum Science Technology B, 25(3), May/June, 2007

2.45) Z. -Q. Fang, et al., "Deep centers in semi-insulating Fe-doped native GaN substrates grown by hydride vapour phase epitaxy”, Physica Status Solidi C, vol. 5, no. 6, pp 1508 1511, 2008 


\section{Chapter 3: Electronic Properties of $4 \mathrm{H}$ and $6 \mathrm{H}-\mathrm{SiC}$ and $2 \mathrm{H}-\mathrm{GaN}$}

This chapter will review the electronic properties of $4 \mathrm{H}, 6 \mathrm{H}-\mathrm{SiC}$ and $2 \mathrm{H}-\mathrm{GaN}$. The creation and transport of photo-generated carriers in a semiconductor is governed by the optical and electronic properties of the particular semiconductor material. Band structure, effective mass, mobility, carrier recombination are but a few of the electronic properties that will affect the operation of wide bandgap, extrinsic photoconductive switches. We are principally interested in the electronic response of $4 \mathrm{H}$ and $6 \mathrm{H}-\mathrm{SiC}$ and $2 \mathrm{H}-\mathrm{GaN}$ to optical excitation at photon energies that are less than the bandgaps of these materials.

\section{1) $4 \mathrm{H}$ and $6 \mathrm{H}-\mathrm{SiC}$ Band Structure and Effective Mass}

The $4 \mathrm{H}, 6 \mathrm{H}-\mathrm{SiC}$ and $2 \mathrm{H}-\mathrm{GaN}$ materials share the hexagonal crystal structure and reciprocal lattice. However, the band structures of $4 \mathrm{H}, 6 \mathrm{H}-\mathrm{SiC}$ and $2 \mathrm{H}-\mathrm{GaN}$ are significantly different. As a result, important electronic parameters like band gap, effective mass, density of states, mobility and recombination will vary significantly between these materials. The band structure determines parameters such as effective mass that have an important impact on carrier transport properties of the material. Correspondingly, the band structure is the best place to start when trying to understand the electronic and optical properties of $4 \mathrm{H}, 6 \mathrm{H}-\mathrm{SiC}$ and $2 \mathrm{H}-\mathrm{GaN}$. The results of band structure calculations are usually expressed in terms of the primitive cell of the reciprocal lattice called the Brillouin zone. The first Brillouin zone (BZ) of the hexagonal lattice is shown in Figure 3.1 [3.1]. This figure shows $\Gamma$ at the center of the BZ, the high symmetry axes of the first hexagonal BZ, and the components of the wave vector $\mathbf{k}$. The importance of the symmetry axes shown in Figure 3.1 becomes apparent upon inspection of the calculated band structures of $4 \mathrm{H}$ and $6 \mathrm{H}-\mathrm{SiC}$ shown in Figures 3.2 and 3.3. The band structures shown for $4 \mathrm{H}$ 
and $6 \mathrm{H}-\mathrm{SiC}$ were calculated using a density functional theory approach [3.2] based on the linear density approximation (LDA). This approach is known to underestimate the SiC bandgaps by $1.0 \mathrm{eV}$ [3.3], however the effective electron masses calculated using these band structures are in good agreement with experimental values. The calculated value for the band gaps for $4 \mathrm{H}-\mathrm{SiC}$ and $6 \mathrm{H}-\mathrm{SiC}$ are $2.1 \mathrm{and} 1.9 \mathrm{eV}$ respectively. The measured room temperature value for these band gaps are 3.26 and $3.02 \mathrm{eV}$, respectively. Figures 3.2 and 3.3 show that both $4 \mathrm{H}$ and $6 \mathrm{H}-\mathrm{SiC}$ have complicated band structures with indirect band gaps. The calculated energy bands for the $6 \mathrm{H}$ polytype are more complex than the $4 \mathrm{H}$ polytype. This is a result of the $6 \mathrm{H}$ polytype containing twelve atoms per unit cell compared to eight for the $4 \mathrm{H}$ polytype and having a unit

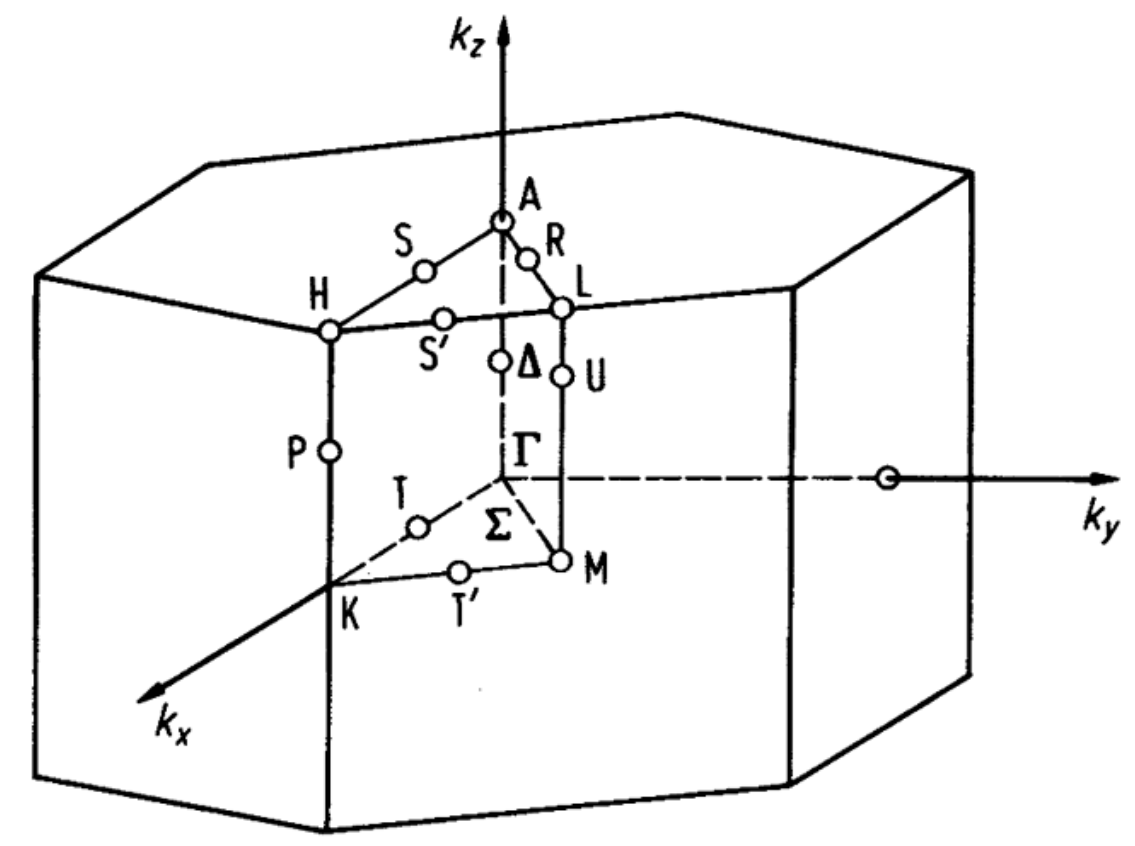

Figure 3.1. First Brillouin zone of the hexagonal lattice [3.1].

cell 50 percent longer in the c direction than the $4 \mathrm{H}$ polytype. The valence band maximums occur at the center of the $\mathrm{BZ}$ at the point $\Gamma$ for both the $4 \mathrm{H}$ and $6 \mathrm{H}-\mathrm{SiC}$ polytypes. The minimum of the conduction band occurs at the boundary of the $\mathrm{BZ}$ for both $4 \mathrm{H}$ and $6 \mathrm{H}-\mathrm{SiC}$, but at different 
locations. The conduction band minimum occurs at the $\mathrm{M}$ point in the $\mathrm{BZ}$ for $4 \mathrm{H}-\mathrm{SiC}$. The conduction band minimum for $6 \mathrm{H}-\mathrm{SiC}$ is located along the $\mathrm{ML}$ line in the $\mathrm{BZ}, 44$ percent out from $\mathrm{M}$ towards $\mathrm{L}$ [3.3]. The lowest conduction band minimum for $6 \mathrm{H}-\mathrm{SiC}$ has a double well shape referred to as a 'camel back' [3.4] with only $6 \mathrm{meV}$ difference between the conduction band minimum along the line $\mathrm{L}-\mathrm{M}$ and the energy at point $\mathrm{M}$. The camels back feature at the minima of lowest conduction band for $6 \mathrm{H}-\mathrm{SiC}$ causes this band to be non-parabolic. The two

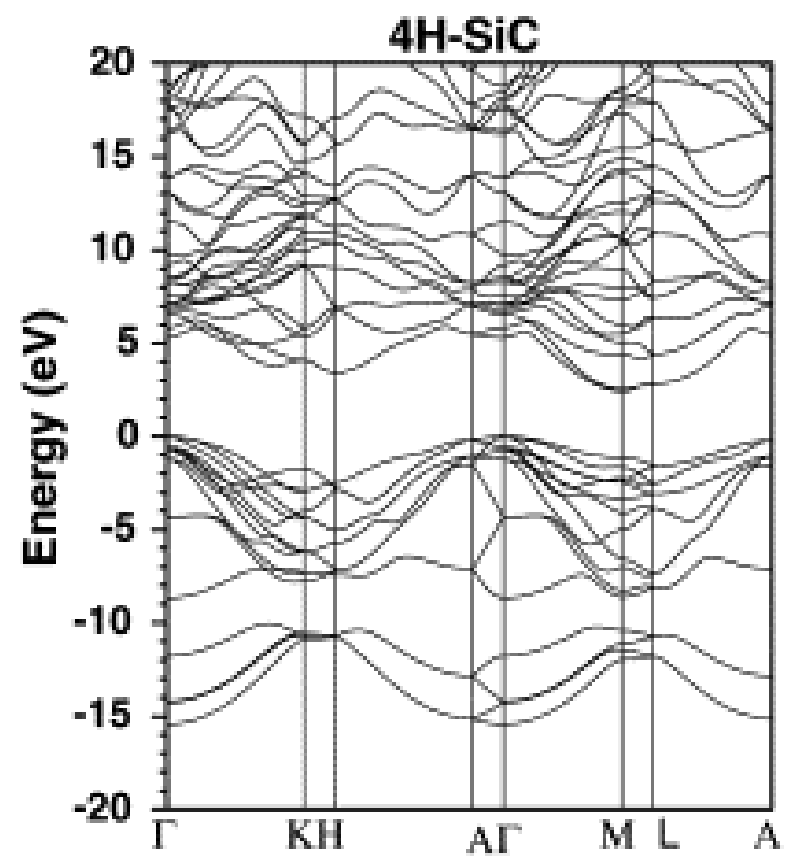

Figure 3.2. Calculated energy bands for $4 \mathrm{H}$-SiC along high symmetry directions of the $\mathrm{BZ}$ [3.2]

lowest $4 \mathrm{H}$ and second lowest $6 \mathrm{H}-\mathrm{SiC}$ conduction bands are parabolic. $6 \mathrm{H}-\mathrm{SiC}$ energy bands both have the two lowest conduction bands degenerate at point $\mathrm{K}$. The second lowest conduction band (cb) minimum is located at point $\mathrm{M}$ for $4 \mathrm{H}-\mathrm{SiC}$, so direct transitions can occur between the two lowest conduction bands at this point in $4 \mathrm{H}-\mathrm{SiC}$. The two lowest cb for $6 \mathrm{H}-\mathrm{SiC}$ are also degenerate along the line from point $\mathrm{M}$ to point $\mathrm{L}$. There are three (six) equivalent conduction 
band minima at the point (line) $\mathrm{M}(\mathrm{L}-\mathrm{M})$ for $4 \mathrm{H}-\mathrm{SiC}(6 \mathrm{H}-\mathrm{SiC})$ polytype. The three highest valence bands are degenerate at the $\Gamma$ point in both the $4 \mathrm{H}$ and $6 \mathrm{H}$ polytypes.

The $4 \mathrm{H}$ and $6 \mathrm{H}-\mathrm{SiC}$ are presented in a simplified schematic form, including corrections to band gap magnitudes, in figures 3.4 and 3.5. These simplified schematics show an idealized parabolic shape for all bands, along with the positions of conduction band minimums and valence band maximums.

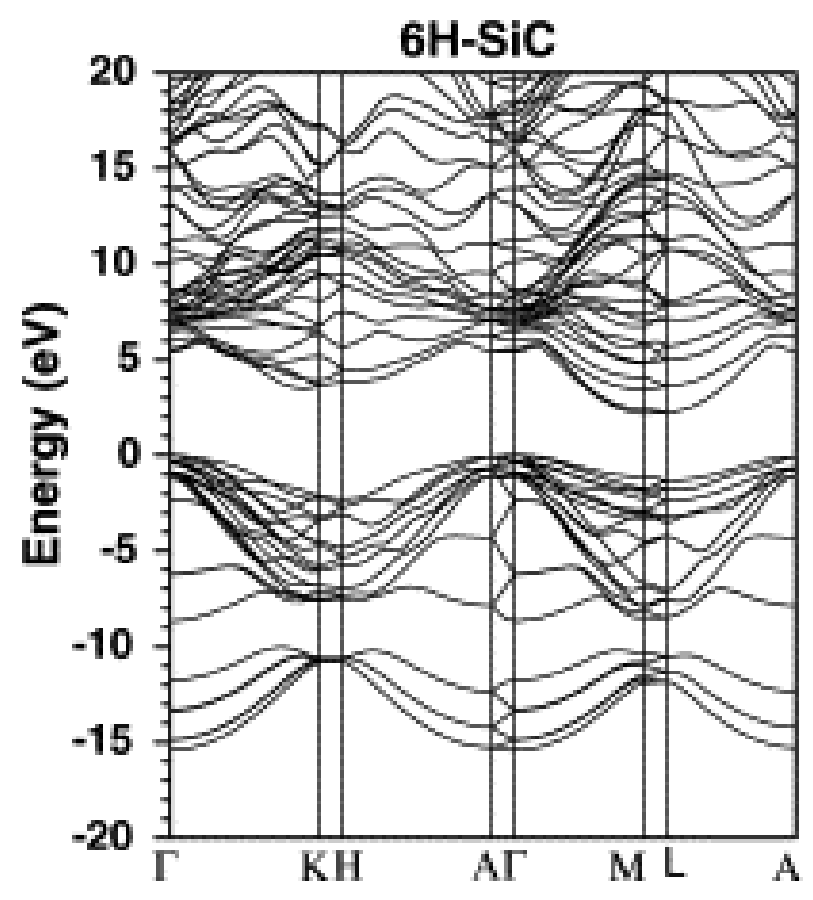

Figure 3.3. Calculated energy bands for $6 \mathrm{H}$-SiC along high symmetry directions of the $\mathrm{BZ}$ [3.2] 


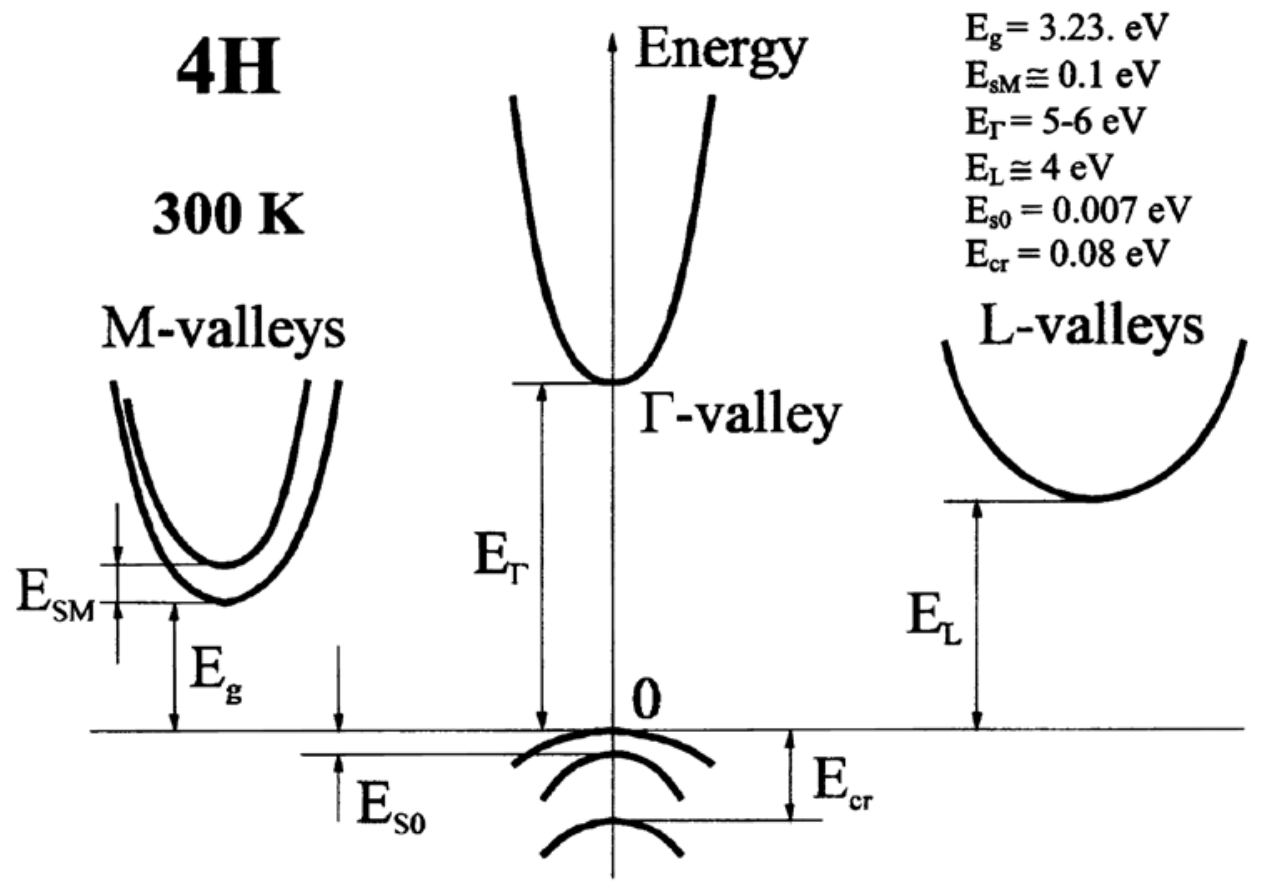

Figure 3.4. Simplified schematic of 4H-SiC energy bands [3.5]

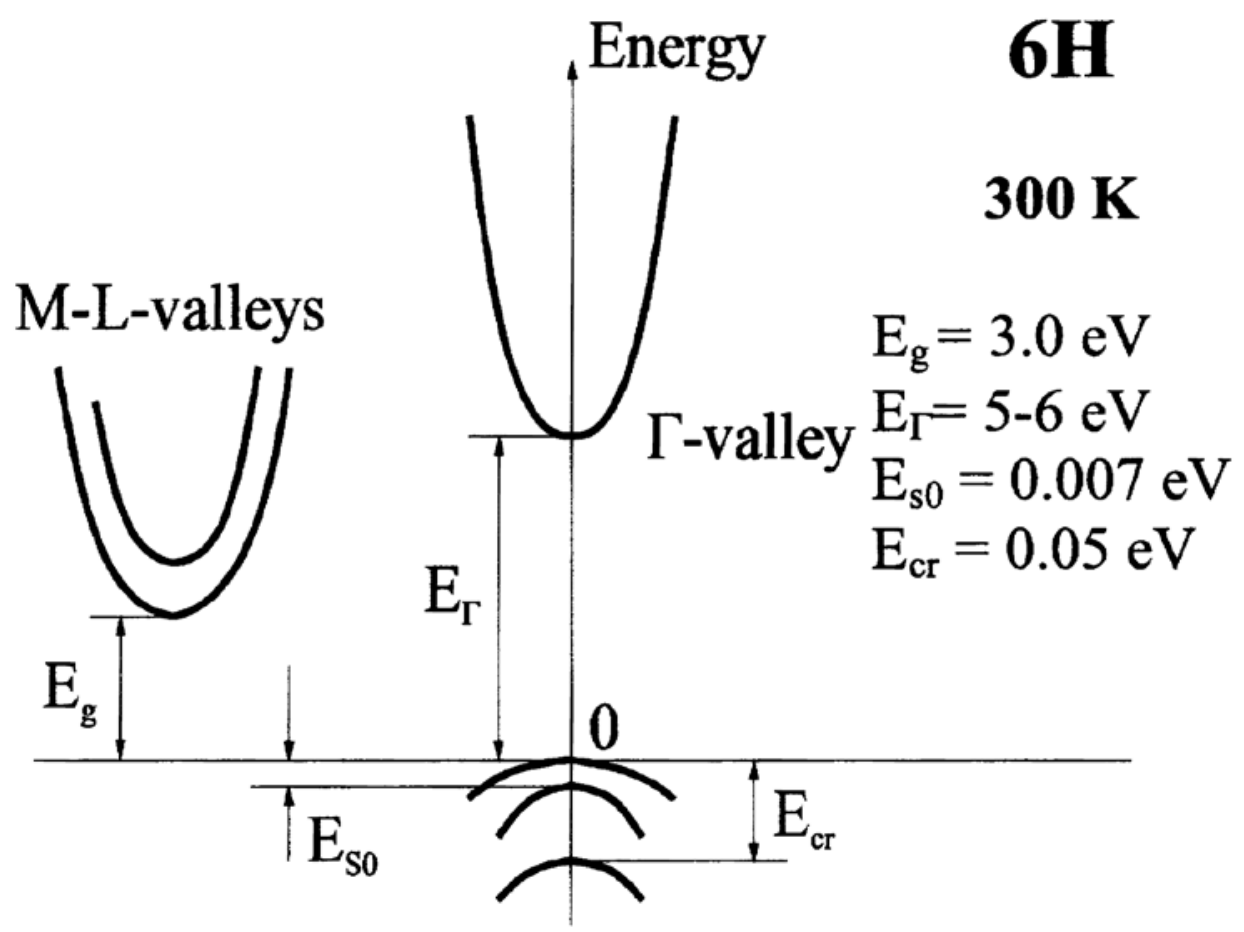

Figure 3.5. Simplified schematic of 6H-SiC energy bands [3.5]

The electron (hole) effective mass can be calculated for parabolic bands from the curvature of the conduction (valence) bands near their minimum (maximum) using equation 3.1. 
The effective mass is a tensor with components $\mathrm{m}_{\mathrm{ij}}{ }^{*}$. The effective electron and effective hole masses calculated from the theoretical $4 \mathrm{H}$ and $6 \mathrm{H}-\mathrm{SiC}$ band structures [3.2, 3.3] are in good

$$
\frac{1}{m_{i j}^{*}}=\frac{1}{\hbar^{2}} \frac{\partial^{2} E(k)}{\partial k_{i} \partial k_{j}}
$$

agreement with experimental measurements [3.6, 3.7]. Table 3.1 summarizes the experimental and theoretical values of electron effective masses at conduction band minimums for $4 \mathrm{H}$ and $6 \mathrm{H}$ $\mathrm{SiC}$ in units of the free electron mass $\left(\mathrm{m}_{0}\right)$. The components of the electron effective mass have been calculated from theoretical band structure along the high symmetry axes of the BZ. The

\begin{tabular}{|l|l|l|l|}
\hline Polytype & $\begin{array}{l}\text { Effective Mass } \\
\text { Component }\end{array}$ & $\begin{array}{l}\text { Theoretical Value } \\
{[3.2,3.3,3.8]}\end{array}$ & $\begin{array}{l}\text { Experimental Value } \\
{[3.6,3.7]}\end{array}$ \\
\hline $4 \mathrm{H}-\mathrm{SiC}$ & $\mathrm{m}_{\mathrm{ML}}$ & $0.30-0.33 \mathrm{~m}_{0}$ & $0.33 \mathrm{~m}_{0}$ \\
\hline & $\mathrm{m}_{\mathrm{M} \Gamma}$ & $0.57-0.66 \mathrm{~m}_{0}$ & $0.58 \mathrm{~m}_{0}$ \\
\hline & $\mathrm{m}_{\mathrm{MK}}$ & $0.28-0.32 \mathrm{~m}_{0}$ & $0.31 \mathrm{~m}_{0}$ \\
\hline $6 \mathrm{H}-\mathrm{SiC}$ & $\mathrm{m}_{\mathrm{ML}}$ & $1.20-2.07 \mathrm{~m}_{0}$ & $2.0-6.0 \mathrm{~m}_{0}$ \\
\hline & $\mathrm{m}_{\mathrm{M} \Gamma}$ & $0.75-0.81 \mathrm{~m}_{0}$ & $\mathrm{~m} \perp=0.48 \mathrm{~m}_{0}$ \\
\hline & $\mathrm{m}_{\mathrm{MK}}$ & $0.23-0.27 \mathrm{~m}_{0}$ & \\
\hline
\end{tabular}

Table 3.1. Theoretical and experimental values of electron effective mass for $4 \mathrm{H}$ and $6 \mathrm{H}$ SiC.

theoretical and experimental values of the effective electron mass for $4 \mathrm{H}-\mathrm{SiC}$ are in good agreement and anisotropic. However, there is a broad range of theoretical and experimental values of the electron effective mass for $6 \mathrm{H}-\mathrm{SiC}$ along the ML axes of symmetry and no experimental results reported for $\mathrm{m}_{\mathrm{MK}}$ and $\mathrm{m}_{\mathrm{M} \Gamma}$. This is a result of the camels back shape of the 
lowest conduction band minimum for $6 \mathrm{H}-\mathrm{SiC}$. The flatness of the conduction band minima for $6 \mathrm{H}-\mathrm{SiC}$ along $\mathrm{ML}$ and general non-parabolic shape results in the broad range of values for the calculated results. Experimental results have been reported for the total transverse component of effective electron mass for $6 \mathrm{H}$-SiC. The general relationships between the effective mass components are shown below. The experimental result reported for the transverse component of the effective electron mass for $6 \mathrm{H}-\mathrm{SiC}$ is the

$$
\begin{gathered}
m_{M L}^{*}=m_{\|}^{*} \\
m_{M K}^{*}=m_{\perp 1}^{*} \\
m_{M \Gamma}^{*}=m_{\perp 2}^{*}
\end{gathered}
$$

geometric mean of the theoretical values for $\mathrm{m}_{\mathrm{MK}}$ and $\mathrm{m}_{\mathrm{M \Gamma}}$. The effective electron mass components of $6 \mathrm{H}-\mathrm{SiC}$ show a much stronger anisotropy compared to the $4 \mathrm{H}-\mathrm{SiC}$ polytype.

The calculated and measured values of the components of the hole effective masses for $4 \mathrm{H}$ and $6 \mathrm{H}-\mathrm{SiC}$ are listed in Table 3.2. The highest valence band maximum for both $4 \mathrm{H}$ and $6 \mathrm{H}-\mathrm{SiC}$ occur in the center of the $\mathrm{BZ}$ at point $\Gamma$. The valence bands are parabolic and well behaved. Comparing figures 3.2 and 3.3, the shape of the highest valence bands for $4 \mathrm{H}$ and $6 \mathrm{H}-\mathrm{SiC}$ are very similar. This is reflected in the close theoretical and experimental values obtained for the components of the hole effective mass for $4 \mathrm{H}$ and $6 \mathrm{H}-\mathrm{SiC}$. The hole effective mass is anisotropic for both the $4 \mathrm{H}$ and $6 \mathrm{H}-\mathrm{SiC}$ polytypes. 


\begin{tabular}{|l|l|l|l|}
\hline Polytype & $\begin{array}{l}\text { Effective Mass } \\
\text { Component }\end{array}$ & $\begin{array}{l}\text { Theoretical Value } \\
{[\mathbf{3 . 2}, \mathbf{3 . 3}, \mathbf{3 . 8}]}\end{array}$ & $\begin{array}{l}\text { Experimental Value } \\
{[\mathbf{3 . 6 , 3 . 7 ]}}\end{array}$ \\
\hline $4 \mathrm{H}-\mathrm{SiC}$ & $\mathrm{m} \|$ & $1.60-1.62 \mathrm{~m}_{0}$ & $1.75 \mathrm{~m}_{0}$ \\
\hline & $\mathrm{m} \perp$ & $0.61-0.66 \mathrm{~m}_{0}$ & $0.66 \mathrm{~m}_{0}$ \\
\hline $6 \mathrm{H}-\mathrm{SiC}$ & $\mathrm{m} \|$ & $1.65-1.80 \mathrm{~m}_{0}$ & $1.85 \mathrm{~m}_{0}$ \\
\hline & $\mathrm{m} \perp$ & $0.60-0.65 \mathrm{~m}_{0}$ & $0.66 \mathrm{~m}_{0}$ \\
\hline
\end{tabular}

Table 3.2. Hole effective mass components for $4 \mathrm{H}$ and $6 \mathrm{H}$ - SiC.

\subsection{1) Band Structure and Effective Mass for $2 \mathrm{H}-\mathrm{GaN}$}

The electronic band structure for $2 \mathrm{H}-\mathrm{GaN}$ is shown in Figure 3.6 [3.8]. 2H-GaN has a

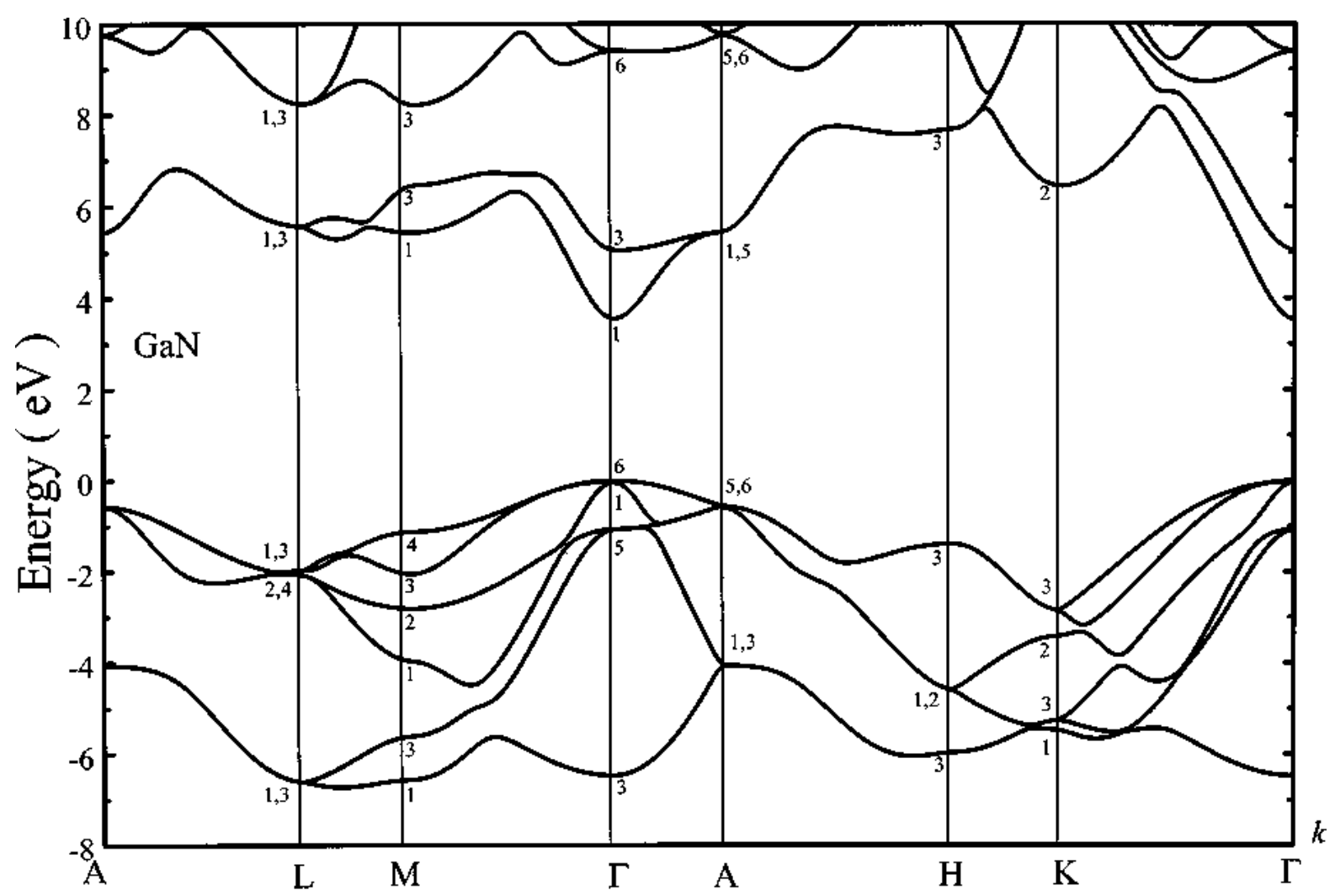

Figure 3.6. Electronic band structure for $2 \mathrm{H}-\mathrm{GaN}$ [3.8] 
direct band gap with the conduction band minimum and valence band maximum both occurring at $\Gamma$ the center of the first BZ. The band structure for $2 \mathrm{H}-\mathrm{GaN}$ was calculated using the empirical pseudopotential method [3.8]. This band gap calculated by this method was adjusted to $3.50 \mathrm{eV}$ in agreement with the measured low temperature $\left(1.6{ }^{\circ} \mathrm{K}\right)$ value of the $2 \mathrm{H}-\mathrm{GaN}$ band gap. The lowest conduction band minimum is parabolic and well behaved close to $\Gamma$. The 3 highest valence bands are the heavy-hole (HH), light-hole (LH) and crystal-field split-hole (CH). The HH and LH bands almost meet at their maximums. The HH band is flatter and broader than the LH, which is the reason it is called the heavy hole. The calculated effective masses for the band structures of Figure 3.6 are listed in Table 3.3 [3.8].

\begin{tabular}{|c|c|c|c|c|c|c|}
\hline $\mathbf{m} \| \mathbf{H H}$ & $\mathbf{m} \| \mathbf{L H}$ & $\mathbf{m}_{\perp_{\mathbf{H H}}}$ & $\mathbf{m} \perp_{\mathbf{L H}}$ & $\mathbf{m}_{\perp_{\mathbf{C H}}}$ & $\mathbf{m} \| \mathbf{C H}$ & $\mathbf{m}_{\mathbf{n}}$ \\
\hline $1.96 \mathrm{~m}_{0}$ & $1.96 \mathrm{~m}_{0}$ & $1.87 \mathrm{~m}_{0}$ & $0.14 \mathrm{~m}_{0}$ & $1.96 \mathrm{~m}_{0}$ & $0.14 \mathrm{~m}_{0}$ & $0.22 \mathrm{~m}_{0}$ \\
\hline
\end{tabular}

Table 3.3. Calculated effective masses for $2 \mathrm{H}-\mathrm{GaN}$ [3.8]

\section{2) Intrinsic Carriers in $4 \mathrm{H}-\mathrm{SiC}, 6 \mathrm{H}-\mathrm{SiC}$ and $2 \mathrm{H}-\mathrm{GaN}$}

We have seen that the energy bands near the conduction band minima and valence band maxima are parabolic for $4 \mathrm{H}-\mathrm{SiC}$ and $2 \mathrm{H}-\mathrm{GaN}$. The valence bands for $6 \mathrm{H}-\mathrm{SiC}$ are also parabolic. The lowest conduction band of $6 \mathrm{H}-\mathrm{SiC}$ has a camel back feature and is not parabolic. However, we will assume parabolic bands and use established expressions for the intrinsic carriers in these materials. We also assume, for the moment, that the semiconductor material contains no impurities. The effective density of states for the conduction and valence bands, for a nondegenerate semiconductor at thermal equilibrium, are given by equations 3.3 and 3.4.

$$
\begin{aligned}
& N_{c}=2\left(\frac{2 \pi m_{d e} k T}{h^{2}}\right)^{\frac{3}{2}} \\
& N_{v}=2\left(\frac{2 \pi m_{d h} k T}{h^{2}}\right)^{\frac{3}{2}}
\end{aligned}
$$


Where $m_{d e}\left(m_{d h}\right)$ is the density of state effective mass for the conduction (valence) band, $k(h)$ is Boltzmann's (Planck's) constant and T is the temperature in degrees Kelvin. The density of state effective mass for electrons and holes are given in Table 3.4 [3.5, 3.8] along with the number of equivalent conduction band minima. Substituting the values for the density of states effective masses in equations (3.3) and (3.4), we obtain the results shown in Table 3.5.

\begin{tabular}{|l|l|l|l|}
\hline Material & $\mathbf{m}_{\mathbf{d e}}$ & $\mathbf{m}_{\mathbf{d h}}$ & $\mathbf{M}_{\mathbf{c}}$ \\
\hline $4 \mathrm{H}-\mathrm{SiC}$ & $0.77 \mathrm{~m}_{0}$ & $1.20 \mathrm{~m}_{0}$ & 3 \\
\hline $6 \mathrm{H}-\mathrm{SiC}$ & 2.34 & $0.80 \mathrm{~m}_{0}$ & 6 \\
\hline $2 \mathrm{H}-\mathrm{GaN}$ & 0.22 & $1.50 \mathrm{~m}_{0}$ & \\
\hline
\end{tabular}

Table 3.4. Density of state effective masses for $4 \mathrm{H}, 6 \mathrm{H}-\mathrm{SiC}$ and $2 \mathrm{H}-\mathrm{GaN}$ [3.5, 3.8]

\begin{tabular}{|c|c|c|}
\hline Material & $\mathbf{N}_{\mathbf{c}}$ & $\mathbf{N}_{\mathbf{v}}$ \\
\hline $4 \mathrm{H}-\mathrm{SiC}$ & $3.25 \mathrm{e} 15 \times \mathrm{T}^{3 / 2}$ & $6.31 \mathrm{e} 15 \times \mathrm{T}^{3 / 2}$ \\
\hline $6 \mathrm{H}-\mathrm{SiC}$ & $1.72 \mathrm{e} 16 \times \mathrm{T}^{3 / 2}$ & $3.43 \mathrm{e} 15 \times \mathrm{T}^{3 / 2}$ \\
\hline $2 \mathrm{H}-\mathrm{GaN}$ & $4.30 \mathrm{e} 14 \times \mathrm{x}^{3 / 2}$ & $8.82 \mathrm{e} 15 \times \mathrm{T}^{3 / 2}$ \\
\hline
\end{tabular}

Table 3.5. Conduction and valence band density of states as function of temperature

We can use the conduction and valence band density of states to calculate the carriers present in the conduction and valence band for non-degenerate semiconductors. The number of electrons (holes) in the conduction (valence) band is given by

$$
\begin{aligned}
& n=N_{c} \exp ^{-\left(\frac{E_{c}-E_{f}}{k T}\right)} \\
& p=N_{v} \exp ^{-\left(\frac{E_{f}-E_{v}}{k T}\right)}
\end{aligned}
$$

Where $E_{c}\left(E_{v}\right)$ is the conduction (valence) band energy and $E_{f}$ is the Fermi level. The number of electrons in the conduction band must equal to the number of holes in the valence band for a pure, intrinsic semiconductor at thermal equilibrium. The intrinsic carrier concentration, $\mathrm{n}_{\mathrm{i}}$, will be equal to both the electrons in the conduction band and holes in the valence band. The intrinsic 
carrier concentration can be calculated using equations $(3.3$ - 3.6) and the value of the band gap $\left(E_{g}\right)$ for the semiconducting material. The intrinsic carrier density is given below. Equations (3.7) and (3.8) are referred to the law of mass action.

$$
\begin{aligned}
n_{i}^{2} & =n p \\
n_{i}{ }^{2} & =N_{c} N_{v} \exp ^{-\left(\frac{E g}{k T}\right)}
\end{aligned}
$$

The room temperature $\left(\mathrm{T}=300^{\circ} \mathrm{K}\right)$ value of the band gap energy $\left(\mathrm{E}_{\mathrm{g}}\right)$ for $4 \mathrm{H}, 6 \mathrm{H}-\mathrm{SiC}$ and $2 \mathrm{H}-\mathrm{GaN}$ is $3.0,3.26$ and 3.42 , respectively. We can calculate the number of free electrons and holes in impurity and flaw free $2 \mathrm{H}-\mathrm{GaN}, 4 \mathrm{H}$ and $6 \mathrm{H}-\mathrm{SiC}$ crystals at room temperature using equations (3.7 - 3.8) and the contents of table 3.5. We will find that the number of free electrons and holes is less than $2 / \mathrm{m}^{3}$ for all three materials. There are virtually no free carriers in these materials at room temperature. This is a result of the large band gap. However, most semiconductors contain impurities (dopants) and flaws. Some dopants are intentional and some unintentional. The unintentional dopants present in $2 \mathrm{H}-\mathrm{GaN}, 4 \mathrm{H}$ and $6 \mathrm{H}-\mathrm{SiC}$ were reviewed in

chapter two. The use of the dopants, vanadium and iron, to compensate Silicon Carbide and Gallium Nitride was also reviewed. We will discuss the compensation mechanisms in more detail in the next section.

\section{3) Compensation in $4 \mathrm{H}-\mathrm{SiC}, 6 \mathrm{H}-\mathrm{SiC}$ and $2 \mathrm{H}-\mathrm{GaN}$}

We saw in the previous section that we should expect virtually no free carriers in ultrapure $2 \mathrm{H}-\mathrm{GaN}, 4 \mathrm{H}$ and $6 \mathrm{H}-\mathrm{SiC}$ at room temperature. Unfortunately, all three of these wide band gap materials, as currently available, contain impurities. The primary unintentional dopants in $4 \mathrm{H}$ and $6 \mathrm{H}-\mathrm{SiC}$ are nitrogen and boron. Nitrogen is a shallow donor in both $4 \mathrm{H}$ and $6 \mathrm{H}-\mathrm{SiC}$. 
Boron is an acceptor in both $4 \mathrm{H}$ and $6 \mathrm{H}-\mathrm{SiC}$. The primary unintentional dopants in $2 \mathrm{H}-\mathrm{GaN}$ are oxygen and silicon, which are both shallow donors. 4H-SiC and 6H-SiC usually contain both nitrogen and boron. $4 \mathrm{H}$ and $6 \mathrm{H}-\mathrm{SiC}$ will be a n-type (p-type) semiconductor if it contains a higher (lower) density of thermally ionized nitrogen atoms compared to thermally ionized boron atoms. If a perfect balance is struck between the electrons donated from the nitrogen donor levels and the electrons accepted by the boron acceptor levels, in either silicon carbide polytype, the material will not only be compensated, but semi-insulating. The compensated material will have few free carriers and a very high resistivity. In practice, there is usually either more nitrogen than boron in silicon carbide, or vice versa. A third impurity is then introduced to compensate the excess nitrogen donors, or boron acceptors. Vanadium is added to compensate either, n or p-type silicon carbide and produce a semi-insulating material. Vanadium is an amphoteric impurity that can act as a donor, or acceptor depending on the position of the Fermi level in the band gap. Iron is used to compensate $2 \mathrm{H}-\mathrm{GaN}$. The compensation method is one technique used to obtain semiinsulating material in $4 \mathrm{H}-\mathrm{SiC}, 6 \mathrm{H}-\mathrm{SiC}$ and $2 \mathrm{H}-\mathrm{GaN}$. Table 3.6 lists the energy levels for the important impurities in 4H-SiC, $6 \mathrm{H}-\mathrm{SiC}$ and $2 \mathrm{H}-\mathrm{GaN}$.

\begin{tabular}{|l|l|l|l|l|l|}
\hline Material & Dopant & Type & $\mathbf{h}$ & $\mathbf{k}_{\mathbf{1}}$ & $\mathbf{k}_{\mathbf{2}}$ \\
\hline $4 \mathrm{H}-\mathrm{SiC}$ & nitrogen & donor & $\mathrm{E}_{\mathrm{C}}-.042 \mathrm{eV}$ & $\mathrm{E}_{\mathrm{C}}-.084 \mathrm{eV}$ & \\
\hline & vanadium & donor & $\mathrm{E}_{\mathrm{C}}-1.7 \mathrm{eV}$ & & \\
\hline & boron & acceptor & $\mathrm{E}_{\mathrm{V}}+.21 \mathrm{eV}$ & $\mathrm{E}_{\mathrm{V}}+.27 \mathrm{eV}$ & \\
\hline & vanadium & acceptor & $\mathrm{E}_{\mathrm{C}}-1.0 \mathrm{eV}$ & & \\
\hline & nitrogen & donor & $\mathrm{E}_{\mathrm{C}}-.080 \mathrm{eV}$ & $\mathrm{E}_{\mathrm{C}}-.137 \mathrm{eV}$ & $\mathrm{E}_{\mathrm{C}}-0.14 \mathrm{eV}$ \\
\hline & vanadium & donor & $\mathrm{E}_{\mathrm{V}}+1.4 \mathrm{eV}$ & & \\
\hline & boron & acceptor & $\mathrm{E}_{\mathrm{V}}+.270 \mathrm{eV}$ & $\mathrm{E}_{\mathrm{V}}+.31 \mathrm{eV}$ & $\mathrm{E}_{\mathrm{V}}+.38 \mathrm{eV}$ \\
\hline & vanadium & acceptor & $\mathrm{E}_{\mathrm{C}}-0.68 \mathrm{eV}$ & $\mathrm{E}_{\mathrm{C}}-0.72 \mathrm{eV}$ & \\
\hline & oxygen & donor & $\mathrm{E}_{\mathrm{C}}-0.02 \mathrm{eV}$ & & \\
\hline & iron & acceptor & $\mathrm{E}_{\mathrm{C}}-0.51 \mathrm{eV}$ & & \\
\hline
\end{tabular}

Table 3.6. Primary donor and acceptor energy levels in $4 \mathrm{H}-\mathrm{SiC}, 6 \mathrm{H}-\mathrm{SiC}$ and $2 \mathrm{H}-\mathrm{GaN}$ 


\subsection{1) Incomplete Ionization}

Most of the energy levels listed in table 3.6 cannot be considered 'shallow' donors, or acceptors. Not all the impurities will be completely ionized at thermal equilibrium at room temperature since their energy levels are located more than a few kT deep in the band gap. This is referred to as incomplete ionization. In general, the donor (acceptor) atoms are neutral until they achieve a high enough thermal energy so that an electron is donated (accepted) to (from) the lattice. As a result, an ionized donor (acceptor) atom becomes positively (negatively) charged. The mathematical expressions for the concentration of ionized donors and acceptors are based on Fermi - Dirac statistics [3.9] and given by equations (3.9) and (3.10).

$$
\begin{aligned}
& N_{d}^{+}=\frac{N_{d}}{1+g_{d} \exp ^{\left(\frac{E_{f}-E_{d}}{k T}\right)}} \\
& N_{a}^{-}=\frac{N_{a}}{1+g_{a} \exp ^{\left(\frac{E_{a}-E_{f}}{k T}\right)}}
\end{aligned}
$$

Where $\mathrm{N}_{\mathrm{d}}\left(\mathrm{N}_{\mathrm{a}}\right)$ is the donor (acceptor) density, $\mathrm{E}_{\mathrm{d}}\left(\mathrm{E}_{\mathrm{a}}\right)$ is the donor (acceptor) energy level, $\mathrm{g}_{\mathrm{d}}$ $\left(\mathrm{g}_{\mathrm{a}}\right)$ is the donor (acceptor) degeneracy factor and $\mathrm{E}_{\mathrm{f}}$ is the Fermi level. The fraction of ionized donors and acceptors are a function of the Fermi level and temperature. The position of the Fermi level will be determined by the number and concentrations of the different impurities that have been added to the semiconductor. The semiconductor must be charge-neutral at equilibrium. The total negative charge must balance the total positive charge present in the semiconductor. This is expressed in equation (3.11). Where it has been assumed that there are

$$
n+\sum_{i=1}^{k} \frac{N_{a i}}{1+g_{a i} \exp \left(\frac{E_{a i}-E_{f}}{k T}\right)}=p+\sum_{j=1}^{m} \frac{N_{d j}}{1+g_{d j} \exp \left(\frac{E_{d j}-E_{f}}{k T}\right)}
$$


$\mathrm{k}$ different ionized acceptor and $\mathrm{m}$ different ionized donor impurities in the semiconductor. We consider a material that contains a single donor impurity to illustrate incomplete ionization. For this case, equation (3.11) simplifies to

$$
n=p+\frac{N_{d}}{1+g_{d} \exp \left(\frac{E_{f}-E_{d}}{k T}\right)}
$$

We can substitute for the Fermi level term in the exponent of (3.12) using equation (3.5). We can neglect the holes in the valence band since the donor density is much higher. These simplifications result in equation (3.13).

$$
n \cong N_{d}^{+}=\frac{N_{d}}{1+g_{d} \frac{n}{N_{c}} \exp \left(\frac{E_{c}-E_{d}}{k T}\right)}
$$

Equation (3.13) results in the quadratic equation for $\mathrm{n}$ shown in (3.14). The only physical solution of equation (3.14) is used to obtain the fraction of ionized donors is listed in (3.15). The analytical result for the fraction of ionized donors can be applied to obtain the degree of ionization of oxygen donors in n-type $\mathrm{GaN}$ using the values for $\mathrm{N}_{\mathrm{c}}$ and $\mathrm{E}_{\mathrm{d}}$ from tables 3.5 and 3.6, respectively. The fraction of ionized oxygen donors in $\mathrm{GaN}$ is plotted in figure 3.7 for a range of temperatures from 25 to $700{ }^{\circ} \mathrm{K}$ for oxygen donor densities of $1 \mathrm{e} 16 / \mathrm{cm}^{3}, 1 \mathrm{e} 17 / \mathrm{cm}^{3}$ and $1 \mathrm{e} 18 / \mathrm{cm}^{3}$.

$$
n^{2} \frac{g_{d}}{N_{c}} \exp \left(\frac{E_{c}-E_{d}}{k T}\right)-n-N_{d}=0
$$




$$
\frac{n}{N_{d}}=\frac{-1+\sqrt{1+\frac{4 g_{d} N_{d} \exp \left(\frac{E_{c}-E_{d}}{k T}\right)}{N_{c}}}}{2 N_{d} \frac{g_{d}}{N_{c}} \exp \left(\frac{E_{c}-E_{d}}{k T}\right)}
$$

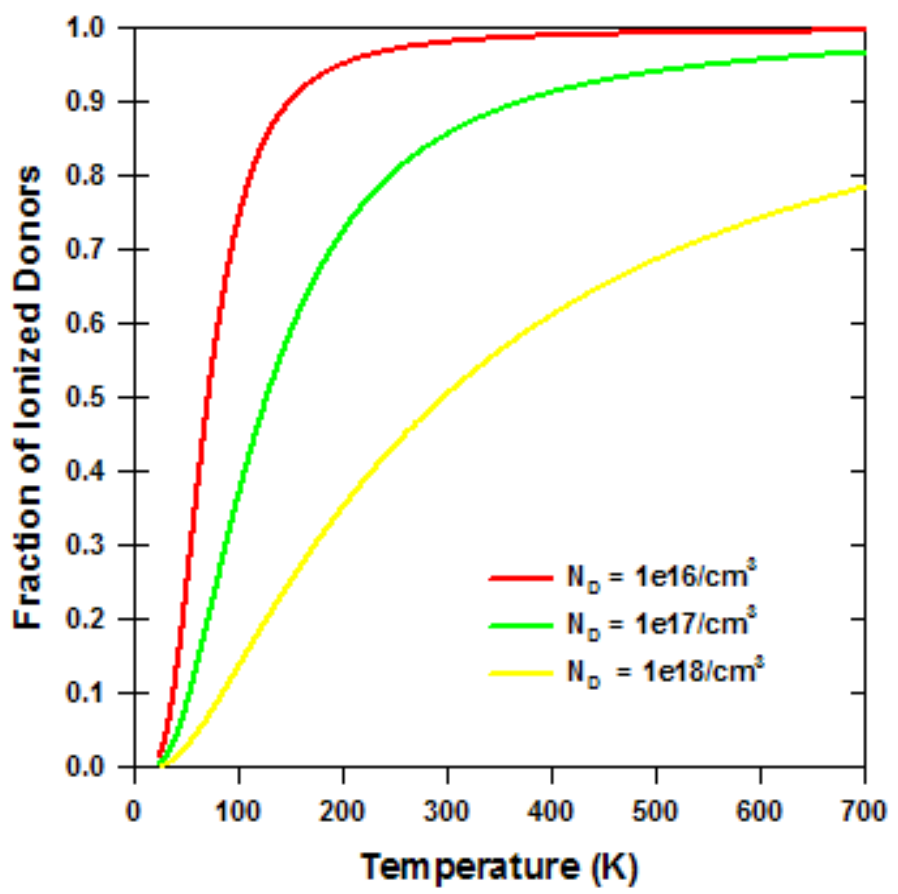

Figure 3.7. Fraction of ionized oxygen donors in $2 \mathrm{H}-\mathrm{GaN}$ for donor densities of $1 \mathrm{e} 16 / \mathrm{cm}^{3}$ and $1 \mathrm{e} 17 / \mathrm{cm}^{3}$

The fraction of ionized oxygen donors is $0.98,0.86$ and 0.50 at room temperature (300 ${ }^{\circ} \mathrm{K}$ ) for donor densities of $1 \mathrm{e} 16 / \mathrm{cm}^{3}, 1 \mathrm{e} 17 / \mathrm{cm}^{3}$ and $1 \mathrm{e} 18 / \mathrm{cm}^{3}$, respectively. It is clear from Figure 3.7 that the fraction of ionized donors decreases with increased donor density. The room temperature value of the fraction of ionized oxygen donors in $2 \mathrm{H}-\mathrm{GaN}$ for a donor density of $1 \mathrm{e} 19 / \mathrm{cm}^{3}$ is 0.20 . Incomplete ionization becomes a significant problem at higher doping densities, but is not significant for donor densities less than $1 \mathrm{e} 17 / \mathrm{cm}^{3}$ in $2 \mathrm{H}-\mathrm{GaN}$ at room temperature, or higher temperatures. The analytical result (equation (3.15) for the fraction of 
ionized donors cannot be applied to calculate the ionization of nitrogen donors in $4 \mathrm{H}$ and $6 \mathrm{H}-\mathrm{SiC}$ since the energy level of the nitrogen donors is different for cubic $(k)$ and hexagonal (h) lattice sites. We assume that the nitrogen donors are evenly split between the cubic and hexagonal lattice sites. The charge neutrality equation for nitrogen doped $4 \mathrm{H}-\mathrm{SiC}$ is written as

$$
n=\frac{N_{d}}{2\left(1+\frac{g_{d} n}{N_{c}} \exp \left(\frac{E_{c}-E_{d k}}{k T}\right)\right)}+\frac{N_{d}}{2\left(1+\frac{g_{d} n}{N_{c}} \exp \left(\frac{E_{c}-E_{d h}}{k T}\right)\right)}
$$

$E_{\mathrm{dk}}$ and $E_{\mathrm{dh}}$ are the nitrogen donor energies for the cubic and hexagonal lattice sites. After some manipulation, equation (3.16) can be rewritten as

$$
\begin{aligned}
& n\left(1+\frac{g_{d} n}{N_{c}} \exp \left(\frac{E_{c}-E_{d k}}{k T}\right)\right)\left(1+\frac{g_{d} n}{N_{c}} \exp \left(\frac{E_{c}-E_{d h}}{k T}\right)\right) \\
= & N_{d}\left(1+\frac{g_{d n}}{2 N_{c}}\left(\exp \left(\frac{E_{c}-E_{d k}}{k T}\right)+\exp \left(\frac{E_{c}-E_{d h}}{k T}\right)\right)\right)
\end{aligned}
$$

Equation (3.17) is solved numerically for the free electron density $n$. The values of $E_{\mathrm{dk}}$ and $\mathrm{E}_{\mathrm{dh}}$ are given in Table 3.6 as 0.42 and $0.84 \mathrm{eV}$ below the conduction band, respectively. The fraction of ionized donors is obtained by dividing the free electron density by the donor density $\mathrm{N}_{\mathrm{d}}$, since the free electron density is approximately equal to the number of ionized nitrogen donors. The fraction of ionized nitrogen donors in $4 \mathrm{H}-\mathrm{SiC}$ and $6 \mathrm{H}-\mathrm{SiC}$ for donor densities of $1 \mathrm{e} 16 / \mathrm{cm}^{3}$ and $1 \mathrm{e} 17 / \mathrm{cm}^{3}$ are shown in Figure 3.8. The fraction of ionized nitrogen donors in $6 \mathrm{H}-$ SiC was obtained by making modifications to equation (3.14). The modifications were based on two assumptions. First, the energy levels of the nitrogen donors at the two cubic lattice sites in 
$6 \mathrm{H}-\mathrm{SiC}$ could be lumped together since the energies were close in value $(0.137$ and $0.140 \mathrm{eV}$ below the conduction band). Second, it was also assumed that two-thirds and one-third of the nitrogen donors in $6 \mathrm{H}-\mathrm{SiC}$ resided at the cubic and hexagonal lattice sites, respectively. The plots

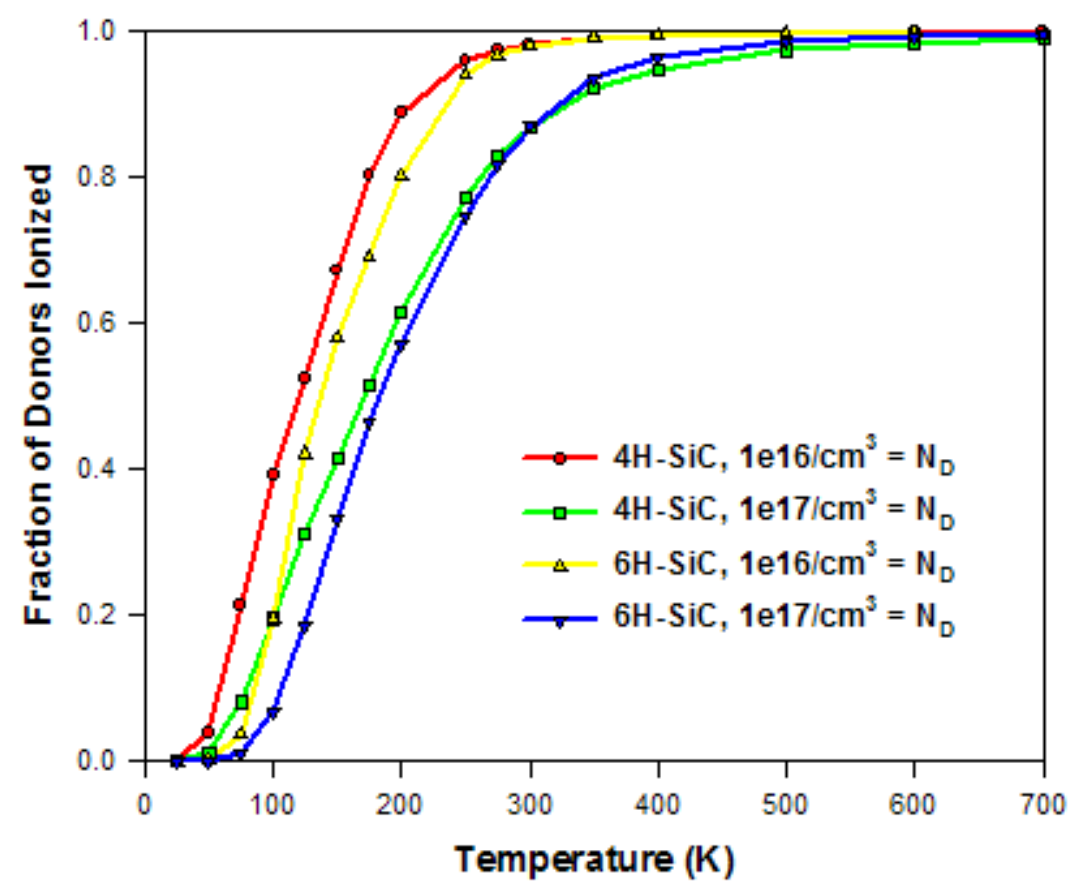

\section{Figure 3,8. Fraction of ionized nitrogen donors in $4 \mathrm{H}$ and $6 \mathrm{H}-\mathrm{SiC}$}

in Figure 3.8 show a decrease in the fraction of ionized nitrogen donors in $4 \mathrm{H}$ and $6 \mathrm{H}-\mathrm{SiC}$ with an increase in donor density similar to the oxygen donors in $2 \mathrm{H}-\mathrm{GaN}$ shown in Figure 3.7. The fraction of ionized nitrogen donors is higher in $4 \mathrm{H}-\mathrm{SiC}$ compared to $6 \mathrm{H}-\mathrm{SiC}$ for equal donor densities at temperatures less than room temperature $\left(300{ }^{\circ} \mathrm{K}\right)$. The fraction of ionized nitrogen donors in $4 \mathrm{H}$ and $6 \mathrm{H}-\mathrm{SiC}$ are approximately equal at temperatures equal to, or greater than, room temperature at equal donor densities. The room temperature values for the fraction of ionized nitrogen donor density are 0.98 and 0.87 for both $4 \mathrm{H}$ and $6 \mathrm{H}-\mathrm{SiC}$ at nitrogen densities of $1 \mathrm{e} 16 / \mathrm{cm}^{3}$ and $1 \mathrm{e} 17 / \mathrm{cm}^{3}$, respectively. Incomplete ionization of nitrogen donors in either $4 \mathrm{H}$, or, 
$6 \mathrm{H}-\mathrm{SiC}$ is not significant for nitrogen densities less than $1 \mathrm{e} 17 / \mathrm{cm}^{3}$ at temperatures greater than, or equal to, room temperature.

\subsection{2) Semi-insulating 4H, $6 \mathrm{H}-\mathrm{SiC}$ and $2 \mathrm{H}-\mathrm{GaN}$ by Compensation with Deep Acceptors}

The primary unintentional dopant in single crystal silicon carbide is nitrogen. Nitrogen, an n-type dopant in silicon carbide, is partially compensated by boron, an unintentional p-type dopant in silicon carbide. This partial compensation of the nitrogen donors in $4 \mathrm{H}$ and $6 \mathrm{H}-\mathrm{SiC}$ results in an n-type semiconductor with low resistivity. We require semi-insulating $4 \mathrm{H}$ and $6 \mathrm{H}-$ SiC substrates to construct high voltage photoconductive switches. Vanadium, a deep acceptor in $4 \mathrm{H}$ and $6 \mathrm{H}-\mathrm{SiC}$, is introduced into $4 \mathrm{H}$ and $6 \mathrm{H}-\mathrm{SiC}$ to compensate the excess nitrogen donors. The density of vanadium acceptor must be greater than the nitrogen density to achieve a semiinsulating $4 \mathrm{H}$, or, $6 \mathrm{H}-\mathrm{SiC}$ material. The following condition is true for the $4 \mathrm{H}$ and $6 \mathrm{H}-\mathrm{SiC}$ material from which we construct a high voltage, photoconducting switch.

$$
N_{d a}>N_{s d}>N_{s a}
$$

Where $\mathrm{N}_{\mathrm{da}}$ is the vanadium density, $\mathrm{N}_{\mathrm{sd}}$ is the nitrogen density and $\mathrm{N}_{\mathrm{sa}}$ is the boron density. The subscripts stand for deep acceptor (da), shallow donor (sd) and shallow acceptor (sa). The charge neutrality requirement mandates that the free holes and positively charged ionized impurities equal the free electrons and negatively charged ionized impurities.

$$
n+N_{s a}^{-}+N_{d a}^{-}=p+N_{s d}^{+}
$$

A semi-insulating material will have a low density of free carriers compared to ionized impurities. We initially assume that the free carrier densities in (3.19) can be neglected. 


$$
N_{s a}^{-}+N_{d a}^{-}=N_{s d}^{+}
$$

Let us apply equation (3.20) to the specific case of $6 \mathrm{H}-\mathrm{SiC}$ containing the impurities nitrogen (shallow donor), boron (shallow acceptor) and vanadium (deep acceptor). Using equations (3.9) and (3.10) we can rewrite equation (3.20) as equation (3.21). We expect the Fermi level to reside in the top half of the band gap since the energy levels of our two dominant impurities, the vanadium acceptor level and nitrogen donor level, are positioned there. As a result, the boron

$$
\frac{N_{s a}}{1+g_{s a} \exp ^{\left(\frac{E_{s a}-E_{f}}{k T}\right)}}+\frac{N_{d a}}{1+g_{d a} \exp ^{\left(\frac{E_{d a}-E_{f}}{k T}\right)}}=\frac{N_{s d}}{1+g_{s d} \exp ^{\left(\frac{E_{f}-E_{s d}}{k T}\right)}}
$$

acceptor level will be almost completely ionized. Equation (3.21) can then be rewritten

$$
N_{s a}+\frac{N_{d a}}{1+g_{d a} \exp ^{\left(\frac{E_{d a}-E_{f}}{k T}\right)}}=\frac{N_{s d}}{1+g_{s d} \exp ^{\left(\frac{E_{f}-E_{s d}}{k T}\right)}}
$$

Equation (3.22) must be solved for a given temperature to determine the location of the Fermi level. Once the Fermi level is obtained the free carrier density and resistivity can be obtained. We will look at the solution of (3.22) for the specific case of $6 \mathrm{H}-\mathrm{SiC}$ at room temperature. We will use $0.7 \mathrm{eV}$ below the conduction band for the vanadium acceptor level. An effective nitrogen donor level is calculated by summing the contributions from the two cubic ( 0.137 and $0.140 \mathrm{eV}$ below the conduction band) and one hexagonal ( $0.08 \mathrm{eV}$ below the conduction band) and matching it to the contribution from a single effective level $(0.1285 \mathrm{eV}$ below the conduction band). The effective nitrogen donor level was calculated to provide the best match to the sum of the three actual nitrogen donor levels at room temperature. This technique has been adopted from the literature [3.11] where it was used to calculate an effective nitrogen donor level for $4 \mathrm{H}-\mathrm{SiC}$. We assign the values of 2 and 4 to the degeneracy factors $\mathrm{g}_{\text {sd }}$ 
and $g_{\text {da. }}$ We assume a temperature of $300{ }^{\circ} \mathrm{K}$. We follow Zucca [3.10] and make the following definitions:

$$
\begin{gathered}
E_{0}=\frac{E_{d a}+E_{s d}}{2} \\
G=\exp ^{\frac{\left(E_{0}-E_{f}\right)}{k T}}
\end{gathered}
$$

We solve for the Fermi level by substituting equations (3.23) and (3.24) into equation (3.22). We obtain the quadratic equation for $G$ listed in equation (3.25). Solving for $G$ in equation

$0=G^{2}+G \frac{\left(\frac{N_{d a}}{N_{s d}}-1+\frac{N_{s a}}{N_{s d}}\left(1+g_{d a} g_{s d} \exp ^{\left.\left(\frac{E_{d a}-E_{s d}}{k T}\right)\right)}\right)\right.}{\left(\frac{N_{s a}}{N_{s d}}-1\right) g_{d a} \exp ^{\left(\frac{E_{d a}-E_{0}}{k T}\right)}}$

$+\frac{\left(\frac{N_{d a}}{N_{s d}}+\frac{N_{s a}}{N_{s d}}\right) g_{s d} \exp ^{\left(\frac{E_{0}-E_{s d}}{k T}\right)}}{\left(\frac{N_{s a}}{N_{s d}}-1\right) g_{d a} \exp ^{\left(\frac{E_{d a}-E_{0}}{k T}\right)}}$

(3.25) allows us to calculate the Fermi level, free carrier densities and material resistivity.

We make the following definitions to allow obtaining the solution of (3.25) as a function

$$
\begin{gathered}
D=\frac{N_{d a}}{N_{s d}} \\
R=\frac{N_{s a}}{N_{s d}}
\end{gathered}
$$

of the compensation ratio, $\mathrm{D}$, and the ratio of shallow acceptors to shallow donors, $\mathrm{R}$. We also use equations (3.5), (3.6) and (3.27) to obtain the Fermi level as a function of D and R.

$$
E_{f}=\left(\frac{E_{0}}{k T}-\ln (G)\right) k T
$$


The solution for $\mathrm{G}$ in equation (3.25) is plotted as a function of $\mathrm{D}$ for the cases of $\mathrm{R}$ equal $0,0.5$ and 0.9. in Figure 3.9.

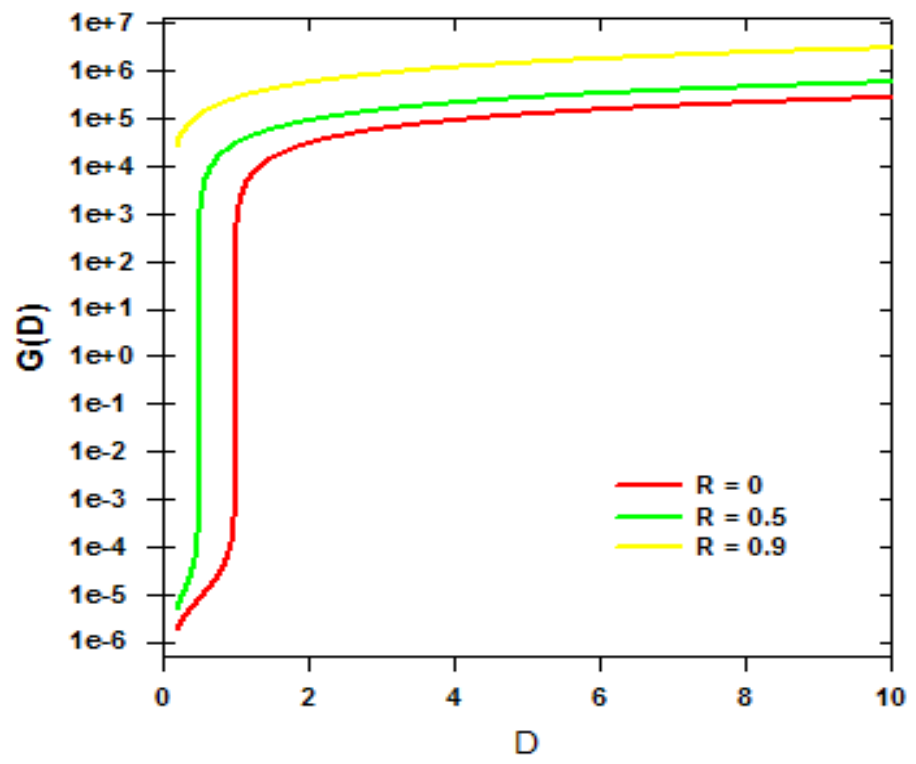

Figure 3.9. The variable $G$ as a function of $D$, the ratio of vanadium to nitrogen densities for $6 \mathrm{H}-\mathrm{SiC}$ at room temperature

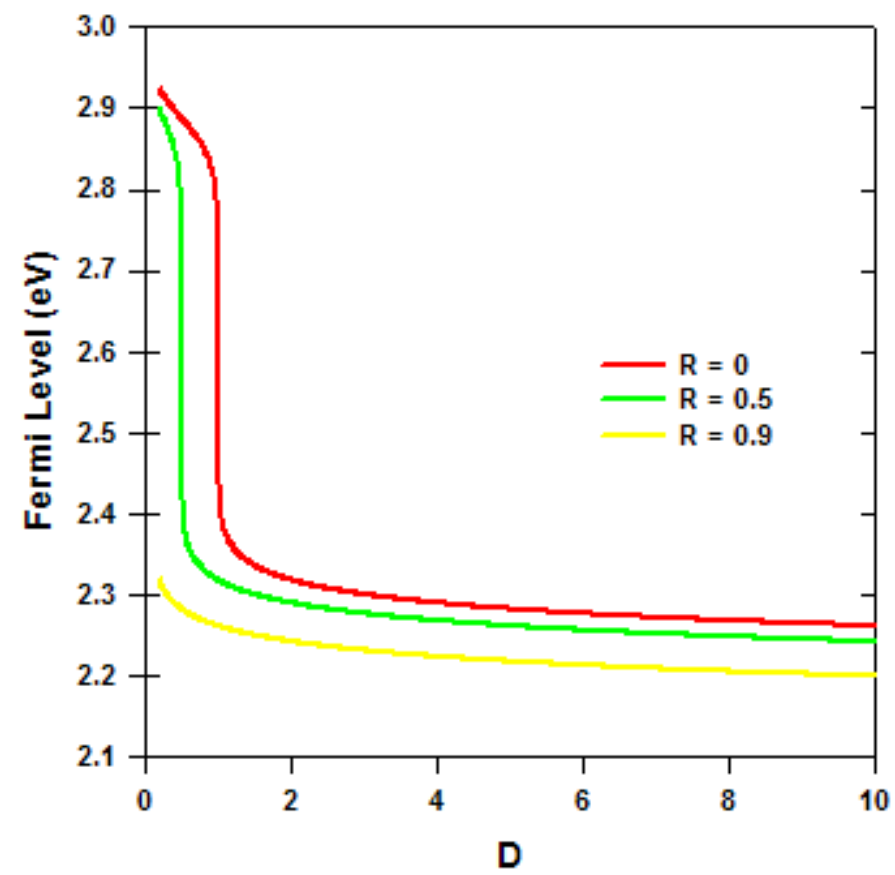

Figure 3.10. Fermi Level $\left(E_{f}\right)$ as a function of $D$ and $R$ for $6 H-S i C$ at room temperature 


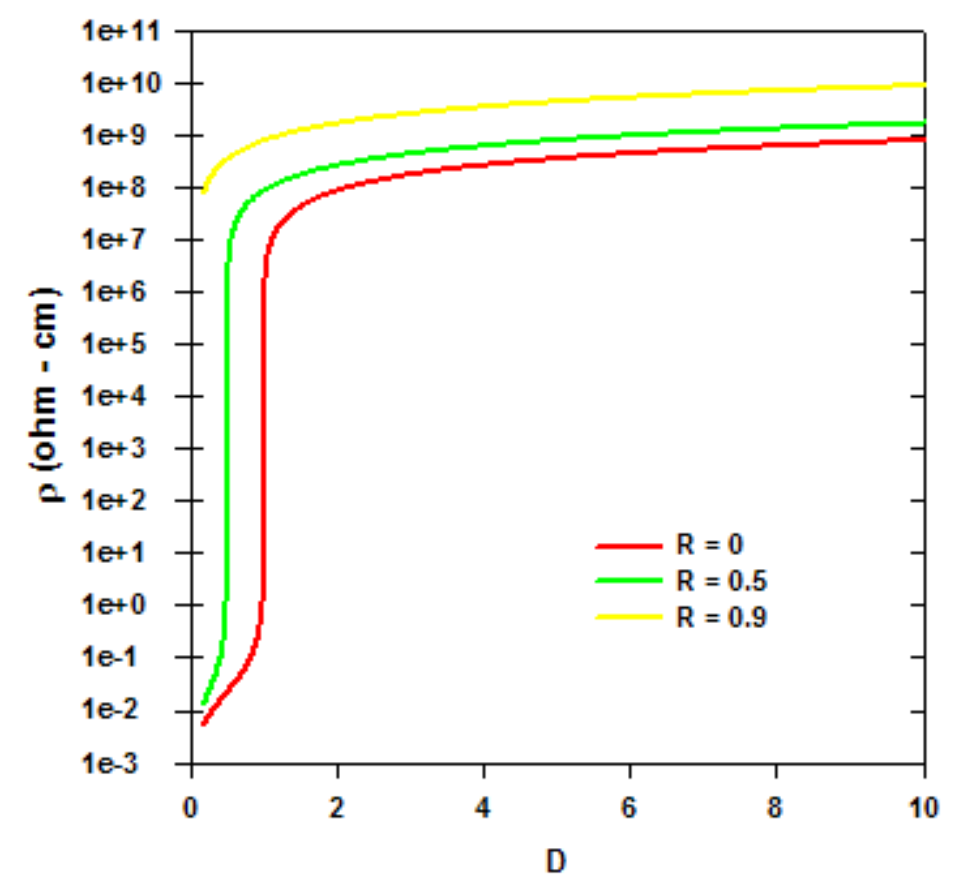

Figure 3.11. The calculated resistivity of $6 \mathrm{H}-\mathrm{SiC}$ at room temperature as a function of $\mathrm{D}$ and $\mathbf{R}$

Figure 3.10 is a plot of the Fermi level, $E_{f}$, as a function of $D$. $E_{f}$ is plotted in units of electron - Volts with the valence band maximum as reference. The Fermi level resides close to the nitrogen donor level in Figure 3.10 until the vanadium density exceeds that of nitrogen (D > 1) for the case of R equals to zero (red trace). The Fermi level then decreases rapidly and approaches that of the vanadium acceptor level when the vanadium density exceeds the nitrogen density. The Fermi level remains close to the vanadium acceptor level $(0.7 \mathrm{eV}$ below the conduction band) for all values of $\mathrm{D}$ greater than one. The Fermi level is pinned at the vanadium acceptor level. The green and yellow plots correspond to when the boron density is half $(\mathrm{R}=0.5)$ and ninety percent $(\mathrm{R}=0.9)$ that of nitrogen, respectively. The effect of the shallow boron acceptor is to reduce the effective number of nitrogen donors. It takes less vanadium to produce semi-insulating $6 \mathrm{H}-\mathrm{SiC}$ as $\mathrm{R}$ increases. That is, the Fermi level is pinned to the vanadium acceptor level a values of $\mathrm{D}$ less than unity. 
Figure 3.11 shows plots of resistivity of the compensated $6 \mathrm{H}-\mathrm{SiC}$ material in units of ohm - cm obtained by substituting the Fermi level results into equations (3.5) and (3.6) to obtain $\mathrm{p}$ and $\mathrm{n}$ as a function of $\mathrm{D}$ and $\mathrm{R}$. The resistivity is then calculated using equation (3.20) by substituting values of 253.5 and $88 \mathrm{~cm}^{2} / \mathrm{V}$-s for the electron $\left(\mu_{\mathrm{n}}\right)$ and hole $\left(\mu_{\mathrm{n}}\right)$ mobilities for $6 \mathrm{H}$ SiC. The electron and hole mobilities were calculated using the mobility models presented in section 3.4. The resistivity is low until the vanadium density approximately equals the nitrogen density $(D=1)$ then there is a rapid increase in resistivity with $D$. The resistivity increases more than ten orders of magnitude as D increases from slightly less than one to a value of two. The material achieves a resistivity of $1 \mathrm{e} 8 \mathrm{Ohm}-\mathrm{cm}$ at $\mathrm{D}$ greater than two. It is clear from these simple calculations that the vanadium is compensating the $6 \mathrm{H}-\mathrm{SiC}$ material by capturing the net

$$
\rho=\frac{1}{q\left(\mu_{n} n+\mu_{p} p\right)}
$$

excess electrons donated by the nitrogen levels. That is, the electrons that were not captured by the boron acceptors. We are interested in putting as many electrons as possible into the vanadium acceptor level, so that we can optically excite them into the conduction band. The semiinsulating $6 \mathrm{H}-\mathrm{SiC}$ substrate at thermal equilibrium serves as the initial condition of the photoconductive switch just prior to optical excitation. Ideally, we would like as little boron, or other shallow acceptors, in the $6 \mathrm{H}-\mathrm{SiC}$ material as possible. We would like to remove all impurities and defects, at energy levels deeper in the band gap, which capture electrons.

We can perform similar calculations for both $4 \mathrm{H}-\mathrm{SiC}$ and $2 \mathrm{H}-\mathrm{GaN}$, but our conclusions would be the same. That is, we would like to minimize the shallow acceptors in the switch 
materials to maximize the electrons in the vanadium acceptor levels in $4 \mathrm{H}$ and $6 \mathrm{H}-\mathrm{SiC}$ and iron acceptor level in 2H-GaN that can be optically switched with above band gap wavelengths. This way, we can achieve the highest possible material conductivity with longer wavelengths that penetrate more deeply into the material.

\section{4) Mobility Models for $4 \mathrm{H}-\mathrm{SiC}, 6 \mathrm{H}-\mathrm{SiC}$ and $2 \mathrm{H}-\mathrm{GaN}$}

The mobility, like the effective mass, for both $4 \mathrm{H}-\mathrm{SiC}$ and $6 \mathrm{H}-\mathrm{SiC}$ is anisotropic. The anisotropy is stronger in $6 \mathrm{H}-\mathrm{SiC}$ than $4 \mathrm{H}-\mathrm{SiC}$. Anisotropy in $2 \mathrm{H}-\mathrm{GaN}$ is expected, but has not yet been detected experimentally. As a practical matter, an electronic device fabricated from $4 \mathrm{H}-$ $\mathrm{SiC}, 6 \mathrm{H}-\mathrm{SiC}$, or, $2 \mathrm{H}-\mathrm{GaN}$ would be constructed to take advantage of the anisotropy of the material. For example, a device fabricated from $6 \mathrm{H}-\mathrm{SiC}$ would be designed so the drift current flows perpendicular to the c direction of the crystal to take advantage of the higher mobility in that direction. As a result, device mobility models are usually isotropic where either the mobility value perpendicular, or, parallel to the crystal c direction are used. Table 3.7 lists the mobility component values for $4 \mathrm{H}-\mathrm{SiC}, 6 \mathrm{H}-\mathrm{SiC}$ and $2 \mathrm{H}-\mathrm{GaN}$. The mobility, besides being anisotropic, is a function of doping concentration and electric field strength. Material modeling requires accurate values for the mobility that accounts for the effects of doping and field levels. The mobility models developed for $4 \mathrm{H}-\mathrm{SiC}$ and $6 \mathrm{H}-\mathrm{SiC}$ are based on empirical models originally developed for silicon. The mobility models have been modified by deriving fitting parameters from experimental results for $4 \mathrm{H}-\mathrm{SiC}[3.11,3.12], 6 \mathrm{H}-\mathrm{SiC}$ [3.11 - 3.13] and 2H-GaN [3.14, 3.15]. The effects of temperature, doping and electric field on mobility have been included. The models have assumed parabolic bands, which is not strictly true for the lowest conduction band in $6 \mathrm{H}-\mathrm{SiC}$. 


\begin{tabular}{|c|c|c|c|}
\hline Mobility & 4H-SiC & 6H-SiC & 2H-GaN \\
\hline$\mu_{\mathrm{n} \perp}$ & $947 \mathrm{~cm}^{2} / \mathrm{V}-\mathrm{s}$ & $415 \mathrm{~cm}^{2} / \mathrm{V}-\mathrm{s}$ & $1000 \mathrm{~cm}^{2} / \mathrm{V}-\mathrm{s}$ \\
\hline$\mu_{\mathrm{n}}$ & $1141 \mathrm{~cm}^{2} / \mathrm{V}-\mathrm{s}$ & $87 \mathrm{~cm}^{2} / \mathrm{V}-\mathrm{s}$ & $1000 \mathrm{~cm}^{2} / \mathrm{V}-\mathrm{s}$ \\
\hline$\mu_{\mathrm{p} \perp}$ & $120 \mathrm{~cm}^{2} / \mathrm{V}-\mathrm{s}$ & $80 \mathrm{~cm}^{2} / \mathrm{V}-\mathrm{s}$ & $30 \mathrm{~cm}^{2} / \mathrm{V}-\mathrm{s}$ \\
\hline$\mu_{\mathrm{p}}$ & $120 \mathrm{~cm}^{2} / \mathrm{V}-\mathrm{s}$ & $80 \mathrm{~cm}^{2} / \mathrm{V}-\mathrm{s}$ & $30 \mathrm{~cm}^{2} / \mathrm{V}-\mathrm{s}$ \\
\hline
\end{tabular}

Table 3.7. Mobility components for $4 \mathrm{H}-\mathrm{SiC}, 6 \mathrm{H}-\mathrm{SiC}$ and $2 \mathrm{H}-\mathrm{GaN}$ [3.11 - 3.15]

The Caughey - Thomas equation [3.11] is used to model the low field electron and hole drift mobility of $4 \mathrm{H}-\mathrm{SiC}$ and $6 \mathrm{H}-\mathrm{SiC}$ including the effects of doping concentration.

$$
\mu_{n, p}=\mu_{n, p}^{\min }\left(\frac{T}{300 K}\right)^{\alpha_{n, p}}+\frac{\left(\mu_{n, p}^{\max }-\mu_{n, p}^{\min }\right)}{1+\left(\frac{N_{d}+N_{a}}{N_{n, p, r e f}}\right)^{\gamma_{n, p}}}\left(\frac{T}{300 K}\right)^{\alpha_{n, p}}
$$

Where $\mu_{n, p}^{\min }$, $\mu_{n, p}{ }^{\max }, N_{\text {ref }}, \alpha_{n, p}$ and $\gamma_{n, p}$ are fitting parameters. $N_{d}$ and $N_{a}$ are the total donor and acceptor doping concentrations. The values for the fitting parameters are listed in Table 3.8. A similar model has been developed for the electron mobility in $2 \mathrm{H}-\mathrm{GaN}$ it is listed in equation (3.30) [3.15]. Temperature dependence is included by using the general relationship shown in equation (3.31) [3.15]. The fitting parameters for 2H-GaN are listed in Table 3.9.

$$
\begin{gathered}
\mu_{n}=\mu_{n}^{\min }+\frac{\left(\mu_{n}^{\max }-\mu_{n}^{\min }\right)}{1+\left(\frac{N_{d}+N_{a}}{N_{n r e f}}\right)^{\alpha}} \\
\operatorname{Parameter}(T)=\operatorname{Parameter}(R T)\left(\frac{T}{300^{\circ} K}\right)^{\alpha_{\text {parameter }}}
\end{gathered}
$$




\begin{tabular}{|c|c|c|}
\hline Parameter & 4H-SiC & 6H-SiC \\
\hline$\mu_{n} \perp$ min & 0 & 20 \\
\hline$\mu_{n} \|^{\min }$ & 0 & 20 \\
\hline$\mu_{n} \perp \max$ & 950 & 415 \\
\hline$\mu_{\mathrm{n}} \|^{\max }$ & 1141 & 87 \\
\hline$\mu_{\mathrm{p}}^{\max }$ & 124 & 99 \\
\hline$\mu_{\mathrm{p}}^{\min }$ & 16 & 6.8 \\
\hline$\alpha_{n}$ & -2.15 & -3.0 \\
\hline$\alpha_{p}$ & -2.15 & -3.0 \\
\hline$\gamma_{n}$ & 0.61 & 0.45 \\
\hline$\gamma_{p}$ & 0.34 & 0.5 \\
\hline $\mathbf{N}_{\mathrm{p}, \text { ref }}$ & $1.76 \mathrm{e} 19$ & $1.0 \mathrm{e} 19$ \\
\hline $\mathbf{N}_{\mathbf{n}, \text { ref }}$ & $1.94 \mathrm{e} 17$ & $4.5 \mathrm{e} 17$ \\
\hline
\end{tabular}

Table 3.8. Mobility model fitting parameters for $4 \mathrm{H}-\mathrm{SiC}$ and $6 \mathrm{H}-\mathrm{SiC}$ [3.11 - 3.13]

\begin{tabular}{|c|c|c|c|c|c|c|c|}
\hline$\mu_{\mathbf{n}}{ }^{\text {min }}$ & $\mu_{\mathbf{n}} \perp^{\mathbf{m a x}}$ & $\mathbf{N}_{\mathbf{n}, \mathbf{r e f}}$ & $\boldsymbol{\gamma}$ & $\boldsymbol{\alpha}_{\boldsymbol{\mu m a x}}$ & $\boldsymbol{\alpha}_{\mu \min }$ & $\boldsymbol{\alpha}_{\mathbf{n r e f}}$ & $\boldsymbol{\alpha}_{\boldsymbol{\gamma}}$ \\
\hline 80 & 1405 & $7.78 \mathrm{e} 16$ & 0.71 & -2.85 & -0.20 & 1.3 & 0.31 \\
\hline
\end{tabular}

\section{Table 3.9. Electron mobility model fitting parameters for $2 \mathrm{H}-\mathrm{GaN}$ [3.14 - 3.15]}

Mobility is also a function of electric field. Again, the basic empirical model for high field mobility developed for silicon has been applied to $4 \mathrm{H}-\mathrm{SiC}$ and $6 \mathrm{H}-\mathrm{SiC}$. The high field mobility model for $4 \mathrm{H}-\mathrm{SiC}$ and $6 \mathrm{H}-\mathrm{SiC}$ is listed in equation (3.32). The mobility $\mu_{\mathrm{n}, \mathrm{p}, \mathrm{low}}$ in equation (3.32) corresponds to the low - field mobility calculated using equation (3.29). Table 3.10 shows the high field mobility fitting parameters for $4 \mathrm{H}-\mathrm{SiC}$ and $6 \mathrm{H}-\mathrm{SiC}$.

$$
\mu_{n, p}=\frac{\mu_{n, p, \text { low }}}{\left(1+\left(\frac{E \mu_{n, p, \text { low }}}{\mathrm{v}_{\text {sat }}}\right)^{\beta}\right)^{\frac{1}{\beta}}}
$$




\begin{tabular}{|c|c|c|}
\hline Parameter & 4H-SiC & 6H-SiC \\
\hline $\boldsymbol{\beta}$ & 0.816 & 0.95 \\
\hline $\mathbf{v}_{\text {sat }}$ & $4.77 \mathrm{e} 7 \mathrm{~cm} / \mathrm{s}$ & $4.60 \mathrm{e} 7 \mathrm{~cm} / \mathrm{s}$ \\
\hline
\end{tabular}

Table3.10. High field mobility fitting parameters for $4 \mathrm{H}-\mathrm{SiC}$ and $6 \mathrm{H}-\mathrm{SiC}$ [3.13]

The mobility for compensated, semi-insulating semiconductors is usually much lower than the mobility for uncompensated semiconductors. This is a result of the addition of impurities that form deep levels in the compensated semiconductors in addition to the impurities that form shallow acceptor and donor levels. The compensating impurities increase the scattering of carriers in the material decreasing the mobility. The mobility of vanadium compensated $4 \mathrm{H}-$ SiC and iron compensated $2 \mathrm{H}-\mathrm{GaN}$ have been measured by the temperature dependent Hall effect method to be 200 and $410 \mathrm{~cm}^{2} / \mathrm{V}$-s, respectively.

\section{5) Recombination}

If a semiconductor in equilibrium is perturbed from equilibrium by the injection of carriers on either side of a p-n junction, or, electron hole pairs are optically generated in the volume of the semiconductor, recombination processes will try to restore the semiconductor to equilibrium. Recombination processes can be radiative, or, nonradiative.

\subsection{1) Radiative Recombination}

Radiative recombination is illustrated in Figure 3.12. A free electron in the conduction band recombines directly with a hole in the valence band and emits a photon with energy equal to the band gap. This process is important for direct band gap materials. The radiative recombination for a direct band to band recombination will be proportional both the electron and hole densities [3.16, 3.20, 3.21] as shown in equation (3.32). Here, $B_{r}$ is the radiative 
recombination coefficient and $\Delta \mathrm{n}(\Delta \mathrm{p})$ and $\mathrm{n}_{0}\left(\mathrm{p}_{0}\right)$ are the excess and equilibrium electron (hole) densities, respectively.

$$
R_{\text {rad }}=B_{r} n p=B_{r}\left(n_{0}+\Delta n\right)\left(p_{0}+\Delta p\right)
$$

For the case of high level injection ( $\Delta \mathrm{n}=\Delta \mathrm{p}$ » $\mathrm{n}_{0}, \mathrm{p}_{0}$ ), equation (3.32) can be rewritten as

$$
R_{\text {rad }}=B_{r} \Delta n\left(n_{0}+p_{0}+\Delta n\right)
$$

The radiative recombination rate and recombination time $\left(\mathrm{t}_{\mathrm{rad}}\right)$ are given by equations (3.34) and (3.35), respectively. The radiative recombination time changes with excess carrier

\section{Radiative Recombination}

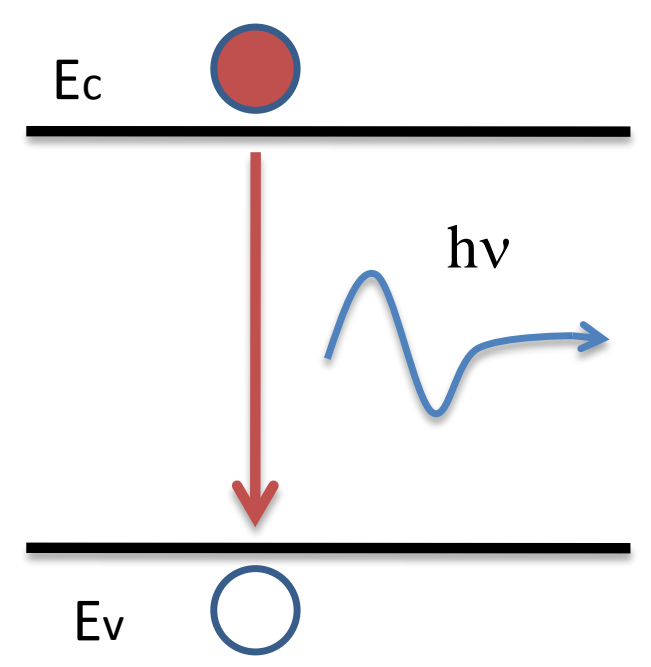

Figure 3.12. Radiative recombination

density $(\Delta \mathrm{n})$ for the high injection limit. Radiative recombination is more likely to occur for direct band gap materials such as GaAs and GaN. However, radiative recombination is unimportant for indirect band gap materials like silicon, $4 \mathrm{H}-\mathrm{SiC}$ and $6 \mathrm{H}-\mathrm{SiC}$.

$$
\begin{aligned}
& R_{\text {rad }}=\frac{\Delta n}{t_{\text {rad }}} \\
& t_{\text {rad }} \cong \frac{1}{B_{r} \Delta n}
\end{aligned}
$$




\subsection{2) Auger and Shockley-Read-Hall (SRH) Recombination}

Important nonradiative recombination processes are Auger and Shockley-Read-Hall (SRH) recombination. The Auger recombination process is illustrated in Figure 3.13. An electron and hole recombine and the energy liberated is transferred to a third carrier that can be either an electron, or, hole. Auger recombination is the inverse of the impact ionization process. The Auger recombination should be proportional to the product of the densities of the participating carriers as written in equation (3.36) [3.17, 3.21].

$$
R=\gamma_{n} n^{2} p+\gamma_{p} p^{2} n
$$

Where $\gamma_{n}$ and $\gamma_{p}$ are the Auger coefficients for electron - electron - hole (eeh) and hole - hole electron (hhe) recombination processes. The Auger recombination should balance the impact ionization at thermal equilibrium when no external generation of carriers is present.

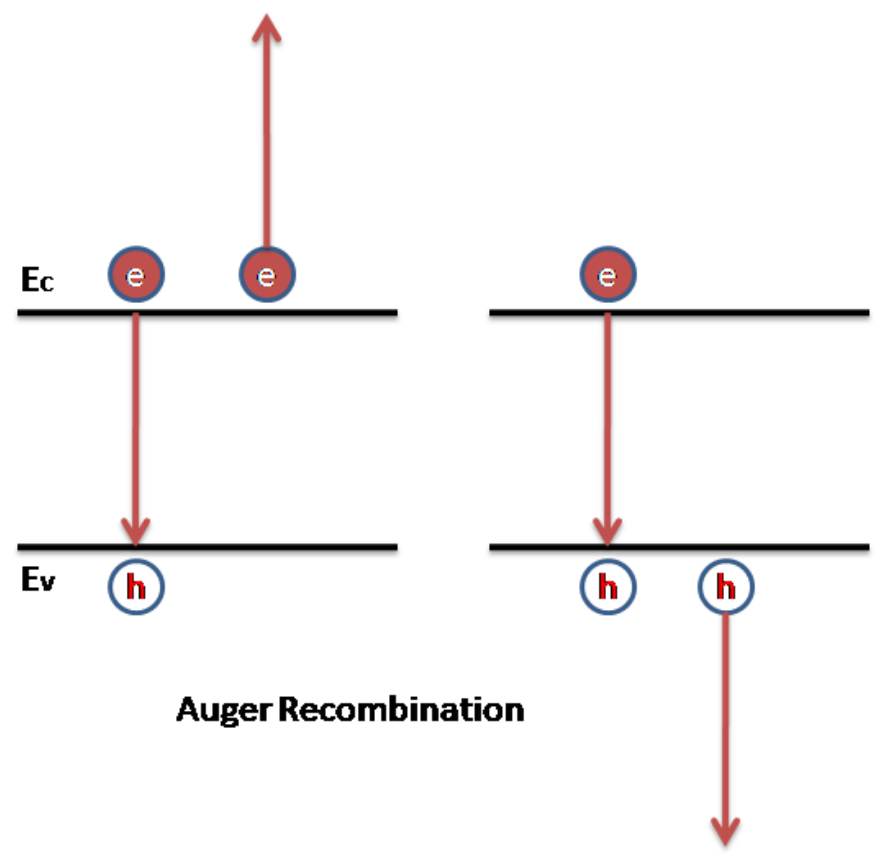

Figure 3.13. Auger Recombination: e-e-h and h-h-e processes 
The balance between Auger recombination and impact ionization at thermal equilibrium is written in equation (3.37). Here the electron (hole) Auger recombination terms must cancel the electron (hole) impact ionization terms. We combine equations (3.37) and (3.38) and obtain equation (3.39).

$$
\begin{gathered}
\beta_{n} n-\gamma_{n} n^{2} p+\beta_{p} p-\gamma_{p} p^{2} n=0 \\
p n=n_{i}^{2} \\
\beta_{n}=\gamma_{n} n_{i}^{2} \\
\beta_{p}=\gamma_{p} n_{i}^{2}
\end{gathered}
$$

Where $\beta_{\mathrm{n}, \mathrm{p}}$ is the impact ionization coefficients by electrons and holes, respectively. The carrier loss due to Auger recombination is obtained by substituting equation (3.39) into (3.37).

$$
\frac{\Delta n}{t_{\text {auger }}}=\gamma_{n} n^{2} p-\gamma_{n} n_{0}^{2} p_{0}+\gamma_{p} p^{2} n-\gamma_{p} n_{0} p_{0}^{2}
$$

Where $n=\Delta n+n_{0} \quad$ and $\quad p=\Delta p+p_{0}$

The Auger lifetime ( $\mathrm{t}_{\text {auger }}$ ) is given by equation (3.41). Equation (3.41) reduces to (3.42) for the case of strong optical pumping of nonequilibrium carriers [3.17]. It is clear from equation (3.41) that Auger recombination is unimportant at low carrier densities. However, Auger recombination becomes the dominant nonradiative recombination mechanism at high carrier, or, high doping densities. The Auger recombination coefficients are physical constants that vary with semiconductor material and temperature. The Auger recombination coefficients at room temperature for $4 \mathrm{H}-\mathrm{SiC}, 6 \mathrm{H}-\mathrm{SiC}$ and $2 \mathrm{H}-\mathrm{GaN}$ are listed in Table 3.11 [3.18 - 3.19].

$$
t_{\text {auger }}=\frac{\Delta n}{\gamma_{n}\left(n^{2} p-n_{0}^{2} p_{0}\right)+\gamma_{p}\left(p^{2} n-p_{0}^{2} n_{0}\right)}
$$




$$
t_{\text {auger }}=\frac{1}{\Delta n^{2}\left(\gamma_{n}+\gamma_{p}\right)}
$$

\begin{tabular}{|c|l|l|l|}
\hline Auger Coefficient $\left(\mathrm{cm}^{6} / \mathrm{s}\right)$ & $4 \mathrm{H}-\mathrm{SiC}$ & $6 \mathrm{H}-\mathrm{SiC}$ & $2 \mathrm{H}-\mathrm{GaN}$ \\
\hline$\gamma_{\mathbf{n}}$ & $5.0 \times 10^{-31}$ & & $1.25 \times 10^{-30}$ \\
\hline$\gamma_{\mathbf{p}}$ & $2.0 \times 10^{-31}$ & & $1.25 \times 10^{-30}$ \\
\hline$\gamma_{\mathbf{p}}$ & $7.0 \times 10^{-31}$ & & $2.50 \times 10^{-30}$ \\
\hline
\end{tabular}

Table 3.11. Room temperature Auger recombination coefficients for $4 \mathrm{H}, 6 \mathrm{H}-\mathrm{SIC}$ and $2 \mathrm{H}$ GaN [3.18 - 3.19]

Shockley-Read- Hall (SRH) recombination is illustrated in Figure 3.14. SRH recombination is the dominant recombination mechanism in indirect band gap semiconductors at low and moderate carrier densities. Electron-hole recombination is facilitated by a single deep trap located at an energy $E_{t}$ below the conduction band. The deep trap can be an impurity, or, defect. SRH recombination is a two-step process whereby an electron (hole) is captured at the trap located at $E_{t}$, then a hole (electron) is captured. In this process, level $E_{t}$ is referred to as a recombination center. The level $E_{t}$ is called a trap when the captured electron (hole) is emitted from the trap before a hole (electron) is captured. Traps will be discussed in more detail below. Electron - hole annihilation does not necessarily occur immediately after the capture of a hole at a recombination center filled with an electron. The possibility exists for an electron - hole pair to be bound to the recombination level. This is called a bound exciton [3.17]. However, this possibility is ignored when generating the SRH recombination time. The SRH recombination time is given in equation (3.43) [3.22]. The energy of the recombining electron - hole pair is released in the form of phonons. 


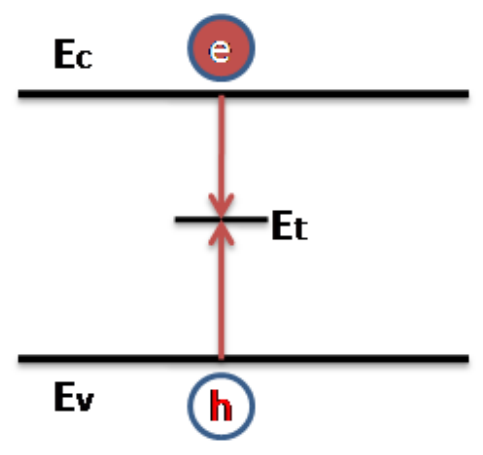

Shockley-Read-Hall Recombination

Figure 3.14. Shockley-Read-Hall recombination

$$
\tau_{S R H}=\frac{\tau_{p 0}\left(n+n_{1}\right)+\tau_{n 0}\left(p+p_{1}\right)}{\left(n p-n_{i}^{2}\right) /\left(n-n_{0}\right)}
$$

Where $\mathrm{n}=\Delta \mathrm{n}+\mathrm{n}_{0}, \mathrm{p}=\Delta \mathrm{p}+\mathrm{p}_{0}$ and

$$
\begin{gathered}
\tau_{p 0}=\frac{1}{\left(\sigma_{p} v_{t h} N_{T}\right)} \\
\tau_{n 0}=\frac{1}{\left(\sigma_{n} v_{t h} N_{T}\right)} \\
n_{1}=n_{i} \exp \left(-\frac{E_{i}-E_{t}}{k T}\right) \\
p_{1}=n_{i} \exp \left(-\frac{E_{t}-E_{i}}{k T}\right)
\end{gathered}
$$

Where $v_{\mathrm{th}}, \mathrm{N}_{\mathrm{T}}, \mathrm{E}_{\mathrm{t}}, \mathrm{E}_{\mathrm{i}}$ and $\sigma_{\mathrm{n}, \mathrm{p}}$ are the carrier thermal velocity, defect density, defect energy level below the conduction band, the intrinsic energy level and electron and hole capture cross section at the defect, respectively. The SRH recombination time, $\tau_{\mathrm{SRH}}$, is minimized when $\mathrm{E}_{\mathrm{t}}$ equals $\mathrm{E}_{\mathrm{i}}$. 
The most efficient recombination centers have energy $E_{t}$ located close to the middle of the band gap. The SRH recombination time is inversely proportional to the electron and hole capture cross sections and the density of traps, $\mathrm{N}_{\mathrm{T}}$. The SRH recombination time for the case of high level injection $\left(\Delta \mathrm{n}=\Delta \mathrm{p}>>\mathrm{n}_{0}, \mathrm{p}_{0}\right)$ is given in (3.48).

$$
\tau_{S R H}=\tau_{p 0}+\tau_{n 0}
$$

All three recombination mechanisms can occur in a semiconductor simultaneously. In general, the carrier recombination time in the bulk material is given by (3.49).

$$
\frac{1}{\tau_{\text {rec }}}=\frac{1}{\tau_{\text {rad }}}+\frac{1}{\tau_{\text {auger }}}+\frac{1}{\tau_{S R H}}
$$

\subsection{3) Traps}

Defects and impurities in semiconductors can act as recombination centers, or traps. A defect, or impurity acts as an electron (hole) trap if, after capturing an electron (hole), the trap is more likely to thermally emit the electron (hole) into the conduction (valence) band than capture a hole (electron). The SRH recombination time was developed based on the assumption of a single recombination center and equal densities of the electron and hole excess carriers $(\Delta n=$ $\Delta \mathrm{p})$. There are numerous defects and impurities present in $4 \mathrm{H}-\mathrm{SiC}, 6 \mathrm{H}-\mathrm{SiC}$ and $2 \mathrm{H}-\mathrm{GaN}$ that provide a broad range of energy levels that can act as either recombination centers, or traps, depending on their location in the band gap. A material with a large number of recombination centers will have a short recombination time. Electron and hole traps, on the other hand, will lengthen the recombination time. Also, the electron and hole capture cross sections at each 
trap/recombination center can have different values leading to the condition that the excess electron and hole densities may not be equal. The quasi-Fermi level approach [3.23 - 3.25] is used to envision the most probable behavior of traps based on their position in the band gap. We assume that carriers have been photo-generated in the volume of a semiconductor containing a large number of traps with energy levels distributed throughout the band gap. The Fermi level has shifted from its position at thermal equilibrium as a result of low level optical excitation of carriers. The quasi-Fermi levels for electrons and holes ( $E_{f n}$ and $E_{f p}$ ) in a nondegenerate semiconductor are the Fermi levels consistent with the free electron and hole densities resulting from the illumination [3.22]. The mathematical definition of the quasi-Fermi levels are listed in equations (3.50) and (3.51).

$$
\begin{aligned}
& n=\Delta n+n_{0}=N_{c} \exp \left(-\frac{\left|E_{f n}-E_{c}\right|}{k T}\right) \\
& p=\Delta p+p_{0}=N_{v} \exp \left(-\frac{\left|E_{f p}-E_{v}\right|}{k T}\right)
\end{aligned}
$$

The defect and impurity levels lying between the quasi-Fermi levels and the band edges will most probably act as traps due to their higher probability of thermal emission into the conduction and valence band. The defect and impurity levels lying between the electron and hole quasi-Fermi levels will most probably act as recombination centers. A quantitative approach to mark the boundary between trap and recombination centers in the band gap is the concept of demarcation levels [3.24, 3.25]. We consider a level, $\mathrm{E}_{\mathrm{T}}$, that has already captured an electron and determine, $\mathrm{k}_{\mathrm{n}}$, the ratio of the level hole capture rate (recombination) to the rate that the level thermally emits the electron back into the conduction band.

$$
k_{n}=\frac{\gamma_{p} p}{\gamma_{n} N_{c} \exp \left(-\left(\frac{E_{c}-E_{T}}{k T}\right)\right)}
$$


Where $\gamma_{p}$ is the hole capture coefficient for an occupied trap and $\gamma_{n}$ is the electron capture coefficient for a free trap. The center located at energy level $\mathrm{E}_{\mathrm{T}}$ will most likely be a recombination center (electron trap) if the value of $k_{n}$ is greater (less) than unity. Similarly for a hole-filled level at $\mathrm{E}_{\mathrm{D}}$, $\mathrm{k}_{\mathrm{p}}$ is the ratio of the level electron capture rate (recombination) to thermal emission rate of holes to the valence band.

$$
k_{p}=\frac{\gamma_{n} n}{\gamma_{p} N_{v} \exp \left(-\left(\frac{E_{D}-E_{v}}{k T}\right)\right)}
$$

The values of $E_{T}$ and $E_{D}$ that cause $k_{n}$ and $k_{p}$ to be equal to one are called the electron and hole demarcation levels, $\mathrm{E}_{\mathrm{Dn}}$ and $\mathrm{E}_{\mathrm{Dp}}$, respectively. The quasi-Fermi and demarcation levels are shown in Figure 3.15. The demarcation levels divide the band gap into regions of electron trapping near the conduction band, hole trapping near the conduction band and the center region where the traps act as recombination centers for both electrons and holes. If the optical

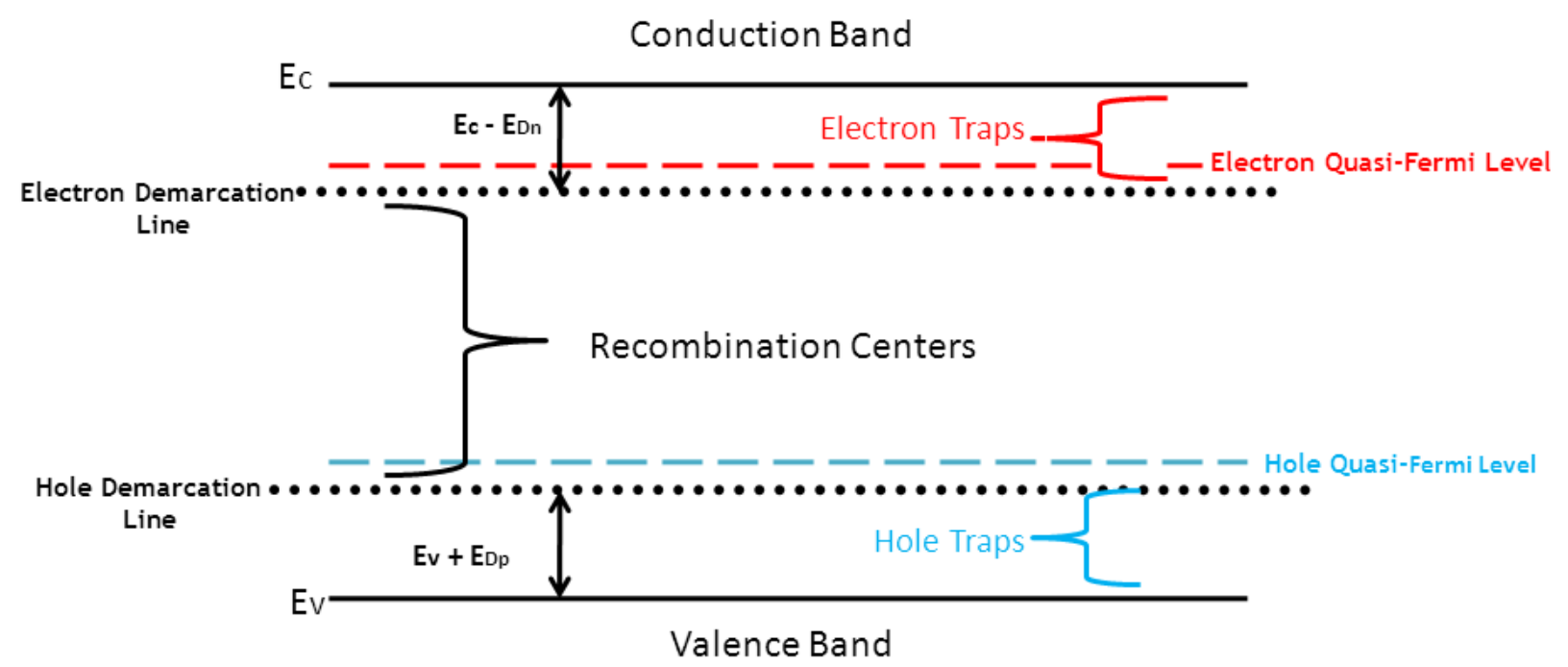

Figure 3.15 Quasi-Fermi and demarcation levels in band gap of a semiconductor with numerous traps 
excitation is increased, the electron and hole quasi-Fermi levels and demarcation lines will move closer to their respective band edges. The motion of the quasi-Fermi and demarcation levels towards their respective band edges will change some electron and hole traps into recombination centers. The effect of the motion of the quasi-Fermi and demarcation level can be compounded by the distribution of the trap levels in the band gap. There can be a rapid decrease in recombination time as the quasi-Fermi and demarcation levels sweep through a volume of the band gap with a high density of traps. The ultimate effect can be a sub-linear dependence of photoconductivity on optical intensity that has been observed in amorphous semiconductors [3.23].

All traps will act as recombination centers under sufficiently high optical excitation conditions. That is, the electron and hole quasi-Fermi levels move into the conduction and valence band edges. We can write a set of coupled rate equations for free carriers (equations 3.54 - 3.56) which includes the effects of a single trap density, $\mathrm{N}_{\mathrm{T}}$, which may act as a recombination center, or, an electron trap [3.26]. We also assume that the remaining traps act as recombination centers which may be modeled using a single electron recombination time $t_{\text {Reff. }}$.

$$
\begin{gathered}
\frac{\partial n}{\partial t}=G_{n}(t)-\frac{n}{t_{R e f f}}+\gamma_{T n} n_{T} N_{c T}-\gamma_{T n} n\left(N_{T}-n_{T}\right) \\
\frac{\partial n_{T}}{\partial t}=\gamma_{T n} n\left(N_{T}-n_{T}\right)-\gamma_{T n} n_{T} N_{c T}-\gamma_{T p} p n_{T}+\gamma_{T p}\left(N_{T}-n_{T}\right) N_{v T} \\
\frac{\partial p}{\partial t}=G_{p}(t)+\gamma_{T p}\left(N_{T}-n_{T}\right) N_{v T}-\gamma_{T p} p n_{T}
\end{gathered}
$$

Where $n(p)$ is the free electron (hole) density, $t_{\text {Reff }}$ is the effective electron recombination time, $N_{T}$ is the trap density, $n_{T}$ is the density of $N_{T}$ traps containing electrons and $G_{n}(t)\left(G_{p}(t)\right)$ is the electron (hole) generation rate. $\gamma_{\mathrm{Tn}}$ and $\gamma_{\mathrm{Tp}}$ are the electron and hole recombination coefficients, 
which are related to the electron and hole capture cross sections as shown below. $\mathrm{N}_{\mathrm{VT}}\left(\mathrm{N}_{\mathrm{CT}}\right)$ is the valence (conduction) band density of states reduced to the trap level $\mathrm{E}_{\mathrm{T}}$.

$$
\begin{gathered}
\gamma_{T n}=\sigma_{T n}\left\langle v_{n}\right\rangle \\
\gamma_{T p}=\sigma_{T n}\left\langle v_{p}\right\rangle
\end{gathered}
$$

Where $\sigma_{\mathrm{Tn}}\left(\sigma_{\mathrm{TP}}\right)$ is the electron (hole) capture cross section of the defect at energy level $\mathrm{E}_{\mathrm{T}}$.

$$
\begin{gathered}
N_{v T}=N_{v} \exp \left(-\left(\frac{E_{T}-E_{v}}{k T}\right)\right) \\
N_{c T}=N_{c} \exp \left(-\left(\frac{E_{c}-E_{T}}{k T}\right)\right)
\end{gathered}
$$

The third and fourth terms on the right hand side (RHS) of equation (3.54) are the free electron capture and trapped electron emission at level $\mathrm{E}_{\mathrm{T}}$, respectively. The four terms on the RHS of equation (3.55) are free electron capture, trapped electron emission, free hole capture by an electron filled level and hole emission into the valence band at level $\mathrm{E}_{\mathrm{T}}$, respectively. The second and third terms on the RHS of equation (3.56) are hole emission into the valence band from level at $\mathrm{E}_{\mathrm{T}}$ and hole capture by an electron filled level at level $\mathrm{E}_{\mathrm{T}}$ (e-h annihilation).

The recombination time will be reduced to the result shown in equation (3.61) if the $\mathrm{N}_{\mathrm{T}}$ levels act like recombination centers. That is, electron emission from $\mathrm{N}_{\mathrm{T}}$ into conduction band is negligible in equation (3.54). We should expect this result to eventually occur if the generation of excess carriers is increased until the electron quasi-Fermi level is moved into the conduction band.

$$
\frac{1}{t_{r}}=\frac{1}{t_{R e f f}}+\gamma_{T n}\left(N_{T}-n_{T}\right)
$$

However, there is another possibility. We have not assumed that $\mathrm{N}_{\mathrm{T}}$ and $\mathrm{n}_{\mathrm{T}}$ are much larger than the doping density and excess carrier density to guarantee equations (3.54 - 3.56) 
remain linear. It is possible to saturate the $\mathrm{N}_{\mathrm{T}}$ minus $\mathrm{n}_{\mathrm{T}}$ terms if the excess carrier generation rate is large enough. We would expect the recombination time to decrease for this case.

We have reviewed the electronic properties of $2 \mathrm{H}-\mathrm{GaN}, 4 \mathrm{H}-\mathrm{SiC}$ and $6 \mathrm{H}-\mathrm{SiC}$ along with compensation, recombination and trapping because these properties all effect the operation of extrinsic photoconductive switches. The electronic properties determine the carrier transport of the material during photoconduction. The compensating impurities provide high resistance in the dark and carriers when illuminated with the proper optical wavelength. Recombination and trapping determine the material carrier recombination time and, in part, switching efficiency.

Optical properties of the photoconductive switch material are also important in determining the operation of an extrinsic photoconductive switch. The optical properties of $2 \mathrm{H}-$ $\mathrm{GaN}, 4 \mathrm{H}-\mathrm{SiC}$ and $6 \mathrm{H}-\mathrm{SiC}$ are reviewed in detail in Chapter 4.

\section{6) References}

3.1) O. Madelung, Semiconductors: Data Handbook, Third Edition, page 60, Springer, 1998

3.2) W. Y. Ching et al, "The electronic structure and spectroscopic properties of 3C, $2 \mathrm{H}, 4 \mathrm{H}$, 6H, 15R, and 21R polymorphs of SiC” Material Science and Engineering A, Vol. 422, pp $147-156,2006$

3.3) C. Persson and U. Lindefelt, "Relativistic band structure calculation of cubic and hexagonal SiC polytypes”, Journal of Applied Physics, Vol. 82, No. 11, 1997

3.4) G. Pensl, et al., "SiC Material Properties", International Journal of High Speed Electronics and Systems, Vol. 15, No. 4, pp. 705 - 745, 2005

3.5) M. E. Levinshtein, S. L. Rumyantsev and M. S. Shur, Properties of Advanced Semiconductor Materials, pages 1 and 93, Wiley - Interscience, 2001

3.6) B. K. Meyer et al., " Optically detected cyclotron resonance investigations on $4 \mathrm{H}$ and $6 \mathrm{H}-$ SiC: Band-structure and transport properties”, Physical Review B, Vol. 61, No. 7, pp. $4844-4849,2000$

3.7) N. T. Son et al., Cyclotron Resonance Studies of Effective Masses and Band Structure in $\mathrm{SiC}$, in Silicon Carbide: Recent Major Advances, W. Choyke, H. Matsunami and G. Pensl, Editors, Springer, 2004

3.8) W. J. Choyke, R. P. Devaty and S. G. Sridhara, "A survey of conduction and valence band edges in SiC”, Physica Scripta, Vol. T79, 9 - 15, 1999

3.9) J. S. Blakemore, Semiconductor Statistics, pp. 117 - 130, Dover Edition, 2000 
3.10) R. Zucca, “Compensation mechanism in GaAs”, Journal of Applied Physics, Vol. , No. , pp. , 1986

3.11) M. Bakowsi, U. Gustafsson and U. Lindefelt, "Simulation of SiC high power devices", Physica Status Solidi (A), Vol. 162, p 421, 1997

3.12) M. Roschke and F. Schwierz, "Electron mobility models for $4 \mathrm{H}, 6 \mathrm{H}$ and $3 \mathrm{C}$ SiC”, IEEE Transactions on Electron Devices, Vol. 48, No. 7, 2001

3.13) M. Ruff, H. Mitlehner and R. Helbig, “ SiC devices: Physics and numerical simulation”, IEEE Transactions on Electron Devices, Vol. 41, No. 6, June 1994

3.14) T. Mnatsakanov et al., "Carrier mobility model for GaN”, Solid State Electronics, Vol. 47, pp. $111-115,2003$

3.15) F. Schwierz, “An electron mobility model for wurtzite GaN”, Solid State Electronics, Vol. 49, pp. 889 - 895, 2005

3.16) P. Bhattacharya, Semiconductor Optoelectronic Devices, page 118, Prentice-Hall, 1994

3.17) V. Abakumov, V. Perel and I. Yassievich, Nonradiative Recombination in Semiconductors, pp. 22 - 30, North-Holland, 1991

3.18) A. Galeckas, et al., "Evolution of Auger recombination rate in 4H-SiC", Materials Science Forum, Volumes 264 - 268, Part 1, pp. 533 - 536, 1997

3.19) S. Onoda, et al., "Impact of Auger recombination on charge collection of a 6H-SiC diode by heavy ions”, IEEE Transactions on Nuclear Science, Vol. 54, no. 6, pp. 2706 - 2713, December, 2004

3.20) E. Rosencher and B. Vinter, Optoelectronics, pp. 261 - 266, Cambridge, 2002

3.21) D. Schroder, Semiconductor Material and Device Characterization, pp. 422 - 426, Wiley Interscience, 1998

3.22) S. Sze and N. Ng, Physics of Semiconductor Devices, page 43, Wiley Interscience, 2007

3.23) R. S. Crandall, Photoconductivity, pp. 252 - 263, Semiconductors and Semimetals, Volume 21, Hydrogenated Amorphous Silicon, Part B: Optical Properties, Jacques Pankove, Editor, Academic Press, 1984

3.24) A. Rose, Concepts in Photoconductivity and Allied Problems, pp. 26 - 33, WileyInterscience, 1963

3.25) S. M. Ryvkin, Photoelectric Effect in Semiconductors, page 123, Consultants Bureau, 1964

3.26) N. V. Joshi, Photoconductivity: Art, Science, and Technology, Chapter 3 - Transient Photoconductivity, Marcel Dekker, 1990 


\section{Chapter 4: Below Bandgap Optical Properties of 2H-GaN, $4 \mathrm{H}$ and 6H-SiC}

\section{1) Introduction}

There are a number of publications reviewing the optical properties of $4 \mathrm{H}-\mathrm{SiC}$ and $6 \mathrm{H}$ $\mathrm{SiC}$ [4.1 - 4.6]. Much of the published material is directed at optical properties near, and above, the band edge. Investigations of the below bandgap optical properties have focused on the photoluminescence properties of crystal defects and impurities [4.7, 4.8] and below bandgap absorption in nitrogen doped $4 \mathrm{H}$ and $6 \mathrm{H}-\mathrm{SiC}[4.9,4.10]$. Most of the published information concerning the optical properties of $2 \mathrm{H}-\mathrm{GaN}, 4 \mathrm{H}$ and $6 \mathrm{H}-\mathrm{SiC}$ is not applicable to the below bandgap photoconductive response of compensated, semi-insulating substrates of these wide bandgap semiconductors. The optical properties we need to consider for a clear understanding of below bandgap photoconduction in semi-insulating $2 \mathrm{H}-\mathrm{GaN}, 4 \mathrm{H}$ and $6 \mathrm{H}-\mathrm{SiC}$ can be obtained by examining the relationship governing the photoconductive switch resistance in equation 4.1. This is the switch on resistance equation (2.11) from Chapter 2 for an optical pulse temporal width much greater than the carrier recombination time $\left(\tau_{\mathrm{r}}\right)$. The optical properties contained in

$$
R(t)=\frac{d^{2} \hbar \omega}{P(t) \tau_{r} \mu_{n}(1-r)\left(1-e^{-\alpha W}\right) q \eta}
$$

equation 4.1 are the applied optical power $(\mathrm{P}(\mathrm{t}))$, photon energy $(\hbar \omega)$, optical transmission through the switch facet (1-r), power reflectance (r), optical power absorbed in the substrate after propagation of a distance $\mathrm{W}\left(1-\mathrm{e}^{-\alpha \mathrm{W}}\right)$, and the quantum efficiency of the switch material $(\eta)$. We define the quantum efficiency as the number of carriers generated per photon transmitted into the switch substrate. We determine the power level and photon energy of the applied optical pulse. The optical transmission, optical power absorbed and quantum efficiency are properties of the material. The photoconductive switch substrate optical properties that we need to consider are 
the index of refraction, optical transitions from impurity levels to the conduction, or valence band, two step optical transitions of electrons from the valence band to the conduction band and two-photon absorption. However, we will begin with a review of above bandgap absorption and the general optical properties of uniaxial crystals.

\section{2) Above Bandgap Optical Absorption in 2H-GaN, 4H and 6H-SiC}

We have discussed in previous chapters the existence of deep acceptor levels that lie at energies between the valence and conduction bands in the wide bandgap materials $2 \mathrm{H}-\mathrm{GaN}, 4 \mathrm{H}$ and 6H-SiC. These deep acceptor levels are sometimes used to compensate the wide bandgap materials by capturing electrons donated by shallow donor levels. The captured electrons can be excited into the conduction band by using below bandgap $\left(E_{g}>\hbar \omega\right)$ light. The main advantage of using below bandgap light to excite carriers from deep levels in the wide bandgap semiconductors is the ability of below bandgap light to penetrate much deeper into the wide bandgap material generating carriers in a large volume of the material. In general, below bandgap light will penetrate millimeters to centimeters into the wide bandgap semiconductor compared to a penetration depth of a few tens of microns for above bandgap light. This is a result of the relatively small density of deep levels occupied by electrons $\left(\leq 1 \times 10^{17} \mathrm{~cm}^{-3}\right)$ that can be excited into the conduction band by below bandgap light, compared to the density of electrons in the valence band $\left(10^{22} \mathrm{~cm}^{-3}\right)$ that can be excited into the conduction band by above bandgap light. In addition, the two photon absorption and two step optical transition coefficients for below bandgap photons with energy greater than $\mathrm{E}_{\mathrm{g}} / 2$ are small in comparison with absorption for photons with energy greater than $\mathrm{E}_{\mathrm{g}}$.

Figures 4.1 and 4.2 show the room temperature (RT) absorption [4.3, 4.13] and penetration depth in $4 \mathrm{H}$ and $6 \mathrm{H}-\mathrm{SiC}$ as a function of wavelength for the electric field vector of 
the optical pulse aligned perpendicular to the c axis of the crystal $(\mathbf{E} \perp \mathbf{c})$. The wavelengths shown in Figures 4.1 and 4.2 correspond to photon energies greater than the RT value of the $6 \mathrm{H}-\mathrm{SiC}$ $(3.0 \mathrm{eV}, 413 \mathrm{~nm})$ and 4H-SiC $(3.26 \mathrm{eV}, 380 \mathrm{~nm})$ bandgap. Figure 4.3 and 4.4 [4.14, 4.15] plot the optical absorption and penetration depth of unpolarized light in $2 \mathrm{H}-\mathrm{GaN}$ for wavelengths less than $350 \mathrm{~nm}$. Subsequently, the penetration depths are shallow for $6 \mathrm{H}-\mathrm{SiC}(\leq 11 \mu \mathrm{m}), 4 \mathrm{H}-\mathrm{SiC}(\leq$ $50 \mu \mathrm{m})$ and $2 \mathrm{H}-\mathrm{GaN}(\leq 100 \mathrm{~nm})$. Photogenerated carriers would be created in a thin region at the surface of the $4 \mathrm{H}, 6 \mathrm{H}-\mathrm{SiC}$ and $2 \mathrm{H}-\mathrm{GaN}$ material at wavelengths less than $350-400 \mathrm{~nm}$. This would necessitate the placement of the switch anode and cathode electrodes on the same side of the switch substrate, while illuminating large substrate surface areas to achieve a low switch on resistance. Exciting the $4 \mathrm{H}, 6 \mathrm{H}-\mathrm{SiC}$ and $2 \mathrm{H}-\mathrm{GaN}$ substrates with below band gap light not only creates carriers in a much larger volume of the material, but also allows the placement of the anode and cathode electrodes on opposing sides of the substrate. This enables us to take advantage of the high critical electric field strengths of the $2 \mathrm{H}-\mathrm{GaN}, 4 \mathrm{H}$ and $6 \mathrm{H}-\mathrm{SiC}$ materials, allowing fabrication of high voltage, compact photoconductive switches.

Comparison of Figures 4.2 and 4.4 shows that the penetration depth of $350 \mathrm{~nm}$ wavelength light is 100 (350) times shallower in $2 \mathrm{H}-\mathrm{GaN}$ compared to $6 \mathrm{H}-\mathrm{SiC}$ (4H-SiC) despite the wider bandgap of $2 \mathrm{H}-\mathrm{GaN}(3.4 \mathrm{eV}$ at $\mathrm{RT}$ ). This is a result of $2 \mathrm{H}-\mathrm{GaN}$ having a direct bandgap compared to the indirect bandgaps of $4 \mathrm{H}$ and $6 \mathrm{H}-\mathrm{SiC}$. 


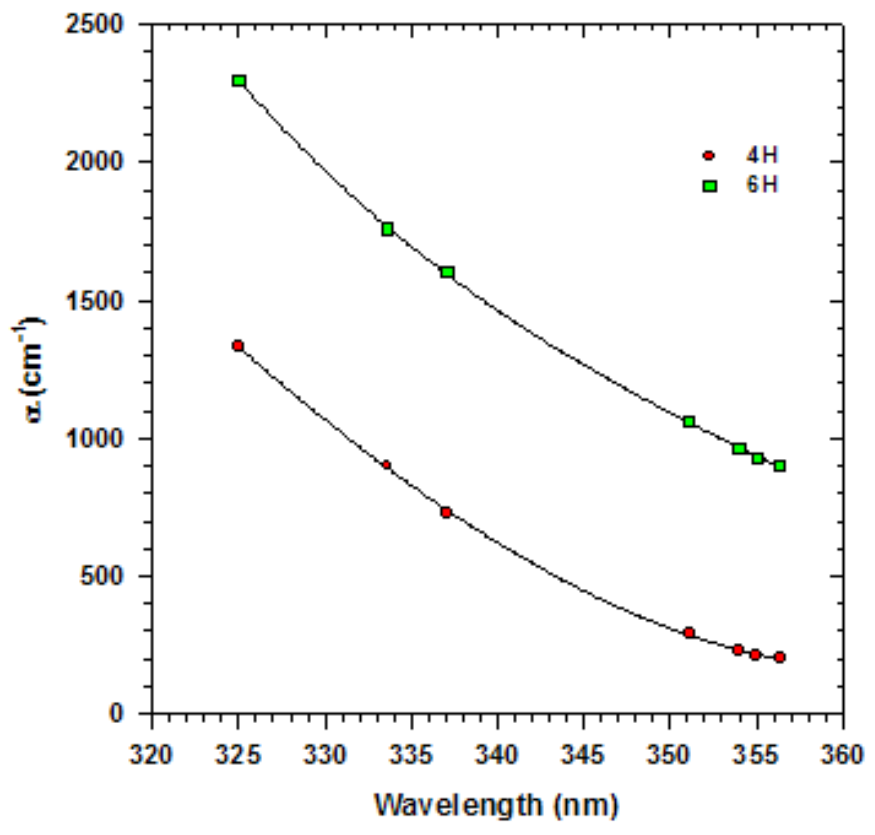

Figure 4.1. Linear optical absorption in $4 \mathrm{H}$ and $6 \mathrm{H}$-SiC for above bandgap wavelengths [4.3, 4.13].

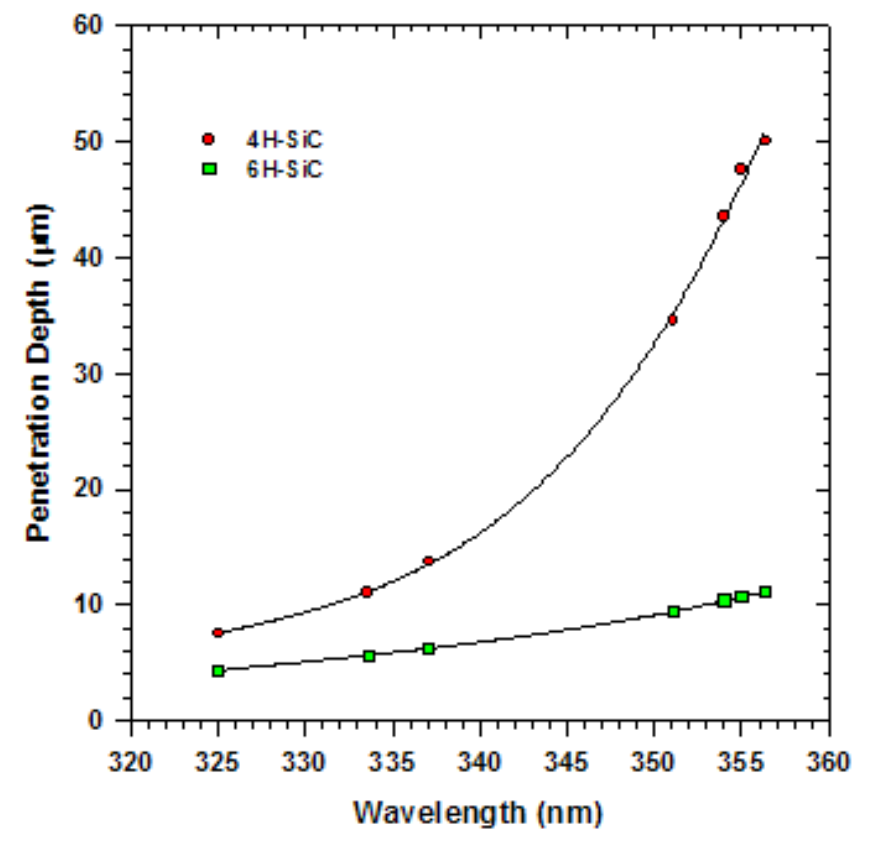

Figure 4.2. Optical penetration depth (1/ $\alpha)$ of above bandgap wavelengths in $4 \mathrm{H}$ and $6 \mathrm{H}-$ SiC. 


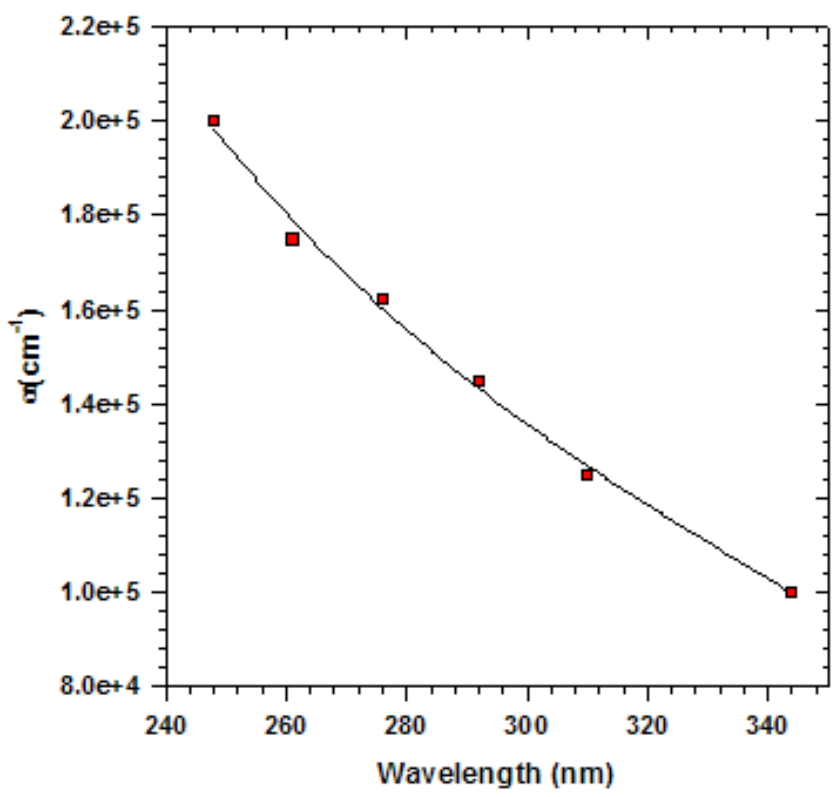

Figure 4.3. Linear optical absorption in $2 \mathrm{H}-\mathrm{GaN}$ for above bandgap wavelengths [4.14, 4.15]

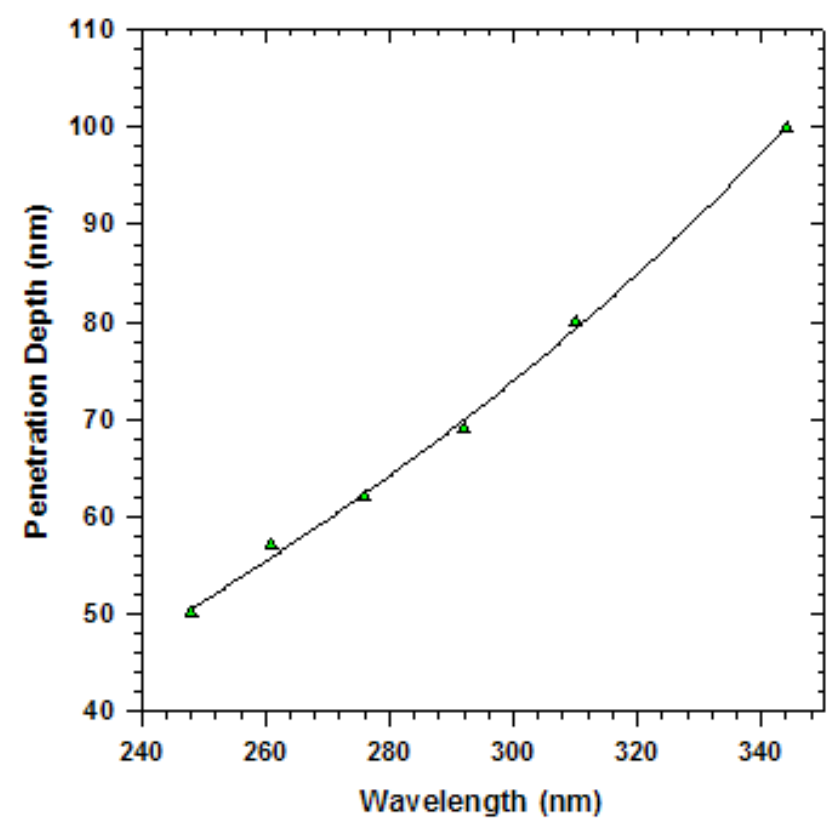

Figure 4.4. Optical penetration depth in $2 \mathrm{H}-\mathrm{GaN}$ plotted versus wavelength. 


\section{3) General Optical Properties of Uniaxial Crystals}

The electronic properties of $2 \mathrm{H}-\mathrm{GaN}, 4 \mathrm{H}$ and $6 \mathrm{H}-\mathrm{SiC}$ differ depending on the orientation of the current density ( $\mathbf{J})$ and electric field (E) with respect to the c axis in the crystal, as we have discussed in Chapter 3. The optical properties of $2 \mathrm{H}-\mathrm{GaN}, 4 \mathrm{H}$ and $6 \mathrm{H}-\mathrm{SiC}$ are also anisotropic. The anisotropy of these materials is due to their hexagonal crystal structure. This is a result of the different spacing and arrangement of atoms along the different axes of the crystal. All three of these materials are classified as uniaxial materials. The dielectric constant $(\varepsilon)$ of these materials depends on the orientation of the electric field (E) with respect to the optical axis of the material. The optical axis of a hexagonal crystal is the c direction (Figure 4.5). The general relationship between the electric displacement (D) and the electric field (E) for uniaxial materials is given in equation 4.2. We have chosen the optical axis of the material to lie along the $\mathrm{z}$ direction. Equation 4.2 can be rewritten as equation 4.3 for an optically transparent material. In equation $4.3, n_{0}$ and $n_{e}$ are the ordinary and extraordinary index of refraction, respectively. The ordinary (extraordinary) index of refraction is the index that a propagating electromagnetic wave "sees” if its electric field is polarized perpendicular (parallel) to the c axis of the $2 \mathrm{H}-\mathrm{GaN}, 4 \mathrm{H}$ and 6H-SiC crystal. Positive (negative) uniaxial materials have $n_{e}>n_{0}\left(n_{0}>n_{e}\right)$. Unpolarized light propagating through uniaxial material will separate into two light rays, the ordinary and extraordinary rays, which are orthogonal to each other, refracted at different angles, propagate at different speeds through the material, and will exit the uniaxial material displaced from one another. This phenomenon is called double refraction [4.11] and is shown in Figure 4.6. A material that has double refractive indices is said to be birefringent [4.12]. 


$$
\begin{aligned}
& {\left[\begin{array}{l}
D_{x} \\
D_{y} \\
D_{z}
\end{array}\right]=\varepsilon_{0}\left[\begin{array}{ccc}
\varepsilon_{1} & 0 & 0 \\
0 & \varepsilon_{1} & 0 \\
0 & 0 & \varepsilon_{2}
\end{array}\right]\left[\begin{array}{l}
E_{x} \\
E_{y} \\
E_{z}
\end{array}\right]} \\
& {\left[\begin{array}{l}
D_{x} \\
D_{y} \\
D_{z}
\end{array}\right]=\varepsilon_{0}\left[\begin{array}{ccc}
n_{0}^{2} & 0 & 0 \\
0 & n_{0}^{2} & 0 \\
0 & 0 & n_{e}^{2}
\end{array}\right]\left[\begin{array}{l}
E_{x} \\
E_{y} \\
E_{z}
\end{array}\right]}
\end{aligned}
$$

The refractive index determines how light is reflected and transmitted at a material boundary. The reflection coefficient of a material with refractive index $n$, and extinction coefficient $\kappa$, for normal incidence of an electromagnetic wave at the air - material interface is given in equation 4.4. The transmission coefficient at the air - material interface is given in 4.5. The extinction coefficient is the complex component of the wave vector and is related to the optical absorption, $\alpha$, of the material as shown in equation 4.6. The light that is transmitted into a uniaxial material is a function of refractive index, extinction coefficient, wavelength and how the light is polarized with respect to the optical axis of the material.

$$
\begin{gathered}
R=\frac{(n-1)^{2}+\kappa^{2}}{(n+1)^{2}+\kappa^{2}} \\
T=1-R \\
\alpha=\frac{4 \pi \kappa}{\lambda}
\end{gathered}
$$




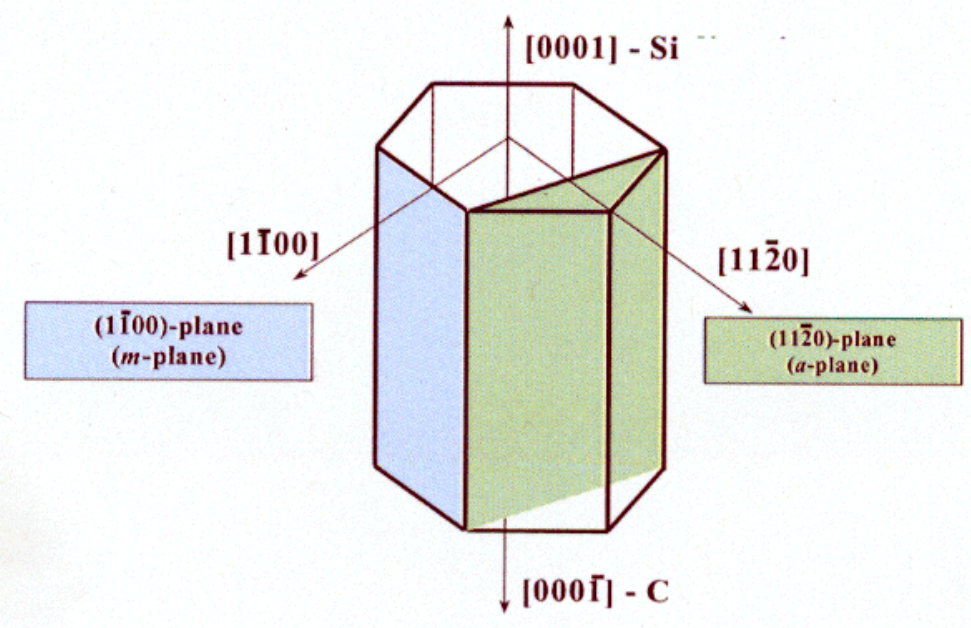

Figure 4.5. Crystal axes for $4 \mathrm{H}$ and $6 \mathrm{H}$-SiC showing the a-plane, m-plane and [0001] vector (c direction) perpendicular to the $c$ plane. The $\mathrm{z}$-axis of equations $X .1$ and $X .2$ has been chosen to lie parallel to the c direction of the crystal.

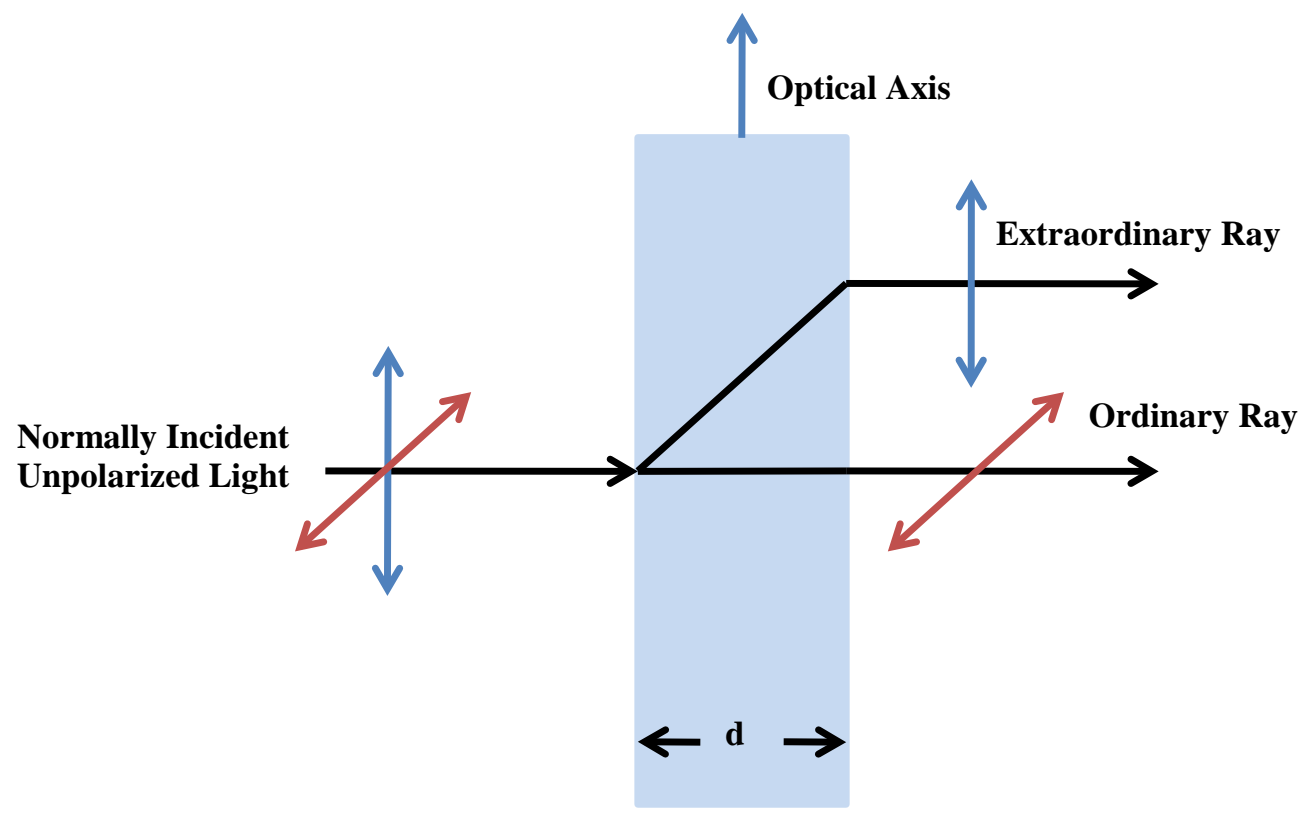

Uniaxial Crystal

Figure 4.6. Double refraction of unpolarized light in a uniaxial crystal 


\section{4) Refractive Index of $2 \mathrm{H}-\mathrm{GaN}, 4 \mathrm{H}-\mathrm{SiC}$ and $6 \mathrm{H}-\mathrm{SiC}$}

The refractive index is important to us since it determines how much light will be transmitted through the photoconductive switch facet into the switching material. The light transmitted into the switch volume will be given by equations 4.4 and 4.5. Measurements have been made of the refractive index for $2 \mathrm{H}-\mathrm{GaN}, 4 \mathrm{H}$ and $6 \mathrm{H}-\mathrm{SiC}$ [4.16 - 4.21]. The refractive index was determined from optical transmission and reflection measurements through thin films and small crystal platelets of undoped material. However, we are interested in constructing photoconductive switches from material that has been doped to increase the generation of carriers via optical transitions in the wide bandgap materials at below bandgap wavelengths. The doping will increase absorption and extinction coefficient at below bandgap wavelengths. However, the increased optical absorption caused by the doping is expected be small enough to allow optical penetration depths of several millimeters to a centimeter. This is a result of expected optical excitation cross sections of $\sim 1 \times 10^{-16} \mathrm{~cm}^{-2}$ and electron occupied deep level densities of $1 \times 10^{16}$ to $1 \times 10^{17} \mathrm{~cm}^{-3}$ [4.22]. As a result, we expect the absorption coefficient $(\alpha)$ to be $10 \mathrm{~cm}^{-1}$, or less. The extinction coefficient is $8.47 \times 10^{-5}$ for an absorption coefficient $(\alpha)$ of 10 at a wavelength of $1064 \mathrm{~nm}$. The extinction coefficient will be negligible compared to the refractive index for the wavelengths and doping levels in which we are interested. The doped silicon carbide and gallium nitride substrates can be treated as transparent materials due to the low extinction coefficient.

The refractive index of $6 \mathrm{H}-\mathrm{SiC}$ is shown in Figure 4.7 [4.16 - 4.19]. Measurements of

both $n_{0}(\mathbf{E} \perp \mathbf{c})$ and $n_{e}(\mathbf{E} \| \mathbf{c})$ are plotted. We can see from the measurements that $6 \mathrm{H}$-SiC is a positive uniaxial material $\left(n_{e}>n_{0}\right)$. The refractive index $n_{e}$ was measured for visible light for the 
range of wavelengths 467 - $691 \mathrm{~nm}[4.16,4.17]$ and $n_{0}$ was measured from ultraviolet [4.18] to visible and infrared wavelengths $[4.16,4.18]$. We show only the refractive index measurements for band edge and below bandgap wavelengths in Figure 4.7. The 6H-SiC ordinary index $\left(\mathrm{n}_{\mathrm{o}}\right)$ is 2.57 at a wavelength of $1250 \mathrm{~nm}$ and slowly increases until the visible wavelengths are reached, where the increase in refractive index with decreasing wavelength becomes rapid. The value of $\mathrm{n}_{\mathrm{o}}$ reaches 2.76 at the band edge $(\lambda \sim 400 \mathrm{~nm})$ and continues to increase for shorter wavelengths. The extraordinary index $\left(n_{e}\right)$ is plotted for the visible wavelengths. The value of $n_{e}$ is 2.66 at a wavelength of $690 \mathrm{~nm}$ and increases to 2.78 at a wavelength of $436 \mathrm{~nm}$. The slope of the increase of $n_{e}$ is almost identical to that $n_{0}$ with $n_{e}$ having a value $\sim 2 \%$ larger than that of $n_{0}$. The $4 \mathrm{H}-\mathrm{SiC}$ ordinary and extraordinary refractive index are plotted as functions of wavelength in Figure 4.8 [4.19]. The plots in Figure 4.8 are for $4 \mathrm{H}-\mathrm{SiC}$ thin film transmission data that have been fitted to a Sellmeier dispersion relationship of the form shown in equation 4.7 [4.19], where the $\mathrm{i}$ subscript corresponds to the ordinary, or extraordinary index.

$$
n_{i}^{2}(E)=1+\frac{f_{i} E_{i}^{2}}{\left(E_{i}^{2}-E^{2}\right)}
$$

Where $f_{o}$ equals $5.63, E_{o}$ equals $7.80, f_{e}$ equals $5.86, E_{e}$ equals 7.52 and $E$ is the optical wavelength expressed in $\mathrm{eV} .4 \mathrm{H}-\mathrm{SiC}$, like $6 \mathrm{H}-\mathrm{SiC}$, is a positive uniaxial material. The refractive index data for $4 \mathrm{H}-\mathrm{SiC}$ is very similar to that of $6 \mathrm{H}-\mathrm{SiC}$. The $4 \mathrm{H}$ and $6 \mathrm{H}-\mathrm{SiC}$ refractive index values vary less than $1 \%$ between each other at a given wavelength. This should be expected since $4 \mathrm{H}$ and $6 \mathrm{H}-\mathrm{SiC}$ are two poly-types of the same material, that vary only by the difference in stacking order in the c direction of the crystal. 


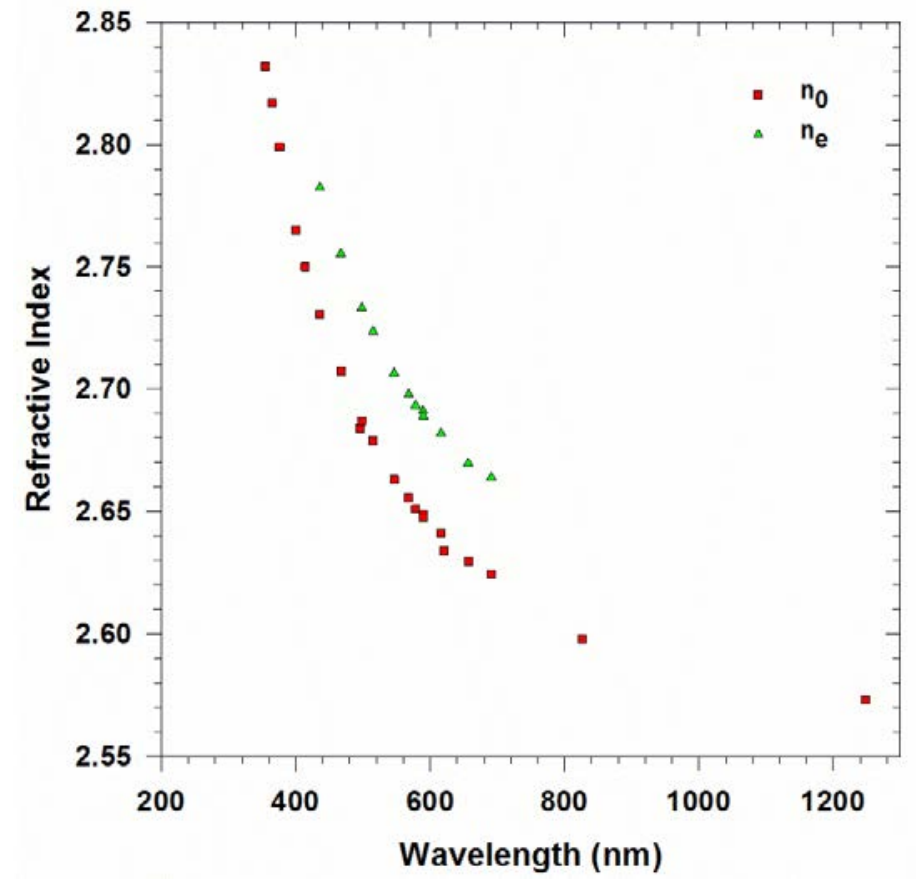

Figure 4.7. $6 \mathrm{H}-\mathrm{SiC}$ ordinary $\left(\mathrm{n}_{\mathbf{0}}\right)$ and extraordinary $\left(\mathrm{n}_{\mathrm{e}}\right)$ refractive index plotted as functions of wavelength $[4.16-4.18]$

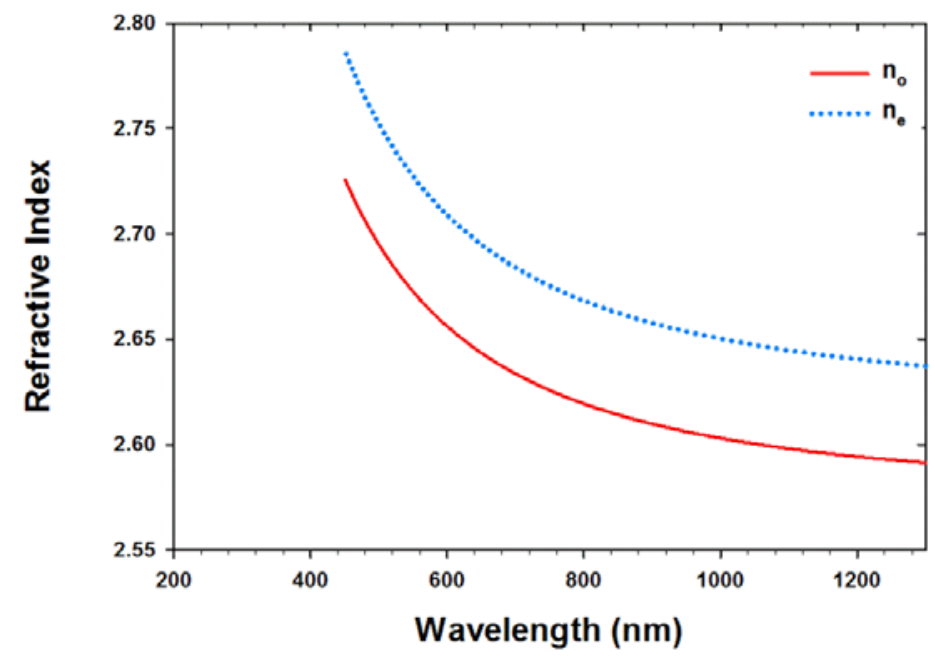

Figure 4.8. 4H-SiC ordinary and extraordinary refractive index plotted as a function of wavelength [4.19] 
The refractive index of $2 \mathrm{H}-\mathrm{GaN}$ is plotted in Figure 4.9 [4.20, 4.21]. Values for the refractive index for $\mathbf{E} \perp \mathbf{C}\left(\mathrm{n}_{0}\right)$ were obtained by Ejder [4.20] using transmission and reflection measurements from undoped, wurtzite, GaN platelets (blue squares). Values for both $n_{o}$ and $n_{e}$ (red and green lines) were obtained by Yu et. al. [4.21] using reflectance spectroscopy and variable angle spectroscopic ellipsometry of thin films of $2 \mathrm{H}-\mathrm{GaN}$ on sapphire. The refractive index results of $\mathrm{Yu}$ et al [4.21] were fitted to a Sellmeier dispersion relationship of the form given in equation 4.8 .

$$
n_{i}^{2}(\lambda)=1+\frac{A_{i} \lambda^{2}}{\lambda^{2}-B_{i}}
$$

Where $A_{o}$ equals 4.14, $B_{0}$ equals $0.038, A_{e}$ equals $4.31, B_{e}$ equals 0.034 and $\lambda$ is the wavelength of light in micrometers. The results of Edjer [4.20] for $n_{o}$ are in better agreement with the data

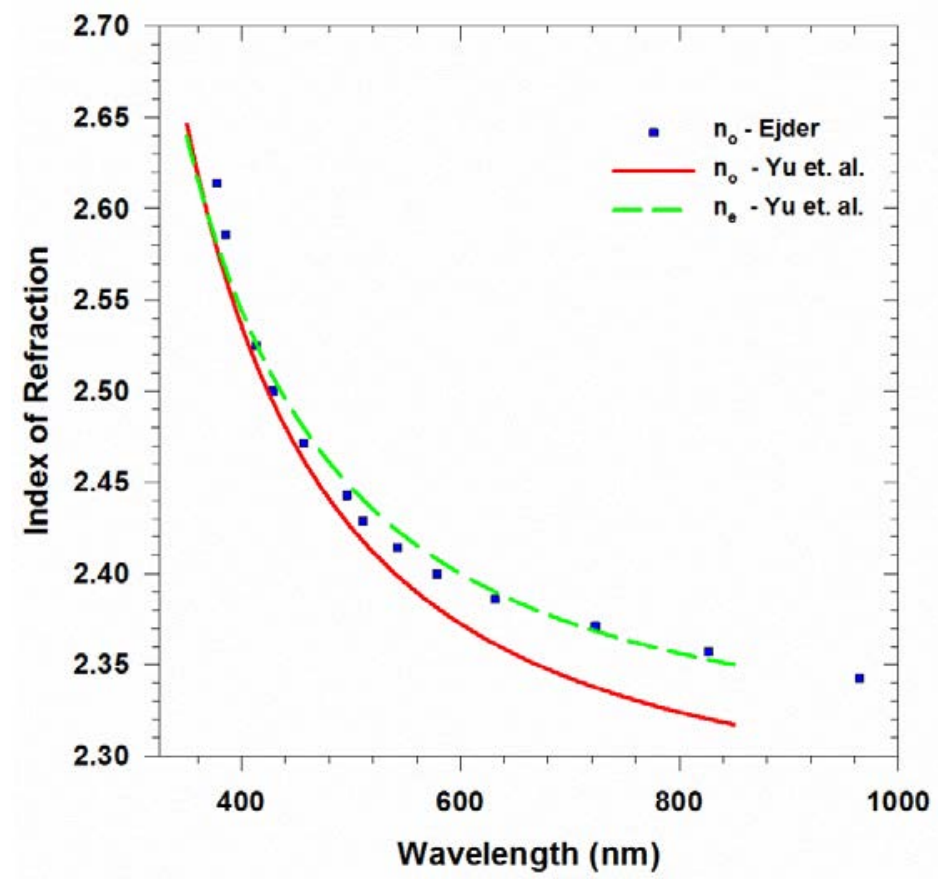

Figure 4.9. Values of $n_{0}[4.20,4.21]$ and $n_{e}[4.21]$ for $2 H-G a N$ 
for $\mathrm{n}_{\mathrm{e}}$ measured by $\mathrm{Yu}$ et al [4.21] at wavelengths longer than $520 \mathrm{~nm}$. The data of $\mathrm{Yu}$ et al identifies $2 \mathrm{H}-\mathrm{GaN}$ as a positive uniaxial material. The values of $\mathrm{n}_{\mathrm{o}}$ and $\mathrm{n}_{\mathrm{e}}$ are almost identical for wavelengths shorter than $400 \mathrm{~nm}$. However, the value of $\Delta n\left(n_{e}-n_{0}\right)$ begins to increase for wavelengths longer than $400 \mathrm{~nm}$ and reaches a value of 0.0328 at $850 \mathrm{~nm}$, a difference of $1.4 \%$ referenced to $\mathrm{n}_{0}$.

The transmission of light through the front facet of photoconductive switches fabricated from substrates of compensated $2 \mathrm{H}-\mathrm{GaN}, 4 \mathrm{H}$ and $6 \mathrm{H}-\mathrm{SiC}$ can be calculated using the refractive index data and equations 4.3 and 4.4 with the value of $\kappa$ assumed to be negligible for the wavelengths of interest. Figures $4.10,4.11$ and 4.12 are plots of the optical transmission, at normal incidence, through the front facets of switch substrates constructed from $6 \mathrm{H}-\mathrm{SiC}, 4 \mathrm{H}-\mathrm{SiC}$

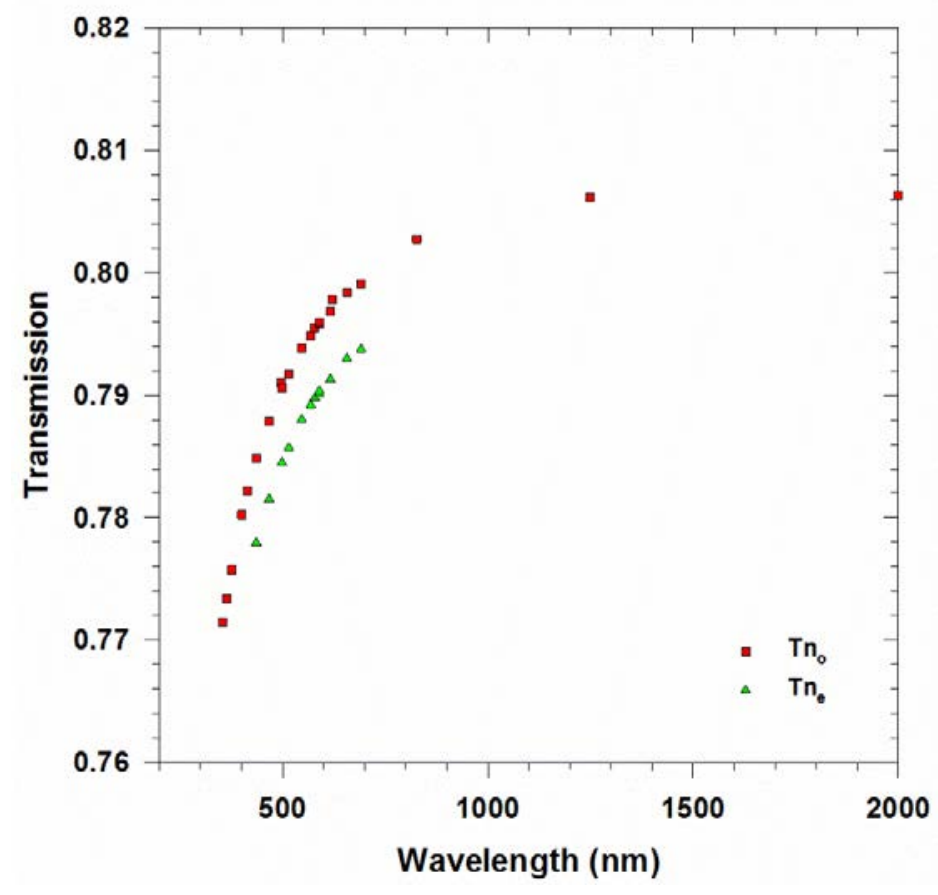

Figure 4.10. Calculated optical transmission through the front facet of a $6 \mathrm{H}-\mathrm{SiC}$ photoconductive switch substrate plotted versus wavelength for light polarized perpendicular and parallel to the optical axis of the material (c direction). 


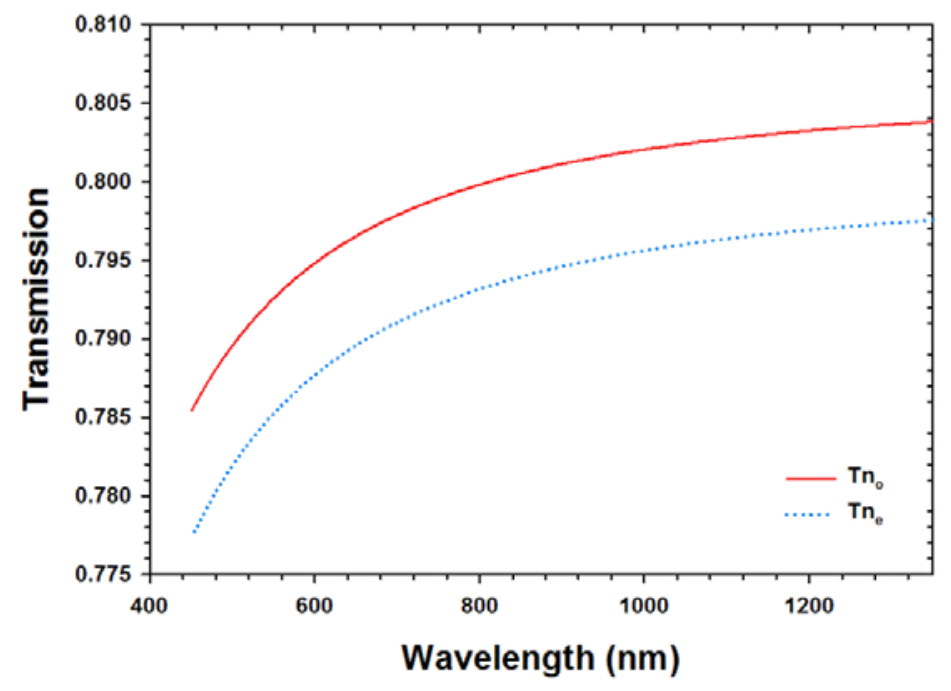

Figure 4.11. Calculated optical transmission through the front facet of a $4 \mathrm{H}-\mathrm{SiC}$ photoconductive switch substrate plotted versus wavelength for light polarized perpendicular and parallel to the optical axis of the material (c direction).

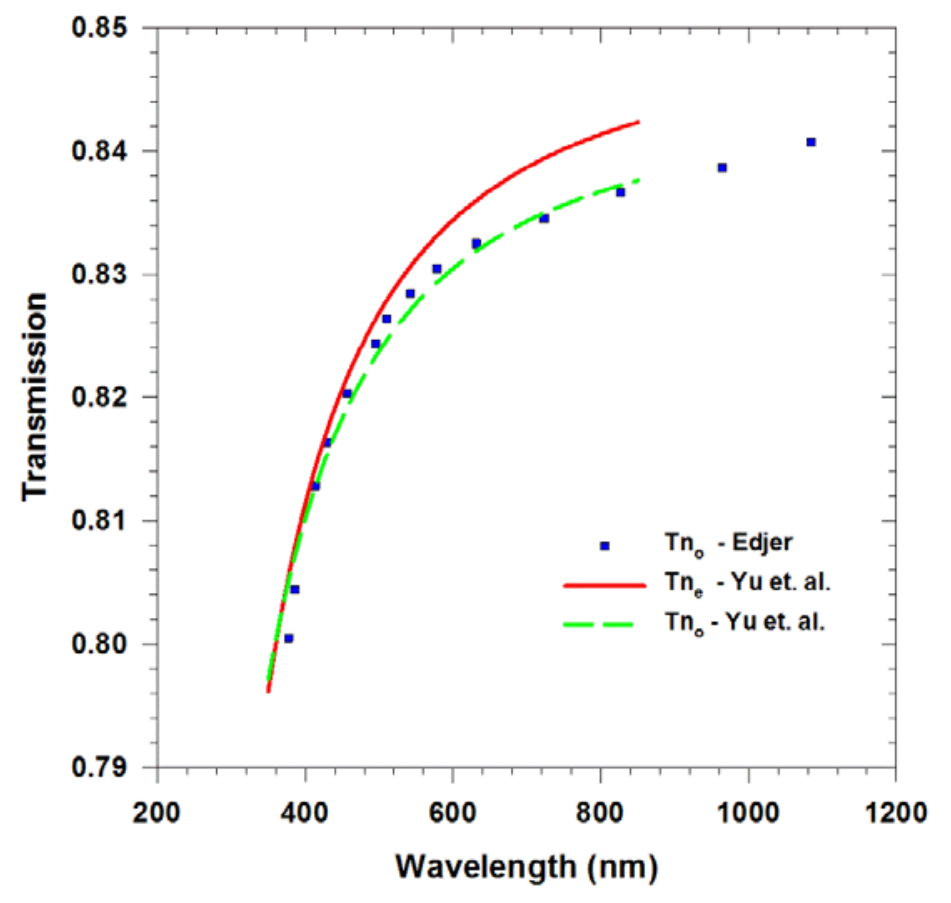

Figure 4.12. Calculated optical transmission through the front facet of a $2 \mathrm{H}-\mathrm{GaN}$ photoconductive switch substrate plotted versus wavelength for light polarized perpendicular and parallel to the optical axis of the material (c direction). 
and $2 \mathrm{H}-\mathrm{GaN}$, respectively. The transmission through the front facet for light polarized perpendicular (parallel) to the optical axis of the material is denoted by $\operatorname{Tn}_{\mathrm{o}}\left(\operatorname{Tn}_{\mathrm{e}}\right)$ on the figure plots. The fraction of normally incident light transmitted into the switch substrates for all three wide bandgap materials is greater than 0.77 and less than 0.85 . The difference in transmission between light polarized perpendicular $\left(\mathrm{Tn}_{0}\right)$ and parallel $\left(\mathrm{Tn}_{\mathrm{e}}\right)$ to the optical axis of $6 \mathrm{H}-\mathrm{SiC}, 4 \mathrm{H}-$ $\mathrm{SiC}$ and $2 \mathrm{H}-\mathrm{GaN}$ is less than one percent. A large fraction of normally incident light is transmitted into all three materials, regardless of the polarization of the incident beam. However, slightly more light polarized perpendicular to the optical axis of the material is transmitted through the front facet of a switch substrate. So we will arbitrarily choose to apply optical pulses polarized perpendicular to the c axis of our switch substrates.

Of course, light can be applied at Brewster's angle so that all light is transmitted into the switch facet. Brewster's angle is given by equation 4.9 [4.23] for the physical situation shown in Figure 4.13.

$$
\tan \theta_{1}=\frac{n_{2}}{n_{1}}
$$

Where $n_{2}$ is the index of refraction of the switch substrate material and $n_{1}$ is the index of air.

We calculate Brewster's angles $\left(\theta_{1}\right)$ of 69.5 and 68.8 degrees for optical pulse wavelengths of 532 and $1064 \mathrm{~nm}$, respectively, incident on the air - 6H-SiC interface. We have used the values of $n_{0}$ for $6 \mathrm{H}-\mathrm{SiC}$ at these two wavelengths for these calculations. This means we would have to apply light at these fairly severe angles to achieve complete transmission of the optical pulse through the front facet of the switch assembly. Applying the optical pulse to the 


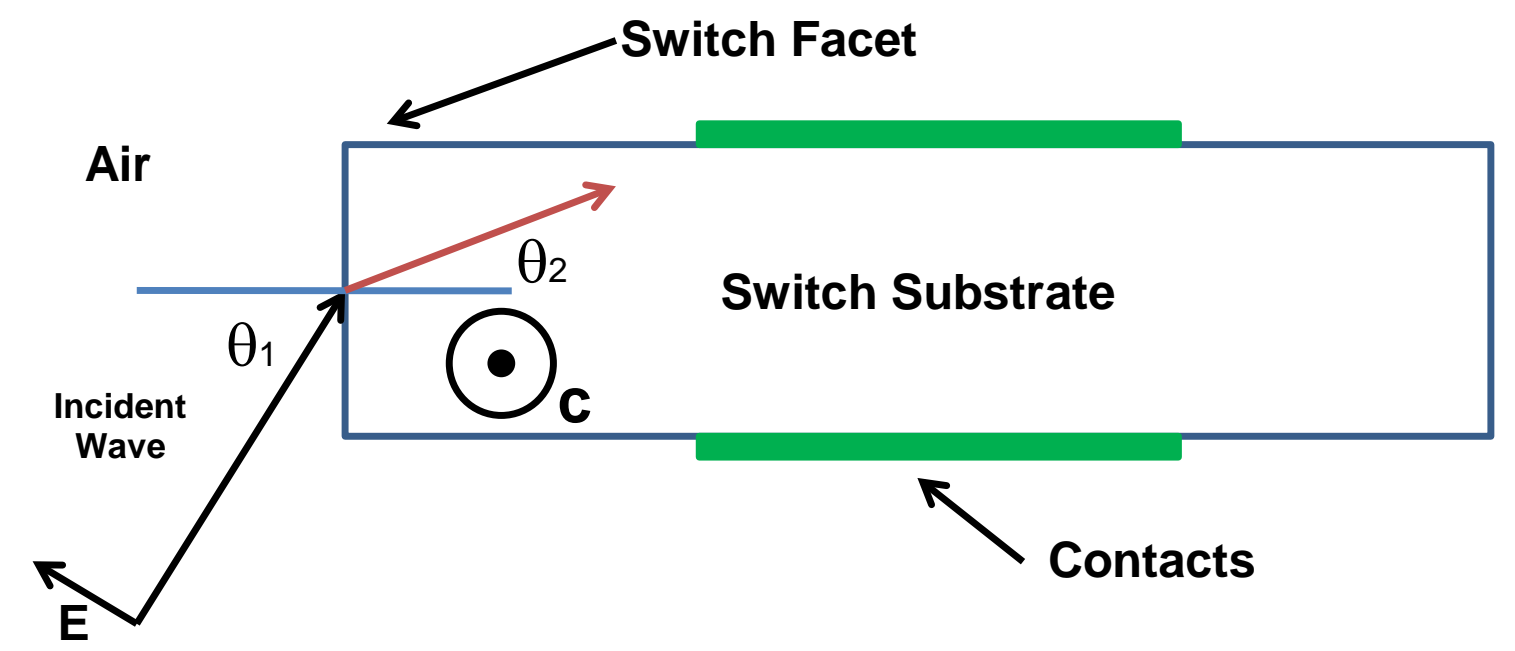

Figure 4.13. We are looking down the edge of the facet that is being illuminated with light that is polarized perpendicular to the $c$ axis of the switch substrate. The applied optical pulse is incident on the switch facet at an angle $\theta_{1}$. All light is transmitted through the front facet at an angle of $\theta_{2}$.

switch at Brewster's angle may make sense if we had a large array of switches and we wanted to reduce the energy/power requirements on the laser system providing the optical trigger pulses. However, in our tests we will apply the optical trigger pulse at normal incidence with the electric field polarized perpendicular to the c plane of the switch substrates. We shall see in section 4.6 that there are additional reasons for applying only light with polarization perpendicular to the optical axis to the switch substrates.

\section{5) Optical Generation of Carriers in Extrinsic Photoconductive Switches}

We have reviewed, in general terms, how electrons are optically excited from deep acceptor levels into the conduction band in compensated, wide bandgap semiconductors [4.20]. However, this is a complicated process that requires a close inspection of the quantum processes 
involved before a clear understanding is obtained. A review of optical excitation of carriers in a semiconductor is the first step towards this understanding.

\subsection{1) Radiative Absorption in Semiconductors}

We consider the optical excitation of an electron from an occupied initial energy level $E_{\mathrm{i}}$ to an empty final energy level $E_{f}$. This requires the absorption of a photon of energy $\hbar \omega$ by an electron occupying energy level $E_{\mathrm{i}}$. The photon energy, $\hbar \omega$, must be greater than, or equal to, the energy difference between the initial and final levels, $E_{f}-E_{i}$. This process is depicted in Figure 4.14. The transition rate $\left(\mathrm{W}_{\mathrm{i}-\mathrm{f}}\right)$ for this absorption process can be calculated using Fermi's Golden Rule [4.24, 4.25, 4.26], which is shown in equations 4.10 and 4.11 .

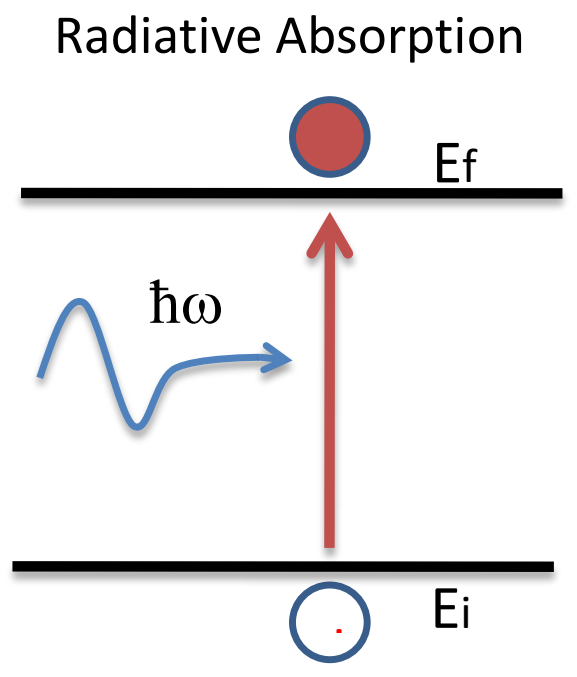

4.14. Absorption of a photon by an electron with the corresponding transition to final level $\mathrm{E}_{\mathrm{f}}$.

$$
W_{i-f}=\frac{2 \pi}{\hbar}\left|\left\langle f\left|H^{\prime}\right| i\right\rangle\right|^{2} \delta\left(E_{f}-E_{i}-\hbar \omega\right)
$$




$$
\left|\left\langle f\left|H^{\prime}\right| i\right\rangle\right|=\int \Psi_{f}^{*}(r) H^{\prime}(r) \Psi_{i} d^{3} r
$$

Where $\Psi_{\mathrm{f}}\left(\Psi_{\mathrm{i}}\right)$ is the wave function for the final (initial) state and $\mathrm{H}^{\prime}(\mathrm{r})$ is the spatial component of the time dependent perturbed Hamiltonian for the photon - electron interaction. The full Hamiltonian for the electron - photon interaction is given in equation $4.12[4.24,4.26]$. Where $\mathbf{p}$

$$
H(r)=\frac{1}{2 m_{0}}(\boldsymbol{p}+e \boldsymbol{A})^{2}+V(r)
$$

is the momentum operator $(\mathrm{h} / \mathrm{i}) \nabla, \mathrm{V}(\mathrm{r})$ is the periodic crystal potential, $\mathrm{m}_{0}$ is the mass of an electron and $\mathbf{A}$ is the vector potential. The Hamiltonian of Equation 4.12 can be expanded into equation 4.13. The perturbed Hamiltonian $\left(\mathrm{H}^{\prime}(\mathrm{r})\right)$ is given by the last two terms on the right hand side of equation 4.13 .

$$
H(r)=H_{0}(r)+H^{\prime}(r)=\frac{\boldsymbol{p}^{2}}{2 m_{0}}+V(r)+\frac{e}{2 m_{0}}(\boldsymbol{p} \cdot \boldsymbol{A}+\boldsymbol{A} \cdot \boldsymbol{p})+\frac{e^{2} \boldsymbol{A}^{2}}{2 m_{0}}
$$

The perturbed Hamiltonian can be further reduced by assuming the Coulomb gauge $(\nabla \cdot \mathbf{A}$ $=0$ ), and neglecting the $\mathbf{A}^{2}$ term compared to the (p. $\left.\mathbf{A}+\mathbf{A} \cdot \mathbf{p}\right)$ term. A more detailed development of obtaining the perturbed Hamiltonian is presented by Miller [4.26]. This results in equation 4.14 for the perturbed Hamiltonian [4.24, 4.25, 4.26]. The vector potential $\mathbf{A}$ is defined in terms of the electric (E) and magnetic (B) fields using equations 4.15 and 4.16.

$$
\begin{aligned}
H^{\prime}(r) & =\frac{e}{m_{0}} \boldsymbol{p} \cdot \boldsymbol{A} \\
\boldsymbol{B} & =\boldsymbol{\nabla} \cdot \boldsymbol{A}
\end{aligned}
$$




$$
\boldsymbol{E}=\frac{\partial \boldsymbol{A}}{\partial t}
$$

Here we have assumed that there are no free charges, so the scalar potential field is zero. The vector potential, $\mathbf{A}$, is aligned with the electric field $\mathbf{E}$, so it carries the information of the polarization of the electric field of the optical pulse.

Equation 4.14 can be substituted into equation 4.10 to obtain equation 4.17, which gives the transition rate for a single electron from a particular initial energy level to a particular final energy level via absorption of a photon. A total upward transition rate, $\mathrm{W}_{\text {uptot, }}$ is the sum of the transition rates from all possible initial energy levels to all possible final energy levels. The total upward transition rate is listed in equation 4.18 .

$$
\begin{gathered}
W_{i-f}=\frac{2 \pi}{\hbar}\left|\left\langle f\left|\frac{e}{m_{0}} \boldsymbol{p} \cdot \boldsymbol{A}\right| i\right\rangle\right|^{2} \delta\left(E_{f}-E_{i}-\hbar \omega\right) \\
W_{\text {uptot }}=\frac{2}{V} \sum_{k i} \sum_{k f} \frac{2 \pi}{\hbar}\left|\left\langle f\left|\frac{e}{m_{0}} \boldsymbol{p} \cdot \boldsymbol{A}\right| i\right\rangle\right|^{2} \delta\left(E_{f}-E_{i}-\hbar \omega\right) f_{i}\left(1-f_{f}\right)
\end{gathered}
$$

Where $\mathrm{V}$ is the volume of the semiconductor crystal, $\mathbf{k}_{\mathbf{i}}\left(\mathbf{k}_{\mathbf{f}}\right)$ is the wave vector of the initial (final) energy level wave functions, and $f_{i}\left(f_{f}\right)$ is the probability that the initial (final) energy level is occupied. Similarly, an expression describing the emission of a photon during the downward transition of an electron from in a final energy level to an initial level can be developed. The total downward transition rate $\left(\mathrm{W}_{\mathrm{dntot}}\right)$ is expressed in equation 4.19. The net upward transition rate

$$
W_{d n t o t}=\frac{2}{V} \sum_{k i} \sum_{k f} \frac{2 \pi}{\hbar}\left|\left\langle f\left|\frac{e}{m_{0}} \boldsymbol{p} \cdot \boldsymbol{A}\right| i\right\rangle\right|^{2} \delta\left(E_{i}-E_{f}+\hbar \omega\right) f_{f}\left(1-f_{i}\right)
$$


$\left(\mathrm{W}_{\text {tot }}\right)$ is $\mathrm{W}_{\text {uptot }}$ minus $\mathrm{W}_{\mathrm{dntot}}$. The net upward transition rate is expressed in equation 4.20. Where we have taken advantage of the fact that the delta function is an even function $(\delta(\mathrm{x})=\delta(-\mathrm{x}))$.

$$
W_{t o t}=\frac{2}{V} \sum_{k i} \sum_{k f} \frac{2 \pi}{\hbar}\left|\left\langle f\left|\frac{e}{m_{0}} \boldsymbol{p} \cdot \boldsymbol{A}\right| i\right\rangle\right|^{2} \delta\left(E_{f}-E_{i}-\hbar \omega\right)\left(f_{i}-f_{f}\right)
$$

A can be expressed as shown in equation 4.21, if we assume that $\mathbf{A}$ is a linearly polarized plane wave with wave vector, $\mathbf{k}_{\mathbf{o p}}$, and angular frequency, $\omega$. Where $\hat{\mathbf{e}}$ is a unit vector in the direction of the optical electric field.

$$
\boldsymbol{A}=\hat{\boldsymbol{e}}\left[\frac{A_{\mathbf{0}}}{2} e^{\left(i\left(\boldsymbol{k}_{\boldsymbol{o p}} \cdot \boldsymbol{r}-\omega t\right)\right)}+\frac{A_{\mathbf{0}}}{2} e^{\left(-i\left(\boldsymbol{k}_{\boldsymbol{o p}} \cdot \boldsymbol{r}-\omega t\right)\right)}\right]
$$

Substituting the spatial component of $\mathbf{A}$ into the expression for the perturbed Hamiltonian in the expression for the total transition rate leads to equation 4.22 .

$$
W_{t o t}=\frac{2}{V} \sum_{k i} \sum_{k f} \frac{2 \pi}{\hbar} \mid\left\langle f\left|\frac{e A_{0} e^{\left(i \boldsymbol{k}_{o p} \cdot \boldsymbol{r}\right)}}{2 m_{0}} \hat{\boldsymbol{e}} \cdot \boldsymbol{p}\right| i||^{2} \delta\left(E_{f}-E_{i}-\hbar \omega\right)\left(f_{i}-f_{f}\right)\right.
$$

Now we have an expression for the total net upward transition rate of electrons from all possible initial energy levels to all possible final energy levels due to photon absorption. The total transition rate, $\mathrm{W}_{\text {tot }}$, also represents the number of photons absorbed per unit volume per second. We now need to relate the total net transition rate, $\mathrm{W}_{\text {tot }}$, to the optical absorption, $\alpha$. The absorption coefficient, $\alpha$, is the fraction of photons absorbed in a material per unit length in the direction of propagation of the applied optical pulse. The absorption coefficient is expressed as a function of $\mathrm{W}_{\text {tot }}$ in equation 4.23 [4.24, 4.26]. 


$$
\alpha=\frac{\hbar \omega W_{t o t}}{I_{o p}}
$$

Where $\mathrm{I}_{\mathrm{op}}$ is the applied optical intensity in units of power per unit area and $\hbar \omega$ is the energy of a photon. Equation 4.23 states mathematically that the absorption coefficient is equal to the ratio of the number of photons absorbed per unit volume per second to the number of photons injected per unit area per second. The optical intensity is obtained from the Poynting vector (ExB), which is calculated using the expressions for the vector potential $\mathbf{A}$ (equations $4.15,4.16,4.21$ ). The absorption can be rewritten as equation 4.24 .

$$
\alpha(\hbar \omega)=\frac{\hbar \omega}{\left(n_{r} \omega^{2} \frac{A_{0}^{2}}{2 \mu_{0} c}\right)} \frac{2}{V} \sum_{k i} \sum_{k f} \frac{2 \pi}{\hbar}|| f\left|\frac{e A_{0} e^{\left(i \boldsymbol{k}_{o p} \cdot \boldsymbol{r}\right)}}{2 m_{0}} \hat{\boldsymbol{e}} \cdot \boldsymbol{p}\right| i||^{2} \delta\left(E_{f}-E_{i}-\hbar \omega\right)\left(f_{i}-f_{f}\right)
$$

Where $n_{r}$ is the index of refraction, $\mu_{0}$ is the permeability of free space and c is the speed of light in free space.

There are physical quantities in equation 4.24 that we can control and some are beyond our control. We can choose the polarization, intensity and wavelength of the applied optical pulse. Generally, $f_{i}$ and $f_{f}$ correspond to Fermi Dirac distributions of the initial and final states occupied by electrons. When dealing with band to band transitions, the $f_{i}$ and $f_{f}$ distributions represent occupied states in the valence and conduction bands, respectively and $\alpha(\hbar \omega)$ corresponds to excitation of electrons from the valence band to the conduction band by photon absorption. In addition, the initial (i) and final ( $f$ ) energy level wave functions are Bloch functions of the form shown in equation 4.25 . 


$$
\psi_{n \boldsymbol{k}}(\boldsymbol{r})=e^{i \boldsymbol{k} \boldsymbol{f} \cdot \boldsymbol{r}} u_{n \boldsymbol{k}}(\boldsymbol{r})
$$

Unfortunately, we are not interested in band to band optical transitions. We are interested in optical transitions from electrons occupying deep acceptor levels to the conduction band by the absorption of photons with below bandgap wavelengths $\left(\hbar \omega<\mathrm{E}_{\mathrm{g}}\right)$. In addition, we have little knowledge of the actual wave functions of the electrons occupying the deep acceptor levels. There is some good news in that similar statistics can be used to calculate the fraction of electrons available to be trapped in the deep acceptor levels. There is better news in that we control the number of donors and acceptors that are present in the semiconductor. However, we need to explore wave function models for the electrons occupying the deep acceptor levels to better understand the nature of absorption at these deep levels.

\subsection{2) Models for Optical Absorption at Deep Impurities in Semiconductors}

Impurity atoms form localized electronic states at energy levels lying between the valence and conduction band. Shallow donors (acceptors) that differ in valency with the host semiconductor atom by plus (minus) one electron, donate an electron (hole) to the conduction (valence) band. These carriers leave behind a fixed positive charge in the case of a donor and a fixed negative charge in the case of an acceptor. In either case, the carrier is moving in the potential of the fixed charge it left behind, similar to the hydrogen atom. Successful models for shallow donor and acceptor impurities were based on Bohr's hydrogen atom model. The hydrogen atom model was modified to model shallow donors by replacing the electron mass with the effective mass $\left(\mathrm{m}_{\mathrm{e}}{ }^{*}\right)$ of an electron in the semiconductor crystal dispersion relation term and by including the dielectric constant $\left(\varepsilon_{\mathrm{r}}\right)$ of the semiconductor in the coulomb potential term. 
These modifications are shown in equations 4.26 and 4.27 . These modifications lead to the effective mass equation for a hydrogenic impurity listed in equation 4.28.

$$
\begin{gathered}
E(k)=\frac{\hbar^{2} k^{2}}{2 m_{e}} \rightarrow \frac{\hbar^{2} k^{2}}{2 m_{e}^{*}} \\
V(r)=\frac{e}{4 \pi \varepsilon_{0} r} \rightarrow \frac{e}{4 \pi \varepsilon_{r} \varepsilon_{0} r} \\
-\frac{\hbar^{2}}{2 m_{e}^{*}} \nabla^{2} \psi+\frac{\mathrm{e}}{4 \pi \varepsilon_{\mathrm{r}} \varepsilon_{0} \mathrm{r}} \psi=\mathrm{E} \psi
\end{gathered}
$$

Solving equation 4.28 yields, $\psi$, the donor envelope function. The donor ground state wave function is the product of the donor envelope function and a Bloch function $\left(u_{k}(r)\right)$ shown in equation 4.29. Where $a_{B}{ }^{*}$ is radius of the radius of the donor ground state, also called the donor Bohr radius.

$$
\begin{gathered}
\psi(r)=\frac{1}{\sqrt{\pi}}\left(\frac{1}{a_{B}^{*}}\right)^{\frac{3}{2}} e^{-\frac{r}{a_{B}^{*}}} u_{k}(r) \\
a_{B}^{*}=\frac{4 \pi \varepsilon \hbar^{2}}{m_{e}^{*} e^{2}}
\end{gathered}
$$

The hydrogenic models for shallow impurities are very successful at predicting impurity ionization energies. However, this approach is limited to substitutional impurities that differ in valency with the host atom by $+/$ - one and the electron (hole) orbits the charged impurity atom with a radius much larger than the lattice constant. These limitations led to the development of other models for impurities in semiconductors. 


\subsection{2) Lucovsky Model for Photoionization of Deep Impurities}

The hydrogenic model predicts that the maximum photoionization cross section occurs when photon energy is equal to the impurity ionization energy. The hydrogenic model also predicts a narrow, sharply peaked photoionization cross section centered at the impurity ionization energy. The photoionization cross section for a hydrogenic impurity center is given by [4.27]

$$
\sigma_{h}(\hbar \omega)=\frac{1}{\sqrt{\varepsilon_{0} \varepsilon_{r}}}\left(\frac{E_{e}}{E}\right) \frac{2^{8} \pi^{2} \hbar e^{2}}{3 m^{*} c E_{i}}\left(\frac{E_{i}}{\hbar \omega}\right)^{4} \frac{e^{\left(-4\left(\frac{\hbar \omega-E_{i}}{E_{i}}\right)^{\frac{1}{2}}\right)} \cot ^{-1}\left(\frac{\hbar \omega-E_{i}}{E_{i}}\right)^{\frac{1}{2}}}{1-e^{\left(-2 \pi\left(\frac{\hbar \omega-E_{i}}{E_{i}}\right)^{\frac{1}{2}}\right)}}
$$

Where $\mathrm{E}$ is the optical field amplitude, $\mathrm{E}_{\mathrm{e}}$ is the effective local field, and $\mathrm{E}_{\mathrm{i}}$ is the ionization energy of the impurity level. The hydrogenic model yields poor results for the wavelength dependence of the photoionization cross section for several shallow dopants in silicon.

Lucovsky [4.28] developed a model for the wavelength dependence of photoionization cross section of deep impurity levels in semiconductors due to the poor agreement between experimental measurements of photoionization cross section and predictions of the hydrogenic model for some impurities in silicon. The impurities, boron, aluminum, gallium and indium, all reside from 45 to $150 \mathrm{meV}$ below the conduction band in silicon.

In general, the photoionization cross section is related to the transition rate $\left(\mathrm{W}_{\mathrm{i}-\mathrm{f}}\right)$ between a localized impurity state and a conduction band state in equations 4.32 and 4.33 [4.24].

$$
\sigma_{o p}=W_{i-f} \frac{V}{n_{p h}\left(\frac{c}{n}\right)}
$$




$$
\sigma_{o p}=\frac{V}{n_{p h}\left(\frac{c}{n}\right)} \frac{2 \pi}{\hbar}\left|\left\langle f\left|\frac{e}{m_{0}} \boldsymbol{p} \cdot \boldsymbol{A}\right| i\right\rangle\right|^{2} \delta\left(E_{f}-E_{i}-\hbar \omega\right)
$$

Where $n_{p h}$ is the number of photons, $\mathrm{V}$ is the volume of the semiconductor, c is the speed of light in vacuum and $\mathrm{n}$ is the refractive index of the semiconductor. The hydrogenic model assumes a potential field that varies as $1 / \mathrm{r}$ between the carriers freed from the impurity and the remaining charged impurity site. The $1 / \mathrm{r}$ potential assumed in the hydrogenic model extends long distances in the semiconductor material. Lucovsky believed that the impurities were more isolated than that assumed in the hydrogenic model. Lucovsky [4.28] assumed that the ground state wave function for an electron associated with a deep impurity will be given by the delta function well of equation 4.34 .

$$
\psi_{i}(r)=\sqrt{\frac{a}{2 \pi}} \frac{e^{-a r}}{r} ; r>0
$$

Where

$$
a=\sqrt{\frac{2 m^{*} E_{i}}{\hbar^{2}}}
$$

Lucovsky assumed that the final state of the electron would be the conduction band and the wave function for electrons in the conduction band can be expressed as a superposition of plane waves. The optical ionization cross section derived for these assumptions is given in equation 4.36 .

$$
\sigma_{L}(\hbar \omega)=\frac{1}{n_{r}}\left(\frac{E_{e}}{E_{0}}\right)^{2} \frac{16 \pi e^{2} \hbar}{3 m^{*} c} \frac{\left(E_{i}\right)^{\frac{1}{2}}\left(\hbar \omega-E_{i}\right)^{\frac{3}{2}}}{(\hbar \omega)^{3}}
$$


Where $n_{r}$ is the index of refraction. The Lucovsky cross section differs significantly from the hydrogenic cross section. The Lucovsky ionization cross section maximum value is not centered at the ionization energy of the impurity $\left(\mathrm{E}_{\mathrm{i}}\right)$, it is centered at $2 \mathrm{E}_{\mathrm{i}}$. In fact, the ionization energy is the threshold energy for the Lucovsky energy cross section. The Lucovsky ionization cross section is much broader and decays much more slowly than the hydrogenic ionization cross section. The Lucovsky photoinonization cross section is in very good agreement with the measured values of photoionization cross sections of indium in silicon. A comparison of the Lucovsky $\left(\sigma_{\mathrm{L}}\right)$ and hydrogenic $\left(\sigma_{\mathrm{h}}\right)$ photoionization cross sections are shown in Figure 4.16. The Lucovsky photoionization cross section has been normalized, while the hyrogenic cross section has not. Normalizing the hydrogenic photoionization cross section would result in what would appear to be a delta function on the scale of Figure 4.16. The actual peak of the hydrogenic

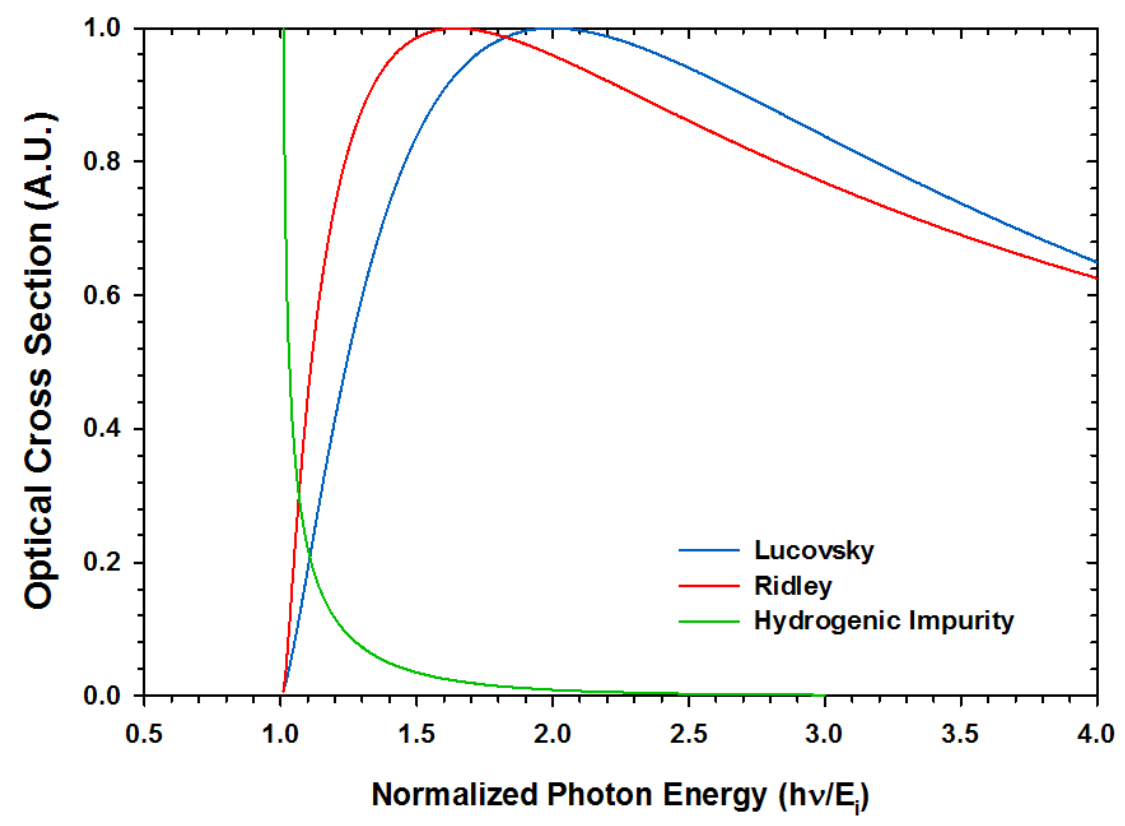

Figure 4.16. Comparison of the optical photoionization cross section for the hydrogenic, Lucovsky and Ridley models. 
photoionization cross section is $\sim 30$ and occurs at $h v / E_{\mathrm{i}}$. Ridley [4.29] also developed a model for the photoionization cross section of deep impurity levels.

\subsection{4) Ridley Model for the Photoionization of Deep Impurities}

Ridley [4.29] believed that existing models for the photoionization of deep impurities all had limitations. Ridley maintained that the effective mass approximation was inapplicable to the photoionization of deep impurities, the effect of the electron scattering strength of the impurity on conduction band electron wave functions should be included and the effect of impurity atom size and charge needed to be considered. These aspects were all included in Ridley's model along with the assumption that the impurity potential is comprised of a core region with an attractive square well potential, an intermediate coulombic region where the potential varies as $1 / \mathrm{r}$, and a third region where the potential is screened and falls to zero. Ridley's calculations are complicated and extensive, so only his results for the spectral function of the photoionization cross section of a deep acceptor level occupied by an electron are shown in equation 4.37.

$$
\begin{gathered}
G\left(\frac{\hbar \omega}{E_{i}}\right)=\left(\frac{1}{\frac{\hbar \omega}{E_{i}}}\right) e^{\left(\frac{-2 \pi \gamma}{\left(\frac{\hbar \omega}{E_{i}}-1\right)^{\frac{1}{2}}}\right)} \\
\gamma=|Z|\left(\frac{R^{*}}{E_{i}}\right)^{\frac{1}{2}}
\end{gathered}
$$

Where $\mathrm{R}^{*}$ is the effective mass Rydberg energy given in equation 4.39.

$$
R^{*}=\frac{1}{2(4 \pi \varepsilon)^{2}} \frac{e^{4} m_{e}^{*}}{\hbar^{2}}
$$


Ridley’s spectral function for the photoionization of a deep acceptor level occupied by an electron is shown in Figure 4.16. Ridley's model prediction is similar to Lucovsky's model result in that both predict a maximum for the photoionization cross section that is $1.6-2.0$ times deeper than the ionization potential of the impurity $\left(\mathrm{E}_{\mathrm{i}}\right)$ and decays rather slowly with normalized photon energy $\left(\hbar \omega / \mathrm{E}_{\mathrm{i}}\right)$. Both the Lucovsky and Ridley model predict that we should expect that photogenerated carriers created by the excitation of electrons occupying deep acceptor levels into the conduction band will peak at photon energies $1.6-2.0$ times the ionization energy of the acceptor level. We should also expect that significant photogeneration of carriers from electron occupied deep acceptor levels will continue for photon energies that are 4 -5 time the ionization energy of the acceptor level.

We can explore this point further using a bit of fiction. Assume we have a semiconductor material that has several different deep acceptor levels of equal densities with ionization energies that are evenly spaced in the energy gap of the semiconductor. This situation is depicted in Figure 4.17. Let us further assume that all acceptor levels are occupied by electrons and that the Lucovsky photoionization cross section model is valid for all three acceptor levels. The optical absorption coefficient for this material is given by the summation over the three acceptor levels shown in Equation 4.40.

$$
\alpha(\hbar \omega)=\sum_{i} \sigma_{i}(\hbar \omega) N_{i}
$$

Where $N_{i}$ is the number of filled acceptor levels and $\sigma_{\mathrm{i}}(\hbar \omega)$ is the photoionization cross section of the acceptor levels. The absorption coefficient as a function of normalized photon energy is shown in Figure 4.18. The absorption threshold is $E_{i}$ and the absorption increases with photon energy with the assumed Lucovsky model dependency. All absorption is due to acceptor level A 


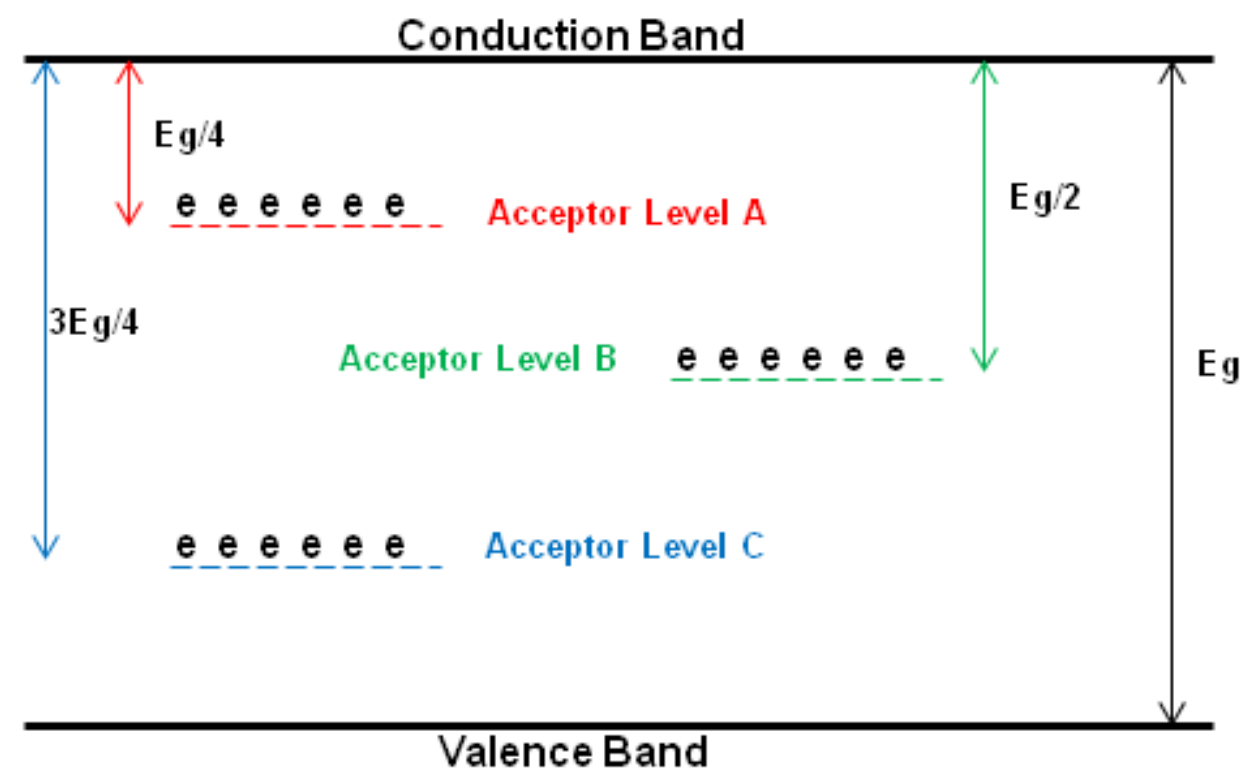

Figure 4.17. Energy band diagram for semiconductor material containing three evenly spaced deep acceptor levels.

until the photon energy reaches $2 \mathrm{E}_{\mathrm{i}}$, the threshold energy for acceptor level B. Absorption due to acceptor level A begins to decrease and absorption due to acceptor level B increases above photon energies of $2 \mathrm{E}_{\mathrm{i}}$. Acceptor level $\mathrm{C}$ starts to contribute to the total absorption at photon energies greater than $3 \mathrm{E}_{\mathrm{i}}$. Eventually the photon energy will reach $4 \mathrm{E}_{\mathrm{i}}\left(\mathrm{E}_{\mathrm{g}}\right)$, when acceptor levels A, B, and C will account for 28,44 and 28 percent of the total absorption, respectively. This simple fictional case highlights some of the predictions of the models for the photoionization cross sections of deep levels. The photoionization cross sections of deep levels have broad spectral width so that these levels absorb over a wide range of applied photon energies. The peak photoionization cross section for a given level occurs at photon energies of $1.6-2.0$ times the level ionization energy, so that very deep levels in the energy gap will make little, or no, contribution to the total absorption. 


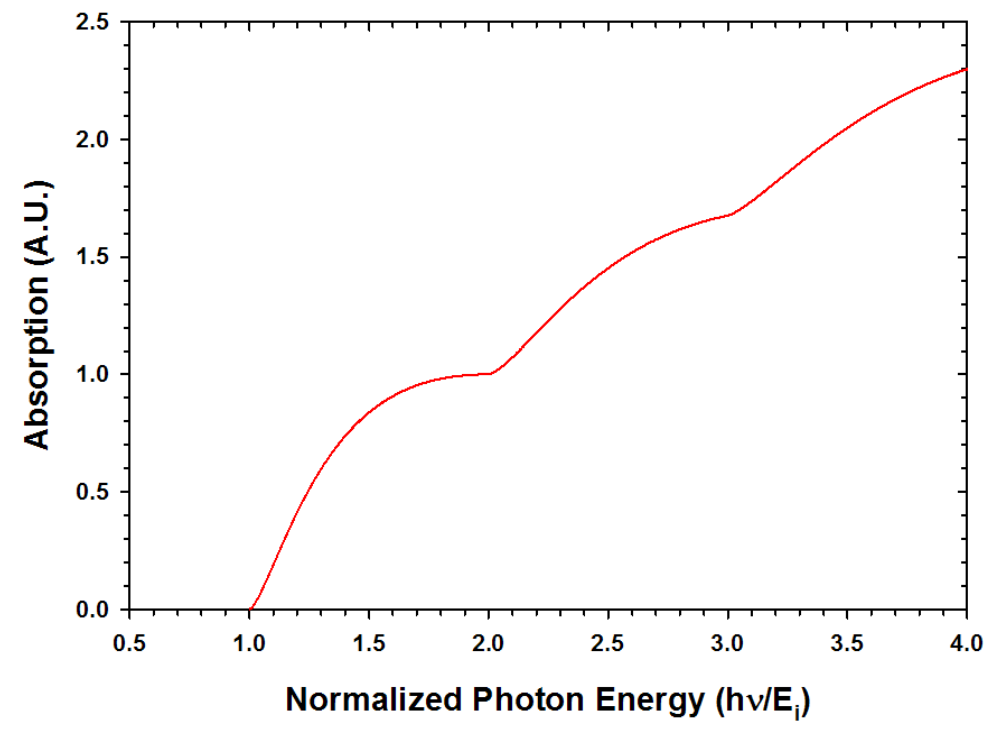

Figure 4.18. Absorption due to deep absorption levels $A, B$, and $C$ for fiction case shown in Figure 4.17.

Let's take our fictional case a step further and imagine that we can increase the density of deep acceptor level A to ten times that of deep acceptor levels B and C. Let's also assume that the Fermi level is pinned at acceptor level A, so we can expect only half the acceptor level A sites are occupied by electrons, while all of acceptor level B and C are occupied by electrons. The absorption for this case is shown in Figure 4.19. Deep acceptor level A dominates the absorption, as expected, for the entire range of photon energies. The percentage of absorption due to acceptor levels $A, B$, and $C$ at the photon energy of $4 \mathrm{E}_{\mathrm{i}}$ is 66,21 and 13 percent, respectively. This can be achieved by doping to increase the density of acceptor level A, while maintaining the densities of acceptor levels B and C. 


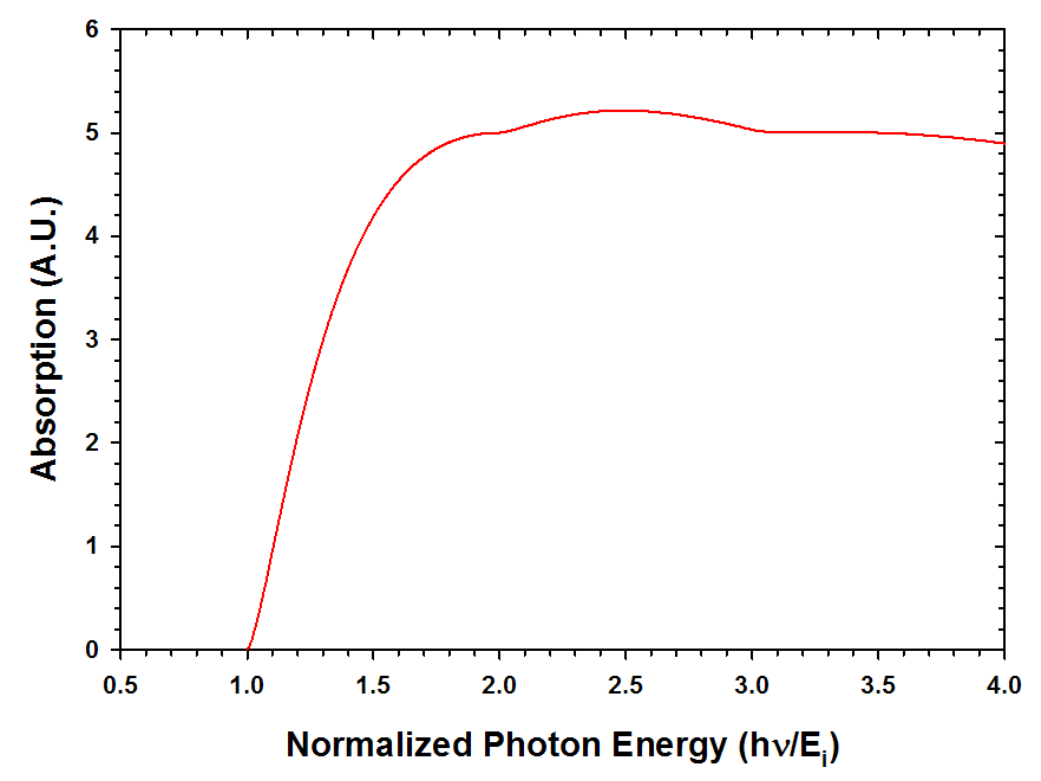

Figure 4.19. Absorption for ten-fold increase of deep acceptor level A density, while maintaining density of deep acceptor levels $B$ and $C$.

The two cases presented above are simple models that assume the position of the acceptor levels and allow only for absorption at the acceptor levels. Excitation of electrons from the valence band to the acceptor levels was not included and neither was excitation from deeper acceptor levels to acceptor levels residing at higher energy levels in the band gap. In addition, our model for the absorption cross section only included excitation of electrons from deep acceptor levels to the conduction band by absorption of a photon of sufficient energy. What we have ignored is the effect of phonons on these transitions. We have assumed that the optical transitions from the deep levels to the conduction band are direct transitions, which they are not. The deep acceptor energy levels will not be aligned with the conduction band minimum in $\mathbf{k}$ (momentum) space. The absorption, or emission, of phonons will be required during the optical transition of an electron from a deep acceptor level to the conduction band to satisfy the 
conservation of momentum. The effect of phonons on these transitions will be addressed in the next section.

\subsection{5) Franck - Condon Shift}

A deep acceptor level is formed when an impurity atom substitutes for a host atom at a lattice site. The impurity atoms we are interested in are vanadium in the semiconductors $4 \mathrm{H}$ and $6 \mathrm{H}-\mathrm{SiC}$ and iron in $2 \mathrm{H}-\mathrm{GaN}$. Substituting an impurity atom for a host atom at a lattice site distorts the lattice in the volume surrounding the impurity [4.30]. The capture of an electron at the deep acceptor level distorts the lattice even further in the immediate volume of the acceptor due to the acquired negative electrostatic charge. The position of the impurity atom in the lattice will be slightly different after the capture of an electron. The lattice will relax to the uncharged acceptor level state when the electron is optically excited into the conduction band. As a result, the eigenstates of the electron trapped in the acceptor level must be described by both electronic and atomic coordinates [4.30, 4.31]. An optical transition of an electron from the acceptor level to the conduction band occurs much more rapidly (several femtoseconds) than the time it takes for the lattice to relax from the charged to uncharged acceptor level position (a few picoseconds). Accordingly, the optical transition is treated as if it occurs with no movement of the lattice. This forms the basis of the Franck - Condon principle illustrated in Figure 4.20.

Figure 4.20 is a configuration diagram showing the ground state energy of an atom and an excited state of the atom as functions of position. The ground state and excited state energies are slightly displaced from one another. The Franck - Condon principle states that no change in lattice configuration can occur during an optical transition of an atom from the ground state to an excited state. This requires the optical transition between states be vertical with respect to the 


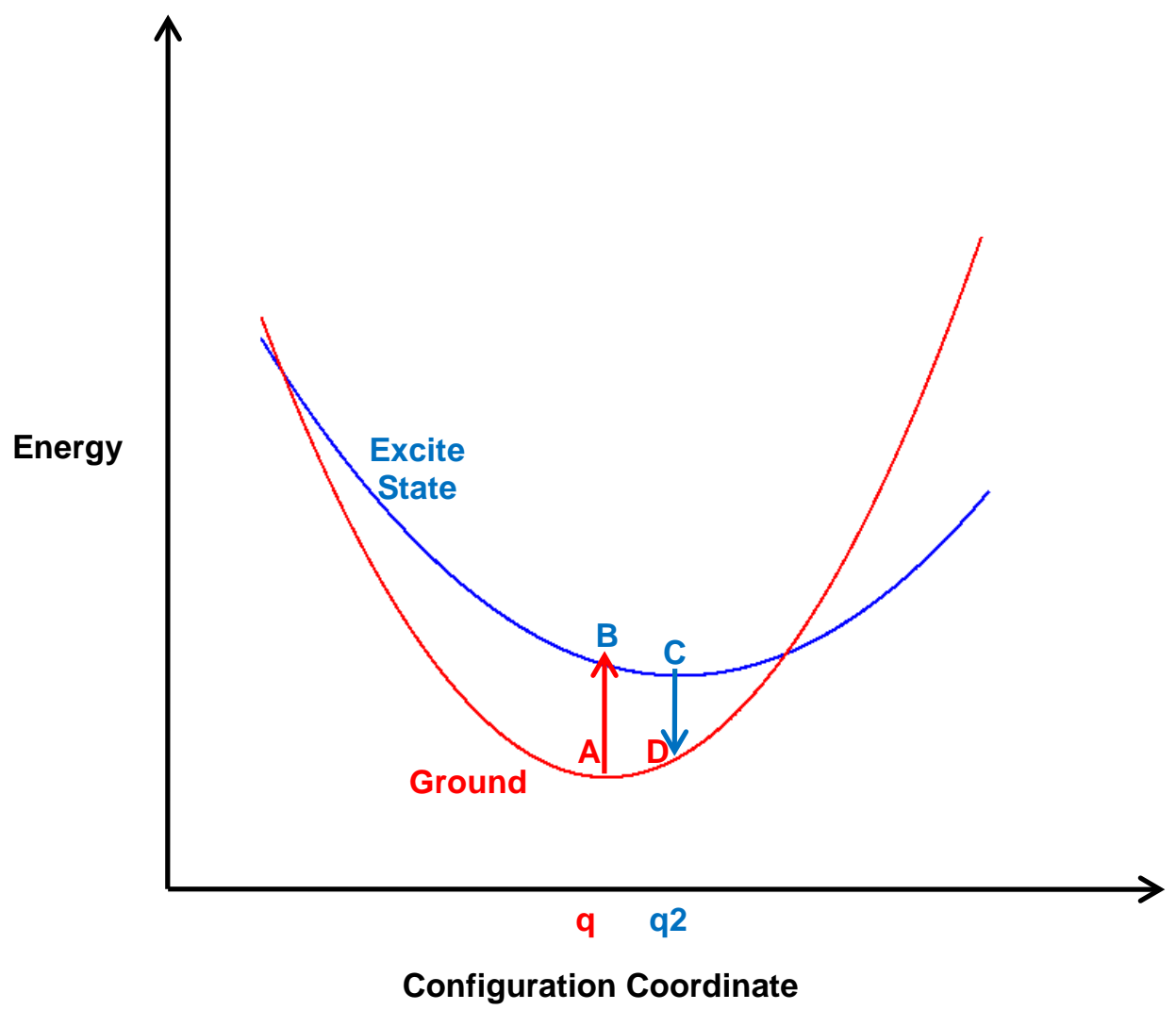

Figure 4.20. Configuration diagram showing position ground state and excited states of an atom

configuration coordinates. The minimum energy of the ground state is at point A. The photon absorption excites an electron from point A to point B located in the excited state. After the optical transition, the system partially relaxes to point $\mathrm{C}$, the energy minimum of the excited state. The electron loses energy during this process $\left(E_{B}-E_{C}\right)$, manifested as phonon emission. Similarly, photon emission occurs and the electron transitions from point $\mathrm{C}$ in the excited state to point $\mathrm{D}$ in the ground state. The system will relax from point $\mathrm{D}$ to point A by phonon emission. The difference between the absorbed photon energy $\left(E_{A}-E_{B}\right)$ and the emitted photon energy $\left(E_{C}\right.$ $\left.-E_{D}\right)$ is called the Franck - Condon Shift. 
We are interested in the particular case of an electron occupying a deep acceptor level and being excited into the conduction band by the absorption of a photon. We construct the configuration coordinate diagram of Figure 4.21 for this physical situation to illustrate the Franck - Condon principle. Here we show an electron at the minimum energy of a deep acceptor level (point A) excited into the conduction band (point B) by absorption of a photon. The electron ends up at point $\mathrm{C}$ after phonon emission. The difference in energy between point $\mathrm{A}$ and point $\mathrm{C}$ $\left(E_{A C}\right)$ is the thermal ionization energy of the deep acceptor level $\left(E_{T}\right)$. The energy difference

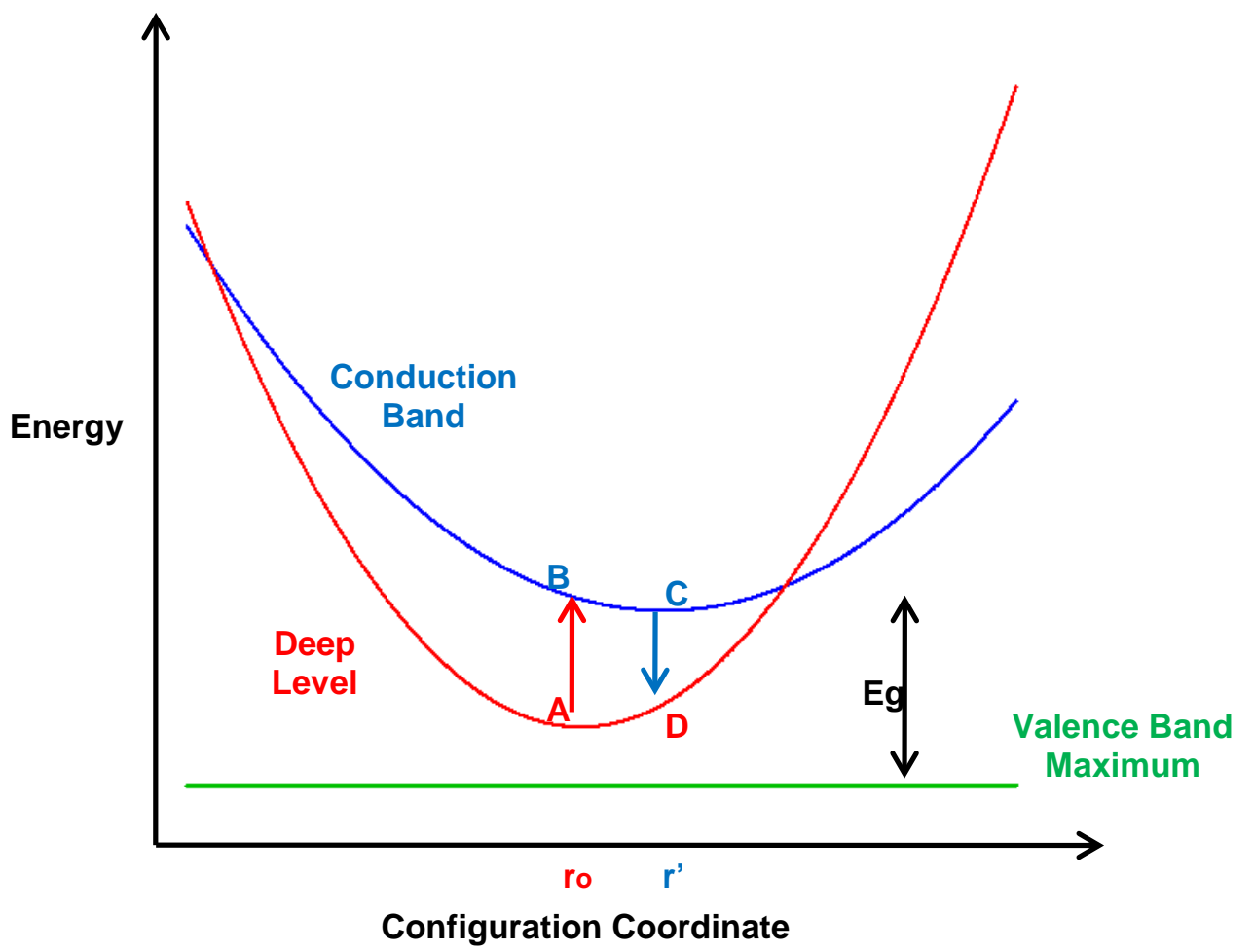

Figure 4.21. Franck - Condon shift is illustrated for the excitation of an electron from a deep acceptor level into the conduction band. The optical energy required for the transition, $E_{O}$, is equal to the sum of the thermal ionization energy $\left(E_{T}\right)$ and the Franck Condon shift (D).

between point $A$ and point $B\left(E_{A B}\right)$ is the optical energy required to excite the electron from the acceptor level into the conduction band $\left(E_{O}\right)$. The energy difference between $E_{O}$ and $E_{T}$ is the Franck - Condon shift for this transition, D. The electron returns to the deep acceptor energy 
level via the nonradiative, multi-phonon, Shockley Read Hall recombination mechanism discussed Chapter 3.

The optical transition of an electron from the deep acceptor level to the conduction band involves the emission of a phonon. This couples the optical transition to the lattice and means that the optical transition will be a function of lattice temperature. A correlation has been found between the depth of the impurity level in the bandgap and the magnitude of the Franck Condon Shift [4.32]. The correlation is believed to be the result of increased localization of the orbit of the electron, or hole, associated with the impurity atom as the ionization energy of the impurity increases [4.32]. The increased localization of the charge associated with the deep impurity leads to increased interaction with neighboring atoms and the lattice. In addition, the electron - phonon coupling of these optical transitions results in broadening of the shape of the long wavelength portion on the photoionization cross section [4.33]. So far, the models that we have reviewed for the optical cross section of the excitation of an electron from a deep acceptor level to the conduction band have only considered the initial and final electronic states of the electron.

Passler [4.34, 4.35, 4.36] has proposed a model for the ionization cross section for deep impurities that includes the coupling to the lattice. Passler's model includes the phonon broadening of the optical transition from a deep level by convolving a temperature dependent Franck - Condon factor with a temperature independent electronic ionization cross section (for example - equations 4.36 and 4.37). Passler's expression for the ionization cross section of a deep level is shown in equation 4.41 .

$$
\sigma(\hbar \omega, T)=\frac{1}{\hbar \omega} \int E_{e} \sigma_{e}\left(E_{e}\right) R\left(\hbar \omega-E_{e}, T\right) d E_{e}
$$


Where $\sigma_{e}$ is the model for the temperature independent ionization cross section and $R\left(\hbar \omega-\mathrm{E}_{\mathrm{e}}, \mathrm{T}\right)$ is the analytical expression for the for the Franck - Condon factor. There are several choices for $\mathrm{R}\left(\hbar \omega-\mathrm{E}_{\mathrm{e}}, \mathrm{T}\right)$. The semi-classical, Gaussian Franck - Condon factor is given in equation 4.42.

$$
R\left(\hbar \omega-E_{e}, T\right)=\frac{1}{\sqrt{2 \pi M^{(2)}(T)}} e^{\left[-\frac{\left(\hbar \omega-E_{e}-D\right)^{2}}{2 M^{(2)}(T)}\right]}
$$

Where D is the Franck - Condon shift and $\mathrm{M}^{(2)}(\mathrm{T})$ is the second moment of the Franck - Condon factor with respect to D [4.34].

Equation 4.40 was used to extract the Franck - Condon shift for several intrinsic defects in CVD grown, thin film, n-type 4H-SiC [4.35]. The thermal ionization energies of the defects were determined using deep level transient spectroscopy (DLTS) and optical capacitance transient spectroscopy (OCTS) was used to measure the optical ionization cross sections. These measurements were made on the $\mathrm{EH}_{6 / 7}, \mathrm{RD}_{1 / 2}, \mathrm{NB}$ and $\mathrm{Z}_{1 / 2}$ deep levels. These are intrinsic defects of unknown origin associated with vacancies and vacancy complexes [4.37, 4.38]. The models for $\sigma_{\mathrm{e}}(\hbar \omega)$ and $\mathrm{R}\left(\hbar \omega-\mathrm{E}_{\mathrm{e}}\right)$ were chosen for the best fit to the OCTS data for the above defects. The results for the thermal ionization energy $\left(E_{T}\right)$, optical ionization energy $\left(E_{0}\right)$ and Franck - Condon shift (D) are listed in Table 4.1 [4.35]. This table lists fairly large values for the Franck - Condon shift (D) for the $\mathrm{RD}_{1 / 2}, \mathrm{NB}$ and $\mathrm{Z}_{1 / 2}$ defect levels. The values of $\mathrm{D}$ exceed $\mathrm{E}_{\mathrm{T}}$ for all three of these defects. $\mathrm{D}>\mathrm{E}_{\mathrm{T}}$ is referred to as the case of large lattice relaxation. 


\begin{tabular}{|c|c|c|c|}
\hline Defect & $\mathrm{E}_{\mathrm{T}}(\mathrm{eV})$ & $\mathrm{E}_{\mathbf{O}}(\mathrm{eV})$ & $\mathbf{D}(\mathrm{eV})$ \\
\hline $\mathrm{Z}_{1 / 2}$ & 0.55 & 1.87 & 1.32 \\
\hline $\mathrm{EH}_{6 / 7}$ & 1.65 & 1.95 & 0.30 \\
\hline $\mathrm{NB}_{1 / 2}$ & 0.80 & 2.08 & 1.28 \\
\hline $\mathrm{RD}_{1 / 2}$ & 1.01 & 2.25 & 1.24 \\
\hline
\end{tabular}

Table 4.1. Thermal ionization, optical ionization and Franck - Condon shift energies for the intrinsic defects $\mathrm{EH}_{6 / 7}, \mathrm{RD}_{1 / 2}, \mathrm{NB}$ and $\mathrm{Z}_{1 / 2}$, in $4 \mathrm{H}-\mathrm{SiC}$ thin films measured using DLTS and OCTS [4.34].

Passler [4.36] also used his expression for $\sigma_{\mathrm{e}}(\hbar \omega)$ (equation 4.39) to determine the Franck - Condon shift for optical ionization cross section data [4.39] for the Trap 1 deep trap in GaN. Passler obtained values of 0.8, 1.9, and 1.1 eV for $\mathrm{E}_{\mathrm{T}}, \mathrm{E}_{\mathrm{O}}$ and $\mathrm{D}$, respectively.

The photon energies required to ionize deep levels in wide bandgap semiconductors are significantly higher than the thermal ionization energies of the deep levels due to electron phonon coupling and the apparent nature of the energy state of the electron occupying the deep level. A conclusion that can be drawn from the exploration of optical transitions from deep levels to the conduction band is that excitation of electrons from the deepest levels in the band gap is unlikely with below bandgap wavelengths. This is a result of the broad predicted transitions which are broadened further and shifted to higher photon energies due to phonon coupling.

\subsection{6) Two Step Optical Transitions}

We have concentrated on the optical excitation of an electron occupying a deep acceptor level into the conduction band. Although we have referred to this process as photoionization, it is actually the photoneutralization of the acceptor level. The acceptor level acquires a negative charge after capturing an electron. The optical transition of the captured electron from the acceptor level to the conduction band results in an electron in the conduction band and a neutral acceptor level. Photoconductive switches based on this type of optical transition will be unipolar devices with one type of charge carrier - electrons. However, there is an optical transition 
process involving the participation of deep levels that results in electrons excited into the conduction band and free holes in the valence band. Photoconductive switches based on this type of optical transition will be bipolar devices with both electron and hole charge carriers participating in electrical conduction. This type of optical transition is called a two-step optical transition [4.33, 4.40, 4.41]. The configuration diagram for this transition is shown in Figure 4.22.

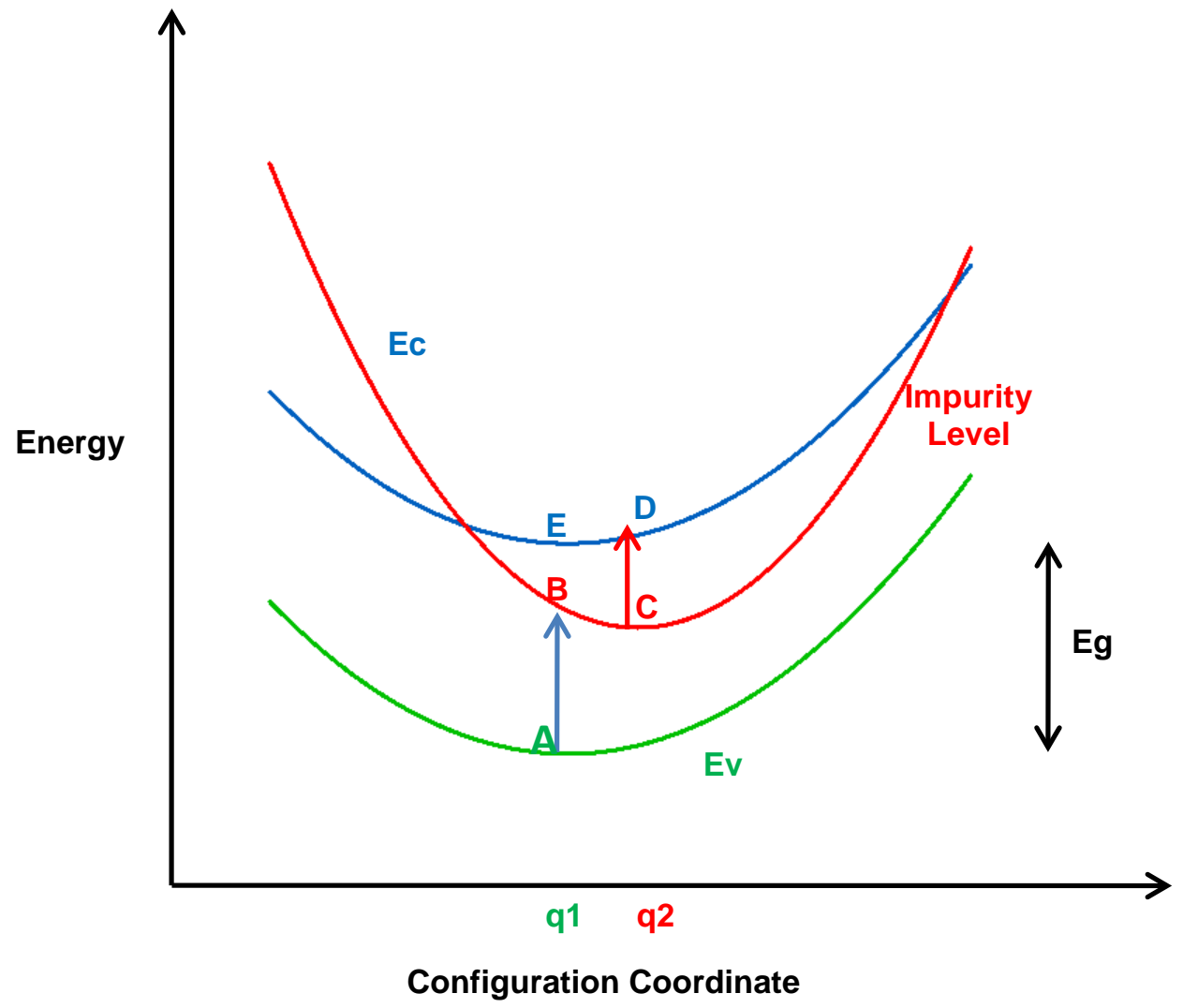

Figure 4.22. Configuration coordinate diagram for two step optical transitions of electrons from the valence band to the conduction band via a deep level

The configuration diagram for the two step optical transition shows an electron at the valence band minimum (point A) optically excited to point B at an impurity energy level by photon absorption. Phonon emission allows the electron to relax to point C, the energy minimum of the impurity level. Absorption of another photon excites the electron to point $\mathrm{D}$ in the 
conduction band. Subsequent phonon emission allows the electron at point D to relax to point E, the energy minimum of the conduction band. The total optical energy $\left(E_{o p}\right)$ required for the two step optical transition portrayed in Figure 4.22 is $E_{B}-E_{A}$ plus $E_{D}-E_{C}$. It is obvious from the configuration diagram that $E_{o p}>E_{g}$. The optical energy required for the two step optical transition is expressed in equation 4.43. $D_{1}$ and $D_{2}$ are the Franck - Condon shifts of the transition from the valence band to the impurity level and the subsequent transition from the impurity level to the conduction band, respectively. The photon energy requirement for this twostep optical transition is shown in equation 4.44. It is clear that the deep levels most likely to

provide significant two step optical transitions will reside in the mid-gap region of the semiconductor.

$$
\begin{gathered}
E_{o p}=E_{g}+D_{1}+D_{2} \\
2 \hbar \omega \geq E_{g}+D_{1}+D_{2}
\end{gathered}
$$

\section{6) Nonlinear Optical Properties of $4 \mathrm{H}-\mathrm{SiC}, 6 \mathrm{H}-\mathrm{SiC}$ and $2 \mathrm{H}-\mathrm{GaN}$}

There are several nonlinear optical properties that can affect the optical absorption of $4 \mathrm{H}$ $\mathrm{SiC}, 6 \mathrm{H}-\mathrm{SiC}$ and $2 \mathrm{H}-\mathrm{GaN}$ photoconductive switches. They are second harmonic generation (SHG), free carrier absorption and two-photon absorption (TPA). We will review these mechanisms to assess their impact on switching performance. 


\subsection{1) Second Harmonic Generation}

4H-SiC, $6 \mathrm{H}-\mathrm{SiC}$ and $2 \mathrm{H}-\mathrm{GaN}$ are uniaxial optical materials with hexagonal crystal structure. As such, they lack inversion symmetry making them capable of second order nonlinear optical interactions of the form shown in equation 4.45 [4.42].

$$
P_{i}^{(2)}\left(\omega_{m}+\omega_{n}\right)=\sum_{j k} \sum_{(m n)} \chi_{i j k}^{(2)}\left(\omega_{m}+\omega_{n}, \omega_{m}, \omega_{n}\right) E_{j}\left(\omega_{m}\right) E_{k}\left(\omega_{n}\right)
$$

Where $P_{i}^{(2)}$ is the second order nonlinear polarization, $\chi_{\mathrm{ijk}}$ is the second order susceptibility tensor, the indices $i, j$, and $k$ refer to the cartesian components of the fields and $E_{j}$ and $E_{k}$ are the electric field amplitudes of the optical waves. Equation 4.44 can be rewritten using the contracted notation $[4.42,4.43]$ to take advantage of symmetries present in the crystal systems of different materials. Equations 4.46 and 4.47 define the second order polarization using the contracted notation.

$$
\begin{gathered}
d_{i j k}=\frac{1}{2} \chi_{i j k}^{(2)} \\
P_{i}^{(2)}\left(\omega_{m}+\omega_{n}\right)=\sum_{j k} \sum_{(m n)} 2 d_{i j k} E_{j}\left(\omega_{m}\right) E_{k}\left(\omega_{n}\right)
\end{gathered}
$$

The tensor $d_{i j k}$ reduces to a 3 X 6 matrix when we apply equations 4.46 and 4.47 to the case of second harmonic generation (SHG) [4.42]. The resulting matrix equation for the nonlinear polarization for the case of SHG is listed in equation 4.48. $4 \mathrm{H}-\mathrm{SiC}, 6 \mathrm{H}-\mathrm{SiC}$ and $2 \mathrm{H}-$ GaN belong to the $C_{6 v}(6 \mathrm{~mm})$ crystal class. The symmetry of the $C_{6 v}$ crystal class results in 5 non-zero components of the $d$ matrix $-d_{15}, d_{24}, d_{31}, d_{32}$, and $d_{33}$. Further, there are only three 
unique components of the $d$ matrix since the symmetry of this crystal class results in $d_{15}$ equal to $\mathrm{d}_{24}$, and $\mathrm{d}_{31}$ equal to $\mathrm{d}_{32}$.

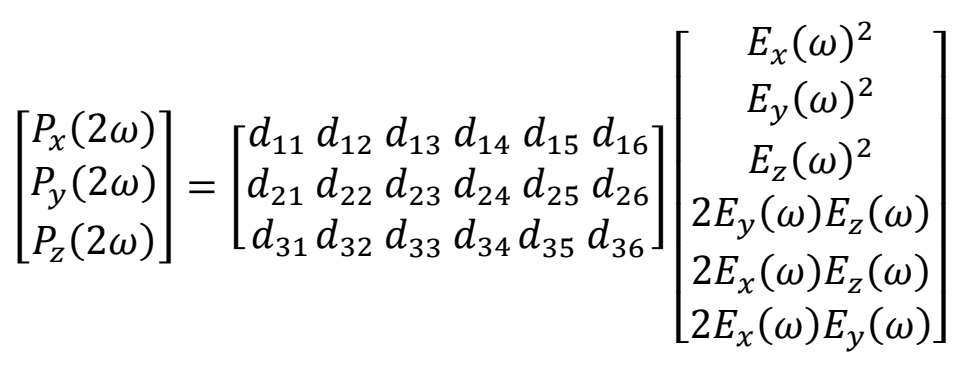

Optical transmission fringe and wedge SHG techniques [4.44 - 4.49] have been used to determine the $d_{15}, d_{31}$ and $d_{33}$ matrix components for powder, thin film and bulk substrate samples of $4 \mathrm{H}-\mathrm{SiC}, 6 \mathrm{H}-\mathrm{SiC}$ and $2 \mathrm{H}-\mathrm{GaN}$. The measured values of $\mathrm{d}_{15}, \mathrm{~d}_{33}$, and $\mathrm{d}_{33}$ for $4 \mathrm{H}-\mathrm{SiC}$, 6H-SiC and 2H-GaN $[4.46,4.48]$ are listed Table 4.2. The measurements were performed at a

\begin{tabular}{|c|c|c|c|c|c|}
\hline & $\mathbf{d}_{\mathbf{1 5}}$ & $\mathbf{d}_{\mathbf{2 4}}$ & $\mathbf{d}_{\mathbf{3 1}}$ & $\mathbf{d}_{\mathbf{3 2}}$ & $\mathbf{d}_{\mathbf{3}}$ \\
\hline $\mathbf{4 H - S i C}$ & $6.7 \mathrm{pm} / \mathrm{V}$ & $6.7 \mathrm{pm} / \mathrm{V}$ & $6.5 \mathrm{pm} / \mathrm{V}$ & $6.5 \mathrm{pm} / \mathrm{V}$ & $-11.7 \mathrm{pm} / \mathrm{V}$ \\
\hline $\mathbf{6 H}-\mathrm{SiC}$ & $6.5 \mathrm{pm} / \mathrm{V}$ & $6.5 \mathrm{pm} / \mathrm{V}$ & $6.7 \mathrm{pm} / \mathrm{V}$ & $6.7 \mathrm{pm} / \mathrm{V}$ & $-12.5 \mathrm{pm} / \mathrm{V}$ \\
\hline $\mathbf{2 H - G a N}$ & $2.5 \mathrm{pm} / \mathrm{V}$ & $2.5 \mathrm{pm} / \mathrm{V}$ & $2.5 \mathrm{pm} / \mathrm{V}$ & $2.5 \mathrm{pm} / \mathrm{V}$ & $-3.8 \mathrm{pm} / \mathrm{V}$ \\
\hline
\end{tabular}

Table 4.2. Second order nonlinear optical coefficients of $4 \mathrm{H}-\mathrm{SiC}, 6 \mathrm{H}-\mathrm{SiC}$ and $2 \mathrm{H}-\mathrm{GaN}$ measured by second harmonic generation methods $[4.46,4.48]$

wavelength of $1064 \mathrm{~nm}$. The independent second order nonlinear optical coefficients were actually measured $\left(\mathrm{d}_{15}, \mathrm{~d}_{32}\right.$, and $\left.\mathrm{d}_{33}\right)$ and the values of $\mathrm{d}_{24}$ and $\mathrm{d}_{32}$ were included in Table 4.2 for completeness. The values for the second order nonlinear optical coefficients shown in Table 4.2 are fairly significant. For comparison purposes, the values of second order nonlinear optical 
coefficients $d_{31}$ and $d_{33}$ at $1064 \mathrm{~nm}$ for Lithium Niobate (crystal symmetry class $3 \mathrm{~m}$ ) are -5.95 and - $33.4 \mathrm{pm} / \mathrm{V}$, respectively.

Second harmonic generation at $1064 \mathrm{~nm}$ will produce a component of light with a wavelength of $532 \mathrm{~nm}$ propagating through the switch substrate. This should not be much cause for concern since the $532 \mathrm{~nm}$ wavelength is still well below the bandgap and is also expected to generate carriers in all the switching materials being considered. However, one study [4.47] observed values of $d_{15}$ and $d_{31}$ that were close in magnitude to the results shown in Table 4.2 for $6 \mathrm{H}-\mathrm{SiC}$ that were fairly uniform over the wavelength range of $400-700 \mathrm{~nm}$. This would cause problems when applying $532 \mathrm{~nm}$ light. Successful second harmonic generation with $532 \mathrm{~nm}$ wavelength light would generate photons at $266 \mathrm{~nm}$. The photon energy at $266 \mathrm{~nm}(4.66 \mathrm{eV})$ is significantly larger than the $6 \mathrm{H}-\mathrm{SiC}$ bandgap $(3.0 \mathrm{eV})$. The $266 \mathrm{~nm}$ photons would be absorbed after propagating only a few microns in the $6 \mathrm{H}-\mathrm{SiC}$ substrate. We would lose the advantage of using below bandgap light if significant second harmonic generation occurs during switch excitation at $532 \mathrm{~nm}$.

The second harmonic conversion efficiency is given in equation 4.49 for the non-depleted pump approximation [4.50].

$$
\eta_{2 \omega}=\frac{8 \pi^{2} d_{e f f}^{2} L^{2} I_{\omega}}{\varepsilon_{0} n_{2 \omega} n_{\omega}^{2} \lambda_{\omega}^{2} c} \frac{\sin ^{2}\left(\frac{\Delta k L}{2}\right)}{\left(\frac{\Delta k L}{2}\right)^{2}}
$$

Where $d_{\text {eff }}$ is the effective value of $d$ obtained from the polarization and propagation direction of the applied optical pulse and orientation of the substrate crystal, $\mathrm{L}$ is the substrate length in the 
direction of propagation, $I_{\omega}$ is the applied optical intensity, and $n_{\omega}\left(n_{2 \omega}\right)$ is the material refractive index at the optical frequency $\omega(2 \omega)$ and $\Delta \mathbf{k}$ is the difference between the sum of the two applied optical wave vectors and the wave vector of the frequency doubled optical wave ( $2 \mathbf{k}_{\omega}$ $\left.\mathbf{k}_{2 \omega}\right)$.

The maximum conversion efficiency is obtained for the condition $\Delta \mathbf{k}$ equal to zero. This is the phased matched condition. For the case of collinear optical beams, the SHG phase matching condition reduces to equation 4.50. The $4 \mathrm{H}-\mathrm{SiC}, 6 \mathrm{H}-\mathrm{SiC}$ and $2 \mathrm{H}-\mathrm{GaN}$ materials cannot be phase matched according to equation 4.50 due to the dispersion of their refractive index. However, since all three materials are positive uniaxial crystals, their refractive index depends on the direction of polarization of the applied optical waves with respect to the optical axis of the crystal. All three materials have an extraordinary index $\left(\mathrm{n}_{\mathrm{e}}\right)$ greater than the ordinary index $\left(\mathrm{n}_{0}\right)$.

$$
n(\omega)=n(2 \omega)
$$

As a result, phase matching can be achieved and equation 4.50 satisfied by selecting Type I, or, Type II phase matching for a positive uniaxial crystal [4.41, 4.42, 4.49]. Figure 4.23 shows the three possible polarization alignments of the applied optical waves with respect to the optical axis of the uniaxial crystal. Figure $4.23 \mathrm{~b}$ ) depicts Type I phase matching which is expressed for the case of SHG in equation 4.51 . Where $\omega_{2}$ is equal to $2 \omega_{1}$ and the $o$ and e superscripts correspond to the ordinary and extraordinary index of refraction of the crystal.

$$
n_{2}^{o} \omega_{2}=n_{1}^{e} \omega_{1}+n_{1}^{e} \omega_{1}
$$



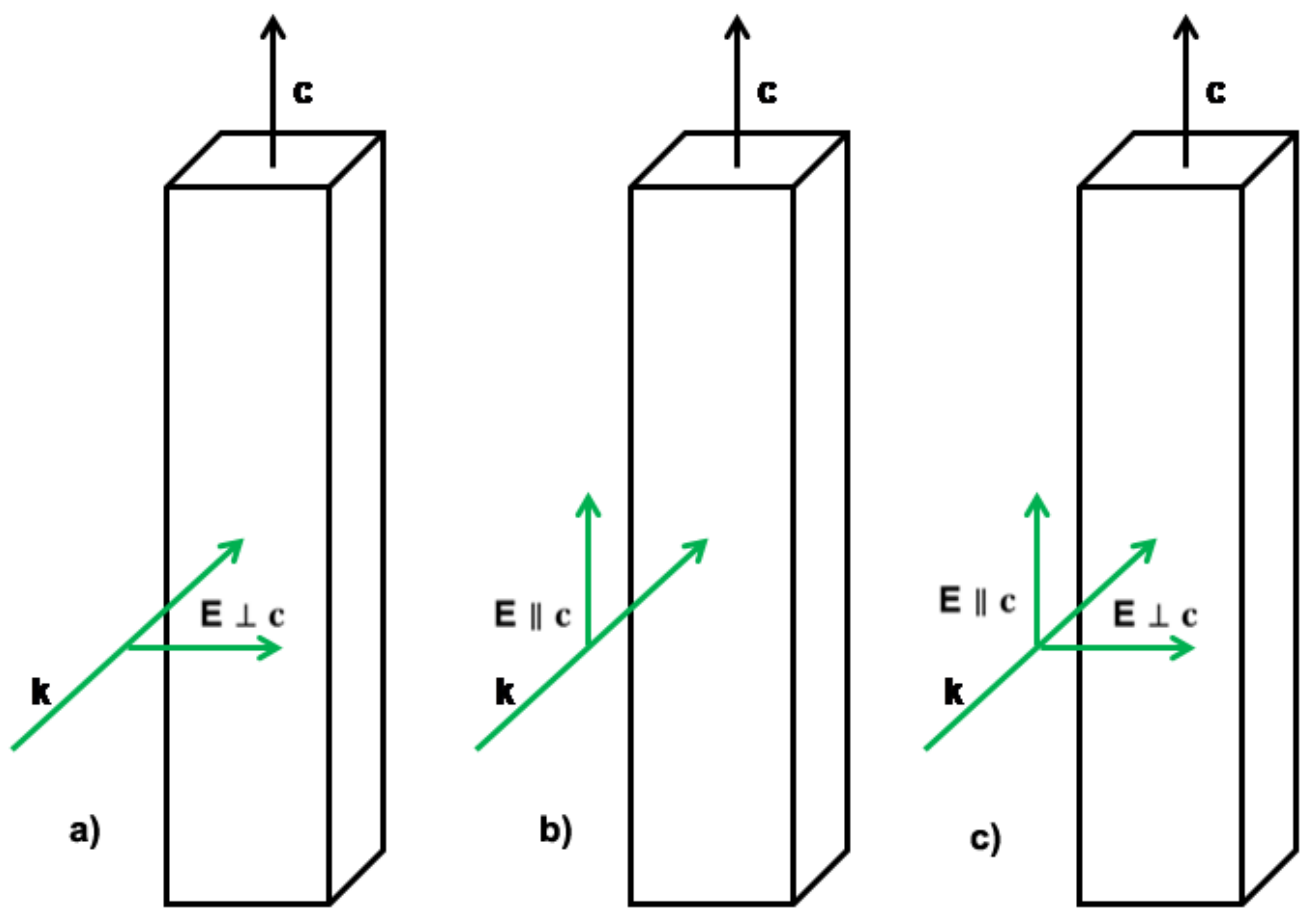

Figure 4.23. Figures $a, b$, and c show the three possible phase-matching alignments for a positive uniaxial crystal. a) Both applied waves are polarized perpendicular to optical axis of crystal. b) Both applied waves are polarized parallel to optical axis of crystal. This is Type I phase matching. c) Two collinear beams, one aligned perpendicular and one aligned parallel, are applied to the crystal. The situation shown in c is Type II phase matching for a positive uniaxial crystal.

The applied optical waves are polarized parallel to the optical axis of the crystal and the second harmonic wave is polarized perpendicular to the optical axis. The phase matching requirement of equation 4.50 can be met using Type I phase matching because $n_{e}>n_{0}$ for all positive uniaxial crystals and both $n_{0}$ and $n_{e}$ monotonically increase with $\omega$. This allows the possibility of $n_{0}(\omega)$ to be equal to $n_{e}(2 \omega)$.

Type II phase matching is illustrated in Figure 4.23 c). Type II phase matching is expressed mathematically in equation 4.52. The two applied optical waves have one polarized parallel and one polarized perpendicular to the optical axis. Type II phase matching allows for the phase matching requirement of equation 4.50 to be met for the reasons described above. 


$$
n_{2}^{o} \omega_{2}=n_{1}^{o} \omega_{1}+n_{1}^{e} \omega_{1}
$$

Figure 4.23 a) shows the two applied optical waves with polarization perpendicular to the optical axis of the crystal. The applied optical waves propagate in the crystal with refractive index $n_{0}$. The phase matching requirement cannot be met for the physical situation depicted in Figure $4.23 c$ ), since $n_{e}>n_{0}$ and both $n_{e}$ and $n_{o}$ monotonically increase with increasing $\omega$. To prevent unwanted second harmonic generation in the photoconductive switch substrates, only normally incident light, polarized perpendicular to the optical axis of the switch material, will be used in switch testing.

\subsection{2) Free Carrier Absorption and Two-Photon Absorption}

The application of below bandgap light to photoconductive switches fabricated from 4H$\mathrm{SiC}, 6 \mathrm{H}-\mathrm{SiC}$ and $2 \mathrm{H}-\mathrm{GaN}$ is expected to generate carriers in the switch volume. The photogenerated carriers can absorb light. Electrons (holes) excited into the conduction (valence) band can be excited into higher (lower) level energy levels by phonon assisted optical absorption [4.50]. The rate of change of the intensity (I) of an optical beam as it propagates through the switch substrate is given in equation 4.53. Where $\alpha$ is the linear absorption, $\sigma_{\mathrm{c}}$ is the free carrier absorption cross section, $\mathrm{N}_{\mathrm{c}}(\mathrm{I})$ is the electron density in the conduction band and $\beta$ is the twophoton absorption coefficient. We are only considering the contribution to free carrier absorption due to electrons in the present discussion. We need to evaluate the free carrier absorption cross-

$$
\frac{\partial I}{\partial z}=-\alpha I-\sigma_{c} N_{c}(I) I-\beta I^{2}
$$

section for electrons to gauge the importance of free carrier absorption compared to the linear absorption during switch operation. A rigorous calculation of $\sigma_{\mathrm{c}}$ requires a quantum mechanical 
approach similar to that of section 4.5.1, since we are evaluating the radiative transition of an electron from one energy state to a higher energy state. A reasonable estimate for $\sigma_{\mathrm{c}}$ can be obtained using the classical formula given in equation 4.54 [4.32]. Where $\lambda$ is the optical wavelength, $\mathrm{m}^{*}$ is the effective electron mass, $\mathrm{n}$ is the index of refraction, $\mathrm{c}$ is the speed of light, $\varepsilon_{0}$ is the permittivity of free space and $\tau$ is the electron scattering time. We can substitute the expression for the electron mobility $\left(\mu_{n}\right)$ at low electric field of equation 4.55 to substitute for the electron scattering time in equation 4.54 to obtain equation 4.56 .

$$
\begin{gathered}
\sigma_{c}=\frac{q^{2} \lambda^{2}}{m^{*} 4 \pi^{2} n \varepsilon_{0} c^{3} \tau} \\
\mu_{n}=\frac{q \tau}{m^{*}} \\
\sigma_{c}=\frac{q^{3} \lambda^{2}}{m^{* 2} 4 \pi^{2} n \varepsilon_{0} c^{3} \mu_{n}}
\end{gathered}
$$

Temperature dependent Hall Effect mobility measurements of semi-insulating 4H-SiC [4.51] and $2 \mathrm{H}-\mathrm{GaN}$ [4.52] substrates yielded the room temperature (300 K) values of 200 and $410 \mathrm{~cm}^{2} / \mathrm{V}$-s, respectively. The semi-insulating, GaN mobility measurements were made on iron compensated substrates almost identical to the GaN substrates used in our switching experiments. The mobility measurements made on semi-insulating $4 \mathrm{H}-\mathrm{SiC}$ material [4.50] were made at the Air Force Research Laboratory on LLNL supplied vanadium and nitrogen co-doped, semi-insulating sample. The free carrier absorption cross section was evaluated for $4 \mathrm{H}-\mathrm{SiC}, 6 \mathrm{H}-\mathrm{SiC}$ and $2 \mathrm{H}-\mathrm{GaN}$ using the Hall mobility values above, an effective electron mass calculated using the tabulated values from Chapter 3, refractive index values at 532 and $1064 \mathrm{~nm}$ from the plots earlier in this chapter, and an assumed mobility value of $100 \mathrm{~cm}^{2} / \mathrm{V}$-s for $6 \mathrm{H}-\mathrm{SiC}$. We have also assumed that 


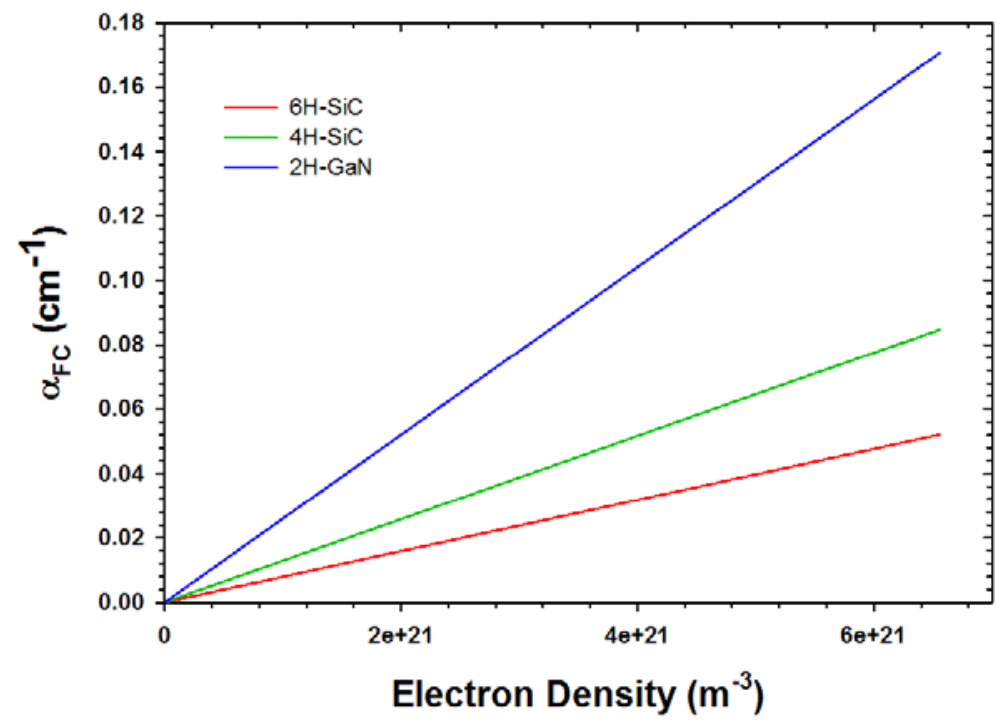

Figure 4.24. Free carrier absorption of $6 \mathrm{H}-\mathrm{SiC}, 4 \mathrm{H}-\mathrm{SiC}$ and $2 \mathrm{H}-\mathrm{GaN}$ plotted as functions of free electrons optically excited into the conduction band with $532 \mathrm{~nm}$ light.

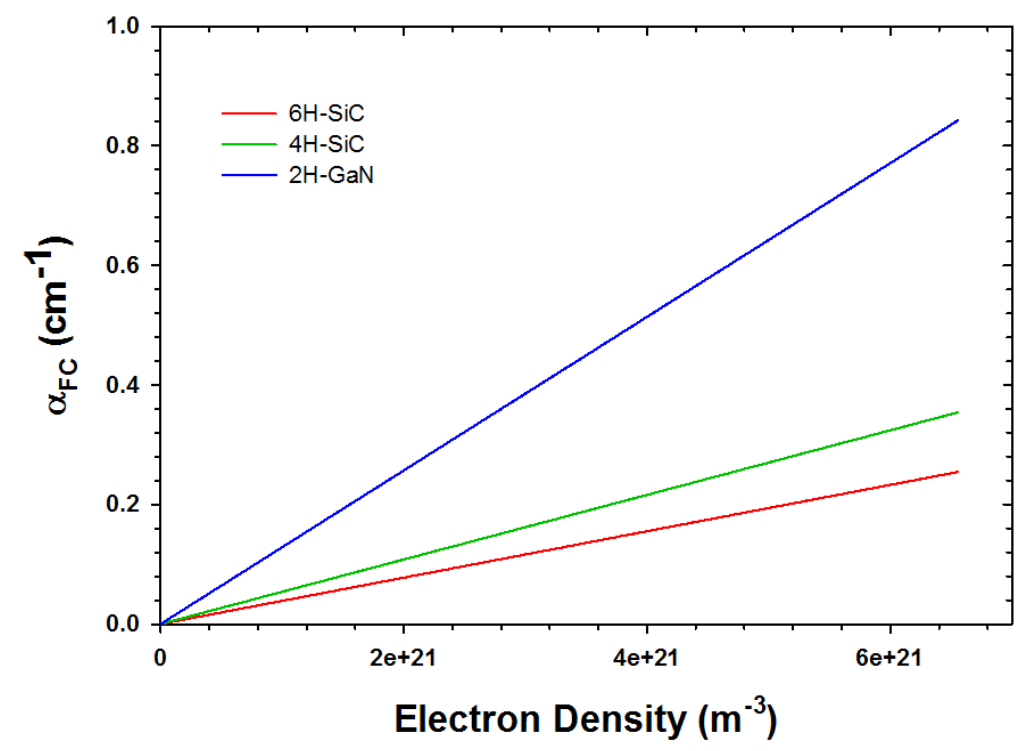

Figure 4.25. Free carrier absorption of $6 \mathrm{H}-\mathrm{SiC}, 4 \mathrm{H}-\mathrm{SiC}$ and $2 \mathrm{H}-\mathrm{GaN}$ plotted as functions of free electrons optically excited into the conduction band with $1064 \mathrm{~nm}$ light.

the Hall factor is $\sim 1.0$ for the three materials $[4.53,4.54]$.The free carrier absorption is given by equation 4.57, where $\mathrm{N}$ is the electron density. The free carrier absorption for $4 \mathrm{H}-\mathrm{SiC}, 6 \mathrm{H}-\mathrm{SiC}$ 
and $2 \mathrm{H}-\mathrm{GaN}$ at wavelengths of 532 and $1064 \mathrm{~nm}$ are plotted as a function of electron density in Figures 4.24 and 4.25, respectively.

$$
\alpha_{F C}=\sigma_{c} N
$$

It is clear from the plots in Figure 4.24 that free carrier absorption is insignificant at 532 $\mathrm{nm}$ compared to the expected linear absorption of $1-2 \mathrm{~cm}^{-1}$. The free carrier absorption increases as expected for the $1064 \mathrm{~nm}$ wavelength excitation shown in Figure 4.25. However, there is no significant free carrier absorption until fairly high photo-generated electron densities are achieved. For example, an electron density of $6 \mathrm{e} 21 / \mathrm{m}^{3}$ in the semi-insulating, $6 \mathrm{H}-\mathrm{SiC}$ corresponds to a resistivity of $10.4 \Omega-\mathrm{cm}$, assuming an electron mobility of $100 \mathrm{~cm}^{2} / \mathrm{V}$-s. This resistivity would result in a $1 \mathrm{ohm}$ on resistance in a switch substrate measuring $1 \mathrm{~cm}^{2}$ and $1 \mathrm{~mm}$ thick. A switch on resistance this low is expected to be obtained at relatively high optical excitation, making free carrier absorption negligible at all but the highest optical excitation powers.

Two-photon absorption occurs when two phase-coherent photons are simultaneously absorbed by an electron, exciting the electron to energy equal to the sum of the photon energies. In our case, the applied photons all have the same energy $(\hbar \omega)$, so the electron energy after twophoton absorption will be $2 \hbar \omega$. However, two-photon absorption in a semiconductor requires $2 \hbar \omega \geq E_{\text {g. }}$. As a result, two-photon absorption need only be considered at $532 \mathrm{~nm}$ excitation. Figure 4.26 shows a simplified energy diagram for the two photon absorption process. Twophoton absorption should not be confused with two step optical transitions discussed previously in this chapter. Two step optical transitions involve an existing energy level that resides within the energy gap. An electron is excited to the existing level and subsequently excited to a higher 
energy level. The mid-level shown in the energy gap in the two-photon absorption diagram is a virtual level. In two-photon absorption, the electron absorbs both photons simultaneously and is excited from its initial level to the final level in a single transition. The two-photon absorption process results with an electron-hole pair being generated in the semiconductor.

\section{Two-Photon Absorption}

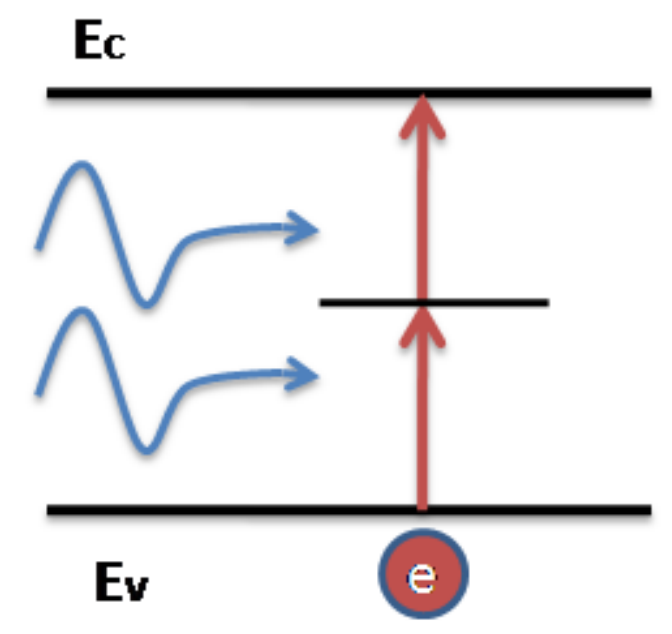

Figure 4.26. A diagram of the two-photon absorption process in a semiconductor that shows the excitation of a valence band electron into the conduction band. It is required that $2 \hbar \omega \geq E_{g}$ for 2 photon absorption.

There are only a few reported measurements of the two-photon absorption coefficient ( $\beta$ ) for $4 \mathrm{H}-\mathrm{SiC}-2.4 \mathrm{~cm} / \mathrm{GW}$ [4.55], $6 \mathrm{H}-\mathrm{SiC}-3.2 \mathrm{~cm} / \mathrm{GW}$ [4.56] and $2 \mathrm{H}-\mathrm{GaN}-20 \mathrm{~cm} / \mathrm{GW}$ [4.57] at $532 \mathrm{~nm}$. Figure 4.27 is a plot of the two-photon absorption ( $\beta \mathrm{I})$ versus optical intensity at the $532 \mathrm{~nm}$ wavelength. Again, we are expecting a linear absorption coefficient of $\sim 1-2 \mathrm{~cm}^{-1}$, so two-photon absorption in $4 \mathrm{H}$ and $6 \mathrm{H}-\mathrm{SiC}$ should be negligible at low optical intensities. GaN on the other hand has significant two photon absorption starting at optical intensities of 10 $\mathrm{MW} / \mathrm{cm}^{2}$. 


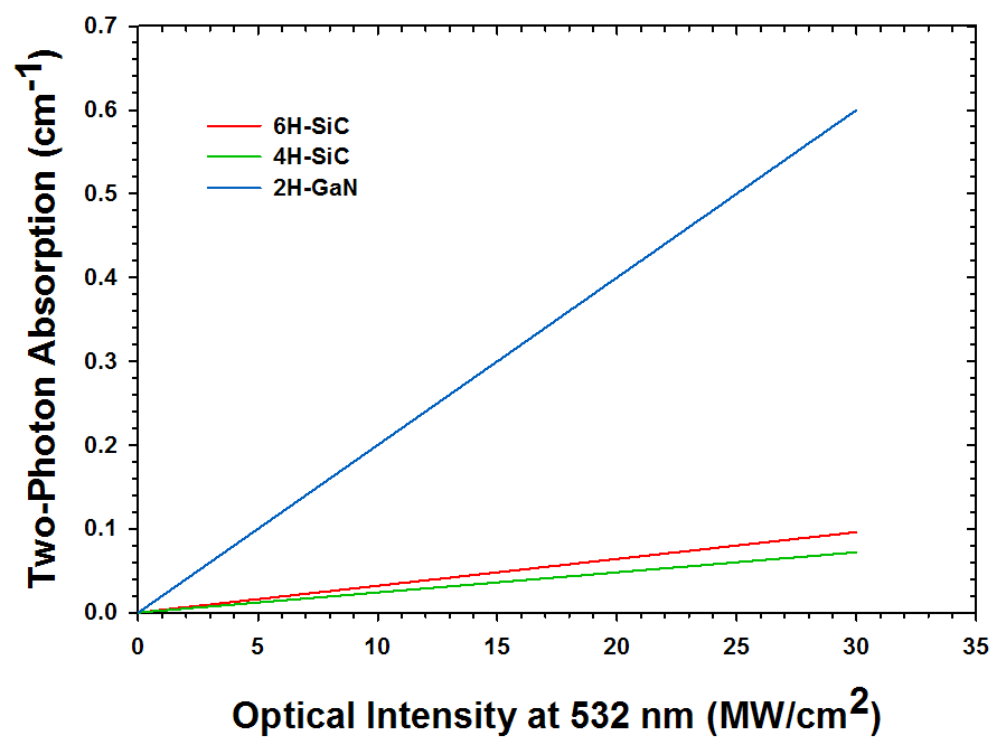

Figure 4.27. Two-photon absorption $(\beta I)$ is plotted versus optical intensity at $532 \mathrm{~nm}$ for $4 \mathrm{H}, 6 \mathrm{H}-\mathrm{SiC}$ and $2 \mathrm{H}-\mathrm{GaN}$.

We want all optical power transmitted through the $6 \mathrm{H}-\mathrm{SiC}$ switch facet to be absorbed exciting electrons from vanadium acceptor levels to the conduction band. Any applied optical signal that that is converted into a second harmonic, or absorbed by free carriers is considered detrimental. Two-photon absorption, or the other hand, generates electron-hole pairs in the switch substrate, which will aid in obtaining a lower switch on resistance. Fortunately, second harmonic generation can be avoided by applying optical pulses with a polarization that is perpendicular to the c-axis of the material. Also, it appears that free carrier absorption is negligible until a large carrier density (low on resistance) is present in the switch substrate.

\section{7) Conclusions}

We have reviewed the optical properties of $2 \mathrm{H}-\mathrm{GaN}, 4 \mathrm{H}-\mathrm{SiC}$ and $6 \mathrm{H}-\mathrm{SiC}$ that are important in determining the performance of an extrinsic photoconductive switch. The refractive index determines how much light is transmitted into the switch material. Selecting an optical 
polarization that is perpendicular to the c axis of $6 \mathrm{H}-\mathrm{SiC}$ switch substrates avoids second harmonic generation (SHG). We have also reviewed models for optical transitions from deep impurities to the conduction band and the effect of the Franck - Condon shift. The optical transition models and the Franck - Condon shift both predict that the maximum of the optical transition cross section will occur at significantly higher energies than the thermal energy of the deep impurity and that the transition threshold occurs at the deep impurity thermal energy. This is significant since it predicts that there will be very few carriers excited from a deep impurity level until the photon energy $(\hbar \omega)$ is sufficiently higher than the thermal energy of the impurity level. This means that optical transitions from the vanadium acceptor level at $0.8 \mathrm{eV}$ below the conduction band in $6 \mathrm{H}-\mathrm{SiC}$ to the conduction band will be much stronger at the $532 \mathrm{~nm}$ than the $1064 \mathrm{~nm}$ wavelength. In addition, we can isolate the response from the vanadium acceptor level in $6 \mathrm{H}$-SiC by exciting the switch with the $1064 \mathrm{~nm}$ wavelength. The $1.17 \mathrm{eV}$ photons of the 1064 nm wavelength have enough energy to excite carriers from the vanadium acceptor level in $6 \mathrm{H}$ $\mathrm{SiC}$, but will not excite carriers from levels deeper than $1.17 \mathrm{eV}$. As a result, we can model the response of a $6 \mathrm{H}-\mathrm{SiC}$ switch substrate to $1064 \mathrm{~nm}$ light by considering the vanadium acceptor levels and levels that are shallower than $1.17 \mathrm{eV}$.

We have concluded from the review of the nonlinear optical properties of $6 \mathrm{H}-\mathrm{SiC}$ and $2 \mathrm{H}-\mathrm{GaN}$ that second harmonic generation can be avoided in $6 \mathrm{H}-\mathrm{SiC}$ by applying light polarized perpendicular to the $\mathrm{c}$ axis of the switch substrate and that free carrier absorption is negligible in both $6 \mathrm{H}-\mathrm{SiC}$ and $2 \mathrm{H}-\mathrm{GaN}$ below carrier densities of $1 \mathrm{e} 16 / \mathrm{cm}^{3}$. Two-photon absorption becomes important at optical intensities greater than $6 \mathrm{MW} / \mathrm{cm}^{2}$ for $2 \mathrm{H}-\mathrm{GaN}$ and $30 \mathrm{MW} / \mathrm{cm}^{2}$ for $6 \mathrm{H}-\mathrm{SiC}$ at the $532 \mathrm{~nm}$ wavelength. 
Chapter 5 will discuss the initial photoconductive switch testing of vanadium compensated $6 \mathrm{H}-\mathrm{SiC}$ and $2 \mathrm{H}-\mathrm{GaN}$.

\section{8) References}

4.1) R.P. Devaty and W.J. Choyke, “Optical characterization of Silicon Carbide Polytypes”, Physica Staus Solidi A, Vol. 162, No. 5, pp. 5-38, 1997.

4.2) G. Pensl and W. J. Choyke, "Electrical and optical characterization of SiC”, Physica B, Vol. 185, pp. 264 - 283, 1993.

4.3) W. J. Choyke, H. Matsunami and G. Pensl, Silicon Carbide: Recent Major Advances, Springer, pp. 413 - 433, 2004

4.4) W. J. Choyke and R. P. Devaty, "Progress in the study of optical and related properties of SiC since 1992”, Diamond and Related Materials, Vol. 6, pp. 1243 - 1248, 1997

4.5) W. J. Choyke, H. Matsunami and G. Pensl, Silicon Carbide, Springer, pp. 136 - 147, 1999

4.6) M. Shur, S. Rumyantsev and M. Levinshtein, Properties of Advanced Semiconductor Materials: GaN, AlN, InN, BN, SiC, SiGe”, Springer, pages 1 and 93, 1999

4.7) A. A. Lebedev, "Deep level centers in Silicon Carbide: A review”, Semiconductors, Vol. 33, No. 2, pp. 107 - 130, 1999.

4.8) A. Henry, T. Egilsson, I. Ivanov, C. Harris, S. Savage, and E. Janzen, "Near band-gap emission in V-implanted and annealed 4H-SiC”, Material Science Forum, Volume 264 268, pp. 497 - 500, 1998

4.9) S. Limpijumnong, W. R. Lambrecht, S. N. Rashkeev and B. Segall, "Optical absorption In the $1-3 \mathrm{eV}$ range of n-type SiC polytypes”, Physical Review B, Vol. 59, No. 30, pp. 12890 - 12899, 1999.

4.10) R. Weingartner, P. Wellmann, M. Bickermann, D. Hofmann, T. Straubinger and A. Winnacker, "Determination of charge carrier concentration in $\mathrm{n}-$ and $\mathrm{p}$ - doped SiC based on optical absorption measurements”, Applied Physics Letters, Vol. 80, No. 1, 2002.

4.11) J. Verdeyen, Laser Electronics, pp. 16 - 20, Third Edition, Prentice Hall, 1995.

4.12) M. Born and E. Wolf, Principles of Optics, pp. 684 - 686, Sixth Edition, Pergamon Press, 1980.

4.13) S. Sridara, T. Eperjesi, R.P. Devaty, and W. J. Choyke, "Penetration depths in the ultraviolet for $4 \mathrm{H}, 6 \mathrm{H}$ and $3 \mathrm{C}$ silicon carbide at seven common laser pumping wavelengths” Materials Science and Engineering B, Vol. 61 - 62, pp. 229 - 233, 1999.

4.14) J. F. Muth, et. al., "Absorption coefficient, energy gap, exciton binding energy and recombination lifetime of GaN obtained from transmission measurements”, Applied Physics Letters, Vol. 71, No. 3, pp 2572 - 2574, 1997.

4.15) J. F. Muth, et. al., "Absorption coefficient and refractive index of GaN, AlN and AlGaN alloys”, Materials Research Society Symposium, “GaN and Related Alloys”, Vol. 537, p. G5.2, 1998.

4.16) P. T. B. Shaffer, "Refractive index, dispersion, and birefringence of silicon carbide polytypes”, Applied Optics, Vol. 10, No. 5, pp 1034 - 1036, 1971. 
4.17) W. J. Choyke and L. Patrick, "Refractive index and low frequency dielectric constant of 6H-SiC”, Journal of the Optical Society of America, Vol. 58, No. 3, pp. 377 - 379, 1968.

4.18) W. J. Choyke and E. D. Palik, "Silicon Carbide” in Handbook of Optical Constants of Solids, pp. 587 - 595, Academic Press, 1985.

4.19) M. Kildemo, F. Hansteen and O.Hunderi, "Details of below bandgap uniaxial dielectric function of $\mathrm{SiC}$ polytypes studied by ellipsometry and polarized light transmission spectroscopy”, Journal of Applied Physics, Vol. 91, No. 9, pp. 5677 - 5685, 2002

4.20) E. Ejder, "Refractive index of GaN”, Physica Status Solidi A, Vol. 6, pp. 445 - 448, 1971

4.21) G. Yu, H. Ishikawa, T. Egawa, T. Soga, J. Watanabe, T. Jimbo and M. Umeno, "Polarized reflectance spectroscopy and spectroscopic ellipsometry determination of the optical anisotropy of Gallium Nitride on Sapphire”, Japanese Journal of Applied Physics, Vol. 36, Pt. 2, pp. 1029 - 1031, 1997.

4.22) Richard H. Bube, Photoelectronic Properties of Semiconductors, page 9, Cambridge, 1992

4.23) Alan Rolf Mickelson, Physical Optics, pp. 62 - 63, Van Nostrand Reinhold, 1992

4.24) Mark Fox, Optical Properties of Solids, pp. 266 - 269, Oxford University Press, 2003

4.25) Shun Lien Chuang, Physics of Optoelectronic Devices, pp. 337 - 343, Wiley Interscience, 1995

4.26) D. A. B. Miller, Course Notes for Semiconductor Optoelectronic Devices, pp. 45 - 52, Stanford University, Winter 2006

4.27) E. Burstein, G. Picu and N. Sclar, "Optical and photoconductive properties of silicon and germanium”, pp. 353 - 413, in Photoconductivity Conference, John Wiley and Sons, 1954

4.28) G. Lucovsky, “On the photoionization of deep impurity centers in semiconductors”, Solis State Communications, Vol. 3, pp. 299 - 302, 1965

4.29) B. K. Ridley, "The photionization cross section of deep level impurities in semiconductors”, Journal of Physics C: Solid State Physics, Vol. 13, pp. 2015 - 2026, 1980

4.30) J. M. Ziman, Principles of the Theory of Solids, pp. 274 - 278, Second Edition, Cambridge University Press, 1995

4.31) Baldassare Di Bartolo, Optical Interactions in Solids, pp. 420 - 423, Second Edition, Cambridge University Press, 2010

4.32) Jacques I. Pankove, Optical Processes in Semiconductors, pages 74 and 140, Dover, 1975

4.33) V. Gavryushin, "On the possibility of the direct study of local electron - phonon interaction in semiconductors” JETP Letters, Vol. 78, No. 5, pp. 309 - 313, 2003

4.34) R. Passler, "Alternative electronic parts for multiphonon - broadened photoionization cross sections of deep levels in SiC”, Journal of Applied Physics, Vol. 97, 113533, 2005

4.35) M. Kato, S. Tanaka, M. Ichimura, E. Arai, S. Nakamura, T. Kimoto and R. Passler, "Optical cross sections of deep levels in 4H-SiC", Journal of Applied Physics, 100, 053708, 2006

4.36) R. Passler, "Photoionization cross-section analysis for a deep trap contributing to current collapse in GaN field-effect transistors", Journal of Applied Physics, Vol. 96, No. 1, pp. $715-722,2001$ 
4.37) A. Kawasuso, M. Weidner, F. Redmann, T. Frank, P. Sperr R. Krause-Rehberg, W. Trifthauser and G. Pensl, "Vacancies in He-implanted $4 \mathrm{H}$ and $6 \mathrm{H} \mathrm{SiC}$ epilayers studied by positron annihilation”, Physica B, Vol. 308 - 310, pp. 660 - 663, 2001

4.38) S. Sasaki, K. Kawahara, G. Feng, G. Alfieri and T. Kimoto, "Major deep levels with the same microstructures observed in n-type $4 \mathrm{H}-\mathrm{SiC}$ and $6 \mathrm{H}-\mathrm{SiC}$ ”, Journal of Applied Physics, Vol. 109, 013705, 2011

4.39) P. Klein, J. Freitas, Jr., S. Binari and A. Wickenden, "Observation of deep traps responsible for current collapse in GaN metal-semiconductor field-effect transistors", Applied Physics Letters, Vol. 75, No. 25, pp. 4016 - 4018, 1999

4.40) V. Gavryushin, "Direct characterization of electron - phonon interaction for deep levels in semiconductors” Lithuanian Journal of Physics, Vol. 42, No. 1, pp. 23 - 29, 2002

4.41) R. Baltramiejunas and V. Gavryushin, "Nonlinear spectroscopy of deep levels in widegap II-VI semiconductors”, Journal of Crystal Growth, Vol. 101, pp. 699 - 704, 1990

4.42) R. W. Boyd, Nonlinear Optics, pages 19 and 39, Second Edition, Academic Press, 2003

4.43) F. Zernike and J. E. Midwinter, Applied Nonlinear Optics, pp. 34 - 41, John Wiley and Sons, 1973

4.44) L. C. Chao and A. J. Steckl, "CW blue-green light emission from GaN and SiC by sumfrequency generation and second harmonic generation”, Journal of Electronic Materials, Vol. 29, No. 9, pp 1059 - 1062, 2000.

4.45) S. Niedermeier, H. Schillinger, R. Sauerbrey, B. Adolph, and F. Bechstedt, "Second harmonic generation in silicon carbide polytypes”, Applied Physics Letters, Vol. 75, pp. $618-620,1999$

4.46) H. Sato, M. Abe, I. Shoji, J. Suda, and T. Kondo, “Accurate measurements of the secondorder nonlinear optical coefficients of $6 \mathrm{H}$ and $4 \mathrm{H}$ silicon carbide”, Journal of the Optical Society of America B, Vol. 26, No. 10, pp. 1892 - 1896, 2009

4.47) P. M. Lundquist, et. al.,"Second harmonic generation in hexagonal silicon carbide", Applied Physics Letters, Vol. 66, No. 15, pp. 1883 - 1885, 1995

4.48) M. Abe, et. al., "Accurate measurement of quadratic nonlinear-optical coefficients of Gallium Nitride”, Journal of the Optical Society of America, Vol. 27, No. 10, 2010

4.49) N. Sanford, et. al., "Measurements of second order susceptibilities of GaN and AlGaN", Journal of Applied Physics, Volume 97, 2005

4.50) R. L. Sutherland, Handbook of Nonlinear Optics, pp. 613 - 615, Second Edition, Marcel Dekker, 2003

4.51) W.C. Mitchel, Air Force Research Laboratory, Wright-Patterson AFB, Ohio

4.52) Z. - Q. Fang, et al, "Deep centers in semi-insulating Fe-doped native GaN substrates grown by hydride vapor phase epitaxy”, Physica Status Solidi C, Vol. 5, No. 6, pp. 1508 -1511 (2008)

4.53) G. Ng, D. Vasileska and D. Schroder, “ Calculation of the electron Hall mobility and Hall scattering factor in 6H-SiC”, Journal of Applied Physics, Vol. 106, 053719 (2009)

4.54) G. Chen, J. Lin, H. Jiang, "Effects of electron mass anisotropy on Hall factors in 6HSiC”, Applied Physics Letters, Vol. 68, No. 10, pp. 1341 - 1343, 1996

4.55) V. Grivickas, P. Grivickas, J. Linros, and A. Galeckas, “Two photon spectroscopy of 4H$\mathrm{SiC}$ by using laser pulses at below bandgap frequencies”, Materials Science Forum, Volumes 457 - 460, Silicon Carbide and Related Materials, 2003 
4.56) A. Borshch, M. Brodyn, and V. Gayvoronsky, "Diagnostic of optical nonlinearities: spatial beam distortion technique and its application to semiconductors and novel materials", Proceedings of SPIE, Vol. 5024, pp.128 - 136, 2003

4.57) V. Pacebutas, A. Krotkus, T. Suski, P. Perlin and M. Leszczynski, "Photoconductive Zscan measurement of multiphoton absorption in GaN", Journal of Applied Physics, Vol. 92, No. 11, pp. 6390 - 6392, 2002 


\section{Chapter 5: Initial Testing of Vanadium Compensated 6H-SiC and Iron Compensated 2H-GaN Extrinsic Photoconductive Switches}

Photoconductive switch assemblies were constructed from initial samples of vanadium compensated $6 \mathrm{H}-\mathrm{SiC}$ and iron compensated $2 \mathrm{H}-\mathrm{GaN}$ substrates. The dark resistance and photoconductive switching response of the $6 \mathrm{H}-\mathrm{SiC}$ and $2 \mathrm{H}-\mathrm{GaN}$ materials was obtained for wavelengths of 532 and $1064 \mathrm{~nm}$. The primary focus of the photoconductive switching tests were to determine the minimum switch on resistance that could be obtained and based on these results, are high power, extrinsic photoconductive switches based on these materials feasible.

\section{1) 6H-SiC Test Results}

Vanadium compensated, a-plane, 6H-SiC substrates grown by the Physical Vapor Transport (PVT) method was used to construct all photoswitch substrates. The vanadium dopant was added to compensate the net n-type $6 \mathrm{H}$-SiC material due to the larger density of nitrogen donors present in the as grown $6 \mathrm{H}-\mathrm{SiC}$ crystal compared to the density of boron acceptors. The nominal vanadium doping was one part per million in the $6 \mathrm{H}-\mathrm{SiC}$ to achieve a minimum semiinsulating resistivity of $1 \times 10^{8} \mathrm{ohm}-\mathrm{cm}$. The nominal substrate dimensions were $1.2 \mathrm{~cm}$ in length, $1.2 \mathrm{~cm}$ in width and $400 \mu \mathrm{m}$ thick. The nominal substrate size and resistivity were the only specifications that were listed in the order for the vanadium compensated, $6 \mathrm{H}-\mathrm{SiC}$ substrates. The bare, semi-insulating, $6 \mathrm{H}-\mathrm{SiC}$ substrates were sent to Semi-South Laboratories for contact metallization and annealing.

\section{H-SiC Switch Assembly Tests}

Four PCSS devices were fabricated from samples of $400 \mu \mathrm{m}$ nominal thickness, $1.2 \mathrm{~cm}$ per side, square substrates of “a” plane, vanadium compensated, semi-insulating 6H-SiC. The 
four facets of the substrates were cleaved. The contacts consist of a $0.8 \mathrm{~cm}$ diameter, circular metalization centered on opposing sides of the substrate. The metalization consisted of layers of nickel, titanium, platinum and gold. The metal deposition and anneal were performed at SemiSouth Laboratories Inc. in Starksville, MS. The metallization process consisted of a $200 \mathrm{~nm}$ deposition of nickel followed by a rapid thermal anneal at $1000{ }^{\circ} \mathrm{C}$ for two minutes. The rapid thermal anneal facilitated the formation of nickel-silicide $\left(\mathrm{Ni}_{2} \mathrm{Si}\right)$ at the nickel/6H-SiC interface. The nickel-silicide layer has been shown to make a low resistivity ohmic contact to n-type, $6 \mathrm{H}$ $\mathrm{SiC}$ [5.1]. The remaining metallization layers of $200 \mathrm{~nm}$ of Ti, $200 \mathrm{~nm}$ of Pt and $500 \mathrm{~nm}$ of $\mathrm{Au}$ were deposited after the rapid thermal anneal. Indium coated, copper electrodes were vacuum brazed to the metalized substrates at LLNL using 0.001 inch thick, 0.8 inch diameter indium solder preforms to facilitate electrical connection. A schematic of the PCSS, a metalized substrate and the finished 6H-SiC PCSS assembly are shown in Figures 5.1 and 5.2. The dark resistance and photoconductive switching response at wavelengths $532 \mathrm{~nm}$ and 1064 were measured for three of the completed 6H-SiC PCSS assemblies.

\subsection{1) 6H-SiC Dark Resistance Measurements}

A schematic for the circuit used to measure the dark resistance of the completed $6 \mathrm{H}-\mathrm{SiC}$ PCSS is shown in Figure 5.3. The dark current of the PCSS was measured using a Keithley 6485 model picoammeter electrically in series with the PCSS while applying a dc bias. The Keithley 6485 picoammeter has a measurement range from $5 \mathrm{~mA}$ to $20 \mathrm{fA}$ with a resolution of $10 \mathrm{fA}$. The voltage across the PCSS was monitored with a precision (1\%) $1000 \mathrm{M} \Omega$, resistive divider constructed with a matched set of Caddock, type MG815, 999.0 M $\Omega$ and 1.0 M $\Omega$ resistors. The dc divider voltage was monitored using a Hewlett-Packard 34401A, 6 1/2 digit multimeter with $0.0035 \%$ dc accuracy. A maximum dc bias voltage of 1000 volts was supplied by a HP 6110A 
dc power supply which was isolated from the PCSS by a $200 \mathrm{k} \Omega$ resistor to limit the maximum possible current into the picoammeter to $5 \mathrm{~mA}$, or less. The resistors and $6 \mathrm{H}$-SiC PCSS were mounted on a printed circuit board and enclosed in a light-tight metal chassis as shown in Figure 5.4 .

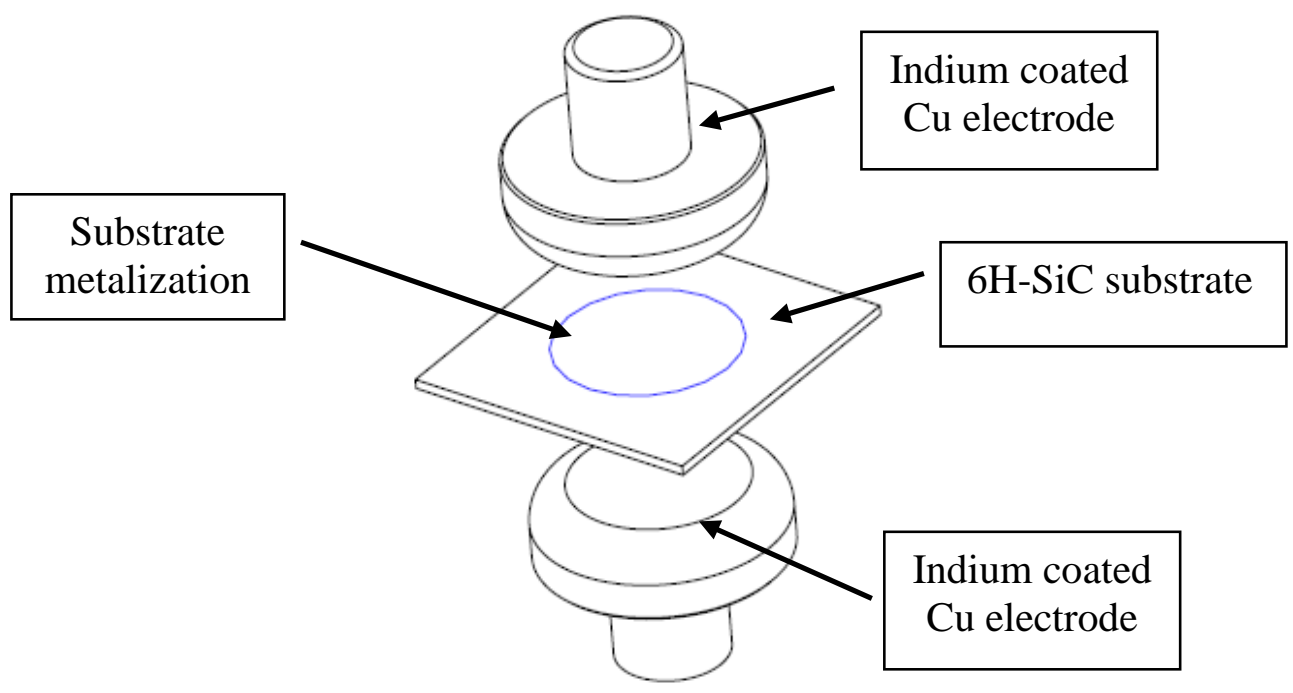

Figure 5.1. Schematic of 6H-SiC PCSS assembly

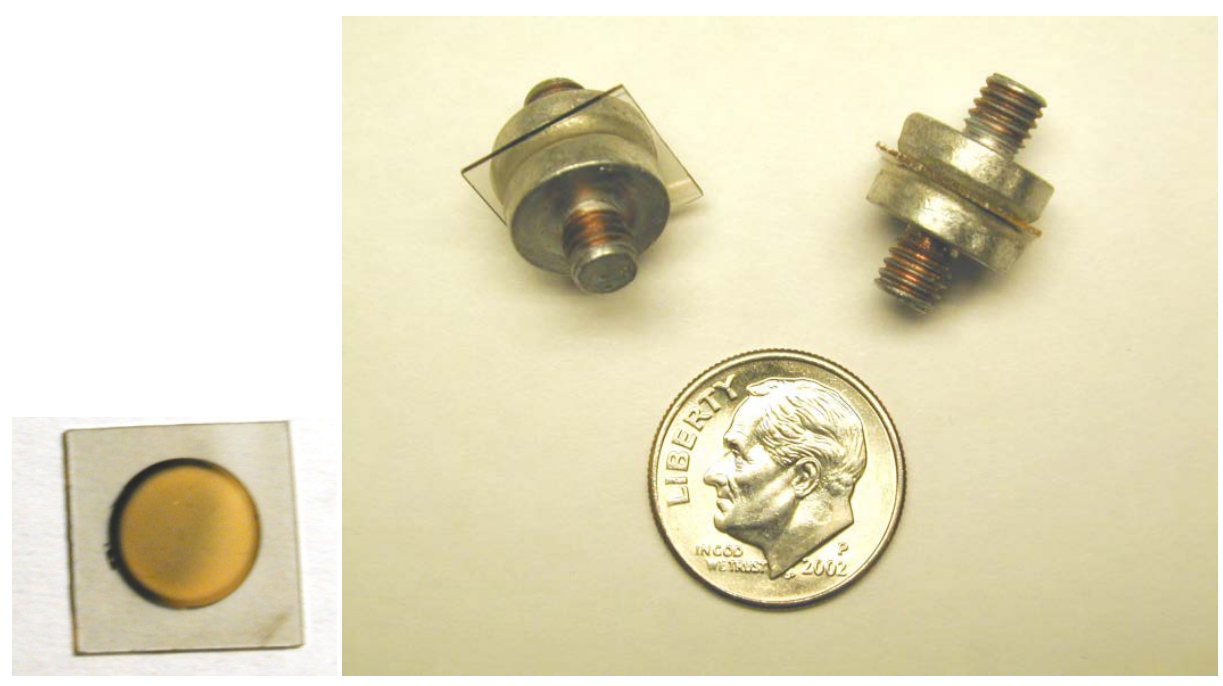

Figure 5.2. Vanadium compensated, semi-insulating, metalized a-plane, $6 \mathrm{H}$-SiC substrate and completed PCSS assemblies 


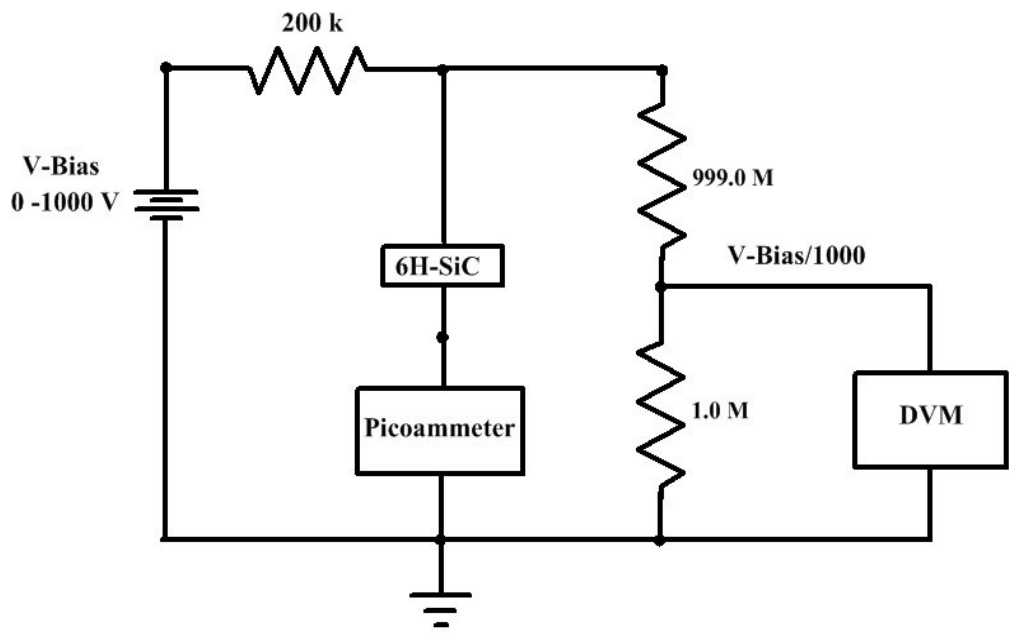

Figure 5.3. Dark current measurement circuit schematic

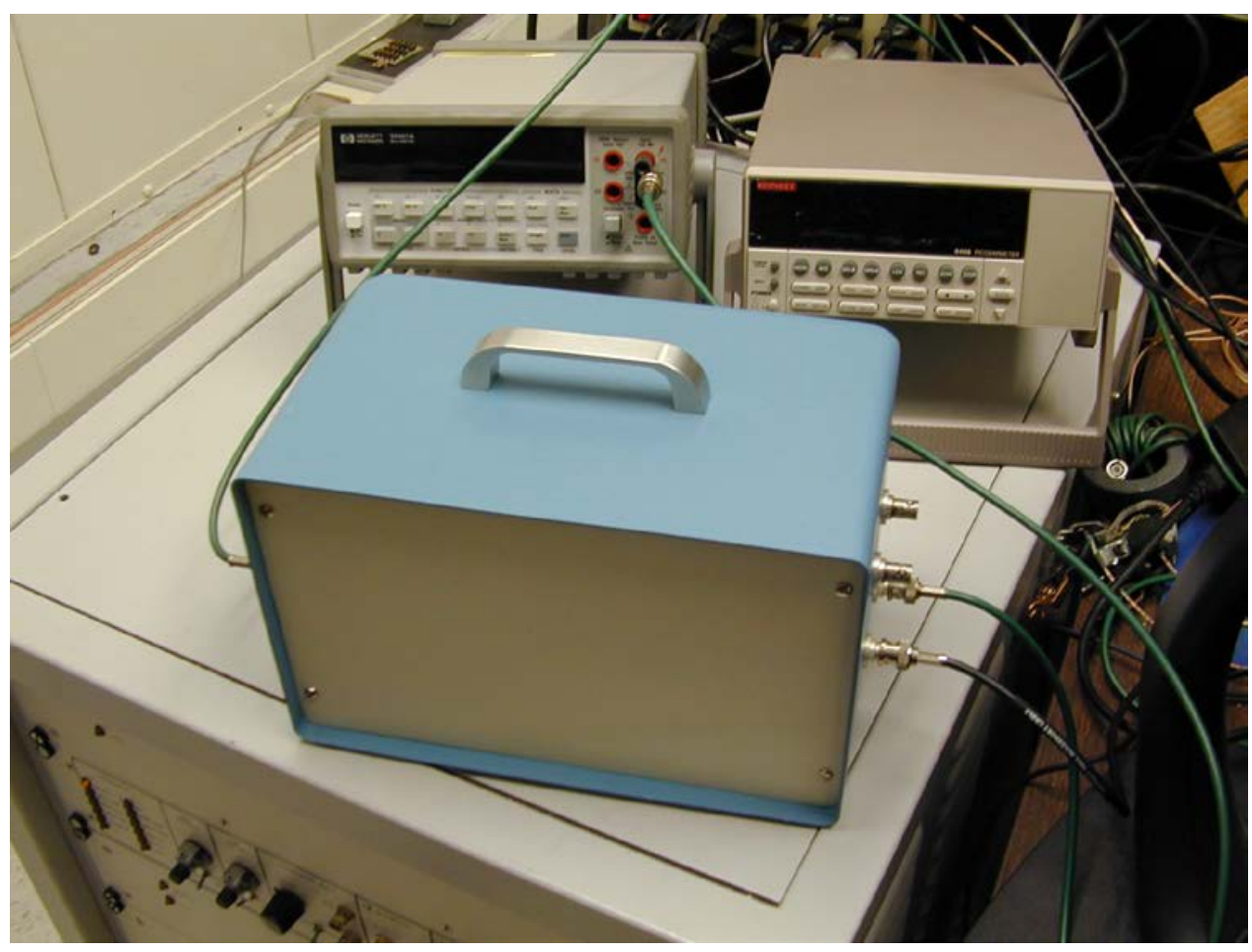

Figure 5.4. Dark current measurement system

The dark current at room temperature for three 6H-SiC PCSS devices were measured using the system shown in Figure 4.4. The three PCSS devices were identified as the 'green', 'black' and 'silver' switches. The green switch measures $1.209 \mathrm{~cm}$ by $1.190 \mathrm{~cm}$ by $401 \mu \mathrm{m}$ thick. The black switch measures $1.222 \mathrm{~cm}$ by $1.207 \mathrm{~cm}$ by $372 \mu \mathrm{m}$ thick. The silver switch 
measures $1.222 \mathrm{~cm}$ by $1.206 \mathrm{~cm}$ by $382 \mu \mathrm{m}$ thick. The dark current for the green switch is shown in Figure 5.5. The green switch dark current varies linearly with the dc bias voltage and ranges from a small fraction of a nano-ampere up to $5.8 \mathrm{nA}$ at the highest dc bias of 1000 Volts. The linear dark current versus bias voltage variation could indicate true ohmic contacts on the PCSS. However, our $\mathrm{Ni}_{2} \mathrm{Si}$ based contacts provide good ohmic contacts to n-type, $6 \mathrm{H}-\mathrm{SiC}$. The $\mathrm{Ni}_{2} \mathrm{Si}$ contacts were selected based on the rationale that the $6 \mathrm{H}$-SiC substrate would become n-type when subjected to optical excitation due to the photo-generation of large numbers of electron carriers. We should not expect our contacts to necessarily be ohmic on semi-insulating 6H-SiC. The linear relationship may be the result of the large number of electron traps present in the material which capture any excess injected carriers. The green switch dark resistance and resistivity were calculated to be $170 \mathrm{G} \Omega$ and $6.1 \times 10^{12} \Omega$-cm using the dark current data of Figure 5.5.

The dark currents versus dc bias voltage for the black and silver switches are shown in Figure 5.6. The dark current for both the black and silver switches, similar to the green switch, are linearly related to the bias voltage. However, the dark currents in the black and silver switches are significantly lower than that of the green switch. The dark current in the black switch ranges from $90 \mathrm{pA}$ at a bias voltage of 100 volts to $550 \mathrm{pA}$ at 500 volts bias. The black switch dark resistance and resistivity were calculated to be $868.5 \mathrm{G} \Omega$ and $3.2 \times 10^{13} \Omega$-cm. The dark current for the silver switch ranges from 17 pA at 50 volts to 254 pA at 500 volts dc bias. The silver switch dark resistance and resistivity are calculated to be $1920 \mathrm{G} \Omega$ and $6.91 \times 10^{13} \Omega$ $\mathrm{cm}$ from these measurements. 


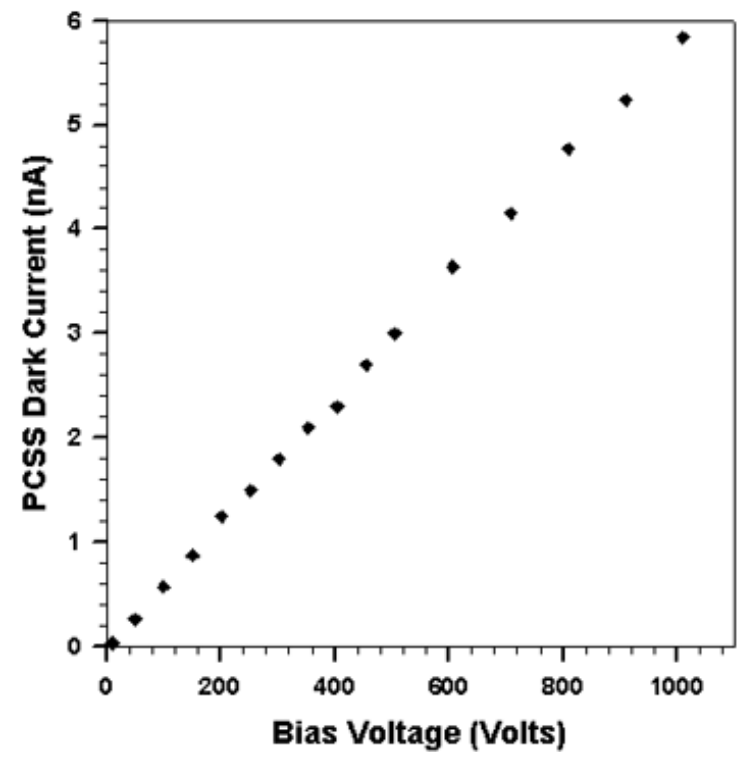

Figure 5.5. Variation of green switch dark current with bias voltage at room temperature

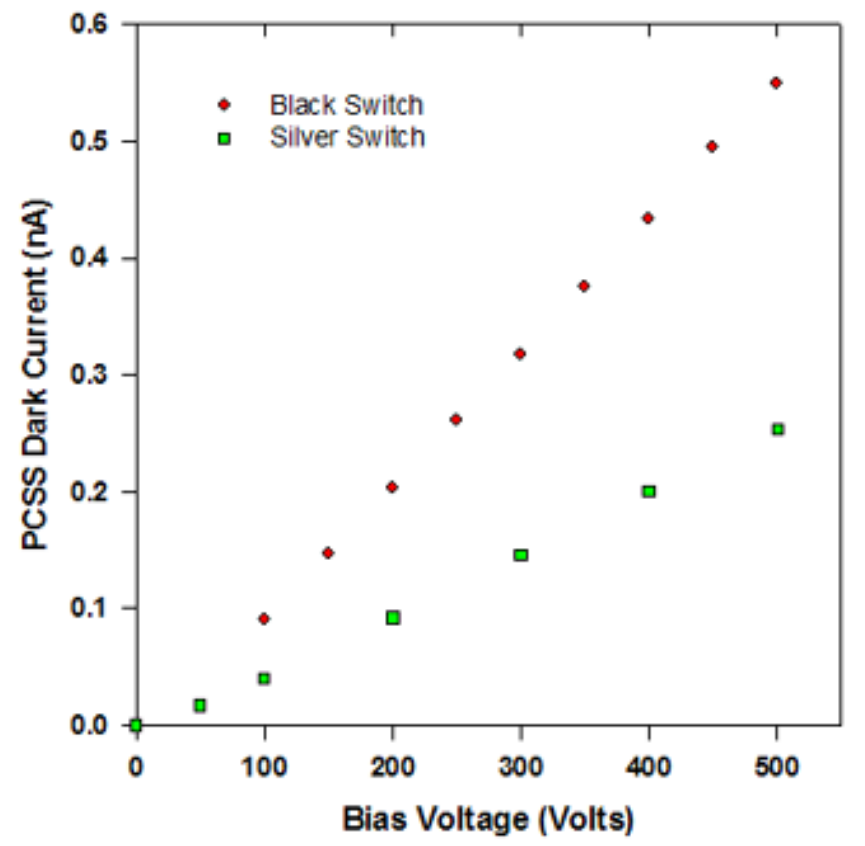

Figure 5.6. Variation of black switch dark current with bias voltage at room temperature

The substrates for the green, black and silver switches were cut from different locations in the same $6 \mathrm{H}-\mathrm{SiC}$ crystal, but the vanadium, nitrogen, boron and defect density vary throughout the crystal. Nitrogen decays during crystal growth (along the c axis) more rapidly 
than either vanadium, or boron. As a result, the compensation ratio and resistivity should be expected to vary along the c axis. The difference in dark resistance in substrates cut from the same crystal is not surprising. What is surprising is the large difference between the measured and calculated (Figure 3.11) values of dark resistivity for vanadium compensated 6H-SiC. The $6 \mathrm{H}-\mathrm{SiC}$ substrates that were tested have expected vanadium, nitrogen and boron densities of $~$ $10^{17}, 3-5 \times 10^{16}$ and $2-3 \times 10^{16}$ per cubic centimeters, respectively. The simple dark resistivity

model we developed predicts a resistivity of between $10^{9}$ and $10^{10} \Omega$-cm for these relative dopant values. The green, black, and silver switches have measured dark resistances two to three orders of magnitude higher. Possible explanations for this discrepancy are: First, the expected values for the vanadium, nitrogen and boron density could be wrong. The expected values were derived from the one part per million ratio of vanadium added to the SiC powder for compensation and typical background levels of nitrogen and boron. Second, the resistivity model assumes that all vanadium, nitrogen and boron are substitutional dopants that are electrically active. This is a first approximation that is not completely accurate. Third, the model assumes that vanadium, nitrogen and boron are the only dopants/defects contained in the $6 \mathrm{H}-\mathrm{SiC}$ crystal. It is possible that other dopants, for instance aluminum, an acceptor, could be present in high enough density to increase the dark resistance. In addition, there are a number of crystal defects and dopant complexes in $6 \mathrm{H}-\mathrm{SiC}$ that act as electron traps and were not accounted for in the simple resistivity model.

\section{2) Photoconductive Switching Test Apparatus}

\subsection{1) Electrical Components}

Three 6H-SiC, switch assemblies, the green, black, and silver switches, were tested in the general test circuit shown in Figure 5.7. The test circuit was constructed on a 2.5 inch long, 1.9 
inch wide, 0.125 inch thick FR-4 printed circuit board with a 2 ounce surface layer of electrodeposited copper. The load resistors and storage capacitors were positioned on opposite sides of the circuit board from a series and parallel combination of components to minimize circuit inductance. The total inductance of the low voltage board was calculated to be $15 \mathrm{nH}$. A maximum $\mathrm{L} / \mathrm{R}$ rise-time of 300 picoseconds is calculated for the low voltage board from the estimated inductance and nominal $50 \mathrm{ohm}$ load resistance.

A low and high voltage version of the switch test circuit were designed and constructed. The low voltage board is used over the voltage range of 150 to 550 volts. The low voltage board consists of a $1.5 \mu \mathrm{F}$ storage capacitor assembly and a $53.0 \mathrm{ohm}$ load resistor assembly. The 1.5 $\mu \mathrm{F}$ capacitor was formed by the series combination of two sets of three, parallel connected, $1 \mu \mathrm{F}$, 250 volt, metalized polyester film capacitors. The $53.00 \mathrm{ohm}$ load resistor was constructed from a parallel combination of ten, $510 \mathrm{ohm}, 1 / 4$ watt, carbon composition resistors. The load and capacitor voltages were monitored using Agilent 10076A, high voltage probes. The Agilent 10076A probes have an attenuation ratio of 100 to 1 , an input impedance of $100 \mathrm{M} \Omega$, a four kilovolt pulse rating, $\pm 2 \%$ accuracy and a bandwidth and rise-time of $250 \mathrm{MHz}$ and $1.4 \mathrm{~ns}$, respectively. A Barth model 2440, high voltage probe was used to monitor the load voltage for faster rising pulse (< a few ns) currents. The Barth 2440, high voltage probe has a nominal attenuation ratio of 20 (10) to 1, an input impedance of 1000 (500) ohms, a $3 \mathrm{GHz}$ bandwidth and a rise-time of 35 ps. The Barth probe, when connected across the load resistor, reduced the load resistance to 50.3 and 47.9 ohms for the $20: 1$ and 10:1 attenuation ratios, respectively. All test circuit signals were monitored using a Tektronix TDS 684C, 4 channel oscilloscope with a 1 GHz bandwidth. Figures 5.8 and 5.9 are photographs of the front and back surfaces of the low voltage board. The charge voltage to the low voltage board is supplied by the Detector Pulse 
Bias (DPB) Chassis. The DPB chassis charges the $1.5 \mu \mathrm{F}$ low voltage board capacitance to full voltage in 20 ms and 60 ms later a crowbar switch in the DPB discharges the board capacitance. A typical 500 volt DPB charge pulse is shown in Figure 5.10. The optical trigger pulse to the switch is timed to arrive during the $6 \mathrm{~ms}$ wide capacitor voltage flat-top.

The high-voltage board replaces the metalized polyester capacitors of the low voltage board with two, parallel sets of $4 \mathrm{kV}, 56 \mathrm{nF}$, ceramic chip capacitors connected in series. The $51.3 \mathrm{ohm}$ load resistor of the high voltage board is constructed from a parallel set of 5, $200 \mathrm{ohm}$, 2 watt carbon composition resistors connected in series with a parallel set of 10, $100 \mathrm{ohm}, 1 / 2$ watt, carbon composition resistors. The high-voltage board is pulse charged using the Silicon Carbide Pulse Charge Chassis (SCPC). The SCPC pulse charges the high voltage board in $\sim 12$ $\mu$ s and the optical trigger pulse is timed to arrive at the switch facet $\sim 10 \mu$ s after charging of the high voltage board has completed. The nominal operating range of the high voltage board is 500 volts to $8 \mathrm{kV}$. The front and back of the high voltage board are shown in Figures 5.11 and 5.12. The high voltage board charge waveform in shown in Figure 5.13. The important point about the

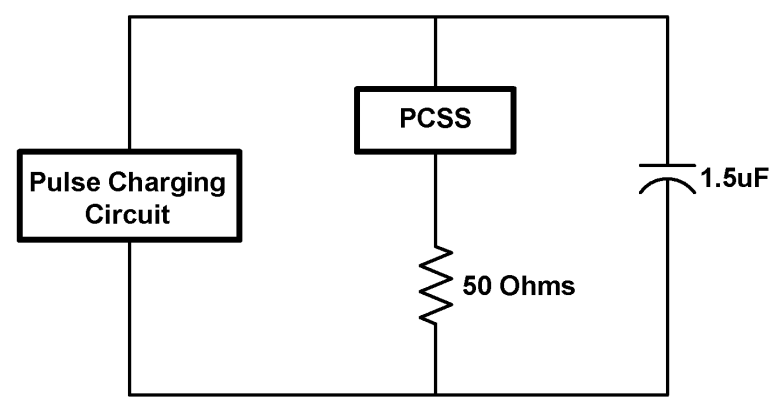

Figure 5.7. Generic test circuit for photoconductivity tests of 6H-SiC PCSS 


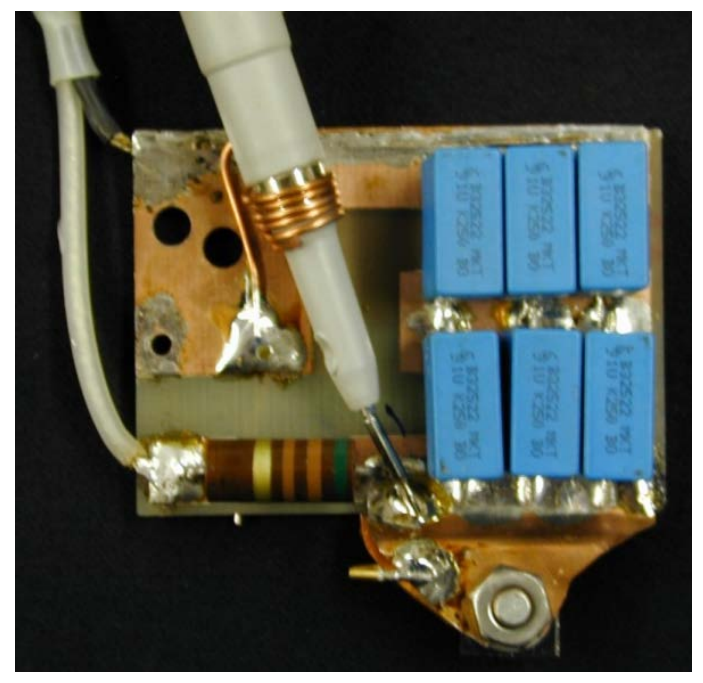

Figure 5.8. Front of low voltage board showing storage capacitors and Agilent probe connections. $6 \mathrm{H}$-SiC switch is mounted between copper tabs at the bottom of photograph.

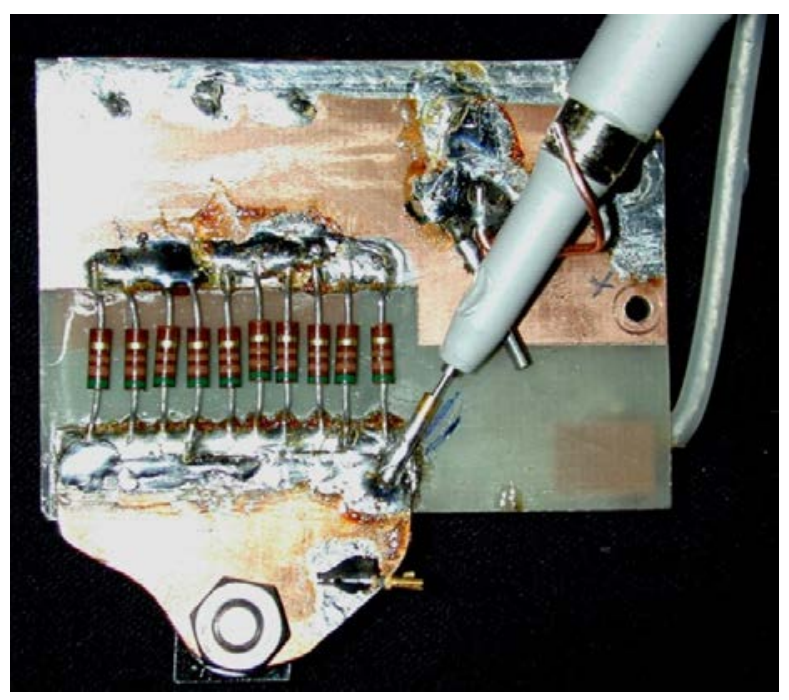

Figure 5.9. Back of low voltage board showing Agilent probe, load resistors and 6H-SiC switch. 


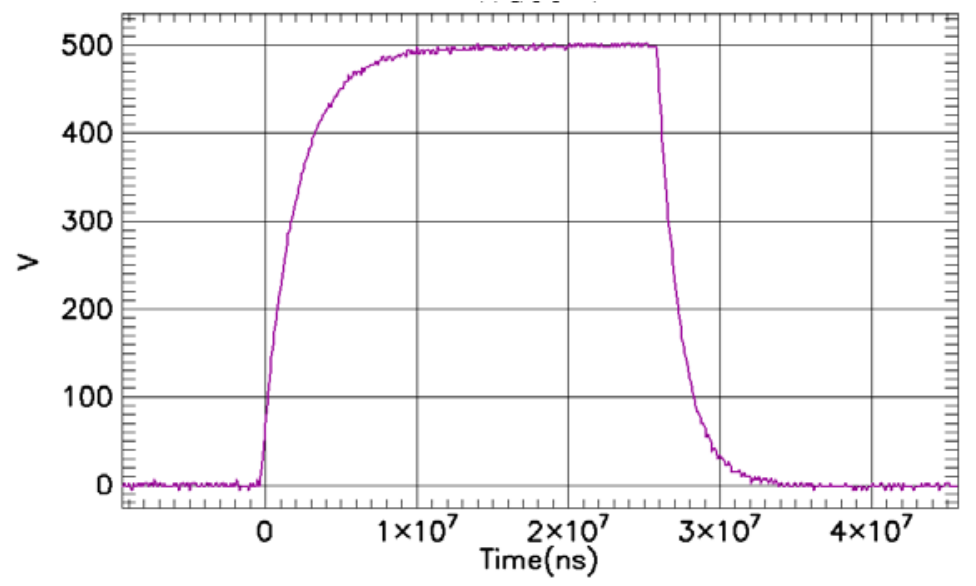

Figure 5.10. Low voltage board 500 volt charging pulse from Detector Pulse Bias Chassis (DPB).

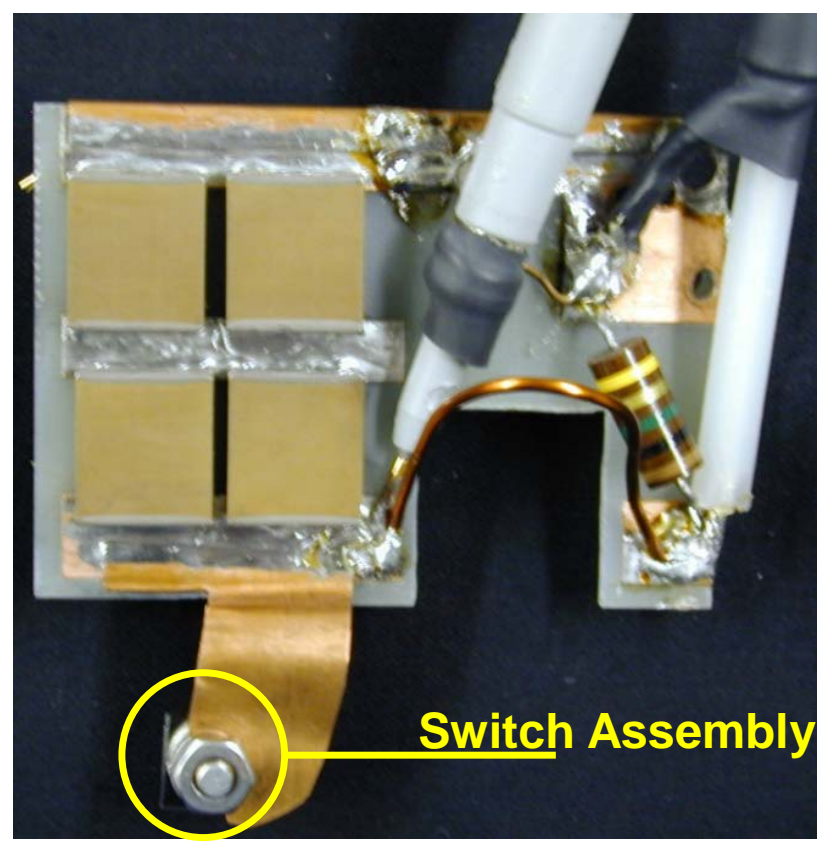

Figure 5.11. Front of high voltage board showing $4 \mathrm{kV}$ ceramic capacitors, Agilent probe and $6 \mathrm{H}-\mathrm{SiC}$ switch assembly. 


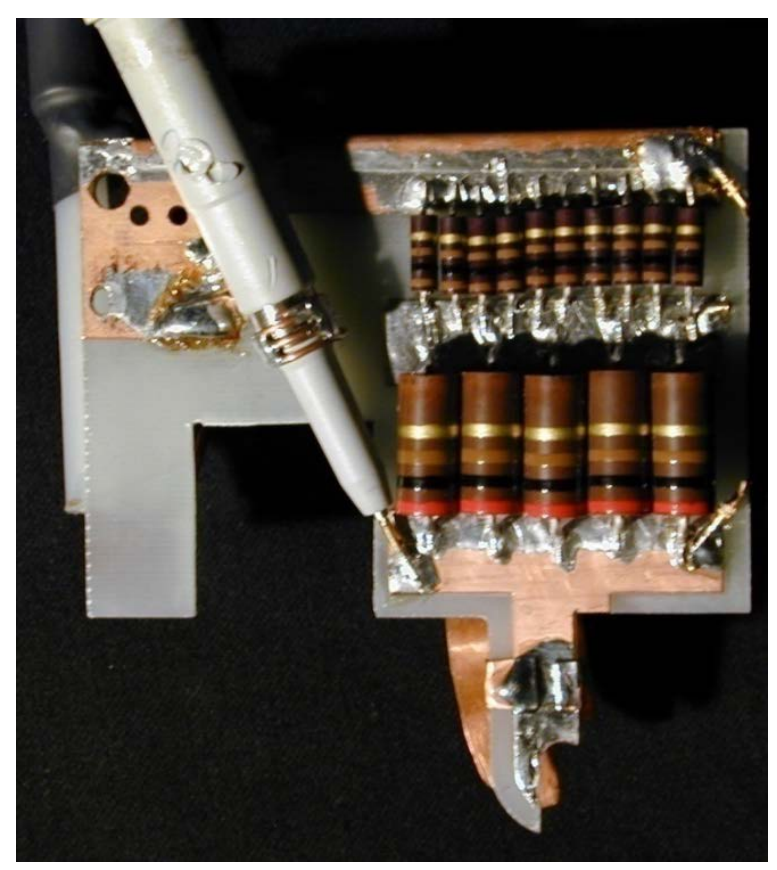

Figure 5.12. Back of high voltage board showing load resistor and Agilent probe.

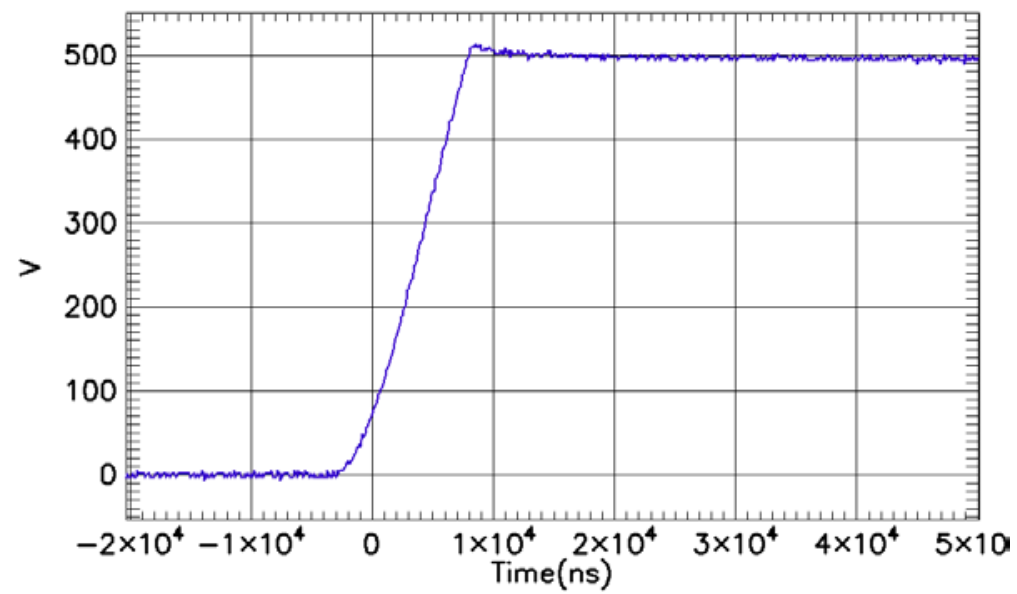

Figure 5.13. High voltage board 500 volt charging pulse from Silicon Carbide Pulse Charge Chassis (SCPC).

low voltage and high voltage board charge waveforms is that the energy storage capacitor voltage is constant during a photoconductive switching event. The charge circuits have been 
designed to produce a 'flat-top' on the energy storage capacitor voltage and the capacitors have been sized so that the voltage droop is negligible during a switching event.

\subsection{2) Optical Components}

Two different lasers were used to supply the optical trigger pulse. Initially a SpectraPhysics, Quanta Ray model Pro - 230, q-switched Nd:YAG laser supplied the optical trigger pulse. This laser has a maximum repetition rate and output energy of $10 \mathrm{~Hz}$ and 2 Joules per pulse (1064 nm), respectively. The spatial profile of this lasers output pulse is $9 \mathrm{~mm}$ in diameter and exhibits concentric rings of high intensity. The temporal profile of this lasers q-switched pulse exhibited large amplitude spikes due to the beat frequencies of competing longitudinal modes. Several switch facets were damaged due to the combination of spatial 'hot spots' and temporal spikes in this lasers output. The spatial and temporal profiles of the Spectra-Physics laser are shown in Figure 5.14. The temporal profile of the Spectra-Physics laser shown in Figure 5.14 is a worst case for longitudinal mode beats. The test data taken with this laser had significantly less mode beats. However, modes beats were present in all laser output pulses at 1064 and $532 \mathrm{~nm}$. As a result, only the green switch was tested with the Spectra-Physics laser. The remaining switches were tested with a Spectron Laser Systems model SL803G - 10, qswitched, Nd:YAG laser. The Spectron laser had a much more uniform spatial profile and far fewer longitudinal modes present in its temporal output. The Spectron laser has peak output energy of $\sim 1 \mathrm{~J}$ at $1064 \mathrm{~nm}$ and maximum repetition rate of 10 Hertz. The optical layout for the initial photoconductive switch tests at 532 and $1064 \mathrm{~nm}$ wavelengths is shown in Figure 5.15. The optical layout is independent of which laser was being used. Optical components AR coated for $532 \mathrm{~nm}$ operation are substituted in for components coated for $1064 \mathrm{~nm}$ and the $1064 \mathrm{~nm}$ 
beam is passed through the lasers second harmonic crystal to apply $532 \mathrm{~nm}$ pulses to the switch facet.
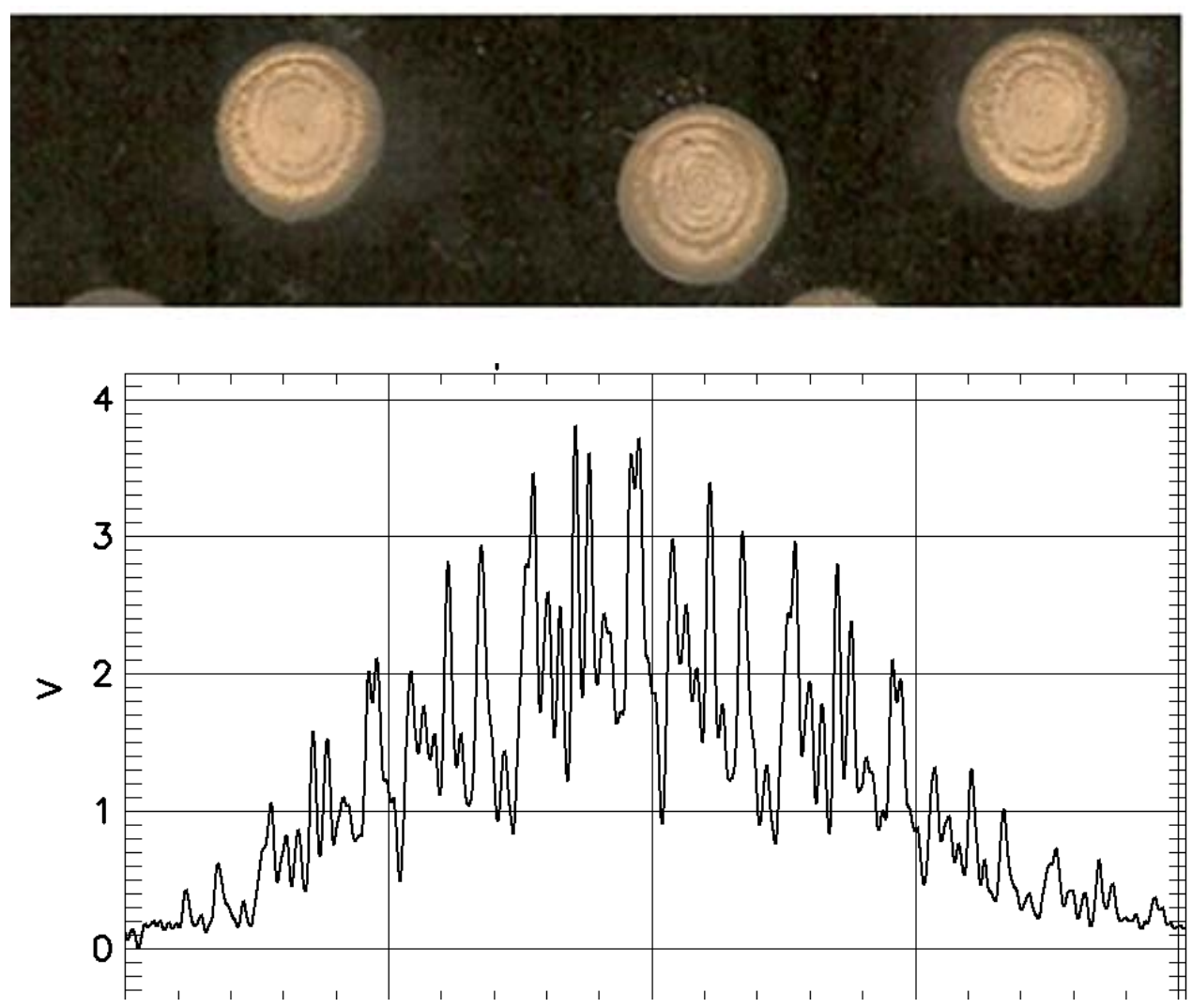

Figure 5.14, Top - burn paper images of output spatial profile of Spectra-Physics Quantaray Pro - 230 Nd:YAG laser showing high intensity rings. Bottom - output pulse temporal of same laser showing longitudinal mode beats. Major division is $\mathbf{5}$ ns on temporal plot.

The laser flashlamps are triggered at a repetition rate of $10 \mathrm{~Hz}$ that is provided by an external clock. The same external clock provides a trigger to the q-switch when required by the test operator. Q-switch pulses with the proper temporal delay are synchronized to the flashlamp pulse and single shot, 1,5 , or $10 \mathrm{~Hz}$ operation is possible.

The temporal diode shown at the top left of Figure 5.15 is a Hamamatsu Model R1328U, Phototube Photodiode. This photodiode has a detection bandwidth ranging from $1100 \mathrm{~nm}$ to 300 


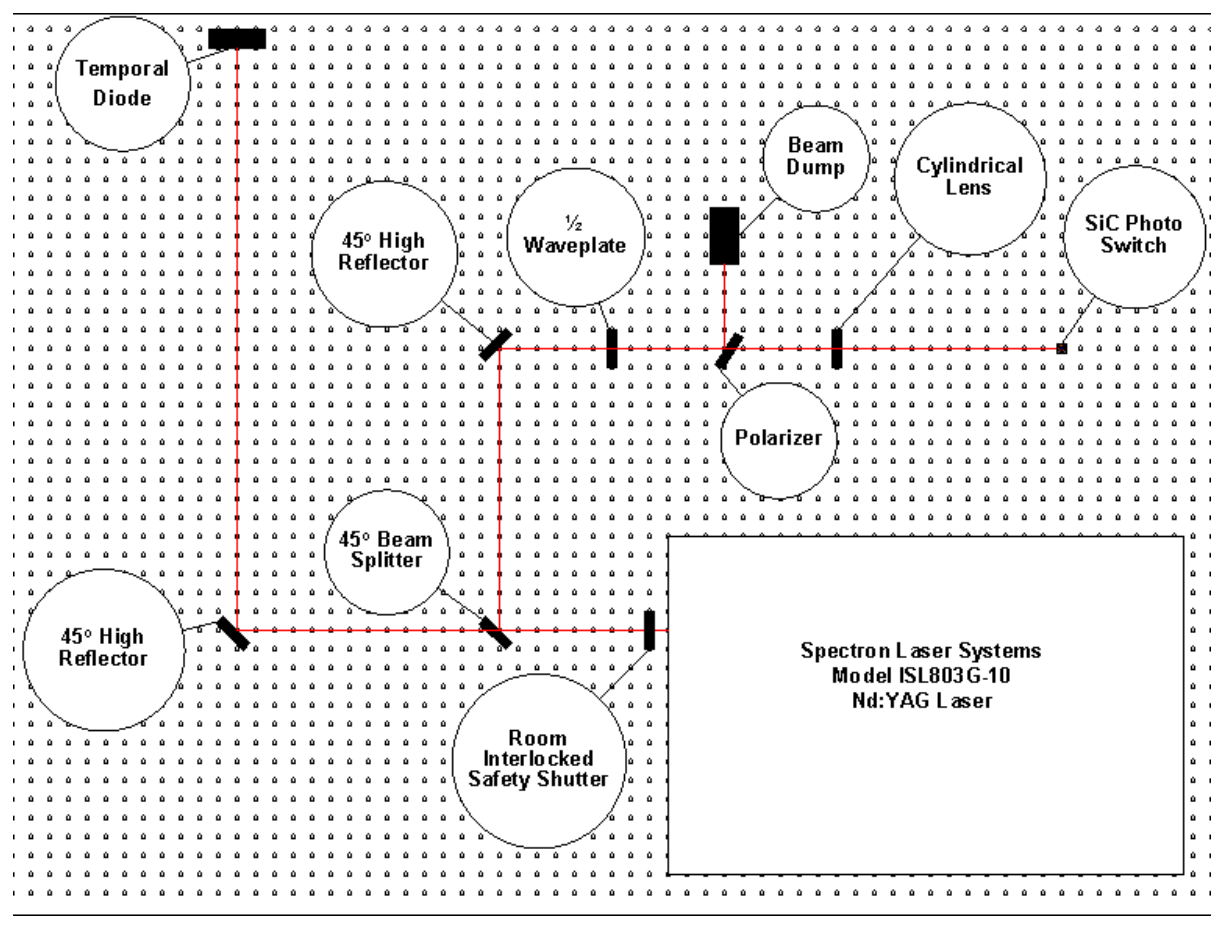

Figure 5.15. Optical system design for initial 6H-SiC switch tests at 532 and 1064 nm.

$\mathrm{nm}$ and rise time of $60 \mathrm{ps}$. The photodiode detector face is positioned at the exact same optical path distance from the laser output mirror as the facet of the $6 \mathrm{H}-\mathrm{SiC}$ switch substrate under test.

The beam splitters and mirrors are 2 inch diameter, circular optics. The laser output pulse energy is maintained at a constant value for greater stability and a waveplate - polarizer pair is used to vary the amount of optical energy being applied to the switch facet. A cylindrical lens is used to create a 'line' focus, $\sim 1 \mathrm{~cm}$ wide and slightly greater than $400 \mu \mathrm{m}$ high at the position of the switch as measured with burn paper. The line focus is centered on the $1.2 \mathrm{~cm}$ dimension of the facet, slightly over-filling the $400 \mu \mathrm{m}$ high facet. The switch facet is positioned $\sim 1.5$ inches past the focal point of the cylindrical lens, so that the beam is expanding when it arrives at the switch facet. Initial optical alignment of the laser output pulse to the switch facet is performed with the laser in long pulse mode. Initially, the flattest, best optical facet of the switch substrates four facets is selected as the facet that will be irradiated by the laser pulse. The optical pulse is 
centered on the switch facet and its reflection from the switch facet is checked to assure it propagates back up the incident beam path. Once the initial alignment is achieved, final alignment of the switch to the optical pulse is performed with the laser in "q-switch" mode. The final alignment is achieved by putting the laser in q-switch mode at a repetition rate of $1 \mathrm{~Hz}$ and $1 \mathrm{~mJ}$ of output energy (at either 532, or $1064 \mathrm{~nm}$ ) and moving the $6 \mathrm{H}$-SiC switch substrate in the $\mathrm{x}$ direction (in and out) and y direction (up and down) in the in the laser beam while observing the pulse to pulse variation in photocurrent. Final alignment is achieved when the photocurrent is maximized. The peak photocurrent at final alignment is recorded and the substrate is rotated 90 degrees and the alignment process is repeated until all four facets have been aligned. The facet that provides the highest photocurrent response during the final alignment process is used to couple light into the substrate during the photoconductive switch testing. Most of the time, this process resulted in the best optical facet being selected to couple light into the switch substrate. Photoconductive switch testing begins after final alignment of the selected facet is achieved.

\section{3) Extrinsic Photoconductive Switch Tests of Semi-Insulating, Vanadium Compensated 6H-SiC Substrates at 532 and 1064 nm}

The primary purpose of the photoconductive switch tests is to determine the minimum switch on resistance as a function of applied optical energy of the initial vanadium compensated $6 \mathrm{H}-\mathrm{SiC}$ material. We believe this testing to be the first demonstration of an extrinsic $6 \mathrm{H}-\mathrm{SiC}$ photoconductive switch and reported our initial results in 2007[5.2]. The 6H-SiC PCSS assemblies are prepared for photoconductivity measurements by cleaning the PCSS facets with acetone and alcohol to remove any organic residue. All PCSS facets are inspected by microscope and given identification marks prior to photoconductivity tests. Photoconductivity tests are performed using $1064 \mathrm{~nm}$ and frequency doubled $532 \mathrm{~nm}$ wavelength light from a Q-switched 
Nd:YAG laser. The green switch was tested using a Spectra-Physics, Quanta Ray Pro 230 Nd:YAG laser and the black and silver switches were tested using a Spectron SL803G - 10 Nd:Yag Laser. Photoconductivity tests were performed using optical pulse energies ranging from 1 to $14 \mathrm{~mJ}$. The pulse to pulse variation in optical energy applied to the switch facet was $\sim 2-$ 3\% at the lower energies for both the 532 and $1064 \mathrm{~nm}$ wavelengths gradually increasing to 7 $8 \%$ at the higher energies $(13-14 \mathrm{~mJ})$. The optical energy was increased in $1 \mathrm{~mJ}$ increments using a polarizer-waveplate pair. PCSS facets were inspected for damage by microscope between optical pulse energy increments. The photoconductivity of the PCSS is measured using the low voltage and high voltage test circuits shown in Figures $5.7-5.9$ and $5.11-5.12$. The $6 \mathrm{H}-\mathrm{SiC}$ photoconductive switches are pulse charged to bias voltages ranging from 300 to 1000 Volts. The PCSS is optically triggered after the charge voltage reaches the flat top interval of the pulse bias voltage. The voltage across the PCSS was measured differentially by using two matched voltage probes to measure the switch anode and cathode voltages with respect to ground and subtracting the cathode voltage from the anode voltage at the oscilloscope. The PCSS current was measured by monitoring the voltage across the nominal 50-Ohm load resistor in series with the PCSS. The storage capacitors on both the high and low voltage test boards are sized so that there is little, or no, voltage-droop in the charge voltage during switch photoconduction. The capacitor behaves as a dc voltage source during the switch test.

Figure 5.16 is a more revealing schematic for both the low and high voltage test circuit. This schematic shows the storage capacitor as a battery, includes the total circuit inductance, stray capacitance across the switch, and shows the switch as a parallel combination of a light controlled resistance and switch capacitance. The switch capacitance is calculated to be $11 \mathrm{pF}$ using the $6 \mathrm{H}-\mathrm{SiC}$ substrate geometry, switch electrode geometry and the dielectric constant for 
$6 \mathrm{H}-\mathrm{SiC}(\sim 10)$. The switch capacitance and stray capacitance across the switch are charged to the source voltage, $\mathrm{V}_{\mathrm{c}}$, prior to illumination of the switch. Current begins to flow in this circuit when the optical intensity is applied to the $6 \mathrm{H}-\mathrm{SiC}$ substrate facet. The switch capacitance and stray capacitance across the switch are discharged through the switch resistance and this current does not pass through the load resistor. The current through the load increases as the intensity applied to the switch increases, decreasing the switch resistance. The switch resistance continues to decrease until the applied optical pulse reaches its peak intensity. The switch resistance will increase at a rate dependent on the decrease of the applied optical pulse intensity and how fast the carriers in the switch substrate volume recombine. The carrier recombination time in vanadium compensated $6 \mathrm{H}-\mathrm{SiC}$ has been measured to be $130 \pm 5$ ps [5.3]. As a result, we should expect the load current pulse to resemble the temporal profile of the applied 5 - 10 ns FWHM optical trigger pulses. The switch resistance will achieve a minimum value and the load current will achieve a maximum value corresponding to the peak applied optical intensity. The following relationships will hold for the point in time when the maximum in load current is achieved:

$$
\begin{gathered}
\frac{\partial i_{L}}{\partial t}=0 \\
V_{c h}=V_{L}+V_{s w} \\
R_{S w}=\frac{V_{c h}-V_{L}}{i_{L}}
\end{gathered}
$$

Where $\mathrm{i}_{\mathrm{L}}$ is the load current, $\mathrm{V}_{\mathrm{L}}$ is the load voltage, $\mathrm{V}_{\mathrm{sw}}$ is the switch voltage, and $\mathrm{V}_{\mathrm{ch}}$ is the voltage on the storage capacitor. 


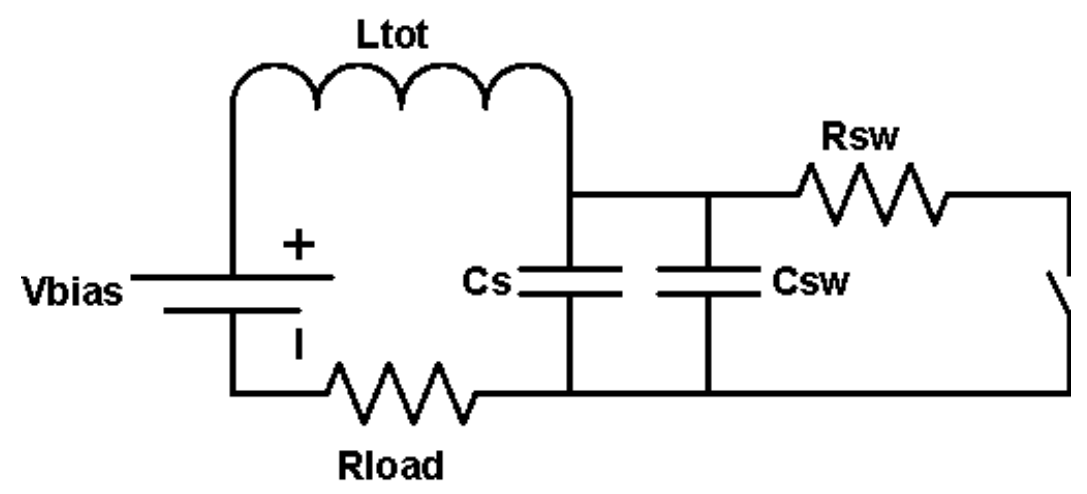

Figure 5.16. Schematic of photoconductive switch test circuit with $6 \mathrm{H}$-SiC photoconductive switch shown as a series combination of ideal switch and switch resistance.

Waveforms for the switch voltage and load current are shown for the green switch for a charge voltage of 4250 volts and optical excitation of $13 \mathrm{~mJ}$ at $1064 \mathrm{~nm}$ using the Spectraphysics laser in Figure 5.17. The PCSS voltage starts at $4.25 \mathrm{kV}$ and collapses to approximately 750 Volts in 10 ns, while the PCSS current increases from zero to 70 Amps. The PCSS current pulse is similar in temporal profile to the $1064 \mathrm{~nm}$ optical trigger pulse. However, the photocurrent has a 16 ns FWHM pulse-width compared to a 10 ns FWHM pulse- width for the optical pulse. Most of the difference in pulse-width between the optical trigger pulse and load current is due to a slightly longer decay in the load current. The load current continues even after the optical trigger pulse has ended. The longer decay in load current is due to displacement current through the switch and test board stray capacitance as the switch impedance increases and these capacitances are recharged to 4250 volts. Displacement currents are always present in the load current as the photoconductive switch recovers to the off state. However, the displacement currents are of greater amplitude and more obvious at the higher charge voltages and optical excitation energies. 


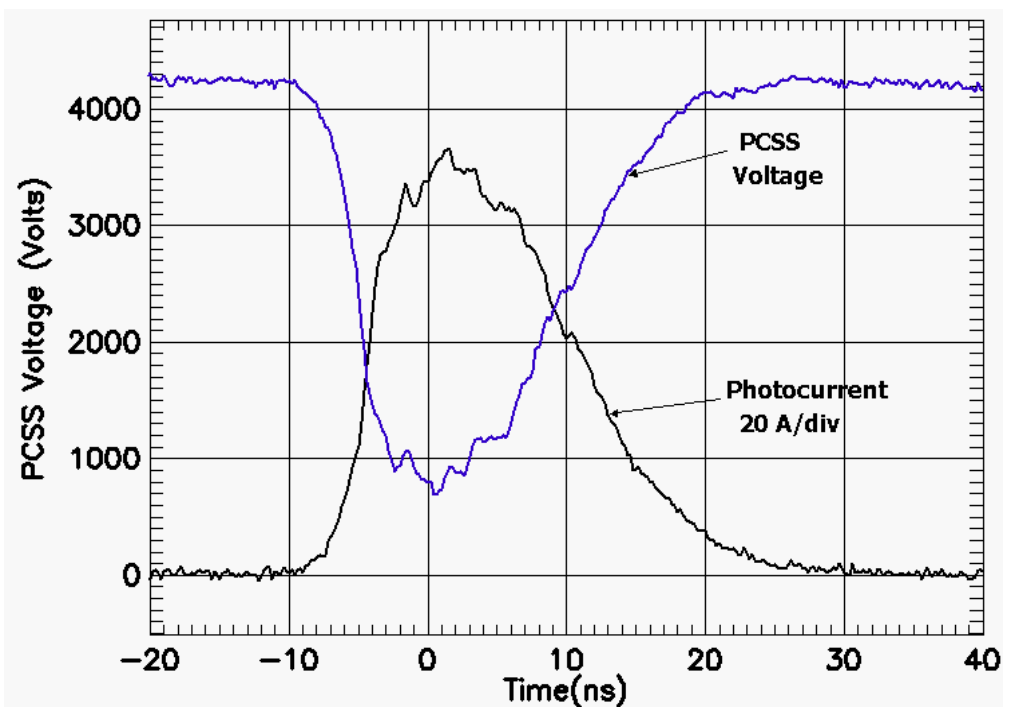

Figure 4.17. Switch voltage and load current for the black switch at 4250 volts charge and $13 \mathrm{~mJ}$ optical excitation at $1064 \mathrm{~nm}$.

\subsection{1) Green Switch Photoconductive Tests}

The green switch was the first, 1.2 by $1.2 \mathrm{~cm}, 400 \mu \mathrm{m}$ thick, switch to be extensively tested. This switch was tested using both the high voltage and low voltage test boards of Figures 5.11 and 5.8. The value of load resistance was 51.28 ohms for these tests. The optical trigger pulse was supplied by the Spectra-Physics Quanta Ray Pro 230 described previously. The qswitched, 532 and 1064 nm wavelength optical pulses from the Quanta Ray Pro 230 laser has 8.0 and 9.6 ns FWHM pulse widths, respectively. Figure 5.18 is an overlay of the photodiode output signal for 4, $1064 \mathrm{~nm}$ wavelength, $14 \mathrm{~mJ}$ optical pulses applied to the facet of the green switch. The peak optical intensity in Figure 4.18 is 1.16 MW. Figure 5.19 shows the photocurrent measurements corresponding to the optical pulses of Figure 5.18. The four photocurrent pulses correspond to charge voltages of 300, 500, 700 and 900 volts at an optical excitation of $14 \mathrm{~mJ}$ at $1064 \mathrm{~nm}$. The peak photocurrent increases linearly with the charge voltage. Figures 5.20 and 5.21 show the optical trigger pulse and corresponding photocurrent pulse for $14 \mathrm{~mJ}$ optical pulses at $1064 \mathrm{~nm}$ for charge voltages of 600 and 900 Volts. It is clear in these figures that the 


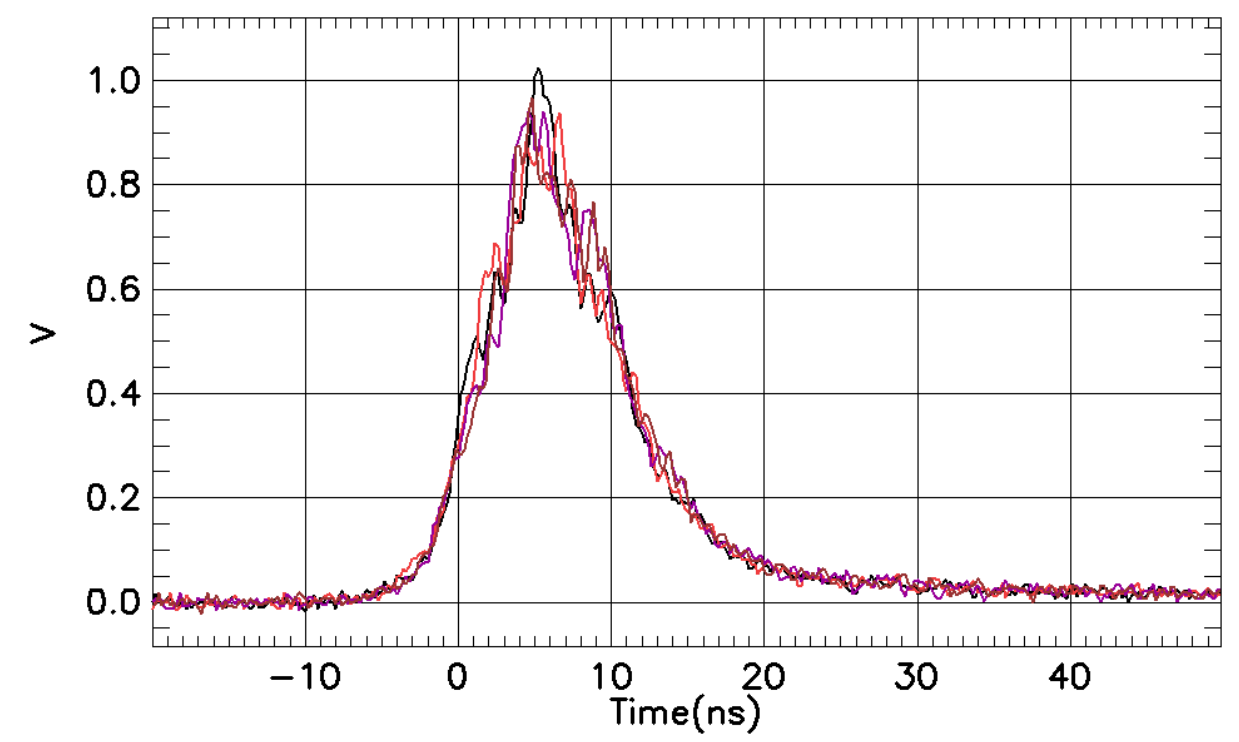

Figure 5.18. Optical pulses (14 mJ) at $1064 \mathrm{~nm}$ applied to green switch facet.

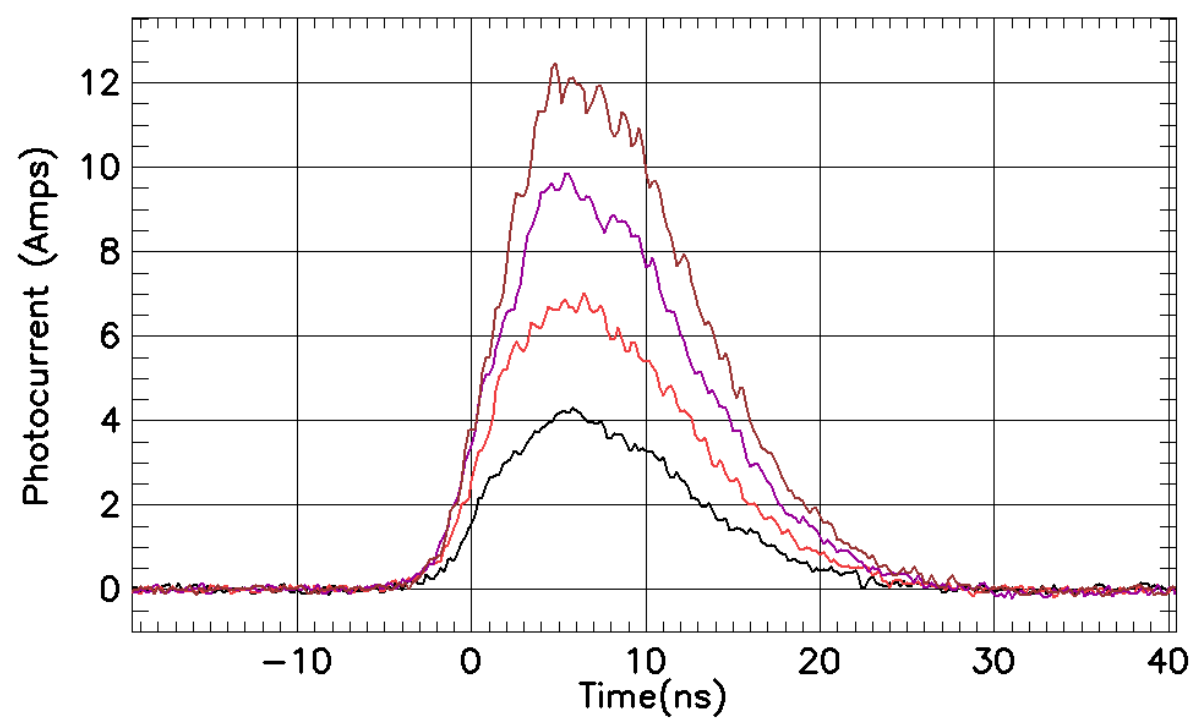

Figure 5.19. Green switch photocurrents excited by the $14 \mathrm{~mJ}, 1064 \mathrm{~nm}$ optical pulses shown in Figure 4.18.

photocurrent temporal pulse follows the optical temporal pulse for excitation at $1064 \mathrm{~nm}$. The correlation between optical pulse and photocurrent pulse becomes closer at lower charge voltages. 
The data shows that the carrier recombination time in the $6 \mathrm{H}-\mathrm{SiC}$ substrate material under test is much shorter than the applied optical pulse width in agreement with the value of $130 \pm 5$ ps for the carrier recombination time for vanadium compensated $6 \mathrm{H}$-SiC cited in the literature [5.3]. Our experimental observations of switch photocurrents in the vanadium compensated $6 \mathrm{H}-\mathrm{SiC}$ switches show the material is capable of fast response. That is, fast turn-on and turn-off. What is also clear from the figures is that the switch is behaving as a controllable switch - it conducts when light is applied and returns to a non-conducting state when light is removed.

The minimum switch on resistance can be calculated using equations $5.1-5.3$. The peak photocurrent (charge voltage) values for the waveforms in Figure 5.19 are 4.26 (300 V), 6.90 (500 V), $9.70(700 \mathrm{~V})$ and 12.2 Amps (900 V). The minimum on resistances calculated for these photocurrents and charge voltages are 20.15, 21.18, 20.88 and 22.49 Ohms, respectively. The calculated minimum resistance results are very consistent, indicating that for a given optical trigger energy (except for variations due to mode beats) the carrier density generated in the $6 \mathrm{H}-$ $\mathrm{SiC}$ substrate is the same. The switch material is behaving as an optically controlled resistor. 


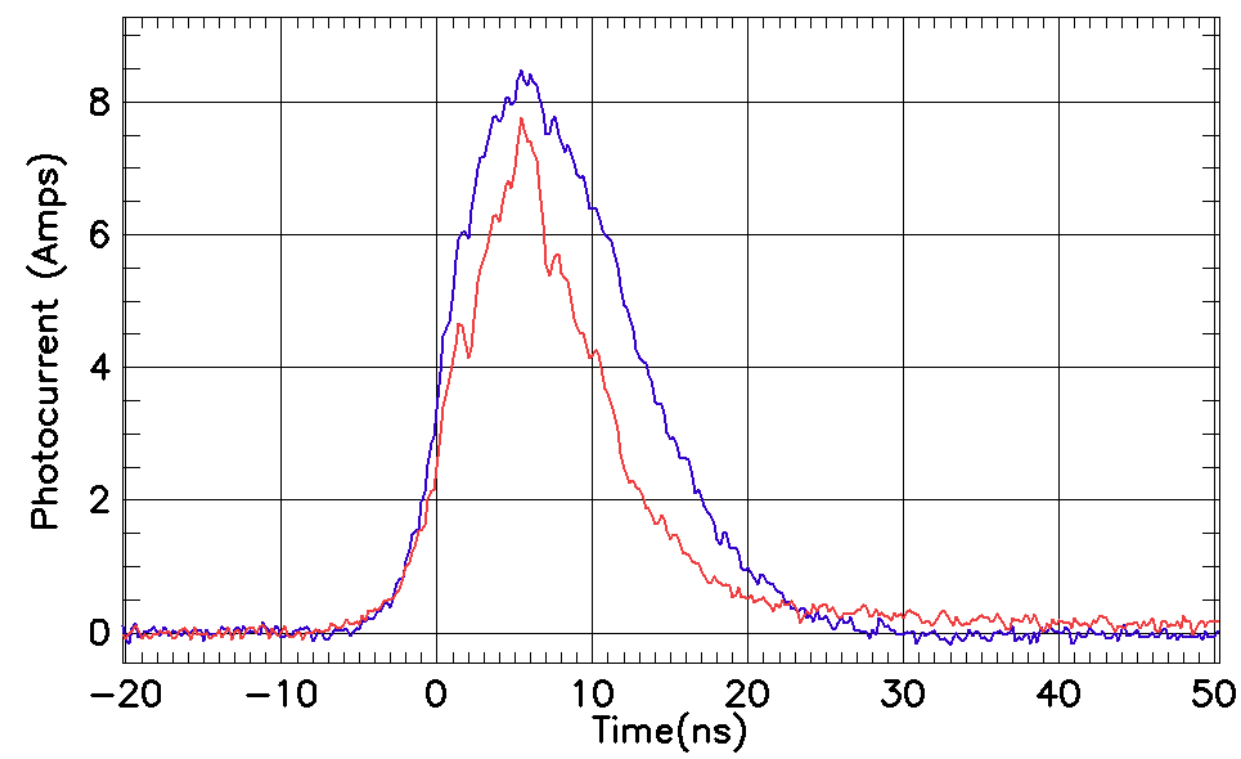

Figure 5.20. Optical trigger (red) and photocurrent pulse (blue) for green switch excited with $14 \mathrm{~mJ}, 1064 \mathrm{~nm}$ wavelength light for charge voltage of 600 volts.

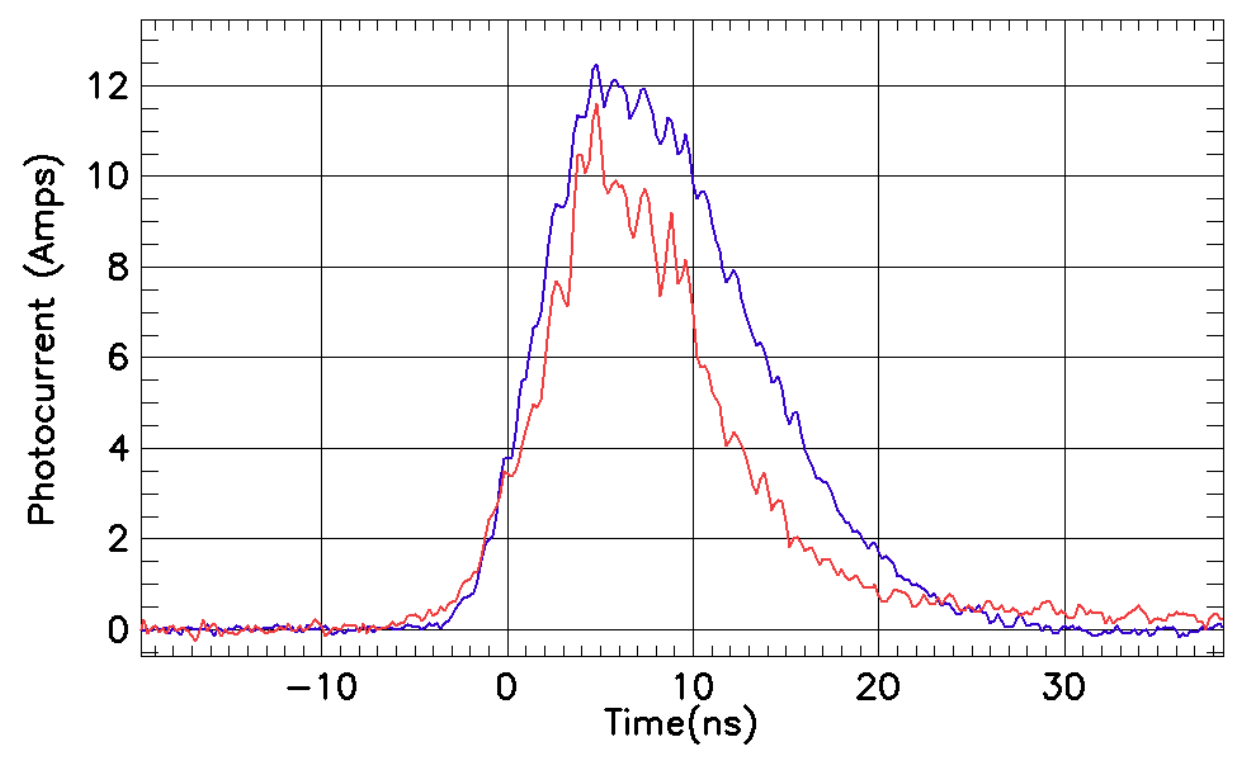

Figure 5.21. Optical trigger (red) and photocurrent pulse (blue) for green switch excited with $14 \mathrm{~mJ}, 1064 \mathrm{~nm}$ wavelength light for a charge voltage of 900 volts. 


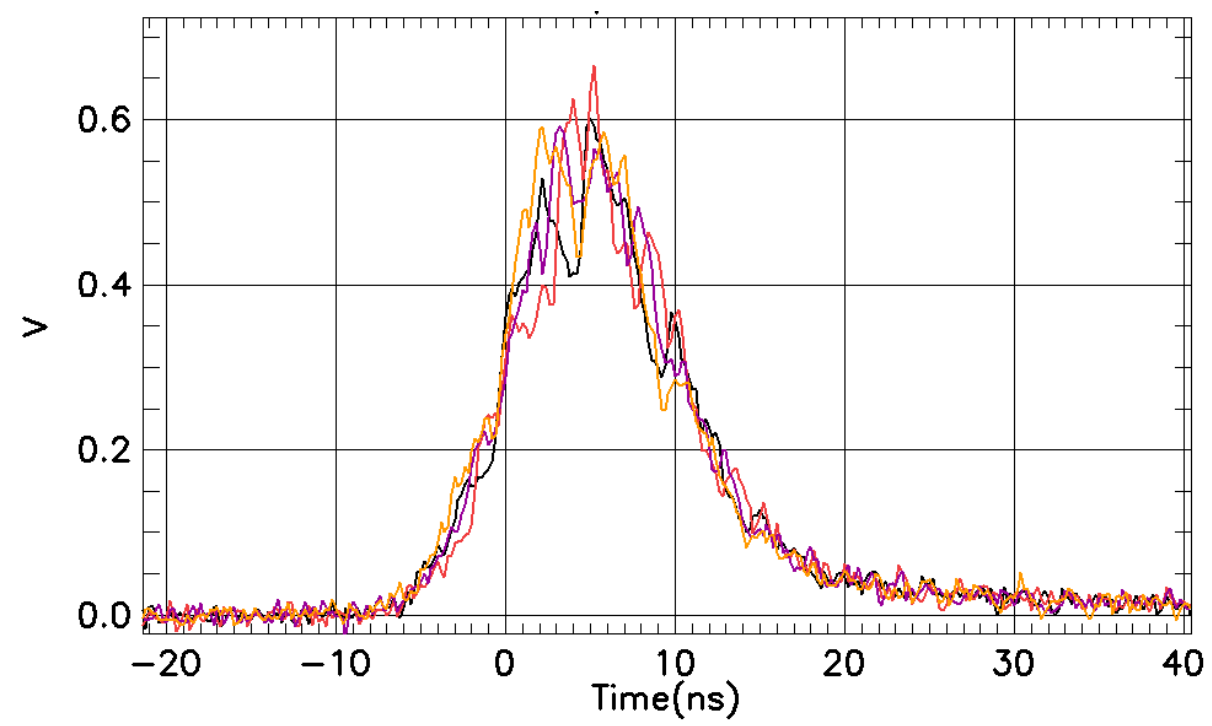

Figure 5.22. Optical pulses (13 mJ, $532 \mathrm{~nm})$ applied to green switch facet.

The green switch was also triggered with $532 \mathrm{~nm}$ light. Figures 5.22 and 5.23 show the $532 \mathrm{~nm}, 13 \mathrm{~mJ}$ optical pulses applied to the green switch facet and the resulting photocurrents for charge voltages of 300, 500, 700 and 900 volts. The peak optical power of the Figure 5.22 waveforms is $\sim 1.04 \mathrm{MW}$. The switching results for the green switch at $532 \mathrm{~nm}$ are qualitatively similar to the results for the green switch triggered with $1064 \mathrm{~nm}$ light. However, there is a striking difference in the photocurrent pulse length for excitation of the green switch at $532 \mathrm{~nm}$. The photocurrent excited with $532 \mathrm{~nm}$ light has a compound recombination time consisting of both a fast and slow decay components. The longer-lived component has amplitude of $8-10 \%$ of the green switch photocurrent excited with $532 \mathrm{~nm}$ wavelength light and decays with a time constant of $\sim 5 \mu$ s. This difference will be discussed in more detail in section 5.4.1. 


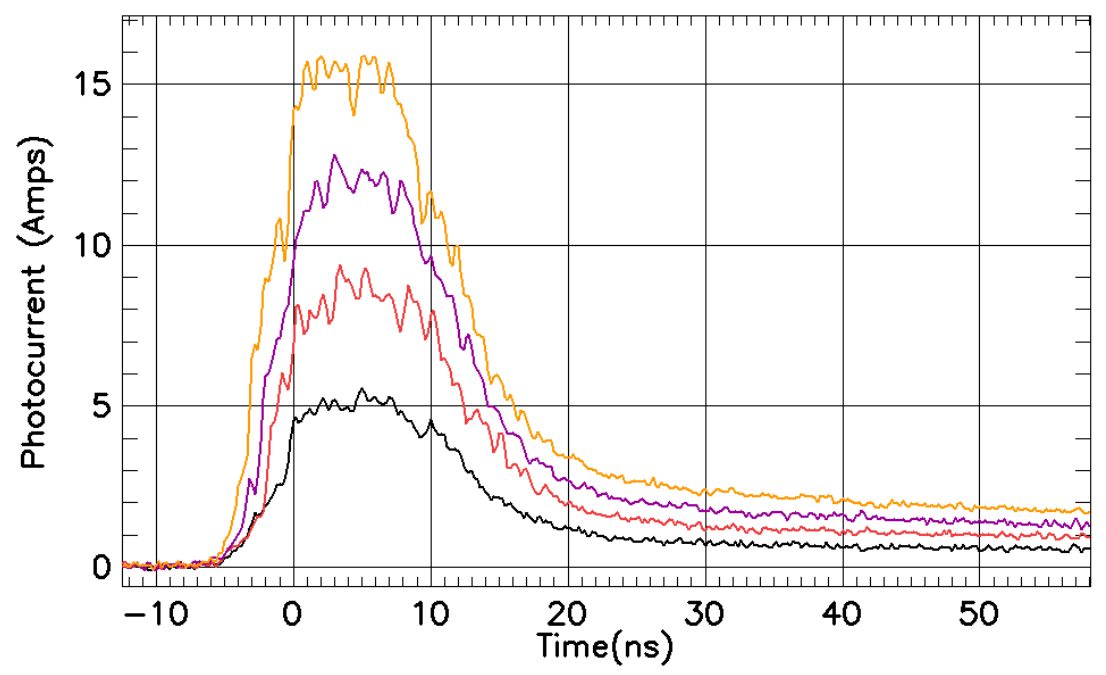

Figure 5.23. Photocurrents excited by optical pulses shown in Figure 4.21 for charge voltages of 300, 500, 700, and 900 volts.

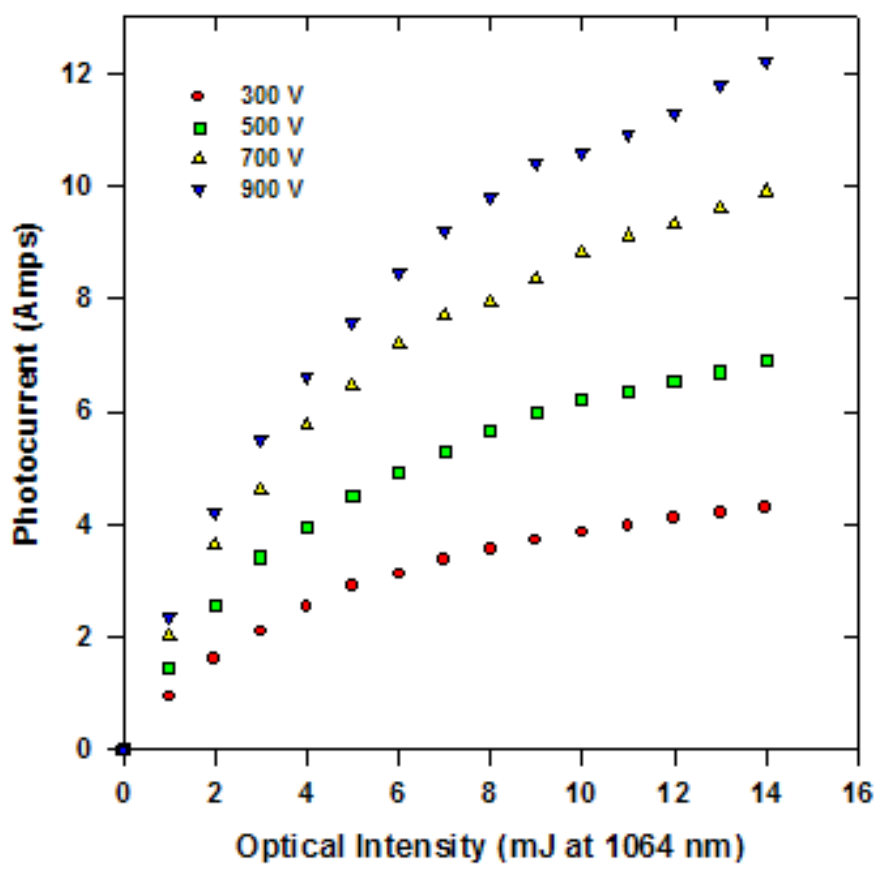

Figure 5.24. Green Switch Peak Photocurrent as a function of optical energy for the 1064 nm wavelength 


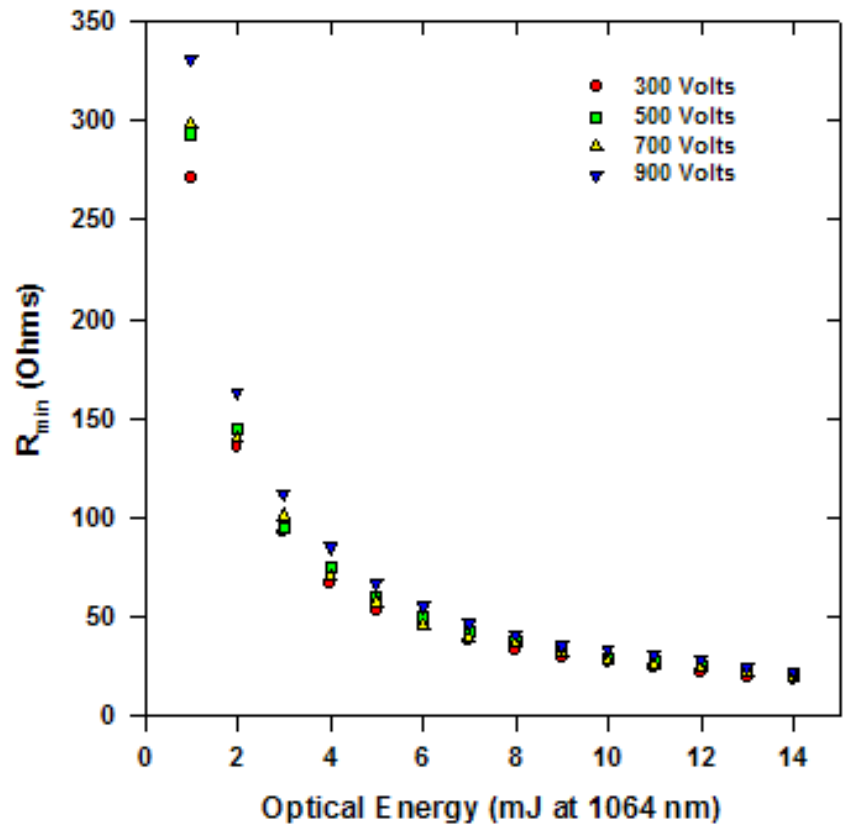

Figure 5.25. Green switch minimum on resistance as a function of optical energy at the $1064 \mathrm{~nm}$ wavelength.

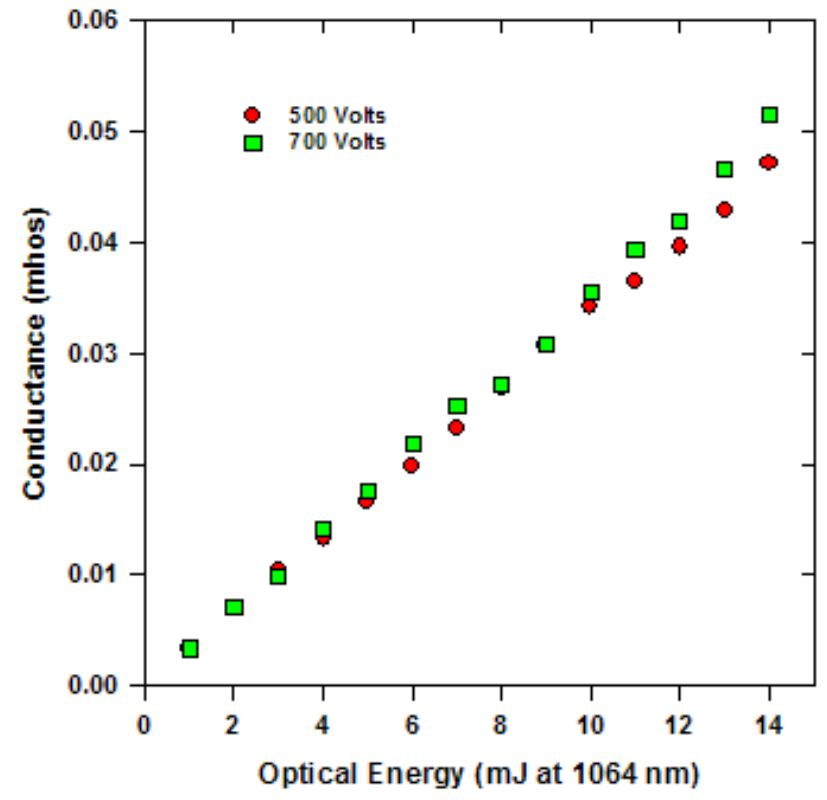

Figure 5.26. Green switch conductance versus optical energy at 1064 nm. 
The overall switching results for the green switch at the $1064 \mathrm{~nm}$ wavelength are contained in Figures 5.24 - 5.27. Each data point contained in Figures $5.24-5.26$ is the average of $4-5$ individual measurements. Figure 5.24 shows the peak photocurrent for the green switch as a function of optical energy at $1064 \mathrm{~nm}$ for charge voltages of 300, 500, 700 and 900 volts. The peak photocurrent increases with charge voltage as expected. The photocurrent increases linearly with optical energy for low optical energies, but appears to saturate at slightly higher optical energies. This is not a result of saturation of the density of photogenerated charge carriers in the switch, but the point at which the switch resistance is no longer the dominant resistance in the circuit. The switch resistance is electrically in series with the load resistance (Figure 5.16). The switch resistance is much higher than the load resistance for low optical excitation energy. Hence, there is an almost linear relationship between the switch resistance and peak photocurrent. The switch resistance decreases with increasing optical excitation energy eventually reaching the point where changes in the switch resistance have much less of an effect on the load current. The minimum switch resistance can be calculated at the photocurrent maximum using equations $5.1-5.3$. The green switch minimum resistance as a function of applied optical energy at $1064 \mathrm{~nm}$ is plotted in Figure 5.25 for charge voltages of 300, 500, 700, and 900 volts. The green switch minimum resistance varies inversely with optical energy, starting at $\sim 300 \Omega$ at $1 \mathrm{~mJ}$ excitation and decreasing to $\sim 20 \Omega$ at $14 \mathrm{~mJ}$. The minimum green switch on resistance of $20 \Omega$ is ten orders of magnitude lower than the green switch dark resistance of $170 \mathrm{G} \Omega$. The decrease in the green switch minimum resistance is close to a hyperbolic decay versus optical energy. This indicates that the optically generated carriers are increasing linearly with applied optical energy. This can be verified by plotting the switch conductance $\left(1 / \mathrm{R}_{\mathrm{sw}}\right)$ as a function of applied optical intensity as shown in Figure 5.26. The linear 
relationship between green switch conductance and applied optical energy shows there is no saturation of optically generated carriers with increased applied optical energy at $1064 \mathrm{~nm}$.

Figure 5.27 is a plot of the green switch peak photocurrent for $1064 \mathrm{~nm}$ optical excitation as a function of charge voltage for applied optical energies of 3, 5, 9, 11, and $14 \mathrm{~mJ}$ at $1064 \mathrm{~nm}$. This plot shows that the peak photocurrent increases linearly with charge voltage for fixed optical excitation. The plot also shows that the test circuit resistance decreases with increasing applied optical energy and is constant at fixed applied optical energy. The straight lines plotted using the data of Figure 5.27 correspond to minimum test circuit resistances of 152, 112, 84, 79, and 70 ohms for excitation at 3, 5, 9, 11, and $14 \mathrm{~mJ}$ of $1064 \mathrm{~nm}$ wavelength light, respectively.

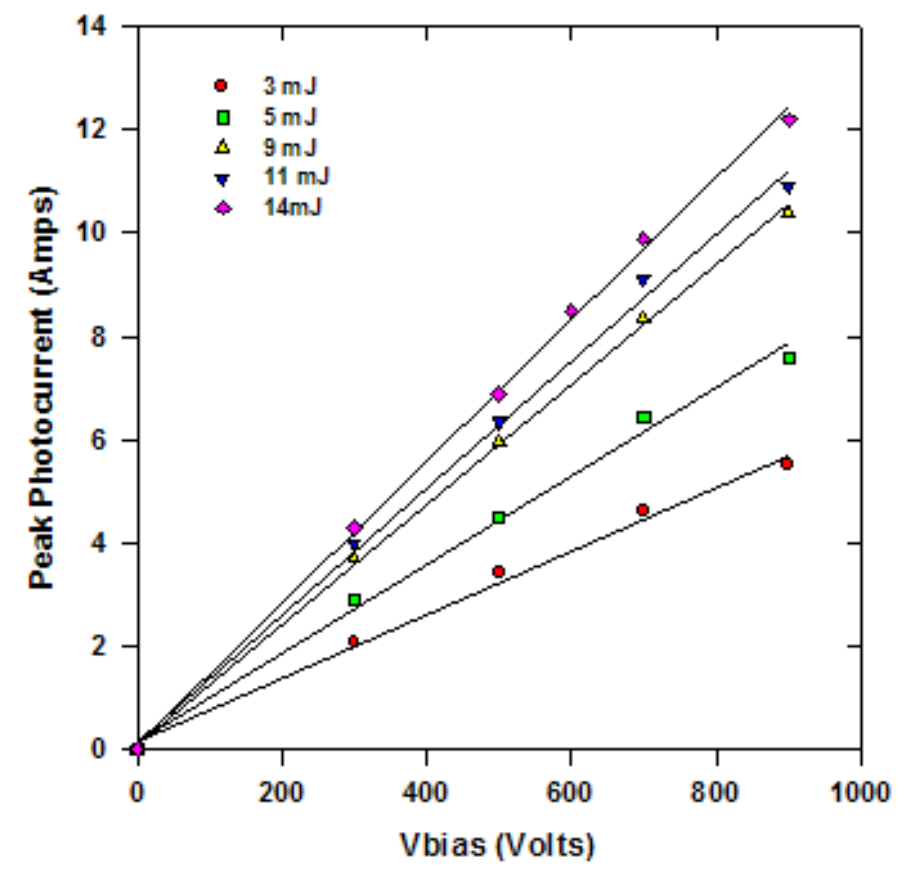

Figure 5.27. Green switch peak photocurrent as a function of charge voltage for the optical energies 3, 5, 9, 11, and $14 \mathrm{~mJ}$ at $1064 \mathrm{~nm}$ (Vbias).

The switch resistance is obtained by subtracting the load resistance (in this case 51.28 ohms), yielding values of 101, 60,33, 27, and 18.5 ohms for the excitation levels listed above. 
The switch consistently reaches the same minimum resistance for a given level of optical excitation. Also, the switch peak photocurrent increases linearly with charge voltage giving no indication that the switch enters a nonlinear avalanche mode similar to that in GaAs switches [5.4].

\subsection{2) Green Switch Photoconductive Switch Results at 532 nm}

The overall photoconductive switching results for the green switch at $532 \mathrm{~nm}$ optical excitation are shown in Figures 5.28 - 5.31. The switching results at $532 \mathrm{~nm}$ are similar to the results at $1064 \mathrm{~nm}$ for the green switch. However, the minimum on resistance of the green switch is $2.5-3.0$ times lower for optical excitation at $1-8 \mathrm{~mJ}$ at the $532 \mathrm{~nm}$ wavelength compared to optical excitation at the same energies at the $1064 \mathrm{~nm}$ wavelength. The minimum on resistance

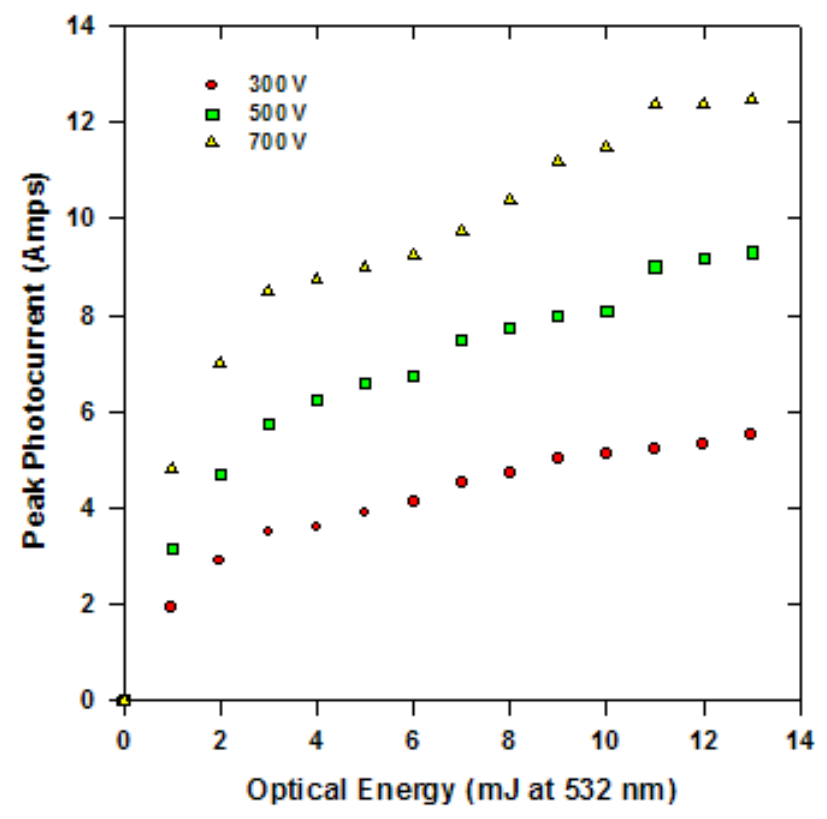

Figure 5.28. Green switch peak photocurrent plotted versus optical energy at $532 \mathrm{~nm}$ for charge voltages of 300,500 , and 700 volts. 


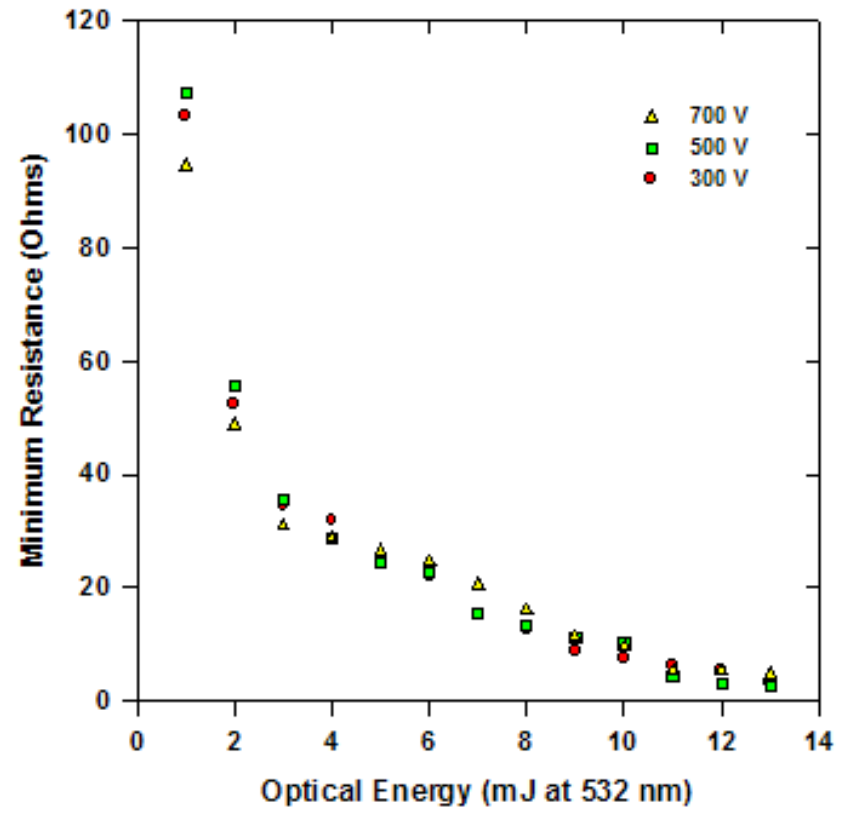

Figure 5.29. Green switch minimum resistance plotted as a function of optical energy at 532 nm for charge voltages of 300,500 , and 700 volts.

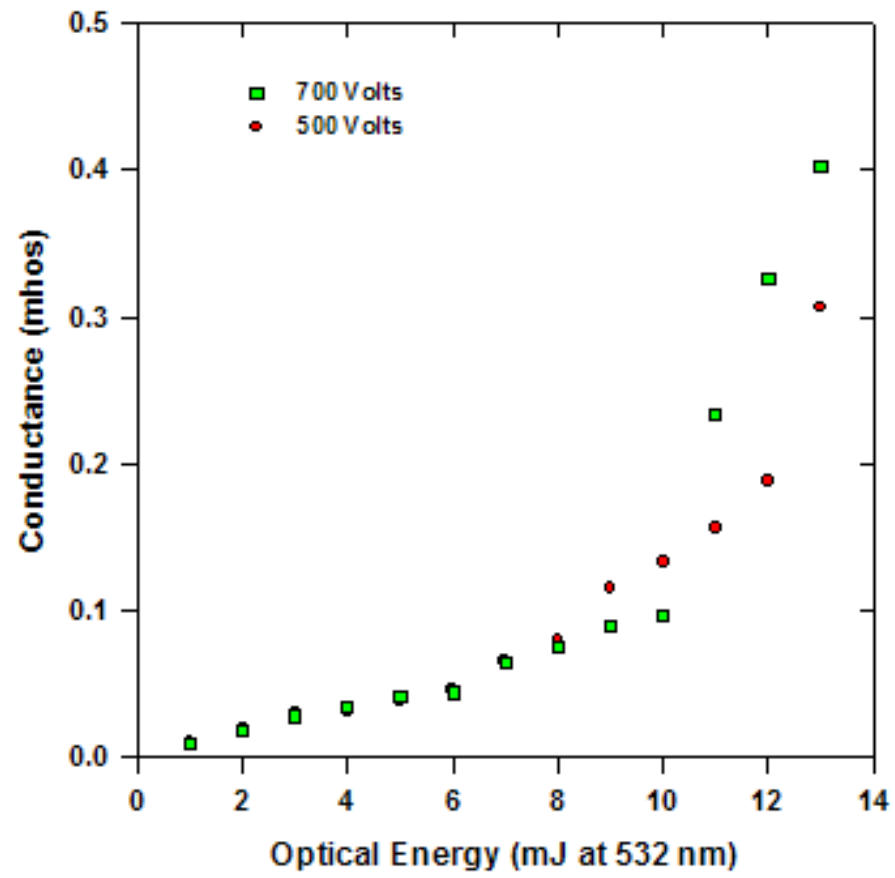

Figure 5.30. Green switch conductance plotted as a function of optical energy at $532 \mathrm{~nm}$ for charge voltages of 500 and 700 volts. 


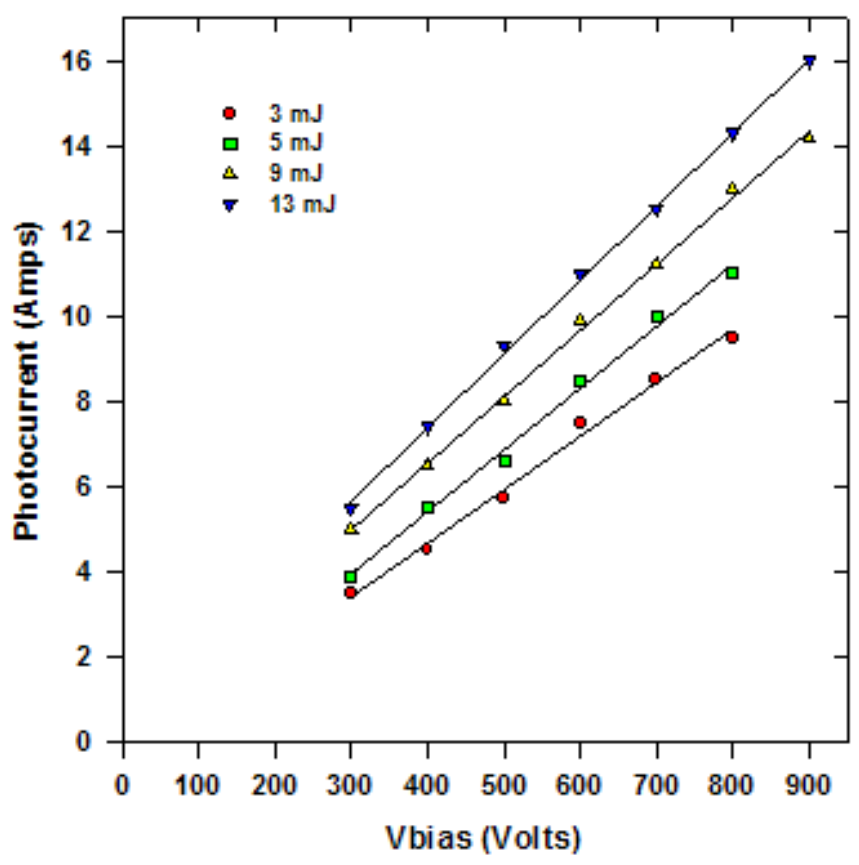

Figure 5.31. Green switch peak photocurrent plotted as a function of charge voltage for optical energies of 3, 5, 9, and $13 \mathrm{~mJ}$ at $532 \mathrm{~nm}$.

for the green switch excited with $1 \mathrm{~mJ}$ of $532 \mathrm{~nm}$ light is $\sim 100 \Omega$ decreasing to a value of $\sim 2-$ $3 \Omega$ at $13 \mathrm{~mJ}$ of $532 \mathrm{~nm}$ light compared to minimum on resistances of $\sim 300$ ohms decreasing to $\sim 20$ ohms for the same excitation levels at $1064 \mathrm{~nm}$.

Two and one half to three times higher carrier densities are generated in the volume of the green switch substrate at low energies of $532 \mathrm{~nm}$ wavelength light compared to $1064 \mathrm{~nm}$ light of the same energy. This occurs despite the fact that there are twice as many $1064 \mathrm{~nm}$ photons striking the green switch facet compared to $532 \mathrm{~nm}$ photons for optical pulses of the same energy. Thus the quantum efficiency of the green switch substrate is $5-6$ times higher for excitation at $532 \mathrm{~nm}$ compared to excitation at $1064 \mathrm{~nm}$, if we define the quantum efficiency as the ratio of optically generated carriers to photons arriving at the substrate facet. Six to seven times higher carrier densities are generated in the green switch at higher optical energies at 532 $\mathrm{nm}$ excitation than for $1064 \mathrm{~nm}$ excitation of the same energy. Further evidence of the improved 
quantum efficiency of the green switch at the $532 \mathrm{~nm}$ wavelength is contained in the straight-line plots contained in Figure 4.31. The straight-line plots correspond to test circuit minimum resistances of 84, 73, 63, and 54 ohms for optical energies of 3, 5, 9, and $13 \mathrm{~mJ}$ at $532 \mathrm{~nm}$ compared to minimum circuit resistances of $150,112,84$, and 73 ohms for excitation at the same energies at $1064 \mathrm{~nm}$.

There are several possible explanations for increased carrier densities at $532 \mathrm{~nm}$ excitation. First, as we have seen, there is a slowly decaying component of photocurrent for excitation at $532 \mathrm{~nm}$ that is not present for excitation at $1064 \mathrm{~nm}$. This slowly decaying component will increase the net recombination time of the switch that should result in increased carrier densities for $532 \mathrm{~nm}$ excitation. However, the slowly decaying photocurrent component is a modest fraction of the total photocurrent at $532 \mathrm{~nm}(\sim 8-10 \%)$. Second, the optical intensity at the switch substrate facet is greater for fixed optical energy excitation for $532 \mathrm{~nm}$ compared to $1064 \mathrm{~nm}$. This is a result of the conversion from 1064 to $532 \mathrm{~nm}$ light. The intensity of $532 \mathrm{~nm}$ wavelength light coming out of the second harmonic crystal is proportional to the square of the $1064 \mathrm{~nm}$ light into the crystal. This causes the temporal pulse of the $532 \mathrm{~nm}$ light to be shorter than the $1064 \mathrm{~nm}$ pulse. However, the temporal profiles of the $532 \mathrm{~nm}$ and $1064 \mathrm{~nm}$ are approximately the 8 and $10 \mathrm{~ns}$ FWHM, respectively. As a result, the $532 \mathrm{~nm}$ light striking the green switch facet will have a higher intensity compared to $1064 \mathrm{~nm}$ light at fixed optical energy. Third, the $532 \mathrm{~nm}$ photons have an energy of $2.34 \mathrm{eV}$ compared to the $1.17 \mathrm{eV}$ photons for the $1064 \mathrm{~nm}$ wavelength. The $2.34 \mathrm{eV}$ photons are capable of exciting carriers from levels much deeper than the vanadium acceptor level. Fourth, it is possible that the photoionization crosssection to excite electrons to the conduction band from filled vanadium acceptor levels may be much larger at $532 \mathrm{~nm}$ compared to $1064 \mathrm{~nm}$. Fifth, we should expect carrier generation via two- 
photon absorption and two-step optical transitions since the photon energy at $532 \mathrm{~nm}(2.34 \mathrm{eV})$ is greater than $\mathrm{E}_{\mathrm{g}} / 2$. It is possible that all five of these possibilities are contributing to the higher quantum efficiency of the green switch at $532 \mathrm{~nm}$ excitation.

Another difference between the green switch performance at 532 and $1064 \mathrm{~nm}$ optical excitation is that the dependence of switch conductance on optical excitation becomes supralinear at energies greater than $8 \mathrm{~mJ}$ at $532 \mathrm{~nm}$. Supralinearity in a photoconductive switch usually indicates that the recombination time is increasing with applied optical intensity. It is possible that the apparent supralinearity is the result of fidelity of the experimental measurements. The minimum on resistance measurements become less accurate as the applied optical energy at $532 \mathrm{~nm}$ is increased to high levels. This is a result of the switch on resistance becoming much lower than the load resistance. The amplitude of the peak load voltage approaches the value of charge voltage when the minimum switch on resistance is much lower than the load resistance. The calculated value for the minimum switch resistance becomes less accurate when the percentage difference between the measured values of the load voltage and charge voltage is on the same order of the accuracy of the voltage probes used to perform the measurements. Hence, accuracy may be lost at high applied optical energies at $532 \mathrm{~nm}$. The voltage measurements were performed with a calibrated set of Agilent 10076A high voltage probes with an accuracy of $\pm 2 \%$. If the probe measuring the charge voltage read $2 \%$ low and the probe measuring the load voltage read $2 \%$ high, we would calculate a switch resistance of zero ohms for an actual value of 2.0 ohms switch resistance. This represents the maximum error due to the probe accuracy. If we add 2.0 ohms to the minimum resistances calculated for the green switch at $13 \mathrm{~mJ}$ excitation at $532 \mathrm{~nm}$, we obtain 5.27 and 4.5 ohms for the charge voltages of 300 and 500 volts, respectively. The inverse of these minimum switch resistances give switch 
conductances of 0.19 and 0.22 siemen. The variation of switch conductance versus optical energy shown in Figure 5.29 will still be supra-linear even if we use the adjusted values of 0.19 and 0.22 siemen to replace the calculated conductances at $13 \mathrm{~mJ}$ at $532 \mathrm{~nm}$. It is unlikely that probe accuracy is the primary cause of the supra-linear behavior in the green switch conductance at high values of optical intensities at $532 \mathrm{~nm}$. We believe the supralinear dependence of switch conductivity with optical input to be a real property of the switch material. However, the supralinear dependence of switch conductivity on optical input is serendipitous. We do not believe the supralinearity is caused by the presence of vanadium, nitrogen, or boron impurities. As a result, we will not actively pursue the cause of the supralinearity of switch on resistance with optical input since our focus is on developing a high power, extrinsic $6 \mathrm{H}-\mathrm{SiC}$ photoconductive switch by manipulating the vanadium, nitrogen and boron densities.

\subsection{3) Black and Silver Switch Photoconductive Tests}

The photoconductive switching tests of the black and silver switches were performed using the Spectron Laser Systems model SL803G - 10, q-switched, Nd:YAG laser. The Spectron laser has a much more uniform spatial profile, far fewer mode beats present in its temporal output and shorter temporal output compared to the Spectra-Physics laser. The Spectron laser has output pulse widths of $\sim 5-6$ and $\sim 7-8$ ns FWHM for the 532 and 1064 nm wavelengths, respectively. The tests performed on the black and silver switches are identical to those performed on the green switch, except a different optical source was used. 


\section{Black Switch Results at 1064 nm}

The black switch 6H-SiC substrate measures $1.222 \mathrm{~cm}$ by $1.207 \mathrm{~cm}$ and is $372 \mu \mathrm{m}$ thick. The black switch was metalized as described and tested as a photo-switch. The optical pulse widths applied to the black switch are 5.0 and 7.0 ns FWHM for the 532 and $1064 \mathrm{~nm}$ wavelengths, respectively. The black switch photocurrent and applied optical intensity waveforms for an $18 \mathrm{~mJ}$ excitation pulse at $1064 \mathrm{~nm}$ and a charge voltage of 700 volts are shown in Figure 5.32. The photocurrent follows the optical pulse, similar to the results of the green switch, indicating a rapid recombination time in the black switch $6 \mathrm{H}-\mathrm{SiC}$ substrate material. The peak optical power for the optical pulses shown in Figures 5.32 and 5.34 is 2.43 MW. Figures 5.33 and 5.34 plot the photocurrent and corresponding optical excitation pulse at $1064 \mathrm{~nm}$ and 18 mJ for bias voltages of 300, 500 and 700 volts. The black switch photocurrents in Figure 5.33 increase linearly with charge voltage as expected and have a pulse width of $~ 12.0$ ns FWHM, which is significantly larger than the corresponding optical pulses of Figure 5.34 ( 7.0 ns FWHM). Most of the difference in the pulse widths between the photocurrent and optical pulses are due to the slower decay in the photocurrent waveform, which we believe is due to displacement current in the switch.

Figure 5.35 shows the plot of peak photocurrent in the black switch as a function of optical energy at $1064 \mathrm{~nm}$ for a number of bias voltages. The black switch peak photocurrent shares the same general saturation with increasing optical energy as the green switch. This is a result of the load resistor becoming the dominant impedance in the test circuit as the switch resistance decreases with applied optical energy. The photoconductive switch test results for the black switch at the $1064 \mathrm{~nm}$ wavelength are summarized in Figures 5.36, 5.37, and 5.38. 


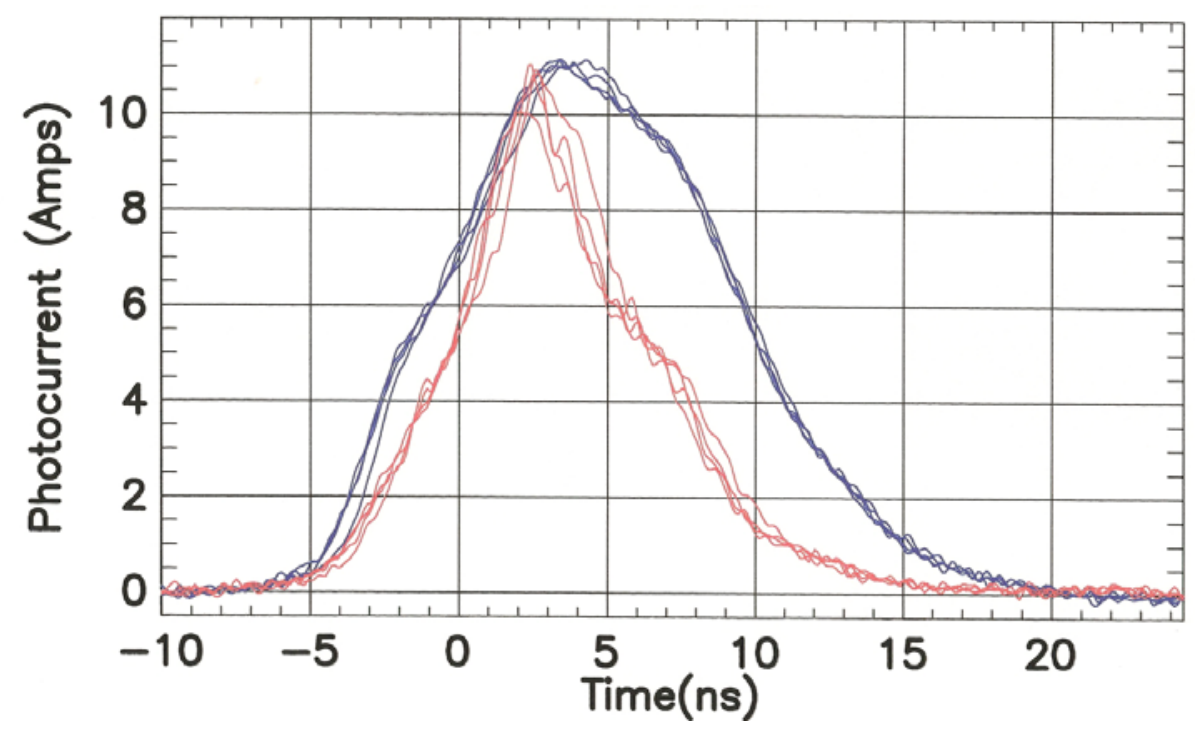

Figure 5.32. Black switch photocurrent (blue) and optical intensity (red) for $18 \mathrm{~mJ}$ excitation at $1064 \mathrm{~nm}$ wavelength and bias voltage of 700 volts.

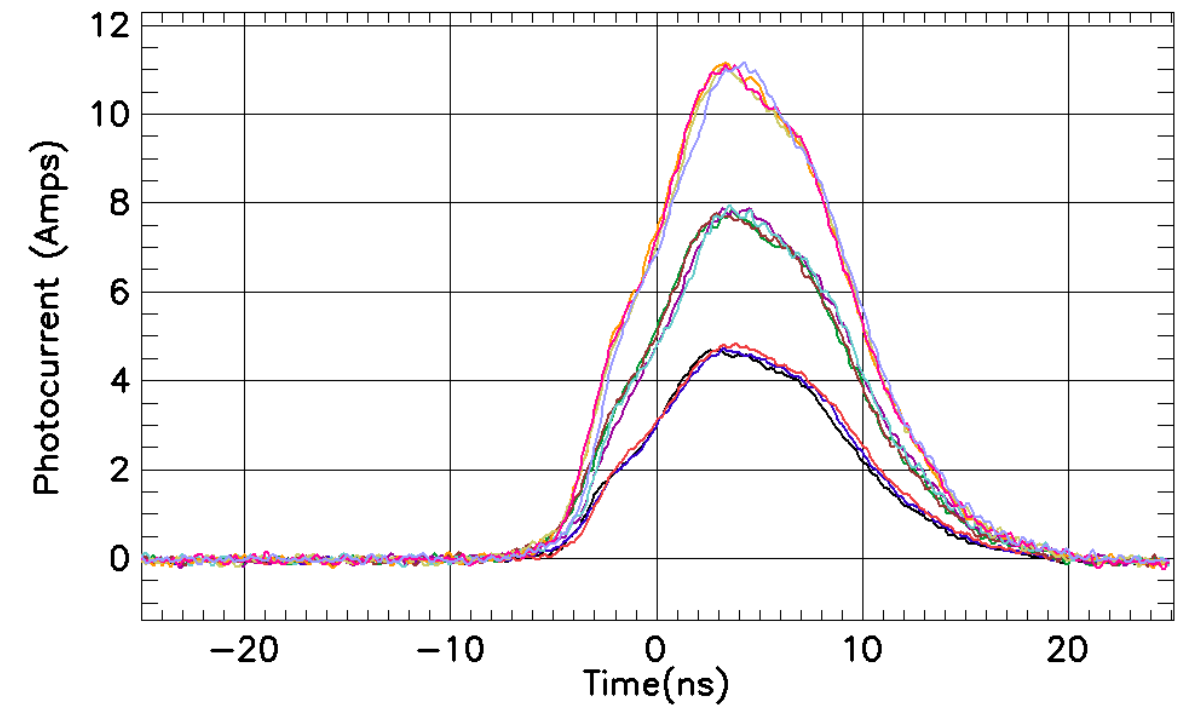

Figure 5.33. Black switch photocurrent for $18 \mathrm{~mJ}$ excitation at $1064 \mathrm{~nm}$ wavelength for bias voltages of 300,500 , and 700 volts. 


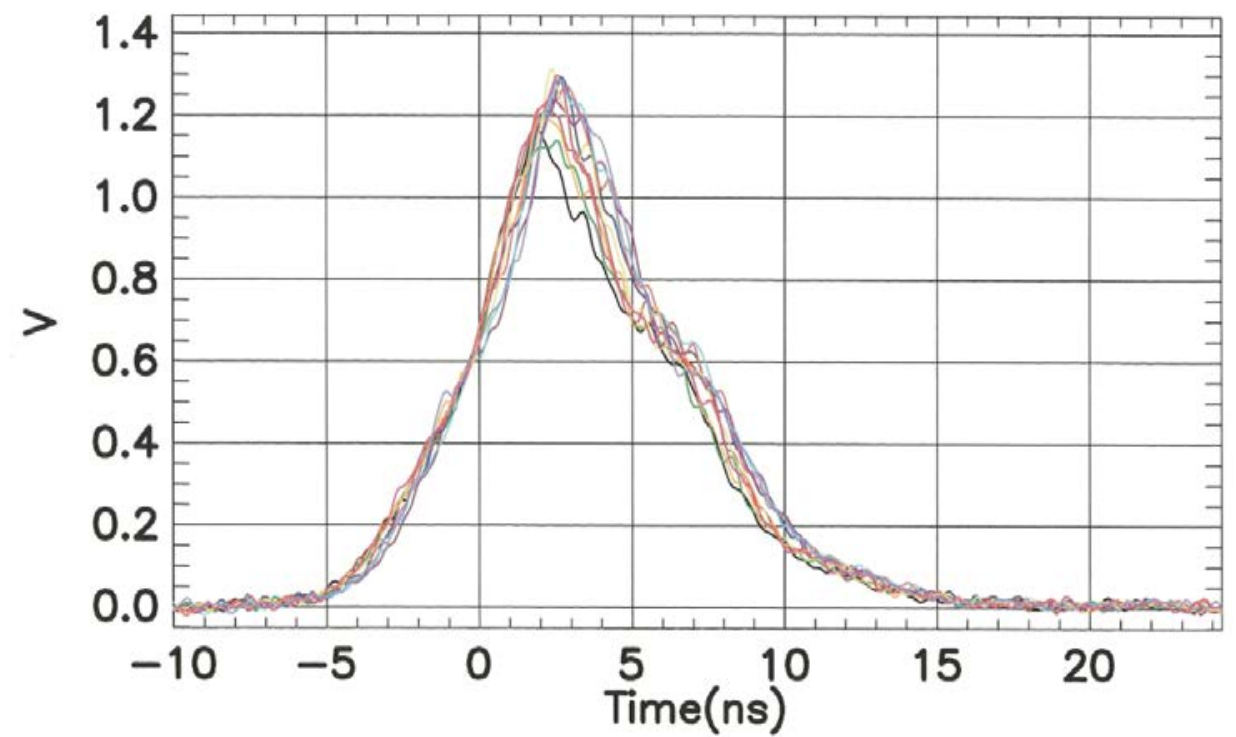

Figure 5.34. $18 \mathrm{~mJ}$ optical excitation pulses applied to black switch corresponding to photocurrents shown in Figure 4.32.

Figure 5.36 is a plot of the black switch minimum on resistance as a function of optical energy at $1064 \mathrm{~nm}$ for a variety of charge voltages. The black switch minimum resistance is 130, 79, 34 and 16 ohms at optical excitation energies of 3, 5, 9 and $14 \mathrm{~mJ}$ at $1064 \mathrm{~nm}$. The green switch has minimum on resistances of 100, 60, 33 and 19 ohms for excitation energies of 3, 5, 9, and $14 \mathrm{~mJ}$ at $1064 \mathrm{~nm}$. The results for the green and black switch are similar at $1064 \mathrm{~nm}$. However, the optical pulse width applied to the black switch is significantly narrower than the green switch (7.5 ns compared to 10 ns FWHM), so the peak optical power and peak photon flux at the black switch facet is higher than that applied to the green switch facet for a given optical energy. As a result, the green switch substrate appears to be constructed with a better switching material since it obtains similar minimum on resistance at a lower photon flux. We should expect some variation in switching properties between the individual switches due to the possible variation in vanadium, nitrogen and boron dopants in the substrates since they are cut from different positions in the $6 \mathrm{H}-\mathrm{SiC}$ crystal. Included in Figure 5.36 are points simulating a 


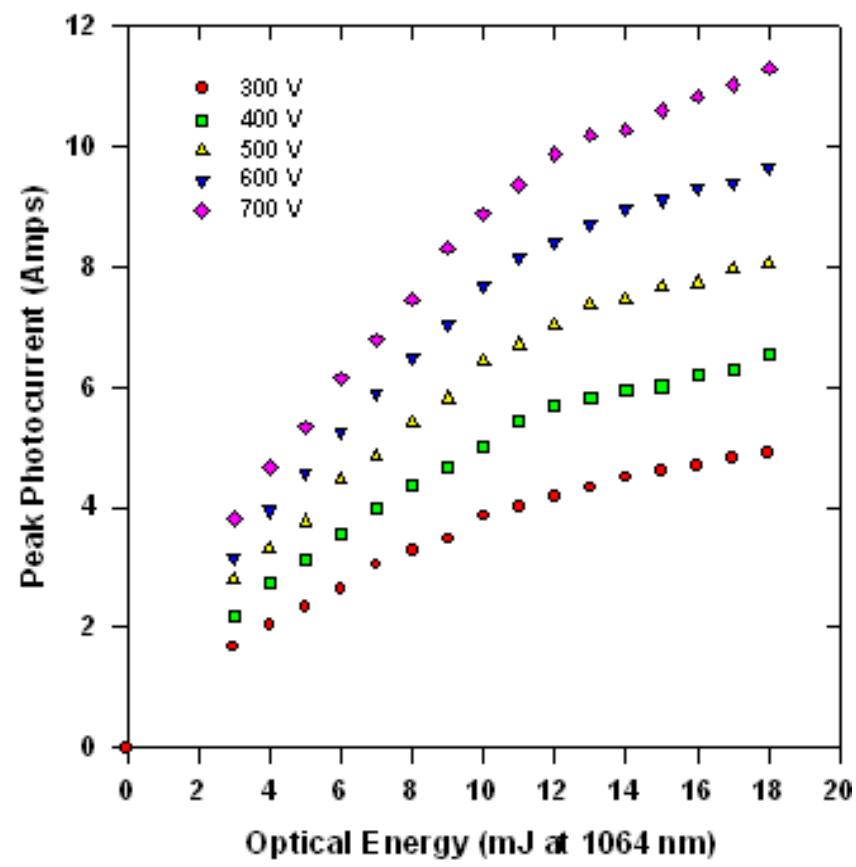

Figure 5.35. Peak photocurrent for the black switch plotted as a function of optical energy at $1064 \mathrm{~nm}$ for bias voltages of $300,400,500,600$ and 700 volts.

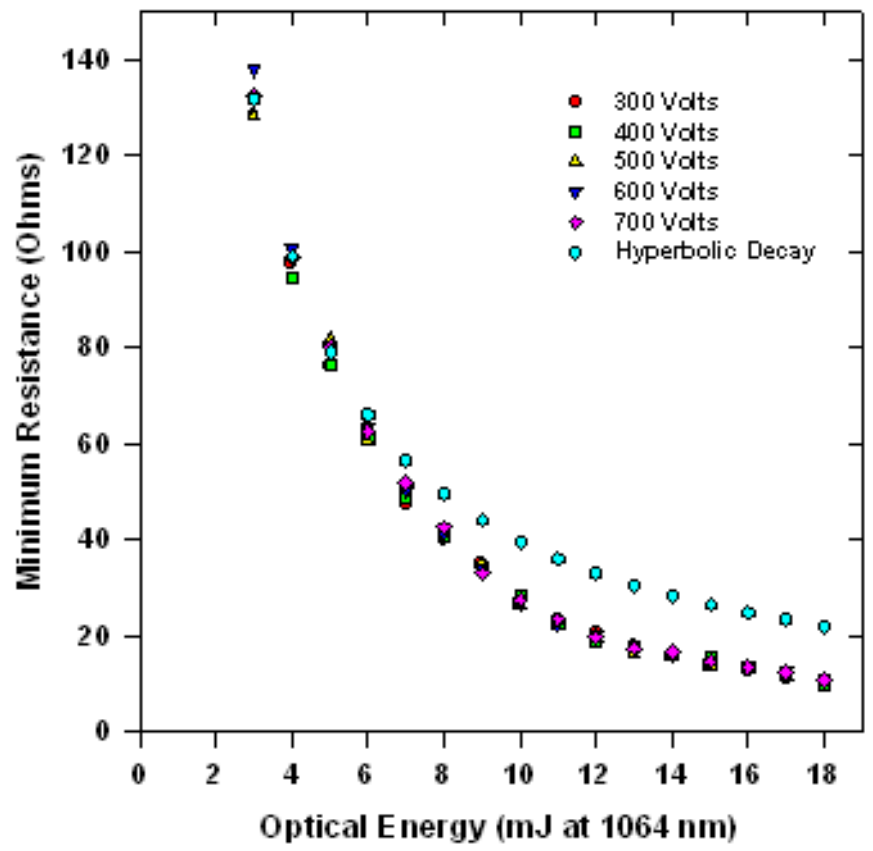

Figure 5.36. Minimum black switch on resistance as a function of optical energy at $1064 \mathbf{n m}$ for charge voltages of 300, 400, 500, 600 and 700 volts. 


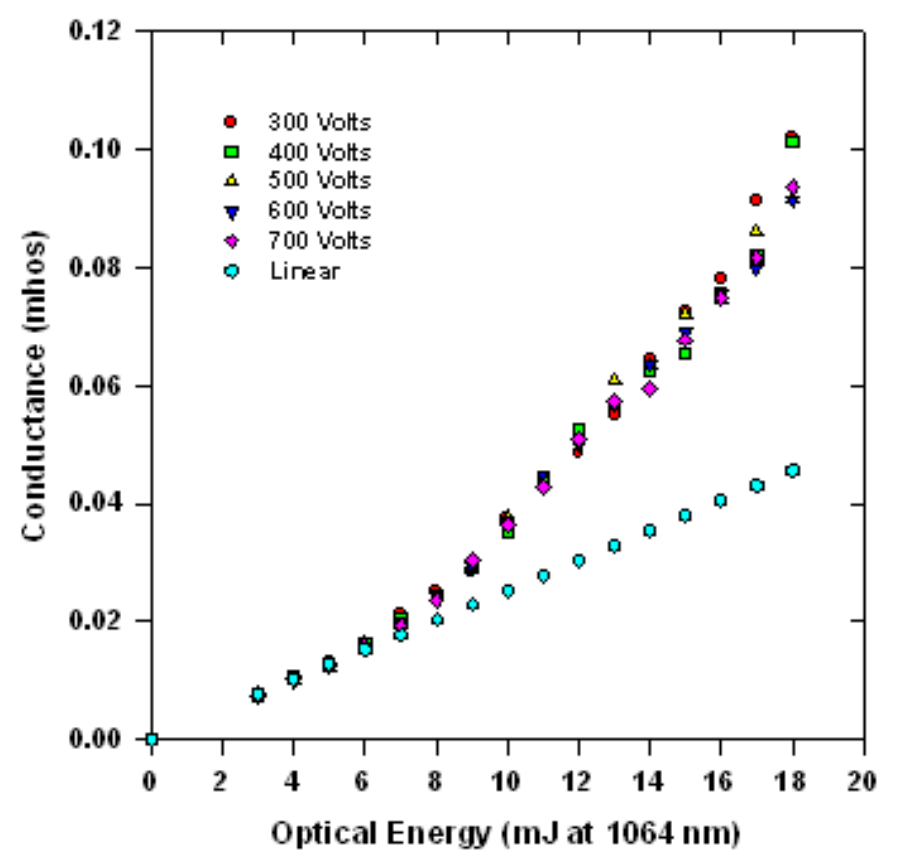

Figure 5.37. Maximum black switch conductance as a function of optical energy at $1064 \mathbf{n m}$ for charge voltages of $300,400,500,600$, and 700 volts.

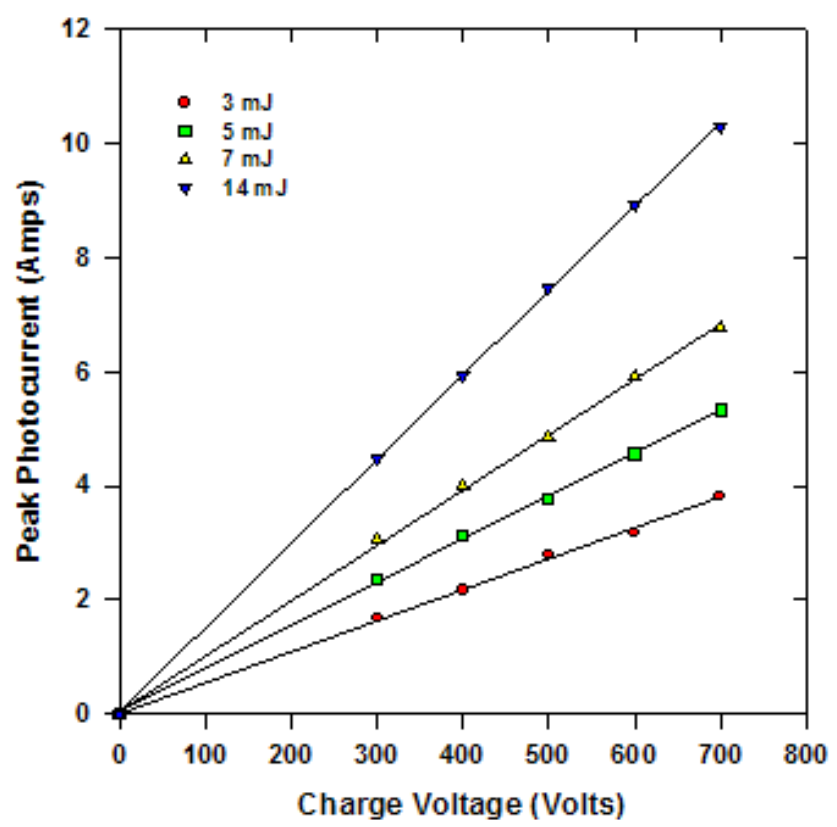

Figure 5.38. Black switch peak photocurrent plotted against switch charge voltage for optical energies of 3, 5, 7, and $14 \mathrm{~mJ}$ at $1064 \mathrm{~nm}$. 
hyperbolic decay of switch on resistance as a function of optical energy. The black switch minimum on resistance begins to decrease more rapidly than the hyperbolic decay at about $7 \mathrm{~mJ}$ of optical energy at $1064 \mathrm{~nm}$ indicating that the black switch on resistance versus optical energy is supralinear. The black switch maximum conductance is plotted as a function of optical energy in Figure 5.37. This data is the inverse of the minimum on resistance data of Figure 5.36. The hyperbolic decay points in Figure 5.36 become the linear points in Figure 5.37. As indicated in Figure 5.36, the black switch maximum conductance becomes supralinear for applied optical energies greater than $7 \mathrm{~mJ}$. This is in comparison to the green switch that had a linear to slightly supralinear variation of switch maximum conductance as a function of optical energy at 1064 $\mathrm{nm}$. The supralinearity of the maximum conductance of the black switch with optical energy is much stronger than that of the green switch. The maximum conductance of the black switch approaches variation as the square of the optical energy. Otherwise, the maximum conductance values of the green switch and black switch are relatively close with the green switch conductance slightly higher at lower optical energies $(<8 \mathrm{~mJ})$ and the black switch higher at higher optical energies (> $8 \mathrm{~mJ}$ ). Figure 5.38 is a plot of peak photocurrent in the black switch versus charge voltage for several different optical energies at $1064 \mathrm{~nm}$. The peak photocurrent increases linearly with charge voltage and optical energy as expected. The straight-line plots in Figure 4.38 correspond to minimum test circuit impedances of 184, 150, 102, and 68 ohms for optical excitation energies of 3, 5, 7, and $14 \mathrm{~mJ}$ at $1064 \mathrm{~nm}$. These results, similar to the green switch results, show that the vanadium compensated $6 \mathrm{H}-\mathrm{SiC}$ substrate material used to construct the black and green switches behave as optically controlled resistors. 


\section{Black Switch Results at 532 nm}

Overlays of five black switch photocurrent and applied optical intensity pulses as functions of time are shown in Figure 5.39 for a charge voltage of 800 volts and $15 \mathrm{~mJ}$ of applied optical energy at $532 \mathrm{~nm}$. The peak optical power for the optical intensity waveforms of Figure 5.39 is $2.61 \mathrm{MW}$. Figures 5.40 and 5.41 show the photocurrent for a range of applied voltages and the applied optical excitation pulses that generated the photocurrents, respectively. The photocurrent waveforms shown in Figure 5.39 and 5.40 include the slowly decaying photocurrent component that was first observed in the green switch results at $532 \mathrm{~nm}$. The black switch also achieves a significantly higher (lower) photocurrent (minimum on resistance) for excitation at a given optical energy at $532 \mathrm{~nm}$ compared to photocurrent for the same applied optical energies at $1064 \mathrm{~nm}$. The slowly decaying component of photocurrent waveforms shown in Figure 5.40 for the black switch represent about $12-13 \%$ of the total photocurrent.

The black switch photoconductive switching results at $532 \mathrm{~nm}$ are summarized in Figures 5.42 to 5.45 . Figure 5.43 is a plot of the peak photocurrent in the black switch as a function of charge voltage for 3, 5, 7, and $14 \mathrm{~mJ}$ optical energies at $532 \mathrm{~nm}$. The straight line plots in Figure 5.43 correspond to test circuit minimum resistances of 74, 63, 59, and 54 ohms (load resistance was 51.3 ohms) for the increasing optical energies. The minimum circuit resistance values correspond to minimum switch on resistance of $22.711 .7,7.7$ and 2.7 ohms for the black switch at $532 \mathrm{~nm}$. These values are significantly lower than the minimum on resistance obtained for the black switch for the same optical energies at $1064 \mathrm{~nm}$, which were 130, 78, 50.7 and 16.7 ohms. There are $6-8$ times as many carriers generated in the volume of the black switch for a given optical energy at $532 \mathrm{~nm}$ compared to $1064 \mathrm{~nm}$. This can be partially explained by the higher intensity applied to the black switch facet at $532 \mathrm{~nm}$ compared to 1064 


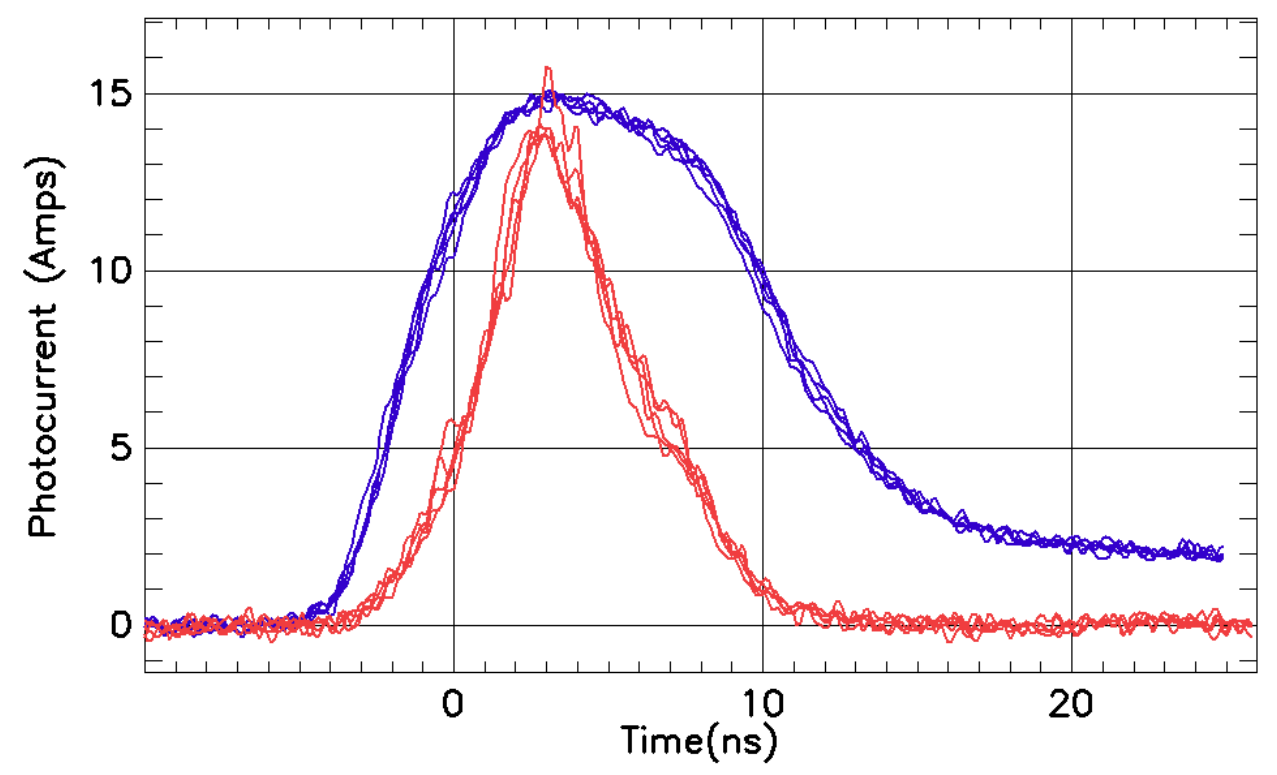

Figure 5.39. Overlay of five Black switch photocurrent and applied optical intensity temporal pulses for a charge voltage of 800 volts and optical energy of $15 \mathrm{~mJ}$ at $532 \mathrm{~nm}$.

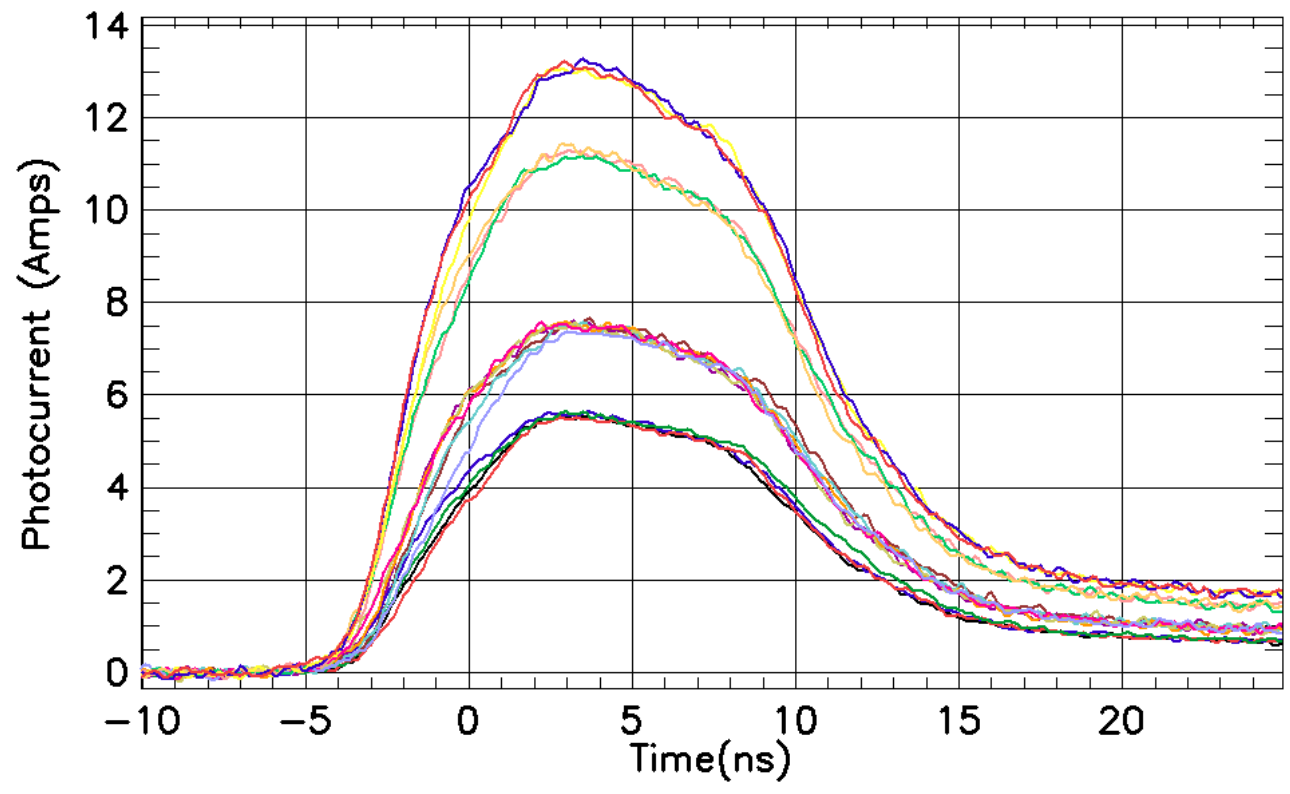

Figure 5.40. Overlay of black switch photocurrents for charge voltages of 300, 400, 600 and 700 volts for an applied optical energy of $15 \mathrm{~mJ}$. 


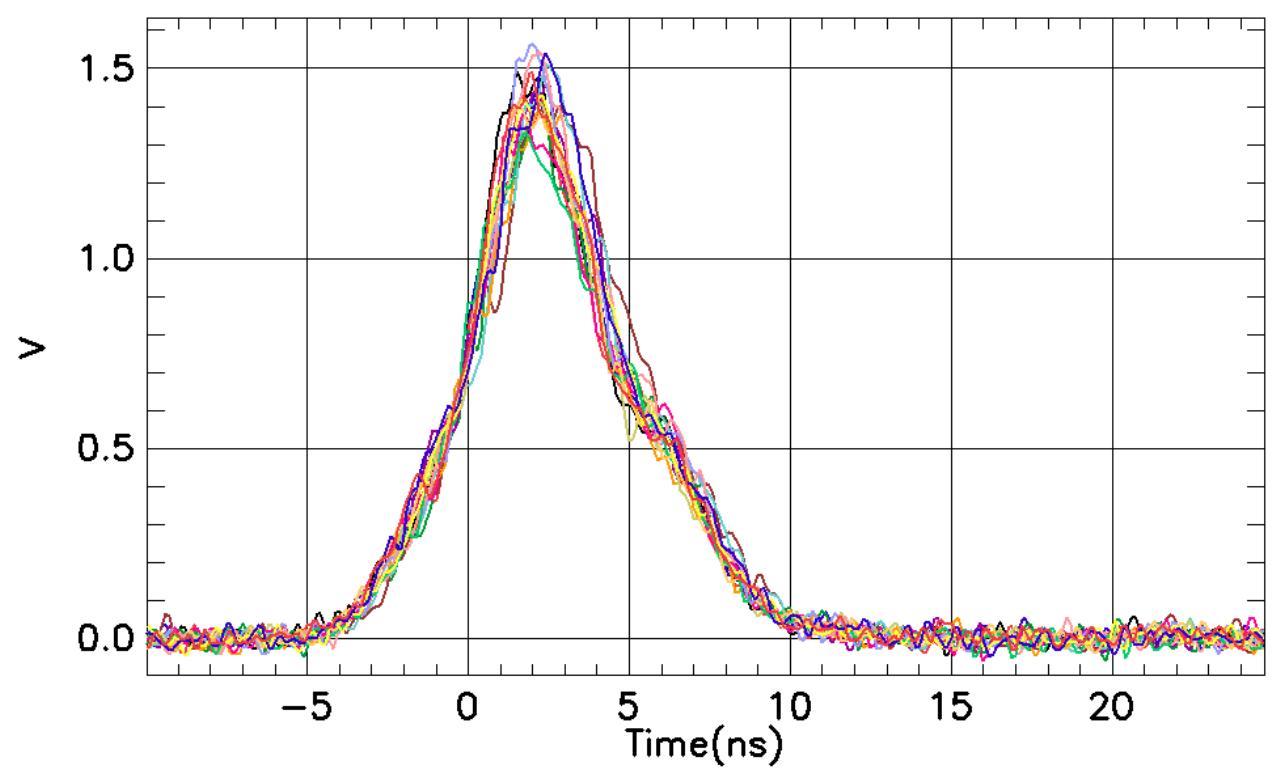

Figure 5.41. Overlay of $15 \mathrm{~mJ}$ optical pulses at $532 \mathrm{~nm}$ applied to the black switch to generate the photocurrents shown in Figure 4.40.

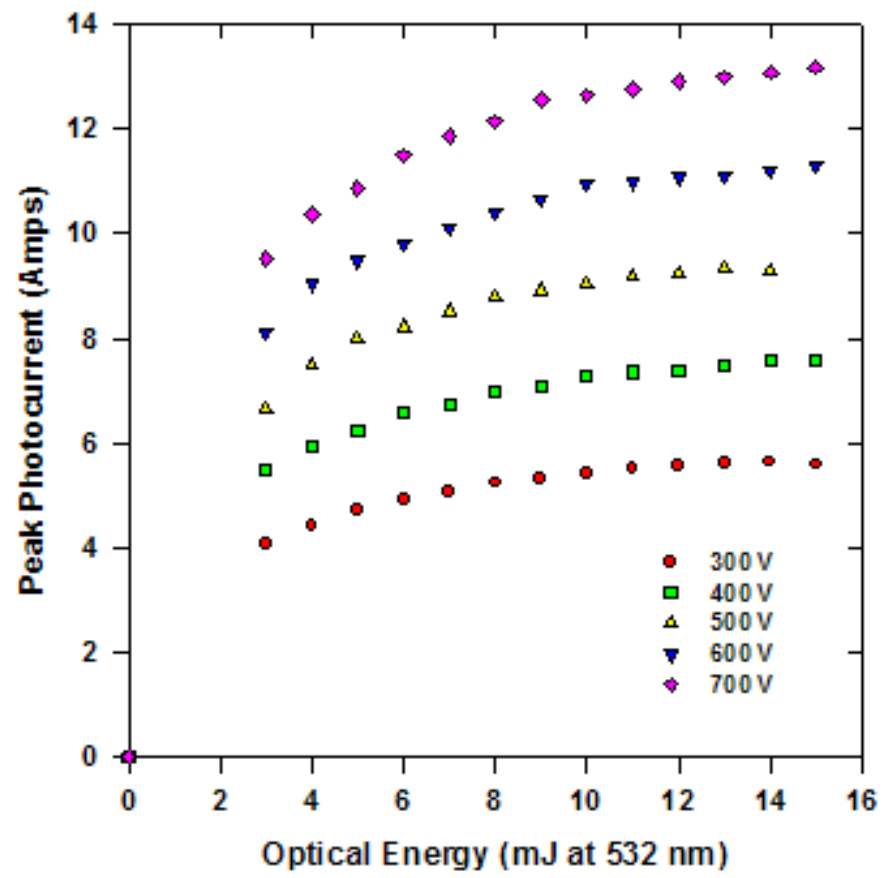

Figure 5.42. Black switch peak photocurrent plotted as a function of optical energy at 532 nm for charge voltages from 300 - 700 volts. 


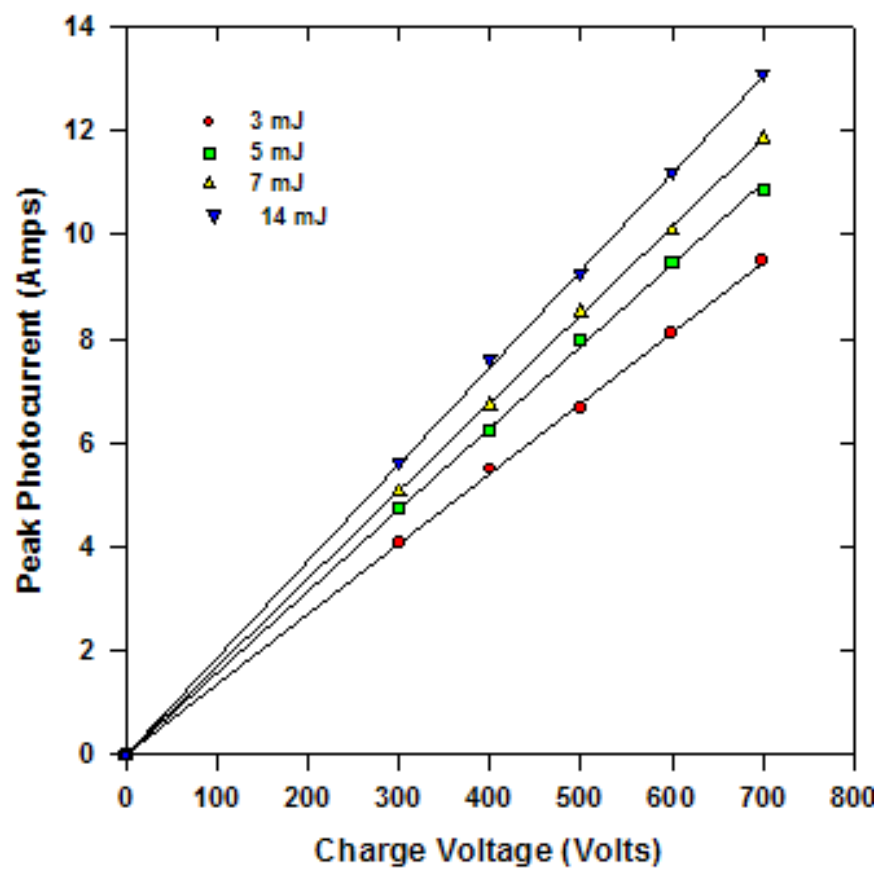

Figure 5.43. Black switch peak photocurrent plotted versus charge voltage for optical energies of $3,5,7$, and $14 \mathrm{~mJ}$ optical at $532 \mathrm{~nm}$

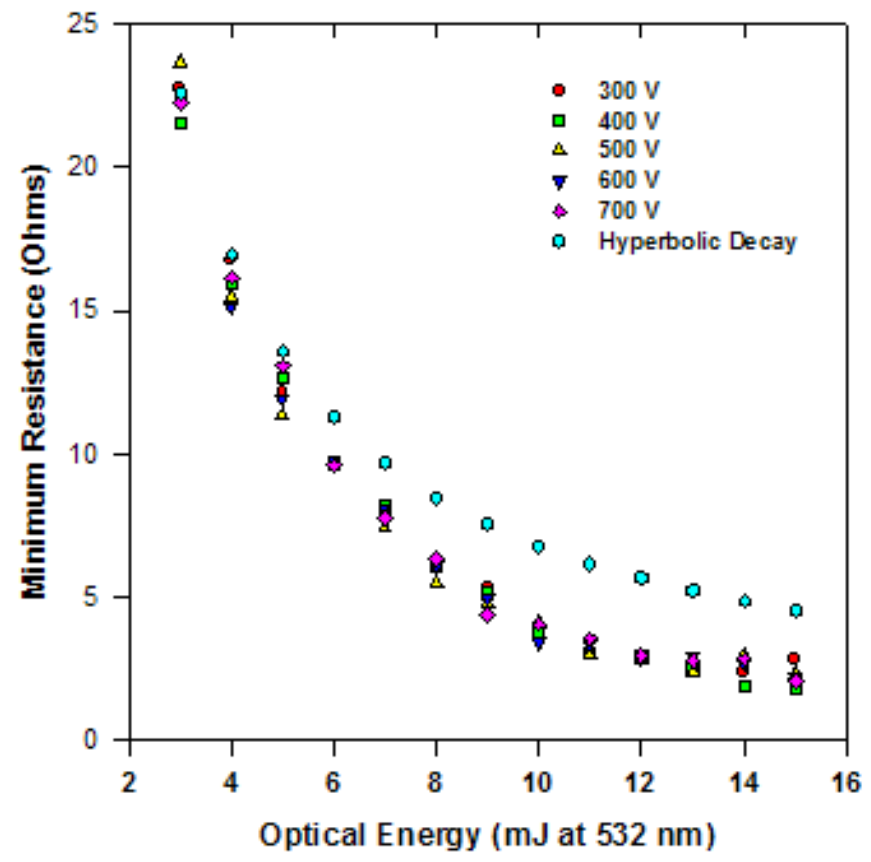

Figure 5.44. Black switch minimum on resistance plotted versus optical energy at $532 \mathbf{~ n m}$ for charge voltages of $300,400,500,600$, and 700 volts. 


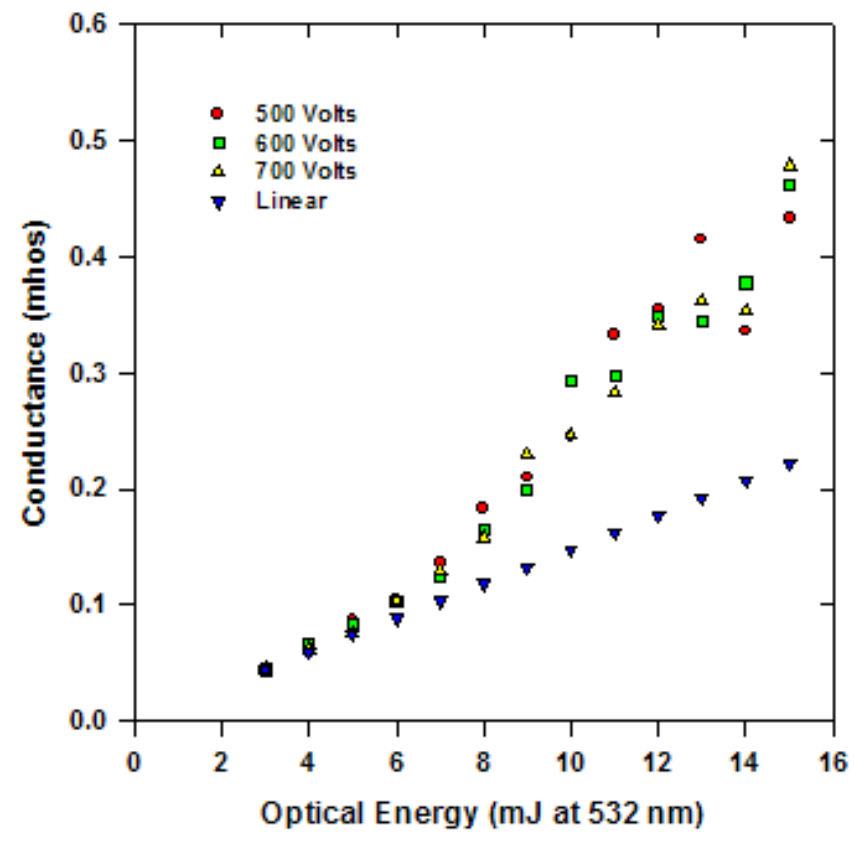

Figure 5.45. Black switch maximum conductance plotted versus optical energy at $532 \mathrm{~nm}$ for charge voltages of 500,600 , and 700 volts.

nm due to the different pulse widths for the two wavelengths. The pulse width for the 532 and $1064 \mathrm{~nm}$ optical pulses are 5.0 and $7.5 \mathrm{~ns}$, respectively, corresponding to an optical power that is $50 \%$ higher for a given optical energy at $532 \mathrm{~nm}$ compared to $1064 \mathrm{~nm}$. This difference can be partially resolved by comparing black switch results at $9 \mathrm{~mJ}$ at $1064 \mathrm{~nm}$ to results at $6 \mathrm{~mJ}$ at 532 $\mathrm{nm}$. The minimum black switch on resistance for $9 \mathrm{~mJ}(6 \mathrm{~mJ})$ at $1064 \mathrm{~nm}(532 \mathrm{~nm})$ is $34(9.5)$ ohms. The $532 \mathrm{~nm}$ wavelength is still $3-4$ times more efficient than the $1064 \mathrm{~nm}$ wavelength in carrier generation, even when the difference in peak optical power is taken into account. We can also compare the black switch minimum resistance at $532 \mathrm{~nm}$ to that of the green switch. However, the $532 \mathrm{~nm}$ optical pulse applied to the green switch has a $7.5 \mathrm{~ns}$ FWHM pulse width compared to the 5.0 ns FWHM pulse applied to the black switch. We can compare the $9 \mathrm{~mJ}$ results for the green switch to the $6 \mathrm{~mJ}$ results for the black switch so that the comparison will be for equal optical intensities at the switch facets. The green (black) switch has a minimum on 
resistance of 10.6 (9.5) ohms for 9 (6) $\mathrm{mJ}$ of $532 \mathrm{~nm}$ light. The results show that the green and black switches respond almost identically to a given optical power of $532 \mathrm{~nm}$ wavelength light.

The maximum conductance of the black switch for the $532 \mathrm{~nm}$ wavelength optical excitation exhibits (Figure 5.45) a fairly strong supralinear dependence on optical energy for optical energies greater than $6 \mathrm{~mJ}$. The black switch also exhibited supralinear dependence of maximum conductance on optical energy at $1064 \mathrm{~nm}$.

\section{Silver Switch Photoconductive Test Results}

The silver switch measures $1.222 \mathrm{~cm}$ by $1.206 \mathrm{~cm}$ by $382 \mu \mathrm{m}$ thick. The silver switch was metalized as described previously and tested as a photoconductive switch at the 532 and $1064 \mathrm{~nm}$ wavelengths. The tests performed on the silver switch were identical to the tests performed on the green and black switches. The optical pulse widths applied to the silver switch facet was 4.9 and 8.0 ns FWHM for the 532 and 1064 nm wavelengths, respectively.

\section{Silver Switch Photoconductive Test Results at 1064 nm}

An overlay of five pulses of the silver switch photocurrent and optical intensity for a charge voltage of 500 volts and an optical energy of $15 \mathrm{~mJ}$ at $1064 \mathrm{~nm}$ is shown in Figure 5.46. The peak optical power for the optical waveforms of Figure 5.46 is $1.68 \mathrm{MW}$. The optical pulse has a 7.5 ns pulse width FWHM compared to a 16 ns pulse width FWHM for the load current. Figure 5.47 and 5.48 show the silver switch load current for 300, 400, and 500 volt charge voltages and applied optical intensities (15 mJ, $1064 \mathrm{~nm})$ to obtain the load currents, respectively. The silver switch photoconductive test results at $1064 \mathrm{~nm}$ are summarized in Figures $5.49-5.52$. 
Figure 5.49 plots the peak photocurrent in the silver switch versus optical energy at 1064 nm for charge voltages of 300, 400, and 500 volts. The plots of Figure 5.48 show the same saturation effect that was obtained for the green and black switches due to the switch on resistance becoming much less than the load resistance. The silver switch peak photocurrent is plotted as a function of charge voltage in Figure 5.50 for optical energies of 3, 5, 9, and $13 \mathrm{~mJ}$ at $1064 \mathrm{~nm}$. The straight-line plots of Figure 5.50 correspond to test circuit resistances of 160.4, 109.5, 77.92, and 70.2 ohms for applied optical energies of 3, 5, 9, and $13 \mathrm{~mJ}$ at $1064 \mathrm{~nm}$. The load resistance for the silver switch tests at $1064 \mathrm{~nm}$ is 51 ohms. Figure 5.51 plots the silver switch minimum resistance as a function of optical energy at $1064 \mathrm{~nm}$ for charge voltages of 400, 400, and 500 volts. The silver switch minimum on resistance at $1064 \mathrm{~nm}$ is somewhat better than that of the black switch at lower optical energies, despite the black switch having higher (0.135 MW/mJ) optical powers applied to its facet compared to the silver switch (0.112 MW/mJ). The silver (black) switch has minimum on resistances of 108.6 (130), 58.5 (78), 16.9 (35) and 16.0 (14.5) ohms for applied optical energies of 3, 5, 9, and $15 \mathrm{~mJ}$. The silver switch also exhibits a supralinear response (Figure 5.52) of conductance versus optical energy. However, the silver switch supralinear response of conductance is not as strong as that of the black switch at $1064 \mathrm{~nm}$. 


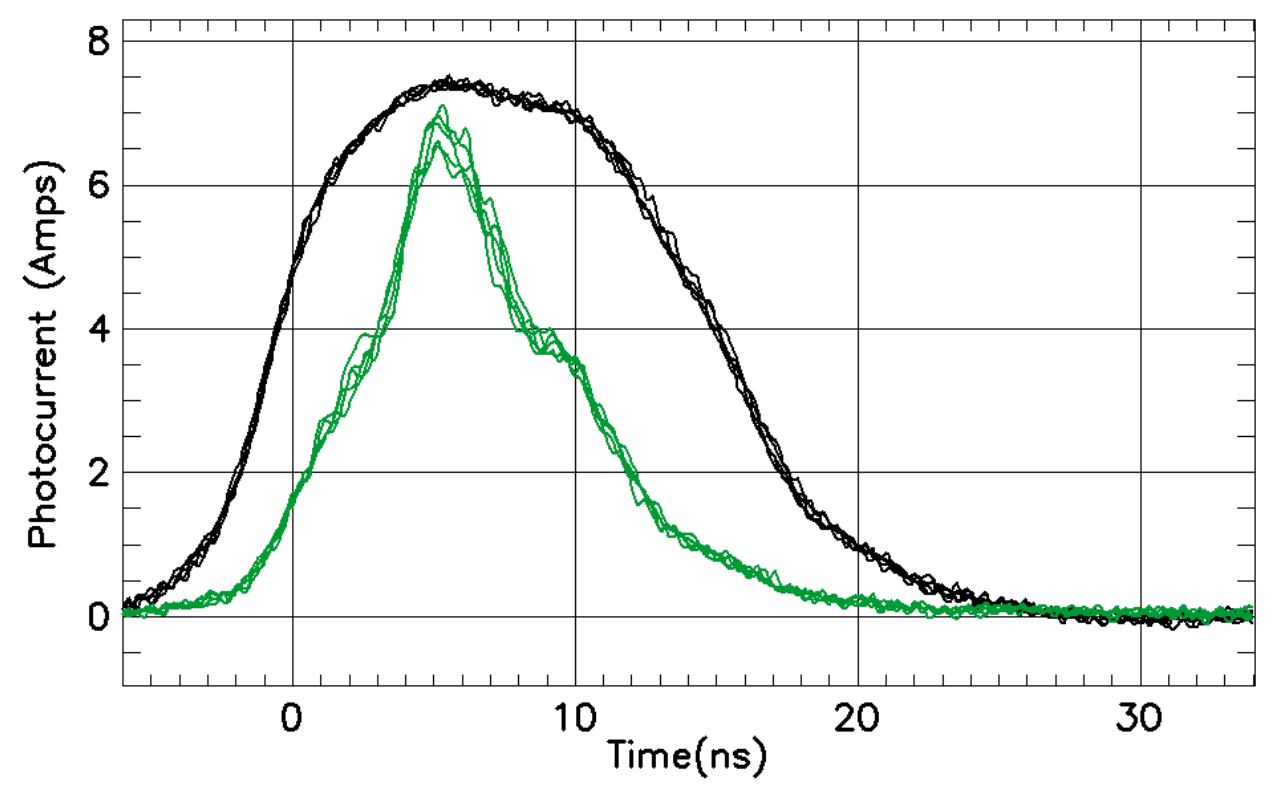

Figure 5.46. Silver switch photocurrent (black trace) and optical intensity (green trace) for charge voltage of 500 volts and applied optical energy of $15 \mathrm{~mJ}$ at $1064 \mathrm{~nm}$

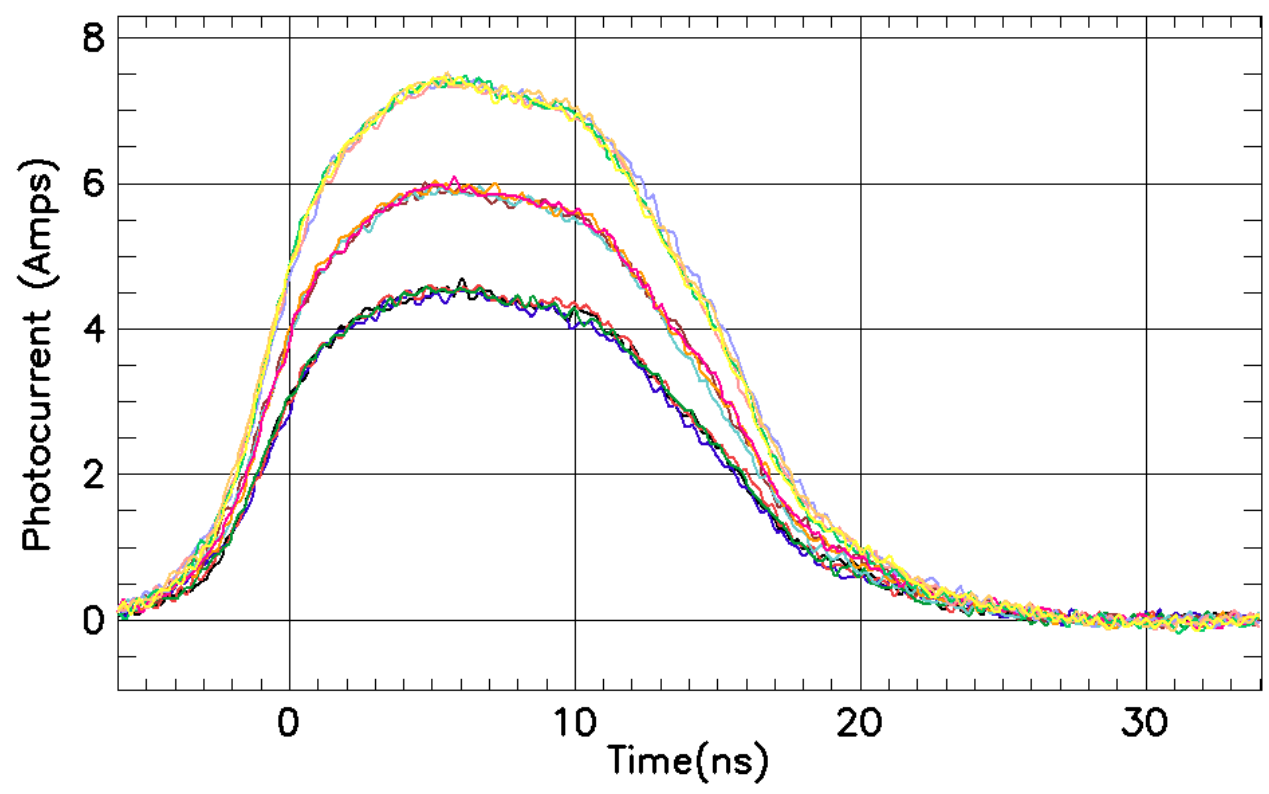

Figure 5.47. Silver switch photocurrents plotted as functions of time for charge voltages of 300, 400 and 500 volts for applied optical energy of $15 \mathrm{~mJ}$. 


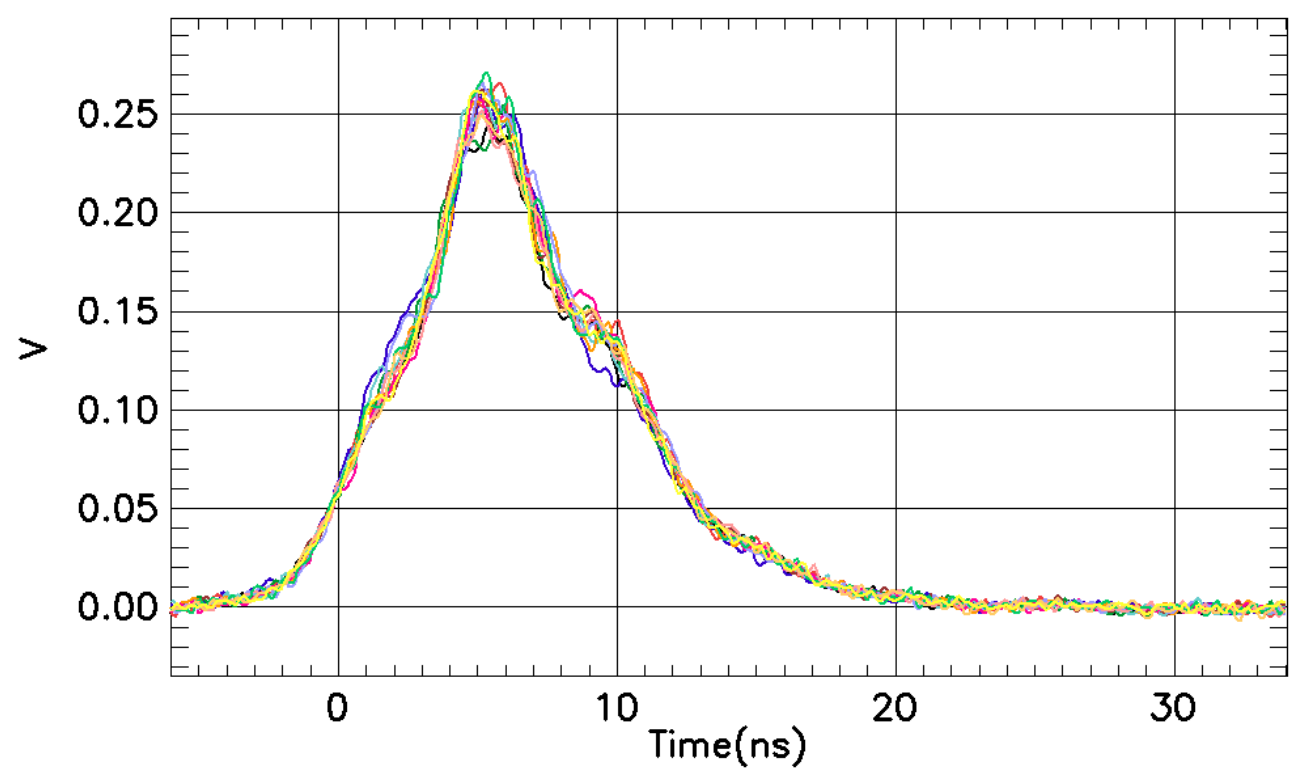

Figure 5.48. Optical intensitiy waveforms $(15 \mathrm{~mJ}$ at $1064 \mathrm{~nm})$ applied to the silver switch to obtain the photocurrents of Figure 4.47.

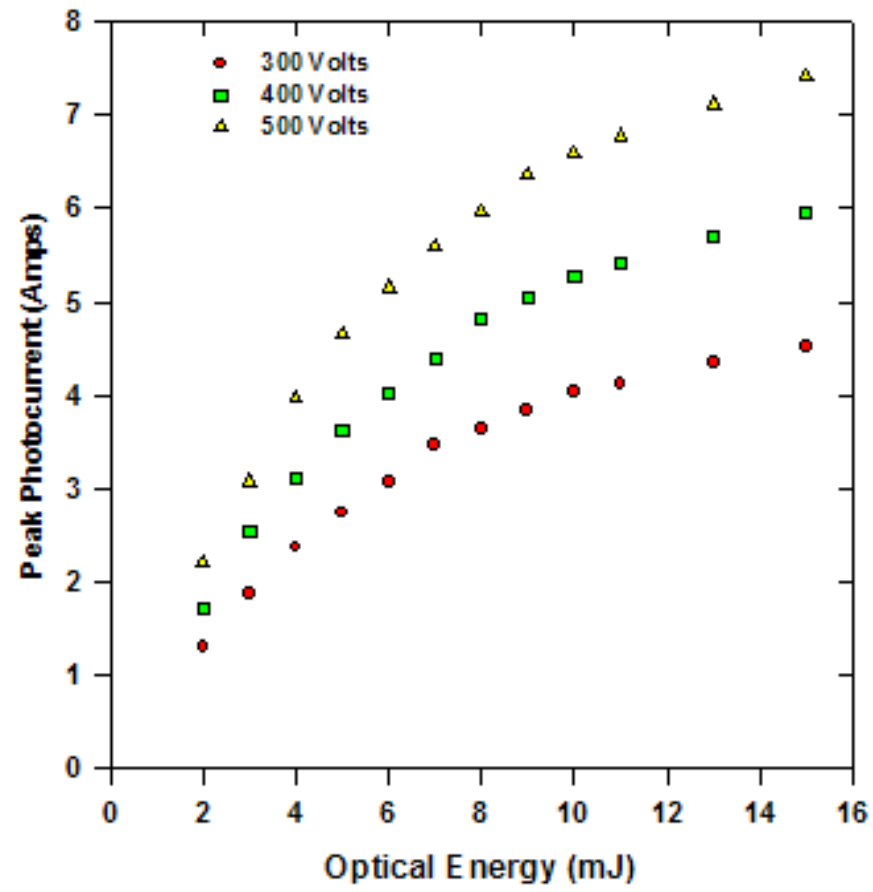

Figure 5.49. Silver switch peak photocurrent plotted versus optical energy at $1064 \mathbf{~ n m}$ for charge voltages of 300,400 , and 500 volts. 


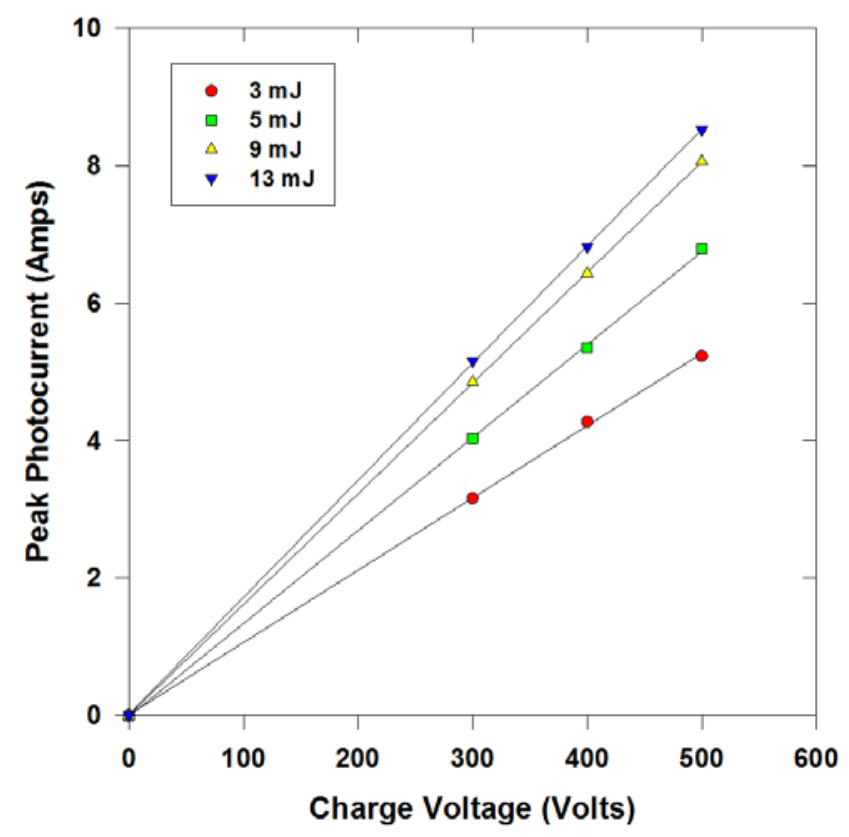

Figure 5.50. Silver switch peak photocurrent plotted versus charge voltage for optical energies of 3, 5, 9, and $13 \mathrm{~mJ}$ at $1064 \mathrm{~nm}$.

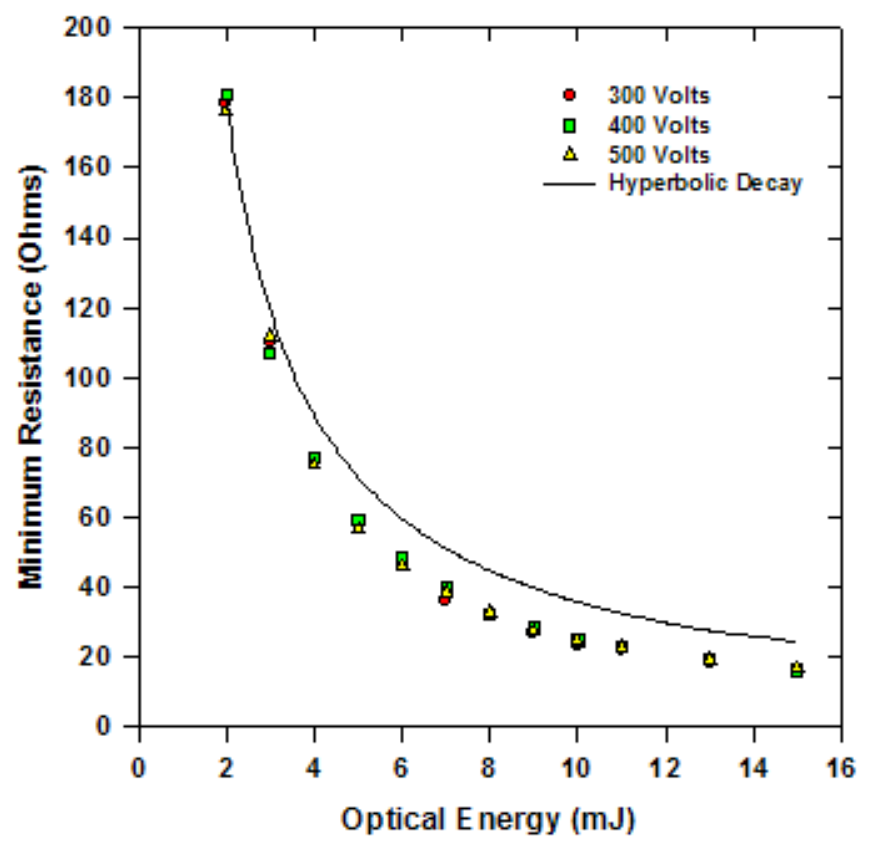

Figure 5.51. Silver switch minimum on resistance plotted versus applied optical energy at $1064 \mathrm{~nm}$ for charge voltages of 300, 400, and 500 volts. 


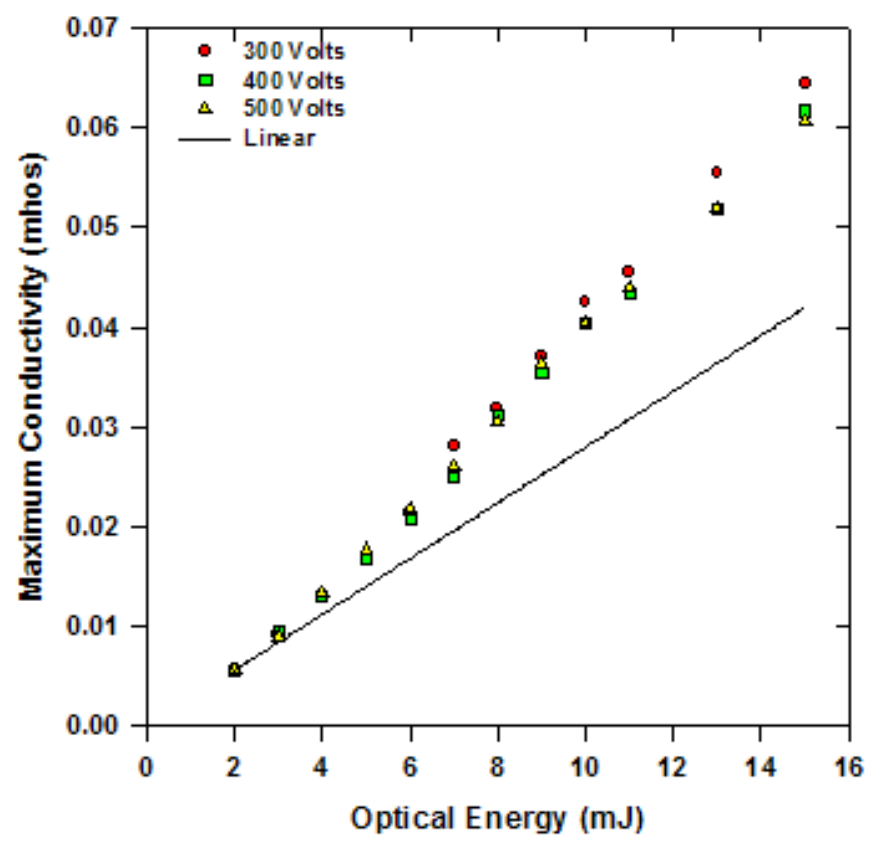

Figure 5.52. Silver switch maximum conductance plotted versus optical energy at $1064 \mathbf{~ n m}$ for charge voltages of 300, 400 and 500 volts.

\section{Silver Switch Photoconductive Test Results at 532 nm}

An overlay of five photocurrent and optical intensity pulses are shown in Figure 5.53 for the silver switch for a charge voltage of 500 volts and applied optical energy of $14 \mathrm{~mJ}$ at $532 \mathrm{~nm}$. The peak optical power for the optical pulses shown in Figure 5.53 is 2.02 MW. The optical intensity and photocurrent pulse widths are 5.0 and 13 ns, respectively. The photocurrent shows the long-lived component that is present in test results for the green and black switches at 532 nm. Figures 5.54 and 5.55 show the silver switch photocurrent and optical intensity pulses for charge voltages of 300, 400 and 500 volts and optical energy of $14 \mathrm{~mJ}$. The silver switch peak photocurrents for charge voltages of 300, 400, and 500 volts are plotted versus applied optical energy at $532 \mathrm{~nm}$ in Figure 5.56. This figure shows the same saturation effect that has been described for all previous switch results. The silver switch peak photocurrents are plotted versus charge voltage for applied optical energies of 3, 5, 9, and $13 \mathrm{~mJ}$ at $532 \mathrm{~nm}$. These optical 


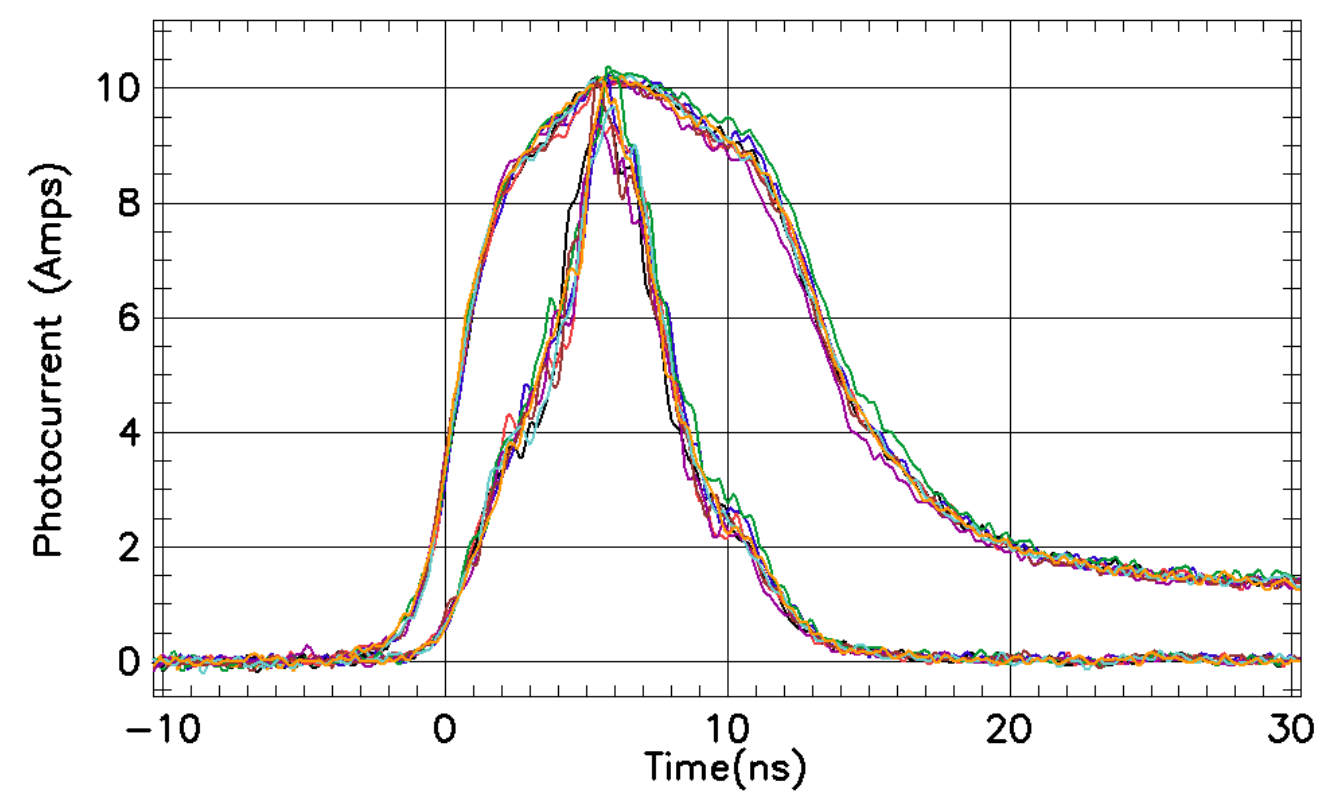

Figure 5.53. Silver switch photocurrent and optical intensity for charge voltage of $\mathbf{5 0 0}$ volts and optical energy of $14 \mathrm{~mJ}$ at $532 \mathrm{~nm}$.

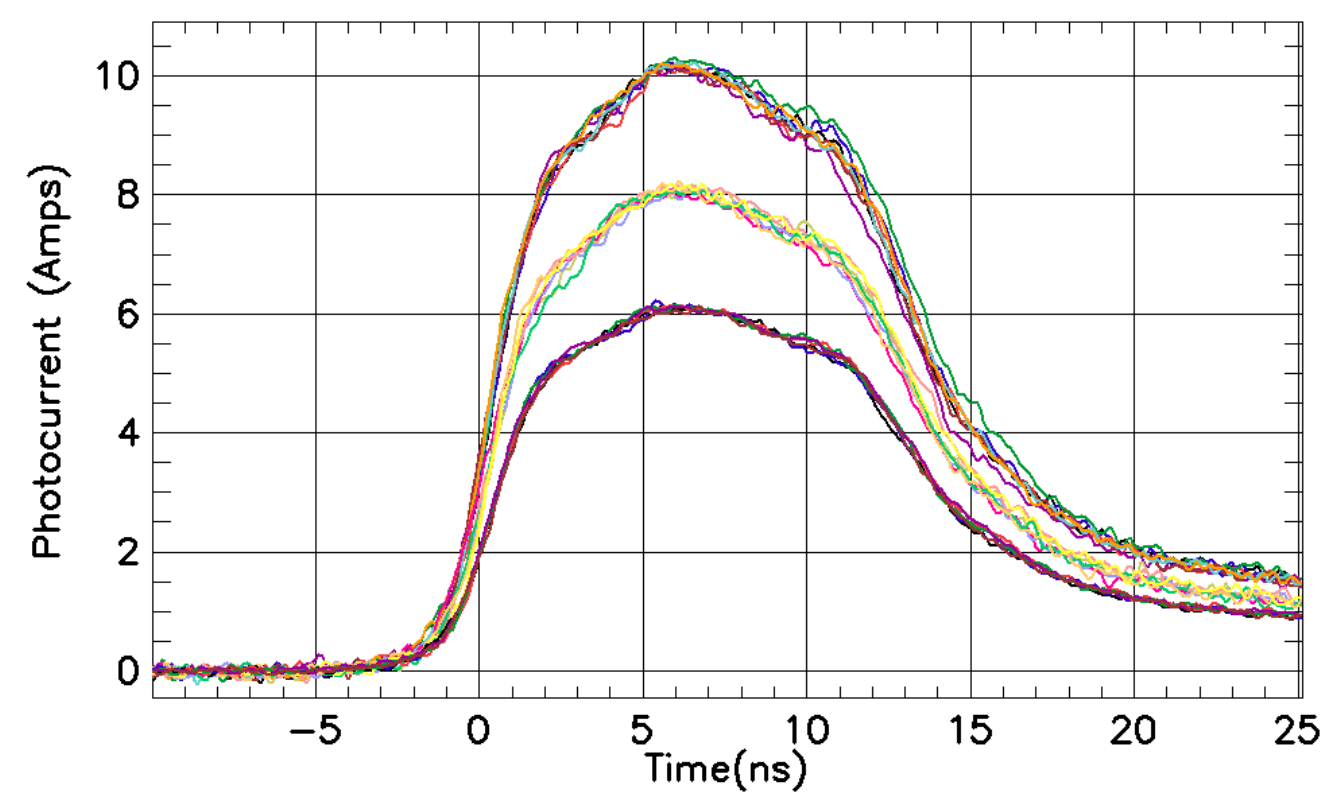

Figure 5.54. Silver switch photocurrent for charge voltages of 300, 400, and 500 volts and optical energy of $14 \mathrm{~mJ}$ at $1064 \mathrm{~nm}$. 


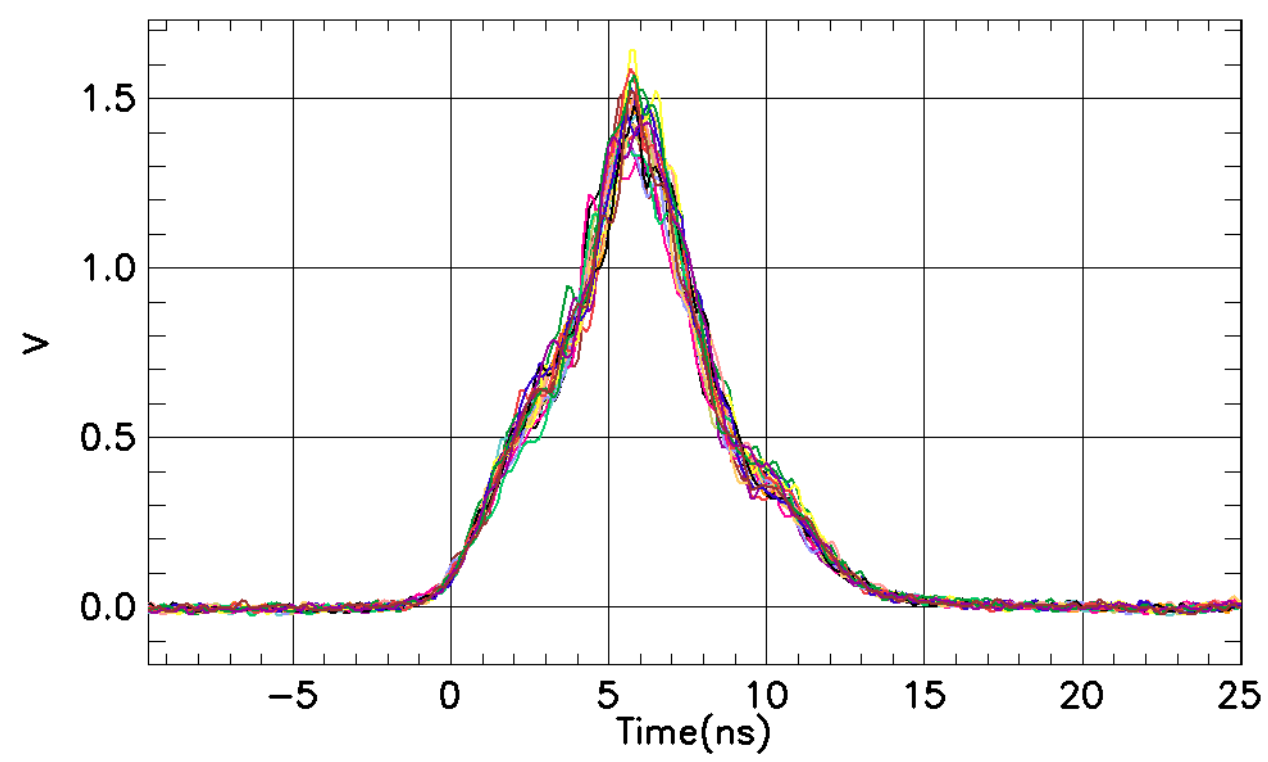

Figure 5.55. Optical intensity waveforms for $14 \mathrm{~mJ}$ at $532 \mathrm{~nm}$ pulses applied to silver switch to generate the photocurrents shown in Figure 4.54.

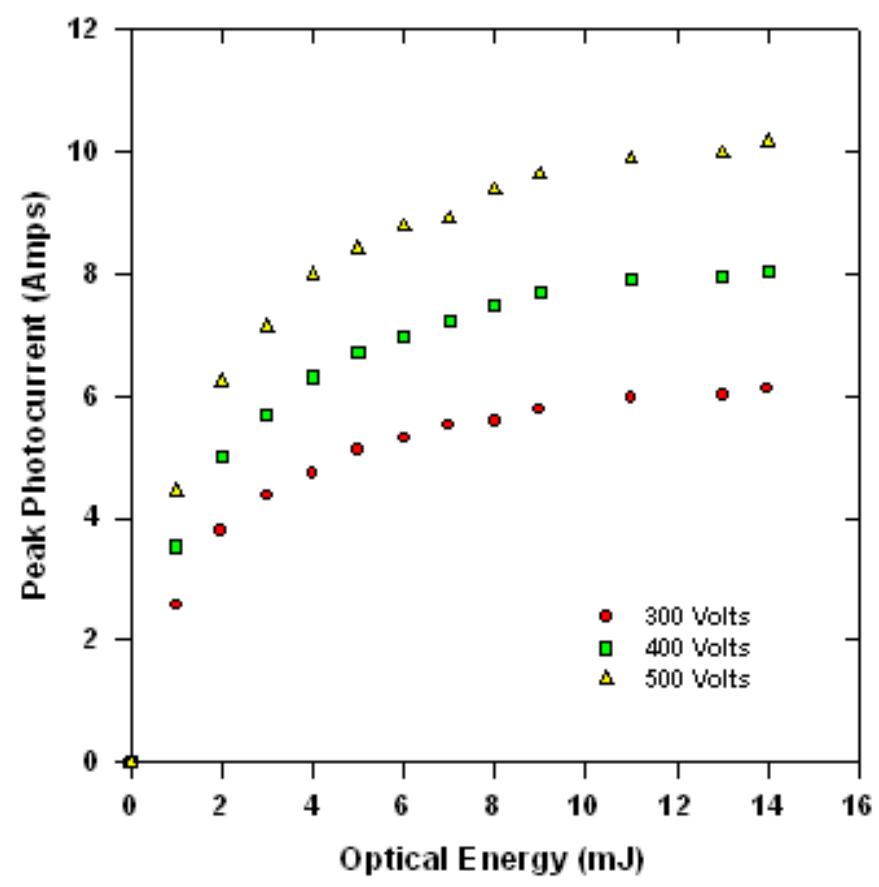

Figure 5.56. Silver switch peak photocurrent plotted versus optical energy at $532 \mathbf{n m}$ for charge voltages of 300,400 , and 500 volts. 


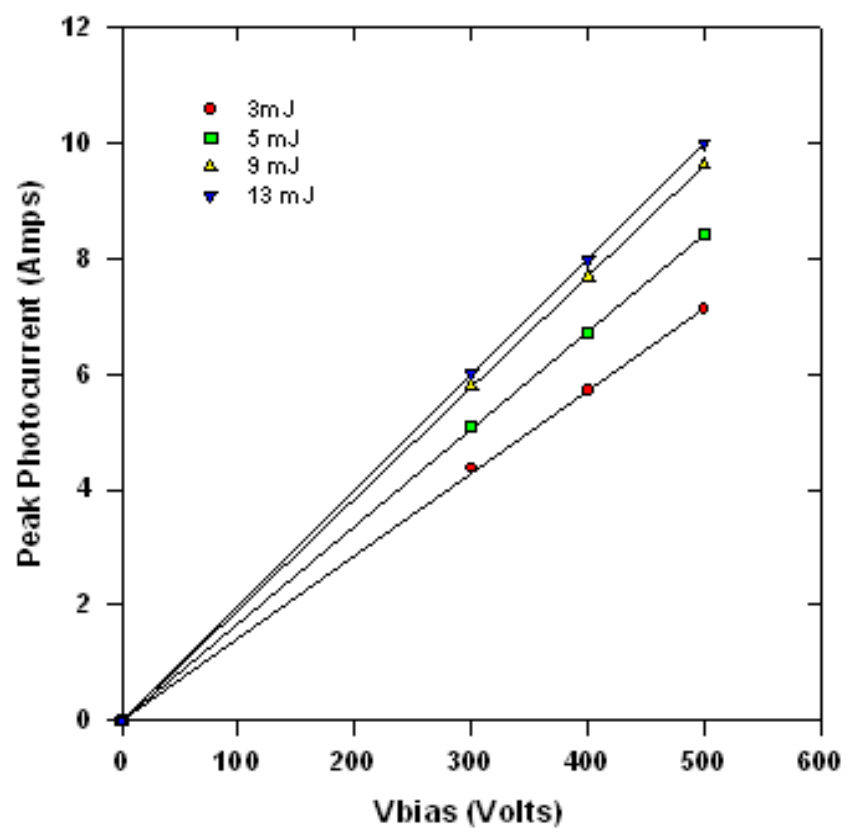

Figure 5.57. Silver switch peak photocurrent plotted versus charge voltage for optical energies of 3, 5, 9, and $13 \mathrm{~mJ}$ at $532 \mathrm{~nm}$.

energies correspond to peak optical powers of $0.432,0.72,1.3$, and $1.87 \mathrm{MW}$. This plot shows a linear relationship of peak photocurrent on charge voltage. The straight-line plots in Figure 5.56 correspond to test circuit resistances of 70, 59.5, 52, and 50 ohms. The test circuit includes a 46.5 ohm load resistance. The silver switch minimum on resistance and maximum conductance are plotted in Figures 5.58 and 5.59. The silver (black) switch minimum on resistance achieves values of 24 (23), 13 (12.2), 5.5 (5.0) and 3.5 (2.6) ohms for applied optical energies of 3, 5, 9 and $13 \mathrm{~mJ}$ at $532 \mathrm{~nm}$. The silver switch has minimum on resistances that are almost identical to that of the black switch at $532 \mathrm{~nm}$. The silver switch achieves these on resistances, despite the fact that the optical power applied to the black switch facet is $22 \%$ higher for all applied optical energies. The silver switch on resistance is $4-5$ times lower for the same optical energies at 532 $\mathrm{nm}$ compared to $1064 \mathrm{~nm}$. The superior performance of the silver switch at $532 \mathrm{~nm}$ compared to $1064 \mathrm{~nm}$ can be partially explained by the applied optical power at $532 \mathrm{~nm}$ being 30\% higher 


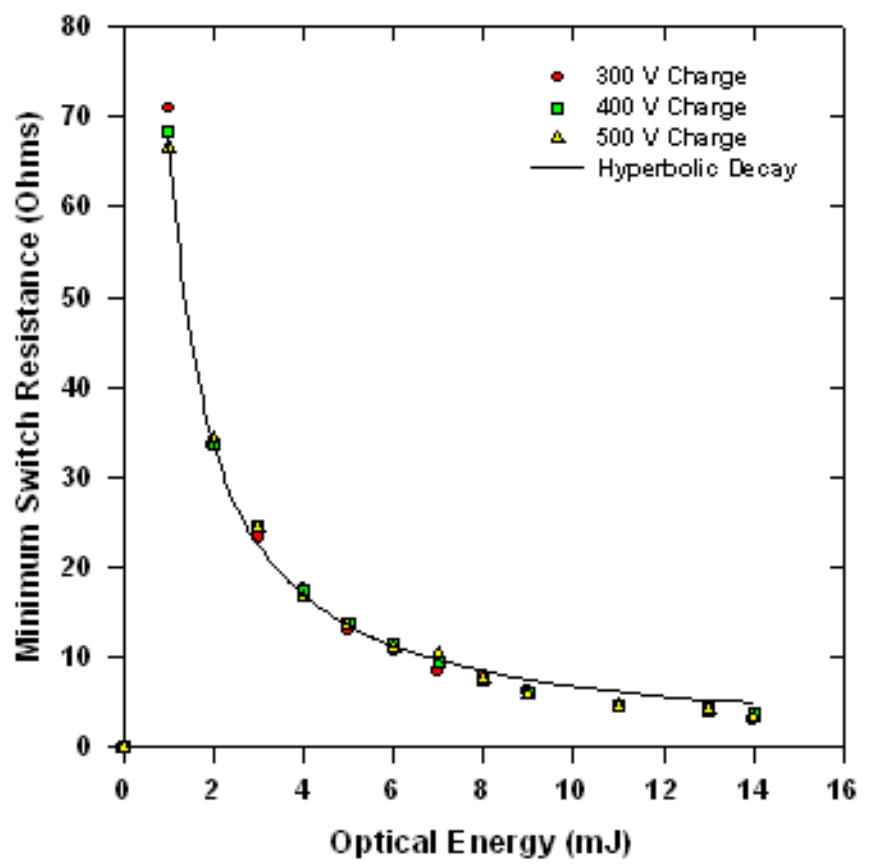

Figure 5.58. Silver switch minimum on resistance plotted versus optical energy at $532 \mathbf{n m}$ for charge voltages of 300,400 , and 500 volts.

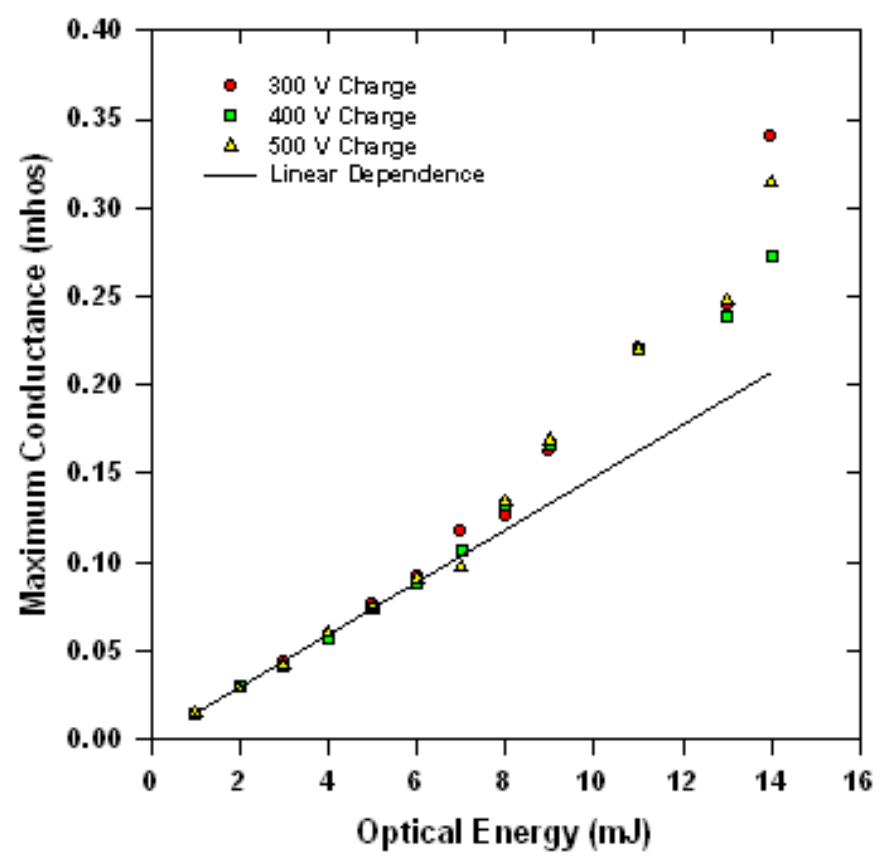

Figure 5.59. Silver switch maximum conductance plotted versus optical energy at $532 \mathbf{n m}$ for charge voltages of 300,400 , and 500 volts.

than that at $1064 \mathrm{~nm}$ for a given optical energy. This is a result of the shorter temporal profiles for the optical pulse at $532 \mathrm{~nm}$ compared to $1064 \mathrm{~nm}$. However, there are still 1.5 times as many 
photons incident on the silver switch facet at $1064 \mathrm{~nm}$ compared to $532 \mathrm{~nm}$. The $532 \mathrm{~nm}$ photons are much more efficient than the $1064 \mathrm{~nm}$ photon in generating carriers in the $6 \mathrm{H}-\mathrm{SiC}$ material. This is true for the green, black, and silver switches.

\section{4) Summary of Initial Photoconductive Switching Results for $6 \mathrm{H}-\mathrm{SiC}$ Material}

The photoconductive switching results for the green, black, and silver switches fabricated using the $6 \mathrm{H}-\mathrm{SiC}$ substrates have strong similarities: All switches have a very strong photoconductive response at the below bandgap wavelengths 532 and $1064 \mathrm{~nm}$; all switches have a very fast response indicating short recombination times; all switches achieve low on resistances (2 - 4 ohms) for excitation at $532 \mathrm{~nm}$ for applied optical energies of $13-15 \mathrm{~mJ}$; the minimum switch on resistances are $\sim 10$ - 11 orders of magnitude lower than the switch dark resistances; all switches show strong switching action at $1064 \mathrm{~nm}$ indicating a large number of carriers being excited from levels $<1.17 \mathrm{eV}$; all switches have a long lived component of photocurrent at $532 \mathrm{~nm}$ excitation; each switch exhibits a supralinear, or linear, dependence of switch conductance on applied optical energy for both the 1064 and 532 nm wavelengths; switch extrinsic photoconductive response is much stronger at the $532 \mathrm{~nm}$ wavelength; all switches behave as optically controlled resistors.

A direct comparison of the performance of the green, black and silver switches is made somewhat difficult due to differences in applied optical pulse width and pulse shape. Some of this difficulty can be removed by taking a closer look at the temporal response of the switches. The fast response of the $6 \mathrm{H}-\mathrm{SiC}$ material used to construct the green, black and silver switches should be expected based on the measured value $130 \mathrm{ps}$ for the recombination time of vanadium compensated $6 \mathrm{H}-\mathrm{SiC}$ [5.3]. A recombination time that is much shorter than the applied optical 
pulse results in a switch on resistance that is inversely proportional to the applied optical intensity. Equation 5.4 gives the theoretical switch on resistance [5.2] for an applied rectangular optical pulse for the case where the carrier recombination time is much shorter than the optical pulse width. Where $d$ is the switch thickness in the direction of current flow, $\tau_{\mathrm{r}}$ is the recombination time, $\mu_{\mathrm{n}}$ is the electron mobility, $\mathrm{r}$ the reflectivity, $\eta$ the material quantum efficiency, $\mathrm{W}$ is the switch width and $\mathrm{P}(\mathrm{t})$ is the applied optical power.

$$
R(t)=\frac{d^{2} \hbar \omega}{P(t)(1-\exp (-\alpha W))(1-r) \eta \tau_{r} \mu_{n} q}
$$

The optical pulses that we have applied to the switches are not rectangular. However, we will see in Chapter 7 that the inversely proportional relationship between switch resistance and optical power holds for other optical pulse shapes, as long as the optical pulse width is much longer than the switch recombination time. As a result, the minimum on resistance of the switches that we have tested is inversely proportional to the peak optical power. Therefore, a more meaningful comparison of the green, black and silver switches is obtained by plotting the minimum switch resistance and maximum conductance as a function of peak applied optical power. This will remove the difficulties in comparing switch performance due to different optical pulse wave shapes and pulse widths.

Figures 5.60 and 5.61 are plots of the green, silver and black switch minimum on resistance and maximum conductance as a function of peak applied optical power at $1064 \mathrm{~nm}$. The green and silver switches have almost identical performance for excitation with the same 
optical power at $1064 \mathrm{~nm}$. The green and silver switch maximum conductance has a linear dependence on peak optical power up to 1.2 MW. The green and silver switches also have a factor of $2-3$ better performance than the black switch at lower applied optical power. The black switch has a higher on resistance compared to the green and silver switch at applied peak powers less than 1.25 MW. The black switch also has a nonlinear dependence of maximum conductance versus peak optical power. Initially, the black switch maximum conductance increases at a rate of $0.02 \mathrm{mhos} / \mathrm{MW}$. The rate of increase of the maximum conductance of the black switch increases significantly at 1MW to a rate of 0.054 mhos/MW.

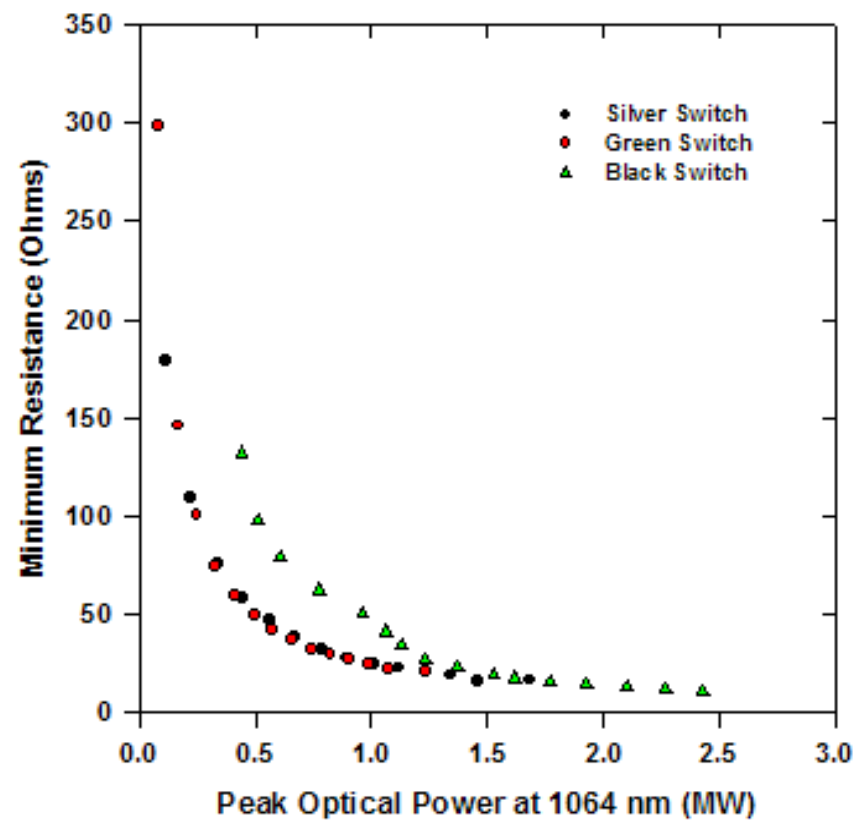

Figure 5.60. Minimum on resistance of silver, green, and black switches plotted as a function of peak optical power at $1064 \mathrm{~nm}$ wavelength. 


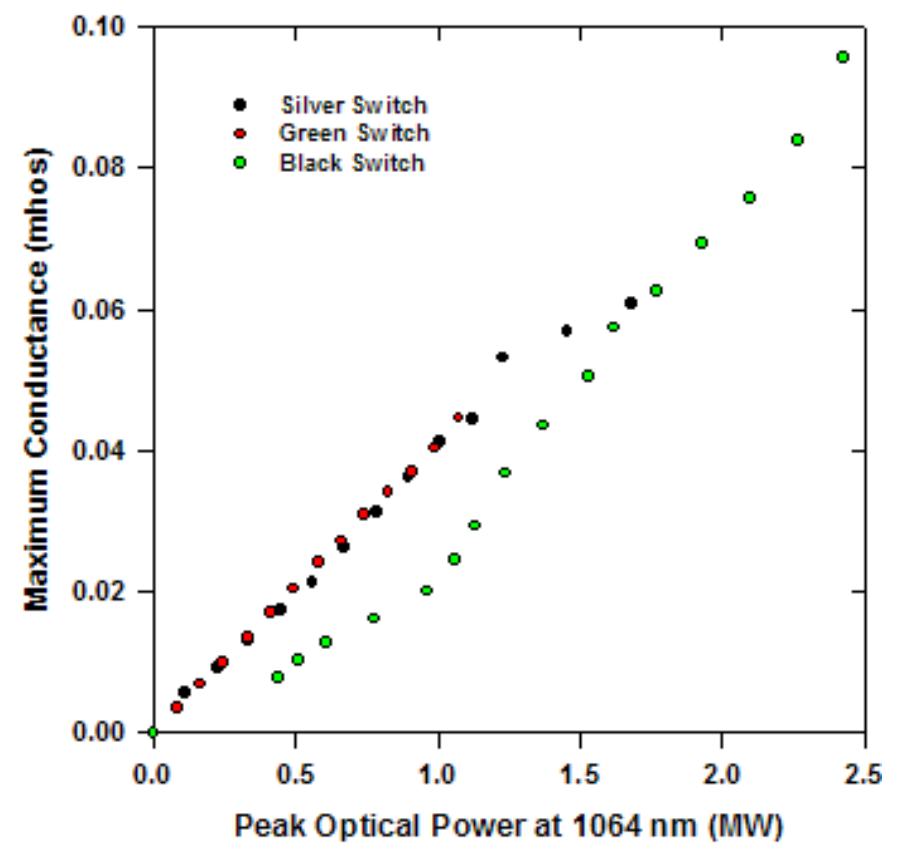

Figure 5.61. Maximum switch conductance of green, silver and black switch plotted versus peak optical power at $1064 \mathrm{~nm}$.

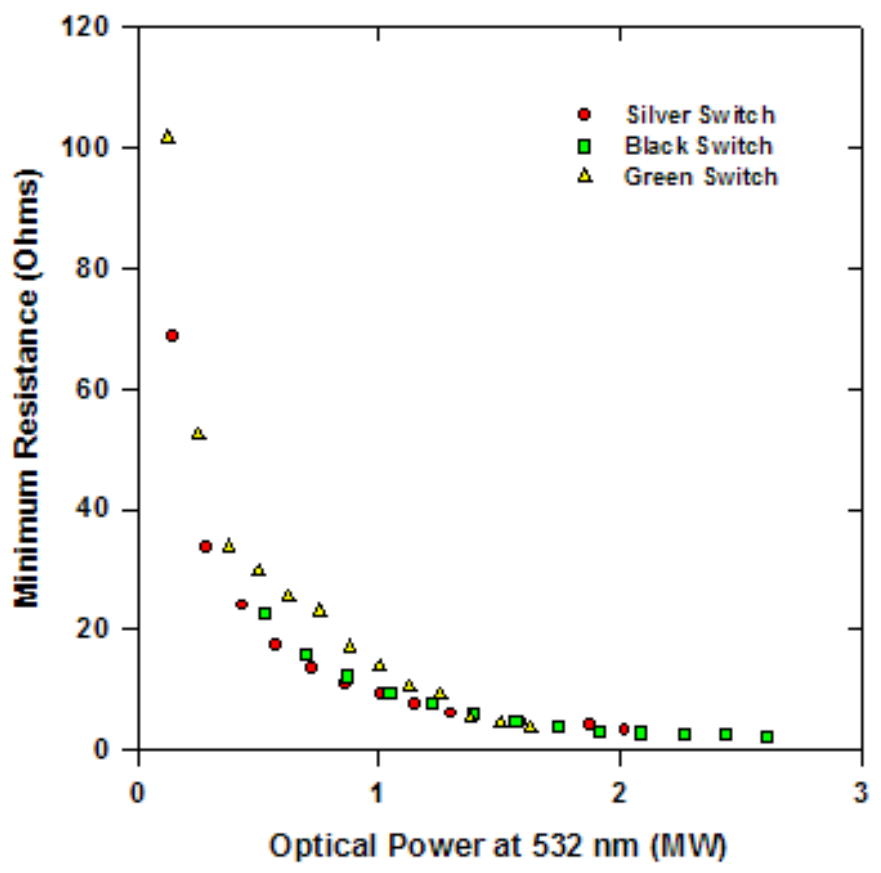

Figure 5.62. Minimum Resistance for silver, black and green switch plotted as a function of peak optical power at $532 \mathrm{~nm}$. 


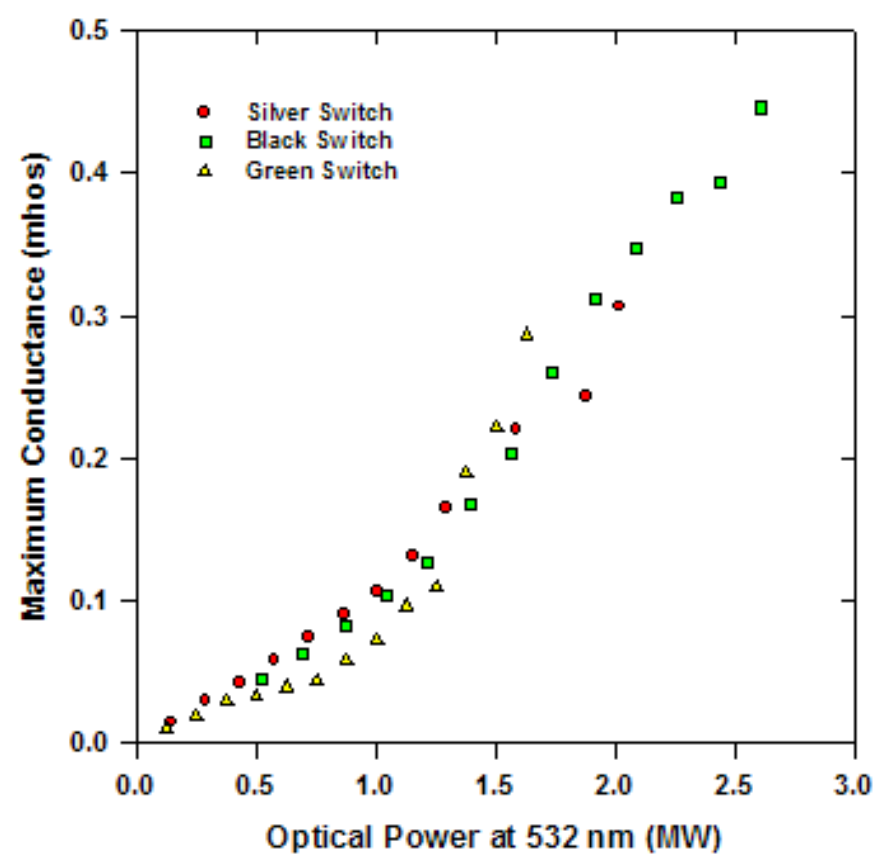

Figure 5.63. Maximum conductance for silver, black and green switch plotted versus peak optical power at $532 \mathrm{~nm}$.

Figures 5.62 and 5.63 show the silver, green and black switch minimum resistance and maximum conductance plotted as a function of peak optical power at $532 \mathrm{~nm}$. The performance of the silver, green and black switches at the $532 \mathrm{~nm}$ wavelength is very similar with the silver and black switches having a slightly lower minimum resistance than the green switch at lower optical power $(<1 \mathrm{MW})$. All three switches exhibit a supralinear dependence of maximum conductance on peak optical power.

\subsection{1) Supralinear Response of 6H-SiC Switches}

The supralinear dependence of conductance on peak optical power indicates that the carrier recombination time in the switch substrate is increasing with optical power. Evidence of an increase carrier recombination time is manifested in the appearance of a long lived component of photocurrent for all three switches for excitation at the $532 \mathrm{~nm}$ wavelength. However, this 
long lived component of photocurrent accounts for ten to fifteen percent of the total photocurrent, which is insufficient to completely explain the nonlinearity in Figure 5.63. In addition, the long lived component of photocurrent is present for all excitation at $532 \mathrm{~nm}$, but the maximum conductance has a steep increase in its rate of rise with respect to optical power at 532 $\mathrm{nm}$ at $1 \mathrm{MW}$. This indicates the presence of a sensitization center [5.5 - 5.7] located in the energy gap between the vanadium acceptor level and the conduction band. The concept of a sensitization [5.5 - 5.7] center can be better understood using Figures 5.64, 5.65 and 5.66.

Figure 5.64 shows the Fermi level position and the positions of the vanadium acceptor, boron acceptor, nitrogen donor, type 1 and type two trap energy levels for a $6 \mathrm{H}-\mathrm{SiC}$ substrate at thermal equilibrium in the dark. The type 1 (2) trap level is located at an energy that is sufficiently above (below) the Fermi level so that this level is devoid (filled) with electrons at equilibrium and that the trap density (both type 1 and 2) is equivalent to the vanadium acceptor level density. Let us assume that trap type 1 has electron and hole capture cross sections that are equivalent to that of the vanadium acceptor level. Let us further assume that trap type 2 has an electron (hole) capture cross section that is much smaller (larger) than that of trap type 1. The situation at equilibrium is altered when low level, $532 \mathrm{~nm}$ wavelength light intensity is applied to the $6 \mathrm{H}-\mathrm{SiC}$ substrate. This situation is depicted in Figure 5.65. We use the concept of quasiFermi levels since thermal equilibrium has been disturbed. The electron and hole densities are given by equations 5.5 and 5.7, respectively. Where $\Delta \mathrm{n}$ and $\Delta \mathrm{p}$ are the photogenerated electrons and holes, $\mathrm{N}_{\mathrm{c}}$ and $\mathrm{N}_{\mathrm{v}}$ are the effective densities of state at the conduction and valence bands, and $E_{c}$ and $E_{v}$ are the conduction band minimum and valence band maximum energies. The intrinsic 


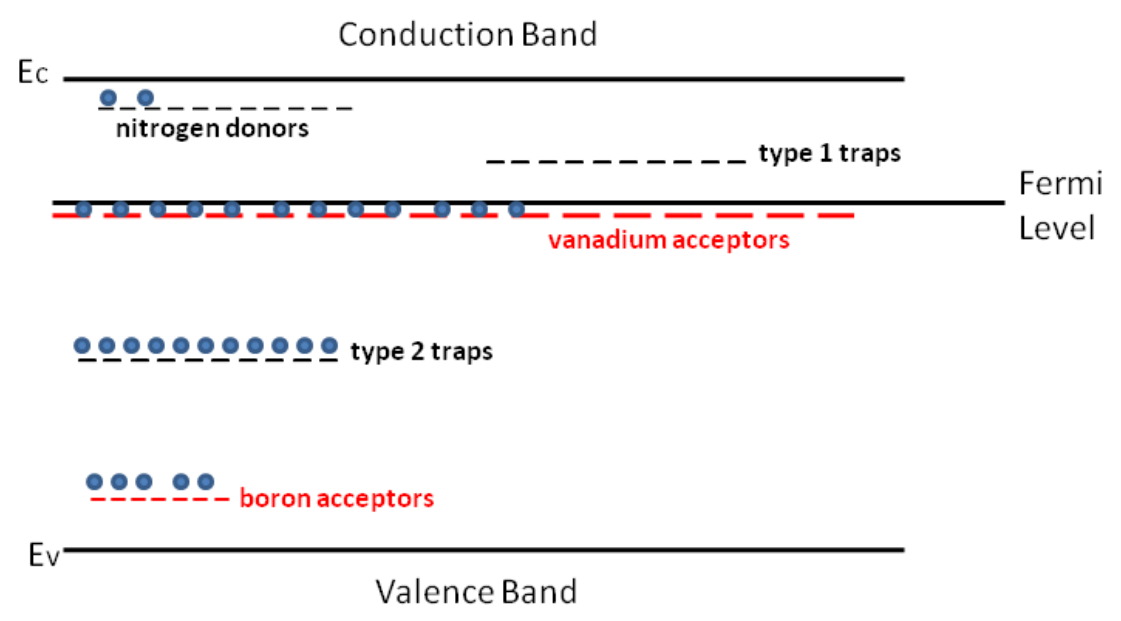

Figure 5.64. Equilibrium position of the Fermi level for vanadium compensated $6 \mathrm{H}$-SiC including type 1 and 2 traps

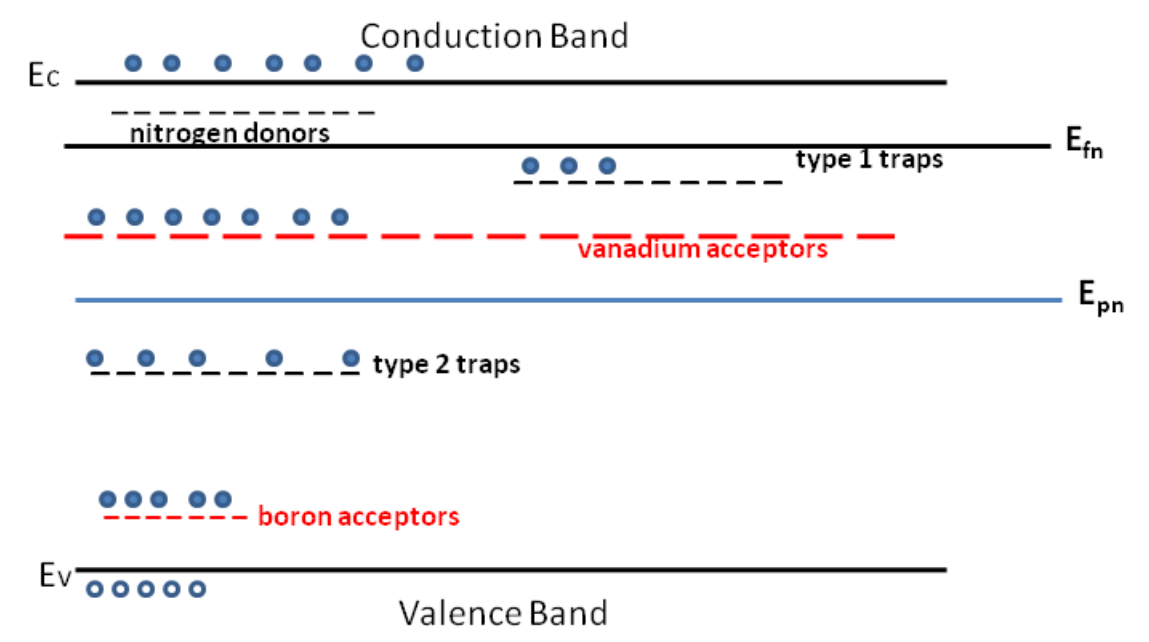

Figure 5.65. Positions of electron $\left(E_{\mathrm{fn}}\right)$ and hole $\left(E_{\mathrm{fp}}\right)$ quasi-Fermi levels for application of low intensity $532 \mathrm{~nm}$ wavelength light

carrier densities are negligible compared to the photogenerated carrier density. The position of the electron $\left(\mathrm{E}_{\mathrm{fn}}\right)$ and hole $\left(\mathrm{E}_{\mathrm{fp}}\right)$ quasi- Fermi levels follow from these equations and are given by equations 5.6 and 5.8. The incident light has created electron and hole carriers in the $6 \mathrm{H}-\mathrm{SiC}$ material. As a result, the quasi-Fermi levels move away from the Fermi level equilibrium 
position (i.e., pinned to the vanadium acceptor level). The electron quasi-Fermi level moves towards the conduction band and the hole quasi-Fermi level moves towards the valence band. This situation is depicted in Figure 5.65. The electron quasi-Fermi level has risen above the type

$$
\begin{gathered}
n=\Delta n+n_{0}=N_{c} \exp \left(-\frac{\left|E_{f n}-E_{c}\right|}{k T}\right) \\
\left|E_{f n}-E_{c}\right|=-k T \ln \left(\frac{n}{N_{c}}\right) \\
p=\Delta p+p_{0}=N_{v} \exp \left(-\frac{\left|E_{f p}-E_{v}\right|}{k T}\right) \\
\left|E_{f p}-E_{v}\right|=-k T \ln \left(\frac{p}{N_{v}}\right)
\end{gathered}
$$

1 trap level changing these traps into recombination centers. However, the hole quasi-Fermi level still lies above the type 2 traps leaving them unchanged. The application of low intensity $532 \mathrm{~nm}$ light have created free carriers and introduced an additional recombination center that behaves a great deal like the vanadium acceptor level.

Figure 5.66 shows the position of the quasi-Fermi levels for the application of higher intensity light on the $6 \mathrm{H}-\mathrm{SiC}$ substrate. Now the hole quasi-Fermi level has moved below the type 2 trap level, transforming it into a recombination center. The type 2 traps capture almost all available holes due to its large hole capture cross section, but recombination at the type 2 trap is slow due to its small electron capture cross section. Recombination at all other recombination centers is slowed down since the type 2 trap is preferentially capturing holes. As a result, the recombination time and free carrier density increases in the $6 \mathrm{H}-\mathrm{SiC}$ substrate material leading to the supralinear increase of conductance with optical intensity. This situation persists until all the 
type 2 traps are filled with holes and a linear, or sublinear, dependence of conductance on optical intensity is restored.

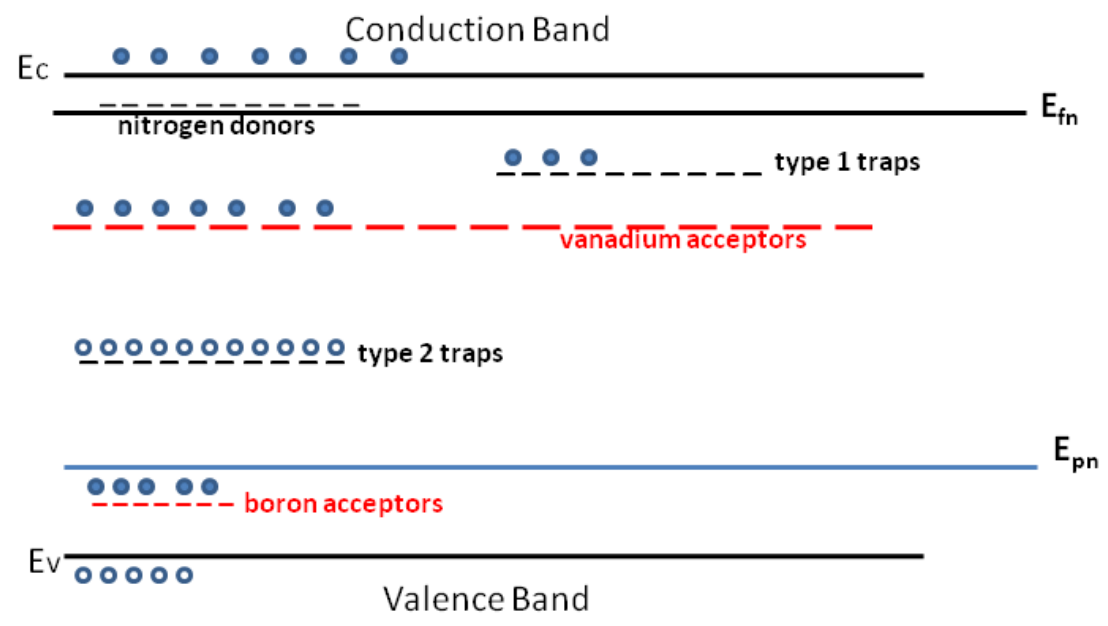

Figure 5.66. Positions of electron $\left(E_{\mathrm{fn}}\right)$ and hole $\left(E_{\mathrm{fp}}\right)$ quasi-Fermi levels for application of higher intensity $532 \mathrm{~nm}$ wavelength light

\section{5) 2H-GaN Photoconductive Switching Results}

We believe that the extrinsic, $2 \mathrm{H}-\mathrm{GaN}$ photoconductive switch constructed and tested in this work represents the first report [5.8] of a high power, GaN photoconductive switch of any kind. The GaN switch assembly was fabricated using a $9 \mathrm{~mm}$ by $9 \mathrm{~mm}, 408 \mu \mathrm{m}$ thick, iron compensated, semi-insulating substrate oriented in the c-plane. Kyma Technologies epitaxially grew the GaN substrates. The manufacturer believes the oxygen donor and iron acceptor densities to be $\sim 1 \mathrm{e} 16 / \mathrm{cm}^{3}$ and $1 \mathrm{e} 18 / \mathrm{cm}^{3}$, respectively. Bare GaN semi-insulating substrates are shown in Figure 5.67. The substrates were prepared for metallization using the steps listed below.

GaN substrate cleaning process:

1) 3, 10 minute baths performed in an ultrasonic cleaner using acetone, methanol and isopropyl alcohol with $\mathrm{N}_{2}$ blow dry between solvent baths. 
2) Wet etch for 5 minutes in $10 \% \mathrm{HCl}: \mathrm{DI} \mathrm{H}_{2} \mathrm{O}$ at room temperature, DI $\mathrm{H}_{2} \mathrm{O}$ rinse for 5 minutes.

3) Wet etch in $10 \% \mathrm{HF}$ :DI $\mathrm{H}_{2} \mathrm{O}$ solution for 5 minutes, DI $\mathrm{H}_{2} \mathrm{O}$ rinse for 5 minutes

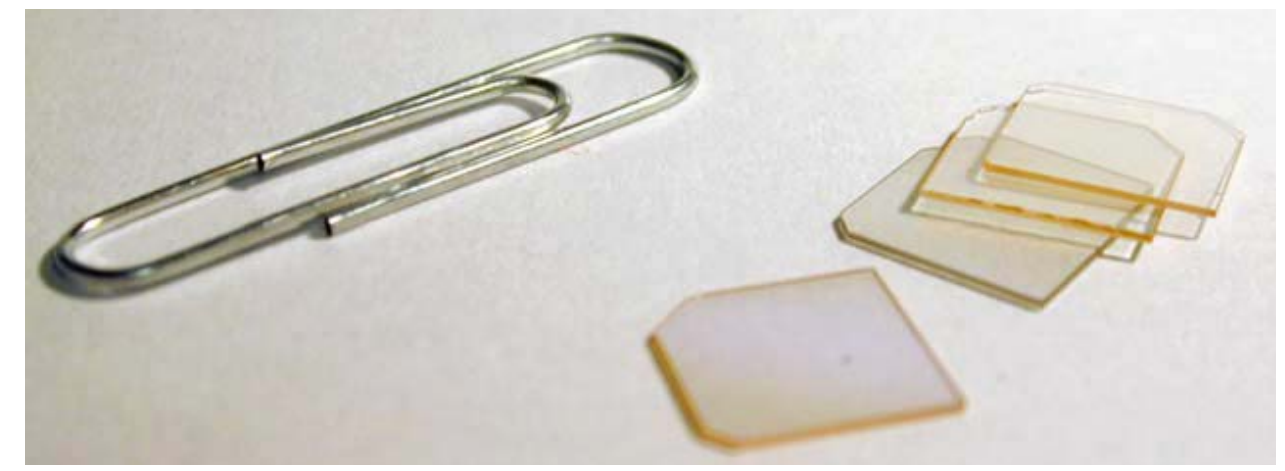

\section{Figure 5.67. Semi-insulating GaN substrates}

A $6.5 \mathrm{~mm}$ diameter circular metallization layer is centered on both sides of the GaN substrate using a lift-off technique to form a switch. The circular metallization consists of vacuum sputter deposited layers of $50 \mathrm{~nm}$ of titanium and $100 \mathrm{~nm}$ of gold. Copper electrodes were soldered to the metalized GaN substrate using indium solder. The GaN switch assembly is shown in Figure 5.68. The GaN photoconductive switch was tested using the same low and high voltage circuits as the $6 \mathrm{H}$-SiC devices. The GaN switch was tested over a range of bias voltages from 150 to 1000 volts. Optical excitation at both 532 and $1064 \mathrm{~nm}$ were applied to the GaN switch using the Spectron Nd:YAG laser used to test the black and silver $6 \mathrm{H}-\mathrm{SiC}$ switches. The optical pulse was applied across the $9 \mathrm{~mm}$ width of the GaN facet with a height that was slightly larger than the $408 \mu \mathrm{m}$ thickness of the substrate. However, only a small amount of photocurrent was generated at $1064 \mathrm{~nm}$. The weak response of the GaN switch to the $1064 \mathrm{~nm}$ wavelength light is shown in the plot of minimum switch resistance versus optical energy of Figure 5.69. The minimum resistance of the GaN switch starts at $~ 4500 \Omega$ and decreases to $1010 \Omega$ as the applied optical energy is increased from 2 to $10 \mathrm{~mJ}$ of $1064 \mathrm{~nm}$ light. The high minimum switch 


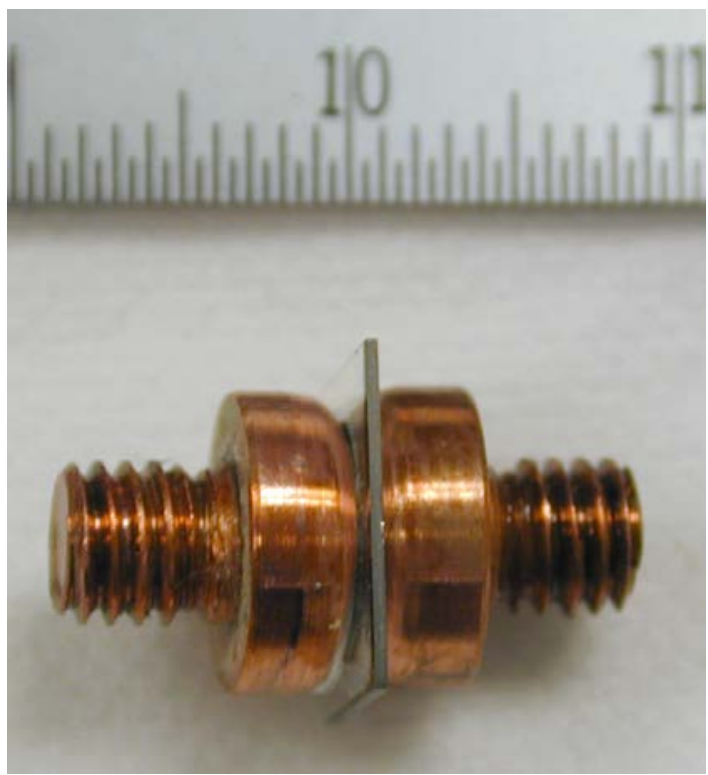

Figure 5.68. GaN photoconductive switch assembly (scale of ruler is cm)

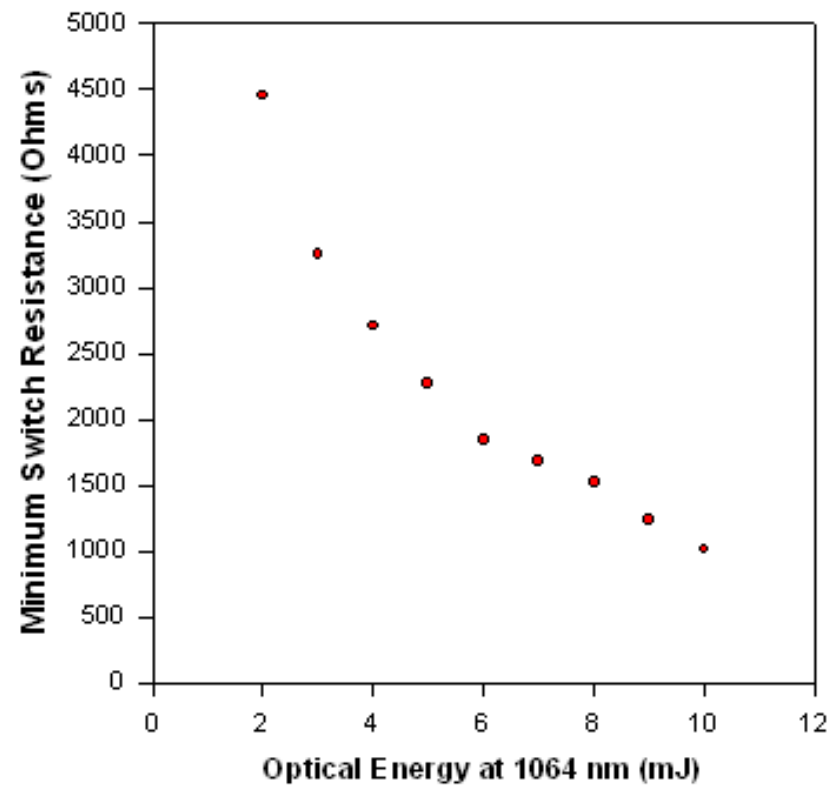

Figure 5.70. Minimum resistance of the GaN switch plotted versus applied optical energy at $532 \mathrm{~nm}$.

resistance of the GaN switch at $1064 \mathrm{~nm}$ indicates that there are not many electrons being excited from the iron acceptor levels in the GaN to the conduction band. The iron acceptor level resides at $\sim 0.58$ to $0.60 \mathrm{eV}$ below the conduction band, so the $1.17 \mathrm{eV}$ photons of the $1064 \mathrm{~nm}$ 
wavelength light pulse should be capable of exciting electrons from the iron acceptor level to the conduction band. The peak free electron density can be estimated for the switch at $10 \mathrm{~mJ}$ excitation using equations 5.9 and 5.10 .

$$
\begin{gathered}
R_{\text {min }}=\frac{d \rho_{\text {min }}}{a_{s w}} \\
\rho_{\text {min }}=\frac{1}{\mu_{n} q n_{\text {max }}}
\end{gathered}
$$

Where $d$ is the switch thickness $(408 \mu \mathrm{m}), a_{s w}$ is the switch active area $\left(0.81 \mathrm{~cm}^{2}\right), R_{\min }$ is the switch minimum on resistance at $10 \mathrm{~mJ}$ excitation $(1010 \Omega)$, $\rho_{\min }$ is the minimum resistivity, and $\mu_{\mathrm{n}}$ is the electron mobility of the iron compensated GaN material. The mobility has been measured [5.7] for similar iron compensated, semi-insulating GaN from this manufacturer by the temperature dependent Hall Effect (TDH) to be $410 \mathrm{~cm}^{2} /(\mathrm{V}-\mathrm{s})$. We obtain a peak free electron density of 7.59e11/ $\mathrm{cm}^{3}$ after substituting the above values into equations 5.9 and 5.10 . We expect the shallow oxygen donors to be completely ionized at room temperature, supplying a density of $1 \mathrm{e} 16 / \mathrm{cm}^{3}$ electrons to be captured by acceptor levels and traps throughout the GaN material. The lack of response of the GaN switch to $1064 \mathrm{~nm}$ wavelength light can be attributed to a high energy shift of the optical ionization cross section of the iron acceptor level (such as Franck Condon Shift) or that most of the electrons donated to the crystal from the oxygen levels have been captured by other levels that are located deeper in the material.

The results for excitation at $532 \mathrm{~nm}$ are shown in Figures 5.71 through 5.77. Figure 5.71 shows the GaN switch voltage, current and optical excitation for a 1000 Volt charge voltage and $12 \mathrm{~mJ}$ of optical excitation at $532 \mathrm{~nm}$. These results were obtained for an optical pulse that measured $~ 5.5 \mathrm{~ns}$ (FWHM), similar to the optical pulses used to excite the black and silver $6 \mathrm{H}$ SiC switches at $532 \mathrm{~nm}$. The switch voltage begins to collapse as soon as the optical pulse is 
applied to the switch facet. The switch voltage decreases from 1005 to 19 Volts in approximately 7 ns. The switch current follows the optical excitation pulse and increases to 19.5 Amps during the same seven nanosecond period. The minimum switch on resistance is just under $1 \mathrm{ohm}$ for this pulse. The switch current pulse width is $11 \mathrm{~ns}$ FWHM, which is approximately twice the

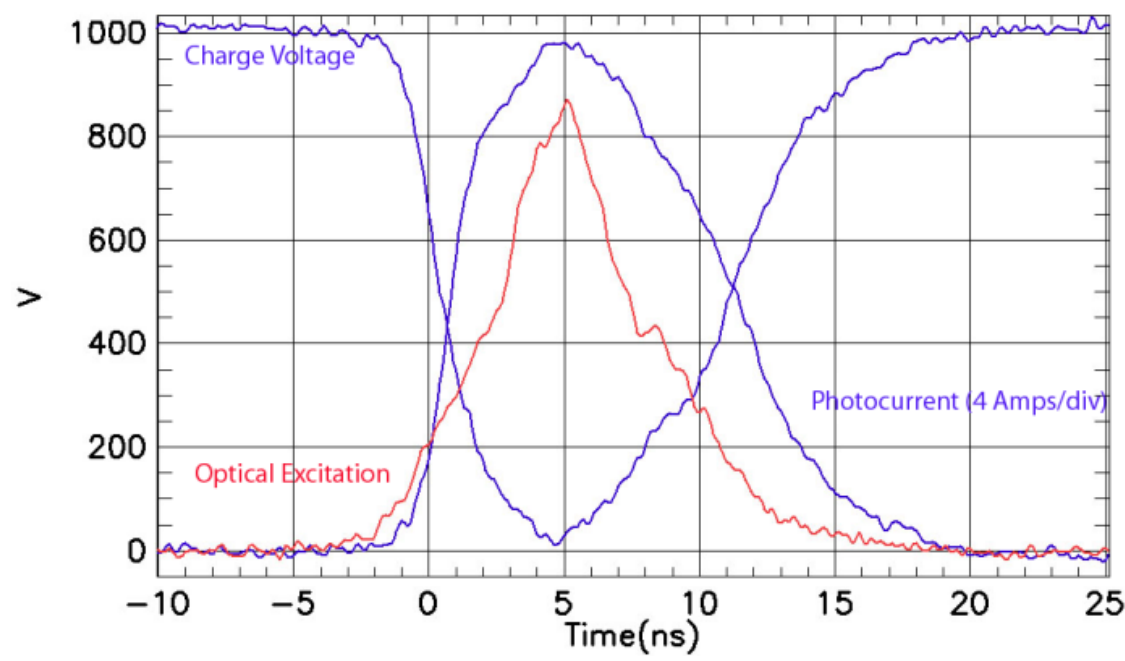

Figure 5.71. Switch voltage, photocurrent and optical excitation waveforms for a charge voltage of 1000 Volts and optical excitation of $12 \mathrm{~mJ}$ at $532 \mathrm{~nm}$

optical pulse width indicating a fast recombination time for this switch. Figure 5.72 shows the peak switch photocurrent as a function of optical excitation energy at a wavelength of $532 \mathrm{~nm}$ for a variety of charge voltages. The saturation of the photocurrent begins at $3-4 \mathrm{~mJ}$ of optical excitation for all charge voltages. This saturation effect is the same that was observed for the $6 \mathrm{H}$ SiC switches. Saturation marks the point at which the load resistance starts to become the dominant resistance in the test circuit.

The data of Figure 5.72 can be recast to show the peak photocurrent as a function of charge voltage for a range of optical excitation energies. The resulting plots are shown in Figure 5.73 and the linear dependence of peak photocurrent with charge voltage of the GaN switch becomes apparent. The peak photocurrent is a linear function of bias voltage for excitation with a 
fixed optical energy. This indicates that the total resistance of the test circuit is constant for a fixed applied optical energy. The circuit resistance calculated for a fixed applied optical energy of 0.5 and $12 \mathrm{~mJ}$ is 693 and 51.7 Ohms, respectively. The minimum GaN switch resistance is calculated by subtracting the $51 \mathrm{Ohm}$ load resistance from the total circuit resistance, resulting in 642 and 0.7 Ohms, respectively for 0.5 and $12 \mathrm{~mJ}$ excitation.

A plot of the GaN switch minimum resistance is plotted as a function of applied optical energy at $532 \mathrm{~nm}$ in Figure 5.74 A and B for the range of 1 to $12 \mathrm{~mJ}$. The switch resistance collapses rapidly from $642 \mathrm{Ohms}$ at $0.5 \mathrm{~mJ}$ to $\sim 10$ ohms at $4 \mathrm{~mJ}$. Figure $5.74 \mathrm{~B}$ shows the same data from Figure 5.74 A over the range of $2 \mathrm{~mJ}$ to $12 \mathrm{~mJ}$ at $532 \mathrm{~nm}$. This is a nonlinear decay of minimum switch resistance with optical energy. We would expect a minimum switch resistance of $26.75 \Omega$ for excitation at $12 \mathrm{~mJ}$ based on the result for excitation at $0.5 \mathrm{~mJ}$ for a linear variation of switch resistance with optical intensity/energy. The nonlinearity becomes more apparent by plotting the GaN switch maximum conductance versus peak optical power in Figure 5.75. The maximum conductance of the GaN switch is proportional to the square of the applied peak optical power at $532 \mathrm{~nm}$. This indicates that the majority of carriers in the GaN material are generated primarily by two photon absorption and, to a lesser extent, two-step photoionization. A better sense of the nonlinear behavior of the GaN switch resistance with applied optical intensity can be obtained by comparing the data of Figure 5.75 to the data for the for the $6 \mathrm{H}$-SiC switches in Figures 5.62 and 5.63. The black and silver switches start with a minimum resistance that is ten times lower than that of the GaN switch at low applied optical energy and end up with a minimum on resistance that is three times higher at an applied optical power of 1.5 MW. 


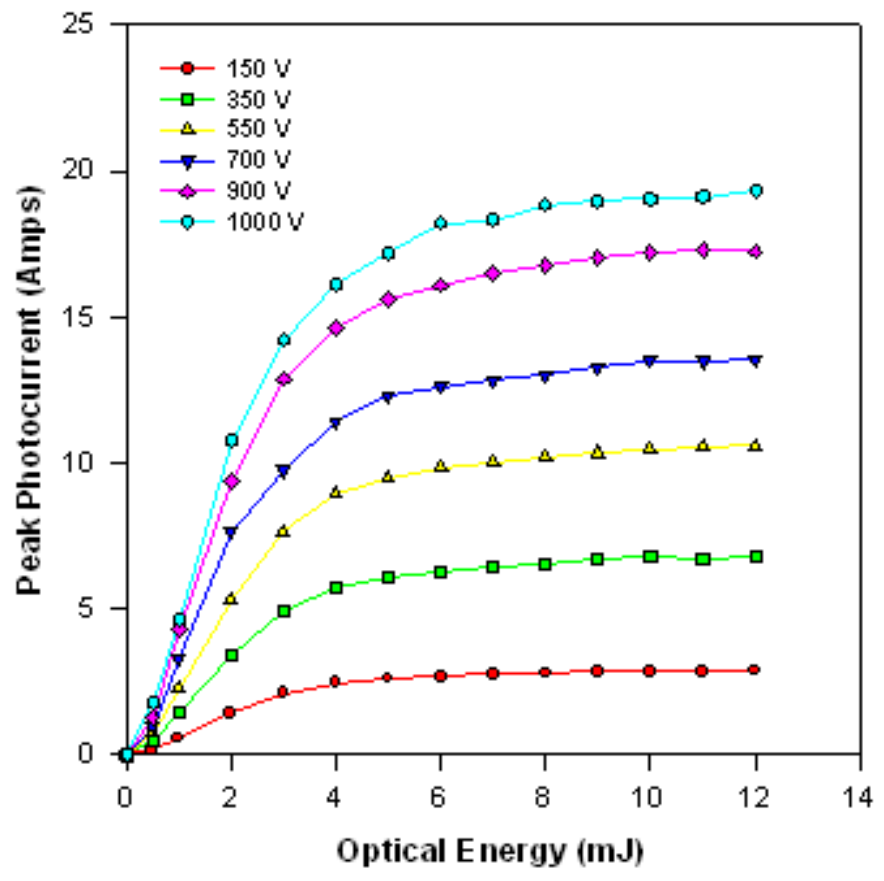

Figure 5.72. GaN switch peak photocurrent plotted as versus optical energy at $532 \mathbf{n m}$ for a range of charge voltages.

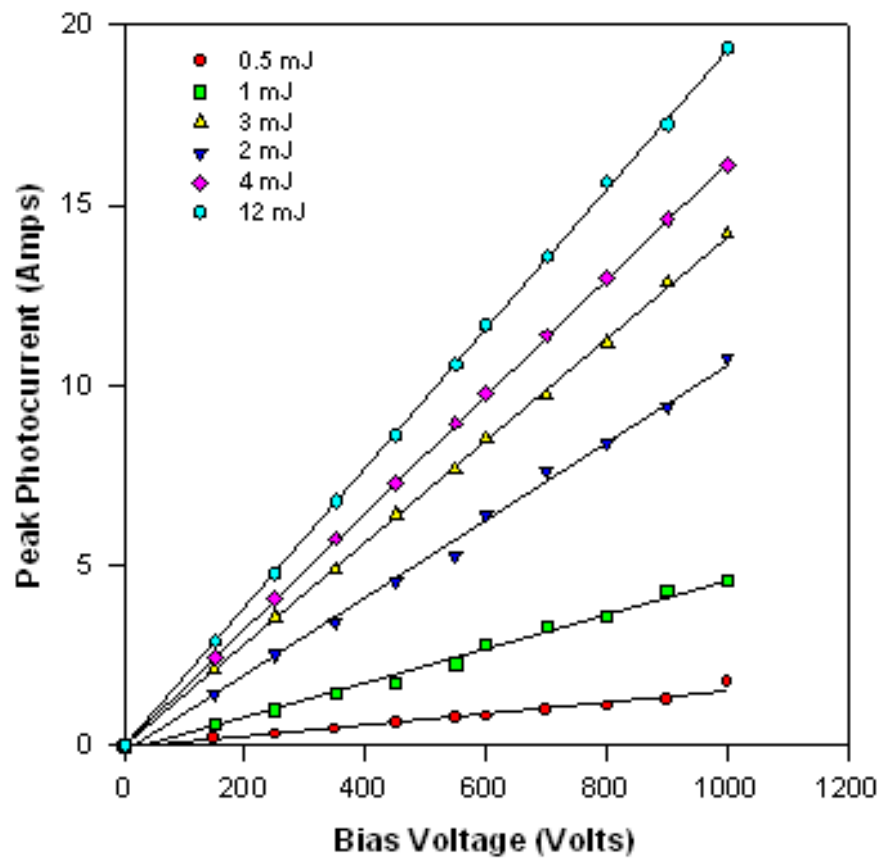

Figure 5.73. GaN switch peak photocurrent plotted as a function of charge voltage for a range of optical energies at $532 \mathrm{~nm}$. 


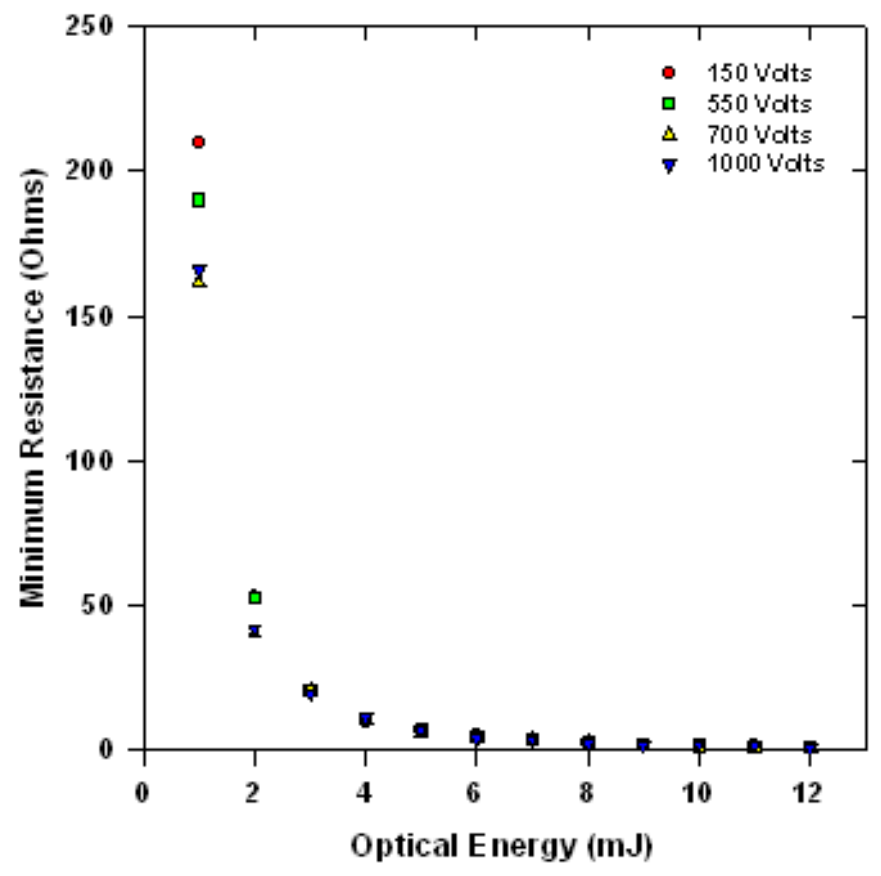

Figure 5.74 A. GaN switch minimum resistance plotted versus optical energy at $532 \mathrm{~nm}$

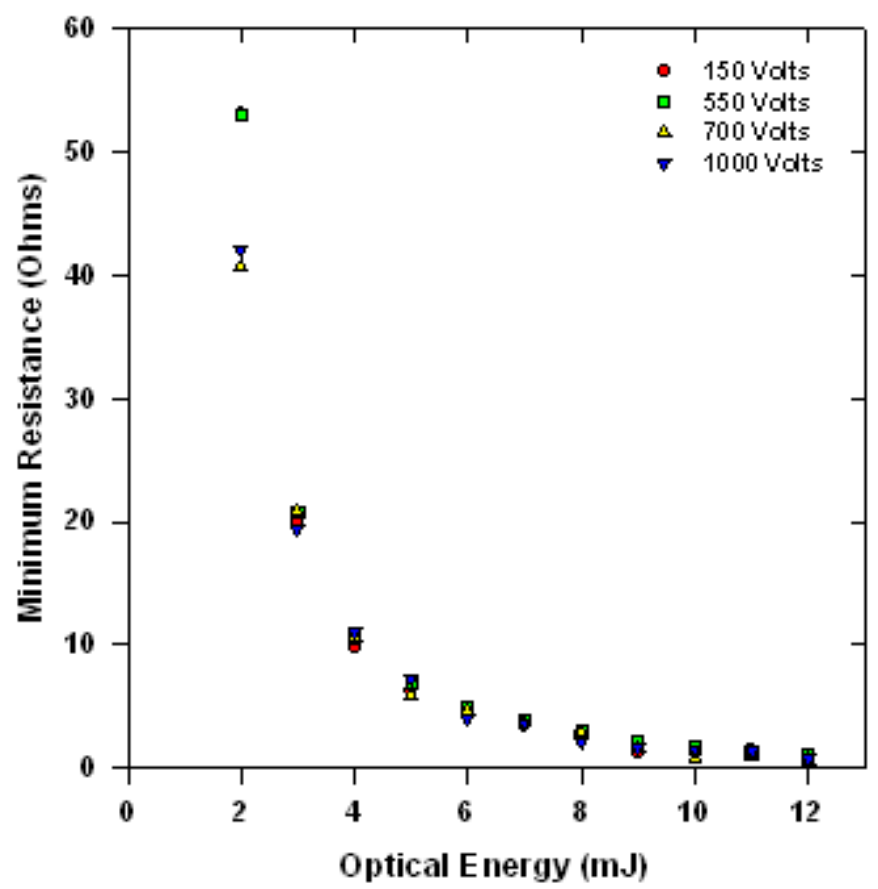

Figure 5.74 B. GaN switch minimum resistance plotted versus optical energy at $532 \mathrm{~nm}$ 


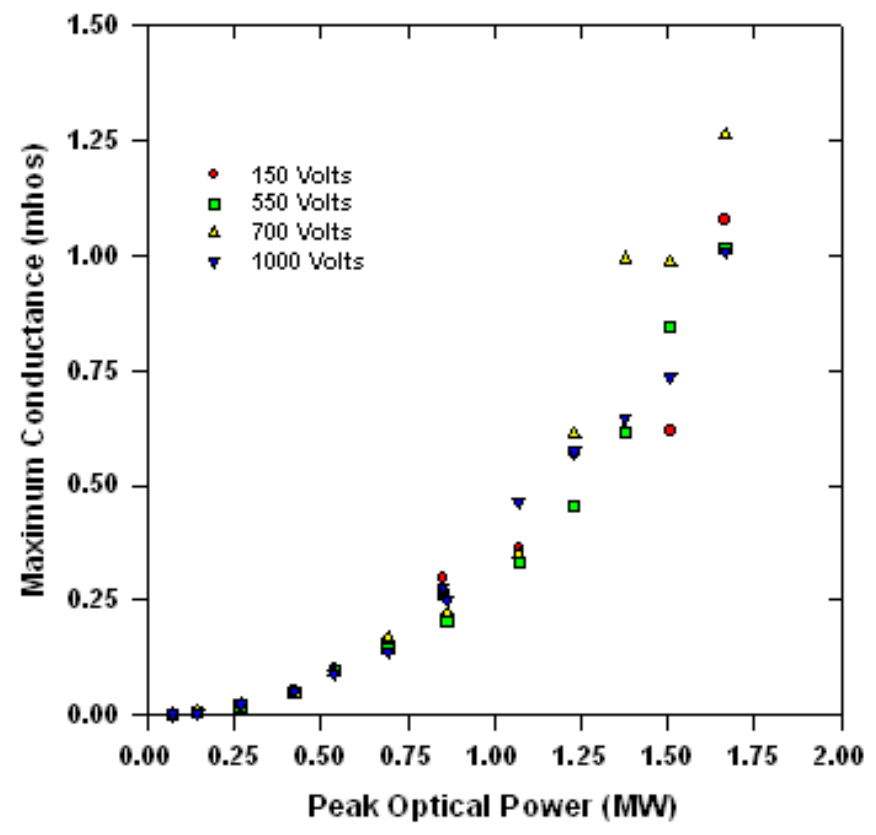

Figure 5.75. GaN Switch maximum conductance plotted versus peak optical power at 532 nm

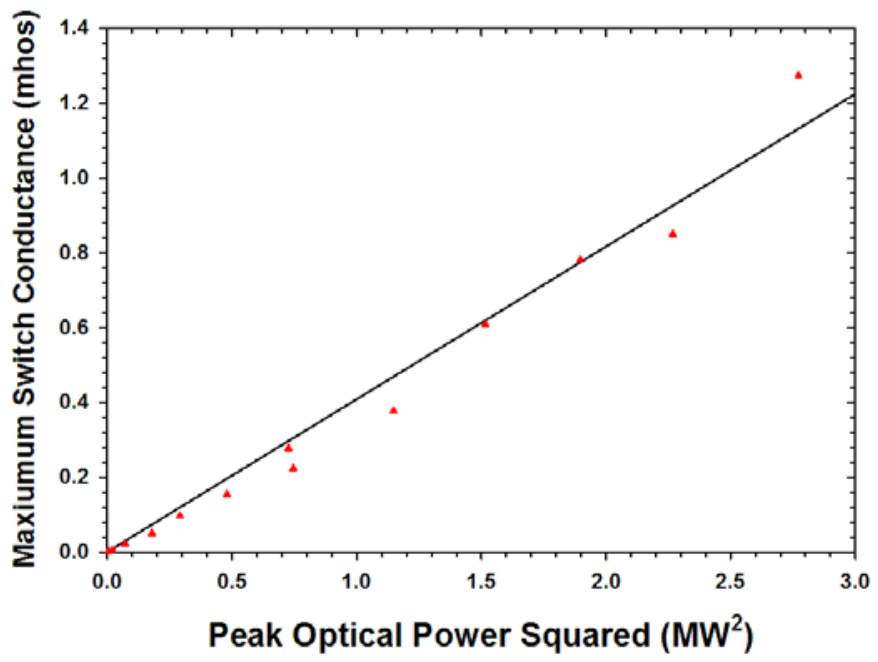

Figure 5.76. Maximum switch conductance plotted as a function of peak optical power squared for $2 \mathrm{H}-\mathrm{GaN}$ photoconductive switch excited with $532 \mathrm{~nm}$ wavelength light and a 550 Volt charge voltage 
Figure 5.76 is a plot of the maximum conductance plotted as a function of the square of the peak applied optical power at $532 \mathrm{~nm}$. This plot shows a primarily linear relationship, confirming that two-photon absorption is responsible for the bulk of the charge carriers optically generated in the $2 \mathrm{H}-\mathrm{GaN}$ substrate at $532 \mathrm{~nm}$.

The photoconductive switching results for the GaN switch at $532 \mathrm{~nm}$ are a clear indication that electrons excited from deep levels make only a small contribution to the switch conductivity during illumination with $532 \mathrm{~nm}$ wavelength light. GaN photoconductive switching results at the $1064 \mathrm{~nm}$ wavelength indicated that very few electrons donated from the oxygen levels were trapped in the iron acceptor level. Electrons may be excited from energy levels deeper than the iron acceptor level at $532 \mathrm{~nm}$, but the number is small compared to generation by two-photon absorption. As a result, its difficult see an approach to engineer the GaN semiinsulating material to optimize its performance as an extrinsic photoconductive switch based on shallow oxygen donors and deep iron acceptors in the near term. However, it is clear that very useful 2H-GaN photoconductive switches based on two-photon absorption can be developed.

\section{6) Conclusions}

This chapter presented the extrinsic photoconductive switch test results for the initial vanadium compensated $6 \mathrm{H}-\mathrm{SiC}$ and iron compensated $2 \mathrm{H}-\mathrm{GaN}$ semi-insulating substrates for pulsed excitation at the 532 and $1064 \mathrm{~nm}$ wavelengths. Both the 6H-SiC and 2H-GaN materials demonstrate their feasibility as extrinsic photoconductive switches. The $6 \mathrm{H}-\mathrm{SiC}$ substrates show a strong photoconductive response at both the 532 and $1064 \mathrm{~nm}$ wavelength. While the 2H-GaN 
substrate has a weak response at $1064 \mathrm{~nm}$, but a very strong response at $532 \mathrm{~nm}$. The strong response of the $6 \mathrm{H}-\mathrm{SiC}$ material at $1064 \mathrm{~nm}$ indicates that a significant number of electrons reside in the vanadium acceptor levels. We expect that the extrinsic photoconductive response of the $6 \mathrm{H}-\mathrm{SiC}$ material can be improved by manipulation of the vanadium, nitrogen and boron dopants present in the material. The weak photoconductive response at $1064 \mathrm{~nm}$ and strong response at $532 \mathrm{~nm}$ of the $2 \mathrm{H}-\mathrm{GaN}$ material indicates that the iron acceptor levels may contain very few electrons and that two photon absorption is responsible for the strong response at 532 $\mathrm{nm}$. As a result, there is no clear path to improving the extrinsic switching performance of the 2H-GaN without establishing a deep acceptor/shallow donor combination that can manipulative to improve switching performance in $2 \mathrm{H}-\mathrm{GaN}$.

The encouraging extrinsic photoconductive response of the $6 \mathrm{H}-\mathrm{SiC}$ and $2 \mathrm{H}-\mathrm{GaN}$ materials is not the only important switching requirement. Voltage hold-off, optical transmission and turn-on and turn-off times are also important switching requirements, which will be explored in the next chapter.

\section{7) References}

5.1) F. La Via, et al, "Structural and electrical characterization of titanium and nickel silicide contacts on silicon carbide”, Microelectronic Engineering, Vol. 60, pp. 269 - 282, 2002

5.2) J. Sullivan and J. Stanley, "6H-SiC photoconductive switches triggered at below bandgap wavelengths", IEEE Transactions on Dielectrics and Electrical Insulation, Vol. 4, No. 4, pp. 980 - 985, August 2007

5.3) G. Tamulaitis, et al., "Carrier lifetime in conductive and vanadium doped 6H-SiC substrates”, Applied Physics Letters, Vol. 84, No. 3, pp. 335 - 337, 2004

5.4) G.M.Loubriel, et al., "Longevity of optically activated, high gain GaAs photoconductive semiconductor switches”, IEEE Transactions on Plasma Science, vol. 26, no. 5, October 1998, pp1393 - 1402 
5.5) A. Rose, Concepts in Photoconductivity and Allied Problems, pp. 43 - 47, WileyInterscience, 1963

5.6) R. Bube, Photoelectronic Properties of Semiconductors, pp. 73 - 83, Cambridge University Press, 1992

5.7) F. Hodge and D. Polder, "Conditions for superlinear intrinsic photoconductivity”, Journal of the Physics of Chemical Solids, Vol. 25, pp. 977 - 984, 1964

5.8) J. Sullivan and J. Stanley, "Wide bandgap extrinsic photoconductives switches", IEEE Transactions on Plasma Science, Vol. 36, No. 5, October 2008 


\section{Chapter 6: Parameters Limiting Switch Performance}

In the previous chapter, the photoconductive switching results of the initial vanadium compensated $6 \mathrm{H}-\mathrm{SiC}$ and iron compensated $2 \mathrm{H}-\mathrm{GaN}$ materials were presented. The photoconductive switching results represent the most important aspect of switch performance. That is, how well the switching material transitions from a high impedance to a low impedance, or, conducting state. Our results show that $6 \mathrm{H}-\mathrm{SiC}$ material collapses $10-11$ orders of magnitude from dark resistance to minimum on resistance, and that both the $6 \mathrm{H}-\mathrm{SiC}$ and $2 \mathrm{H}$ GaN material achieve minimum on resistances of a few ohms, or less, for excitation at $12-14$ $\mathrm{mJ}$ of $532 \mathrm{~nm}$ light. While these results are encouraging, there are several other important switching parameters that will determine whether the compensated $6 \mathrm{H}-\mathrm{SiC}$ and $2 \mathrm{H}-\mathrm{GaN}$ materials can be used to fabricate a high power, extrinsic photoconductive switch. An ideal switch should hold off high voltage in the off state, have negligible resistance in the on state, be easy to trigger and as small as practical. Voltage hold-off, optical transmission and switch closing and opening times are all important switching parameters that will be considered in this chapter.

\section{1) Switch High Voltage Breakdown}

Extrinsic PCSS fabricated from 2H-GaN and 6H-SiC offer hold off voltages limited only by the critical fields of the materials $(3.0-3.5 \mathrm{MV} / \mathrm{cm})$, the enhancement factor of the switch electrode geometry, and the switch substrate thickness. Switches constructed from compensated $6 \mathrm{H}-\mathrm{SiC}$ and $2 \mathrm{H}-\mathrm{GaN}$ were tested to catastrophic electrical failure. The $6 \mathrm{H}-\mathrm{SiC}$ switch assembly identified as the blue switch was fabricated from a $401 \mu \mathrm{m}$ thick, $1.22 \mathrm{~cm}$ by $1.21 \mathrm{~cm}$ substrate. The metallization and electrode geometry are the same as shown in Figures 5.1 and 5.2. The blue 
switch was tested using a modified version of the high voltage board (Figures 5.11 and 5.12). The modification consisted of replacing the two series connected set of two parallel connected, 4 $\mathrm{kV}$, $56 \mathrm{nF}$, ceramic chip capacitors with a set of 3 , series connected sets of six parallel connected, $6 \mathrm{kV}, 100 \mathrm{pF}$, radial leaded ceramic capacitors. The resulting value of high voltage board effective capacitance changed from $56 \mathrm{nF}(8 \mathrm{kV})$ to $200 \mathrm{pF}(18 \mathrm{kV})$. The reduction in capacitance on the modified high voltage board had two effects. First, the capacitor voltage now has a significant droop during conduction of the $6 \mathrm{H}-\mathrm{SiC}$ switch. Second, a resonant charge now takes place between the storage capacitors located in the Silicon Carbide Pulse Charge Chassis

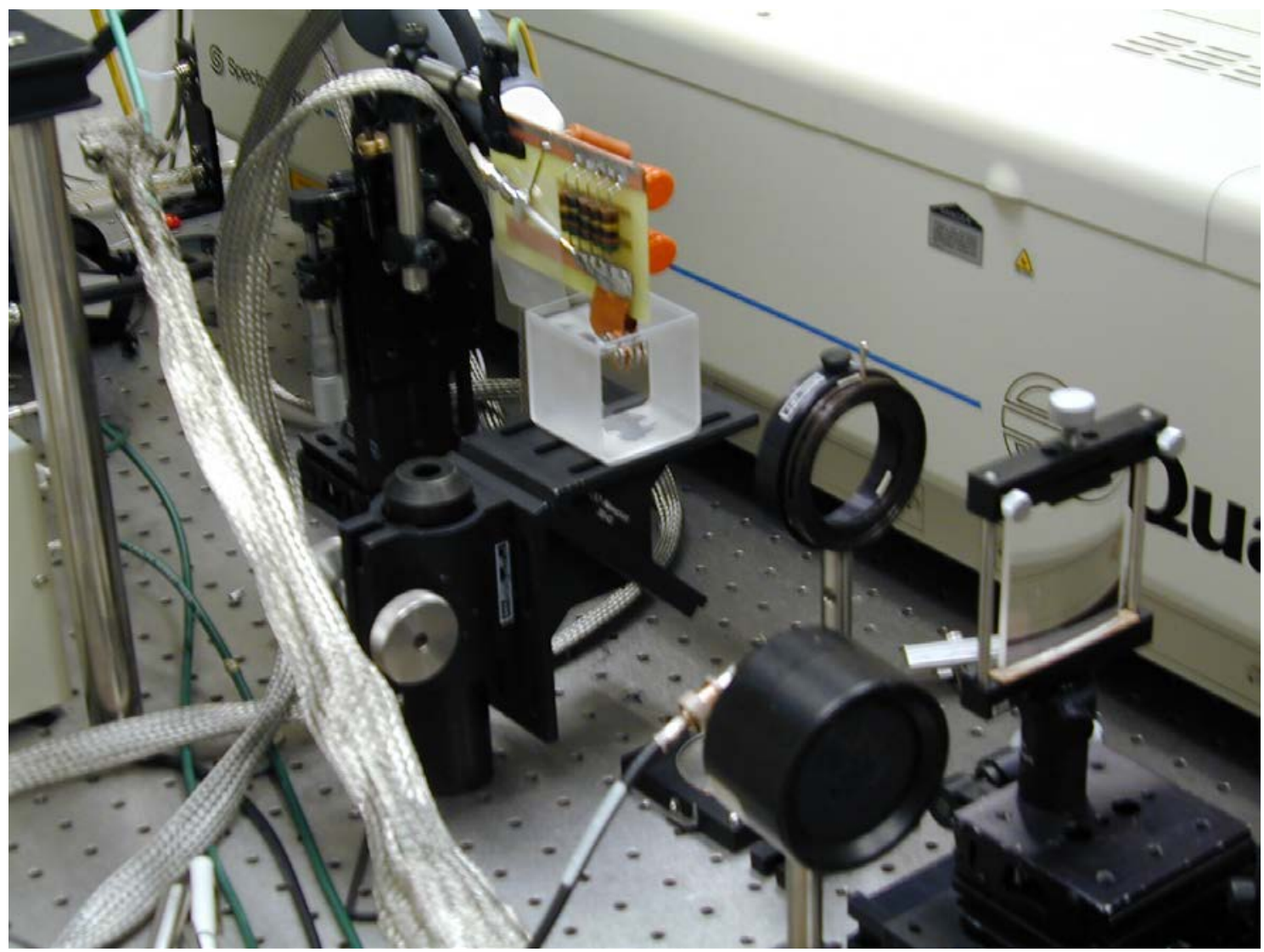

Figure 6.1. Modified high voltage test board with $6 \mathrm{H}-\mathrm{SiC}$ switch immersed in FC 77, liquid dielectric.

(SCPC) and the $200 \mathrm{pF}$ high voltage board storage capacitor. This allows for charge voltages up to $16 \mathrm{kV}$. The modified high voltage board was mounted so that the blue switch was immersed in 
a Flourinert FC 77 liquid dielectric bath contained in an optical test cell to prevent surface flashover. The high voltage test set-up is pictured in Figure 6.1.

The switch was operated with $9 \mathrm{~mJ}$ pulses at $1064 \mathrm{~nm}$ centered across a $1 \mathrm{~cm}$ portion of a single facet. The Spectra-Physics Nd:YAG laser was the source of the optical trigger pulses. The operating voltage was raised slowly with several pulses taken at each voltage step. Catastrophic electrical failure of the $6 \mathrm{H}-\mathrm{SiC}$ switch occurred at charge voltage of $11 \mathrm{kV}$. Figure 6.2 shows the blue switch assembly after high voltage breakdown. The shock from the punch through of the $6 \mathrm{H}-\mathrm{SiC}$ material fractured the substrate along two crystal planes, which is visible in Figure 6.2.

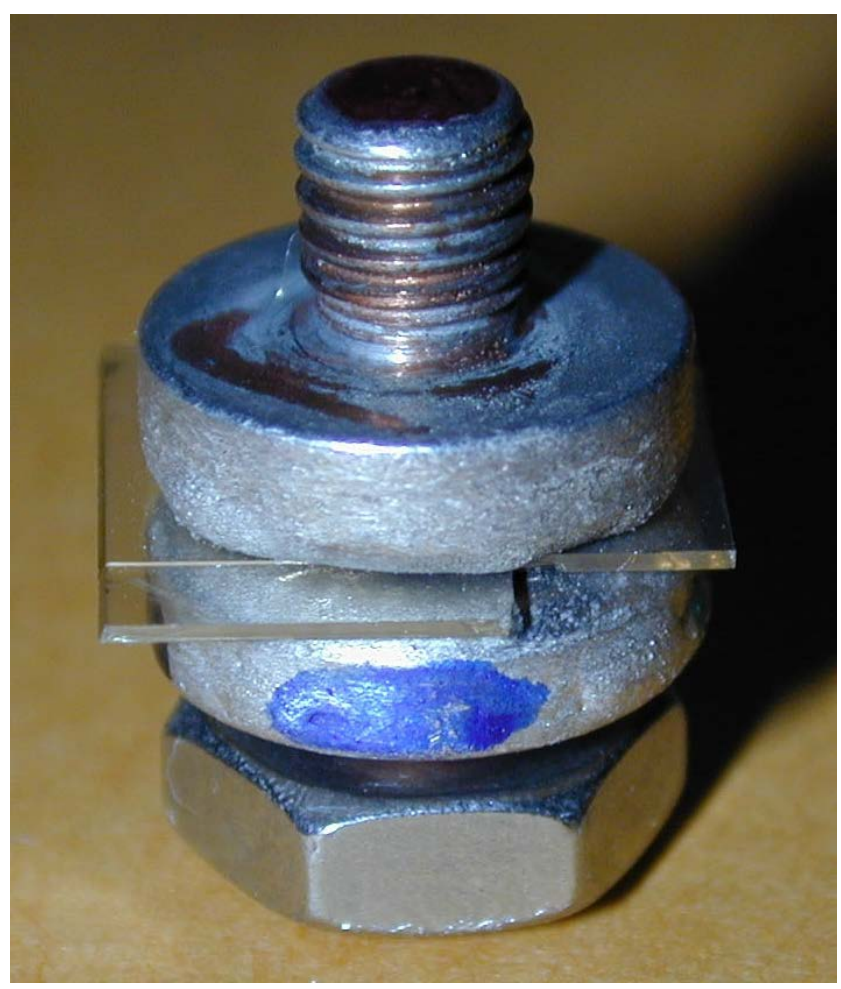

Figure 6.2. Blue switch assembly after high voltage breakdown at $11 \mathrm{kV}$

In fact, the corner of the substrate is missing. The breakdown occurred at the point where the switch metallization ends and the copper electrode is no longer in contact with the switch 
substrate. The exact point of failure is visible on the left-hand side of the micro-photograph in Figure 6.3. This location is the expected point of maximum electric field enhancement.

The electrostatic field of the switch assembly has been modeled using the FlexPDE5 [6.1] finite element software. Figure 6.4 is a plot of the modeled electric field magnitude starting at point 1 on the surface of the copper electrode (see inset of Figure 6.4) and moving along the electrode-substrate interface to point 3 at the center of the switch metallization. The model includes the switch electrodes, 6H-SiC substrate, substrate metallization and dielectric liquid. The failure point shown in Figure 6.3 corresponds to position of maximum electric field in Figure 6.4. The peak electric field predicted for the blue switch at a charge voltage of $11 \mathrm{kV}$ is

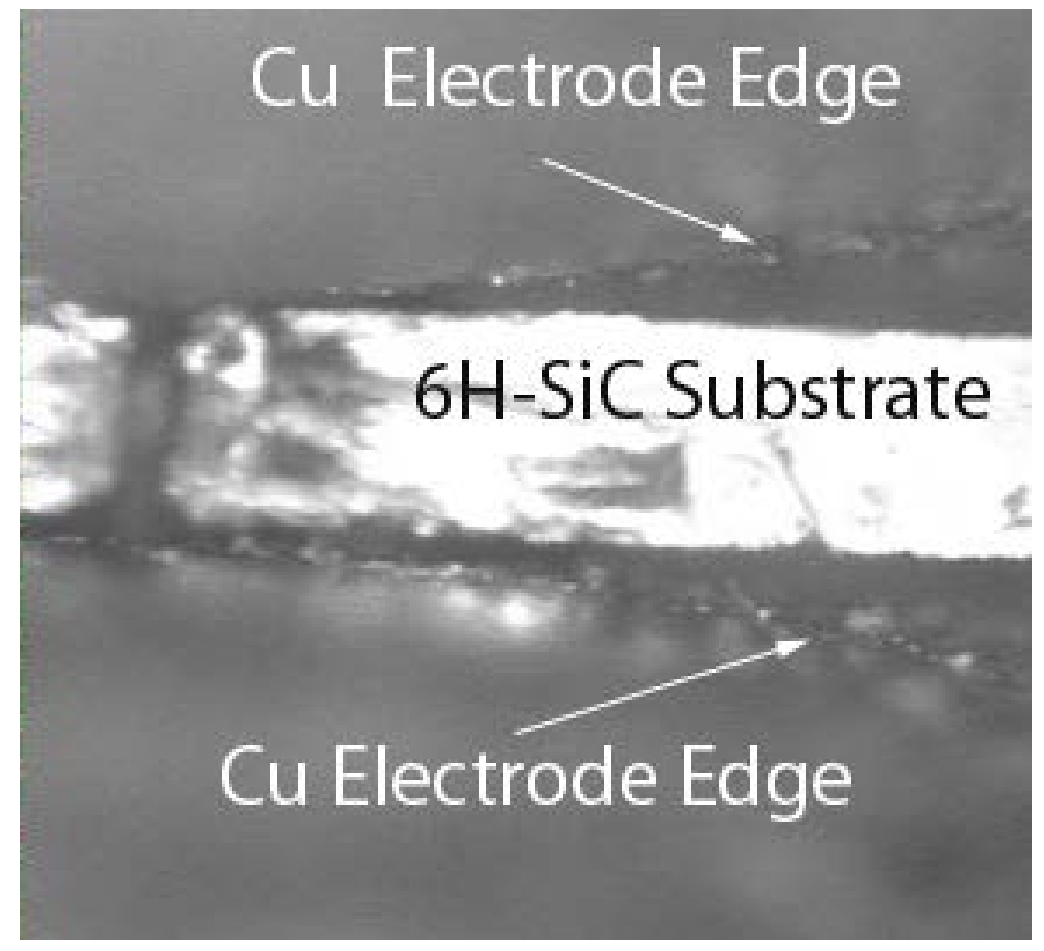

Figure 6.3. Micro-photograph of the electrical breakdown point of the blue switch 
$175 \mathrm{MV} / \mathrm{m}$ and the average field at the center of the substrate is $27.4 \mathrm{MV} / \mathrm{m}$. This corresponds to an electric field enhancement factor ( $E_{\max } / E_{\text {ave }}$ ) of 6.39 . Fields as high as $235 \mathrm{MV} / \mathrm{m}$ have been calculated for the blue switch at a charge voltage of $11 \mathrm{kV}$ when enhancement due to the indium

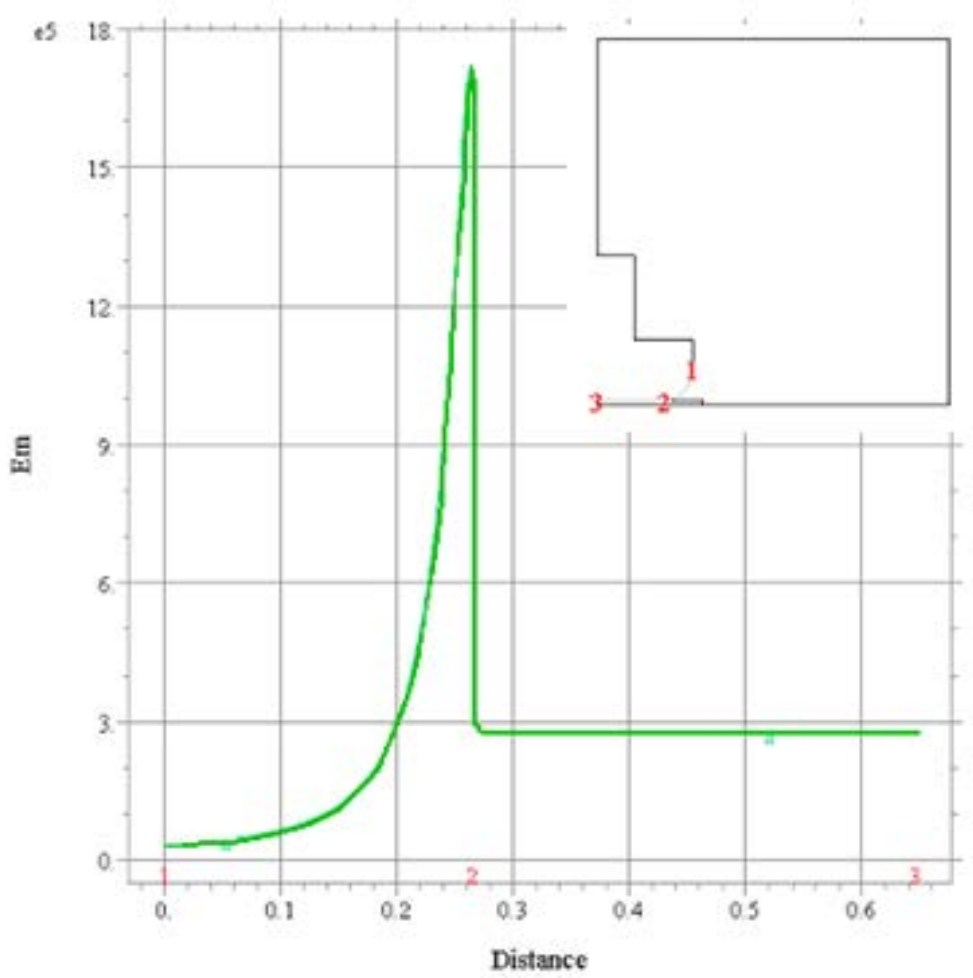

Figure 6.4. Finite element model of electric field magnitude in blue switch assembly for charge voltage of $11 \mathrm{kV}$

solder layer are included. The critical field for the vanadium compensated 6H-SiC material lies somewhere in the range of $175-235 \mathrm{MV} / \mathrm{m}$. This range corresponds to 58 to $78 \%$ of the critical field strength of high purity $6 \mathrm{H}-\mathrm{SiC}$ (300 MV/m). The reduction in critical field strength of our vanadium compensated $6 \mathrm{H}-\mathrm{SiC}$ material compared to high purity material is assumed to be due to the vanadium, nitrogen and boron dopants.

A $2 \mathrm{H}-\mathrm{GaN}$ switch assembly with a $400 \mu \mathrm{m}$ thick substrate was tested to catastrophic electrical failure in an identical fashion as the blue $6 \mathrm{H}-\mathrm{SiC}$ switch. The $2 \mathrm{H}-\mathrm{GaN}$ switch failed at a voltage of $8 \mathrm{kV}$ resulting in an average field of $20 \mathrm{MV} / \mathrm{m}$. The failure point in the $2 \mathrm{H}-\mathrm{GaN}$ 
substrate was in the same location as that for the blue switch. The failed $2 \mathrm{H}-\mathrm{GaN}$ switch is shown in Figure 6.5. The electric field enhancement factors for the $2 \mathrm{H}-\mathrm{GaN}$ switch are the same as that calculated for the $6 \mathrm{H}-\mathrm{SiC}$ blue switch $(6.48-8.7)$. This corresponds to a peak field at the point of breakdown in the $2 \mathrm{H}-\mathrm{GaN}$ switch of 129.6 to $174 \mathrm{MV} / \mathrm{m}$, which represents 37 to $50 \%$ of the published critical field strength (350 MV/m) of high purity 2H-GaN material.

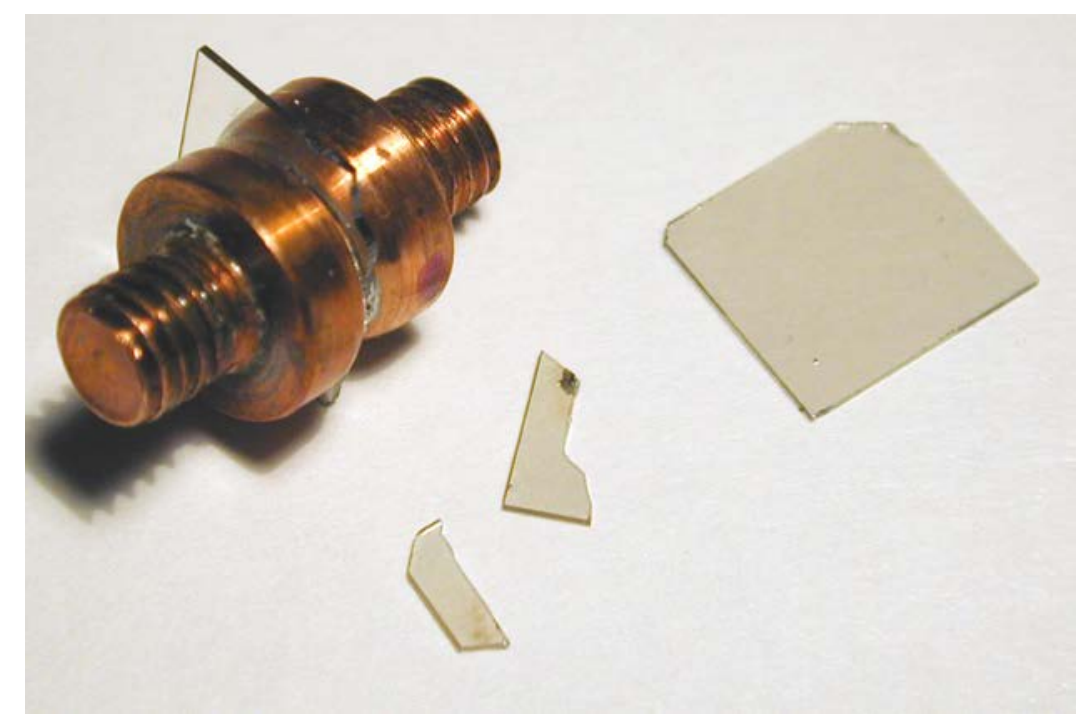

Figure 6.5. $2 \mathrm{H}-\mathrm{GaN}$ switch assembly that failed at a charge voltage of $8 \mathrm{kV}$

We can take the conservative approach and assume that the critical field strength of our vanadium compensated, $6 \mathrm{H}-\mathrm{SiC}$ and iron compensated, $2 \mathrm{H}-\mathrm{GaN}$ are 175 and $129.6 \mathrm{MV} / \mathrm{m}$, respectively. We can determine the maximum hold-off voltage for a switch design as shown below.

$$
V_{\max }=\frac{d E_{c r i t}}{\beta}
$$

Where $d$ is the switch substrate thickness and $\beta$ is the electric field enhancement factor. Based on equation 6.1, we have to double the switch thickness if we want to double the maximum hold-off voltage for a given switch geometry and material. We know from the photoconductive switch 
measurements presented in Chapter 5 that both the vanadium compensated $6 \mathrm{H}-\mathrm{SiC}$ and iron compensated $2 \mathrm{H}-\mathrm{GaN}$ have fast recombination times. An expression for switch resistance in response to a rectangular optical pulse is given in equation (6.2) for switch material with a fast recombination time. If we wish to double the switch hold-off voltage but keep the same minimum switch resistance, while maintaining all material parameters constant, the peak optical power will have to be quadrupled. This is certainly possible, but this approach increases the laser output requirement, particularly if there are a number of switches being triggered simultaneously. Also, this approach may lead to peak intensities that can exceed the damage threshold of the $6 \mathrm{H}-$ SiC, or, 2H-GaN switch facets.

$$
R(t)=\frac{d^{2} \hbar \omega}{P(t)(1-\exp (-\alpha W))(1-r) \eta \tau_{r} \mu_{n} q}
$$

There is an alternate approach to increasing the switch hold-off voltage other than arbitrarily increasing switch substrate thickness. We can concentrate on reducing the field enhancement factor, $\beta$. The electrode profile could be contoured into a Rogowski [6.2], Bruce [6.3], Chang [6.4], or similar profile. These electrode profiles produce a maximum in the electric field at the switch center and reduce the electric field at the edge of the metallization. However, these profiles would require the capability of machining their profile into the $6 \mathrm{H}$-SiC substrate which is unlikely considering the hardness of the material. In addition, these profiles would require the $6 \mathrm{H}-\mathrm{SiC}$ substrate to increase in width and length to enable the lowering of the electric field at the electrode radius. These two factors make employing this field enhancement reduction technique on the $6 \mathrm{H}-\mathrm{SiC}$ switches impractical. However, the conservative value of critical field 
for the initial $6 \mathrm{H}-\mathrm{SiC}$ material, $175 \mathrm{MV} / \mathrm{m}$, allows for us to construct switches with a thickness of $1 \mathrm{~mm}$ that should operate at charge voltages of $15 \mathrm{kV}$.

An alternate approach to our problem is to explore the possibility of reducing the intensity required to achieve low on resistances in thicker substrate switches by improving the quantum efficiency of the switching material. This requires that we know the density of dopants in our initial switch material and what levels are filled with electrons, or holes.

\section{2) Improving the Quantum Efficiency of Vanadium Doped 6H-SiC}

We define the quantum efficiency as the carrier density generated in the volume of the photoconductive switch substrate divided by the photon density injected into the switch substrate. If we consider only electrons, the quantum efficiency is mathematically expressed in equation (6.3). Where $\mathrm{n}(\mathrm{t})$ is the electron density, $\mathrm{P}(\mathrm{t})$ is the incident optical power, $\hbar \omega$ is the photon energy, $\tau_{\mathrm{r}}$ is the recombination time and $\mathrm{L}, \mathrm{W}$ and $\mathrm{d}$ are the substrate length, width and thickness, respectively.

$$
\eta=\frac{n(t) \hbar \omega L W d}{P(t) \tau_{r}}
$$

The carriers being excited in the $6 \mathrm{H}-\mathrm{SiC}$ and $2 \mathrm{H}-\mathrm{GaN}$ are coming from extrinsic levels in the bandgap. In particular, the electrons being excited into the conduction band in the $6 \mathrm{H}-\mathrm{SiC}$ material are primarily coming from filled vanadium acceptor levels. A photon is absorbed at a filled vanadium acceptor level and an electron is excited into the conduction band. Bube [6.5] gives the optical absorption coefficient for impurities $\left(\alpha_{E}\right)$ in the gap as

$$
\alpha_{E}=\sigma_{E} N_{t}
$$


Where $\mathrm{N}_{\mathrm{t}}$ is the density of filled extrinsic levels of type $t$ and $\sigma_{\mathrm{E}}$ is the optical absorption cross section for level t. If we can increase the optical absorption at the filled vanadium acceptor levels, we can increase the quantum efficiency. The quantity, $\sigma_{\mathrm{E}}$, is a quantum mechanical function dependent on the host material and the position of the extrinsic levels in the gap. We have no engineering control over $\sigma_{\mathrm{E}}$. We do have control over the intentional impurity density in the host material. We need to know the densities of the dopants in the initial 6H-SiC material to access if improvements can be made to the quantum efficiency by changing the dopant densities. The densities of the vanadium, nitrogen, boron and aluminum impurities in our initial $6 \mathrm{H}-\mathrm{SiC}$ were measured using Secondary Ion Mass Spectroscopy (SIMS). The SIMS measurements were

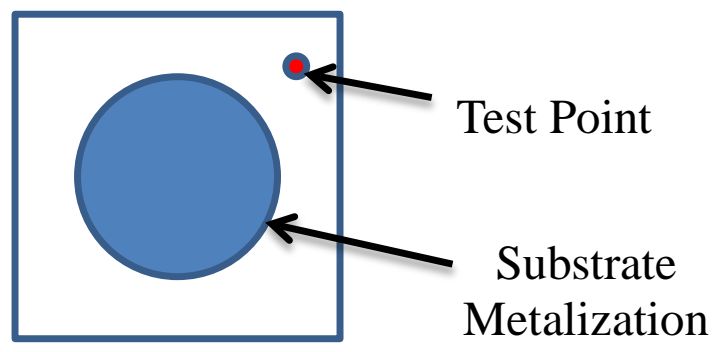

Figure 6.6. SIMS test point location on metalized 6H-SiC substrates

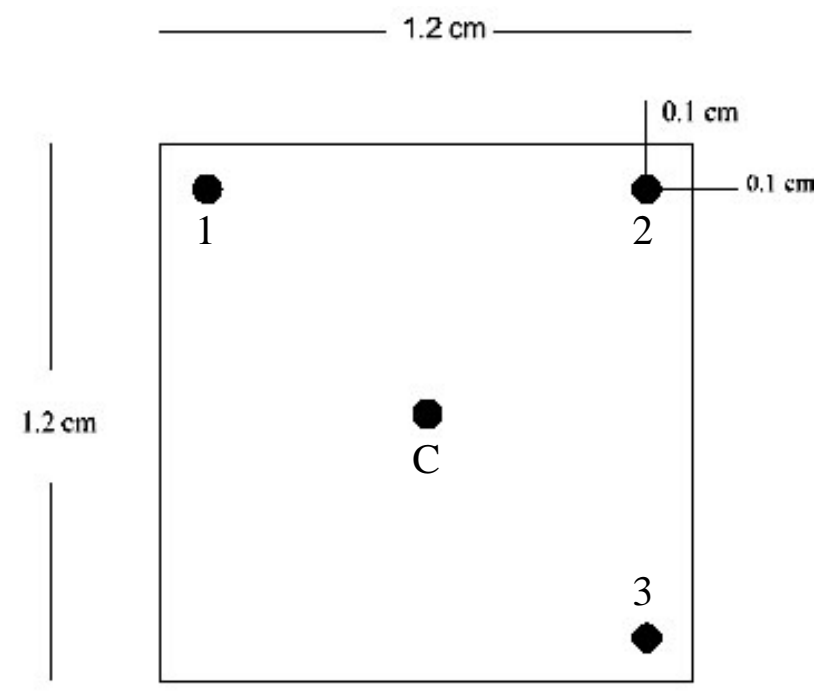

Figure 6.7 Bare 6H-SiC substrate SIMS test points 
performed on a total of 3, $6 \mathrm{H}-\mathrm{SiC}$ substrates, one bare and two metalized. All three $6 \mathrm{H}-\mathrm{SiC}$ substrates come from the same batch of material that was used to construct the green, black and silver switches. The metalized 6H-SiCsubstrates had the SIMS measurement performed at one test point on the substrate located $\sim$ halfway between the end of the switch metallization and the corner of the substrate as shown in Figure 6.6. The bare 6H-SiC substrate had SIMS measurements performed at four different locations on the bare substrate to determine the impurity densities and how they vary across the substrate. The measurements were made at the points labeled 1, 2, 3 and $\mathrm{C}$ on the same face of the substrate as shown in Figure 6.7. The SIMS measurements for all three substrates are summarized in Table 6.1.

\begin{tabular}{|c|c|c|c|c|c|c|}
\hline & \multicolumn{3}{|c|}{ Bare 6H-SiC Substrate Test Point Dopant } & Metalized \\
Densities $\mathbf{( c m}^{-3}$ ) & $\begin{array}{c}\text { Metalized } \\
\text { Substrate } \\
\mathbf{1}\end{array}$ & $\begin{array}{c}\text { Substrate } \\
\mathbf{2}\end{array}$ \\
\hline Dopant & 1 & 2 & 3 & C & Corner & Corner \\
\hline Vanadium & $2.3 \mathrm{e} 17$ & $2.1 \mathrm{e} 17$ & $1.4 \mathrm{e} 17$ & $2.0 \mathrm{e} 17$ & $1.61 \mathrm{e} 17$ & $1.97 \mathrm{e} 17$ \\
\hline Nitrogen & $6.6 \mathrm{e} 16$ & $6.1 \mathrm{e} 16$ & $6.4 \mathrm{e} 16$ & $6.4 \mathrm{e} 16$ & $5.8 \mathrm{e} 16$ & $3.5 \mathrm{e} 16$ \\
\hline Boron & $4.4 \mathrm{e} 16$ & $4.4 \mathrm{e} 16$ & $4.0 \mathrm{e} 16$ & $4.5 \mathrm{e} 16$ & $4.7 \mathrm{e} 16$ & $4.9 \mathrm{e} 16$ \\
\hline Aluminum & $7.4 \mathrm{e} 14$ & $7.4 \mathrm{e} 14$ & $7.4 \mathrm{e} 14$ & $7.4 \mathrm{e} 14$ & $7.3 \mathrm{e} 14$ & $7.5 \mathrm{e} 14$ \\
\hline
\end{tabular}

Table 6.1. SIMS measurements for bare and metalized $6 \mathrm{H}$-SiC substrates

The SIMS measurements performed on the bare substrate show very little variation in the nitrogen, boron, or aluminum densities. The nitrogen (boron) has an average density of $6.375 \mathrm{e} 16 / \mathrm{cm}^{3}\left(4.325 \mathrm{e} 16 / \mathrm{cm}^{3}\right)$ with a variation of $8 \%(9.2 \%)$ peak to peak across the substrate. The aluminum average density is very low at $7.4 \mathrm{e} 14 / \mathrm{cm}^{3}$ and its contribution is assumed to be negligible. The average vanadium density is $1.95 \mathrm{e} 17 / \mathrm{cm}^{3}$ with a variation of $46 \%$ peak to peak. The vanadium has the largest variation of the intentional dopants based on the bare $6 \mathrm{H}-\mathrm{SiC}$ substrate SIMS measurements. The metalized substrates are cut from the same crystal as the bare 
substrate. We can obtain a crude picture of how much the dopants can vary throughout the crystal if we take the measurements for all three substrates as a whole. The vanadium and boron densities for the metalized substrates are close in value to the values obtained for the bare $6 \mathrm{H}$ SiC substrate. The nitrogen densities of the metalized substrates are somewhat lower than the values obtained for the bare substrate. If we average all the SIMS measurements together for each dopant we obtain the results in table 5.2. There is a larger variation in both vanadium and nitrogen compared to boron. This is in agreement with the literature [6.6] that claims the boron density varies much slower during crystal growth than the vanadium and nitrogen density.

\begin{tabular}{|c|c|c|}
\hline Dopant & $\begin{array}{c}\text { Average Value } \\
\left(\mathrm{cm}^{-3}\right)\end{array}$ & $\begin{array}{c}\text { Variation } \\
(\text { peak to peak) }\end{array}$ \\
\hline Vanadium & $1.90 \mathrm{e} 17$ & $47 \%$ \\
\hline Nitrogen & $5.80 \mathrm{e} 16$ & $53 \%$ \\
\hline Boron & $4.48 \mathrm{e} 16$ & $18 \%$ \\
\hline
\end{tabular}

Table 6.2. Variation of vanadium, nitrogen, and boron dopants when all $6 \mathrm{H}-\mathrm{SiC}$ SIMS measurements are included.

The nitrogen and boron dopant values determine the maximum number of electrons $\left(\mathrm{N}_{T \max }\right)$ that will be available to be trapped in the vanadium acceptor levels. An expression for $\mathrm{N}_{\text {Tmax }}$ is given in equation 6.5. Where $\mathrm{N}_{\mathrm{nd}}$ and $\mathrm{N}_{\mathrm{ba}}$ are the ionized nitrogen donor and ionized boron acceptor levels, respectively. The boron acceptors are completely ionized. That is,

$$
N_{\text {Tmax }}=\left(N_{n d}-N_{b a}\right)
$$

filled with electrons donated from the nitrogen donor levels. The average values of $\mathrm{N}_{\text {nd }}$ and $\mathrm{N}_{\mathrm{ba}}$ taken from the SIMS measurements for the bare $6 \mathrm{H}-\mathrm{SiC}$ substrate are $6.375 \mathrm{e} 16 / \mathrm{cm}^{3}$ and $4.325 \mathrm{e} 16 / \mathrm{cm}^{3}$, respectively. If we substitute these two values into equation 6.5 , we obtain a value 
of $2.05 \mathrm{e} 16 / \mathrm{cm}^{3}$ for $\mathrm{N}_{\text {Tmax }}$. We can increase the number of electrons that can be excited into the conduction band and decrease the minimum possible switch resistance if we increase the value of $\mathrm{N}_{\text {Tmax }}$. We can increase the value of $\mathrm{N}_{\text {Tmax }}$ by removing the boron from the substrate. The value of $\mathrm{N}_{\text {Tmax }}$ will increase from $2.05 \mathrm{e} 16 / \mathrm{cm}^{3}$ to $6.375 \mathrm{e} 16 / \mathrm{cm}^{3}$ by removing the boron from the bare $6 \mathrm{H}-\mathrm{SiC}$ substrate. This triples the maximum value of $\alpha_{\mathrm{E}}$, the absorption coefficient for the vanadium acceptor level. However, care must be taken in making this change. We must be sure that the vanadium density is still large enough to compensate the net donor level to maintain a semi-insulating $6 \mathrm{H}-\mathrm{SiC}$ substrate. We can determine the amount of vanadium required to compensate an increased net donor level by examining Figure 6.8 (originally Figure 3.11). Figure 6.8 is a plot of our analytical model for the resistivity of $6 \mathrm{H}-\mathrm{SiC}$ as a function the ratios of vanadium to nitrogen (D) and boron to nitrogen (R). We are interested in the red trace which assumes that there is no boron present in the material. The red trace has a sharp knee for a value

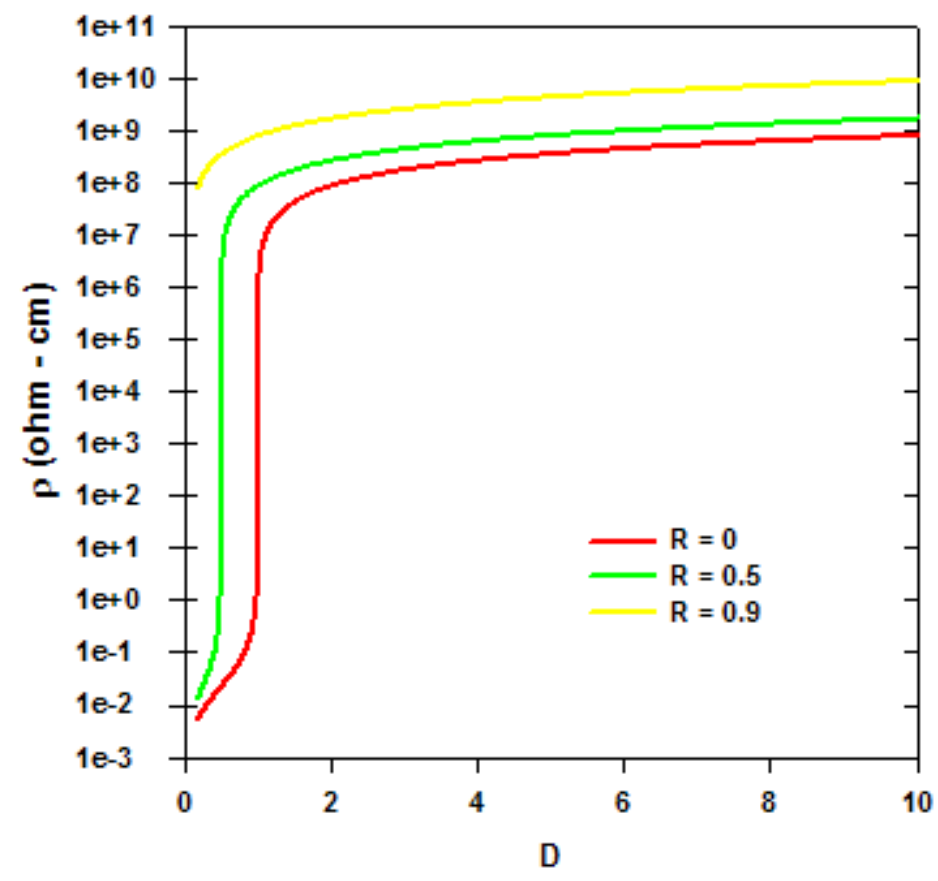

Figure 6.8. The calculated resistivity of $6 \mathrm{H}-\mathrm{SiC}$ at room temperature plotted as a function of $D$, the ratio of vanadium to nitrogen, and $R$, the ratio of boron to nitrogen. 
of D slightly greater than one. The red trace rounds off and starts to flatten out as D increases. It appears that a minimum value of $\mathrm{D}$ equal to two is required to assure that the $6 \mathrm{H}-\mathrm{SiC}$ material is a stable semi-insulating material. This corresponds to a minimum value of vanadium density of $1.275 \mathrm{e} 17 / \mathrm{cm}^{3}$ to compensate the net donor density of the bare $6 \mathrm{H}-\mathrm{SiC}$ substrate if we could magically remove the boron. The bare $6 \mathrm{H}-\mathrm{SiC}$ substrate already has a high enough vanadium density to remain semi-insulating if its boron density is reduced to zero. So, it is possible to decrease the minimum on resistance of our initial $6 \mathrm{H}-\mathrm{SiC}$ material by reducing the boron density from its present values.

Why not remove the boron from the compensated $6 \mathrm{H}-\mathrm{SiC}$ material and continue to increase $\mathrm{N}_{\operatorname{Tmax}}$ and decrease the minimum possible switch resistance by arbitrarily increasing the vanadium and nitrogen densities, while maintaining a ratio of 2 to 1 ? We are limited in how much we can increase the amounts of nitrogen and vanadium by the solubility limit of vanadium in $6 \mathrm{H}-\mathrm{SiC}$. The solubility limit of vanadium is $3-5 \mathrm{e} 17 / \mathrm{cm}^{3}$ in $6 \mathrm{H}-\mathrm{SiC}[6.6,6.7]$. Vanadium precipitates form in the $6 \mathrm{H}-\mathrm{SiC}$ crystal if the vanadium density is at, or exceeds this limit. Vanadium precipitates can act as initiation points for micropipes [6.6]. In addition, exceeding the vanadium solubility level creates mechanical stress problems in the $6 \mathrm{H}-\mathrm{SiC}$ crystal [6.7]. The highest value of $\mathrm{N}_{\text {Tmax }}$ we can obtain is achieved by increasing the densities of vanadium and nitrogen up to the vanadium solubility limit while maintaining the 2:1 ratio of vanadium to nitrogen. This approach results in maximum values of $1.5 \mathrm{e} 17 / \mathrm{cm}^{3}$ and $3.0 \mathrm{e} 17 / \mathrm{cm}^{3}$ for the nitrogen and vanadium densities to obtain the largest practical value of $\mathrm{N}_{\text {Tmax }}$ for vanadium and nitrogen doped 6H-SiC. This value of $\mathrm{N}_{\text {Tmax }}$ represents a factor of 6 - 7 increase over the initial $6 \mathrm{H}-\mathrm{SiC}$ material that we have tested. We should obtain $6 \mathrm{H}-\mathrm{SiC}$ material with the increased 
values of nitrogen and vanadium densities and minimized levels of boron and aluminum for improved switch performance.

Increasing the absorption of the filled vanadium acceptor levels $\left(\alpha_{\mathrm{E}}\right)$ decreases the optical penetration depth of the 532 and $1064 \mathrm{~nm}$ wavelength light in the 6H-SiC material. A decreased optical penetration depth reduces the physical extent (length and width) of 6H-SiC switch that can be fully turned on. It is important to know the optical transmission of our initial $6 \mathrm{H}-\mathrm{SiC}$ material at the 532 and $1064 \mathrm{~nm}$ wavelengths to understand the effect of increasing optical absorption in 6H-SiC material with improved dopant levels.

\section{3) Optical Transmission Measurements in $6 \mathrm{H}-\mathrm{SiC}$ and $2 \mathrm{H}-\mathrm{GaN}$}

The optical absorption of the switch material is important since it determines the penetration depth of the optical trigger pulse. The optical penetration depth is a limitation on switch active area, electrode position, electrode size and overall switch geometry since the optical trigger pulse has to penetrate into the switch material and generate carriers throughout the switch volume. Two different optical transmission measurements were performed on $6 \mathrm{H}-\mathrm{SiC}$ and $2 \mathrm{H}-\mathrm{GaN}$ substrates to determine the optical absorption. A Cary 5000 spectrophotometer was used to measure transmission through the $400 \mu \mathrm{m}$ dimension of the $6 \mathrm{H}-\mathrm{SiC}$ and $2 \mathrm{H}-\mathrm{GaN}$ substrates at low intensities over the spectral range of 300 to $1200 \mathrm{~nm}$. It is reasonable to neglect all but linear optical absorption in the bulk $\mathrm{SiC}$ and $\mathrm{GaN}$ substrates at the low intensities applied by the spectrophotometer. The optical transmission at room temperature of the semi-insulating $6 \mathrm{H}-\mathrm{SiC}$ and $2 \mathrm{H}-\mathrm{GaN}$ substrates is shown in Figure 6.8. The transmission through $\mathrm{GaN}$ is higher and persists to shorter wavelengths compared to the $6 \mathrm{H}$-SiC sample due to its wider bandgap and lower index of refraction. The $6 \mathrm{H}-\mathrm{SiC}$ has a sharper knee in its transmission that occurs at $~ 430$ 
$\mathrm{nm}$ (55 \% transmission). The knee of the optical transmission through GaN occurs at $\sim 460 \mathrm{~nm}$ (65 \% transmission). The conduction band edge is assumed to reside at the wavelength where the transmission equals one half the value the transmission of the knee of the curves. This occurs at $385 \mathrm{~nm}$ and $405 \mathrm{~nm}$ for the $\mathrm{GaN}$ and $6 \mathrm{H}-\mathrm{SiC}$ samples, respectively. These wavelengths correspond to $3.22 \mathrm{eV}$ for the $\mathrm{GaN}$ substrate and $3.06 \mathrm{eV}$ for the $6 \mathrm{H}-\mathrm{SiC}$ substrate.

We are particularly interested in transmission through the $6 \mathrm{H}-\mathrm{SiC}$ and $2 \mathrm{H}-\mathrm{GaN}$ substrates at the 532 and $1064 \mathrm{~nm}$ wavelengths. The transmission at $532 \mathrm{~nm}(1064 \mathrm{~nm})$ is $61.25 \%$

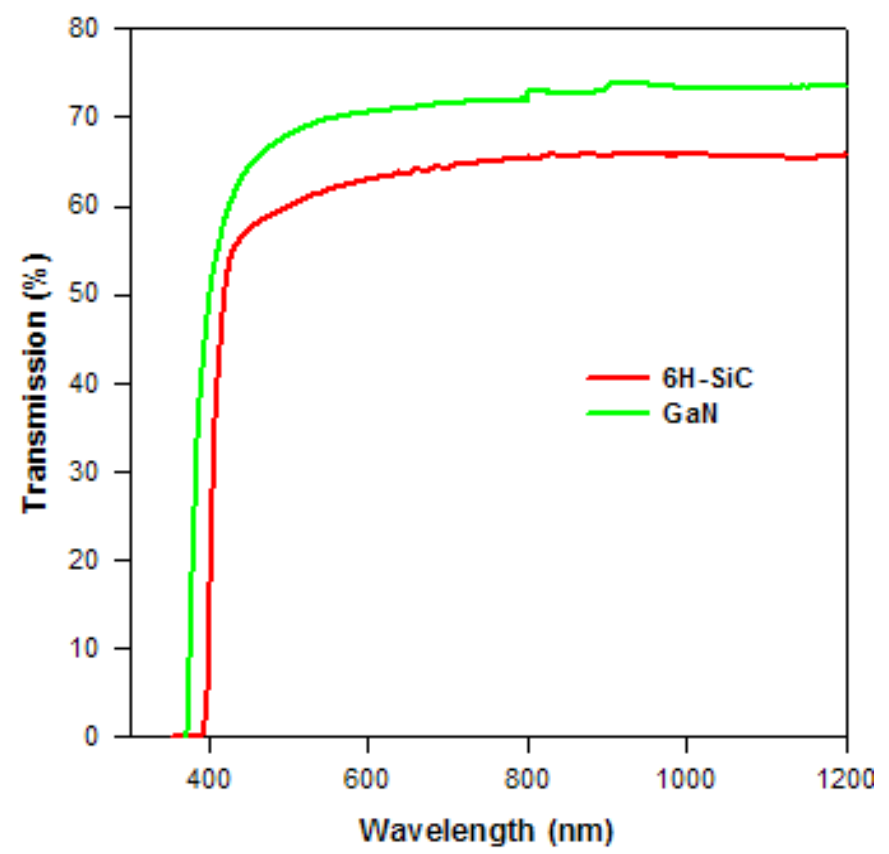

Figure 6.8. Optical transmission through $400 \mu \mathrm{m}$ thick, semi-insulating, $6 \mathrm{H}-\mathrm{SiC}$ and $2 \mathrm{H}$ GaN substrates

(65.67\%) and $69.30 \%$ (73.27 \%) for the $6 \mathrm{H}-\mathrm{SiC}$ and $2 \mathrm{H}-\mathrm{GaN}$ substrates, respectively. The spectrophotometer results were used to calculate the effective linear absorption of the $6 \mathrm{H}-\mathrm{SiC}$ and 2H -GaN at 532 and $1064 \mathrm{~nm}$ using equation (6.6), assuming linear absorption and measured values of the index of refraction [6.8,6.9] for both materials. We calculated the values of effective linear absorption from the spectrophotometer measurements to be 1.61 and $0.63 \mathrm{~cm}^{-1}$ for $6 \mathrm{H}-\mathrm{SiC}$, and 0.43 and $0.10 \mathrm{~cm}^{-1}$ for $\mathrm{GaN}$ at the 532 and $1064 \mathrm{~nm}$ wavelengths, respectively. 


$$
T=\frac{\left(1-\left(\frac{n-1}{n+1}\right)^{2}\right)^{2} \exp (-\alpha l)}{1-\left(\exp (-\alpha l)\left(\frac{n-1}{n+1}\right)^{2}\right)^{2}}
$$

Where $\mathrm{T}$ is the transmission, $\alpha$ is the effective linear absorption, $\mathrm{l}$ is the length traveled through the substrate and $n$ is the index of refraction of either $6 \mathrm{H}-\mathrm{SiC}$ or $\mathrm{GaN}$. The length travelled through the substrates for the spectrophotometer measurements was d, the substrate thickness ( $400 \mu \mathrm{m})$. We need to know the transmission through the $6 \mathrm{H}-\mathrm{SiC}$ and $\mathrm{GaN}$ substrates at the same optical energy and peak power that was applied to the green, black and silver switches during photoconductive switch tests. This requires measuring the optical transmission at peak optical powers up to a few MW.

If sufficiently high optical intensities are applied, sources of nonlinear optical absorption need to be considered. The differential equation describing the optical loss in a bulk material is given by equation (6.7) if we ignore third order and higher effects.

$$
\frac{d I}{d z}=-\alpha I-\beta I^{2}-\sigma_{c} N_{c}(I) I
$$

Where I is the optical intensity, $\beta$ is the two photon absorption coefficient, $\sigma_{\mathrm{c}}$ is the free carrier cross-section and $\mathrm{N}_{\mathrm{c}}$ is the free carrier density.

High power optical transmission measurements were performed on a $7.2 \mathrm{~mm}$ by $3.7 \mathrm{~mm}$, $400 \mu \mathrm{m}$ thick, $6 \mathrm{H}-\mathrm{SiC}$ substrate and a $9.0 \mathrm{~mm}$ by $9.0 \mathrm{~mm}, 408 \mu \mathrm{m}$ thick, $2 \mathrm{H}-\mathrm{GaN}$ substrate. The layout of the optical transmission measurement is shown in Figure 6.9. The optical pulse passes through a 50/50 beam splitter and half the pulse is applied to the $\mathrm{SiC}$, or $\mathrm{GaN}$, substrate facet and the other half is applied to an optical energy meter. Optical pulses were applied to the $3.7 \mathrm{~mm}$ by $400 \mu \mathrm{m}$ facet of the $6 \mathrm{H}-\mathrm{SiC}$ substrate with the optical $\mathrm{E}$ field perpendicular to the c direction of 
the $\mathrm{SiC}$ growth crystal. For the GaN substrate, the optical pulse was applied to the $9.0 \mathrm{~mm}$ by $408 \mu \mathrm{m}$ facet with the optical E field vector parallel to the c direction of the GaN crystal. Both substrates were masked so that only light striking the facet was transmitted to

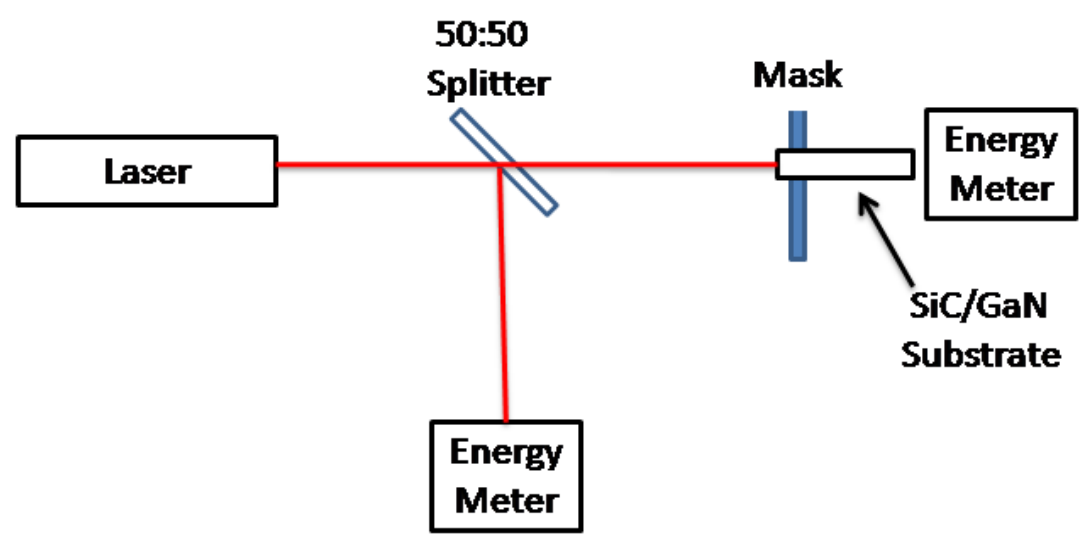

\section{Figure 6.9. Optical transmission experiment layout}

an optical energy meter positioned behind the substrate. The mask is shown in Figures 6.10 and 6.11. The effective absorption was calculated from the transmission measurements using equation (6.6) assuming only linear absorption in the bulk material. We expect the effective absorption coefficient to be constant as the applied optical energy is increased, if only linear absorption were taking place in the material. If the effective optical absorption coefficient increases with applied intensity, this indicates that the two photon, or, free carrier absorption terms in equation (2) are contributing to the optical loss. Plots of the effective absorption coefficient as a function of optical energy are shown in Figure 6.12. Each data point in Figure 6.12 is the average of twenty-five individual measurements. We observe a variation of five percent, or less, in each set of the twenty-five transmission measurements, which is within the error of the energy meters. The calculated optical penetration depth $(1 / \alpha)$ from the Figure 6.12 data for a $10 \mathrm{~mJ}$ optical pulse at $532 \mathrm{~nm}$ is 0.47 and $0.57 \mathrm{~cm}$ for $6 \mathrm{H}-\mathrm{SiC}$ and $2 \mathrm{H}-\mathrm{GaN}$, respectively. It is apparent from the plots that the effective linear absorption is constant in the 


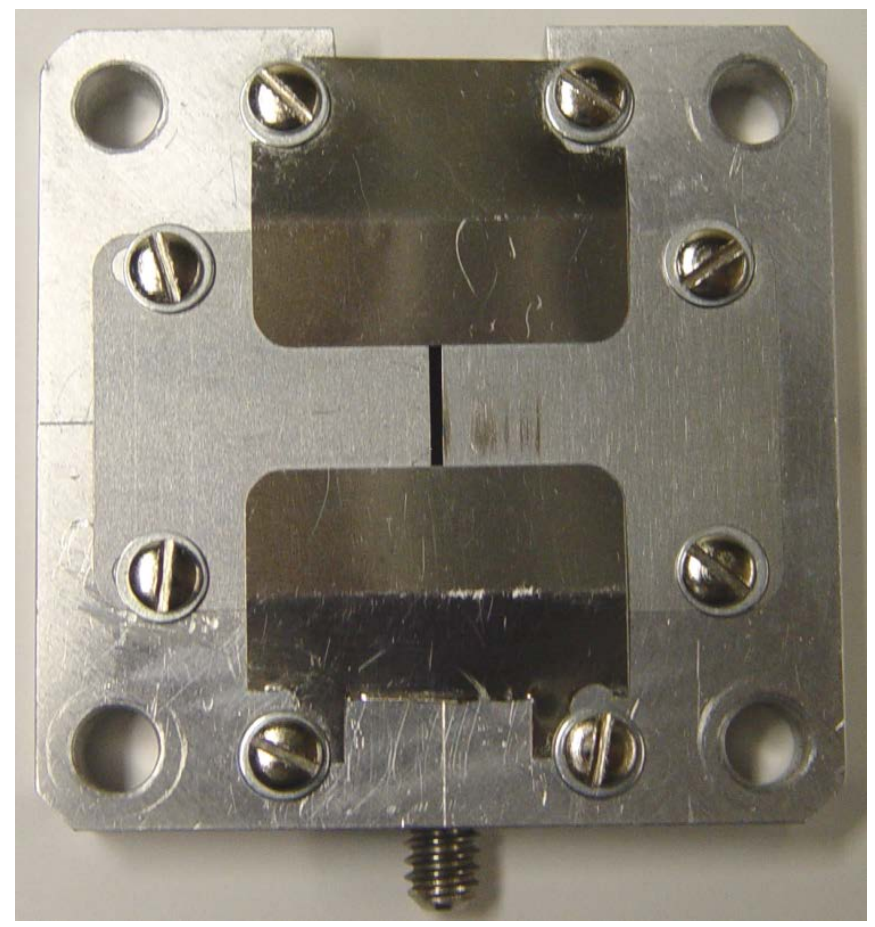

Figure 6.10. Front of stainless steel mask. Substrates are positioned in the slot and held in place by thin stainless steel sheets. Stainless steel sheets can be positioned to accommodate various sized substrates.

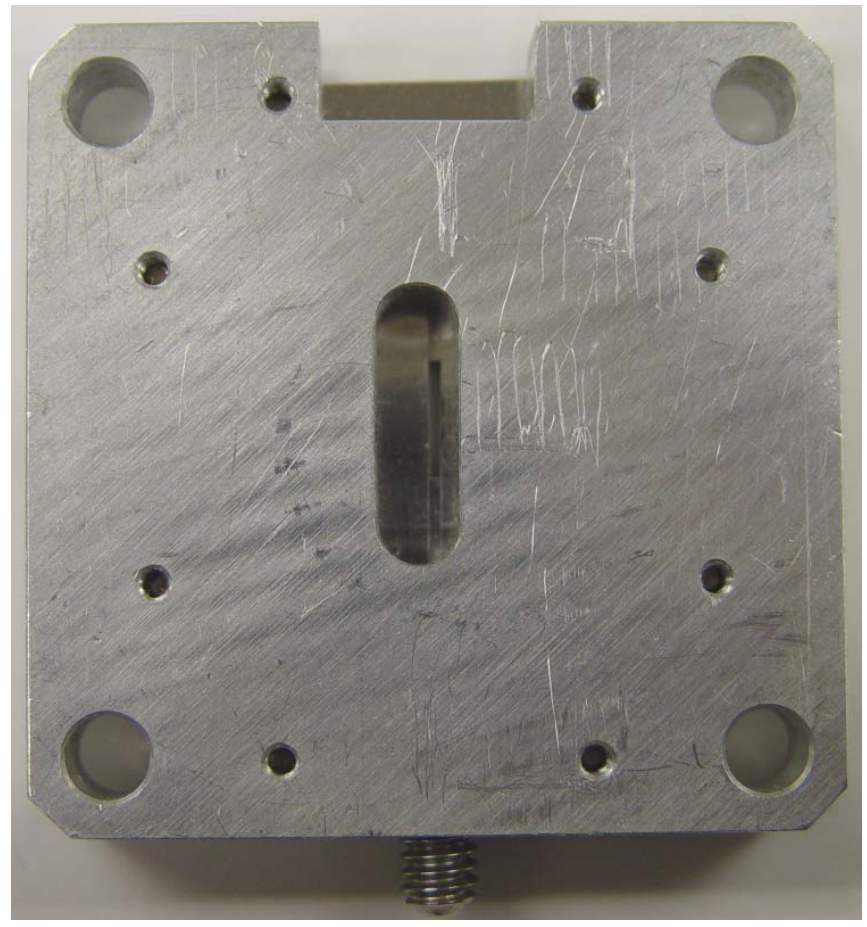

Figure 6.11. Back side of stainless steel mask. Energy meter is butted against mask surface. 


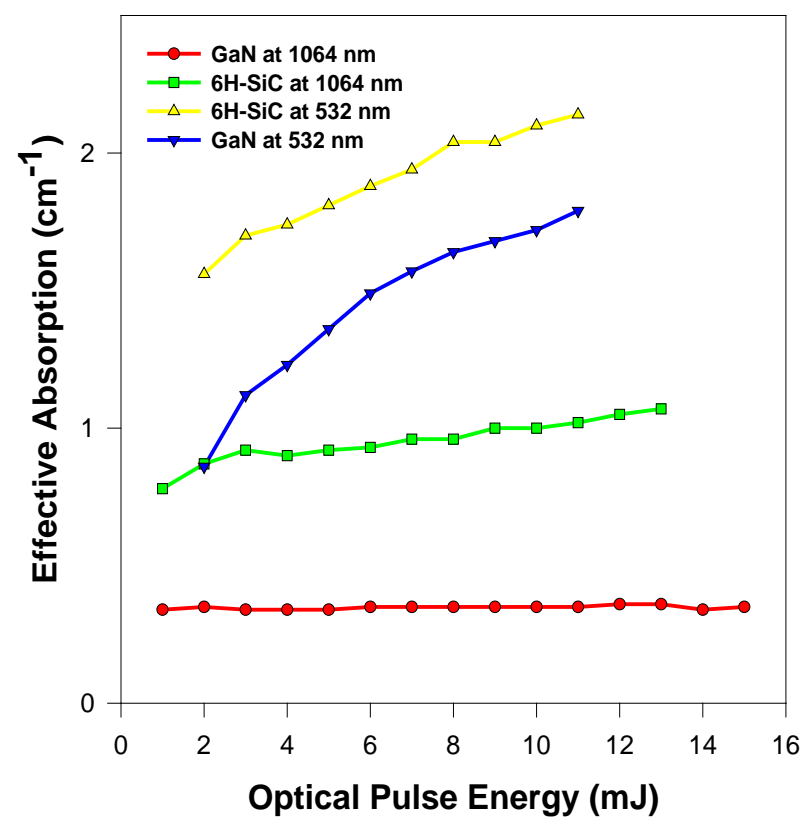

Fig. 6.12. Effective optical absorption in $\mathrm{GaN}$ and $\mathrm{SiC}$ at 532 and $1064 \mathrm{~nm}$

bulk $2 \mathrm{H}-\mathrm{GaN}\left(0.35 \mathrm{~cm}^{-1}\right)$ at $1064 \mathrm{~nm}$, indicating only linear absorption occurs. However, the $2 \mathrm{H}-$ GaN has a significant nonlinear optical loss component at $532 \mathrm{~nm}$. 6H-SiC exhibits a significant increase in optical loss with applied optical energy at the $532 \mathrm{~nm}$ wavelength and a milder increase in optical loss with applied optical energy at the $1064 \mathrm{~nm}$ wavelength. We determined that the magnitude of free carrier absorption in $6 \mathrm{H}-\mathrm{SiC}$ and $2 \mathrm{H}-\mathrm{GaN}$ is negligible for carrier densities less than $1 \times 10^{16} \mathrm{~cm}^{-3}$ in Chapter 4. As a result, we expect that the nonlinear optical loss component for the both the $2 \mathrm{H}-\mathrm{GaN}$ and $6 \mathrm{H}-\mathrm{SiC}$ material is due to two-photon absorption (TPA) at the $532 \mathrm{~nm}$ wavelength. We have re-plotted the measured optical absorption for $6 \mathrm{H}-\mathrm{SiC}$ and $2 \mathrm{H}-\mathrm{GaN}$ along with the calculated value for TPA based on values of $\beta$ for $6 \mathrm{H}-\mathrm{SiC}(3.2 \mathrm{~cm} / \mathrm{GW})$ and 2H-GaN $(20 \mathrm{~cm} / \mathrm{GW})$ in Figure 6.13.

Figure 6.13 shows the measured optical absorption (data points) for $6 \mathrm{H}-\mathrm{SiC}$ and $2 \mathrm{H}-\mathrm{GaN}$ at $532 \mathrm{~nm}$ along with the absorption calculated from the sum of TPA and linear absorption (solid 
lines) plotted as a function of the peak optical intensity at the substrate facet. The peak optical intensities are very high for the $6 \mathrm{H}-\mathrm{SiC}$ substrate since the facet area is $0.0148 \mathrm{~cm}^{2}(0.04 \times 0.37$ $\mathrm{cm}^{2}$ ) compared to our nominal facet area of $0.04 \mathrm{~cm}^{2}$. The peak optical intensity of $124 \mathrm{MW} / \mathrm{cm}^{2}$ used in the optical transmission tests on $6 \mathrm{H}-\mathrm{SiC}$ would be reduced to $45.9 \mathrm{MW} / \mathrm{cm}^{2}$ when spread over the nominal facet area used in our photoconductive switch tests. The TPA for the 6H-SiC substrate would account for $\sim 10 \%$ of the total absorption for our switch tests at the $532 \mathrm{~nm}$ wavelength. However, we should expect that two-photon absorption in 6H-SiC contributes significantly to the supralinear photoconductivity in $6 \mathrm{H}-\mathrm{SiC}$ at the higher optical powers at 532 nm.

The facet area of the substrate used for the transmission experiments is only 10 percent smaller than the facet of the $2 \mathrm{H}-\mathrm{GaN}$ switch substrate. As a result, the optical intensities used for the $2 \mathrm{H}-\mathrm{GaN}$ transmission and switching tests are almost identical. The absorption plot for $2 \mathrm{H}-$ $\mathrm{GaN}$ in Figure 6.13 shows that two-photon absorption is the dominant carrier generating mechanism in $2 \mathrm{H}-\mathrm{GaN}$ at $532 \mathrm{~nm}$. This is in good agreement with the switch testing that revealed the switch conductance of the $2 \mathrm{H}-\mathrm{GaN}$ substrate decreased with the square of the applied optical intensity.

Both materials have higher optical absorption at $532 \mathrm{~nm}$. However, higher optical absorption translates to shallower optical penetration depths and limits on the size and geometry of switch structures. The optical penetration depth of $0.47 \mathrm{~cm}$ calculated for $6 \mathrm{H}-\mathrm{SiC}$ for operation at $10 \mathrm{~mJ}$ at $532 \mathrm{~nm}$ indicates that the $6 \mathrm{H}$-SiC substrate length and width should be limited to $1 \mathrm{~cm}$ by $1 \mathrm{~cm}$ and light should applied to more than just a single facet. The response of a $6 \mathrm{H}-\mathrm{SiC}$ substrate to triggering at multiple facets and by different optical spatial profiles is the subject of section 6.4. 


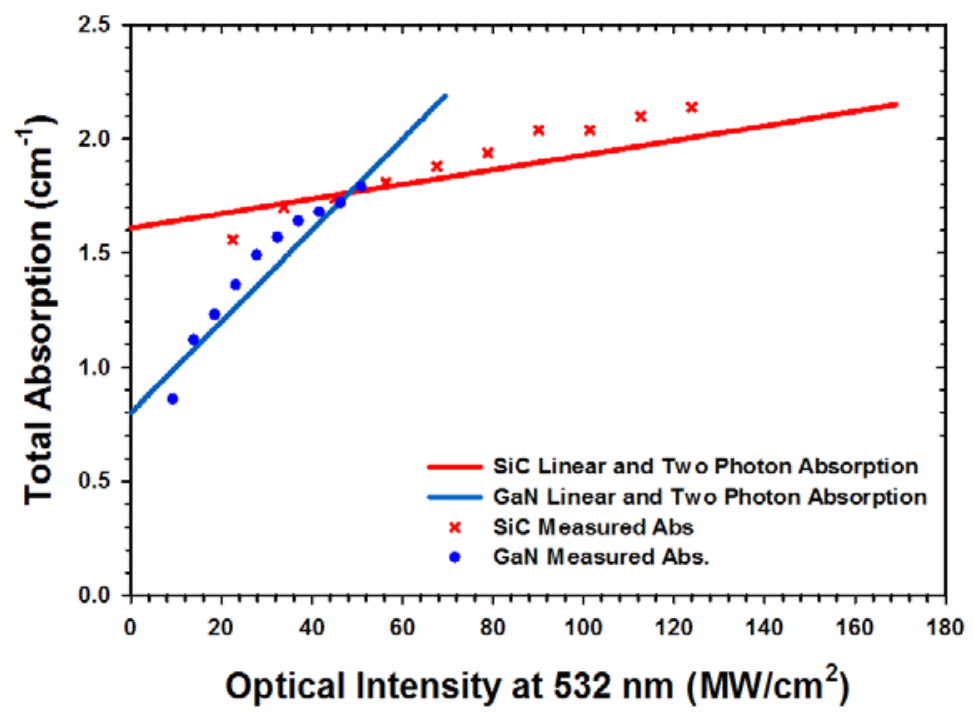

Figure 6.13. Measured optical absorption and calculated optical absorption at the $532 \mathrm{~nm}$ wavelength for $6 \mathrm{H}-\mathrm{SiC}$ and $2 \mathrm{H}-\mathrm{GaN}$ substrates used in the high power optical transmission tests.

\section{4) Switch Triggering with Different Optical Profiles and at Multiple Facets}

The $6 \mathrm{H}-\mathrm{SiC}$ and $2 \mathrm{H}-\mathrm{GaN}$ photoconductive switches are switched on by applying an optical pulse that is $1 \mathrm{~cm}$ wide and slightly overfills the $400 \mu \mathrm{m}$ tall facet. However, the spatial profile of the applied optical pulse is not uniform across the $400 \mu \mathrm{m}$ dimension of the facet. The profile of the optical pulse in the $400 \mu \mathrm{m}$ dimension is closer to Gaussian. We will denote Gaussian optical intensity profile across the switch facet as I(x). The electron density in the switch volume, $\mathrm{n}(\mathrm{x})$, will assume the same profile of the optical pulse $\mathrm{I}(\mathrm{x})$ because of the rapid carrier recombination time of both the $6 \mathrm{H}-\mathrm{SiC}$ and $2 \mathrm{H}-\mathrm{GaN}$ materials. The switch will have a higher density of electrons at the switch center and a reduced density closer to the switch metallization. As a result, the switch will have a section of lower resistivity at the switch center sandwiched by portions of higher resistivity closer to the switch metallization. The portions of 
higher resistivity will dominate the on resistance of the switch. A uniform spatial profile of the applied optical pulse should correct this problem.

The situation can be visualized with the help of Figure 6.14. Figure 6.14 shows an experiment that was constructed to explore the difference in switch minimum resistance obtained for the application of the typical optical pulse $(\mathrm{I}(\mathrm{x}))$ and a pulse profile that was spread out to provide a uniform intensity across the $400 \mu \mathrm{m}$ tall switch facet $\left(\mathrm{I}_{\text {spread }}(\mathrm{x})\right)$. The experiment was set up using a mask to assure that that same total optical energy is applied to the switch facet by either the standard, or spread, optical pulse. The tests were performed at the $532 \mathrm{~nm}$ wavelength. Figure 6.15 shows a plot of minimum resistance obtained for the standard pulse applied to one facet, the spread pulse applying a uniform optical profile across a single facet and a standard pulse that was divided into two pulses of equal energy applied at two opposing facets. The results show no improvement in minimum on resistance for the spread uniform pulse compared to the standard pulse. The results also show a marginal improvement in minimum resistance for applying half the optical intensity at two opposing switch facets. We believe that the uniform optical pulse provided no real improvement in minimum switch resistance due to the surface condition the switch facet. All the switches tested from the initial batches of $6 \mathrm{H}-\mathrm{SiC}$ have either cleaved, or finely ground facets. The ground facets are flat, but have a rough surface. The cleaved facets are either flat, or have steps. Cleaved facets parallel to the c- direction of the crystal were likely to have steps. Cleaved facets perpendicular to the c-direction were likely to be flat. However, the $6 \mathrm{H}-\mathrm{SiC}$ substrate used in this test had ground facets. These rough facets scatter the applied light and homogenize it, effectively making it a uniform optical pulse. 


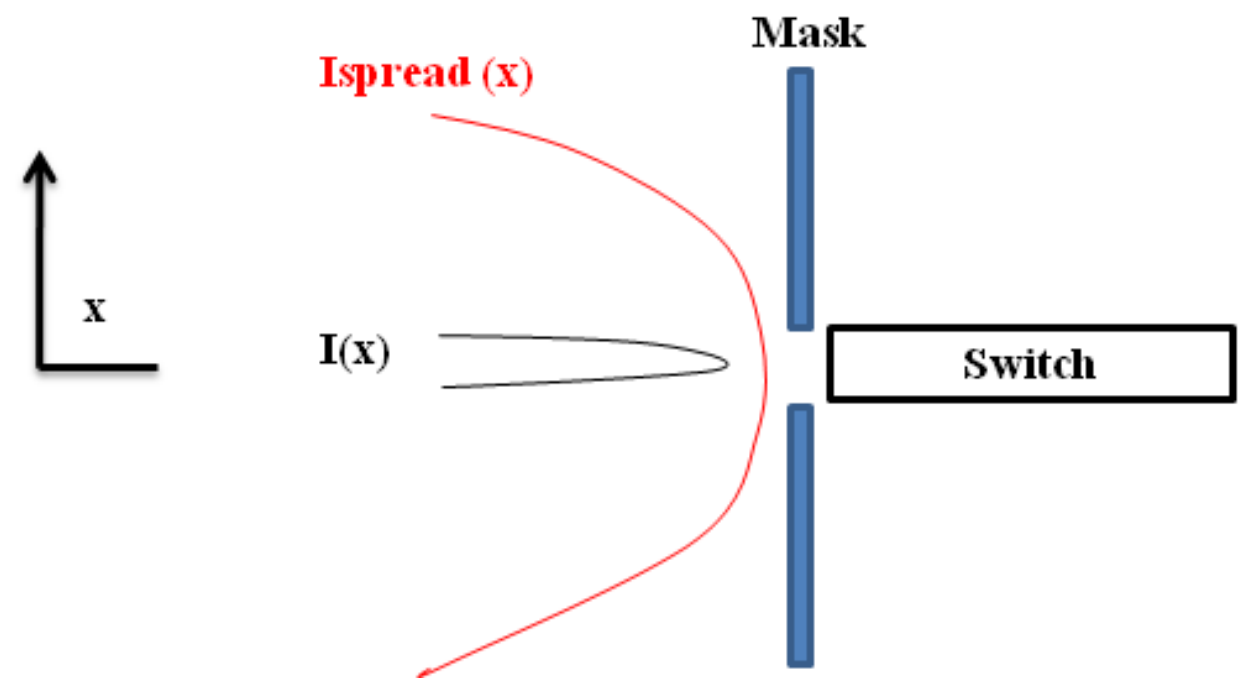

Figure 6.14. Experiment to explore the effect of optical pulse profile on the minimum resistance of a $6 \mathrm{H}-\mathrm{SiC}$ switch.

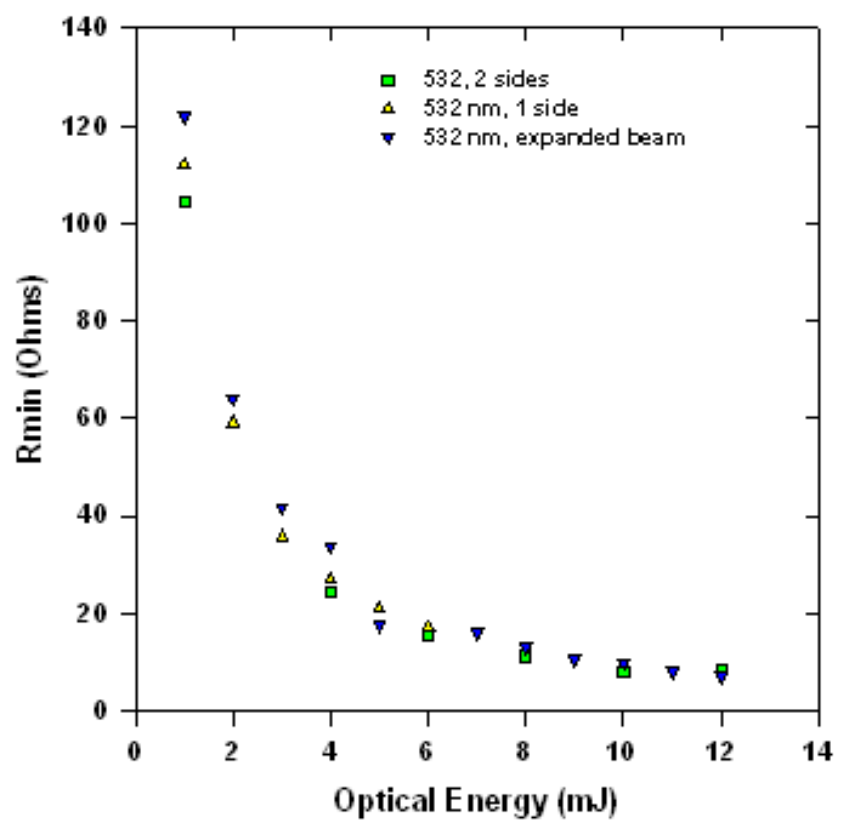

Figure 6.15. Minimum resistance plotted for standard pulse applied to one facet, standard pulse split in half with each half applied at opposing facets and the spread pulse applied at a single facet. 
The results for the split optical pulse are attractive since they provide a slight improvement in minimum switch resistance for half the peak intensity applied to opposing facets. This will require an additional optical path. However, the reduced optical intensity at the switch facets reduces the possibility of any optical damage occurring there. The carrier density uniformity in the volume of the switch will also be improved by applying half the optical intensity at opposing facets of the switch. This can be visualized with the aid of Figure 6.16 and Beer's law (equation 6.8). Neglecting the nonlinear optical absorption, the optical intensity will decay exponentially in the direction of propagation (z) in an absorbing medium as shown in Equation 6.8. We also expect that the photogenerated carrier density, and hence the conductivity, will be proportional to $\mathrm{I}(\mathrm{z})$ as expressed in equation 6.9. Figure 6.16 is a plot of the switch conductivity that results from applying I(z) at a single switch facet, or, applying half of $\mathrm{I}(\mathrm{z})$ at two opposing facets for linear absorption coefficients $(\alpha)$ of 3 and $6 \mathrm{~cm}^{-1}$. It is clear from the plot that the conductivity, and as a result the switch current density, is more uniform when the optical pulse is applied at opposing electrodes. Another interesting point is that the switch conductance,

$$
\begin{gathered}
I(z)=I_{0} e^{-\alpha z} \\
\sigma(z)=\sigma_{0} e^{-\alpha z} \\
\sigma_{\text {switch }}=\int_{0}^{1} \sigma(z) d z
\end{gathered}
$$

as given by equation 6.10 , will be equal for either case of applying the optical pulse at single facet, or, dividing it between two facets. This agrees with the experimental results shown in Figure 6.15 that show little difference in switch on resistance for applying the optical pulse at one facet, or, dividing the optical pulse between opposing facets. However, it is clear that for 


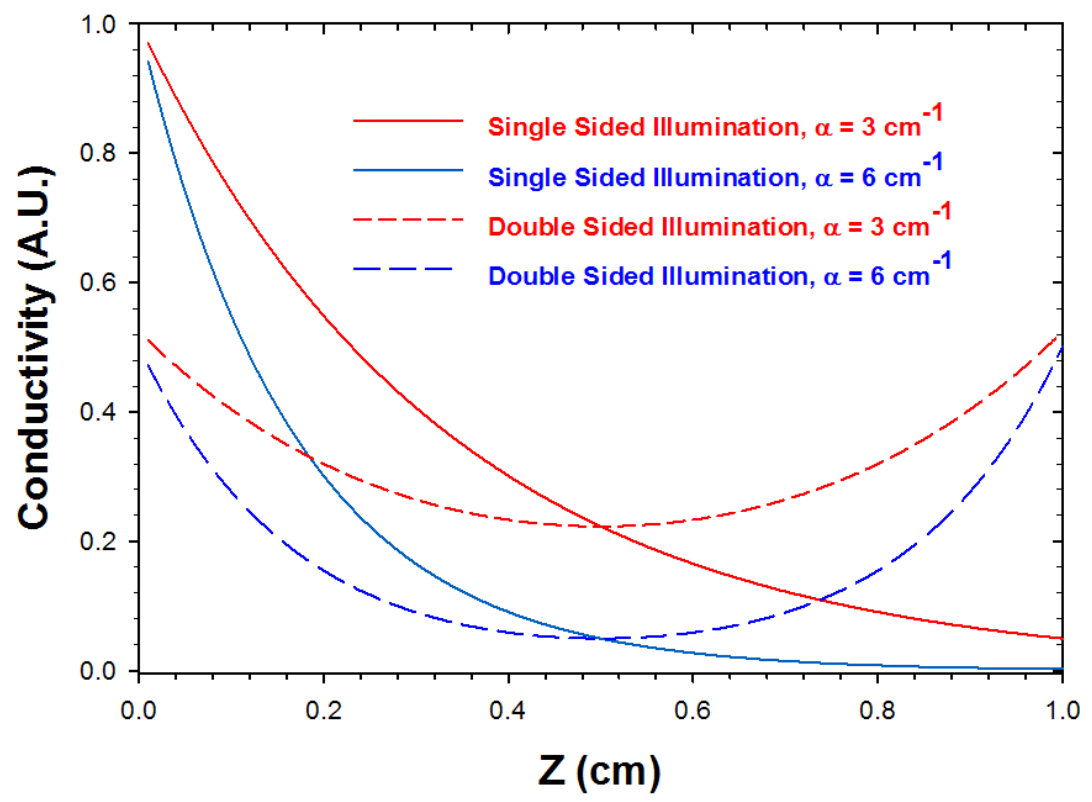

Figure 6.16. Conductivity is plotted as a function of switch length (z) for the cases when the optical pulse is applied at a single facet, or divided between opposing facets. The two cases are shown for a material with linear absorption of 3 and $6 \mathrm{~cm}^{-1}$.

more uniform switch current density and less optical intensity at the switch facet the light should be divided between opposing facets.

\section{5) Switch Turn-on and Turn-off Times}

The medical DWA application discussed in Chapter 1 requires switch turn-on times of 1 ns, or less. Switch turn-off time are not as stringent. The main requirement for switch turn-off is that is does not prevent operating at the desired DWA application repetition rate. Optical pulses with rise and fall times of 1 ns, or less, are required to determine the switch turn-on and turn-off limitations. The Spectron Nd:YAG laser that is used in our photoconductive switching tests has a 
5 - 6 ns and $7.5-8 \mathrm{~ns}$ FWHM pulse width at $532 \mathrm{~nm}$ and $1064 \mathrm{~nm}$ wavelengths, respectively.

We decided to obtain faster rise and fall times of our optical pulse by "chopping” off the

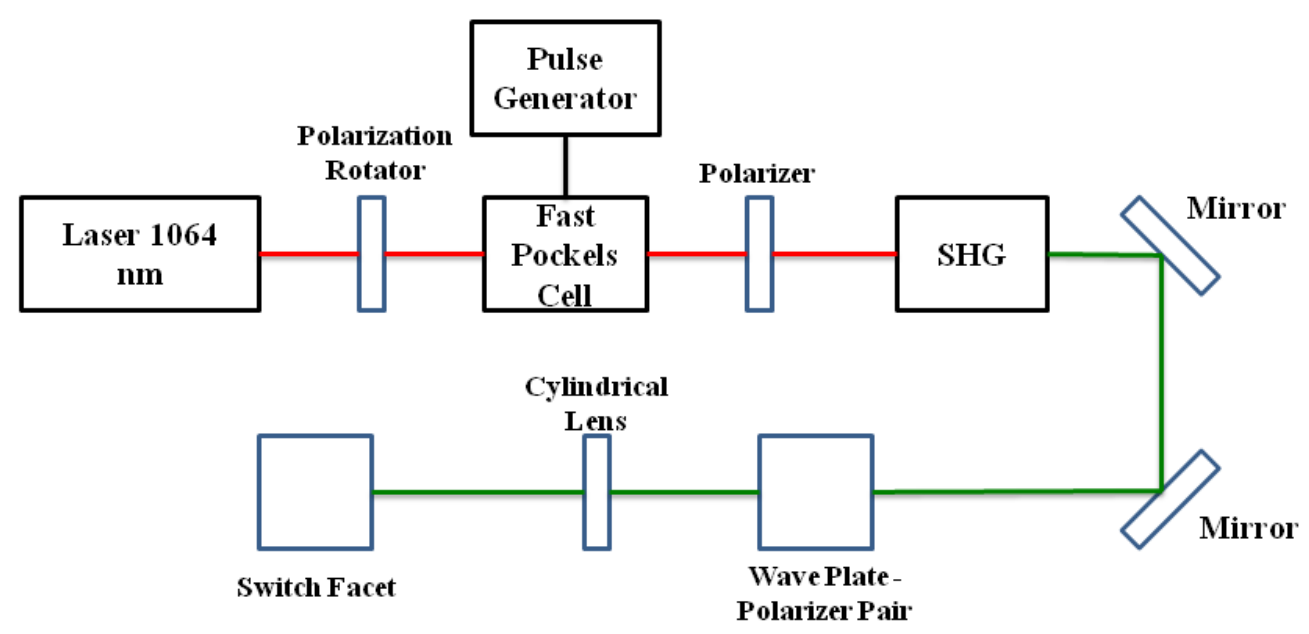

Figure 6.17. Schematic diagram of the optical layout including a "pulse-slicing” Pockels cell to sharpen the rise or fall time of the optical pulse applied to the $6 \mathrm{H}-\mathrm{SiC}$ switch facet

rising and falling edges of our typical optical pulse using a pulse slicing Pockels cell. The pulse slicing Pockels cell is a fast transition Pockels cell constructed to be a $50 \mathrm{ohm}$ transmission line structure. The fast Pockels cell is installed between the output of the Spectron laser amplifier and the input of the Spectron laser second harmonic generating (SHG) module, as shown in Figure 6.17. The purpose of the fast Pockels cell is to "chop" off the slower rising and falling portions of the laser pulse.

The effect of chopping the rising and falling edges of the optical pulse is shown in Figures 6.18 and 6.19. The optical pulse exiting the laser amplifier is blocked from reaching the SHG module until a voltage pulse is applied to the fast Pockels cell. A fast rising voltage pulse is applied to the fast Pockels cell, just prior to the time that laser pulse will be at its peak intensity, allowing the laser pulse to be passed through to the SHG module on its way to the switch facet. 
This results in the red rising edge being carved into the normal laser pulse shown in Figure 6.17. This is all accomplished by taking advantage of the polarization of the laser pulse. The majority

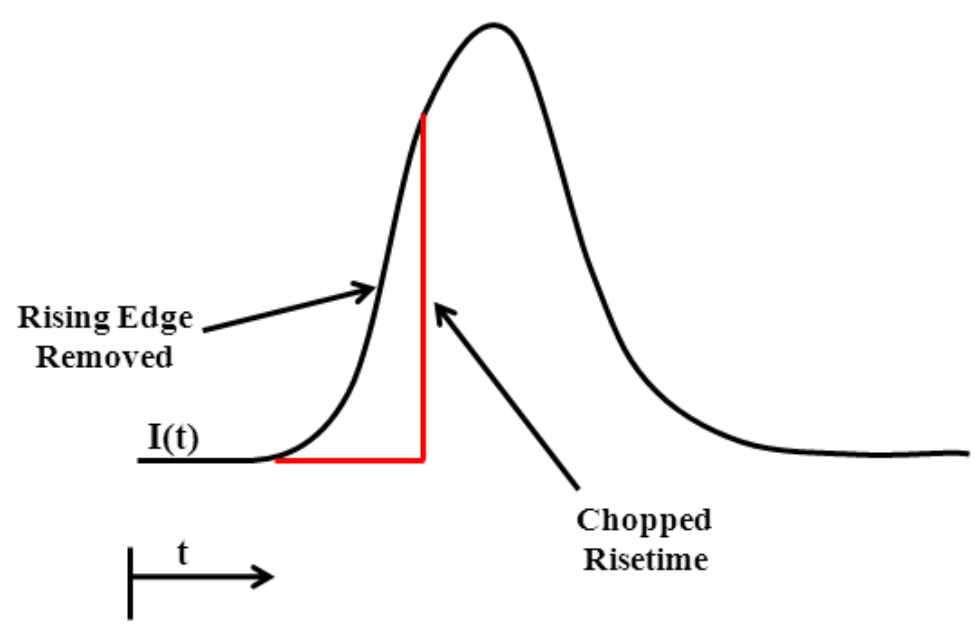

Figure 6.18. Fast rising edge (red) is carved out of the slower rising edge of the typical laser pulse.

of the laser pulse shown on the far left of Figure 6.17 is S-polarized and becomes P-polarized after passing through the polarization rotator. The optical pulse is rotated back to being Spolarized by the Pockels cell, if the fast voltage pulse is not applied to the Pockels cell. The Spolarized optical pulse then reaches the P-polarizer where the S-polarized optical pulse is rejected. The process is changed when a voltage pulse is applied to the Pockels cell while the laser pulse is incident on it. The Pockels cell does not rotate the P-Polarized light to S-polarized light while full voltage is applied to it, so P-polarized light passes through the Pockels cell unchanged. The P-polarized light then passes through the P-polarizer and is converted in the SHG module and eventually applied to the switch facet. The speed of the rise time that is carved into the laser pulse is determined primarily by the rise time of the voltage pulse applied to the Pockels cell. We need a voltage rise time of $\sim 1$ ns to obtain laser pulse rise time of 1 ns. 
The fall time of the laser pulse is chopped by inserting another polarization rotator between the fast Pockels cell and the P-polarizer. The laser pulse will pass through the SHG module to the switch facet while no voltage is applied to the fast Pockels cell. The application of

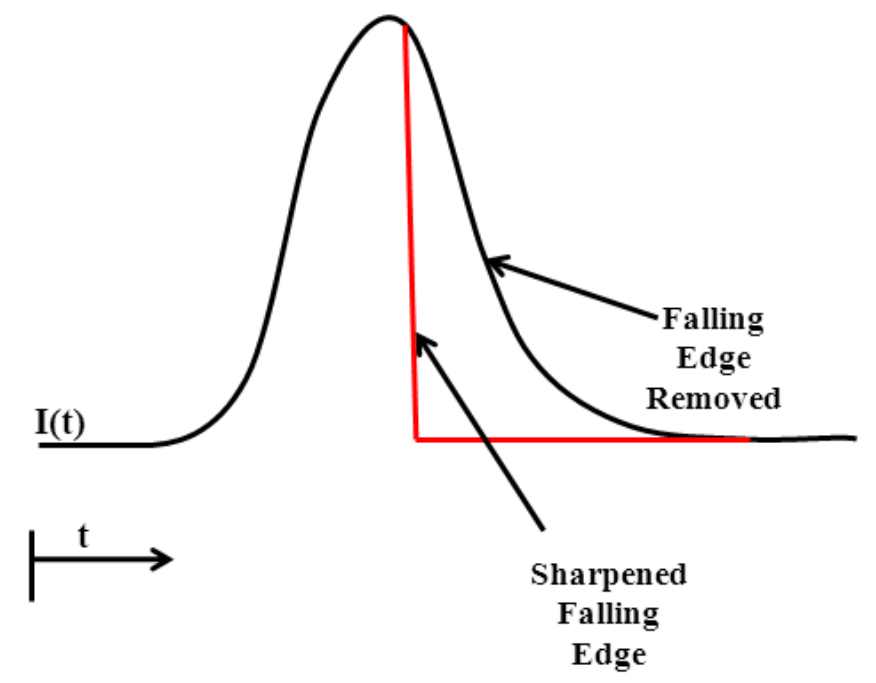

Figure 6.19. Fast falling edge is carved into the slower falling edge of an optical pulse.

a fast rising voltage pulse to the fast Pockels cell just after the peak of the optical pulse arrives will carve a fast fall time into the optical pulse similar to Figure 6.19.

This technique was put into practice using a Leysop UPC069/2 pulse slicing Pockels cell [6.10] designed for operation at $1064 \mathrm{~nm}$, an avalanche transistor based, high voltage pulse generator for chopping the rising edge of the optical pulse and a commercial FID Technology model FDS high voltage pulser [6.11] to chop the falling edge of the optical pulse. The pulse slicing Pockels cell is designed as a $50 \mathrm{ohm}$ structure capable of rise times as fast as 500 ps. The avalanche transistor based, high voltage pulser used to chop the rising edge of the optical pulse is based on three, 8-high, series stacks of avalanche transistors arranged in the Marx geometry [6.12]. The avalanche transistor high voltage pulser is designed to drive a $50 \mathrm{ohm}$ load with a 3.0 to $3.5 \mathrm{kV}$ pulse with a rise time of $\sim 1$ ns. The FID Technology model FDS pulser provides a 
rectangular output pulse with up to $16 \mathrm{kV}$ voltage amplitude pulse into a $50 \mathrm{ohm}$ load with subnanosecond rise and fall times.

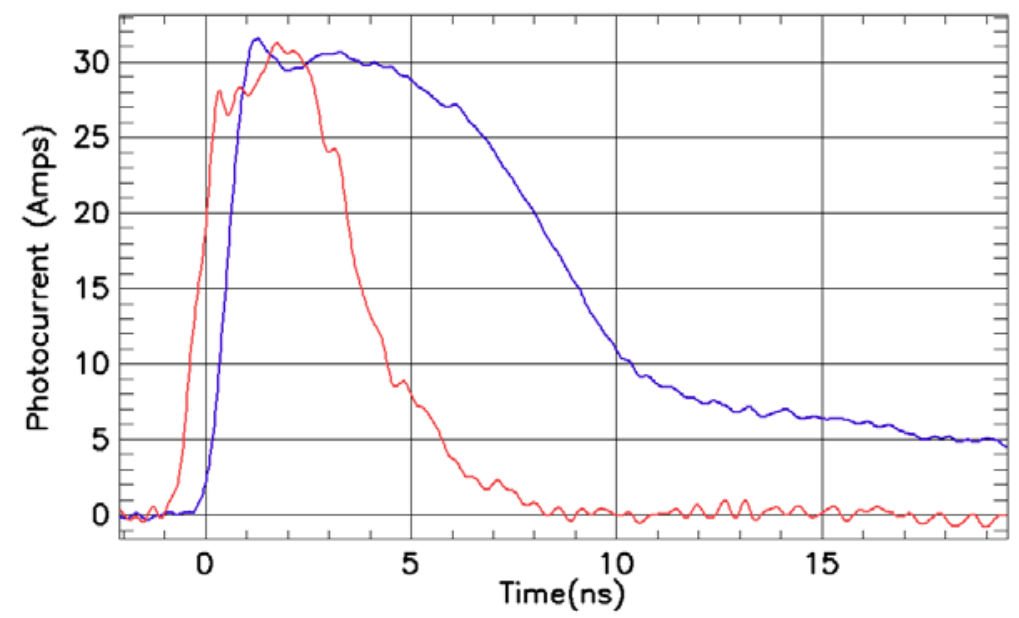

Figure 6.20. Photocurrent (blue) and optical intensity (red) for a chopped rise time optical pulse applied to the silver switch. The charge voltage and pre-chop optical energy are 1550 Volts and $13 \mathrm{~mJ}$ at $532 \mathrm{~nm}$.

The optical pulse with chopped rise time and resulting photocurrent for the silver switch at a charge voltage of 1550 Volts for $13 \mathrm{~mJ}$ of $532 \mathrm{~nm}$ light tested in the high voltage test circuit (Figures 5.11 - 5.12) is shown in Figure 6.20. The $13 \mathrm{~mJ}$ optical energy at $532 \mathrm{~nm}$ represents the pre-chop energy contained in the optical pulse. The optical energy contained in the chopped pulse is less, but the peak optical intensity is the same. The chopped photocurrent follows the rise time of the optical pulse and has a 10 - $90 \%$ rise time of 850 picoseconds. The temporal displacement between the optical pulse and photocurrent pulse is due to differences in cable lengths. The chopped optical rise time for this pulse is as fast as we could obtain with the pulse slicing Pockels cell and avalanche pulser combination.

Chopping the fall time of an optical pulse at the $532 \mathrm{~nm}$ wavelength presented somewhat of a problem since all the $6 \mathrm{H}-\mathrm{SiC}$ switches we have tested have a long tail of photocurrent that persists well after the optical pulse decays. The long lived component of the switch current represents 10 - 15 percent of the total current, but has a decay time of $5 \mu$ s and would dominate 
switch recovery time. Most of the carriers excited using $532 \mathrm{~nm}$ light recover rapidly, similar to carrier recombination at $1064 \mathrm{~nm}$. It was decided to use $1064 \mathrm{~nm}$ light in the chopped fall time tests to remove any complications due to the long lived component in switch current at $532 \mathrm{~nm}$. A chopped fall time optical pulse and resulting photocurrent is shown in Figure 6.21. The FID Technology model FDS pulser was used in this test. The output of the pulser was reduced to 4 $\mathrm{kV}$ so as not to damage the pulse slicing Pockels cell. The rise time of the FID pulser was so fast as to cause the $50 \mathrm{ohm}$ structure of the Pockels cell to resonate causing oscillations on the rising edge of the optical pulse. However, the chopped fall time was relatively well behaved with a fall time on the order of $300-500$ ps. The switch current in Figure 6.21 persists $\sim 15$ ns longer than the optical pulse. The conduction current in the switch is expected to subside much faster than

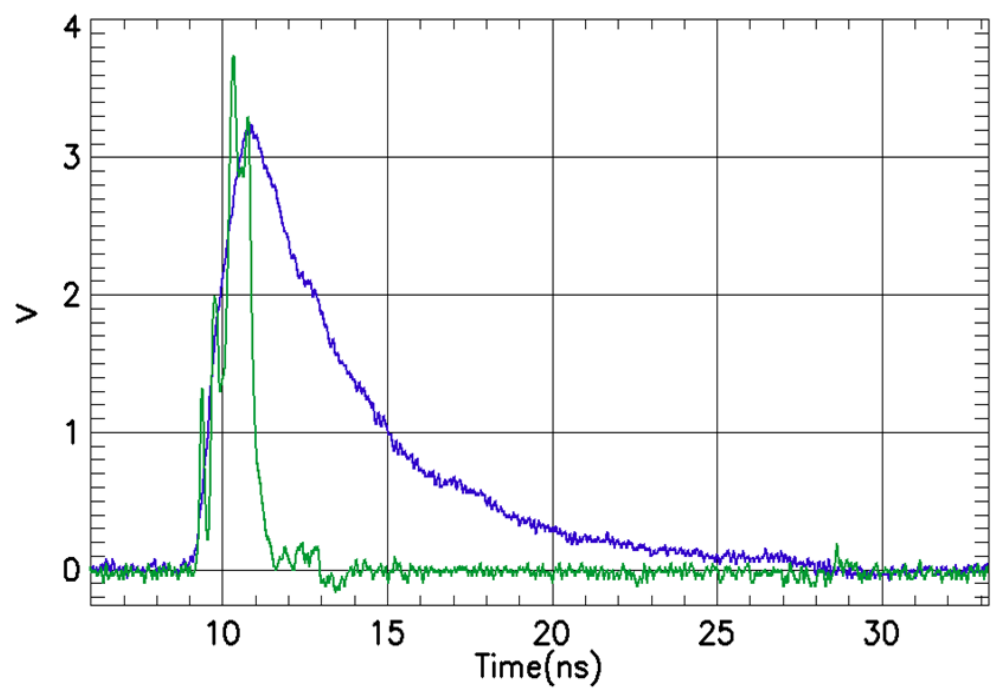

Figure 6.21. Optical intensity (green trace) and photoconductive switch current for a chopped fall time optical pulse appled to the green switch. The charge voltage and pre-chop optical energy were 400 Volts and $18 \mathrm{~mJ}$ at $1064 \mathrm{~nm}$.

this based on the fast recombination time of this material. However, the switch current during turn-off will be comprised of both conduction and displacement currents. The long decay of switch current appears to be displacement current through the green switch due to the recharging of the switch capacitance and stray capacitance in parallel with the green switch. The calculated 
values of switch capacitance and the stray capacitance are 11 and $14.5 \mathrm{pF}$, respectively. The capacitance calculations are made using formula for a parallel plate capacitor and using the switch and test board geometry and dielectric constants.

The temporal decay of switch current as a function of time plotted for four different load resistances is shown in Figure 6.22. The green, red, blue and black waveforms are the normalized switch current for load resistances of 48.5, 89, 137 and 215 ohms. The e-fold decay times of the switch current waveforms are 1.5, 2.5, 3.5 and 5.25 ns for the 48.5, 89, 137, and 215 ohm loads, respectively. The switch current decay times correspond to the R-C time constant of the test circuit formed by the load resistance and parallel combination of switch and stray capacitance. The sum of the switch and stray capacitances can be estimated by dividing the efold decay times by the load resistance. This calculation results in estimated capacitances of 31, 28.1, 25.5 and $24.4 \mathrm{pF}$ for the sum of the switch and stray capacitances for the 48.5, 89, 137, and $215 \mathrm{ohm}$ load resistances, respectively. This is in good agreement with the sum of the switch and test board stray capacitance, $25.5 \mathrm{pF}$, which was obtained from the calculations based on the switch and board geometry. It is clear from these measurements that the recovery time, or, the time it takes re-establish of full voltage across the switch is dominated by the switch capacitance and stray capacitance across the switch, not the material recombination time. The stray capacitance can be minimized, but the switch capacitance is a function of switch geometry and dielectric constant ( $\varepsilon=10$ for $6 \mathrm{H}-\mathrm{SiC}$ and $2 \mathrm{H}-\mathrm{GaN}$ ). The switch capacitance and load resistance will determine how fast the operating voltage is re-established across the switch until the RC time constant approaches the $6 \mathrm{H}-\mathrm{SiC}$ recombination time of the switch material. 


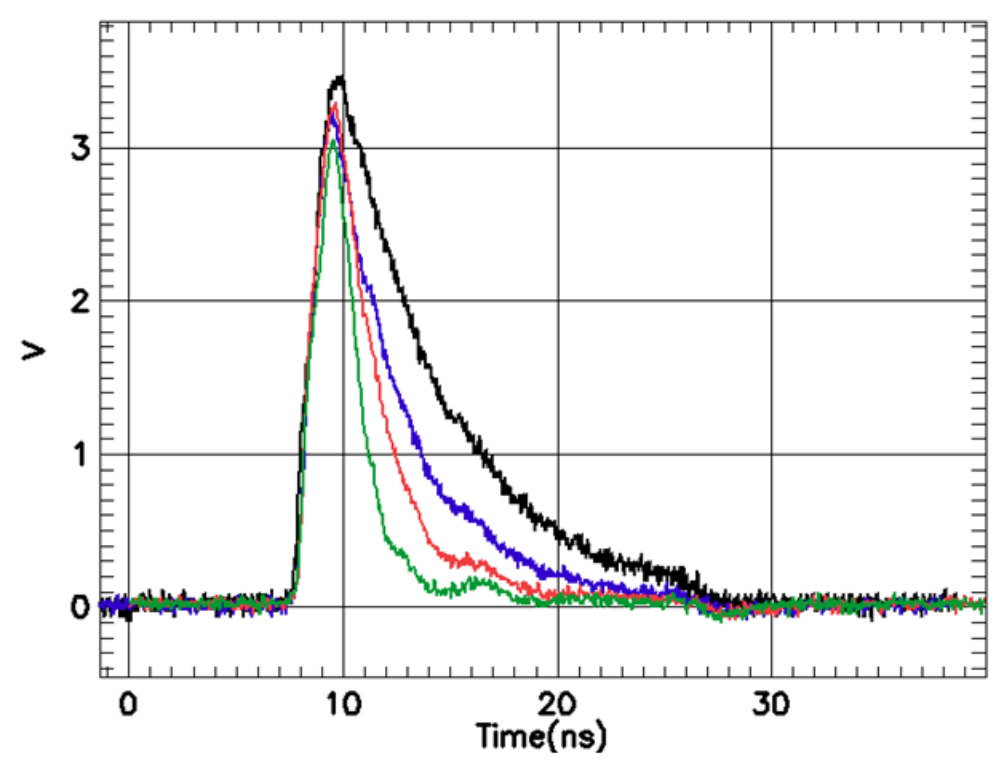

Figure 6.22. Green switch current for load resistances of 48.5 (green), 89 (red), 137 (blue) and 215 (black) ohms. The charge voltage is 400 volts and the applied optical energy is 18 $\mathrm{mJ}$ at $1064 \mathrm{~nm}$.

\section{6) Conclusions}

In the previous chapter, we obtained switch on resistances of $1-3$ ohms for $400 \mu \mathrm{m}$ thick, $1.2 \mathrm{~cm}$ by $1.2 \mathrm{~cm}$, compensated 6H-SiC and 2H-GaN switch substrates excited at $532 \mathrm{~nm}$. These results demonstrated the viability of semi-insulating, vanadium doped $6 \mathrm{H}-\mathrm{SiC}$ and iron doped 2H-GaN as extrinsic photoconductive switches. In this chapter, we explored other important parameters of the $6 \mathrm{H}-\mathrm{SiC}$ switch.

The critical field strength of $175 \mathrm{MV} / \mathrm{m}$ obtained for a $6 \mathrm{H}-\mathrm{SiC}$ switch is sufficient to construct $1 \mathrm{~mm}$ thick switches that will operate at $15 \mathrm{kV}$. Higher hold-off voltages can be obtained by exploring novel switch packaging geometries.

SIMS measurements of the vanadium, nitrogen, boron and aluminum dopant densities led to an understanding of how the dopant densities can be changed to decrease the switch 
minimum on resistance, while still maintaining a semi-insulating substrate of good crystal quality.

Optical absorption measurements ( $\alpha \sim 2.0$ at $532 \mathrm{~nm}$ ) set the practical overall dimensions of a $6 \mathrm{H}-\mathrm{SiC}$ switch substrate at $1 \mathrm{~cm}$ by $1 \mathrm{~cm}$. In addition, optical absorption measurements determined that two-photon absorption is the dominant absorption mechanism in GaN at $532 \mathrm{~nm}$ and important in $6 \mathrm{H}-\mathrm{SiC}$ at high optical intensities. We also determined that a line focused optical pulse sized to slightly overfill the switch facet across its thickness yielded the same minimum switch on resistance as a uniform optical profile of the same optical energy and temporal shape. Also, dividing the applied optical pulse between opposing facets enables more uniform switch current density and lowers the optical intensity at the facets.

Switch turn-on and turn-off were explored to determine minimum switch current rise and fall times. The current rise through the $6 \mathrm{H}-\mathrm{SiC}$ silver switch followed the optical rise time and achieved a 10 - 90\% current rise of 850 ps. The current fall time through the $6 \mathrm{H}-\mathrm{SiC}$ switches is dominated by a long-lived component of switch current $(\tau=3 \mu \mathrm{s})$ for excitation at $532 \mathrm{~nm}$ and by the load resistance and the sum of switch and stray capacitance at $1064 \mathrm{~nm}$. The time constants for switch current decay for excitation at $1064 \mathrm{~nm}$ are 1.5, 2.5, 3.5 and $5.25 \mathrm{~ns}$ for load resistances of 48.5, 89, 137 and 215 ohms, respectively. The switch current fall times can be reduced by decreasing switch electrode area, switch stray capacitance or, reducing the load resistance. The switch current fall time will decrease linearly with decreased electrode area, or, decreased load resistance. However, there are penalties that are incurred for either of these changes. Switch current density is inversely proportional to electrode area. Switch current density doubles if the electrode area is halved. The peak switch current is inversely proportional to load resistance. Halving the load resistance will double the peak current in the switch. These 
changes can be made in an effort to decrease current fall time in the switch, but their adverse effect on switch requirements must be taken into account.

The results of this chapter show that $1 \mathrm{~cm}$ by $1 \mathrm{~cm}, 1 \mathrm{~mm}$ thick, vanadium compensated, $6 \mathrm{H}$-SiC substrate with improved doping densities can be used to fabricate a high power, extrinsic photoconductive switch for operation reliably at $15 \mathrm{kV}$.

We will explore 6H-SiC extrinsic photoconductive switch operation in Chapter 7 and present models for switch performance for materials with low, and high, densities of nitrogen and vanadium. In particular, we will calculate the expected increase in switch performance for idealized nitrogen, vanadium and boron doping compared to the initial 6H-SiC material.

\section{6) References}

6.1) FlexPDE 5.0.7, PDE Solutions Inc., www.pdesolutions.com

6.2) J. D. Cobine, Gaseous Conductors - Theory and Engineering Applications, pp. 177 180, Dover, 1958

6.3) F. M. Bruce, "Calibration of uniform field spark gaps for high volume measurement at power frequencies", Journal of the Institute of Electrical Engineers, Vol. 94, pp. 138 149, 1947

6.4) T. J. Chang, "Improved uniform field electrode profiles for TEA lasers and high voltage applications”, Review of Scientific Instruments, Vol. 44, pp. 405 - 407, 1973

6.5) R. H. Bube, Photoelectronic Properties of Semiconductors, pp. 9 - 10, Cambridge University Press, 1992

6.6) M. Bickermann et. al., "Incorporation of boron and vanadium during PVT growth of $6 \mathrm{H}-$ SiC crystals”, Journal of Crystal Growth, Vol. 233, pp. 211 - 218, 2001

6.7) J. R. Jenny, et. al., "Deep level transient spectroscopic and Hall effect investigation of the position of the vanadium acceptor level in $4 \mathrm{H}$ and $6 \mathrm{H}-\mathrm{SiC}$ ", Applied Physics Letters, Vol. 68, Issue 14, page 1963, 1996

6.8) W. J. Choyke and E. D. Palik, "Silicon Carbide" in Handbook of Optical Constants of Solids, pp. 587 - 595, Academic Press, 1985.

6.9) G. Yu, H. Ishikawa, T. Egawa, T. Soga, J. Watanabe, T. Jimbo and M. Umeno, "Polarized reflectance spectroscopy and spectroscopic ellipsometry determination of the optical anisotropy of Gallium Nitride on Sapphire”, Japanese Journal of Applied Physics, Vol. 36, Pt. 2, pp. 1029 - 1031, 1997.

6.10) Leysop Model UPC069/2 Pulse Slicing Pockel Cell, www.leysop.com

6.11) FID Technology Model FDS Pockel Cell Pulser, www.fidtechnology.com 
6.12) A. I. Bishop and P. F. Barker, "Subnanosecond Pockels' cell switching using avalanche transistors", Review of Scientific Instruments, Vol. 77, Issue 4, 2006

6.13) G. Tamulaitis, et al., "Carrier lifetime in conductive and vanadium doped 6H-SiC substrates”, Applied Physics Letters, Vol. 84, No. 3, pp. 335 - 337, 2004 


\section{Chapter 7: Analysis of Initial Test Results of Vanadium Compensated, Semi- insulating, 6H-SiC Extrinsic Photoconductive Switches}

The initial photoconductive switching tests of vanadium compensated, semi-insulating, $6 \mathrm{H}-\mathrm{SiC}$ and iron compensated, semi-insulating, $2 \mathrm{H}-\mathrm{GaN}$ substrates established both materials as viable candidates for use as high voltage, high current, extrinsic photoconductive switches. Both materials are semi-insulating $\left(10^{12}-10^{13} \Omega-\mathrm{cm}\right)$, but rapidly transition to a resistivity of $30-$ $100 \Omega$-cm when 2 - $3 \mathrm{MW}$ of optical power at a wavelength of $532 \mathrm{~nm}$ is applied to a switch facet. The $6 \mathrm{H}-\mathrm{SiC}$ switch substrates respond well to $1064 \mathrm{~nm}$ light reaching resistivities of 400 $800 \Omega$-cm at optical powers of 1.25 to $2.4 \mathrm{MW}$. The $2 \mathrm{H}-\mathrm{GaN}$ switch attained a resistivity of 20.2 $\mathrm{k} \Omega-\mathrm{cm}$ at an applied optical power of $1.5 \mathrm{MW}$ at $1064 \mathrm{~nm}$. The $6 \mathrm{H}-\mathrm{SiC}$, vanadium compensated, semi-insulating material has a switch conductance with a linear, or supralinear, dependence on applied optical intensity at both the 1064 and 532 nm wavelengths. The iron compensated, semiinsulating GaN material has a switch conductance that is proportional to the square of the applied optical intensity at $532 \mathrm{~nm}$ and a poor response at $1064 \mathrm{~nm}$. Both materials have recombination times that appear to be much shorter than the applied optical pulse-width of 7 - 10 ns at 1064 nm. The current decay of the initial $6 \mathrm{H}-\mathrm{SiC}$ photoconductive switch material has a compound recombination time consisting of a fast and slow component for excitation at $532 \mathrm{~nm}$. The fast component of the $6 \mathrm{H}-\mathrm{SiC}$ recombination time appears to be much shorter than the $5.0-10.0 \mathrm{~ns}$ pulse-widths of the applied optical pulses. The slow component comprises 10 - 15 percent of the

peak switch current and decays with a time constant of $\sim 5 \mu \mathrm{s}$. The GaN switch material has a recombination time that appears to be much shorter that the applied optical pulses at the $532 \mathrm{~nm}$ wavelength. 
The poor response of the iron compensated, GaN photoconductive switch to $1064 \mathrm{~nm}$ wavelength light is puzzling since the iron acceptor level lies at $0.58-0.60 \mathrm{eV}$ below the conduction band. The $1.17 \mathrm{eV}$ photons of the $1064 \mathrm{~nm}$ wavelength optical excitation are twice the thermal ionization energy of the iron acceptor level, close to the peak optical transition cross section predicted by the Lucovsky and Ridley models [7.1, 7.2]. These photons are also energetic enough to generate a reasonable photoconductive response even if this transition has a significant Frank-Condon shift. So, the lack of a significant photoconductive response to the $1064 \mathrm{~nm}$ optical excitation leads one to believe that there are few electrons residing in the iron acceptor level of our compensated GaN material. This conclusion is supported by activation energy measurements [7.3] that were conducted on iron compensated, semi-insulating GaN substrates grown under the same nominal conditions as our GaN switch substrates. The activation energy indicates the energy level of the dominant impurity in the GaN material. In our case, the activation energy corresponds to the energy level where the electrons donated from oxygen impurities are trapped. These measurements [7.3] revealed that some iron compensated, semiinsulating GaN substrates have activation energies of $0.95 \mathrm{eV}$, not the expected $0.58-0.6 \mathrm{eV}$ of the iron acceptor level. The difference in activation energies between different iron compensated $2 \mathrm{H}$-Gan substrates was attributed to a reduced iron density $\left(7.5 \times 10^{17} \mathrm{~cm}^{-3}\right.$ as opposed to $2.0 \times 10^{18}$ $\mathrm{cm}^{-3}$ ) in the $\mathrm{GaN}$ substrates with the $0.95 \mathrm{eV}$ activation energy. A weak photoconductive response would be expected for a GaN substrate with an activation energy of $0.95 \mathrm{eV}$, since the $1.17 \mathrm{eV}$ photons of the $1064 \mathrm{~nm}$ optical excitation are just above the optical transition energy threshold. In addition, the GaN switch conductance is proportional to the square of the applied optical intensity at $532 \mathrm{~nm}$. This is a clear indication that most of the carriers are generated primarily by two photon absorption and, to a lesser extent, two step optical transitions at $532 \mathrm{~nm}$. 
The photoconductive switching results at the 1064 and $532 \mathrm{~nm}$ wavelengths for the GaN switch demonstrate that there are not many electrons being excited from deep levels to the conduction band. Most of the switch carriers at the $532 \mathrm{~nm}$ wavelength are the result of the photogeneration of electron - hole pairs by two photon absorption. As a result, there is no clear path forward for the engineering development of an improved iron compensated, $2 \mathrm{H}-\mathrm{GaN}$, extrinsic photoconductive switch. The establishment of a controllable set of shallow donor and deep acceptor impurities in $2 \mathrm{H}-\mathrm{GaN}$ need to be established to enable extrinsic photoconductive switch development. However, development of $2 \mathrm{H}-\mathrm{GaN}$ photoconductive switch based on two-photon absorption appears to be straight-forward.

The vanadium compensated, $6 \mathrm{H}-\mathrm{SiC}$ material has a clearer development path for use as an extrinsic photoconductive switch compared to the iron compensated, $2 \mathrm{H}-\mathrm{GaN}$, due to the greater control of dopants, which contribute to switch performance, during crystal growth. As a result, the analysis presented in this chapter will focus solely on the 6H-SiC material.

While the bulk of this chapter will be devoted to the analysis of the vanadium compensated, $6 \mathrm{H}-\mathrm{SiC}$ photoconductive switches, there are some aspects of the interaction between the switch and test circuit that were mentioned during the presentation of switching results in Chapter 5 that need additional explanation. This topic will be addressed in section 7.1 before proceeding on to switch analysis.

\section{1) Photoconductive Switch and Test Circuit Interaction}

The initial photoconductive switching results of Chapter 5 show that the $6 \mathrm{H}-\mathrm{SiC}$ carrier recombination time is small compared to the pulse-widths of the applied optical pulses, since the switch current temporal pulses resemble the applied optical pulses. There is a long-lived 
component of switch conduction current at $532 \mathrm{~nm}$ that accounts for $10-15$ percent of the current, but most of the carriers decay rapidly. The resemblance between the temporal profiles of the applied optical pulse and switch current is particularly true at low optical power. This would indicate that the recombination time for $1064 \mathrm{~nm}$ excitation and the fast component of the recombination time for $532 \mathrm{~nm}$ excitation is $\leq 1 \mathrm{~ns}$, since the applied pulse widths ranged from 8 $-12 \mathrm{~ns}$ at $1064 \mathrm{~nm}$ and $5-8 \mathrm{~ns}$ at 1064 . The short recombination time enables us to evaluate the switch electron density during optical excitation using the convolution integral shown in 7.1. $\mathrm{G}(\mathrm{s})$ is the Laplace transform of the carrier optical generation rate shown in equation 7.2. Where $P(t)$ is the optical power applied to the switch facet, $r$ is the reflectance of the switch facet, $\eta$ is the quantum efficiency, $\alpha$ is the absorption coefficient of the $6 \mathrm{H}-\mathrm{SiC}$ switch material, $\mathrm{d}$ is the switch thickness and $\mathrm{W}$ and $\mathrm{L}$ are the width and length of the switch substrate (Figure 7.1).

$$
\begin{gathered}
n(t)=\int_{-\infty}^{t} G(s) e^{\frac{(s-t)}{\tau_{r}}} d s \\
G(t)=\frac{P(t) \eta}{W d L \hbar \omega}(1-r)\left(1-e^{-\alpha W}\right)
\end{gathered}
$$

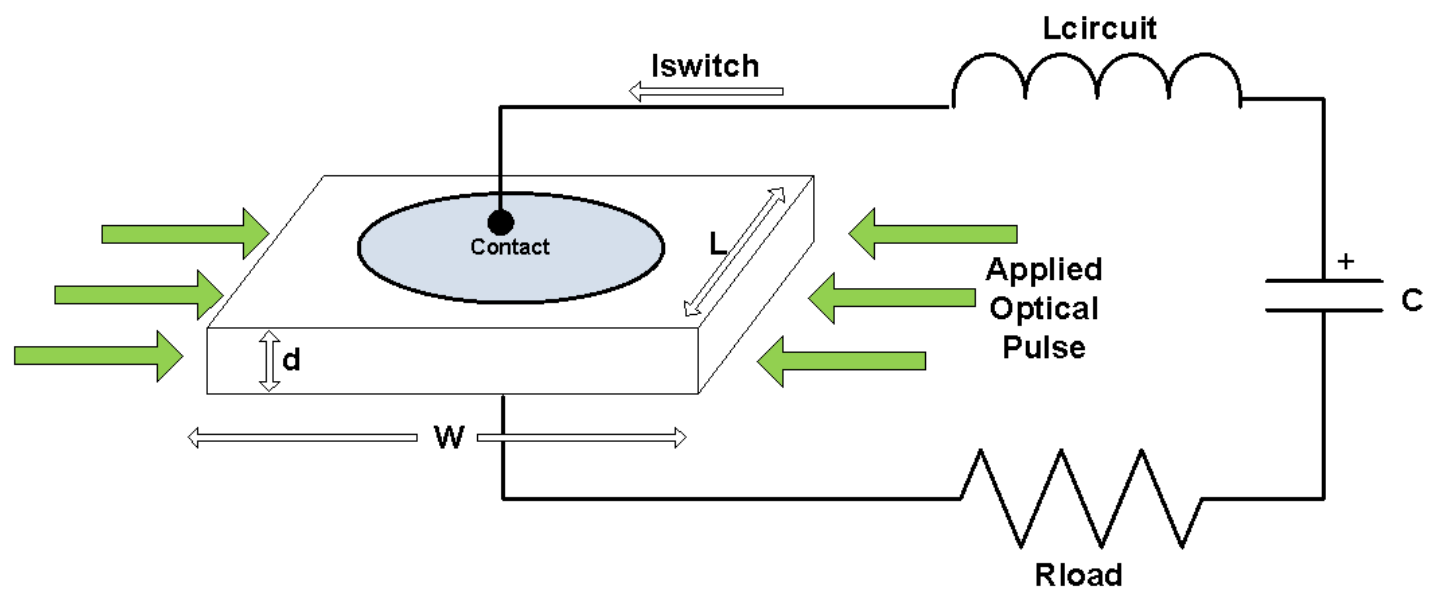

Figure 7.1. Photoconductive switch substrate and test circuit 
The solution of equation 7.1 is given in Chapter 2 [7.4] for an applied optical step pulse with an amplitude and duration of $\mathrm{P}_{0}$ and $\mathrm{T}_{0}$, respectively (Figure 7.2).

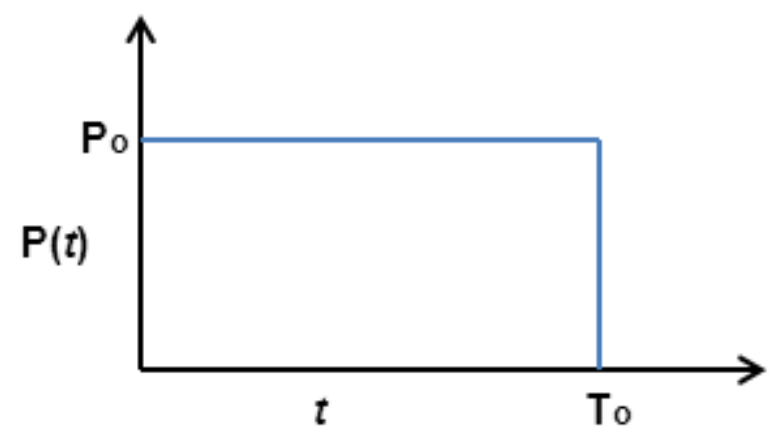

Figure 7.2. Temporal profile and amplitude for optical step pulse.

The solution for the switch carrier density and resistance for the optical step pulse are given in equations 7.3 and 7.4 for $t$ greater than zero, but less than $\mathrm{T}_{0}$.

$$
\begin{gathered}
n(t)=\frac{P_{0}(1-r)\left(1-e^{-\alpha W}\right) \eta t_{r}}{\hbar \omega W L d} \quad \text { for } 0 \leq t \leq T_{0} \\
R(t)=\frac{d^{2} \hbar \omega}{P_{0} t_{r} \mu_{n}(1-r)\left(1-e^{-\alpha W}\right) q \eta}
\end{gathered}
$$

Solutions for carrier density and switch resistance are also provided below for an optical ramp pulse and a 1-cos( $\omega t)$ pulse (Figure 7.3 and 7.4, respectively). The solutions for $n(t)$ and $\mathrm{R}(t)$ for the optical ramp and 1-cos $(\omega \mathrm{t})$ for $\mathrm{T}_{\max }>>t_{\mathrm{r}}$ are given in equations 7.5 , 7.6 (optical ramp) and 7.7, $7.8(1-\cos (\omega t))$. 


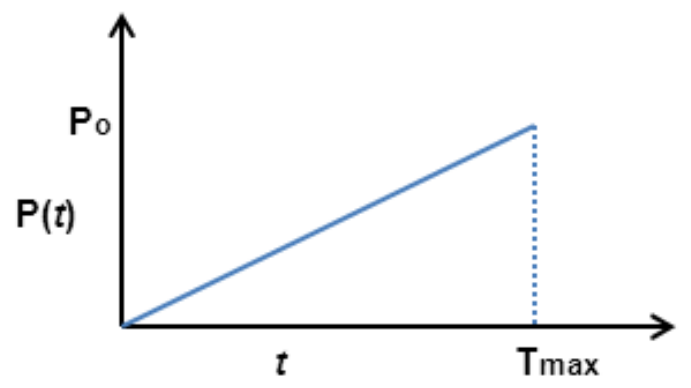

Figure 7.3. Temporal profile of optical ramp pulse.

Solutions for the switch electron density and on resistance for the applied optical ramp shown in Figure 7.3 are:

$$
n(t)=\eta P_{0}(1-r)\left(1-e^{-\alpha W}\right) \frac{\left(t_{r}^{2}\left(e^{\frac{-t}{t_{r}}}+\frac{t}{t_{r}}-1\right)\right)}{T_{\max } \hbar \omega L W d} \quad \text { for } 0 \leq t \leq T_{\max }
$$

The $(1-\cos (\omega t))$ waveform is shown in Figure 7.4.

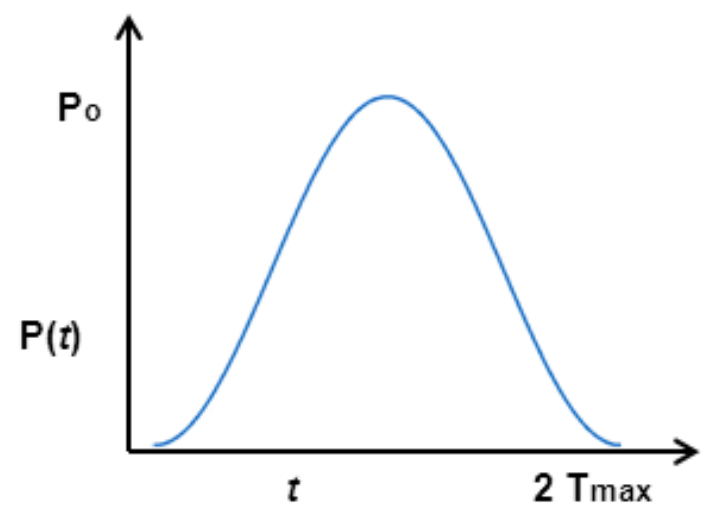

Figure 7.4. Temporal profile of $1-\cos (\omega t)$ optical pulse.

Solutions for the $1-\cos (\omega t)$ applied optical pulse shown in Figure 7.4 are: 


$$
\begin{aligned}
& n(t)=\frac{P_{0} \eta}{2 \hbar \omega L W d}(1-r)\left(1-e^{-\alpha W}\right)\left(t_{r}\right. \\
& \left.-\frac{t_{r}^{2} \pi^{2}}{T_{\max }^{2}}\left[\frac{T_{\max } \sin \left(\frac{\pi t}{T_{\max }}\right)}{\pi}+\frac{T_{\max }^{2} \cos \left(\frac{\pi t}{T_{\max }}\right)}{t_{r} \pi^{2}}+t_{r} e^{\frac{-t}{t_{r}}}\right]\right) \text { for } 0 \leq t \leq 2 T_{\max }
\end{aligned}
$$

$R(t)$

$$
=\frac{2 \hbar \omega d^{2}}{(1-r)\left(1-e^{-\alpha W}\right) P_{0} \eta \mu_{n} q} \frac{1}{\left(t_{r}-\frac{t_{r}^{2} \pi^{2}}{T_{\max }^{2}}\left[\frac{T_{\max } \sin \left(\frac{\pi t}{T_{\max }}\right)}{\pi}+\frac{T_{\max }^{2} \cos \left(\frac{\pi t}{T_{\max }}\right)}{t_{r} \pi^{2}}+t_{r} e^{\frac{-t}{t_{r}}}\right]\right)}
$$

In all cases, the switch resistance is proportional to the square of the switch thickness and inversely proportional to the quantum efficiency, recombination time and the number of photons applied to the switch facet.

The carrier density has the same temporal profile of the applied optical pulse for $\mathrm{T}_{\max }>>$ $t_{\mathrm{r}}$. In turn, the switch resistance has the inverse temporal profile of the applied optical pulse. In general, we would like the switch dark resistance to be much greater than the load resistance and decay rapidly to a resistance much lower than the load resistance when light is applied. The optical pulse wavelength, amplitude and pulse shape determines the switch transition from high to low resistance. We can explore the application of voltage across the load resistor as the photoconductive switch transitions from high to low resistance using the idealized circuit of Figure 7.5. The voltage division between the time-varying switch resistance and the fixed load resistance in the switch test circuit lead to some interesting results. The load voltage for the idealized test circuit is given by equation 7.9. If we assume the applied optical pulse has a (1$\cos (\omega t) / 2$ ) temporal profile, $\mathrm{R}_{\mathrm{sw}}(t)$ will be given by equation 7.8 and the load voltage waveforms 


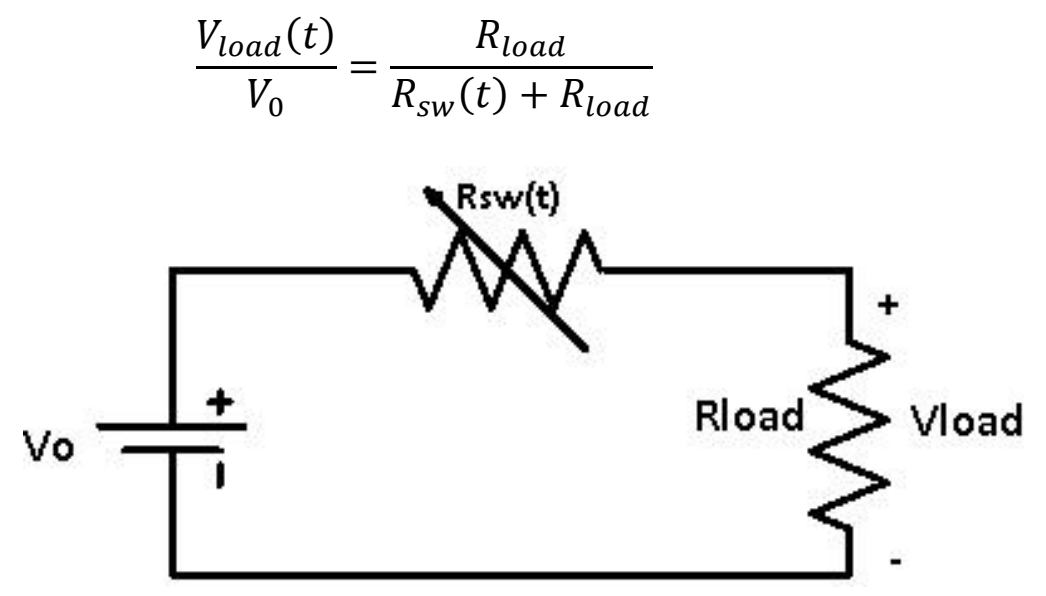

Figure 7.5. Idealized switch test circuit is a time varying switch resistance $\left(\mathbf{R}_{\mathrm{sw}}(t)\right)$ in series with a fixed load resistor $R_{\text {load. }}$ The load voltage $\left(V_{\text {load }}\right)$ is determined by the resistive divider formed by $R_{s w}(t)$ and $R_{\text {load. }}$

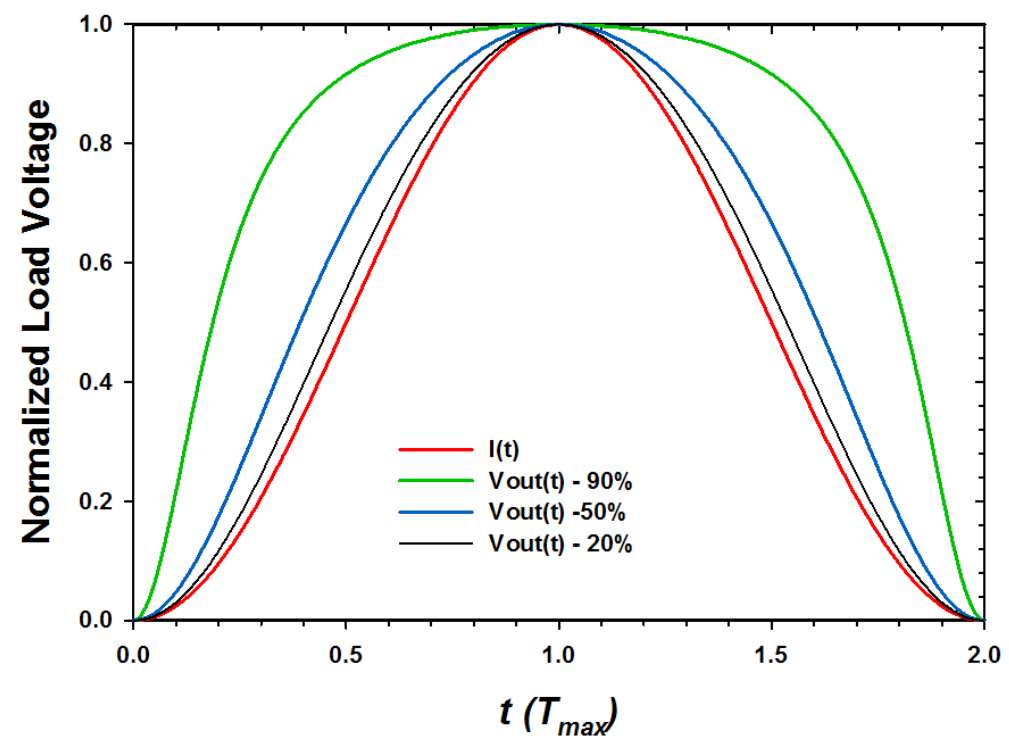

Figure 7.6. Normalized load voltage is plotted as a function of time. Red waveform is the applied optical pulse. The black, blue and green waveforms correspond to the peak optical excitation required to obtain 20,50 and 90 percent of the voltage $V_{0}$ to appear across the $\mathbf{R}_{\text {load }}$.

are shown in Figure 7.6. The red waveform in Figure 7.6 is the $(1-\cos (\omega t) / 2)$ applied optical pulse. The black waveform designated $\mathrm{V}_{\text {out }}(t)-20 \%$ in Figure 7.6 is the normalized $\mathrm{V}_{\text {load }}(t)$ waveform for a peak optical intensity that will reduce the switch resistance $\left(R_{s w}(t)\right)$ to a minimum value of $4 R_{\text {load. }}$ As a result, only 20 percent of the voltage $V_{0}$ appears across $R_{\text {load }}$. The 
$\mathrm{V}_{\text {load }}(t)$ waveform is slightly broader, but closely follows the applied optical pulse, I $(t)$. This corresponds to the case of low optical excitation of a $6 \mathrm{H}-\mathrm{SiC}$ switch. Figure 7.7 is a plot of the load voltage and applied optical pulse for the silver switch for a charge voltage of 300 Volts and a peak optical power of $0.1533 \mathrm{MW}$. This is a fairly low optical excitation and the load voltage follows the optical pulse quite closely.

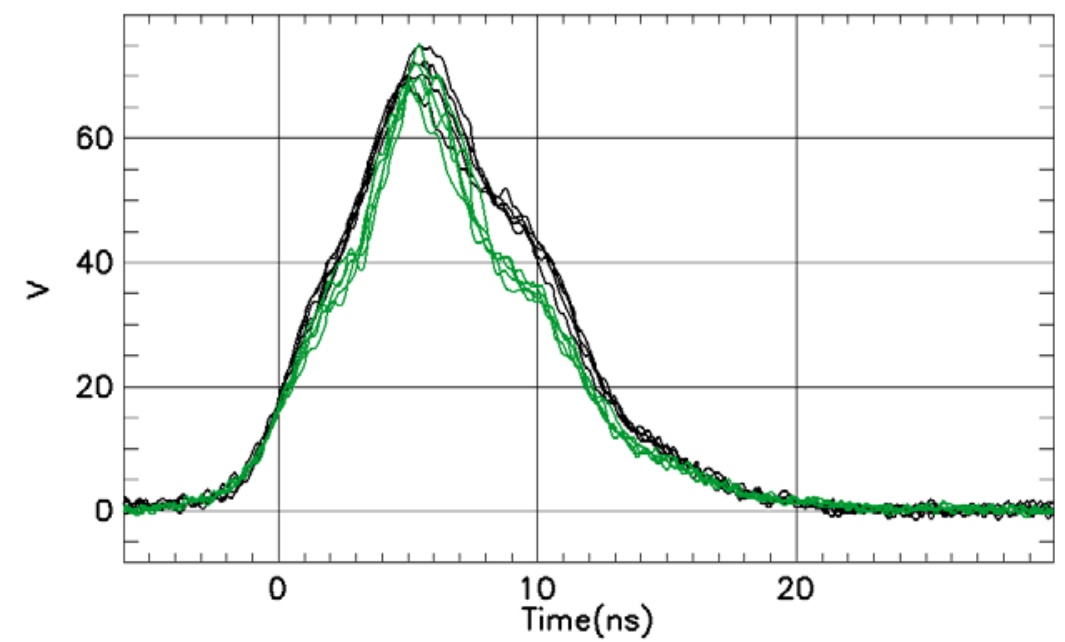

Figure 7.7. The load voltage (black traces) for silver switch is plotted for a charge voltage of 300 Volts, peak optical power (green traces) of $0.1533 \mathrm{MW}$ at $1064 \mathrm{~nm}$, and a load resistance of $50 \mathrm{Ohms}$ (overlay of 5 pulses).

Figure 7.6 also shows the normalized $\mathrm{V}_{\text {load }}(t)$ waveforms for the cases where the minimum value of $R_{s w}(t)$ equals $R_{\text {load }}$ (blue trace) and $R_{\text {load }} / 9$. These waveforms are designated $\mathrm{V}_{\text {out }}(t)-50 \%$ (blue trace) and $\mathrm{V}_{\text {out }}(t)-90 \%$ (green trace). The $\mathrm{V}_{\text {out }}(t)-90 \%$ corresponds to a high intensity optical pulse that reduces the switch resistance to a value of $1 / 9$ of $\mathrm{R}_{\text {load, }}$, so that $90 \%$ of the voltage $V_{0}$ appears across the load resistor. The shape of $V_{\text {out }}(t)-90 \%$ is not the same as the applied optical pulse $\mathrm{I}(t)$. The load voltage pulse shape has ballooned to a much wider shape compared to the optical pulse. The $\mathrm{V}_{\text {out }}(t)$ - $90 \%$ load voltage pulse shape gives the impression that it precedes the applied optical pulse in time when it does not. Figure 7.8 is a plot of the load current and applied optical pulse for the silver switch for a charge voltage of 500 
Volts and a peak applied optical power of 2.30 MW. The load voltage may be obtained by multiplying the load current times the load resistance, 51 Ohms. The apparent pulse distortion is the result of the nonlinearity of the voltage division between the time varying switch resistance and the fixed load resistance.

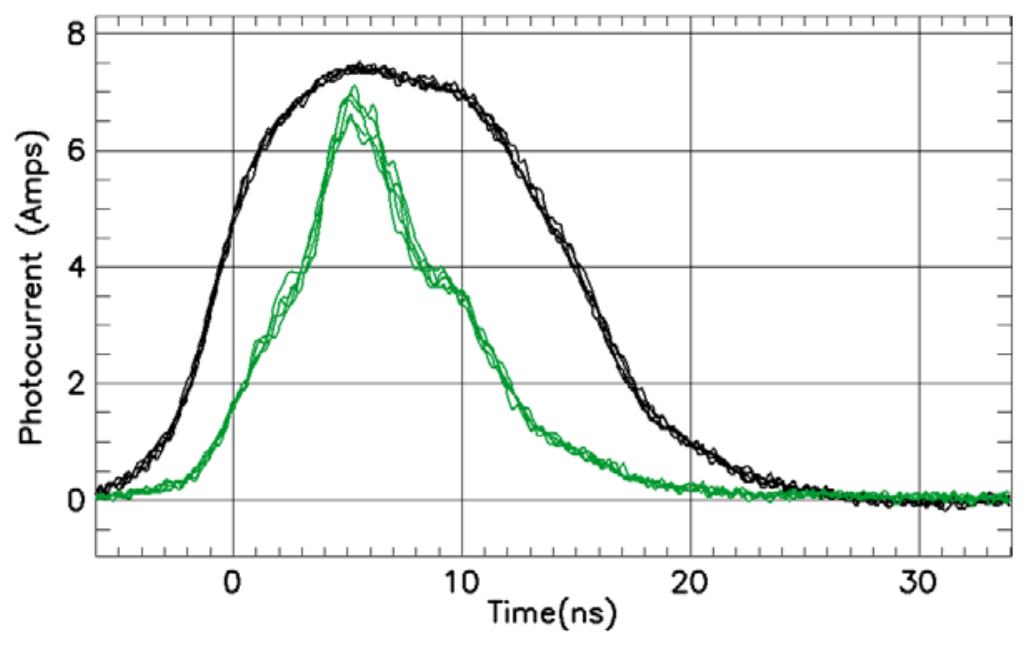

Figure 7.8. The load current (black traces) for the silver switch is plotted for a charge voltage of 500 Volts, peak optical power (green traces) of $2.30 \mathrm{MW}$ at $1064 \mathrm{~nm}$, and a load resistance of 51 Ohms (overlay of 5 pulses).

Another effect of the nonlinear voltage division of our switch test circuit is the apparent saturation of switch current with applied optical intensity. The peak switch current is calculated using the peak load voltage and dividing by the value of the load resistor. The peak switch current for a given charge voltage increases rapidly at low optical intensities, decreases less rapidly as the optical intensity increases and increases very slowly for high optical intensity. This gives the false impression that the switch resistance is no longer decreasing at high optical intensity. We can explore this effect using the $(1-\cos (\omega t)) / 2$ optical waveform and our simplified test circuit (Figure 7.5). Figure 7.9 has two plots of the normalized load voltage (Voltage Ratio) versus the normalized peak applied optical intensity. The peak applied optical intensity is in units of the intensity required to reduce the switch resistance to the value of $\mathrm{R}_{\text {load }}$. One plot of Figure 
7.9 is for a photoconductive switch conductance $\left(1 / \mathrm{R}_{\mathrm{sw}}(t)\right)$ that increases linearly with optical intensity (red trace) and one plot for a photoconductive switch conductance that increases as the square of optical intensity (blue trace). These two cases correspond to the $6 \mathrm{H}-\mathrm{SiC}$ and $2 \mathrm{H}-\mathrm{GaN}$ photoconductive switches, since the $6 \mathrm{H}-\mathrm{SiC}$ switch conductance increases linearly with optical intensity, while the $2 \mathrm{H}-\mathrm{GaN}$ switch conductance increases with the square of optical intensity. We expect the red trace of Figure 7.9 to give a fair approximation to the peak photocurrent plotted as a function of peak optical intensity for the $6 \mathrm{H}-\mathrm{SiC}$ switches. The red trace of

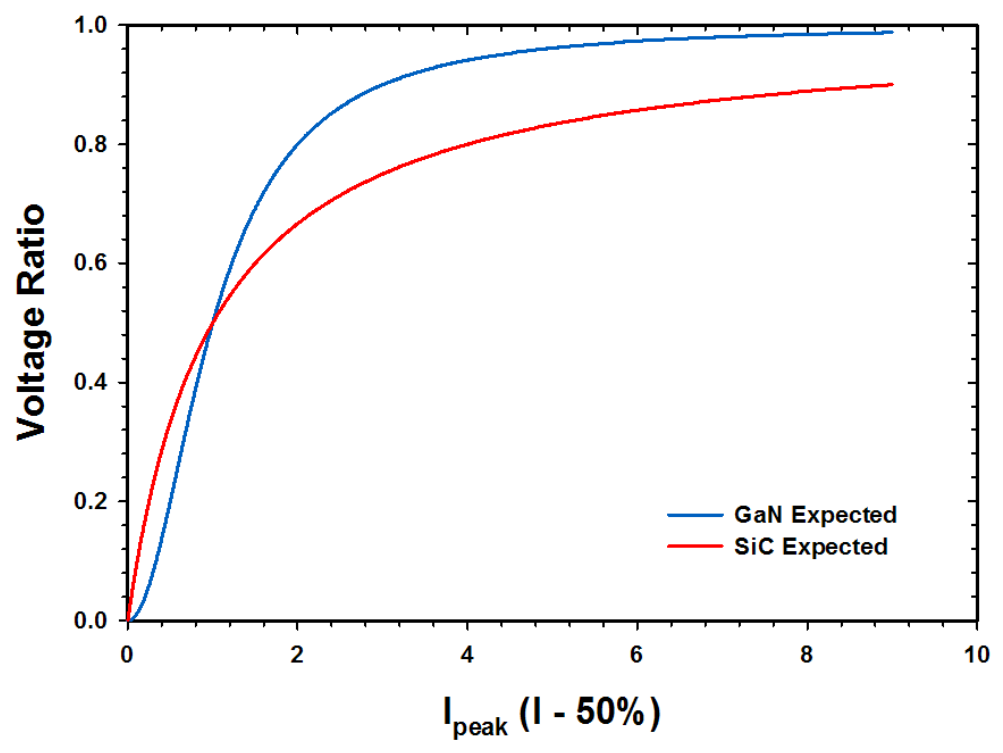

Figure 7.9. Normalized peak load voltage of simplified circuit plotted as a function of peak applied optical intensity. Optical intensity is in units of intensity required to reduce the minimum photoconductive switch resistance to the value of $R_{\text {load. }}$. Red (blue) plot is for a switch conductance that increases linearly (as the square of) optical intensity. We should expect the red and blue traces to mimic $6 \mathrm{H}-\mathrm{SiC}$ and $2 \mathrm{H}-\mathrm{GaN}$ switch results, respectively.

Figure 7.9 is compared to measurements of the $6 \mathrm{H}-\mathrm{SiC}$ silver switch peak normalized load voltage plotted as a function of normalized intensity in Figure 7.10 for excitation at $1064 \mathrm{~nm}$. There is good agreement between the calculated and measured normalized load voltage. It is 


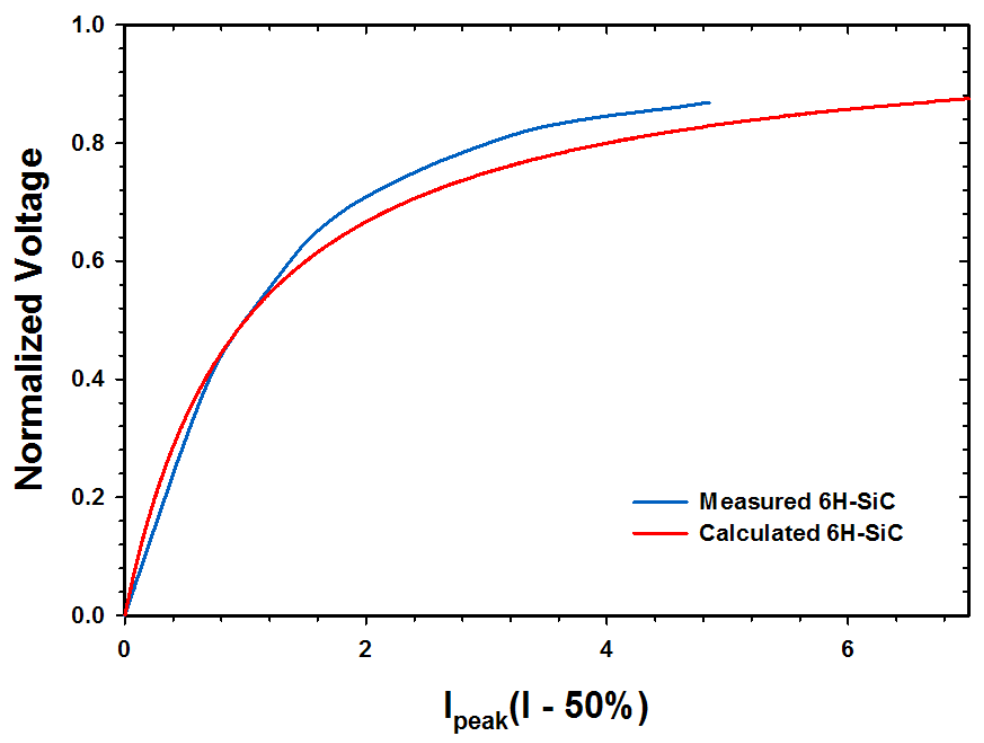

Figure 7.10. Measured normalized output voltage for the silver switch (blue trace) is compared to the calculated normalized voltage (red trace) for a switch with an on resistance that decreases linearly with increasing optical intensity.

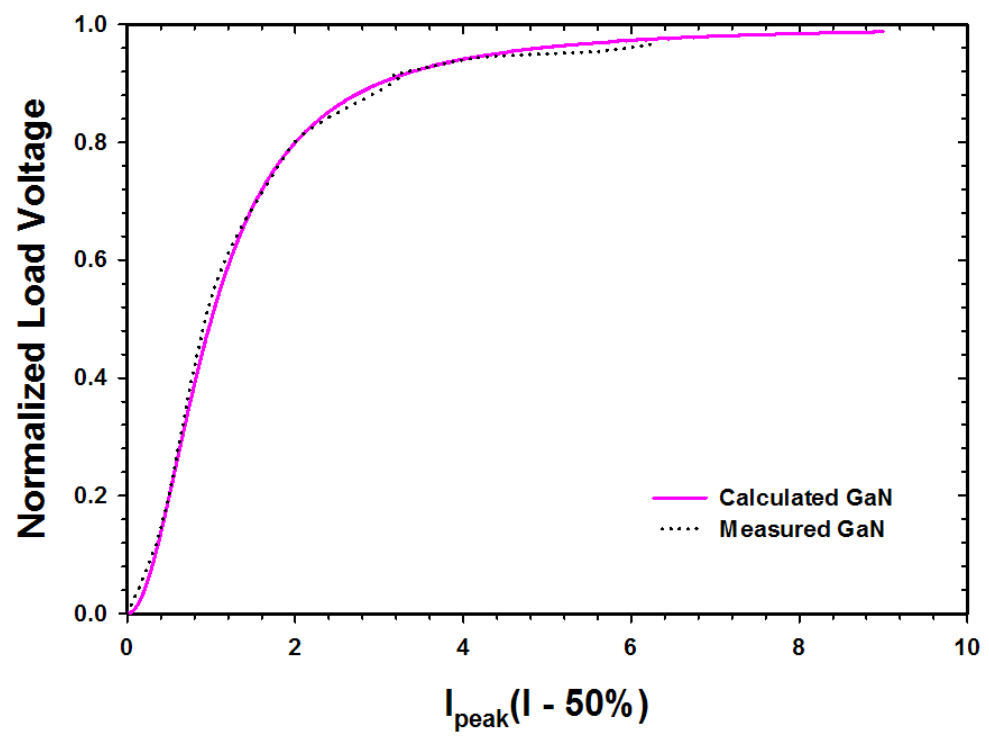

Figure 7.11. Measured normalized output voltage for the $2 \mathrm{H}-\mathrm{GaN}$ switch (black dotted trace) is compared to the calculated normalized voltage (red trace) for a switch with an on resistance that decreases as the optical intensity squared.

clear that the appearance of saturation of the plotted silver switch measured normalized load voltage data is due to the nonlinearity of the test circuit, rather than saturation of the switch on 
resistance. Also, it is clear that the $6 \mathrm{H}-\mathrm{SiC}$ switch on resistance is linearly dependent on applied optical intensity.

The blue trace of Figure 7.9 is compared to the measured 2H-GaN switch normalized peak load voltage plotted as a function of normalized peak optical intensity in Figure 7.11 for excitation at $532 \mathrm{~nm}$. There is excellent agreement between the calculated and measured normalized load voltage for the $2 \mathrm{H}-\mathrm{GaN}$ switch. It is clear that the $2 \mathrm{H}-\mathrm{GaN}$ switch on resistance varies as the inverse of optical intensity squared. It is interesting to note that there is a portion on the rising edge of the $2 \mathrm{H}-\mathrm{GaN}$ normalized load voltage plots (Figure 7.11) where the normalized load voltage increases linearly with normalized optical intensity at $532 \mathrm{~nm}$. This linear portion of the normalized load voltage plot occurs despite the fact that we have a switch with a nonlinear dependence of on resistance with optical intensity installed in a nonlinear circuit.

\section{2) Switch Analysis}

Chapter 6 outlined an intuitive approach to improving the switching performance of the $6 \mathrm{H}-\mathrm{SiC}$ material based on manipulating the densities of the vanadium, nitrogen and boron dopants. The intuitive approach called for removing, or reducing, the boron density to a negligible level, while increasing the nitrogen and vanadium levels to $1.25 \mathrm{e} 17$ and $2.5 \mathrm{e} 17 \mathrm{~cm}^{-3}$, respectively. The remainder of this chapter will present analytical models for the photoconductive switching process in $6 \mathrm{H}-\mathrm{SiC}$ at the $1064 \mathrm{~nm}$ wavelength. The analytical model will be used to evaluate the intuitive doping changes proposed in Chapter 6. The common starting point for the analytical models of switch operation at $1064 \mathrm{~nm}$ is the thermal equilibrium condition of the $6 \mathrm{H}-\mathrm{SiC}$ switch substrate material just prior the application of light to a switch facet. 
Thermal equilibrium at room temperature has been the initial condition for each photoconductive switch prior to the application of light. As a result, the thermal equilibrium condition defines the initial conditions for the densities of free electrons in the conduction band, electrons trapped in the vanadium acceptor level, unoccupied vanadium acceptor levels and the position of the Fermi level. The simple model we use to calculate the Fermi level and resistivity of the $6 \mathrm{H}-\mathrm{SiC}$ substrates includes the densities of the nitrogen donors, shallow boron acceptors and deep vanadium acceptors. These represent the main intentional and unintentional impurities in $6 \mathrm{H}-\mathrm{SiC}$. Calculations predict that the nitrogen donors will be completely ionized at room temperature. The electrons donated from the nitrogen levels will be captured at the boron and vanadium acceptor levels. The boron acceptor levels will be filled and the vanadium acceptor

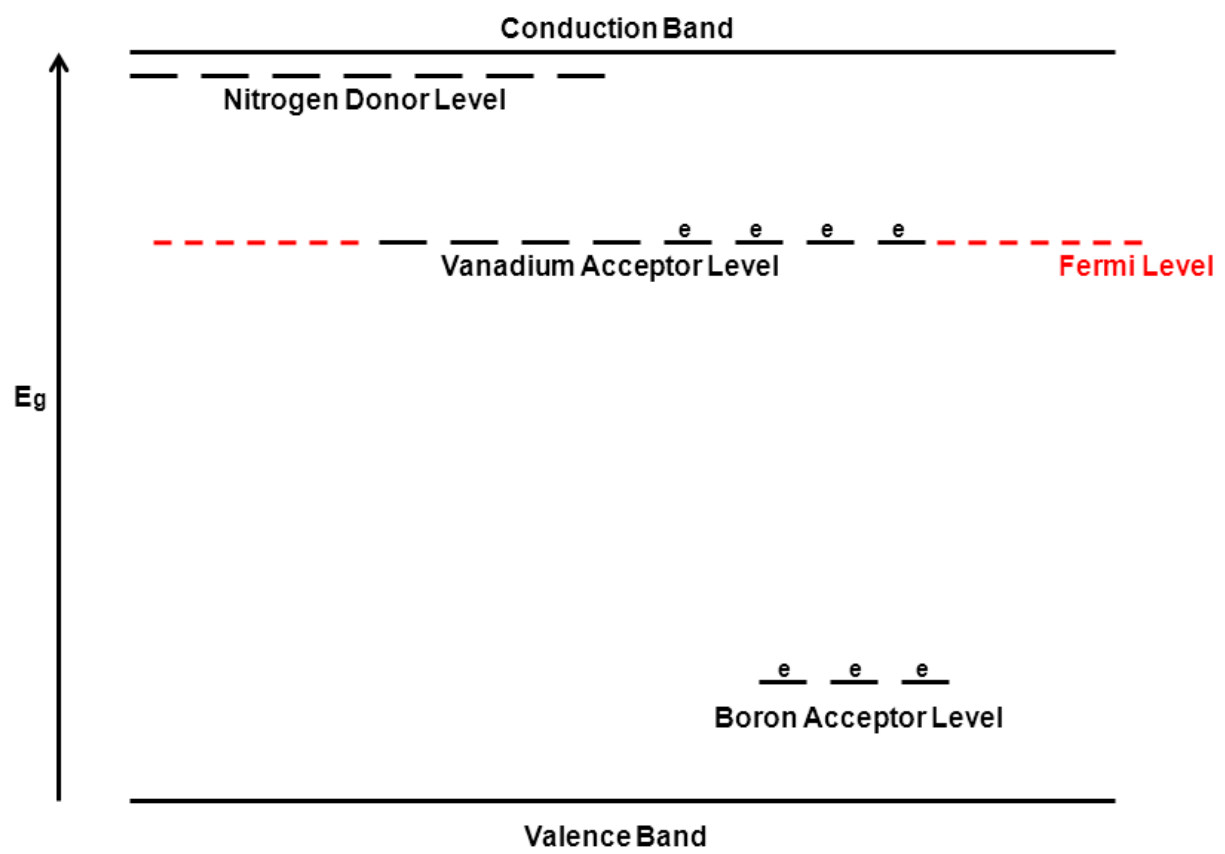

Figure 7.12. 6H-SiC impurity energy levels and position of Fermi level prior to optical excitation $\left(E_{g}=3.0 \mathrm{eV}\right)$.

levels will be partially filled if $\mathrm{N}_{\mathrm{V}}>\mathrm{N}_{\mathrm{N}}>\mathrm{N}_{\mathrm{B}}$. This situation is depicted in Figure 7.12. This is an idealized picture of the initial conditions that exist in the $6 \mathrm{H}-\mathrm{SiC}$ substrate prior to optical 
excitation, but it includes all the impurity levels under our control. We are now in a position to compare our initial switch test results with our idealized model of the $6 \mathrm{H}$-SiC substrates.

\section{3) Vanadium and Nitrogen Co-doped 6H-SiC in Thermal Equilibrium at Room Temperature}

The 6H-SiC photoconductive switch substrate has an initial condition of thermal equilibrium at room temperature prior to the application of optical excitation. The position of the Fermi level in the bandgap of our switch material will determine the density of free electrons (holes) in the conduction (valence) band. The position of the Fermi level is determined by the presence of dopants, intentional and unintentional, in our $6 \mathrm{H}-\mathrm{SiC}$ switch material. We know that the vanadium density exceeds the nitrogen density, which in turn, exceeds the boron density in our $6 \mathrm{H}-\mathrm{SiC}$ substrates from SIMS measurements. This condition is stated mathematically in equation 7.10. The net donor density for our substrate is given in equation 7.11.

$$
\begin{gathered}
N_{V}>N_{N}>N_{B} \\
N_{\text {Dnet }}=N_{N}-N_{B}
\end{gathered}
$$

In Chapter 3 we saw how the relative values of vanadium, nitrogen and boron impurity densities stated in equation 7.10 leads to the Fermi level being pinned close to the vanadium acceptor energy level. We can calculate the position of the Fermi level for the initial vanadium and nitrogen co-doped 6H-SiC switch substrates using the SIMS measurement results presented in Chapter 5 and equations 3.23 - 3.27. The following substitutions have to be made in equations

3.23 - 3.27: $N_{V}=N_{d a}, N_{N}=N_{s d}, N_{B}=N_{s a}, E_{V}=E_{d a}, E_{N}=E_{s d}, E_{B}=E_{s a}, D=\frac{N_{V}}{N_{N}}$, and $R=\frac{N_{B}}{N_{N}}$. The effective values for the vanadium, nitrogen and boron energy levels are 2.2, 2.86 and $0.3 \mathrm{eV}$ with respect to the valence band. 
The vanadium, nitrogen, boron and aluminum densities were measured at the points shown in Figure 7.13 for a bare $6 \mathrm{H}$-SiC substrate cut from the same crystal as our initial switch substrates. The SIMS measurements results are shown in Table 7.1. We obtain values of $1.85 \times 10^{17} \mathrm{~cm}^{-3}, 6.37 \times 10^{16} \mathrm{~cm}^{-3}$, and $4.27 \times 10^{16} \mathrm{~cm}^{-3}$ for the vanadium, nitrogen and boron density, respectively, if we take the average value of the impurity densities measured along the substrate diagonal (points 1, C, and 3). The Fermi level of the $6 \mathrm{H}-\mathrm{SiC}$ substrate is plotted as a function of $\mathrm{D}$ in Figure 7.14. The value of the Fermi level is $2.165 \mathrm{eV}$ in our $6 \mathrm{H}-\mathrm{SiC}$ substrate for the specific values of $\mathrm{D}=2.9$ and $\mathrm{R}=0.67$ obtained from the measured impurity densities. The Fermi level is predicted to reside just below the vanadium acceptor level $(0.8 \mathrm{eV})$ at 0.835 $\mathrm{eV}$ below the conduction band. The calculated value of the $6 \mathrm{H}-\mathrm{SiC}$ substrate resistivity is $7.48 \times 10^{10} \Omega$-cm based on the impurity density measurements. The calculated value of $7.48 \times 10^{10}$ $\Omega$-cm for our $6 \mathrm{H}$-SiC substrates is two to three orders of magnitude below our measured dark

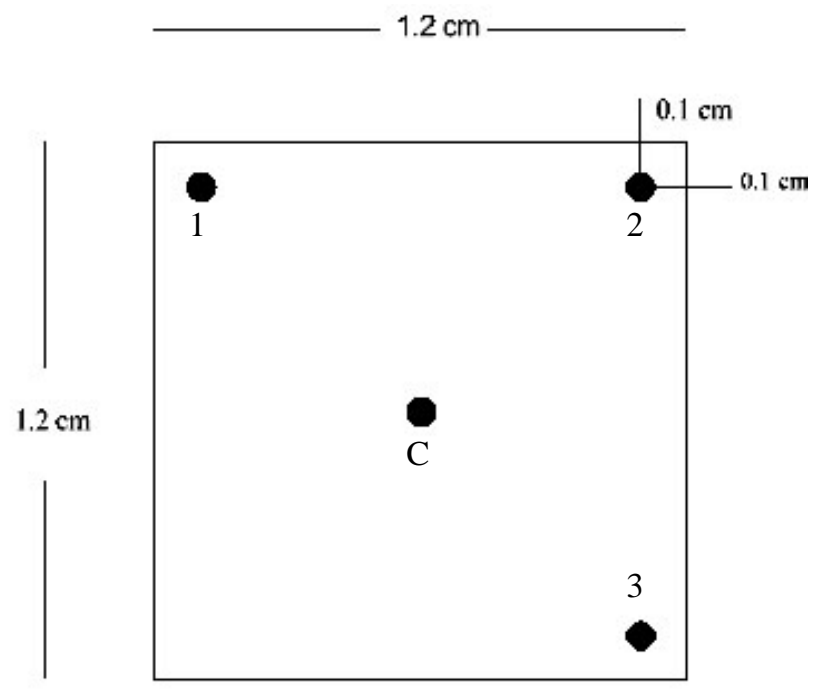

Figure 7.13. SIMS measurement positions on bare 6H-SiC substrate 


\begin{tabular}{|l|l|l|l|l|}
\hline & \multicolumn{5}{|c|}{$\begin{array}{c}\text { Bare 6H-SiC Substrate Test Point } \\
\text { Dopant Densities (cm } \\
\left(\mathbf{c m}^{-3}\right)\end{array}$} \\
\hline Dopant & & \multicolumn{2}{|c|}{2} & \multicolumn{2}{|c|}{3} & C \\
\hline Vanadium & $2.3 \mathrm{e} 17$ & $2.1 \mathrm{e} 17$ & $1.4 \mathrm{e} 17$ & $2.0 \mathrm{e} 17$ \\
\hline Nitrogen & $6.6 \mathrm{e} 16$ & $6.1 \mathrm{e} 16$ & $6.4 \mathrm{e} 16$ & $6.4 \mathrm{e} 16$ \\
\hline Boron & $4.4 \mathrm{e} 16$ & $4.4 \mathrm{e} 16$ & $4.0 \mathrm{e} 16$ & $4.5 \mathrm{e} 16$ \\
\hline Aluminum & $7.4 \mathrm{e} 14$ & $7.4 \mathrm{e} 14$ & $7.4 \mathrm{e} 14$ & $7.4 \mathrm{e} 14$ \\
\hline
\end{tabular}

Table 7.1. SIMS measurements results for vanadium, nitrogen, boron and aluminum densities at the points shown in Figure 7.12.

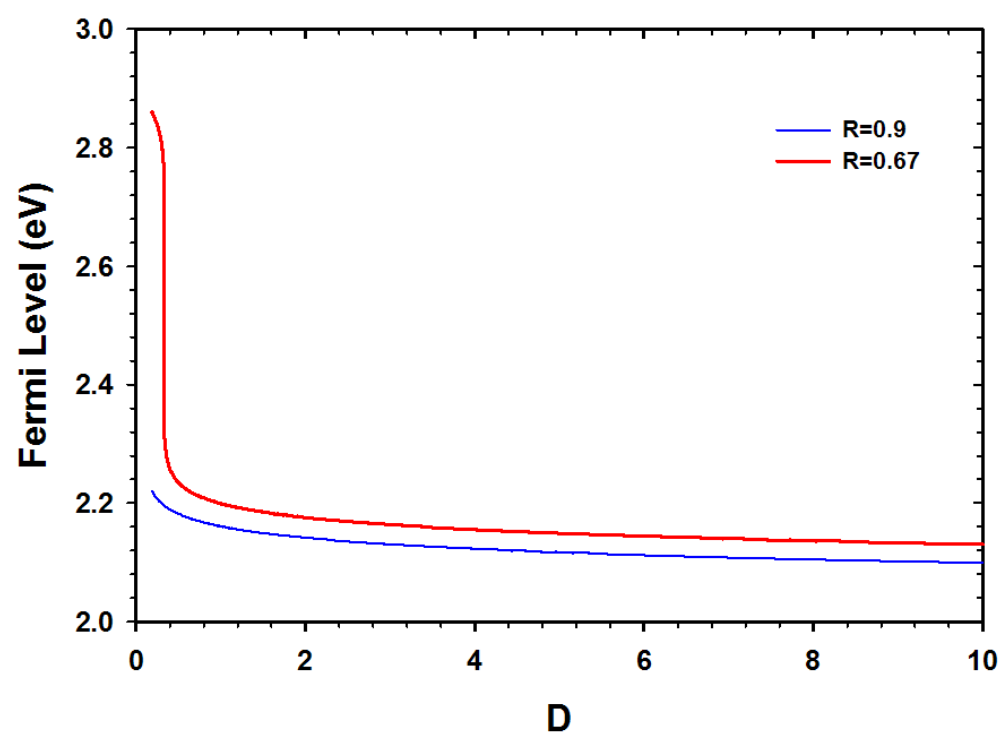

Figure 7.14. Fermi level plotted as a function of $\mathrm{D}$ for the $6 \mathrm{H}$-SiC substrate using SIMS measurement values for vanadium, nitrogen and boron densities. $D$ equals the ratio of vanadium to nitrogen density $\left(\mathrm{N}_{\mathrm{V}} / \mathrm{N}_{\mathrm{N}}\right)$. $R$ is the ratio of boron to nitrogen density $\left(\mathrm{N}_{\mathrm{B}} / \mathrm{N}_{\mathrm{N}}\right)$. 


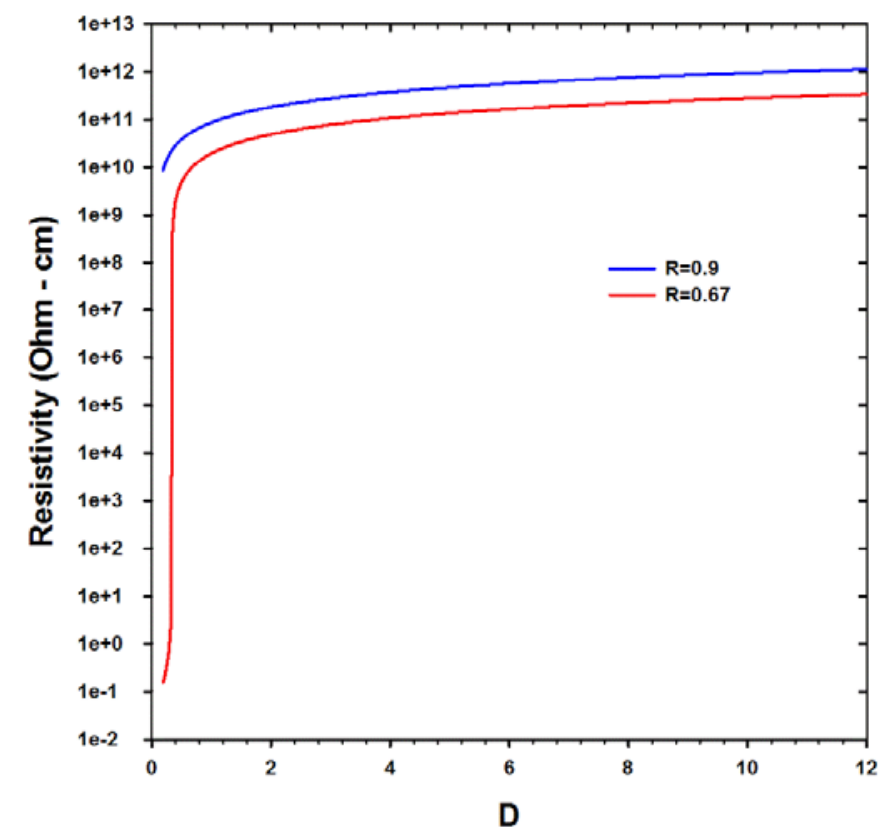

Figure 7.15. 6H-SiC substrate resistivity plotted as a function of $D$ using SIMS measurement values for vanadium, nitrogen and boron densities. $D$ equals the ratio of vanadium to nitrogen density $\left(\mathrm{N}_{\mathrm{V}} / \mathrm{N}_{\mathrm{N}}\right) . \mathrm{R}$ is the ratio of boron to nitrogen density $\left(\mathrm{N}_{\mathrm{B}} / \mathrm{N}_{\mathrm{N}}\right)$.

resistance for the green $\left(6.1 \times 10^{12} \Omega-\mathrm{cm}\right)$, black $\left(3.2 \times 10^{13} \Omega\right.$-cm) and silver $\left(6.9 \times 10^{13} \Omega\right.$-cm) switch substrates. The equilibrium (RT) free electron and hole densities can be calculated using our calculated Fermi level value of $2.165 \mathrm{eV}$ and equations 7.12 - 7.15. The free electron and hole densities are calculated to be $8.393 \times 10^{5} \mathrm{~cm}^{-3}$ and zero, respectively.

$$
\begin{gathered}
n=N_{c} \exp ^{-\left(\frac{E_{c}-E_{f}}{k T}\right)} \\
p=N_{v} \exp ^{-\left(\frac{E_{f}-E_{v}}{k T}\right)} \\
N_{c}=2\left(\frac{2 \pi m_{d e} k T}{h^{2}}\right)^{\frac{3}{2}}=1.72 \times 10^{16} T^{\frac{3}{2}} \\
N_{v}=2\left(\frac{2 \pi m_{d h} k T}{h^{2}}\right)^{\frac{3}{2}}=3.43 \times 10^{15} T^{\frac{3}{2}}
\end{gathered}
$$


The free electron densities for the green, black and silver switches can be estimated from the dark resistance measurements using equation 7.16. We realize that the application of an electric field across the switch substrates during the dark current measurements will disturb the thermal equilibrium. The joule heating due to the applied voltage and dark current will increase the electron, and eventually, the switch substrate lattice temperature. However, the dark current varied linearly with applied voltage. If significant joule heating was taking place, we would expect the dark current to have a greater than linear increase with applied voltage. This was not observed. In addition, it is possible that parallel leakage current paths in the ancillary circuitry used to perform the dark current measurements could lead to inaccurate measurements. Our confidence in our dark current measurements is based on measurements performed on vanadium and nitrogen co-doped $4 \mathrm{H}-\mathrm{SiC}$ substrates. The $4 \mathrm{H}-\mathrm{SiC}$ substrate dark currents were measured in the same test circuit as the $6 \mathrm{H}-\mathrm{SiC}$ measurements with identical annealed contacts. Dark current measurements on $15,4 \mathrm{H}-\mathrm{SiC}$, metalized substrates yielded a room temperature average value of dark resistivity of $5.23 \times 10^{14} \Omega$-cm with a standard deviation of $2.1 \times 10^{14} \Omega$-cm. An un-metalized 4H-SiC cut from the same crystal was provided to the Air Force Research Lab [7.5] for Temperature Dependent Hall Effect (TDH) measurements. A measurement of the 4H-SiC material resistivity as a function of temperature was included in the TDH measurements. The results are shown in Figure 7.16. Figure 7.16 is an Arrhenius plot of the $4 \mathrm{H}-\mathrm{SiC}$ resistivity measured using $\mathrm{TDH}$. The room temperature value of the $4 \mathrm{H}-\mathrm{SiC}$ substrate resistivity extrapolated from high temperature measurements is $5.395 \times 10^{14} \Omega$-cm, which is in excellent agreement with the average of our measured resistivity values. 


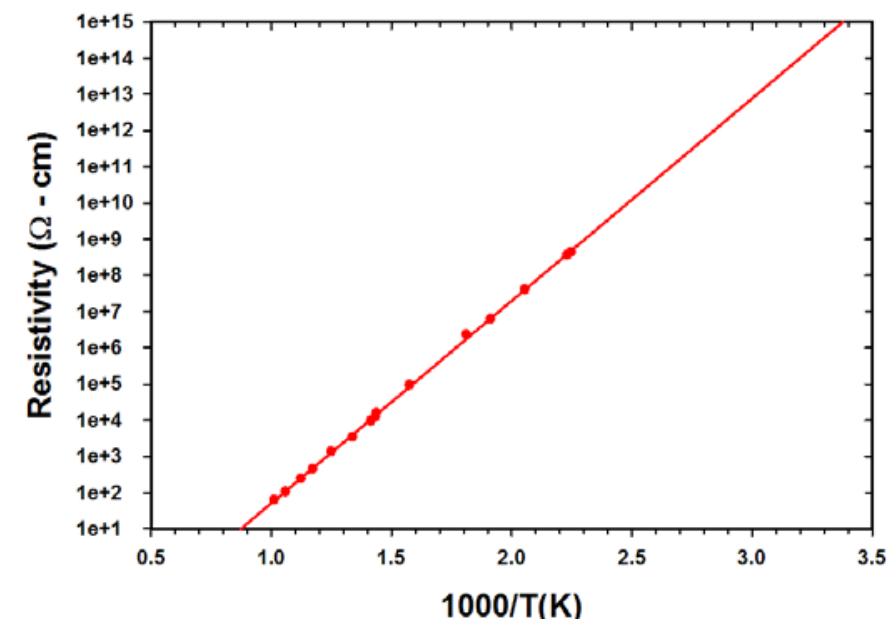

Figure 7.16. Resistivity plotted as a function of inverse temperature for a vanadium and nitrogen co-doped, $4 \mathrm{H}$-SiC substrate [7.5]. Filled red circles are data points. Red line is straight line fit to data extrapolated to room temperature $(T / 1000=3.33)$.

We believe that the differences in dark currents between the green, black and silver switches are a result of differences in the bulk resistivity of the $6 \mathrm{H}$-SiC substrates, not differences in the test circuit, or substrate contacts. We also acknowledge that the application of voltage certainly disturbs thermal equilibrium. As a result, the free electron densities calculated using dark resistance measurements are equal to the free electron densities calculated using the electron quasi-Fermi level (equation 7.17).

The free electron densities for the green, black, and silver $6 \mathrm{H}-\mathrm{SiC}$ switches are calculated using the measured dark resistance/resistivity and equation 7.16. The free hole density is assumed to be negligible since the Fermi level is expected to be located in the upper half of the band gap. The free electron densities calculated using the dark resistance measurements for the green, black, and silver switches are $10.2 \times 10^{3}, 1.95 \times 10^{3}$ and $9.0 \times 10^{2} \mathrm{~cm}^{-3}$, respectively, assuming an electron mobility equal to $100 \mathrm{~cm}^{2} / \mathrm{V}$-s for current flowing perpendicular to the caxis. The free electron densities calculated from the switch dark current measurements are

$$
\rho_{\text {dark }}=\frac{1}{q \mu_{n} n_{\text {dark }}}
$$


considerably lower than the free electron density calculated from our simple model using SIMS measurements. This discrepancy can be the result of some of the nitrogen in the $6 \mathrm{H}-\mathrm{SiC}$ substrate being interstitial, rather than substitutional, impurities. This would result in fewer donated electrons from the nitrogen levels leading to a higher compensation ratio and higher substrate resistivity. However, SIMS measurements of nitrogen and boron impurity densities in compensated 6H-SiC substrates have been in reasonable agreement with nitrogen and boron densities calculated from Temperature Dependent Hall Effect (TDH) measurements made on similar $6 \mathrm{H}-\mathrm{SiC}$ substrates [7.6]. So, we expect most of the nitrogen impurity atoms to be substitutional.

We can estimate the position of the quasi-Fermi level $\left(E_{\mathrm{fn}}\right)$ in the green, black, and silver switches using the free electron densities calculated from the measured dark resistances and equation 7.17. Equation 7.17 is rearranged to give the quasi-Fermi level as a function of free electron density in equation 7.18. The positions of the quasi-Fermi level in the green, black and silver switches are estimated to be $0.95,0.99$ and $1.01 \mathrm{eV}$ below the conduction band. These values range from 4.45 to $6.77 \mathrm{kT}$ below the Fermi level of $0.835 \mathrm{eV}$ calculated using the impurity densities from SIMS measurements. We expect the quasi-Fermi levels to be located slightly closer to the conduction band than the true equilibrium position of the Fermi level. We conclude that the values calculated for the quasi-Fermi levels are an upper bound for the room temperature position of the Fermi level in our initial $6 \mathrm{H}-\mathrm{SiC}$ material, with the true position slightly deeper in the bandgap.

$$
\begin{gathered}
n_{\text {dark }}=n_{0}+\Delta n=N_{c} e^{\left(-\left(\frac{E_{c}-E_{f n}}{k T}\right)\right)} \\
\ln \left(\frac{n}{N_{c}}\right) k T=-\left(E_{c}-E_{f n}\right)
\end{gathered}
$$


There is the possibility that the electron density calculated from the dark current measurements are being underestimated using too large a value for the electron mobility. It is possible that the electron mobility could be lower than $100 \mathrm{~cm}^{2} / \mathrm{V}$-s. The electron mobility value of $100 \mathrm{~cm}^{2} / \mathrm{V}$-s is an educated guess based on mobility measurements performed on vanadium and nitrogen co-doped 4H-SiC substrates [7.5]. The Fermi levels calculated based on the green, black and silver switch dark current measurements would be in better agreement with the Fermi level calculated using the impurity densities if we have overestimated the electron mobility. We can allow the electron mobility to be a variable in equation 7.16 to determine if a reasonable value of electron mobility exists that will reconcile the difference in measured resistivity for the green, black, and silver switches and the resistivity/Fermi level predicted using our model. Our model predicts an equilibrium electron density of $8.39 \times 10^{5} \mathrm{~cm}^{-3}$ at room temperature. We recast equation 7.16 as equation 7.19 to express the free electron density as a function of mobility. We plot $n\left(\mu_{n}\right)$ as a function of electron mobility in Figure 7.16. Included in Figure 7.16 is a black line at the value of the free electron density predicted by our simple model. The point at which the free electron densities calculated from the switch dark resistances equal our predicted value of free electron density is the value of electron mobility that would need to be used in equation 7.19 to force the equality. This occurs at electron mobility values of 1.22 (green switch), 0.23 (black switch) and $0.21 \mathrm{~cm}^{2} / \mathrm{V}$-s (silver switch). These values are exceedingly low. They would be low for an amorphous, or polycrystalline, semiconductor. As a result, we can dismiss the possibility

$$
n_{\text {dark }}\left(\mu_{n}\right)=\frac{1}{q \mu_{n} \rho_{\text {dark }}}
$$




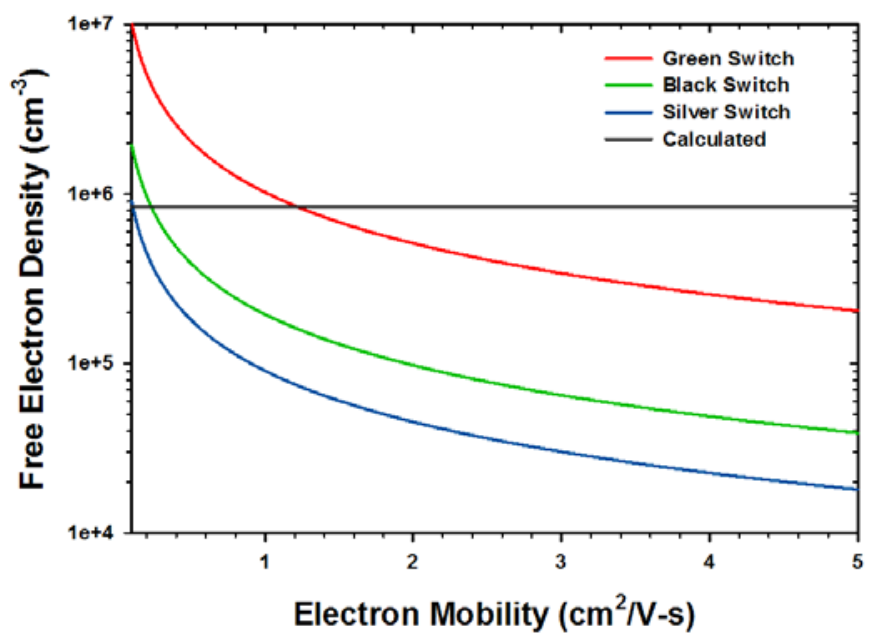

Figure 7.16. Free electron densities calculated from dark current measurements for the green, black and silver switches plotted as a function of electron mobility.

that an overestimate in the value of electron mobility is responsible for the difference between the free electron density calculated using the impurity densities and switch dark currents.

It seems clear that the discrepancy between the measured $6 \mathrm{H}-\mathrm{SiC}$ switch substrate dark resistivity and our calculated resistivity is not due to overestimates of electron mobility, or, discrepancies in the density of active nitrogen dopants. We believe that the discrepancy is caused by what has been left out of our simple model used to calculate the Fermi level and material resistivity. Our simple model only includes nitrogen, boron and vanadium impurities. It does not include the intrinsic point defects present in the $6 \mathrm{H}-\mathrm{SiC}$ substrates.

\subsection{1) Point Defects}

Vacancies, antisites and complexes are point defects that will have an effect on the properties of the $6 \mathrm{H}-\mathrm{SiC}$ semiconductor substrates [7.7]. A vacancy is created by an atom that is missing from its normal lattice position in a semiconductor crystal. $\mathrm{V}_{\mathrm{C}}$ denotes a carbon vacancy in the $6 \mathrm{H}-\mathrm{SiC}$ crystal. An antisite is a defect created by a host atom, silicon, occupying the site of 
another host atom, carbon. This antisite is denoted by $A_{\text {s-c. }}$ Complexes are defects that are comprised by a small number of point defects, or, a small number of point defects and an impurity [7.7]. Vacancies and antisites are classified as intrinsic defects since no foreign atoms are involved. Complicated point defects such as divacancies, vacancy - antisite pairs are also present. Point defects act as traps. Traps capture charge carriers. An electron (hole) trap will capture a free electron (hole) and either thermally emit it to the conduction (valence) band, or, capture a hole (electron) leading to recombination. A filled electron (hole) trap, that is more likely to capture a hole (electron) than thermally emit the electron (hole) to the conduction (valence) band, is a recombination center. Electron (hole) traps with energy levels located in the top (bottom) half of the bandgap are unlikely to behave as recombination centers. Electron and hole traps located near the middle of the bandgap will likely behave as recombination centers.

Numerous point defects have been observed in both $4 \mathrm{H}$ and $6 \mathrm{H}-\mathrm{SiC}$. The most highly studied deep defects in $6 \mathrm{H}-\mathrm{SiC}$ are the $\mathrm{E}_{1} / \mathrm{E}_{2}, \mathrm{Z}_{1} / \mathrm{Z}_{2}, \mathrm{~V}_{1}, \mathrm{~V}_{3}$ and $\mathrm{R}$ centers $[7.8,7.9]$. The $\mathrm{E}_{1} / \mathrm{E}_{2}$, $\mathrm{Z}_{1} / \mathrm{Z}_{2}$ and $\mathrm{R}$ centers are deep defects associated with the carbon vacancy point defect and $\mathrm{V}_{1}$ and $V_{3}$ are deep defects associated with the silicon vacancy. The deep defects can have plus, minus and neutral charge states and can be located at the hexagonal or, either of the two quasi-cubic non-equivalent positions in $6 \mathrm{H}-\mathrm{SiC}$ lattice. Point defects with multiple charge states are also possible. For example, the carbon vacancy can be doubly charged positive, or negative, making it amphoteric. The different charge states and lattice positions lead to a large array of deep levels that can behave as electron, or hole, traps. Different deep defects are present in n-type, $6 \mathrm{H}-\mathrm{SiC}$ and vanadium compensated, semi-insulating 6H-SiC. Table 7.2 lists only two of many electron traps present in n-type, $6 \mathrm{H}$-SiC $[7.8,7.9]$. The important point to notice about Table 7.2 is the electron capture cross section. Table 7.3 lists several important deep level electron traps that 
occur in vanadium compensated, semi-insulating, $6 \mathrm{H}-\mathrm{SiC}$ along with the vanadium acceptor level. Note that the electron capture cross section for the traps is much larger than the vanadium acceptor level capture cross section. This means that that in a one for one comparison, the point defect electron traps capture electrons more easily than the vanadium acceptor level. However, the density of the vanadium acceptor levels is much larger than the individual trap densities. The individual point defect traps are present in densities ranging from $10^{13} \mathrm{~cm}^{-3}$ up to $10^{16} \mathrm{~cm}^{-3}$. The total number of traps present in the $6 \mathrm{H}-\mathrm{SiC}$ material can reach a few times $10^{16} \mathrm{~cm}^{-3}$. Electron traps located a few kT below the Fermi level will be filled with electrons, while electron traps located at, or above the Fermi level, will be partially filled, or empty.

\begin{tabular}{|c|c|c|}
\hline Electron Trap Designation & $\begin{array}{c}\text { Energy Level Below } \\
\text { Conduction Band (eV) }\end{array}$ & $\begin{array}{c}\text { Electron Capture Cross } \\
\left.\text { Section } \mathbf{( c m}^{\mathbf{2}}\right)\end{array}$ \\
\hline $\mathrm{E}_{1} / \mathrm{E}_{2}$ & 0.45 & $1 \times 10^{-15}$ \\
\hline $\mathrm{R}$ Center & 1.25 & $1 \times 10^{-13}$ \\
\hline
\end{tabular}

Table 7.2. Energy level and electron capture cross section for two important electron traps in n-type, $6 \mathrm{H}-\mathrm{SiC}[7.8]$

\begin{tabular}{|c|c|c|}
\hline Electron Trap Designation & $\begin{array}{c}\text { Energy Level } \\
\text { BelowConduction Band (eV) }\end{array}$ & $\begin{array}{c}\text { Electron Capture Cross } \\
\left.\text { Section } \mathbf{( c m}^{\mathbf{2}}\right)\end{array}$ \\
\hline Vanadium Complex & 0.905 & $2 \times 10^{-14}$ \\
\hline Silicon Vacancy & 0.93 & $1.85 \times 10^{-13}$ \\
\hline UD - 1 & 1.0 & $3.2 \times 10^{-14}$ \\
\hline Vanadium Acceptor & 0.8 & $5.77 \times 10^{-16}$ \\
\hline
\end{tabular}

Table 7.3. Energy level and electron capture cross section for several electron traps present in vanadium compensated, semi-insulating, 6H-SiC [7.9] 
A better sense of the effect of traps in the $6 \mathrm{H}-\mathrm{SiC}$ switch substrates can be obtained by exploring the effect of deep traps in high purity, semi-insulating (HPSI), 4H-SiC. HPSI 4H-SiC is a semi-insulating form of $4 \mathrm{H}-\mathrm{SiC}$ that is grown without the use of a deep level dopant, such as vanadium, to pin the Fermi level deep in the bandgap [7.10]. HPSI 4H-SiC makes use of intrinsic defects, such as point defects, to pin the Fermi level deep in the bandgap. As a result, the location and nature of the point defects contributing to the semi-insulating behavior of HPSI $4 \mathrm{H}-\mathrm{SiC}$ is a subject of great interest. Room temperature resistivities of $10^{9} \Omega$-cm are achieved despite the fact that the HPSI 4H-SiC samples have nitrogen and boron densities of $2 \times 10^{16} \mathrm{~cm}^{-3}$ and $7 \times 10^{15} \mathrm{~cm}^{-3}$, respectively [7.11].

Resistivity versus temperature measurements were performed on five different HPSI 4H$\mathrm{SiC}$ samples revealing that each sample had different activation energies [7.11]. Subsequent measurements on $16 \mathrm{HPSI} 4 \mathrm{H}-\mathrm{SiC}$ samples identified activation energies of $0.55,0.95,1.1,1.2$, 1.4, and $1.65 \mathrm{eV}$ distributed among the test pieces [7.11] with defect densities ranging from $1 \times 10^{15}-4 \times 10^{15} \mathrm{~cm}^{-3}$. All defect levels exhibited acceptor-like behavior (electron traps) [7.11]. No individual point defect was present in high enough density to be solely responsible for the semi-insulating behavior of the HPSI 4H-SiC [7.11]. The understanding that resulted from these measurements is that there are a significant number of point defects in HPSI-4H-SiC, distributed throughout the bandgap, which all can contribute to the semi-insulating behavior of HPSI-4HSiC. The deepest traps, including any shallow acceptors, are filled with electrons first and higher level traps are filled in order of their position in the bandgap until the electrons donated from the nitrogen levels are exhausted. This process will result with different point defects acting as the dominant impurity and different activation energies being measured for different HPSI 4H-SiC samples depending on the effective donor density $\left(\mathrm{N}_{\mathrm{N}}-\mathrm{N}_{\mathrm{B}}\right)$. We should expect an identical 
process to be at work in the vanadium compensated $6 \mathrm{H}-\mathrm{SiC}$. The same point defects in HPSI$4 \mathrm{H}-\mathrm{SiC}$ will also be present in $6 \mathrm{H}-\mathrm{SiC}$ at energy levels that will be slightly shifted towards the conduction band [7.8]. The filling process of deep traps in vanadium compensated $6 \mathrm{H}-\mathrm{SiC}$ can be visualized with the aid of Figure 7.17.

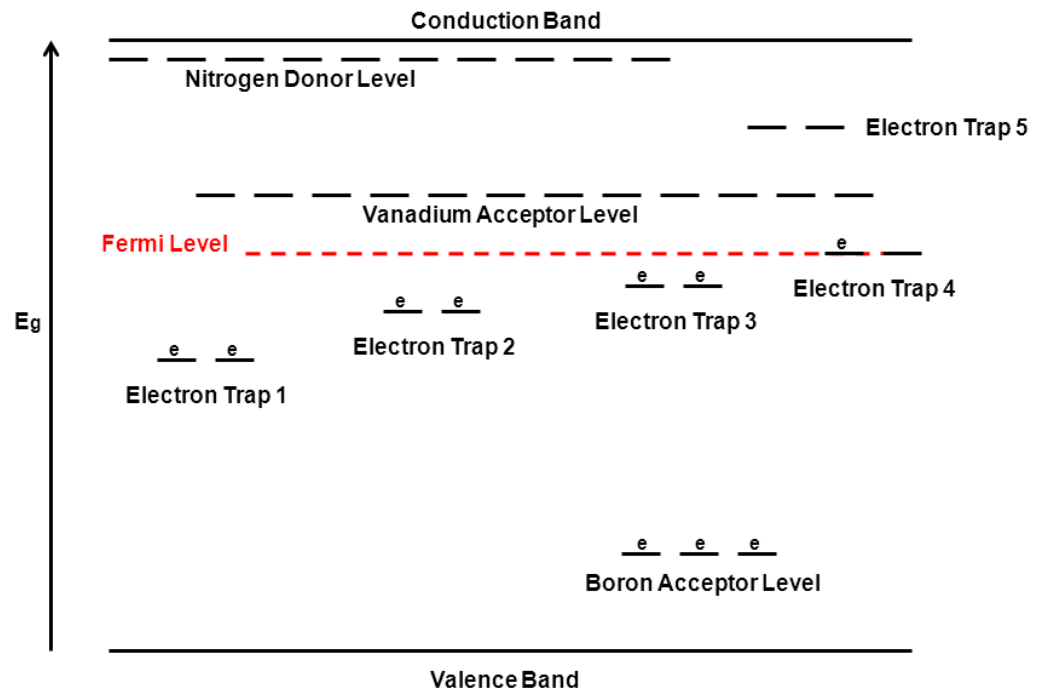

Figure 7.17. The energy bandgap diagram is shown for vanadium compensated $6 \mathrm{H}-\mathrm{SiC}$ material including electron traps, shallow donor and shallow and deep acceptors. This diagram depicts the case when the net donor density (nitrogen density - boron density) is less than the sum of the densities of Electron Traps $1-4$. The Fermi level is pinned at Electron Trap 4, which is below the vanadium acceptor level.

Figure 7.17 is a diagram of the energy gap for a vanadium compensated, $6 \mathrm{H}-\mathrm{SiC}$ including five electron traps, nitrogen donor level, boron acceptor level and the vanadium acceptor level. Figure 7.17 depicts the case where the net donor density is less than the sum of the densities of electron traps $1-4$. All the electrons donated by the nitrogen donors are captured by the boron acceptor level, electron trap 1, electron trap 2, electron trap 3 and electron trap 4. As a result, the Fermi level is pinned close to electron trap 4 and levels more than a few kT higher than electron trap 4 are unoccupied by electrons. This leaves a large number of empty acceptors and electron traps, including half the electron trap 4 levels, all of the vanadium acceptor levels, electron trap 5 levels and nitrogen donor levels, available to trap electrons. This 
will result in a very short electron recombination time. $6 \mathrm{H}-\mathrm{SiC}$ material that can be depicted by the diagram in Figure 7.17 will have the following physical attributes: semi-insulating, very high room temperature resistivity, very fast carrier recombination time and weak, or no, photoconductive response to photons with energy $\leq \mathrm{E}_{\mathrm{T} 4}$. The physical situation depicted in Figure 7.17 appears to be close to the reality of our initial $6 \mathrm{H}-\mathrm{SiC}$ substrates. Our measured dark resistance for the green, black and silver switches range from $6.1 \times 10^{12} \Omega$-cm to $6.9 \times 10^{13} \Omega$-cm compared to the value $7.48 \times 10^{10} \Omega$-cm predicted by our model that only included the effects of impurity levels. Also, the green, black, and silver switches have a very fast recombination time for excitation at $1064 \mathrm{~nm}$. We believe that the Fermi level is pinned at $\sim 1.0 \mathrm{eV}$ below the conduction band based on the room temperature resistivity measured for our initial vanadium compensated $6 \mathrm{H}-\mathrm{SiC}$ substrates. This is $\sim 0.2 \mathrm{eV}$ below the vanadium acceptor level. The number of electrons trapped at the vanadium acceptor level for this $6 \mathrm{H}-\mathrm{SiC}$ material at thermal equilibrium can be calculated using equation 7.20. Here the vanadium acceptor level ( $\left.\mathrm{E}_{\mathrm{VA}}\right)$ is 0.8 $\mathrm{eV}$ below the conduction band the Fermi level is $1.0 \mathrm{eV}$ below the conduction band, $\mathrm{N}_{\mathrm{VA}}$ is $1.75 \times 10^{17} \mathrm{~cm}^{-3}$ and $\mathrm{g}_{\mathrm{a}}$ is $4 . \mathrm{N}_{\mathrm{VA}}{ }^{-}$is equal to $1.9 \times 10^{13} \mathrm{~cm}^{-3}$. A small fraction of acceptor levels ( 1/10,000) are occupied by electrons.

$$
N_{V A}^{-}=\frac{N_{V A}}{1+\frac{1}{g_{a}} e^{\left(\frac{E_{V A}-E_{f}}{k T}\right)}}
$$

We believe that the Fermi level is pinned below the vanadium acceptor level in our initial 6H-SiC switch material, leaving the vanadium acceptor level virtually empty and an electron trap at $\sim 1.0 \mathrm{eV}$ below the conduction band partially filled. This belief is based on total deep trap densities of $\sim 2 \times 10^{16} \mathrm{~cm}^{-3}$ present in HPSI 4H-SiC [7.11] that are related to carbon vacancies, 
di-vacancies and complexes. Similar total trap densities should also be present in our vanadium and nitrogen co-doped $6 \mathrm{H}$-SiC substrates. The effect of the trap density in our vanadium and nitrogen co-doped material will depend on the total nitrogen and excess donor densities $\left(\mathrm{N}_{\mathrm{N}}-\right.$ $\left.\mathrm{N}_{\mathrm{B}}\right)$. If $\mathrm{N}_{\mathrm{N}}>\left(\mathrm{N}_{\mathrm{B}}+\Sigma \mathrm{N}_{\mathrm{TD}}\right)$, then all the deep traps at energy levels lower than the vanadium acceptor level ( $\mathrm{N}_{\mathrm{TD}}$ ) and boron acceptors will be filled with electrons and the vanadium acceptor level will be partially filled with electrons. The material will be semi - insulating and the Fermi level will be pinned close to the vanadium acceptor level. If $\mathrm{N}_{\mathrm{B}}<\mathrm{N}_{\mathrm{N}} \leq\left(\mathrm{N}_{\mathrm{B}}+\Sigma \mathrm{N}_{\mathrm{TD}}\right)$, the boron acceptors and the deepest electron traps will be filled with electrons, a deep trap will be partially filled with electrons and the vanadium acceptor level will be empty. This material will be semiinsulating with the Fermi level pinned close to the partially filled electron trap level.

The average values for the nitrogen and boron densities for our vanadium and nitrogen co-doped $6 \mathrm{H}$-SiC are $6.375 \times 10^{16} \mathrm{~cm}^{-3}$ and $4.325 \times 10^{16} \mathrm{~cm}^{-3}$. This results in a net donor density $\left(\mathrm{N}_{\mathrm{N}}-\mathrm{N}_{\mathrm{B}}\right)$ of $2.05 \times 10^{16} \mathrm{~cm}^{-3}$. This is about the same expected density of traps in $6 \mathrm{H}-\mathrm{SiC}$. As a result, we should not necessarily expect the Fermi level to be pinned at the vanadium acceptor level. A model for the carrier density in our initial $6 \mathrm{H}-\mathrm{SiC}$ material would have to include the effect of the deep traps resulting from the intrinsic point defects and complexes.

A model for the optically generated carrier density in our initial $6 \mathrm{H}-\mathrm{SiC}$ material will have to account for the carriers excited from, and trapped at, each energy level residing in the bandgap shown in Figure 7.17. This would require rate equations for electron and hole densities written for each energy level including two-step optical transitions of electrons from the valence band to the conduction band via mid-gap levels and creation of electron-hole pairs via twophoton absorption for photon energies $>E_{g} / 2$ (i.e., $532 \mathrm{~nm}$ ). This would require values of the electron and hole capture cross-section and optical transition cross sections at the applied 
wavelength for each energy level. The carrier capture and optical transition cross-section values are not available for all of these levels. This would amount to an extensive and complicated system of simultaneous, differential equations. We can simplify this set of equations by making some judicious assumptions. First, we will assume that the empty nitrogen donor levels lie close enough to the conduction band that any electrons that are captured are immediately re-excited to the conduction band, so that the nitrogen levels can be ignored. Second, we will replace all the mid-band traps by a single mid-band trap level with effective trap density and capture cross section. Third, the traps located just below the vanadium acceptor level can be represented by a single trap level with the proper effective density and capture cross section based on measured parameters [7.12, 7.13]. Fourth, we will ignore electron traps residing above the vanadium acceptor level for the moment. Incorporating these assumptions result in the energy bandgap diagram of Figure 7.18.

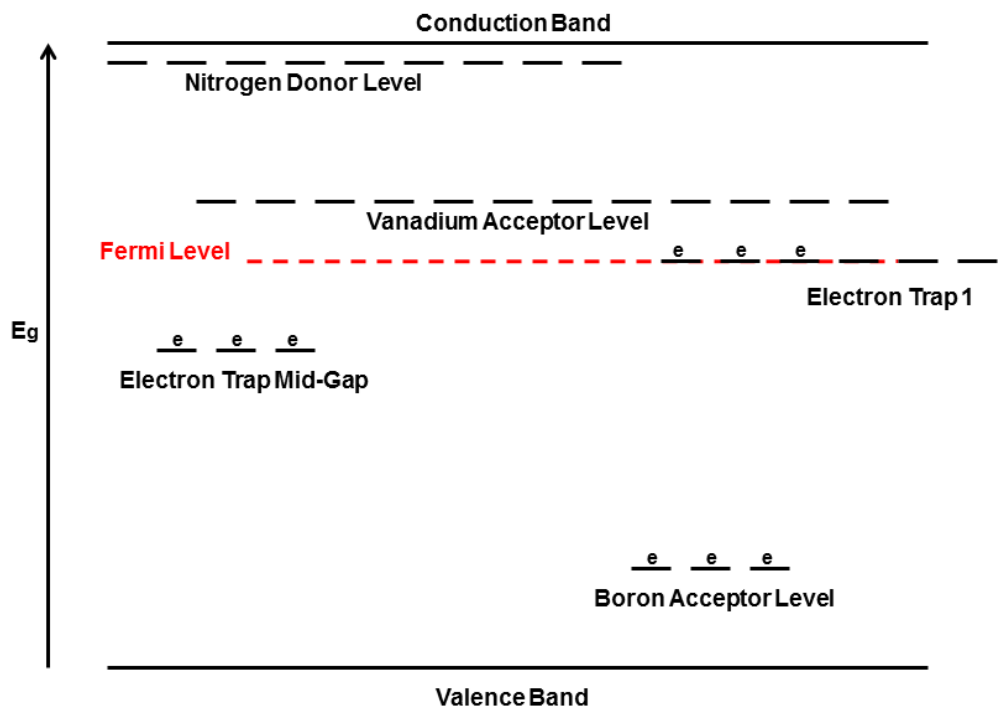

Figure 7.18. . The energy bandgap diagram is shown for vanadium compensated $6 \mathrm{H}-\mathrm{SiC}$ material including the effective electron traps electron trap mid-gap and electron trap 1, shallow donor and shallow and deep acceptors. This diagram depicts the case when the net donor density (nitrogen density - boron density) is less than the sum of the densities of the effective electron traps. The Fermi level is pinned at effective Electron Trap 1, which is below the vanadium acceptor level. 
Figure 7.18 is the energy bandgap diagram of what we believe to be the initial conditions existing in our initial $6 \mathrm{H}-\mathrm{SiC}$ material just prior to optical excitation. All levels lying more than a few kT below the Fermi level are filled with electrons and levels a few kT above the Fermi level are empty. Electron Trap 1 is an effective trap level located $1.00 \mathrm{eV}$ below the conduction band with a number density of $5 \times 10^{15} \mathrm{~cm}^{-3}$ and an electron capture cross section of $7.9 \times 10^{-14} \mathrm{~cm}^{-2}$ [7.12, 7.13]. The effective electron capture cross section for the $1.0 \mathrm{eV}$ trap was calculated by assuming an equal number of vanadium complex, silicon vacancy and UD-1 electron traps and averaging the capture cross sections listed in Table 7.3. Electron Trap Mid-Gap is an effective trap level located at $1.5 \mathrm{eV}$ below the conduction band with a number density $1.5 \times 10^{16} \mathrm{~cm}^{-3}$ and an electron capture cross section of $5 \times 10^{-14} \mathrm{~cm}^{-2}$. The vanadium acceptor level number density and electron capture cross section are $1.75 \times 10^{17} \mathrm{~cm}^{-3}$ and $5.77 \times 10^{-16} \mathrm{~cm}^{-2}$, respectively.

\section{4) Switch Model for Operation at $1064 \mathrm{~nm}$}

Developing a model for switch operation for optical excitation at $1064 \mathrm{~nm}$ is less difficult than a switching model for operation at $532 \mathrm{~nm}$. First, two-step optical transitions and twophoton absorption need not be considered. Second, the photon energy is $1.17 \mathrm{eV}$ for the $1064 \mathrm{~nm}$ wavelength optical excitation. This photon energy is just above the threshold energies for optical transitions of electrons from Electron Trap 1 and the vanadium acceptor level. As a result, we can expect optical transitions of electrons from these two levels to the conduction band, but no optical transitions from electrons trapped at levels located deeper in the 6H-SiC bandgap. We are effectively isolating these two levels by using the $1064 \mathrm{~nm}$ wavelength excitation.

All the information on impurity densities is included in the switch model since we know the doping densities from SIMS measurements. We assume that the total point defect - trap 
densities are $\sim 2 \times 10^{16} \mathrm{~cm}^{-3}$, similar to trap densities determined for HPSI 4H-SiC [7.11]. An accurate switch model needs to include both impurity and trap levels. First, we will take a step backward and include only the impurity levels in our switch model at $1064 \mathrm{~nm}$.

\subsection{1) Switch Model at $1064 \mathrm{~nm}$ Including Only the Effects of Nitrogen, Vanadium and Boron}

Our initial switch model will include only the effects of the nitrogen, vanadium and boron impurities. This physical situation is represented in Figure 7.12. Only the electrons trapped in the vanadium acceptors levels will respond to $1064 \mathrm{~nm}$ wavelength illumination. The switch will be a unipolar device at $1064 \mathrm{~nm}$ since the only carriers will be electrons excited from the filled vanadium acceptor levels to the conduction band. Equation 7.21 is the general rate equation describing the rate of change of electrons $(n(t))$ in the conduction band. Where $G(t)$ is

$$
\frac{d n(t)}{d t}=G(t)-R(t)
$$

the generation rate of electrons into the conduction band and $R(t)$ is the recombination rate for

electrons. In our case, the generation term includes the thermal emission and optical transition of electrons from the filled vanadium acceptor levels to the conduction band. The recombination term includes the trapping of free electrons at unfilled vanadium acceptor levels. True recombination does not take place during optical excitation at $1064 \mathrm{~nm}$ since there are no free holes in the valence band. True recombination requires the trapping of an electron at a recombination center and then the capture of a hole (or, vice versa) at the same recombination center before the electron is emitted. As a result, the recombination term is the electron trapping rate at unfilled vanadium acceptor levels. Equation 7.22 is the general rate equation 7.21 
rewritten for the case of when recombination/re-trapping is proportional to the number density of free electrons. $\tau_{\text {eff }}$ is the effective re-trapping, or recombination, time in (7.22).

$$
\frac{d n(t)}{d t}=G(t)-\frac{n(t)}{\tau_{e f f}}
$$

Equation 7.23 is the rate equation for free electrons in the conduction band including the expanded expressions for the thermal and optical generation terms and electron re-trapping term. The variable/parameters of equation 7.24 are defined in Table 7.4.

$$
\begin{aligned}
\frac{d n(t)}{d t}= & \sigma_{n} v_{t h} N_{c v}\left(\left(N_{n}-N_{b}\right)-n(t)\right)+K \sigma_{o p} I(t)\left(\left(N_{n}-N_{b}\right)-n(t)\right) \\
& -\sigma_{n} v_{t h} n(t)\left(N_{v}-\left(N_{n}-N_{b}\right)+n(t)\right)
\end{aligned}
$$

Where

$$
K=\frac{\eta(1-r)\left(1-e^{-\alpha W}\right)}{L d \hbar \omega}
$$

The first term on the right hand side (RHS) of equation (7.23) is the expression for the thermal emission of electrons from vanadium acceptor levels occupied by electrons. The term $\left(\mathrm{N}_{\mathrm{n}}-\mathrm{N}_{\mathrm{b}}-\right.$ $\mathrm{n}(t))$ is the number density of electrons occupying vanadium acceptor levels and the terms $v_{t h}$ and $N_{c v}$ are the electron thermal velocity and effective conduction band density of states reduced to the vanadium acceptor level, respectively.

$$
\begin{gathered}
v_{t h}=\sqrt{\frac{3 k T}{m_{e}}} \\
N_{c v}=N_{c} e^{\left(-\left(\frac{E_{c}-E_{v a}}{k T}\right)\right)}
\end{gathered}
$$




\begin{tabular}{|c|c|c|}
\hline Variable/Parameter & Definition & Units \\
\hline $\mathrm{n}(t)$ & Electrons in conduction band & $\mathrm{cm}^{-3}$ \\
\hline$\sigma_{\mathrm{n}}$ & $\begin{array}{c}\text { Vanadium acceptor level } \\
\text { electron capture cross section }\end{array}$ & $\mathrm{cm}^{2}$ \\
\hline$v_{t h}$ & $\begin{array}{c}\text { Electron thermal velocity } \\
\text { Effective conduction band } \\
\text { density of states reduced to the } \\
\text { vanadium acceptor level }\end{array}$ & $\mathrm{cm}^{-3}$ \\
\hline $\mathrm{N}_{c v}$ & Nitrogen density & $\mathrm{cm}^{-3}$ \\
\hline $\mathrm{N}_{\mathrm{n}}$ & Boron density & $\mathrm{cm}^{-3}$ \\
\hline $\mathrm{N}_{\mathrm{b}}$ & $\begin{array}{c}\text { Vanadium acceptor level } \\
\text { optical transition cross section }\end{array}$ & $\mathrm{cm}^{2}$ \\
\hline$\sigma_{\mathrm{op}}$ & Applied optical power & $\mathrm{Watts}^{-3}$ \\
\hline $\mathrm{I}(t)$ & Vanadium density & $\mathrm{cm}^{-3}$ \\
\hline $\mathrm{N}_{\mathrm{v}}$ & Switch facet area & $\mathrm{cm}^{2}$ \\
\hline $\mathrm{Ld}$ & Quantum efficiency & \\
\hline$\eta$ & Reflectance & $\mathrm{cm}^{-1}$ \\
\hline$\alpha$ & Total optical absorption of & 6H-SiC substrate \\
\hline
\end{tabular}

Table 7.4. Parameter definitions for equations 7.22 and 7.24

The second term on the RHS of equation 7.23 is the expression for optical excitation of electrons from vanadium acceptor levels to the conduction band. $\sigma_{\mathrm{op}}$ and $\mathrm{I}(t)$ are the vanadium acceptor level optical transition cross section at the applied wavelength and applied optical power at the switch facet. The third term on the RHS of equation 7.23 is the vanadium acceptor level electron re-trapping term which is proportional to the number of free electrons and the number of vacant vanadium acceptor levels. The number of vacant vanadium acceptor levels is given by $\left(\mathrm{N}_{\mathrm{v}}-\left(\mathrm{N}_{\mathrm{n}}-\mathrm{N}_{\mathrm{b}}\right)+\mathrm{n}(t)\right)$.

Equation 7.23 can be rewritten in the form of equation 7.22 by collecting terms.

$$
\begin{aligned}
\frac{d n(t)}{d t}=\left(N_{n}-\right. & \left.N_{b}\right)\left(\sigma_{n} v_{t h} N_{c v}+K \sigma_{o p} I(t)\right) \\
& -\sigma_{n} v_{t h} n(t)\left(\left(N_{v}-\left(N_{n}-N_{b}\right)+n(t)\right)+N_{c v}+\frac{K \sigma_{o p} I(t)}{\sigma_{n} v_{t h}}\right)
\end{aligned}
$$




$$
\tau_{e f f}=\left[\sigma_{n} v_{t h}\left(\left(N_{v}-\left(N_{n}-N_{b}\right)+n(t)\right)+N_{c v}+\frac{K \sigma_{o p} I(t)}{\sigma_{n} v_{t h}}\right)\right]^{-1}
$$

Comparing equations 7.27 and 7.23 , the effective carrier decay time will be given by equation 7.28. We know from SIMS measurements that $\mathrm{N}_{\mathrm{v}} \sim 1.75 \times 10^{17}$ and $\left(\mathrm{N}_{\mathrm{n}}-\mathrm{N}_{\mathrm{b}}\right) \sim 2.0 \times 10^{16} \mathrm{~cm}^{-3}$. The electron density calculated from switching experiments for the highest optical intensities at $1064 \mathrm{~nm}$ is $\sim 4 \times 10^{14} \mathrm{~cm}^{-3}$, and $N_{c v}$ is $3.25 \times 10^{6} \mathrm{~cm}^{-3}$ at room temperature. These two terms are negligible compared the impurity density terms. The effective carrier decay time will be given by equation 7.29 and 7.30 at low optical intensities. The generation term of equation 7.27 is proportional to the effective donor density as written in equation 7.31. We want to develop a switch with a large generation rate and carrier decay time. We can obtain a large generation rate by making $N_{n}$ large and $N_{b}$ zero in equation 7.31. The decay rate is given by 7.32, when $N_{b}$ is set to zero. A large carrier decay time can be obtained if $\mathrm{N}_{\mathrm{v}}$ and $\mathrm{N}_{\mathrm{n}}$ are approximately equal.

$$
\begin{gathered}
\tau_{e f f=}\left[\sigma_{n} v_{t h}\left(N_{v}-\left(N_{n}-N_{b}\right)\right)\right]^{-1} \\
\tau_{e f f} \sim \frac{1}{N_{v}-\left(N_{n}-N_{b}\right)} \\
G \sim\left(N_{n}-N_{b}\right) \\
\tau_{e f f} \sim \frac{1}{N_{v}-N_{n}}
\end{gathered}
$$

In Chapters 3 and 6 we defined the variables $\mathrm{D}$ and $\mathrm{R}$ as the ratios shown below. The

$$
D=\frac{N_{v}}{N_{n}}, R=\frac{N_{b}}{N_{n}}
$$

conclusions reached above on achieving large generation and carrier decay rates require $\mathrm{R}$ to be zero and $\mathrm{D}$ to be unity. The results from the electron rate equation agree with the intuitive requirements for the value of $\mathrm{R}$ and $\mathrm{D}$ from Chapter 6 . The intuitive requirements were for the boron impurity $\left(\mathrm{N}_{\mathrm{b}}\right)$ to be set to zero $(\mathrm{R}=0)$ and the nitrogen density to be set as close in value 
to the vanadium density while still maintaining a semi-insulating $6 \mathrm{H}-\mathrm{SiC}$ substrate. Ideally, this would result in D equal to unity. The value of $\mathrm{D}$ equal to two was selected in Chapters 3 and 6 to provide some thermal stability of the semi-insulating property of the substrates.

We can calculate the low intensity carrier decay time using the vanadium acceptor level electron capture cross section from Table 7.3, the average impurity densities and equations 7.25, 7.26 and 7.29. The room temperature value of the low intensity carrier decay time is 960 ps. This is much larger than the value of 140 ps from the literature [7.14]. What we must keep in mind is that our calculated carrier decay time is for an idealized case that ignores the presence of electron traps. The 140 ps recombination time [7.14] was measured for a vanadium compensated material with nominal impurity densities of $\mathrm{N}_{\mathrm{v}}=1 \times 10^{17} \mathrm{~cm}^{-3}, \mathrm{~N}_{\mathrm{n}}=1 \times 10^{16} \mathrm{~cm}^{-3}$ and $\mathrm{N}_{\mathrm{b}}=1 \times 10^{15} \mathrm{~cm}^{-3}$. We have seen that the trap density in $4 \mathrm{H}-\mathrm{SiC}$ and $6 \mathrm{H}-\mathrm{SiC}$ is $\sim 1-2 \times 10^{16} \mathrm{~cm}^{-3}$. This means that it is likely than the 140 ps recombination time was measured for a trap dominated $6 \mathrm{H}$-SiC substrate with virtually no electrons occupying the vanadium acceptor level and the Fermi level pinned at a deep trap level. This brings us full circle back to a model that includes the effect of traps on $6 \mathrm{H}-\mathrm{SiC}$ photoconductive switch operation. However, we must remember that if we can dope the material so that the Fermi level is pinned at the vanadium acceptor level and have a much larger number of electrons trapped in vanadium acceptor levels than at deep traps, we can improve both the quantum efficiency and increase the carrier decay time. We can transform the $6 \mathrm{H}-\mathrm{SiC}$ semiinsulating material from a trap-dominated material to an impurity-dominated material with properties that can be controlled. 


\subsection{2) Switch Model at $1064 \mathrm{~nm}$ Including the Effects of Impurities and a Deep Trap Located Close to the Vanadium Acceptor Level}

Let's return to the more realistic switch model that includes the effects of deep traps. The physical situation is depicted in Figure 7.18. The Electron Trap 1 and Electron Trap Mid-Gap levels have been added to the impurity levels included in the previous switch model. Electron Trap 1 is assumed to be located at $1.0 \mathrm{eV}$ below the conduction band with a number density of $5 \times 10^{15} \mathrm{~cm}^{-3}$ and an electron capture cross section of $7.9 \times 10^{-14} \mathrm{~cm}^{2}$. The Fermi level is pinned at Electron Trap 1, so half the Electron Trap 1 levels are occupied by electrons at equilibrium. Electron Trap Mid-Gap is assumed to be located at $1.5 \mathrm{eV}$ below the conduction band. Electron Trap Mid-Gap is filled with electrons. The vanadium acceptor levels are empty. Only the Electron Trap 1 and the Fermi acceptor levels will participate in switching at the $1064 \mathrm{~nm}$ wavelength. The photoconductive switch will be a unipolar device at the $1064 \mathrm{~nm}$ wavelength excitation with the only carriers being electrons optically excited from Electron Trap 1. The rate equation for the number density of electrons in the conduction band is given in equation 7.34.

$$
\begin{aligned}
\frac{d n(t)}{d t}= & \sigma_{n T 1} v_{t h} N_{c T 1}\left(N_{T 10}-n(t)\right)+K \sigma_{o p T 1} I(t)\left(N_{T 10}-n(t)\right) \\
& -\sigma_{n T 1} v_{t h} n(t)\left(\frac{\sigma_{n V a}}{\sigma_{N T 1}} N_{v a}+\left(N_{T 1}-N_{T 10}\right)+n(t)\right)
\end{aligned}
$$

Where $\sigma_{\mathrm{NT1}}$ is the electron capture cross section of Electron Trap1, $\mathrm{N}_{\mathrm{cT} 1}$ is the effective conduction band density of states reduced to the Electron Trap 1 level, $\mathrm{N}_{\mathrm{T} 10}$ is the number density of electron occupied Electron Trap 1 levels prior to optical excitation, $\mathrm{N}_{\mathrm{T} 1}$ is the total number density of Electron Trap 1 levels, $\sigma_{\text {opT1 }}$ is the optical transition cross section for an 
electron excited from Electron Trap 1 to the conduction band, $\sigma_{\mathrm{nVa}}$ is the vanadium acceptor electron capture cross section and $\mathrm{N}_{\mathrm{va}}$ is the vanadium acceptor level number density.

The first two terms on the RHS of equation 7.34 are the thermal and optical generation terms. The generation terms only include contributions from the Electron Trap 1 level. This is because we have assumed that there are no electrons residing in the vanadium acceptor levels. The third term on the RHS of equation 7.24 includes electron capture terms from both the vanadium acceptor and Electron Trap 1 levels. The vanadium acceptor electron capture term must be included despite the fact that the Electron Trap 1 level has a much larger electron capture cross section. This is due to the much greater number of empty vanadium acceptor levels $\left(1.75 \times 10^{17} \mathrm{~cm}^{-3}\right)$ compared to the number of empty Electron Trap 1 levels $\left(\sim 2.5 \times 10^{15} \mathrm{~cm}^{-3}\right)$. We can rearrange terms in equation 7.34 to put it in the form of equation 7.22.

$$
\begin{aligned}
\frac{d n(t)}{d t}=N_{T 10} & \left(\sigma_{n T 1} v_{t h} N_{c T 1}+K \sigma_{o p T 1} I(t)\right) \\
& \quad-\sigma_{n T 1} v_{t h} n(t)\left(\frac{\sigma_{n v a}}{\sigma_{n T 1}} N_{v a}+\left(N_{T 1}-N_{T 10}\right)+n(t)+N_{c T 1}+\frac{K \sigma_{o p T 1} I(t)}{\sigma_{n T 1} v_{t h}}\right)
\end{aligned}
$$

We can extract the effective carrier decay time from equation 7.35. The effective carrier decay time is given by equation 7.36. The $\mathrm{N}_{\mathrm{cT} 1}$ term is negligible compared to the number density of empty Electron Trap 1 Levels $\left(\mathrm{N}_{\mathrm{T} 1}-\mathrm{N}_{\mathrm{T} 10}\right)$. We can neglect the $\mathrm{n}(t)$ and $\mathrm{I}(t)$ terms compared to the electron capture terms at low optical intensity. The effective carrier decay at low optical intensity is given in equation 7.37.

$$
\tau_{e f f T 1}=\sigma_{n T 1} v_{t h}\left(\frac{\sigma_{n v a}}{\sigma_{n T 1}} N_{v a}+\left(N_{T 1}-N_{T 10}\right)+n(t)+N_{c T 1}+\frac{\beta K \sigma_{o p T 1} I(t)}{\sigma_{n T 1} v_{t h}}\right)
$$




$$
\tau_{e f f T 1}=\sigma_{n T 1} v_{t h}\left(\frac{\sigma_{n v a}}{\sigma_{n T 1}} N_{v a}+\left(N_{T 1}-N_{T 10}\right)\right)
$$

The low intensity effective carrier decay time can be calculated be substituting the values of $\sigma_{\mathrm{nT} 1}\left(7.9 \times 10^{-14} \mathrm{~cm}^{2}\right), \sigma_{\mathrm{nva}}\left(5.77 \times 10^{-16} \mathrm{~cm}^{2}\right), \mathrm{N}_{\mathrm{va}}\left(1.75 \times 10^{17} \mathrm{~cm}^{-3}\right)$ and the number of empty Electron Trap 1 levels $-\mathrm{N}_{\mathrm{T} 1}-\mathrm{N}_{\mathrm{NT10}}\left(2.5 \times 10^{15} \mathrm{~cm}^{-3}\right)$. The room temperature value of the low intensity effective carrier decay time is 287 ps. This value of effective carrier decay time is much less than the 960 ps value calculated for the rate equation that included only the effects of the vanadium, nitrogen and boron impurities. The decrease in carrier decay time is the result of including the Electron Trap 1 levels. The large electron capture cross section makes the Electron Trap 1 levels more efficient at trapping electrons, even though the Electron Trap 1 number density is two orders of magnitude lower than the vanadium acceptor number density.

The effective low intensity decay time calculated from the rate equation including the effects of Electron Trap 1 is 3.35 times smaller than the decay time calculated for the model including the effects of impurities only. The smaller carrier decay time is the result of the empty trap levels. The carrier decay value including the effective trap level has decreased to a value much closer to the measured recombination time of 140 ps [7.14]. This is further indication that the measured 140 ps recombination time was made on a trap-dominated material.

We can assume that the thermal generation and capture terms are negligible compared to the optical generation and trapping terms in equation (7.35). In addition, the electron density term located within the parenthesis on the RHS of equation is negligible compared to the density of empty Electron Trap 1 and empty vanadium acceptors. Removing these terms from equation (7.35) yields equation (7.38). 


$$
\begin{array}{rl}
\frac{d n(t)}{d t}=N_{T 10} & K \sigma_{o p T 1} I(t) \\
& -\sigma_{n T 1} v_{t h} n(t)\left(\frac{\sigma_{n v a}}{\sigma_{n T 1}} N_{v a}+\left(N_{T 1}-N_{T 10}\right)+\frac{K \sigma_{o p T 1} I(t)}{\sigma_{n T 1} v_{t h}}\right)
\end{array}
$$

Equation 7.38 can be put into a non-dimensionalized form by making the following substitutions:

$$
\begin{gathered}
n^{*}(t)=\frac{n(t)}{N_{T 10}} \\
t^{*}=\frac{t}{T_{0}} \\
T_{0}=\frac{1}{\sigma_{n T 1} v_{t h}\left(\frac{\sigma_{n v a}}{\sigma_{n T 1}} N_{v a}+\left(N_{T 1}-N_{T 10}\right)\right)}
\end{gathered}
$$

Equation 7.42 is the non-dimensionalized form of equation 7.38.

$$
\begin{aligned}
\frac{\partial n^{*}\left(t^{*}\right)}{\partial t^{*}} & =\frac{K \sigma_{o p T 1} I\left(t^{*}\right)}{\sigma_{n T 1} v_{t h}\left(\frac{\sigma_{n v a}}{\sigma_{n T 1}} N_{v a}+\left(N_{T 1}-N_{T 10}\right)\right)} \\
& -n^{*}\left(\begin{array}{c}
\left.1+\frac{K \sigma_{o p T 1} I\left(t^{*}\right)}{\sigma_{n T 1} v_{t h}\left(\frac{\sigma_{n v a}}{\sigma_{n T 1}} N_{v a}+\left(N_{T 1}-N_{T 10}\right)\right)}\right)
\end{array}\right)
\end{aligned}
$$

With the initial condition of $\mathrm{n}^{*}\left(\mathrm{t}^{*}=0\right)=0$

The parameter values for equation 7.42 are listed in Table 7.5 and $\mathrm{K}$ is given in equation 7.24. 


\begin{tabular}{|c|c|c|}
\hline Variable/Parameter & Definition & Value \\
\hline $\mathrm{n}(t)$ & Electrons in conduction band & \\
\hline$\sigma_{\text {nva }}$ & $\begin{array}{c}\text { Vanadium acceptor level } \\
\text { electron capture cross section }\end{array}$ & $5.77 \mathrm{e}-16 \mathrm{~cm}^{2}$ \\
\hline$\sigma_{\mathrm{nt} 1}$ & $\begin{array}{l}\text { Electron Trap } 1 \text { electron } \\
\text { capture cross section }\end{array}$ & $7.9 \mathrm{e}-14 \mathrm{~cm}^{2}$ \\
\hline$v_{\text {th }}$ & Electron thermal velocity & $1.16796 \mathrm{e} 7 \mathrm{~cm} / \mathrm{s}$ \\
\hline $\mathrm{N}_{c t 1}$ & $\begin{array}{l}\text { Effective conduction band } \\
\text { density of states reduced to the } \\
\text { Electron Trap } 1 \text { level }\end{array}$ & $1.4193 \mathrm{e} 3 \mathrm{~cm}^{-3}$ \\
\hline$N_{n}$ & Nitrogen density & $6.375 \mathrm{e} 16 \mathrm{~cm}^{-3}$ \\
\hline $\mathrm{N}_{\mathrm{b}}$ & Boron density & $4.325 \mathrm{e}^{2} 6 \mathrm{~cm}^{-3}$ \\
\hline $\mathrm{N}_{\mathrm{T} 1}$ & Electron Trap 1 density & $5 \mathrm{e} 15 \mathrm{~cm}^{-3}$ \\
\hline $\mathrm{N}_{\mathrm{T} 10}$ & $\begin{array}{c}\text { Density of Electron Trap } 1 \\
\text { levels occupied by an electron }\end{array}$ & $2.5 \mathrm{e} 15 \mathrm{~cm}^{-3}$ \\
\hline$\sigma_{\mathrm{opT} 1}$ & $\begin{array}{l}\text { Electron Trap } 1 \text { optical } \\
\text { transition cross section }\end{array}$ & $1 \mathrm{e}-17 \mathrm{~cm}^{2}$ \\
\hline $\mathrm{I}(t)$ & Applied optical power & 1.8 MW maximum \\
\hline $\mathrm{N}_{\mathrm{va}}$ & Vanadium acceptor density & $1.75 \mathrm{e} 17 \mathrm{~cm}^{-3}$ \\
\hline Ld & Switch facet area & $0.04 \mathrm{~cm}^{2}$ \\
\hline$\eta$ & Quantum efficiency & 0.1 \\
\hline $\mathrm{r}$ & Reflectance & 0.804 \\
\hline$\alpha$ & $\begin{array}{c}\text { Total optical absorption of } \\
6 \mathrm{H}-\mathrm{SiC} \text { substrate }\end{array}$ & $0.8 \mathrm{~cm}^{-1}$ \\
\hline$\hbar \omega$ & Photon energy at $1064 \mathrm{~nm}$ & 1.87434e-19 Joules \\
\hline
\end{tabular}

Table 7.5. Parameter values for equations 7.38 and 7.42

$\mathrm{I}(\mathrm{t})$ is the optical power applied to the switch facet. Equation 7.43 gives the assumed temporal pulse for I(t).

$$
I(t)=\frac{4 I_{0} e\left[-\left(\frac{t-b}{c}\right)\right]}{\left(1+e\left[-\left(\frac{t-b}{c}\right)\right]\right)^{2}}
$$

Where $\mathrm{I}_{0}$ is the peak optical power, $\mathrm{b}$ is $17.0 \mathrm{~ns}$ and $\mathrm{c}$ is $1.98554 \mathrm{~ns}$. The values of $\mathrm{b}$ and $\mathrm{c}$ were chosen so that the pulse center (17.0 ns) and full width half maximum (FWHM) (7 ns) matched the $1064 \mathrm{~nm}$ optical pulses applied to the silver switch facet. Similarly, the maximum applied optical power was taken from optical waveforms applied to the facet of the silver switch. A plot 
of $\mathrm{I}(\mathrm{t})$ is shown in Figure 7.19. $\mathrm{I}(\mathrm{t})$ can be transformed into $\mathrm{I}^{*} \mathrm{t}^{*}$ by dividing $\mathrm{b}$ and c by $\mathrm{T}_{0}(287$ ps). The transformation of $\mathrm{I}(\mathrm{t})$ into $\left.\mathrm{I} \mathrm{t}^{*}\right)$ results in values of $\mathrm{b}^{*}$ and $\mathrm{c}^{*}$ equal to 59.233 and 6.918, respectively.

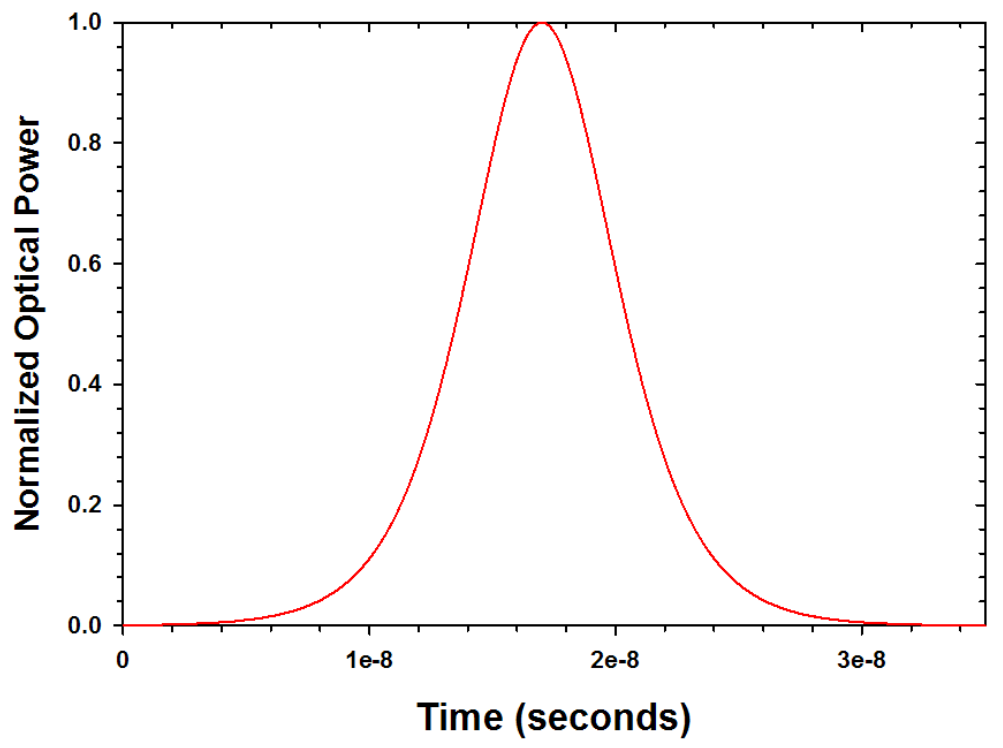

Figure 7.19. Temporal plot of optical pulse used in modeling $6 \mathrm{H}-\mathrm{SiC}$ switch at $1064 \mathrm{~nm}$.

MATLAB [7.15] is used to solve for $\mathrm{n}^{*}\left(\mathrm{t}^{*}\right)$ in equation 7.42 for peak applied optical powers ranging from 0.12 to $1.8 \mathrm{MW}$. Values of the electron density as a function of time are extracted from the solutions of the normalized equation and are shown in Figure 7.20. This model will be referred to as the Low Doping Model (LD). Electron density results for the LD model are slightly lower than electron densities calculated from measurements of silver switch on resistance at $1064 \mathrm{~nm}$.

Figure 7.21 is a plot comparing the model electron density calculation to the applied optical waveform. The model result for the peak value of electron density lags the peak applied optical applied power by 292 picoseconds and its pulse width has increased by 129 picoseconds compared to the applied optical pulse. This is to be expected due to the electron decay time of 287 picoseconds $\left(\mathrm{T}_{0}\right)$. It is also clear that the assumption that the temporal pulse of the electron 
density, and hence the photocurrent, in the switch will follow the temporal shape of the optical pulse is reasonable.

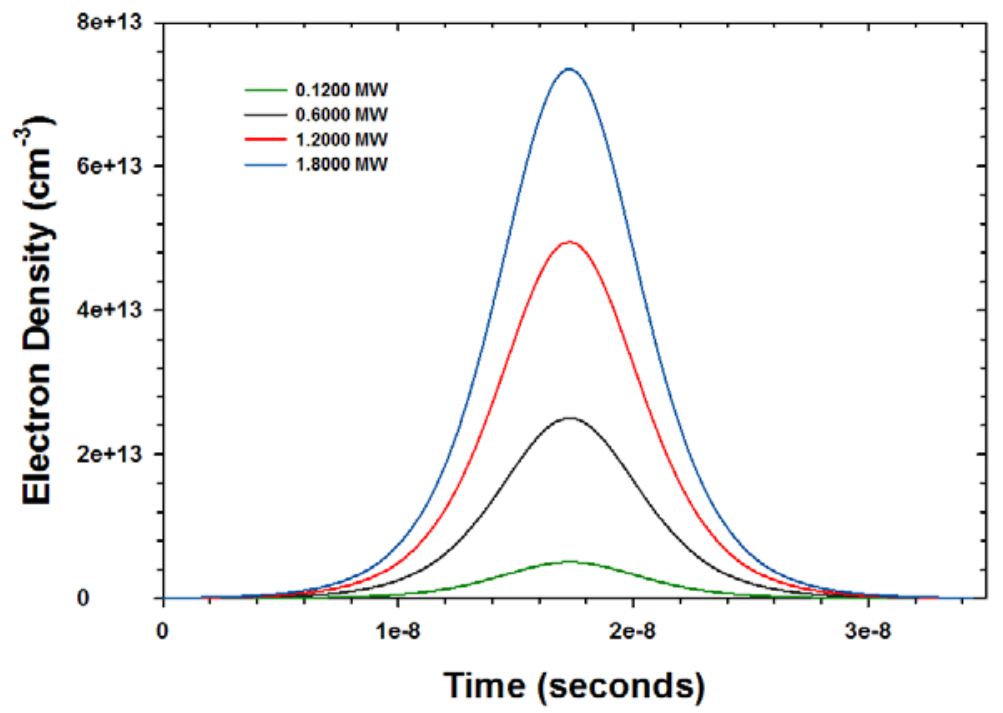

Figure 7.20. Plots of calculated model results for electron density for $\mathbf{6 H}-\mathrm{SiC}$ material with Fermi level pinned at an electron trap (Electron Trap 1) located at $1.0 \mathrm{eV}$ below the conduction band

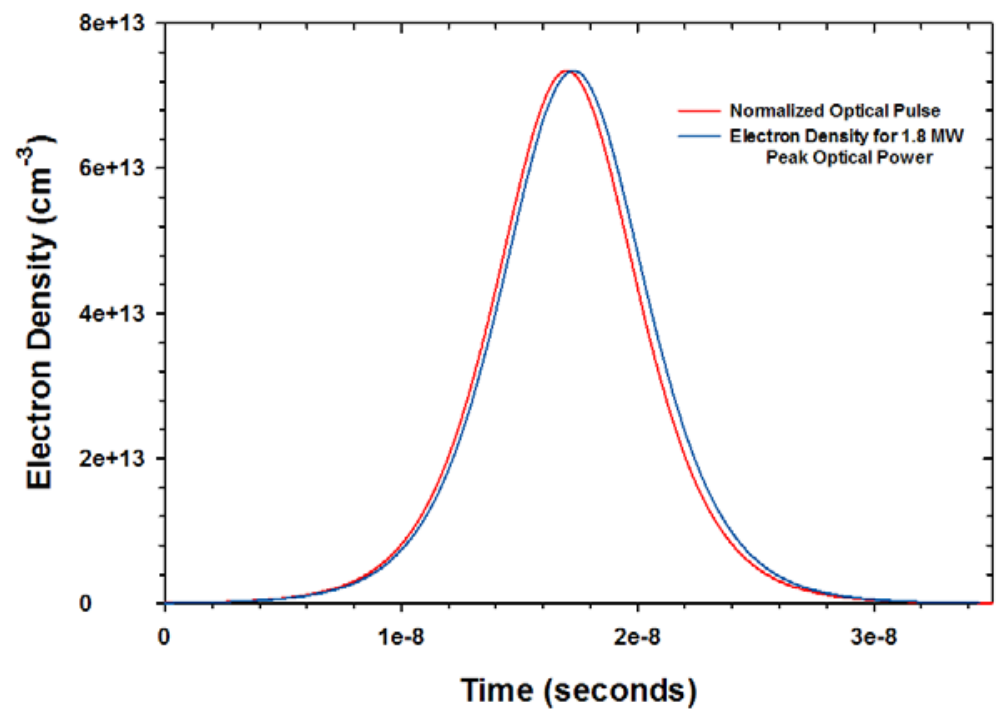

Figure 7.21. Calculated electron density temporal pulse for a peak applied optical power of 1.8 MW is compared to normalized optical pulse.

The peak electron density is plotted versus the peak applied optical power from 0.12 to 1.8 MW in Figure 7.22. The electron density increases linearly with peak optical power which 
should be expected since the peak electron densities are a small fraction of the $2.5 \mathrm{e} 15 \mathrm{~cm}^{-3}$ electrons residing in Electron Trap 1 levels. The electron density increases from 5.04e12 to $7.35 \mathrm{e} 13 \mathrm{~cm}^{-3}$ in Figure 7.22 which corresponds to a decrease in switch on resistance from 495 to 34 Ohms.

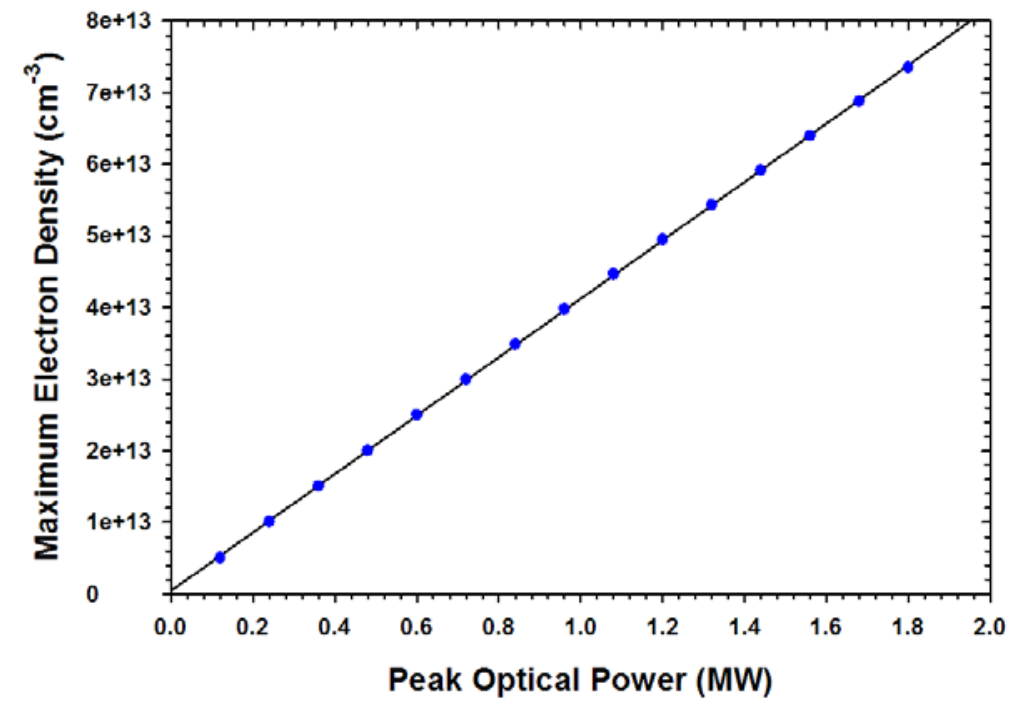

Figure 7.22. Calculated peak electron density plotted as a function peak applied optical power for the LD model at $1064 \mathrm{~nm}$.

The agreement between the LD model and the silver switch measurements are good at low optical intensity. The switch resistance predicted by the LD model at low optical intensity $(\leq$ $0.5 \mathrm{MW}$ ) are about 12 - $15 \%$ higher then the measured minimum resistances for the silver switch. This indicates that there are slightly more available electrons to excite into the conduction band at $1064 \mathrm{~nm}$ in the silver switch material at low optical intensities at $1064 \mathrm{~nm}$. This is most likely the result of perhaps a slightly lower density of deep electron traps in the $6 \mathrm{H}$ SiC switch material possibly resulting in a higher density of occupied Electron Trap 1 levels and vanadium acceptor levels occupied by electrons. The switch resistance predicted by the LD model is $50-70 \%$ higher at the highest applied optical intensities. This is a result of of the 
supralinear increase of switch conductivity measured at high intensities for the silver switch (Figure 5.52). The supralinear increase in the silver switch conductivity is most likely the result of the electron decay time increasing at higher optical intensities at $1064 \mathrm{~nm}$.

The LD model can then serve as a worst case which we can compare to measured results and a best case model. The best case model would be constructed for the the case of high nitrogen and vanadium doping outlined in Chapter 6 section 6.2 .

\subsection{3) Switch Model at $1064 \mathrm{~nm}$ for Increased Nitrogen and Vanadium Doping}

This model is based on the increased nitrogen and vanadium doping levels described in Chapter 6. The reasoning in Chapter 6 called for the complete removal of shallow boron acceptors while increasing the nitrogen and vanadium densities. The upper limit of the nitrogen and vanadium densities is bounded by the vanadium solubility level in $6 \mathrm{H}-\mathrm{SiC}$ and the requirement that the ratio of vanadium to nitrogen is sufficient to maintain a stable, semiinsulating, $6 \mathrm{H}-\mathrm{SiC}$ material. The vanadium and nitrogen densities were selected to be $2.5 \mathrm{e} 17$ and $1.25 \mathrm{e} 17 \mathrm{~cm}^{-3}$ based on this reasoning. As a result we will refer to this model as the High Doping Model (HD). Our HD model also includes the effects of deep traps and the concession that the boron density will be reduced, but not completely removed. Table 7.6 lists the parameters of importance for the HD model. 


\begin{tabular}{|c|c|c|}
\hline Variable/Parameter & Definition & Value \\
\hline $\mathrm{n}(t)$ & Electrons in conduction band & \\
\hline$\sigma_{\text {nva }}$ & $\begin{array}{l}\text { Vanadium acceptor level } \\
\text { electron capture cross section }\end{array}$ & $5.77 \mathrm{e}-16 \mathrm{~cm}^{2}$ \\
\hline$\sigma_{\text {nt1 }}$ & $\begin{array}{l}\text { Electron Trap } 1 \text { electron } \\
\text { capture cross section }\end{array}$ & $7.9 \mathrm{e}-14 \mathrm{~cm}^{2}$ \\
\hline$v_{t h}$ & Electron thermal velocity & $1.16796 \mathrm{e} 7 \mathrm{~cm} / \mathrm{s}$ \\
\hline $\mathbf{N}_{c t 1}$ & $\begin{array}{l}\text { Effective conduction band } \\
\text { density of states reduced to the } \\
\text { vanadium acceptor level }\end{array}$ & $1.4193 \mathrm{e} 3 \mathrm{~cm}^{-3}$ \\
\hline $\mathrm{N}_{\mathrm{n}}$ & Nitrogen density & $1.25 \mathrm{e} 17 \mathrm{~cm}^{-3}$ \\
\hline $\mathrm{N}_{\mathrm{b}}$ & Boron density & $1 \mathrm{e} 16 \mathrm{~cm}^{-3}$ \\
\hline $\mathrm{N}_{\mathrm{T} 1}$ & Electron Trap 1 density & $5 \mathrm{e} 15 \mathrm{~cm}^{-3}$ \\
\hline $\mathrm{N}_{\mathrm{T} 10}$ & $\begin{array}{c}\text { Density of Electron Trap } 1 \\
\text { levels occupied by an electron }\end{array}$ & $5 \mathrm{e} 15 \mathrm{~cm}^{-3}$ \\
\hline$\Sigma \mathrm{N}_{\mathrm{DT}}$ & Total deep trap density & $2 \mathrm{e} 16 \mathrm{~cm}^{-3}$ \\
\hline$\sigma_{\mathrm{opT} 1}$ & $\begin{array}{c}\text { Electron Trap } 1 \text { optical } \\
\text { transition cross section at } 1064 \\
\mathrm{~nm}\end{array}$ & $1 \mathrm{e}-17 \mathrm{~cm}^{2}$ \\
\hline$\sigma_{\text {opnva }}$ & $\begin{array}{c}\text { Vanadium acceptor level } \\
\text { optical transition cross section } \\
\text { at } 1064 \mathrm{~nm}\end{array}$ & $1 \mathrm{e}-17 \mathrm{~cm}^{2}$ \\
\hline $\mathrm{I}(t)$ & Applied optical power & 1.8 MW maximum \\
\hline $\mathrm{N}_{\mathrm{va}}$ & Vanadium acceptor density & $2.5 \mathrm{e} 17 \mathrm{~cm}^{-3}$ \\
\hline $\mathrm{N}_{\text {vao }}$ & $\begin{array}{l}\text { Density of vanadium } \\
\text { acceptors occupied by } \\
\text { electrons }\end{array}$ & $0.95 \mathrm{e} 17 \mathrm{~cm}^{-3}$ \\
\hline Ld & Switch facet area & $0.04 \mathrm{~cm}^{2}$ \\
\hline$\eta$ & Quantum efficiency & 0.1 \\
\hline $\mathrm{r}$ & Reflectance & 0.804 \\
\hline$\alpha$ & $\begin{array}{c}\text { Total optical absorption of } \\
6 \mathrm{H}-\mathrm{SiC} \text { substrate }\end{array}$ & $0.8 \mathrm{~cm}^{-1}$ \\
\hline$\hbar \omega$ & Photon energy at $1064 \mathrm{~nm}$ & 1.87434e-19 Joules \\
\hline
\end{tabular}

Table 7.6. Parameter values for increased nitrogen and vanadium $6 \mathrm{H}$-SiC material

The HD model for the $6 \mathrm{H}$-SiC material with increased nitrogen and vanadium densities makes the following assumptions:

1) Vanadium acceptor density is $2.5 \mathrm{e} 17 \mathrm{~cm}^{-3}\left(\mathrm{~N}_{\mathrm{VA}}\right)$.

2) Nitrogen density is $1.25 \mathrm{e} 17 \mathrm{~cm}^{-3}\left(\mathrm{~N}_{\mathrm{N}}\right)$. 
3) Deep trap density is $2 \mathrm{e} 16 \mathrm{~cm}^{-3}\left(\Sigma \mathrm{N}_{\mathrm{TD}}\right)$.

4) Boron density is $1 \mathrm{e} 16 \mathrm{~cm}^{-3}\left(\mathrm{~N}_{\mathrm{B}}\right)$.

5) Nitrogen density is high enough so that Electron Trap 1 is completely filled. The Fermi level is located a few kT above the Electron Trap 1 level.

6) The effective donor density is $\mathrm{N}_{N}-\mathrm{N}_{\mathrm{B}}-\Sigma \mathrm{N}_{\text {DT }}$.

7) The number of vanadium acceptor levels occupied by electrons at thermal equilibrium is equal to the effective donor density $\left(0.95 \mathrm{e} 17 \mathrm{~cm}^{-3}\right)$.

8) The optical transition cross sections for the vanadium acceptor level and Electron Trap Level 1 are equal $\left(1 \mathrm{e}-17 \mathrm{~cm}^{2}\right)$.

9) Electrons optically excited from Electron Trap Level 1 represent only five percent of the total electron density, so they will be assumed negligible.

10) Recombination at Electron Trap Level 1 can only take place after electrons are excited from this level. It will be assumed that electrons excited from Electron Trap Level 1 are rapidly trapped trapped back at Electron Trap Level 1.

11) Thermal generation and recombination terms can be neglected while light is being applied to the switch.

12) The optical transition cross sections for the vanadium acceptor level and Electron Trap Level 1 are equal $\left(1 \mathrm{e}-17 \mathrm{~cm}^{2}\right)$.

Rate equation 7.44 can be written for the electron density in the conduction band based on the above assumptions.

$$
\begin{aligned}
\frac{d n(t)}{d t}=K \sigma_{o p n v a} I(t)\left(N_{N}-N_{B}\right. \\
\left.\quad-\Sigma N_{D T}\right) \\
\quad-\sigma_{n v a} v_{t h} n(t)\left(N_{v a}-\left(N_{N}-N_{B}-\Sigma N_{D T}\right)+\frac{K \sigma_{o p T 1} I(t)}{\sigma_{n T 1} v_{t h}}\right)
\end{aligned}
$$




$$
N_{\text {vao }}=\left(N_{N}-N_{B}-\Sigma N_{D T}\right)
$$

Equation 7.44 can be normalized by making the following substitutions:

$$
\begin{gathered}
n^{\wedge}(t)=\frac{n(t)}{N_{v a o}} \\
t^{\wedge}=\frac{t}{T_{0}} \\
T_{0}=\frac{1}{\sigma_{n v a} v_{t h}\left(N_{v a}-N_{v a o}\right)} \\
\frac{\partial n^{\wedge}\left(t^{\wedge}\right)}{\partial t^{\wedge}}=\frac{K \sigma_{o p T 1} I\left(t^{\wedge}\right)}{\sigma_{n v a} v_{t h}\left(N_{v a}-N_{v a o}\right)}-n^{\wedge}\left(1+\frac{K \sigma_{o p T 1} I\left(t^{\wedge}\right)}{\sigma_{n v a} v_{t h}\left(N_{v a}-N_{v a o}\right)}\right)
\end{gathered}
$$

The normalized conduction band electron density (equation 7.49) is solved using MATLAB [7.15] with the initial condition of $n^{\wedge}\left(t^{\wedge}\right)=0$. The applied optical power is again assumed to be given by equation 7.43. The electron densities calculated using the HD model for a range of applied optical powers is shown in Figure 7.23. The calculated electron density

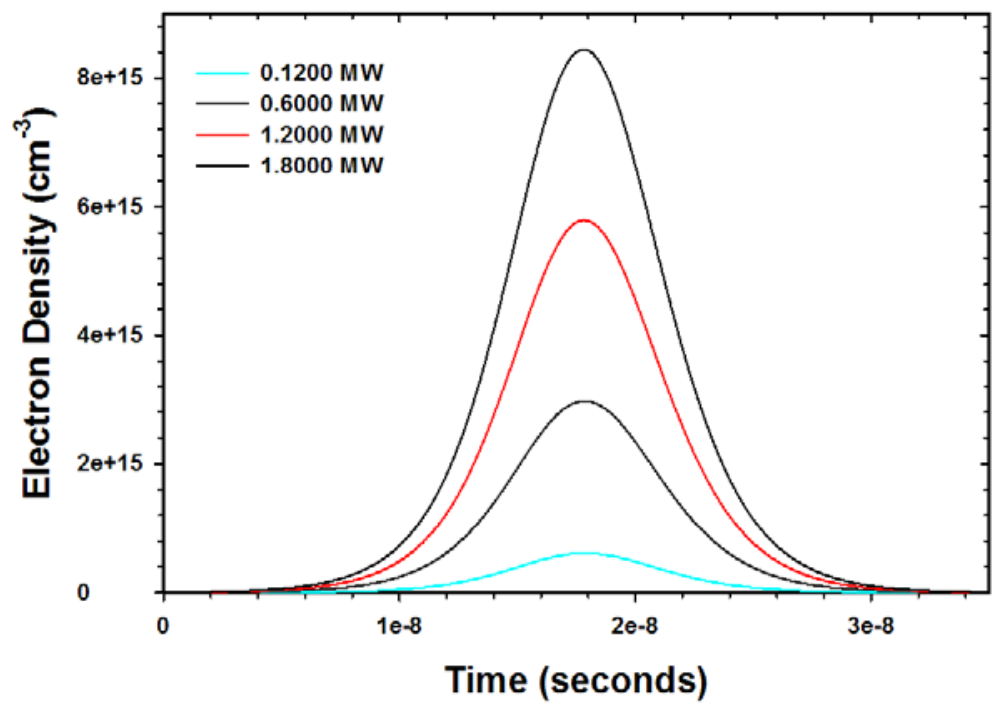

Figure 7.23. Electron densities calculated using the HD model for a range of peak applied optical powers of 0.12 to $1.8 \mathrm{MW}$. 
temporal pulse closely resembles the applied optical pulse and increases linearly with optical power. A direct comparison of the applied optical pulse and calculated electron density using the HD model is shown in Figure 7.24. The electron density pulse lags the applied optical pulse by 806 picoseconds and has broadened by 600 picoseconds (FWHM) to 7.6 ns. The time lag and spread of the electron density pulse calculated with the HD model has increased compared to our initial model (low doping, or LD model). This is a result of the electron decay time, $\mathrm{T}_{0}$, increasing from 287 picoseconds for the LD model to 957 picoseconds for the HD model. The increase in $\mathrm{T}_{0}$ is a result in the increased nitrogen doping. The additional donated electrons from the increased nitrogen density fill the empty Electron Trap 1 levels and a large portion of the empty vanadium acceptors until the filled vanadium acceptor levels greatly outnumber the total Electron Trap 1 levels. The filled vanadium acceptor levels become the dominant source of electrons optically excited into the conduction band and the empty vanadium acceptor levels become the dominant electron trap. Hence, the electron decay time increases.

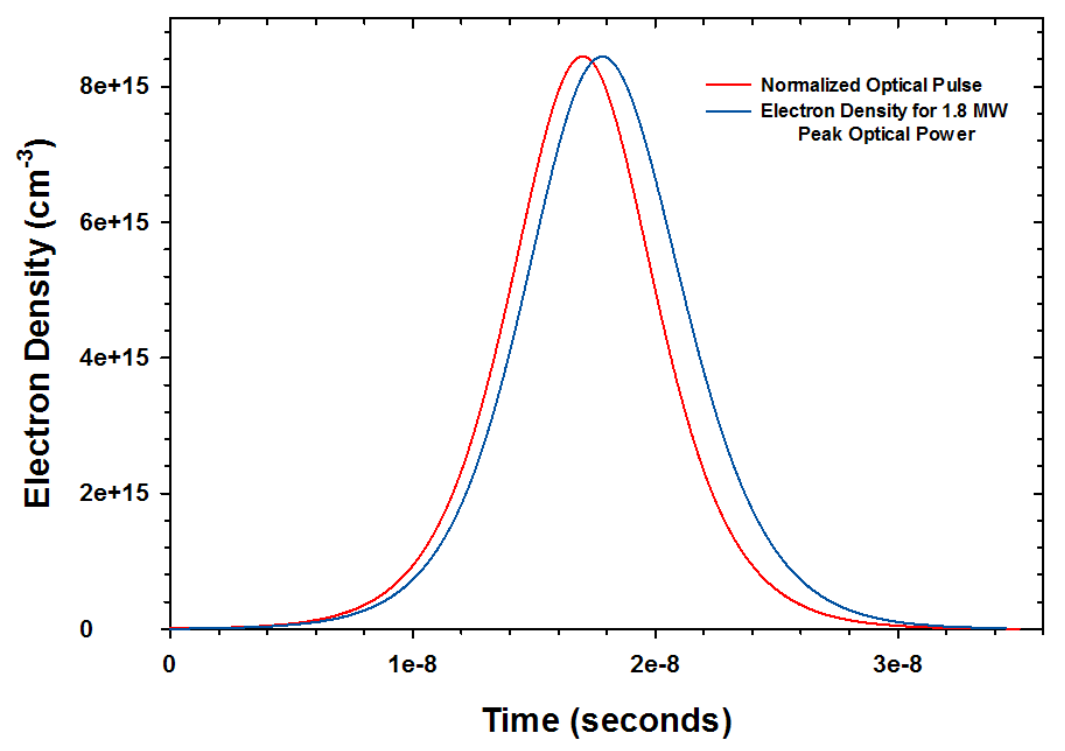

Figure 7.24. Comparison of an applied optical pulse and calculated electron density for the peak applied optical power of $1.8 \mathrm{MW}$ 
The number of electrons available for optical excitation into the conduction band has also increased in the HD model. The number of vanadium acceptor levels occupied by electrons is $0.95 \mathrm{e} 17 \mathrm{~cm}^{-3}$ for the HD model compared to $2.5 \mathrm{e} 15 \mathrm{~cm}^{-3}$ occupied Electron Trap 1 levels of the LD model. As a result, the number of electrons excited into the conduction band per watt of optical power at $1064 \mathrm{~nm}$ has greatly increased. Figure 7.25 is a plot of peak electron density calculated using the HD model plotted as a function of peak applied optical energy. The electron density calculated using the HD model is $~ 115$ times that calculated using the LD model This large increase is a direct result of increasing the number of electrons available for excitation into the conduction band and the increase in electron decay time. The electron densities plotted in Figure 7.25 correspond to the switch on resistance decreasing from 4.09 to 0.296 Ohms. The results of the HD model indicate that sub-ohm switch on resistances can be obtained for

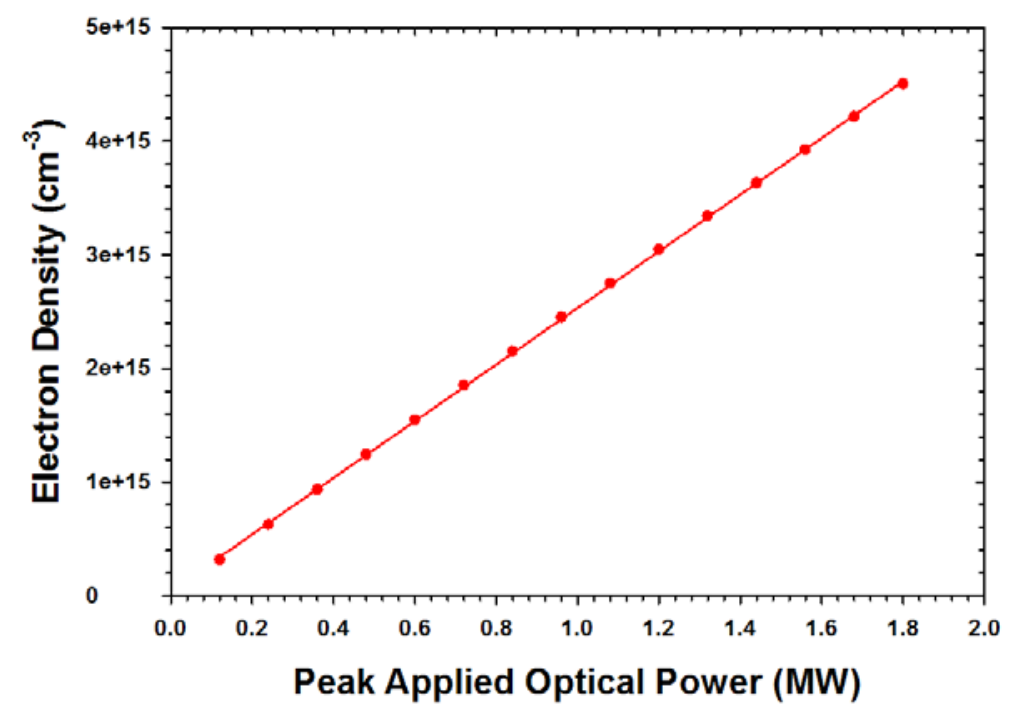

Figure 7.25. Peak electron density calculated using the HD model plotted as a function of peak applied optical power.

operation at $1064 \mathrm{~nm}$ if we increase the $6 \mathrm{H}-\mathrm{SiC}$ vanadium and nitrogen doping to $2.5 \mathrm{e} 17$ and $1.25 \mathrm{e} 17 \mathrm{~cm}^{-3}$, respectively. These simple models reinforce the intuitive argument of Chapter 6 for increasing the nitrogen and vanadium doping to increase quantum efficiency. The models 
indicate that along with increasing the quantum efficiency by increasing the nitrogen and vanadium doping, the electron decay time will also be increased - an added bonus.

The LD and HD models show that large improvements in switch on resistance can be obtained by increasing the nitrogen and vanadium densities as prescribed. However, the model results need to be compared to measured data. Figure 7.26 shows plots of the maximum conductivity calculated using the LD and HD models compared to switching results for the silver switch at $1064 \mathrm{~nm}$ as functions of peak optical intensity. The conductivity results for the silver switch lie much closer to the LD model results as expected. However, the difference between the LD model and measured silver switch conductivity increases with optical intensity. The HD model results show conductivities that are 100 times greater than that measured for the silver switch at low optical intensities. This factor decreases to 70 at high optical intensities. Large improvements in switch performance that can be obtained at $1064 \mathrm{~nm}$ by increasing the nitrogen and vanadium dopants to the prescribed levels.

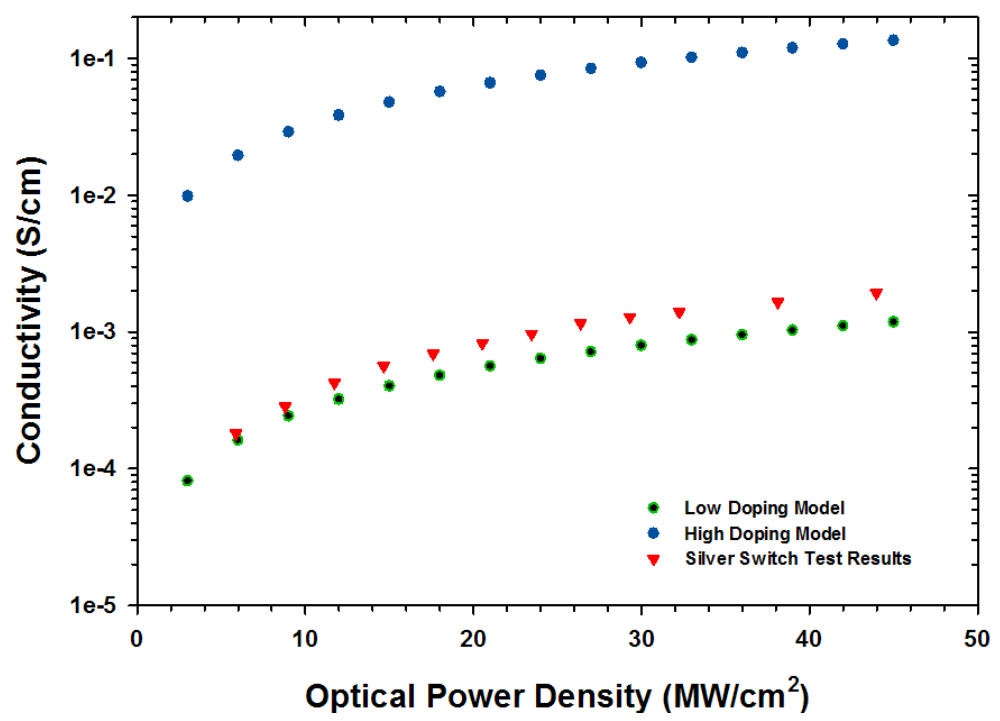

Figure 7.26. Maximum switch conductivity calculated using the LD and HD models is compared to switch results for the silver switch. 


\section{5) Conclusions}

Physical intuition and rate equation modeling both indicate that switch performance can be improved by factors of 70 to 100 over that of our initial $6 \mathrm{H}-\mathrm{SiC}$ switch material for operation at $1064 \mathrm{~nm}$. The LD and HD rate equation model results for switch operation at $1064 \mathrm{~nm}$ are applicable to switch operation at $532 \mathrm{~nm}$. Switch modeling at $1064 \mathrm{~nm}$ contains all the important physics resulting from increasing the nitrogen and vanadium densities to the prescribed levels. In fact, the rate equation models at $1064 \mathrm{~nm}$ allow us to focus on only the changes in operation brought about by the increased nitrogen and vanadium doping since the $1064 \mathrm{~nm}$ photons only reach $1.17 \mathrm{eV}$ into the bandgap. This allows us to include the vanadium acceptor level and electron traps lying just below the vanadium acceptor level in the models. In addition, the switch is a unipolar device at $1064 \mathrm{~nm}$ where only electrons take part in conduction. Modeling at 532 $\mathrm{nm}$ requires electron and hole rate equations to be constructed for levels lying within $2.34 \mathrm{eV}$ of the conduction band and the inclusion of generation terms that include both two - step optical transitions and two-photon absorption. Considerable difficulty is added to the calculation with no added clarity to how increasing the nitrogen and vanadium dopants improve switch performance.

Improved switch performance at $1064 \mathrm{~nm}$ indicates that switch performance at $532 \mathrm{~nm}$ will also improve. We expect that the optical transition cross section for electrons occupying vanadium acceptor levels to be larger at $532 \mathrm{~nm}$ compared to $1064 \mathrm{~nm}$. As a result, the optical generation rate of electrons from the vanadium acceptor level to the conduction band will increase at $532 \mathrm{~nm}$ compared to $1064 \mathrm{~nm}$. In addition, two-photon absorption and two-step optical transitions which occur at $532 \mathrm{~nm}$, but not $1064 \mathrm{~nm}$, produce electron - hole pairs and further reduce switch on resistance. We expect lower switch on resistances for operation at 532 $\mathrm{nm}$ compared to switch operation at $1064 \mathrm{~nm}$. However, modeling improvements in switch 
performance at $1064 \mathrm{~nm}$ for increases in nitrogen and vanadium densities is an excellent indicator of how switch performance will improve at $532 \mathrm{~nm}$.

The next chapter compares three generations of $6 \mathrm{H}-\mathrm{SiC}$ switch materials. The first generation of $6 \mathrm{H}-\mathrm{SiC}$ material is comprised of the green, black, and silver switches. The switching results for these switches is covered in Chapter 5 . The second and third generation $6 \mathrm{H}-$ $\mathrm{SiC}$ materials have increased nitrogen and vanadium densities compared to the first generation material. The nitrogen and vanadium densities along with the performance of switches constructed from second and third generation $6 \mathrm{H}-\mathrm{SiC}$ material is covered in Chapter 8.

\section{6) References}

7.1) G. Lucovsky, "On the photoionization of deep impurity centers in semiconductors”, Solid State Communications, Vol. 3, pp. 299 - 302, 1965

7.2) B. K. Ridley, "The photionization cross section of deep level impurities in semiconductors”, Journal of Physics C: Solid State Physics, Vol. 13, pp. 2015 - 2026, 1980

7.3) Z. -Q. Fang, et al, "Deep centers in semi-insulating Fe-doped native GaN substrates grown by hydride vapour phase epitaxy”, Physica Status Solidi C, Vol. 5, No. 6, pp. 1508 $-1511,2008$

7.4) A. Rosen and F. Zutavern, High-Power Optically Activated Solid State Switches, page 245, Artech House, 1994

7.5) W. C. Mitchel, Air Force Research Lab, Personal Communication

7.6) W. C. Mitchel, et al, "Electrical properties of unintentionally doped semi-insulating and conducting 6H-SiC”, Journal of Applied Physics, Vol. 100, Article 043706, 2006

7.7) P. Y. Yu and M. Cardona, Fundamentals of Semiconductors: Physics and Material Properties, Second Edition, Springer, 1999

7.8) S. Sasaki, K. Kawahara, G. Feng, G. Alfieri and T. Kimoto, "Major deep levels with the same mocrostructures observed in n-type $4 \mathrm{H}-\mathrm{SiC}$ and $6 \mathrm{H}-\mathrm{SiC}$ ", Journal of Applied Physics, Vol. 109, Paper 013705, 2011

7.9) C. Longneaud, J. Kleider, P. Kaminski, R. Koslowski and M. Miczuga, "Characterization of the defect levels in semi-insulating $6 \mathrm{H}-\mathrm{SiC}$ by means of photoiinduced transient spectroscopy and modulated photocurrent technique”, Journal of Physics: Condensed Matter, Vol. 21, Paper 045801, 2009

7.10) J. R. Jenny, et al, "High-purity semi-insulating 4H-SiC for microwave device applications”, Journal of Electronic Materials, Vol. 32, No. 5, pp. 432 - 436, 2003

7.11) W. C. Mitchel, et al, "Compensation mechanism in high purity semi-insulating 4H-SiC', Journal of Applied Physics, Vol. 101, Paper 053716, 2007 
7.12) P. Kaminski, et al, "Investigation of defect levels in 6H-SiC single crystals", Physica Status Solidi C, Vol. 4, No. 8, pp. 2967 - 2971, 2007

7.13) P. Kaminski, et al, "Compensating defect centres in semi-insulating 6H-SiC", Optoelectronics Review, Vol. 17, No. 1, pp. 1- 7, 2009

7.14) G. Tamulaitus, et al, "Carrier lifetime in conductive and vanadium-doped $6 \mathrm{H}-\mathrm{SiC}$ substrates”, Applied Physics Letters, Vol. 84, No. 3, pp. 335 - 337, 2004

7.15) $\quad$ MATLAB ${ }^{\circledR}$, www.mathworks.com 


\section{Chapter 8: Comparison of Switch Assemblies Fabricated with Improved Doping Profile 6H-SiC to Switches Fabricated with Initial 6H-SiC Material}

\section{1) Introduction}

Vanadium (V), nitrogen (N) and boron (B) are the dominant impurities in vanadium compensated, semi-insulating 6H-SiC. Carriers are photo-generated in vanadium compensated $6 \mathrm{H}-\mathrm{SiC}$ by exciting electrons captured by the vanadium acceptor levels into the conduction band by below bandgap light. The photoconductive switch tests of initial $6 \mathrm{H}-\mathrm{SiC}$ substrates presented in Chapter 5 demonstrated encouraging extrinsic photoconductivity with material that did not have a good doping profile of $\mathrm{V}, \mathrm{N}$ and $\mathrm{B}$. The doping profiles for the initial substrates that were used to construct switches are not known precisely, but SIMs measurements were performed on another substrate from the same batch of $6 \mathrm{H}-\mathrm{SiC}$ material. The dopant densities for this initial substrate are $\mathrm{V}=2.3 \mathrm{e} 17 \mathrm{~cm}^{-3}, \mathrm{~N}=6.6 \mathrm{e} 16 \mathrm{~cm}^{-3}$, and $\mathrm{B}=4.4 \mathrm{e} 16 \mathrm{~cm}^{-3}$ at one end of the substrate and $\mathrm{V}=1.4 \mathrm{e} 17 \mathrm{~cm}^{-3}, \mathrm{~N}=6.1 \mathrm{e} 16 \mathrm{~cm}^{-3}$, and $\mathrm{B}=4.4 \mathrm{e} 16 \mathrm{~cm}^{-3}$ at the opposite end of the substrate.

The intuitive argument presented in Chapter 6 and the analysis results of Chapter 7 both indicate that the extrinsic photoconductive switch performance of the vanadium and nitrogen doped $6 \mathrm{H}-\mathrm{SiC}$ can be greatly improved by increasing the densities of the vanadium and nitrogen and decreasing boron density in the $6 \mathrm{H}-\mathrm{SiC}$ crystal. We would like to have as high a vanadium density as possible and have a large fraction of the vanadium acceptor levels filled with electrons in the $6 \mathrm{H}-\mathrm{SiC}$. Unfortunately, there are physical considerations that limit this possibility. The maximum density of vanadium in $6 \mathrm{H}-\mathrm{SiC}$ is limited by its solubility. The solubility limit of

vanadium in $6 \mathrm{H}-\mathrm{SiC}$ is $3-5 \mathrm{e} 17 / \mathrm{cm}^{3}$ [8.1]. Above this density the vanadium precipitates out of the $6 \mathrm{H}-\mathrm{SiC}$ and forms inclusions in the crystal that can act as micropipe initiation sites. This 
establishes a practical upper limit of $<3 \mathrm{e} 17 / \mathrm{cm}^{3}$ for the vanadium density in $6 \mathrm{H}$-SiC for an extrinsic photoconductive switch material.

Nitrogen and boron are the remaining dopants in semi-insulating, vanadium compensated $6 \mathrm{H}-\mathrm{SiC}$. Boron is a shallow acceptor that captures electrons donated by the nitrogen levels. The boron acceptors limit the number of electrons available to be captured by the vanadium acceptors. As a result, we would like to remove all the boron and any other shallow acceptor from the semi-insulating $6 \mathrm{H}-\mathrm{SiC}$. This is not physically realizable in $6 \mathrm{H}-\mathrm{SiC}$ grown by the Physical Vapor Transport (PVT), since boron is an unintentional dopant present in the growth furnace materials. However, boron can be limited to below a density of $1 \mathrm{e} 16 / \mathrm{cm}^{3}$ using high purity materials during PVT growth.

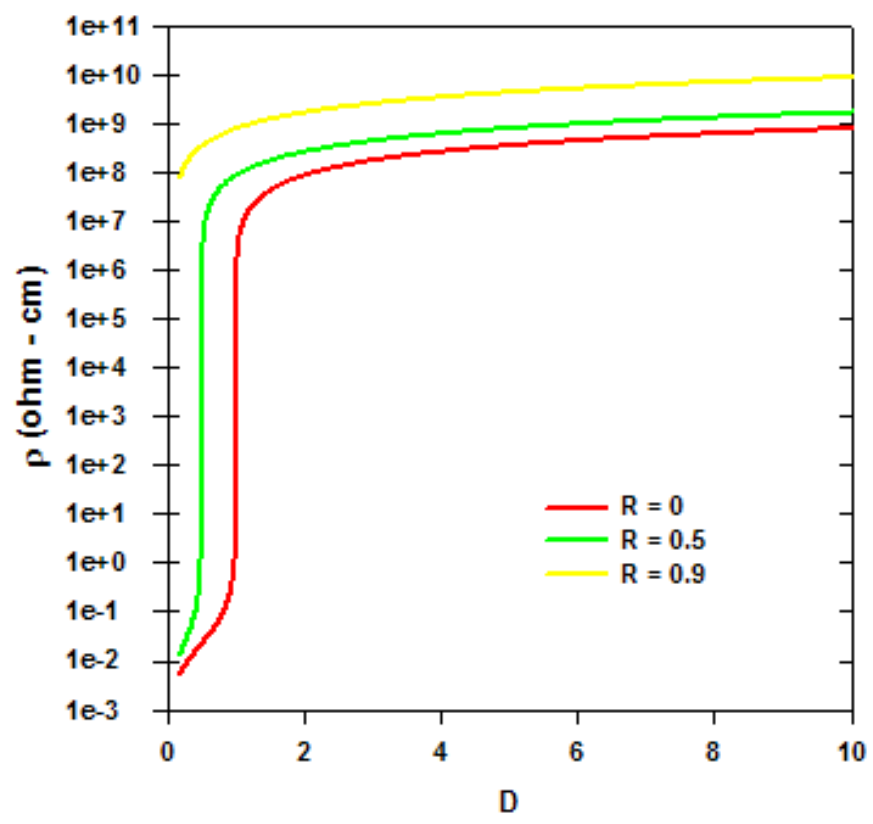

Figure 8.1. Resistivity of $6 \mathrm{H}$-SiC versus the ratio of vanadium to nitrogen (D) for different values of the ratio of boron to nitrogen.

We would like as much nitrogen as possible to fill the vanadium acceptor levels, while still maintaining the semi-insulating property of the $6 \mathrm{H}-\mathrm{SiC}$ substrates. We can obtain an initial estimate for the nitrogen density using Figure 8.1. Figure 8.1 is a plot of material resistivity for 
$6 \mathrm{H}-\mathrm{SiC}$ based on the requirement of charge neutrality in $6 \mathrm{H}-\mathrm{SiC}$ doped with vanadium, nitrogen and boron in thermal equilibrium at room temperature. The parameters $\mathrm{D}$ and $\mathrm{R}$ are the ratios of vanadium to nitrogen and boron to nitrogen, respectively. Stability of the $6 \mathrm{H}-\mathrm{SiC}$ resistivity requires that the ratio $\mathrm{D}$ be sufficiently greater than unity. We select a value of $\mathrm{D}$ equal to two as we did in Chapter 6. Semi-insulating $6 \mathrm{H}-\mathrm{SiC}$ doped with $\mathrm{V}=2.5 \mathrm{e} 17 / \mathrm{cm}^{3}, \mathrm{~N}=1.25 \mathrm{e} 17 / \mathrm{cm}^{3}$ and B $<1 \mathrm{e} 16 / \mathrm{cm}^{3}$ should show much improved extrinsic photoconductive switching response compared to our initial 6H-SiC material based upon the considerations listed above.

Changing the densities of the vanadium, nitrogen and boron can change other material properties [8.2] of the $6 \mathrm{H}-\mathrm{SiC}$ substrates besides the number of electrons residing in vanadium acceptor levels. The carrier recombination time, dislocation density, micropipe density and the electron and hole mobility can also change with dopant densities. The modeling results of Chapter 7 showed that we should also expect an increase in electron decay (recombination time) as the nitrogen doping is increased. Changes in the carrier recombination time and mobility can mask improvements in the quantum efficiency of the $6 \mathrm{H}-\mathrm{SiC}$ material. For example, if the carrier recombination time $\left(\tau_{\mathrm{r}}\right)$ decreases with increasing vanadium density due to an associated increase in intrinsic defects, the loss term $\left(n / \tau_{r}\right)$ in the rate equation (8.1) for the photogeneration of free electrons in the $6 \mathrm{H}-\mathrm{SiC}$ substrate increases with increasing vanadium density.

$$
\frac{\partial n(t)}{\partial t}=\frac{\eta^{*} P(t)}{W L d \hbar \omega}-\frac{n}{\tau_{r}}
$$

Where $\mathrm{P}(\mathrm{t})$ is the optical power applied to the switch facet, $\tau_{\mathrm{r}}$ is the electron recombination time, Ld is the illumination area of the switch facet, $\mathrm{W}$ is the length of the switch in the direction of optical propagation, $\hbar \omega$ is the photon energy in Joules and $\eta^{*}$ is the effective quantum efficiency. Equation (8.1) can be rewritten as (8.2) to explicitly include the optical absorption coefficient of 
the filled vanadium acceptor levels, if we assume that $\mathrm{V}, \mathrm{N}$, and $\mathrm{B}$ are the only impurities in the 6H-SiC material.

$$
\frac{\partial n}{\partial t}=\frac{\alpha_{V}(\hbar \omega) \eta^{*} P(t)}{L d \hbar \omega}-\frac{n}{\tau_{r}}
$$

Where $\alpha_{V}(\hbar \omega)$ is the optical absorption of the filled vanadium acceptor levels and we now define the effective quantum efficiency in equation (8.3).

$$
\eta^{*}=\eta(1-R)\left(1-e^{-\alpha W}\right)
$$

Here $\mathrm{R}$ is the optical reflectivity between air and $6 \mathrm{H}-\mathrm{SiC}$ substrate facet, $\alpha$ is the total optical absorption coefficient of the $6 \mathrm{H}-\mathrm{SiC}, \mathrm{W}$ is the length of the $6 \mathrm{H}-\mathrm{SiC}$ substrate in the direction of optical propagation and $\eta$ is the quantum efficiency of a photon exciting an electron from a filled vanadium acceptor level to the conduction band. The extrinsic optical absorption of the vanadium acceptor level is written as [8.3]

$$
\alpha_{v}(\hbar \omega)=N_{V n} \sigma(\hbar \omega)
$$

Where $N_{V n}$ is the density of vanadium acceptor levels occupied by electrons and $\sigma(\hbar \omega)$ is the phonon broadened photo-ionization cross section of the filled vanadium acceptor level. We assume that all electrons excited into the conduction band have come from filled vanadium acceptor levels, so the density of filled vanadium acceptor levels will be reduced by the density of electrons in the conduction band. The density of filled vanadium acceptor levels is given in equation (8.5).

$$
N_{V n}=N_{V n}^{*}-n=N_{N}-N_{B}-\Sigma N_{D T}-n
$$

Where $\mathrm{N}_{\mathrm{Vn}}{ }^{*}$ is the density of filled vanadium acceptor levels prior to illumination of the switch facet. Equation (8.2) is rewritten using equation (8.4) and (8.5).

$$
\frac{\partial n}{\partial t}=\frac{\left(N_{V n}^{*}-n\right) \sigma(\hbar \omega) \eta^{*} P(t)}{L d \hbar \omega}-\frac{n}{\tau_{r}}
$$


The first term on the RHS of equation (8.6) represents the generation rate of electrons excited from the filled vanadium acceptor levels into the conduction band per unit time. The terms included in the generation rate concerning switch geometry $(\mathrm{L}, \mathrm{d})$ and applied optical power and wavelength $(\mathrm{P}(\mathrm{t}), \hbar \omega)$ are selected during the switch design. The terms $\sigma(\hbar \omega)$ and $\eta$ are functions of the $6 \mathrm{H}-\mathrm{SiC}$ material and cannot be explicitly manipulated as part of the design. The term $\mathrm{N}_{\mathrm{Vn}}^{*}$ in the generation rate is the density of vanadium acceptor levels that are filled with electrons prior to illumination. This term can be controlled by the density of the vanadium, nitrogen and boron dopants. However, if the recombination time decreases with increase in the vanadium and nitrogen density the increase in the generation rate can be offset by an increase in the loss term and will result in minimal gain in switch performance.

\section{2) Substrate Doping Profile, Physical Dimensions and Micropipe Density for Second Generation, Vanadium and Nitrogen Doped 6H-SiC Material}

Four substrates of $6 \mathrm{H}-\mathrm{SiC}$ were obtained that had improved doping levels. These four substrates constitute the second generation of $6 \mathrm{H}-\mathrm{SiC}$ material for the extrinsic photoconductive switch application. The four substrates were cut from the a-plane of the same crystal of $6 \mathrm{H}-\mathrm{SiC}$. SIMs measurements performed on the crystal put the doping profile at $\mathrm{V}=3.5 \mathrm{e} 17 \mathrm{~cm}^{-3}, \mathrm{~N}=$ $1.5 \mathrm{e} 17 \mathrm{~cm}^{-3}$, and $\mathrm{B}<1.0 \mathrm{e} 16 \mathrm{~cm}^{-3}$ at one end of the substrates and $\mathrm{V}=1.0 \mathrm{e} 17 \mathrm{~cm}^{-3}, \mathrm{~N}=1.0 \mathrm{e} 17$ $\mathrm{cm}^{-3}$, and $\mathrm{B}<1.0 \mathrm{e} 16 \mathrm{~cm}^{-3}$ at the opposite end of the substrates along the c-plane. The variation in the vanadium and nitrogen density across the $6 \mathrm{H}-\mathrm{SiC}$ substrate is a result of the decay of these two dopants during crystal growth. The density of $\mathrm{V}$ and $\mathrm{N}$ will be at a maximum during early stages of the $6 \mathrm{H}-\mathrm{SiC}$ crystal growth and will decrease along the direction of crystal growth. The dopant decay during crystal growth manifests itself as a variation in dopants across the $6 \mathrm{H}-\mathrm{SiC}$ 
a-plane substrates since the $6 \mathrm{H}-\mathrm{SiC}$ is grown in the c direction of the crystal. The substrates with improved doping profile are nominally $1.0 \mathrm{~cm}$ by $1.0 \mathrm{~cm}, 1 \mathrm{~mm}$ thick. The four substrates are designated A, B, C, and D. All four substrates measured $0.958 \mathrm{~mm}$ thick. The length and width measurements for the substrates A (0.995 by 0.997$)$, B (1.00 by 1.001$), C$ (1.002 by 1.01$)$, and D (1.003 by 0.997$)$ are given in centimeters and enclosed in the parentheses. The second term in the parentheses is the width of the substrate in the c direction of the crystal. The substrates A, B, C, and D have polished facets on the c faces of the substrates. The remaining two facets were ground to a planar finish. The initial $400 \mu \mathrm{m}$ thick substrates have cleaved facets. Figures 8.2 and 8.3 show the polished and ground facets of substrate A. Figures 8.4 and 8.5 are

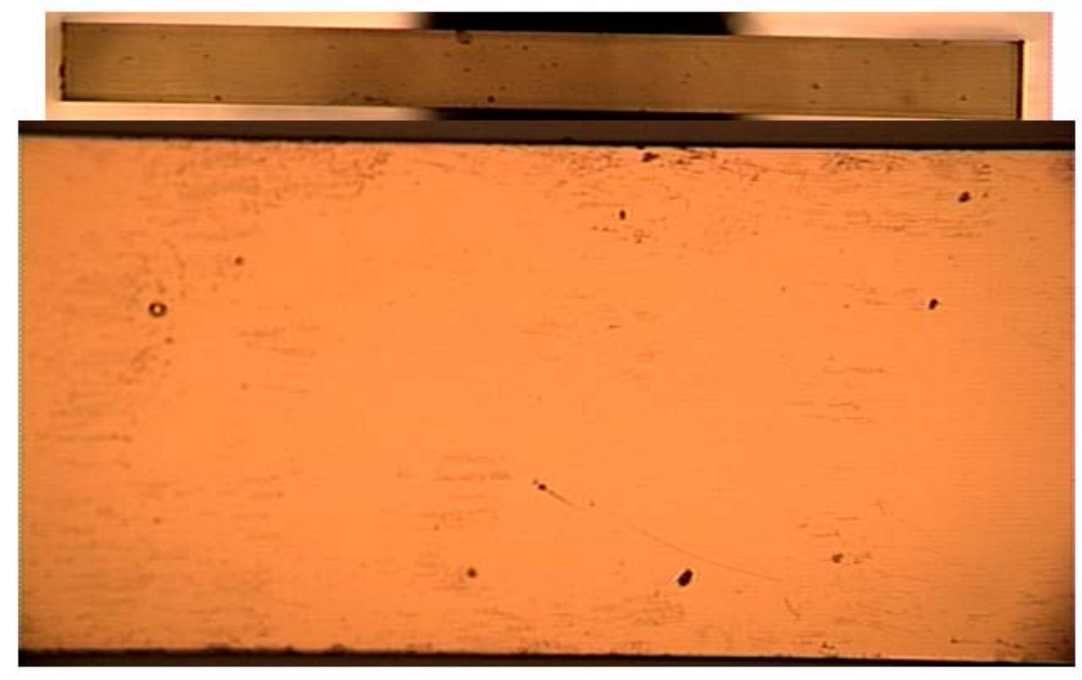

Figure 8.2. Polished facet of A substrate. Top - full facet view 1.5 magnification. Bottom partial facet view magnification of 10.

microphotographs of the cleaved facets of the $400 \mu \mathrm{m}$ thick black switch from the initial $6 \mathrm{H}-\mathrm{SiC}$ substrates. In general, the polished facets of the substrates A, B, C and D offer the flattest and cleanest surfaces from an optical point of view. The cleaved c facets on the initial $6 \mathrm{H}-\mathrm{SiC}$ substrates are optically superior to the ground facets of substrates A, B, C, and D. The ground 
facets of substrates $\mathrm{A}, \mathrm{B}, \mathrm{C}$, and $\mathrm{D}$ are flat, but somewhat rough. The cleaved $\mathrm{m}$ facets of the black switch have steps and are very rough.

The substrates A, B, C, and D have a higher density of micropipes than the initial batch of $6 \mathrm{H}-\mathrm{SiC}$ substrates. Four substrates of the first generation of $6 \mathrm{H}-\mathrm{SiC}$ material were inspected for micropipes by microscope. The four original $400 \mu \mathrm{m}$ thick substrates have a very low micropipe density. Only a total of three micropipes were discovered in the four substrates with

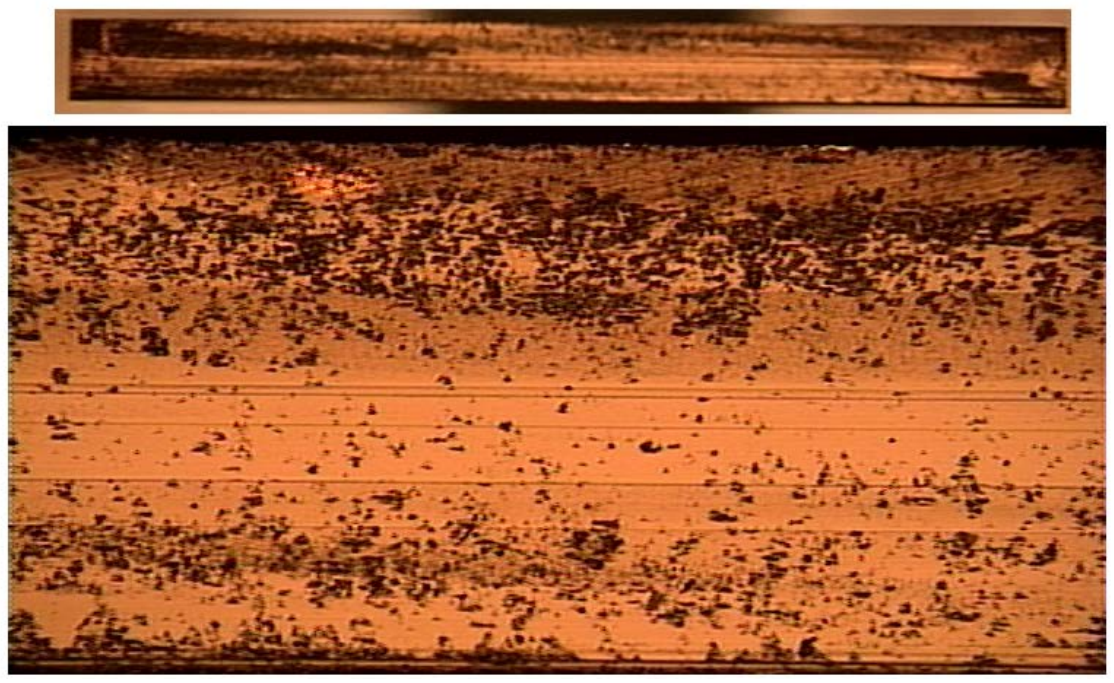

Figure 8.3. Ground facet of A substrate. Top - full facet view 1.5 magnification. Bottom partial facet view magnification of 10 .
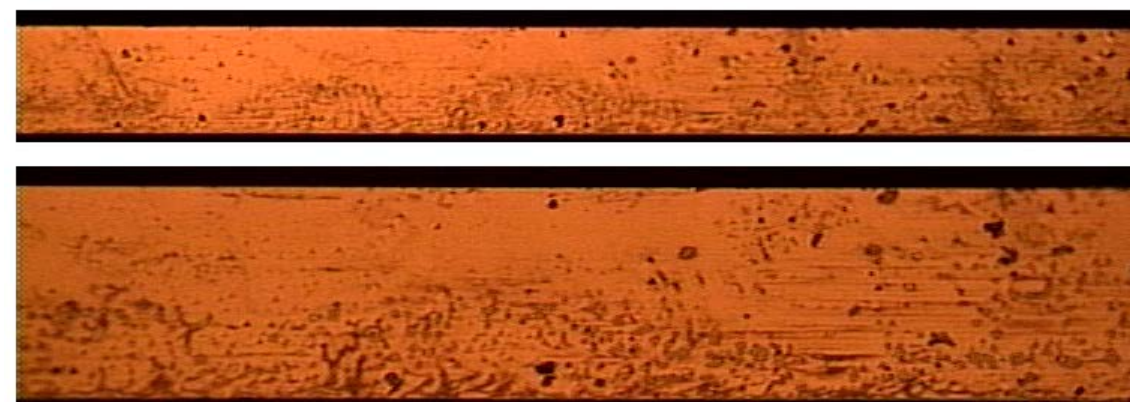

Figure 8.4. Cleaved c facet of $400 \mu \mathrm{m}$ thick Black switch. Top - partial facet view magnification of 5. Bottom - partial facet view magnification of 10. 
no more than one micropipe in any substrate. Figure 6.6 shows a $1 \mathrm{~mm}$ by $1 \mathrm{~mm}$ corner section of a substrate from the initial batch of $6 \mathrm{H}-\mathrm{SiC}$ substrates. This corner is free of micropipes and inclusions. A micropipe that was discovered in one of the four original substrates is shown in Figures 8.7 and 8.8 . It is located at the very edge of the $1.2 \mathrm{~mm}$ by $1.2 \mathrm{~mm}$ substrate. The figures show that the micropipe is initiated at an inclusion in the substrate. The substrates A, B, C, and D

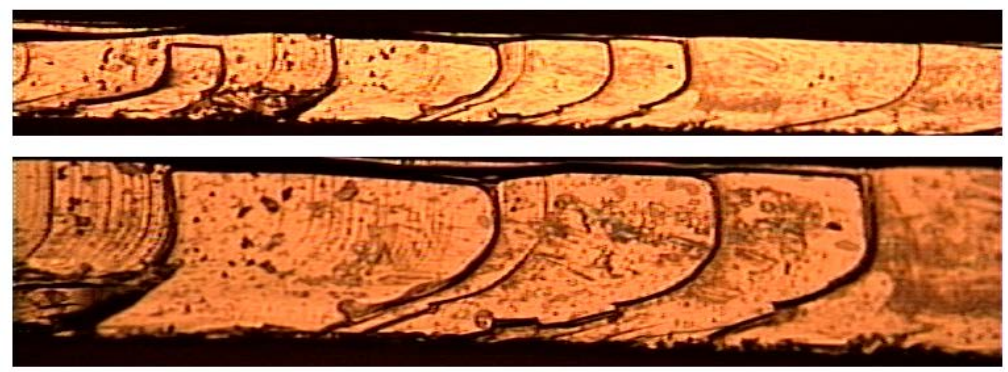

Figure 8.5. Cleaved facet of $400 \mu \mathrm{m}$ thick Black switch. Top - partial facet view magnification of 5 . Bottom - partial facet view magnification of 10.

have a higher micropipe density than the initial batch of $6 \mathrm{H}-\mathrm{SiC}$ substrates. The micropipe density varies greatly between substrates A, B, C and D. Substrate A has the lowest micropipe density and the micropipe density increases in substrates $\mathrm{B}$ and $\mathrm{C}$, with the greatest density residing in substrate D. Micropipes in substrates A, B, C, and D are shown in Figures 7.9 through

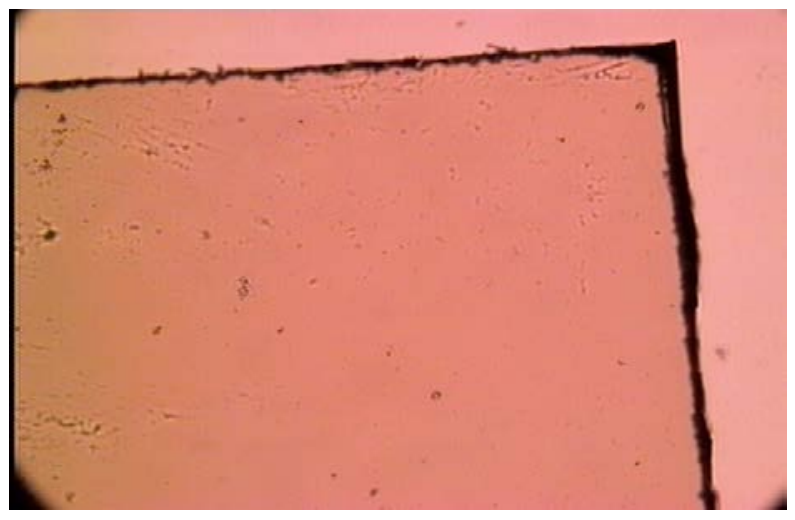

Figure 8.6. $1 \mathrm{~mm}$ by $1 \mathrm{~mm}$ corner of an original $400 \mu \mathrm{m}$ thick, $6 \mathrm{H}$-SiC substrate showing area free of micropipes 


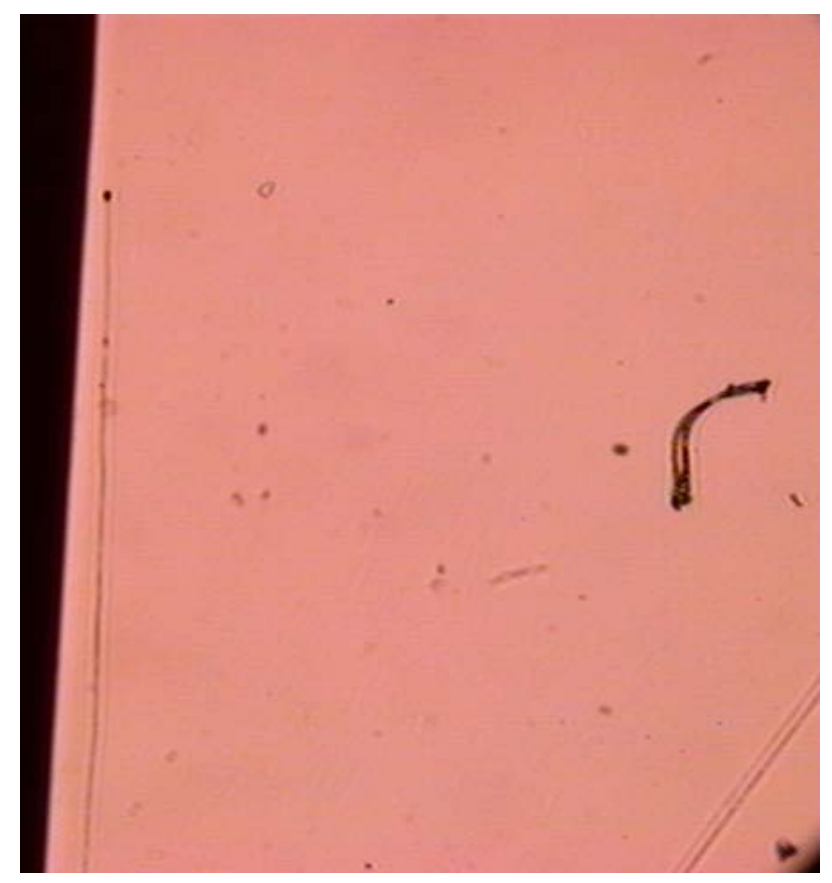

Figure 8.7. Micropipe located at the edge of an original, $400 \mu \mathrm{m}$ thick, $6 \mathrm{H}$-SiC substrate (5X magnification)

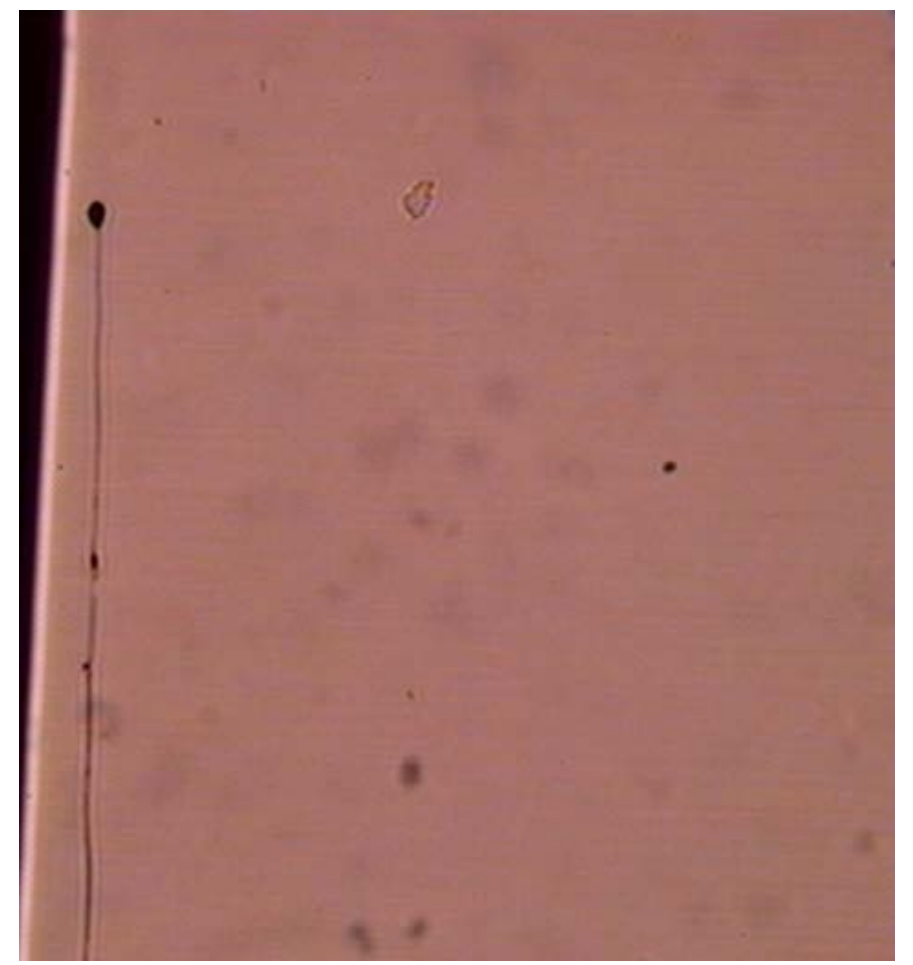

Figure 8.8. Micropipe located at the edge of an original, $400 \mu \mathrm{m}$ thick, $6 \mathrm{H}$-SiC substrate (10X magnification) 


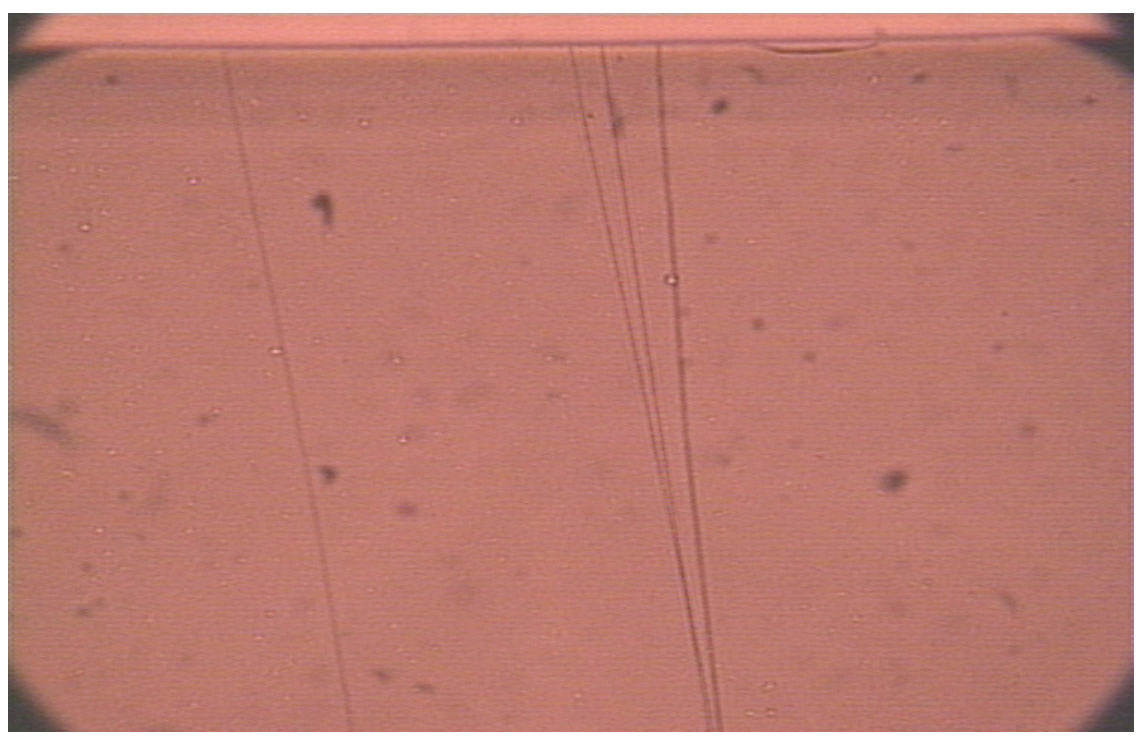

Figure 8.9. Micropipes in $6 \mathrm{H}-\mathrm{SiC}$ substrate A with improved doping profile (5X magnification)

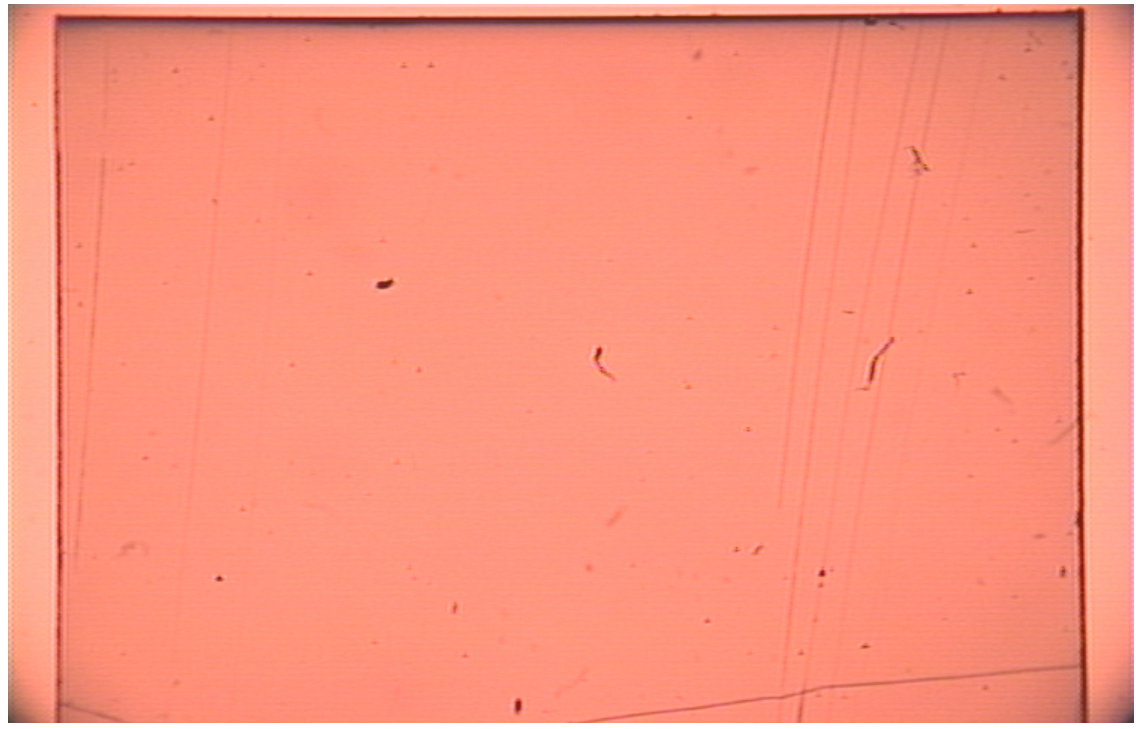

Figure 8.10. Micropipes in $6 \mathrm{H}-\mathrm{SiC}$ substrate $\mathrm{B}$ with improved doping profile (1.5X magnification) 


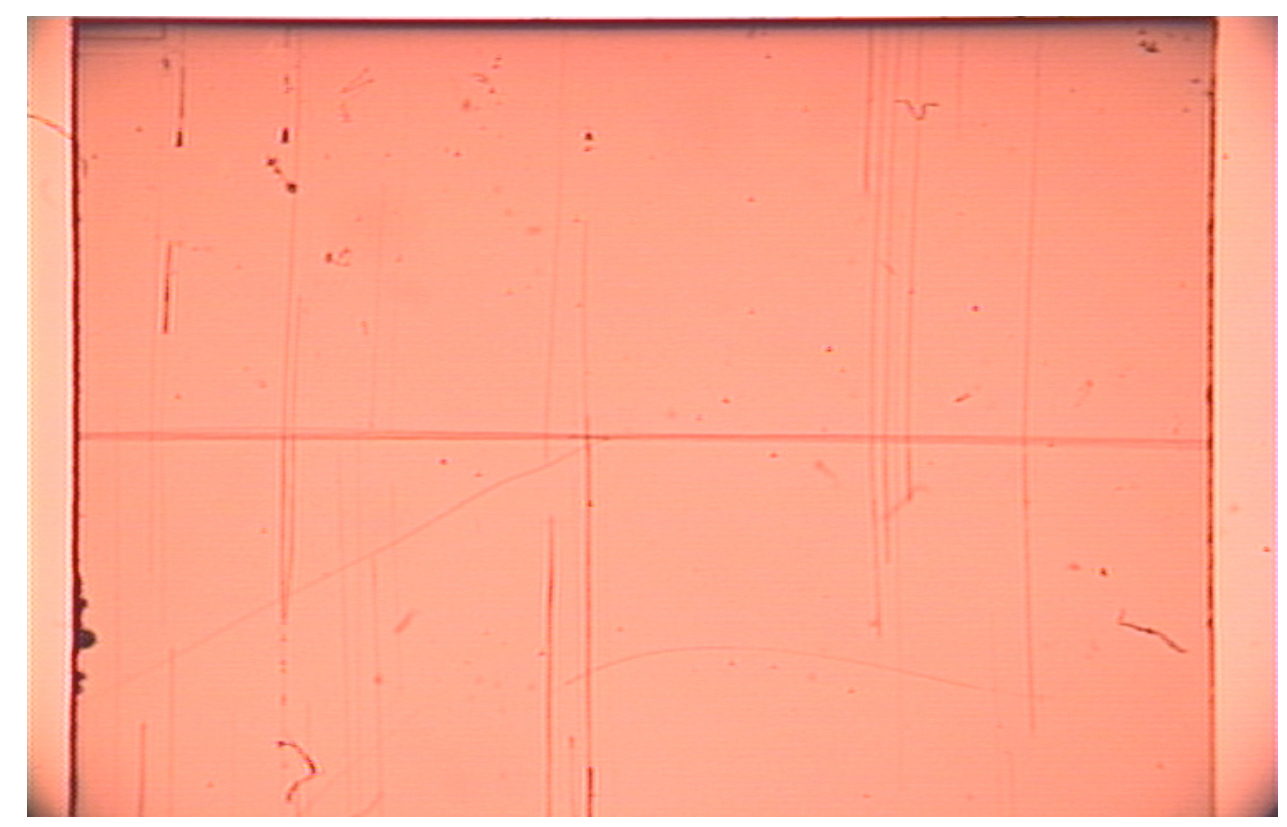

Figure 8.11. Micropipes in $6 \mathrm{H}-\mathrm{SiC}$ substrate $\mathrm{C}$ with improved doping profile (1.5X magnification

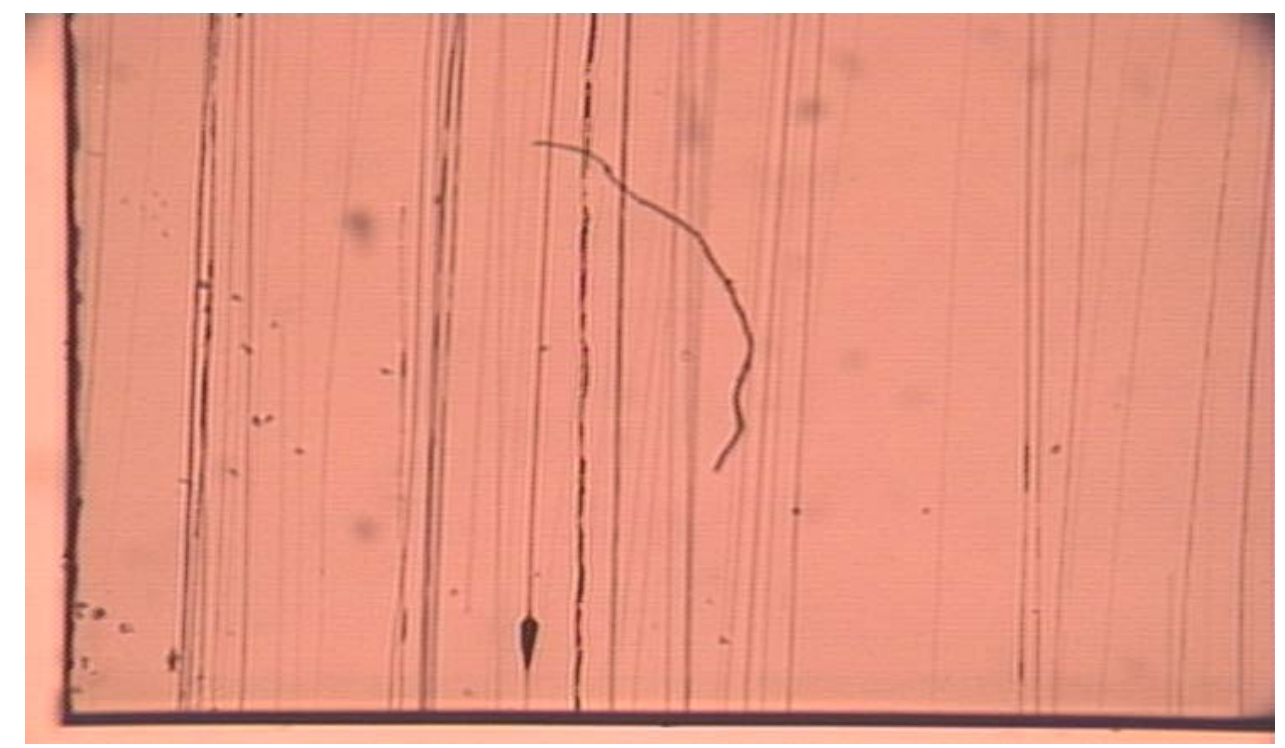

Figure 8.12 . Micropipes in 6H-SiC substrate D with improved doping profile (5X magnification

8.12, respectively. Substrate A (B) has 5 - 10 (10 - 20) micropipes, Substrate C has 20 - 40 micropipes and substrate $\mathrm{D}$ has $>50$ micropipes. The increase in vanadium density to > 
$3 e 17 / \mathrm{cm}^{3}$ is in part to blame for the increase in micropipes in the second generation $6 \mathrm{H}-\mathrm{SiC}$ materials.

\section{3) Dark Current in Improved Doping Profile, Second Generation, 6H-SiC Substrates}

Four photoconductive switch assemblies were constructed with the second generation $6 \mathrm{H}-\mathrm{SiC}$ material with improved vanadium, nitrogen and boron doping profiles. The substrates with improved doping profile are nominally $1.0 \mathrm{~cm}$ by $1.0 \mathrm{~cm}$, and $1 \mathrm{~mm}$ thick. The substrates were cleaned, etched and metalized at LLNL. Circular electrodes with a diameter of 7.8 millimeters were deposited on opposing sides of the substrates by electron beam evaporation. The metal stack consisted of a nickel base layer $100 \mathrm{~nm}$ thick, $100 \mathrm{~nm}$ of titanium, $100 \mathrm{~nm}$ of platinum and a $250 \mathrm{~nm}$ cap layer of gold. The nickel base layer was annealed at a temperature of $1000{ }^{\circ} \mathrm{C}$ for two minutes. The dark resistances of the completed switch assemblies were 2.63e9, 2.53e9, 2.21e9, and 2.96e9 ohms for the A, B, C, and D metalized substrates, respectively. Figure 8.13 shows plots of dark current versus bias voltage for the metalized substrates fabricated from the $6 \mathrm{H}-\mathrm{SiC}$ second generation material. The dark resistance measurements of the improved doping profile material are very consistent (2.58 $\pm 0.37 \mathrm{e} 9$ Ohms).

The dark resistance of the A, B, C, and D metalized substrates are two to three orders of magnitude lower than that of the 'green', 'black' and 'silver' switches constructed from the 400 $\mu \mathrm{m}$ thick, first generation $6 \mathrm{H}-\mathrm{SiC}$ substrates. The dark resistances for the green, black, and silver switches were 170e9, 868.5e9, and 1920e9 ohms, respectively. The values of dark resistance for the green, black and silver switches increase by a factor of 2.5 to $425 \mathrm{e} 9,2171.3 \mathrm{e} 9$ and $4800 \mathrm{e} 9$ if 


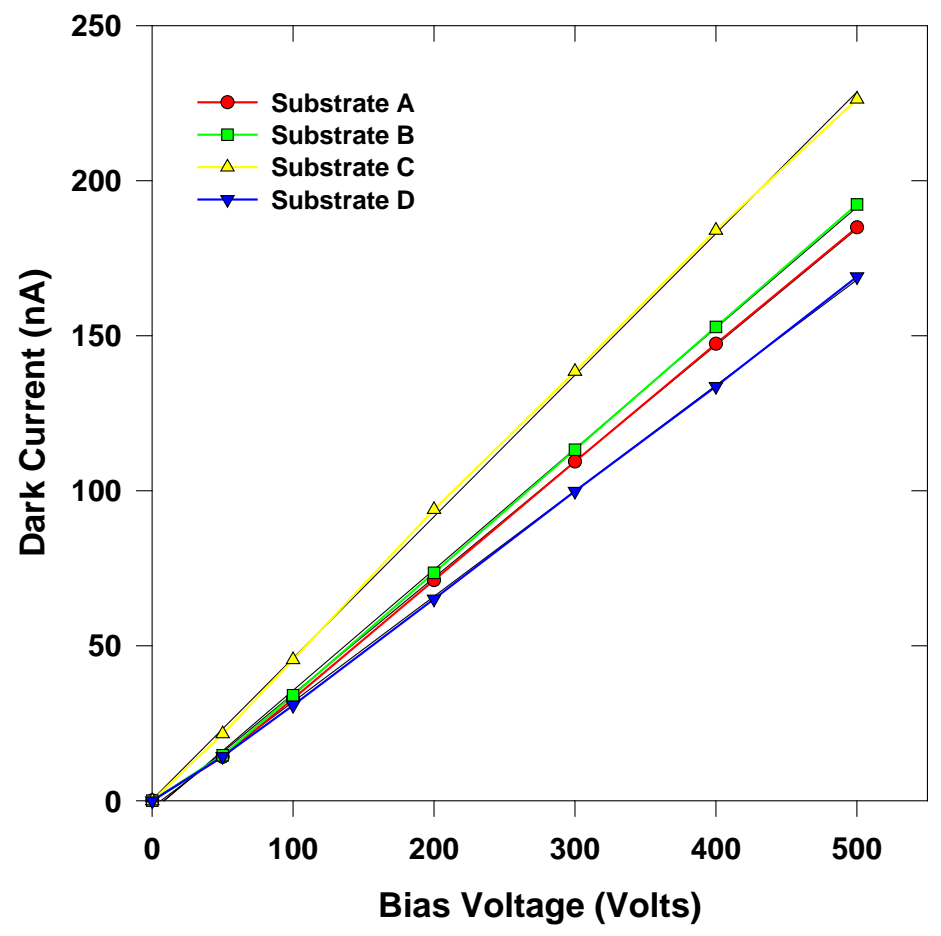

Figure 8.13. Dark currents for the improved doping profile substrates A, B, C, and D

they are normalized to a nominal $1 \mathrm{~mm}$ thickness. The large discrepancy in dark resistance among the initial $6 \mathrm{H}-\mathrm{SiC}$ substrates indicates possible variations in dopant profile, density of unintentional dopants and crystal defects in the substrates. The large difference in dark resistance between the second generation and first generation $6 \mathrm{H}-\mathrm{SiC}$ material can be partially explained by the difference in doping profiles. The doping profiles for the 'green', 'black' and 'silver' substrates are taken to be $\mathrm{V}=2.3 \mathrm{e} 17 \mathrm{~cm}^{-3}, \mathrm{~N}=6.6 \mathrm{e} 16 \mathrm{~cm}^{-3}$, and $\mathrm{B}=4.4 \mathrm{e} 16 \mathrm{~cm}^{-3}$ at one end of the substrate and $\mathrm{V}=1.4 \mathrm{e} 17 \mathrm{~cm}^{-3}, \mathrm{~N}=6.1 \mathrm{e} 16 \mathrm{~cm}^{-3}$, and $\mathrm{B}=4.4 \mathrm{e} 16 \mathrm{~cm}^{-3}$ at the opposite end of the substrate. The vanadium acceptors compensate the 'net' nitrogen donors $(\mathrm{N}-\mathrm{B})$. If we compute the compensation ratio of vanadium to the net nitrogen donors for the improved doping profile $6 \mathrm{H}-\mathrm{SiC}$ substrates we obtain 2.5 at one end and 1.11 at the opposite end of the substrates. The compensation ratio computed for the initial $6 \mathrm{H}-\mathrm{SiC}$ substrate material is 10.45 at one end and 8.24 at the opposite end of the substrate. The higher compensation ratio of the initial $6 \mathrm{H}-\mathrm{SiC}$ 
substrate material indicates that there are many more vanadium acceptor levels available to scavenge any remaining electrons from the nitrogen donor levels leading to a higher material resistivity. In addition, the net impurity donor density $\left(\mathrm{N}_{\mathrm{N}}-\mathrm{N}_{\mathrm{B}}\right)$ is $\sim 2 \mathrm{e} 16 \mathrm{~cm}^{-3}$, which close to the value of deep traps due to intrinsic defects $\left(1-2 \mathrm{e} 16 \mathrm{~cm}^{-3}\right)$. Our model for the resistivity of vanadium compensated 6H-SiC (Figure 8.1) shows that we should expect the initial 6H-SiC material to have a resistivity that is $\sim$ a factor of ten higher than that of the $6 \mathrm{H}-\mathrm{SiC}$ material with the improved doping profile. Other factors such as additional dopants (unintentional) and crystal defects acting as electron traps could explain the remaining difference in dark resistance between the initial $6 \mathrm{H}-\mathrm{SiC}$ material substrates and the substrates with improved doping profile. However, what can be said is the improved doping profile $6 \mathrm{H}-\mathrm{SiC}$ substrates have a much more repeatable material resistivity that is in closer agreement with the model predicting the resistivity of vanadium compensated $6 \mathrm{H}-\mathrm{SiC}$. Also, the consistency in material resistivity appears to be a direct result of a consistent doping profile among the four improved doping profile $6 \mathrm{H}-\mathrm{SiC}$ substrates.

The improved doping profile $6 \mathrm{H}-\mathrm{SiC}$ material should also offer better photoconductive switching performance compared to the initial $6 \mathrm{H}-\mathrm{SiC}$ material. Improved performance is expected since a larger number of vanadium acceptor levels will be filled with electrons in the second generation $6 \mathrm{H}-\mathrm{SiC}$ material and a possible increase in recombination time is expected.

\section{4) Photoconductive Switching Tests of Second Generation 6H-SiC Substrates}

Low voltage, photoconductive switch tests were performed on metalized substrates from the second generation $6 \mathrm{H}-\mathrm{SiC}$ material. The purpose of the low voltage photoconductive switch 
testing of the second generation $6 \mathrm{H}-\mathrm{SiC}$ material was to determine if changes in the material dopant profiles did, in fact, improve switching performance.

\section{Comparing Switching Results for Switches Constructed with Different Generations of 6H-SiC Substrates}

We need to perform photoconductive switching tests on switches fabricated using the second generation $6 \mathrm{H}-\mathrm{SiC}$ material to make a useful comparison of this material to the original $6 \mathrm{H}-\mathrm{SiC}$ material. However, care must be taken to make a useful comparison between the first and second generation materials due to the different lengths, widths and thicknesses of the substrate, differences in applied optical pulse temporal shapes, and possible differences in carrier

mobility and recombination time. We know that the switch resistance is proportional to the switch thickness squared and inversely proportional to the applied optical power, carrier recombination time and mobility for a variety of applied optical temporal pulses when the carrier recombination time is much shorter than the optical pulse width. The switch resistance for the specific case of an applied step pulse of optical power, $\mathrm{P}_{0}$, is given in

$$
R(t)=\frac{d^{2} \hbar \omega}{P_{0}(1-\exp (-\alpha W))(1-r) \eta \tau_{r} \mu_{n} q}
$$

The switch resistance is related to the switch conductivity by equation (8.8). The maximum switch conductivity $\left(\sigma_{\max }\right)$ is given in equation (8.9). We can substitute equation (8.7) into equation (8.9) to

$$
\begin{gathered}
R(t)=\frac{\rho(t) d}{a_{s w}}=\frac{d}{a_{s w} \sigma(t)} \\
\sigma_{\max }=\frac{d}{a_{s w} R_{\min }}
\end{gathered}
$$

obtain a more revealing expression for the maximum conductivity in equation (8.10). 


$$
\sigma_{\max }=\frac{q \eta^{*} \tau_{r} \mu_{n} P_{\max }^{*}}{W \hbar \omega}
$$

Where

$$
P_{\max }^{*}=\frac{P_{\max }}{d L}
$$

It is clear in equation (8.10) that the maximum conductivity $\left(\sigma_{\max }\right)$ is proportional to the peak optical power density at the switch facet $\left(\mathrm{P}^{*}{ }_{\text {max }}\right)$ multiplied by the factor of the effective quantum efficiency, carrier mobility and carrier recombination time. We can compare the photoconductive switch test results from switches of different sizes constructed with different generations of $6 \mathrm{H}-\mathrm{SiC}$ materials by plotting the maximum conductivity $\left(\sigma_{\max }\right)$ as a function of the peak optical power density at the switch facet $\left(\mathrm{P}_{\max }{ }^{*}\right)$. The resulting plots for switches fabricated with the different $6 \mathrm{H}-\mathrm{SiC}$ materials will reveal information regarding the effective quantum efficiency, carrier recombination time and carrier mobility of each 6H-SiC material and how one material compares to the other.

\section{Photoconductive Switch Tests}

Photoconductive switching tests were performed at low voltage on switch assemblies fabricated using substrates A, B, C, and D at wavelengths of 532 and $1064 \mathrm{~nm}$. The generic low voltage test circuit shown in Figure 8.14 was used in these tests. However, the low voltage test board was modified so that metalized substrates could be tested without having to solder electrodes on them. The modified low voltage board is 4.5 inches long, 2.3125 inches wide and 0.07 inches thick, including copper cladding. The components mounted on the modified low voltage board are identical to the original low voltage board (Figures 5.8 and 5.9). The energy storage capacitor is an assembly of metalized polyester capacitors with an effective capacitance and operating voltage of $1.5 \mu \mathrm{F}$ and 500 Volts, respectively. The load resistance was a parallel 
combination of ten, $510 \mathrm{ohm}, 0.25$ watt, carbon composition resistors. Contact is made to the metalized switch substrate electrodes using Bal Seal Inc [8.2] canted coil spring contacts. The top, bottom and coiled spring contacts of the modified low voltage test board are shown in Figures 8.15 - 8.17. There is a difference in the layout of current path in the modified low voltage board. The switch current follows a more serpentine path in the modified low voltage board resulting in a slightly higher value of stray inductance for this circuit.

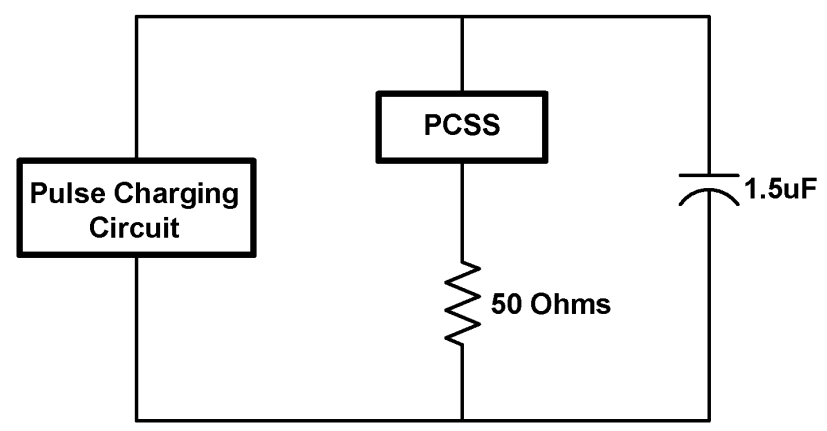

Figure 8.14. Low voltage test circuit used in photoswitch testing of substrates A, B, C, and D.

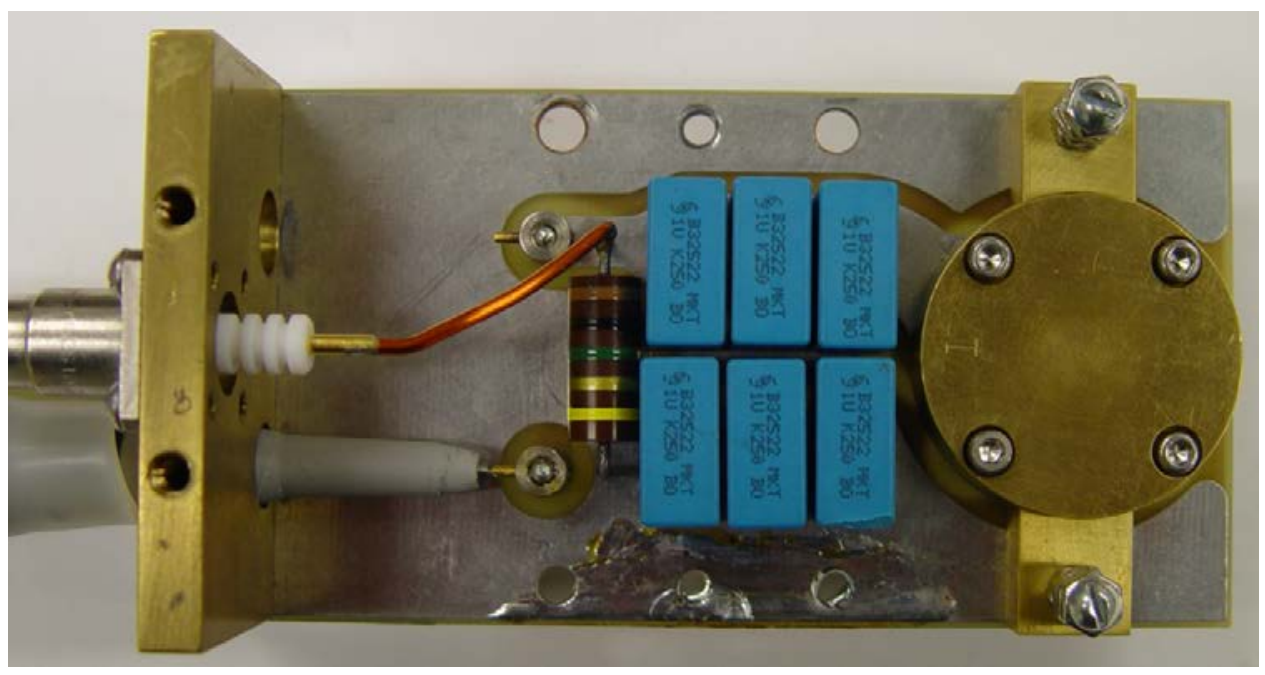

Figure 8.15. Top of modified low voltage test board showing storage capacitors, $1 \mathrm{M} \Omega$ bleeder resistor and brass switch holder assembly. 


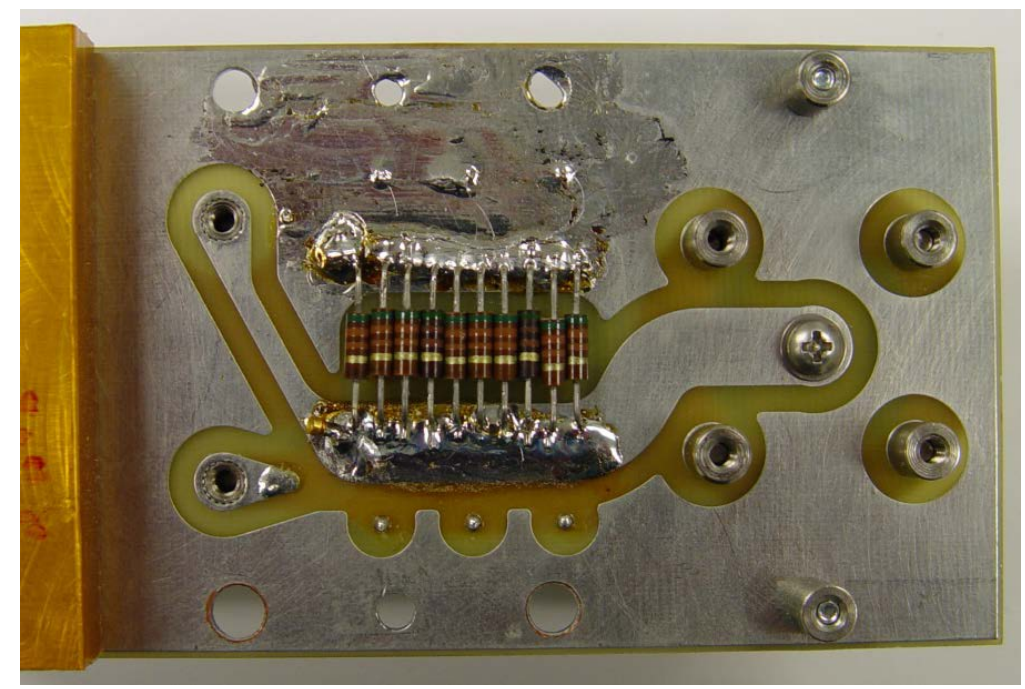

Figure 8.16. Load resistor assembly is mounted on bottom of modified low voltage test board.

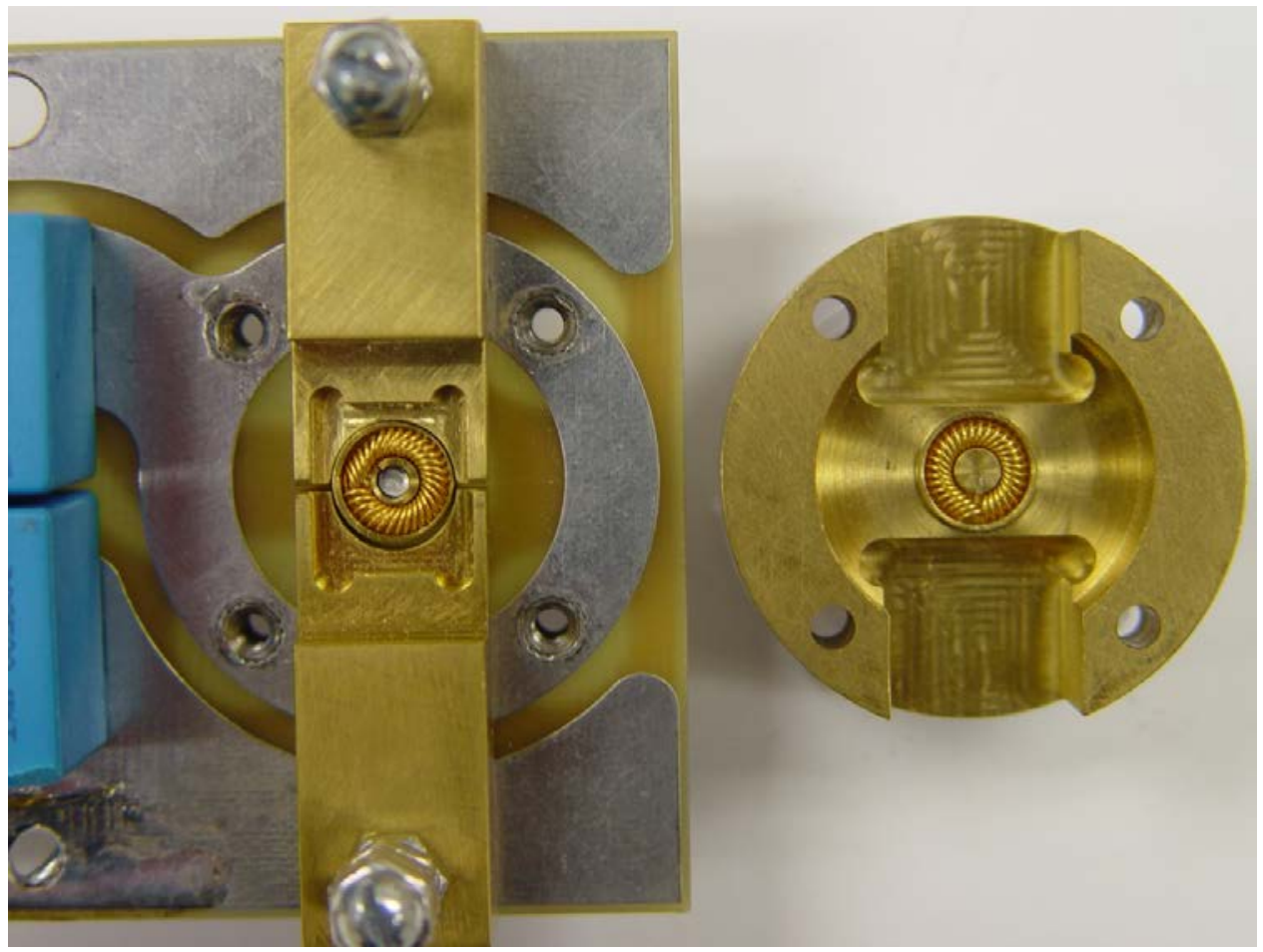

Figure 8.17. Brass switch-holder assembly shows switch mounting pocket (left) and switch assembly cap. The metalized $6 \mathrm{H}-\mathrm{SiC}$ substrate is centered in the switch mounting pocket and the cap is lowered and tightened down. Compression of spring contacts makes electrical connection. 
Tests were performed at a charge voltage of 266 Volts with a load impedance of 50.8 ohms. The optical trigger pulse was applied across a single facet area $(1 \mathrm{~cm}$ by $0.958 \mathrm{~mm})$ of the second generation $6 \mathrm{H}-\mathrm{SiC}$ switches with the lowest micropipe densities, A and $\mathrm{B}$.

\section{Second Generation Material Switch Test Results at 1064 nm}

An overlay of 5 optical pulses applied to the A switch facet and their corresponding switch currents are shown in Figure 8.18. The optical energy of the pulses is $1 \mathrm{~mJ}$ at the $1064 \mathrm{~nm}$ wavelength. The charge voltage for all switching events is 266 Volts. The pulse width of the optical pulses is $11.0 \mathrm{~ns}$ (FWHM). The peak optical power of the $1 \mathrm{~mJ}$ pulses is $0.0907 \mathrm{MW}$. The switch current waveform has a peak value of 2.7 amps, lags the optical pulse by $\sim 2$ ns and is $\sim 7$ ns wider than the optical pulse. However, the switch current follows the rise of the optical pulse and its waveform indicates that the second generation $6 \mathrm{H}-\mathrm{SiC}$ material has a fast recombination time. Figure 8.19 shows the A switch photocurrent corresponding to $20 \mathrm{~mJ}$ optical pulses at 1064 $\mathrm{nm}$. The peak optical power of the $20 \mathrm{~mJ}$ pulses is $1.736 \mathrm{MW}$. The A switch peak current is 4.7 amps and has a pulse width of 34 ns FWHM compared to the 11 ns pulse width of the applied optical pulse and lags the optical pulse by 2.8 ns.

The photocurrent waveform shown in Figure 8.19 gives the impression that it precedes the applied optical pulse in time. It does not. This is due to the circuit - switch interaction described in Chapter 7. The peak optical power of the $20 \mathrm{~mJ}$ pulses shown in Figure 8.19 is 19.1 times greater than the peak optical power of the $1 \mathrm{~mJ}$ pulse of Figure 7.18. As a result, the peak optical power of the $1 \mathrm{~mJ}$ pulse (0.0907 MW), which reduced the switch on resistance to 47.4 ohms, occurs early in the foot of the $20 \mathrm{~mJ}$ optical pulse. Consequently, fairly large switch currents occur for what appear to be very low levels of optical excitation giving the false 
impression that the switch current precedes the optical excitation. However, this effect is testament to the improved switching performance of the second generation $6 \mathrm{H}-\mathrm{SiC}$ material.

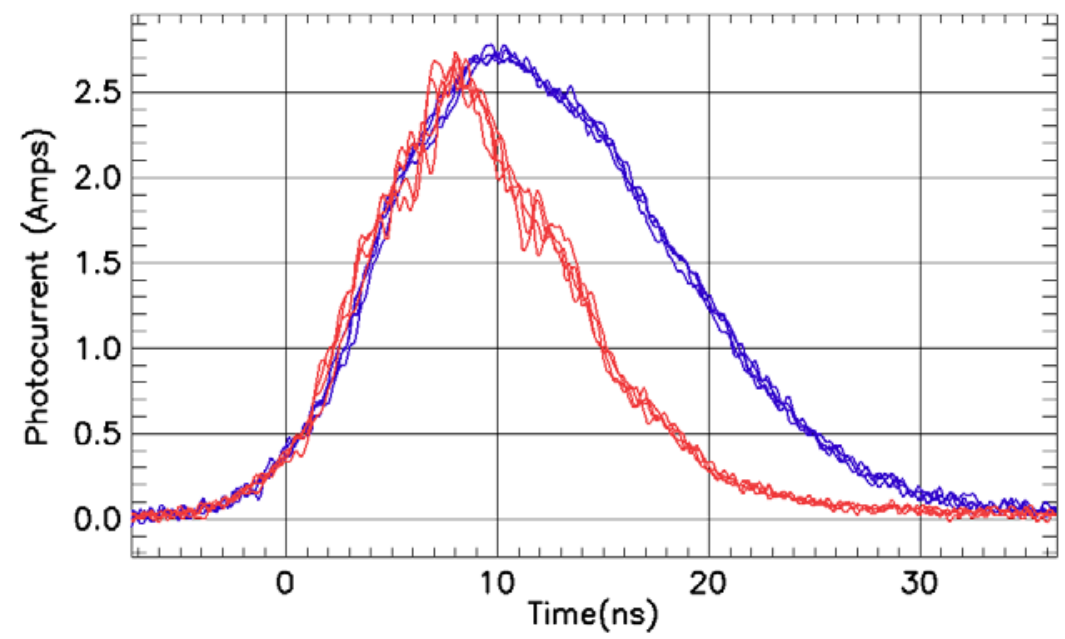

Figure 8.18. Five pulse overlay of applied optical power (red trace) and A switch photocurrent (blue trace) plotted versus time for $1 \mathrm{~mJ}$ optical pulses at $1064 \mathrm{~nm}$.

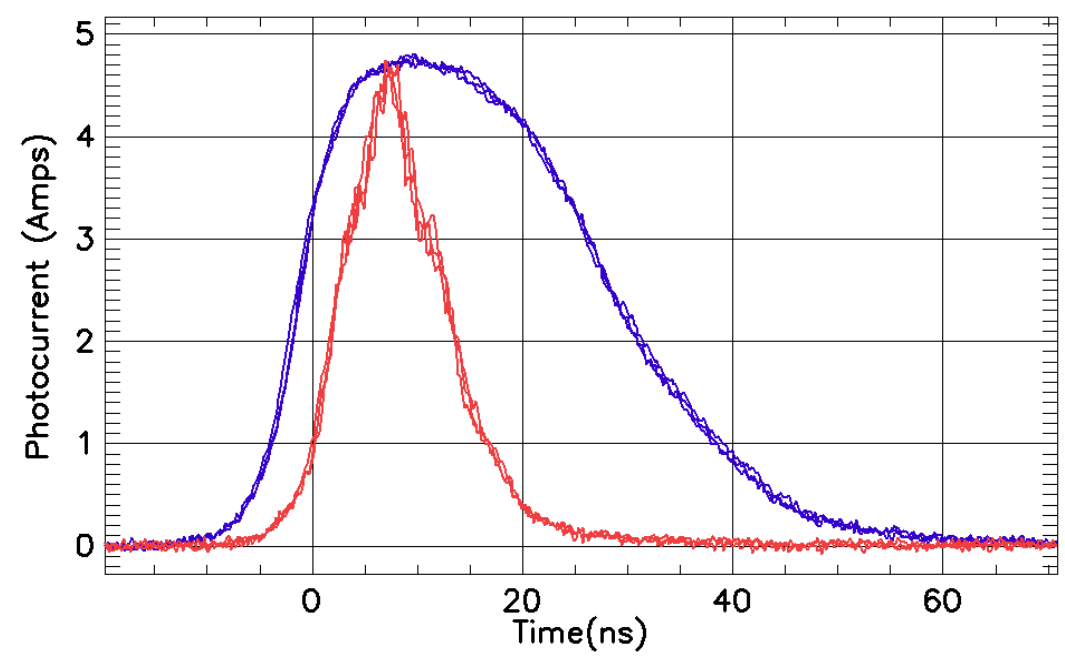

Figure 8.19. Four pulse overlay of applied optical power (red trace) and A switch photocurrent (blue trace) plotted versus time for $20 \mathrm{~mJ}$ optical pulses at $1064 \mathrm{~nm}$.

The performance of the A and B switches are summarized in Figures 8.20 and 8.21. These figures show plots of the minimum switch resistance and maximum conductance as functions of the peak applied optical power at $1064 \mathrm{~nm}$. The results of Figure 8.20 show that the 
A switch achieves a minimum resistance of $\sim 1 / 2$ that of the B switch for all peak applied optical powers at the $1064 \mathrm{~nm}$ wavelength. The minimum on resistance of the A (B) switch is 24.28 (41) ohms for peak applied powers of $\sim 0.17 \mathrm{MW}$, which corresponds to a pulse of $2 \mathrm{~mJ}$ optical energy for the 11 ns pulse width, and decreases to 3.47 (7.0) ohms for peak optical powers of 3.0 MW. The maximum conductance of the A and B switches are plotted as functions of the peak applied optical power in Figure 8.21. These plots show signs of saturation of the maximum conductance with increasing peak optical power, something that was not observed in the green, black and silver switch test results at $1064 \mathrm{~nm}$.

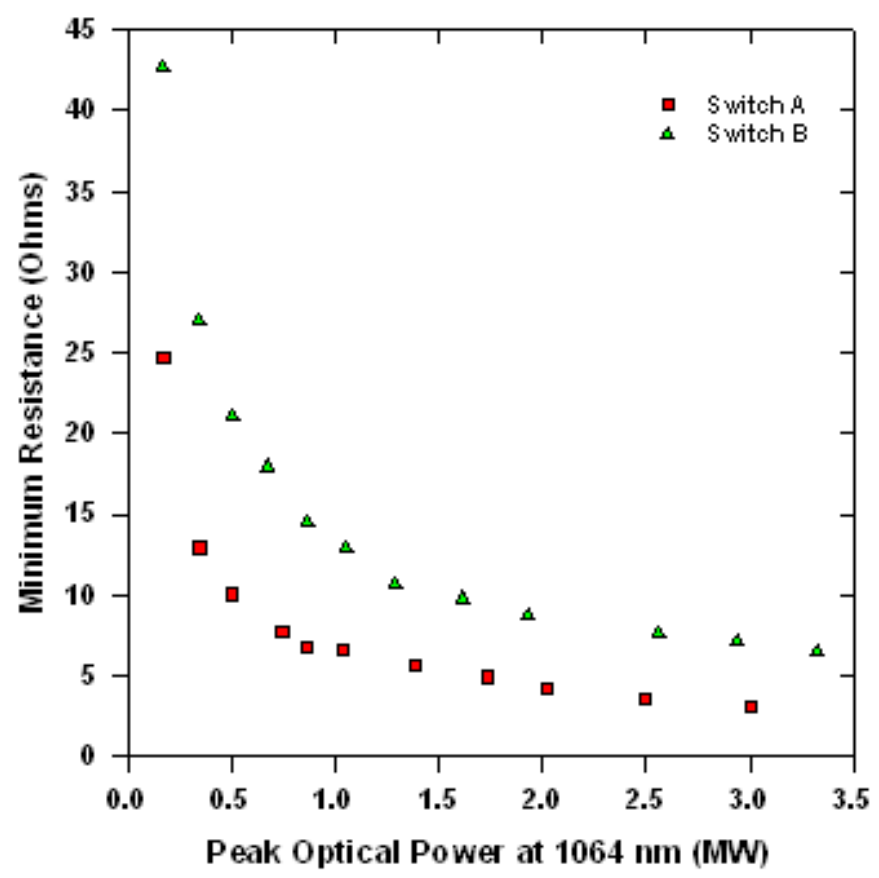

Figure 8.20. Minimum switch on resistance plotted versus peak applied optical power at $1064 \mathrm{~nm}$ for the $A$ and $B$ switches.

The test results for the A and B switch show much lower (higher) minimum resistance (maximum conductance) than the green, black, and silver switches. The log of the maximum conductivity of the A, B, green, black and silver switches is plotted as a function of the peak 


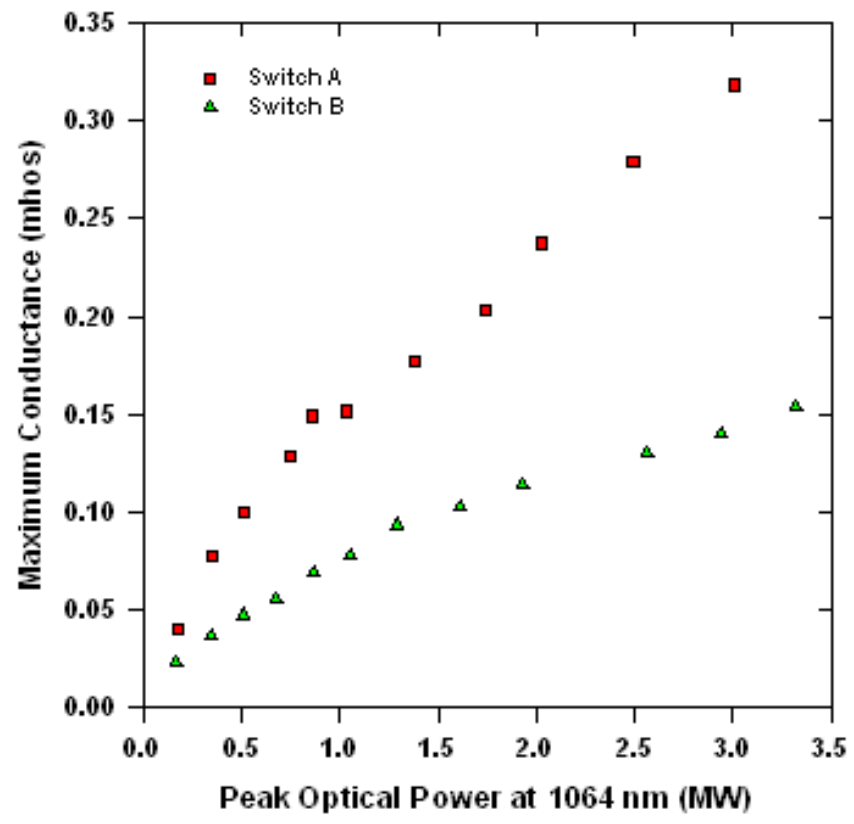

Figure 8.21. Maximum conductance of $A$ and $B$ switch plotted as function of peak applied optical power at $1064 \mathrm{~nm}$.

optical power density at the switch facet in Figure 8.22. This should give an accurate comparison of the switches constructed with the first and second generation $6 \mathrm{H}-\mathrm{SiC}$ material that takes differences in switch thickness, length and pulse shape of the applied optical pulse into consideration. The maximum conductivity $\left(\sigma_{\max }\right)$ is calculated using equation (8.8). The peak optical power density $\left(\mathrm{P}_{\max }^{*}\right)$ is given in equation (8.11). $\mathrm{P}_{\max }$ is the peak optical power in (8.11). The photoconductive switching performance of the switches (A and B) constructed with the second generation $6 \mathrm{H}-\mathrm{SiC}$ material is much better than the switches constructed from the original 6H-SiC material (green, black and silver switches). The A switch (B switch) shows a maximum conductivity that is thirty (twenty) times higher than the best of the original switches at low optical power density (a few MWs $/ \mathrm{cm}^{2}$ ) and twenty (ten) times higher at several tens of $\mathrm{MWs} / \mathrm{cm}^{2}$. If we assume that the carrier recombination time and mobility are the same for the 


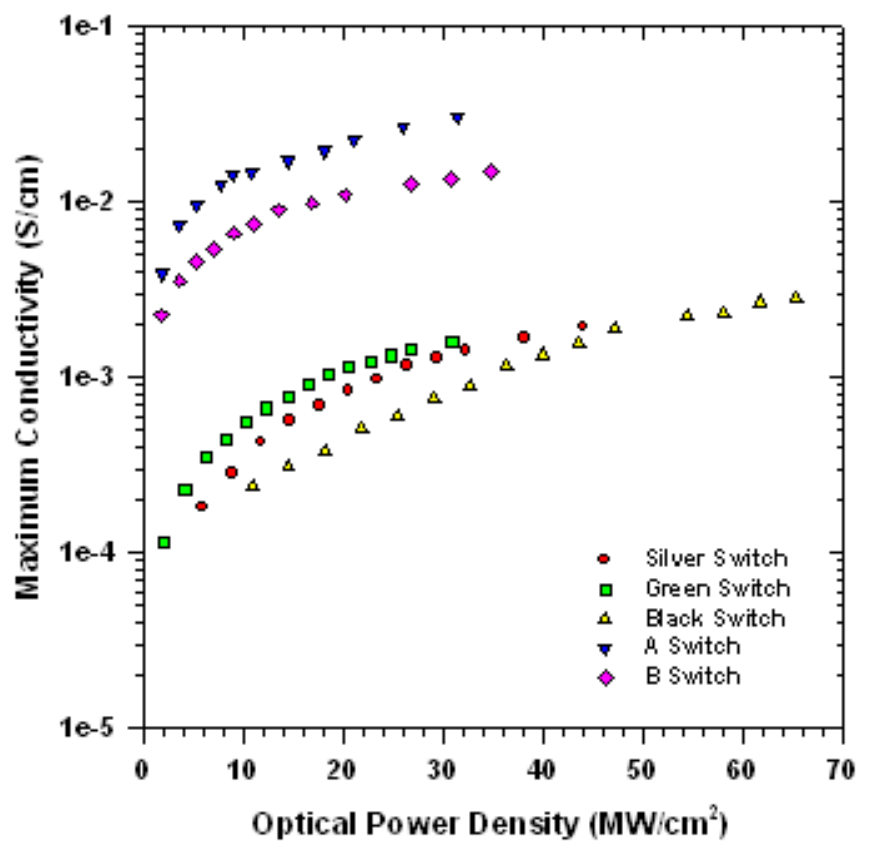

Figure 8.22. Maximum switch conductivity plotted as function of optical power density at the switch facet for the A, B, green, black, and silver switches at $1064 \mathbf{~ n m}$.

original and second generation $6 \mathrm{H}-\mathrm{SiC}$ materials, the improved switching performance at the $1064 \mathrm{~nm}$ wavelength of the second generation $6 \mathrm{H}-\mathrm{SiC}$ material represents a substantial increase in quantum efficiency of this material. The best results for the A switch at $32 \mathrm{MW} / \mathrm{cm}^{2}$ optical energy compared to the best results for any of the original switches corresponds to an increase in quantum efficiency of a factor of thirty.

In actuality, we expect an increase in the carrier recombination time between the original and second generation $6 \mathrm{H}-\mathrm{SiC}$ material based on the LD and HD model results of Chapter 7. We expect that the vanadium acceptor levels and intrinsic defects to act as traps, or, recombination centers during photoswitch conduction. The original $6 \mathrm{H}-\mathrm{SiC}$ substrates have an average vanadium density of $1.85 \mathrm{e} 17 \mathrm{~cm}^{-3}$ and net donor density of $1.95 \mathrm{e} 16 \mathrm{~cm}^{-3}$. The second generation $6 \mathrm{H}-\mathrm{SiC}$ material has average vanadium and net donor densities of $2.25 \mathrm{e} 17 \mathrm{~cm}^{-3}$ and $1.15 \mathrm{e} 17$ $\mathrm{cm}^{-3}$, respectively. If we assume that all the net-donated electrons have been captured by vanadium acceptor levels, then there will be $1.655 \mathrm{e} 17 \mathrm{~cm}^{-3}$ and $1.1 \mathrm{e} 17 \mathrm{~cm}^{-3}$ still available to 
capture electrons for the original and second generation materials, respectively. If we include the effect of the $1-2 \mathrm{e} 16 \mathrm{~cm}^{-3}$ electron traps this effect is even more pronounced. As a result, we should expect a longer carrier lifetime for the second generation material, particularly at lower optical excitation levels. The expected increase in carrier lifetime for the second generation material will also contribute to its improved switch efficiency.

\section{Second Generation Material Switch Test Results at 532 nm}

The modified low voltage test board is also used to test the second generation $6 \mathrm{H}-\mathrm{SiC}$ material switches (A and B) at the $532 \mathrm{~nm}$ wavelength for a charge voltage of 265 Volts and a load resistance of 50.8 Ohms. Figure 8.23 shows an overlay of three optical pulses and the resulting switch photocurrents for applied optical pulse energy of $1 \mathrm{~mJ}$ at $532 \mathrm{~nm}$. The peak switch current is 2.95 Amps corresponding to a peak optical power of $0.1135 \mathrm{MW}$ at the switch facet. The optical and photocurrent pulse widths are 8.0 ns and 12.8 ns FWHM, respectively. The peak of the switch photocurrent lags the peak of the optical trigger pulse by 1.24 ns.

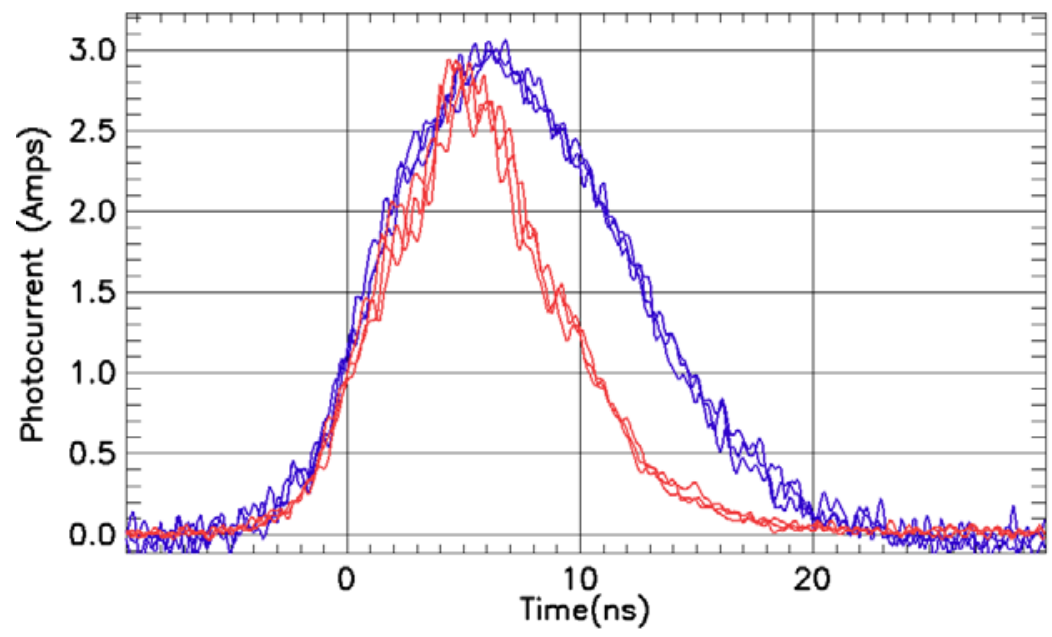

Figure 8.23. Three pulse overlay of optical power pulses (red) and corresponding switch photocurrents (blue) plotted versus time for charge voltage of 265 Volts and optical pulse energy of $1 \mathrm{~mJ}$ at $532 \mathrm{~nm}$. 


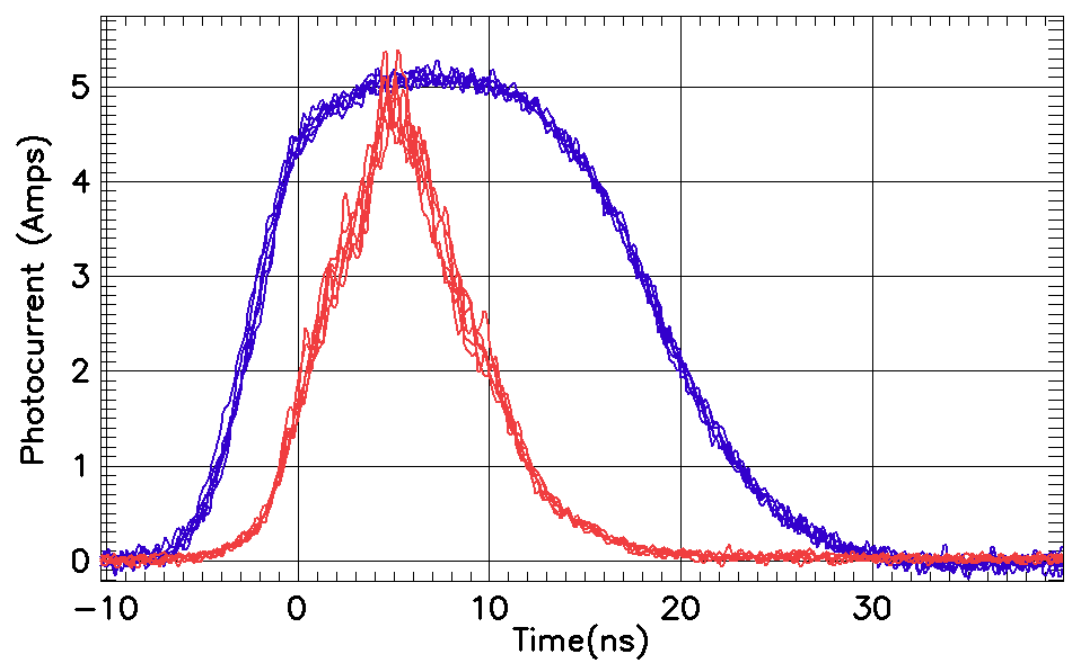

Figure 8.24. Seven pulse overlay of optical power pulses (red) and corresponding switch photocurrents (blue) plotted versus time for a charge voltage of 265 Volts and optical pulse energy of $20 \mathrm{~mJ}$ at $532 \mathrm{~nm}$.

Figure 8.24 shows a seven pulse overlay of the applied optical power pulses and the corresponding switch photocurrents for $20 \mathrm{~mJ}$ optical pulses at $532 \mathrm{~nm}$. The peak switch photocurrent is 5.1 amps and corresponds to a peak optical power of $2.3733 \mathrm{MW}$ at $532 \mathrm{~nm}$. The optical and switch current pulse widths are 8.0 and 21.2 ns FWHM, respectively. The peak of the switch current lags the peak optical power by 1.9 ns. Figure 8.24 gives the impression that the switch photocurrent precedes the optical power waveform. However, this is only emblematic of the improved second generation $6 \mathrm{H}-\mathrm{SiC}$ switch performance as explained in the switching results for the second generation $6 \mathrm{H}-\mathrm{SiC}$ material at $1064 \mathrm{~nm}$.

An important point to notice about the switch photocurrent waveforms in Figures 8.23 and 8.24 is the absence of the long-lived component of photocurrent that produced the long tails in the photocurrent waveforms for the black, green and silver switches when excited with 532 nm wavelength light. All switches fabricated from the second generation $6 \mathrm{H}-\mathrm{SiC}$ material did not exhibit a long-lived component of switch photocurrent when excited with $532 \mathrm{~nm}$ light. This 


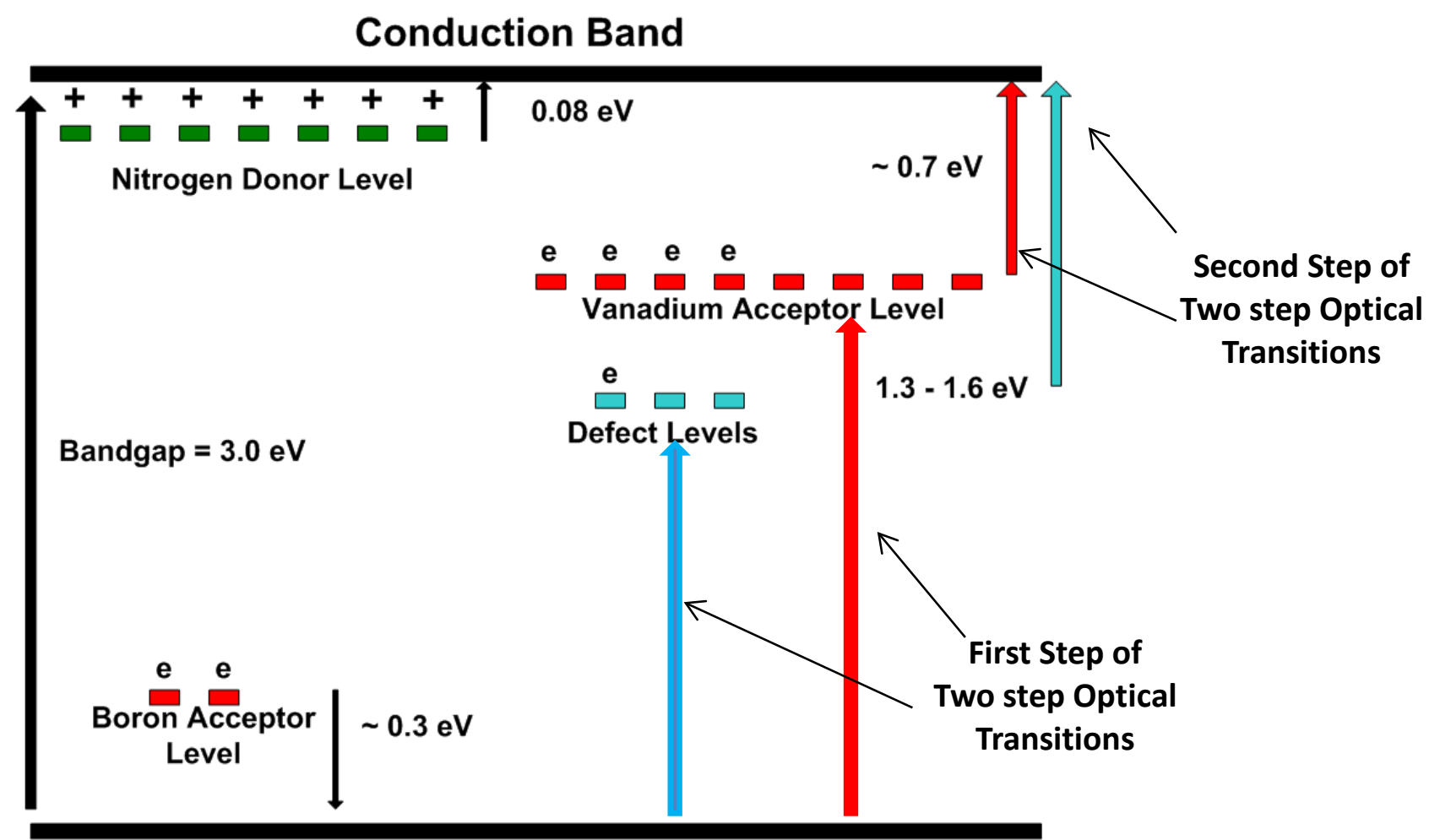

\section{Valence Band}

Figure 8.25. Bandgap diagram for $6 \mathrm{H}$-SiC shows the position of the vanadium, nitrogen, boron and defect energy levels. The figure shows the possible two-step optical transitions of electrons from the valence band to the conduction band.

indicates that the source of the long-lived photocurrent component is not associated with the vanadium and nitrogen levels, or any of the complexes formed by these levels. In addition, the long-lived switch photocurrent cannot be hole current generated by two step optical transitions $[8.4,8.5]$ of electrons from the valence band to the conduction band as shown in Figure 8.25. Holes can be created in the valence band if electrons are optically excited to empty vanadium acceptor levels, or defect levels that act as acceptors. The electrons at the acceptor/defect levels can then be excited into the conduction band by the absorption of another photon. The holes that are left behind in the valence band contribute to the switch current and do not necessarily have the same recombination time as the electrons. The requirement for two-step optical absorption is that twice the photon energy $\left(2 \hbar \omega>\mathrm{E}_{\mathrm{g}}\right)$ is greater than the $6 \mathrm{H}-\mathrm{SiC}$ bandgap. This is true at 532 
$\mathrm{nm}$, but not at $1064 \mathrm{~nm}$ excitation. As a result, there are no two-step optical transitions, or twophoton absorption occurring at $1064 \mathrm{~nm}$, but two-step optical transitions and two-photon absorption both occur at $532 \mathrm{~nm}$.

The performance of the A and B switches are summarized in Figures 8.26 and 8.27. These figures show plots of the minimum switch resistance and maximum conductance as functions of the peak applied power at $532 \mathrm{~nm}$. The individual results for the minimum resistance and maximum conductance for the A and B switches are much closer at $532 \mathrm{~nm}$ then they were at $1064 \mathrm{~nm}$. The switches have a minimum resistance of $\sim 40$ ohms at a peak optical power of $0.1135 \mathrm{MW}$ at $532 \mathrm{~nm}$, corresponding to $1 \mathrm{~mJ}$ optical energy at the $8.0 \mathrm{~ns}$ FWHM pulse width, that decreases to 1.5 ohms at $2.38 \mathrm{MW}$ (20 mJ optical energy). The A and B switch maximum conductance are plotted against peak optical power in Figure 8.27. The maximum conductance of switch A appears to become slightly supralinear for peak applied

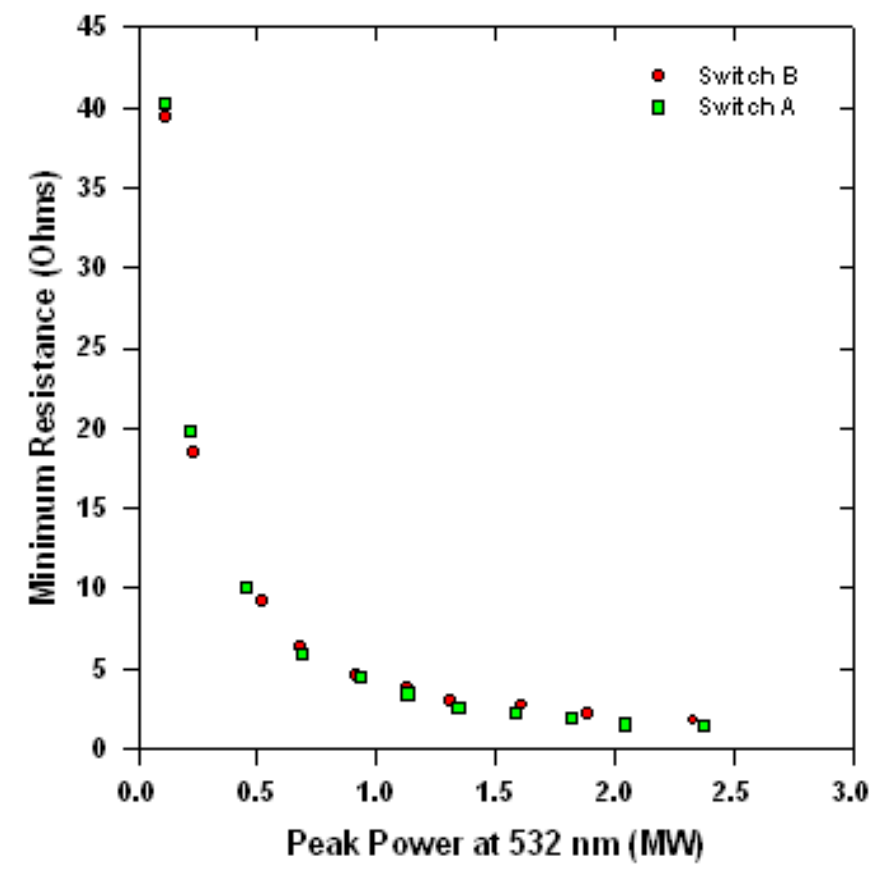

Figure 8.26. Minimum switch resistance of the $A$ and $B$ switches plotted as a function of peak applied optical power at $532 \mathrm{~nm}$ for a charge voltage of 265 Volts. 


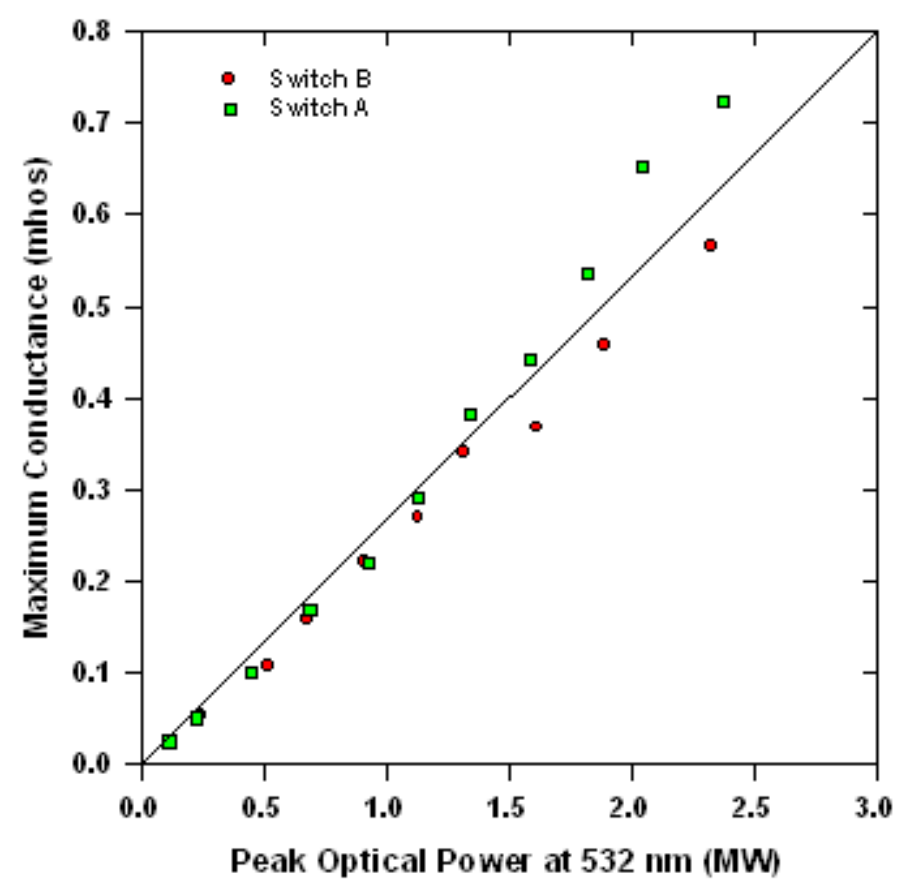

Figure 8.27. Maximum switch conductance of the $A$ and $B$ switches plotted as a function of peak applied optical power at $532 \mathrm{~nm}$ for a charge voltage of 265 Volts.

optical power greater than $1.5 \mathrm{MW}$, while the maximum conductance of switch B appears to be linear. The minimum switch on resistance of switch A (B) is $\leq 2.25$ (3.0) ohms for peak optical energies greater than 1.5 MW. The A and B switch maximum conductance is $1.5-2.0$ times higher than that of the green, black, and silver switches (see Figure 5.63) for a given peak optical power despite the A and B switches being 2.5 times thicker. A direct comparison of the A and B switches with the green, black, and silver switches is given in Figure 8.28. The maximum conductivity of the two second generation $6 \mathrm{H}-\mathrm{SiC}$ switches (A and $\mathrm{B}$ ) and the three first generation switches (green, black and silver) is plotted against the peak optical power density applied to the switch facets. The second generation switches are clearly better switches. 
We can compare the performance at $532 \mathrm{~nm}$ of the weaker of the two second generation switches (B switch) to the best performing first generation switch (silver). It takes a factor of fifteen higher power density applied to the silver switch to obtain the maximum conductivity

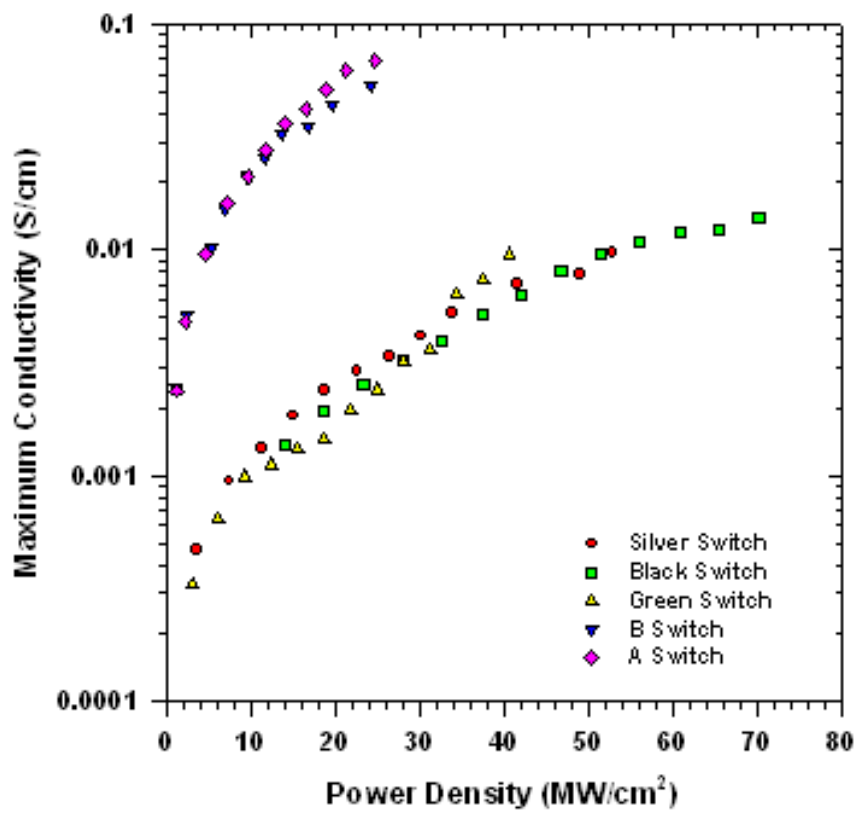

Figure 8.28. Maximum conductivity of the A ad B switches is compared to the maximum conductivity of green, black and silver switches as a function of peak applied optical power density at $532 \mathrm{~nm}$.

obtained for the B switch $(2.43 \mathrm{e}-3 \mathrm{~S} / \mathrm{cm})$ at an applied power density of $1.225 \mathrm{MW} / \mathrm{cm}^{2}$. In addition, the highest value of maximum conductivity obtained for the B switch is $0.0541 \mathrm{~S} / \mathrm{cm}$ at an applied power density of $24.3 \mathrm{MW} / \mathrm{cm}^{2}$. The maximum conductivity for the green switch at a power density of $\sim 24 \mathrm{MW} / \mathrm{cm}^{2}$ is 17 times lower. The switching performance of the A and B switches is at least 15 - 20 times better than that of the first generation $6 \mathrm{H}-\mathrm{SiC}$ switches for excitation at $532 \mathrm{~nm}$.

The maximum conductivity of switches A and B is much higher than that of the green, black, and silver switches at all optical power densities at both the 532 and $1064 \mathrm{~nm}$ wavelengths. This is true at $1064 \mathrm{~nm}$ excitation despite the fact that the conductance of the A and 
B switches show signs of saturation with increasing applied peak optical power, while the green, black and silver switch maximum conductance is linear, or supralinear, with increasing peak applied optical power. The maximum conductivity of the second generation switches is significantly larger than the first generation $6 \mathrm{H}-\mathrm{SiC}$ switches, despite the long-lived component $(10-15 \%)$ of photocurrent present in the first generation switches and the apparent increase in carrier recombination with peak applied optical power for excitation at $532 \mathrm{~nm}$. All test results show that the switching performance of the second generation $6 \mathrm{H}-\mathrm{SiC}$ switches is from $15-20$ times higher than that of the first generation switches.

\section{5) Third Generation 6H-SiC Material}

The doping profile for the second generation $6 \mathrm{H}-\mathrm{SiC}$ substrates had $\mathrm{V}=3.5 \mathrm{e} 17 \mathrm{~cm}^{-3}, \mathrm{~N}=$ $1.5 \mathrm{e} 17 \mathrm{~cm}^{-3}$, and $\mathrm{B}<1.0 \mathrm{e} 16 \mathrm{~cm}^{-3}$ at one end of the substrates and $\mathrm{V}=1.0 \mathrm{e} 17 \mathrm{~cm}^{-3}, \mathrm{~N}=1.0 \mathrm{e} 17$ $\mathrm{cm}^{-3}$, and $\mathrm{B}<1.0 \mathrm{e} 16 \mathrm{~cm}^{-3}$ at the opposite end of the substrates along the c-plane. The second generation $6 \mathrm{H}-\mathrm{SiC}$ doping densities are an improvement over the dopant levels in the first generation $6 \mathrm{H}-\mathrm{SiC}$, but were different from the proposed levels of $\mathrm{V}=2.5 \mathrm{e} 17 / \mathrm{cm}^{3}, \mathrm{~N}=$ $1.25 \mathrm{e} 17 / \mathrm{cm}^{3}$ and $\mathrm{B}<1 \mathrm{e} 16 / \mathrm{cm}^{3}$. The second generation $6 \mathrm{H}-\mathrm{SiC}$ substrates have values of vanadium in excess of $3.0 \mathrm{e} 17 / \mathrm{cm}^{3}$, possibly leading to increased crystal defects and micropipes. All second generation $6 \mathrm{H}-\mathrm{SiC}$ substrates have visible micropipes. Substrates with improved doping profiles and free from micropipes are required for high power switching devices. As a result, we continued to obtain $6 \mathrm{H}-\mathrm{SiC}$.

A third generation $6 \mathrm{H}-\mathrm{SiC}$ material was obtained with the doping densities of $\mathrm{V}=$ $3 \mathrm{e} 17 / \mathrm{cm}^{3}, \mathrm{~N}=1.6 \mathrm{e} 17 / \mathrm{cm}^{3}$, and $\mathrm{B}=1.7 \mathrm{e} 15 / \mathrm{cm}^{3}$ at one end of the substrate and $\mathrm{V}=1.6 \mathrm{e} 17 / \mathrm{cm}^{3}$, $\mathrm{N}=1.24 \mathrm{e} 17 / \mathrm{cm}^{3}$, and $\mathrm{B}=1.9 \mathrm{e} 15 / \mathrm{cm}^{3}$. However, the third generation $6 \mathrm{H}-\mathrm{SiC}$ material substrates 
were cut from the m-plane as opposed to the first and second generation material that were cut from the a-plane. The difference in the a-plane and m-plane of the $6 \mathrm{H}-\mathrm{SiC}$ crystal is illustrated in Figure 8.29. We know that $6 \mathrm{H}-\mathrm{SiC}$ is a uniaxial crystal with optical and electrical properties that are anisotropic. The $6 \mathrm{H}-\mathrm{SiC}$ crystal has different optical properties depending on whether the polarization of an applied optical pulse is parallel, or perpendicular, to the c-axis of the crystal.

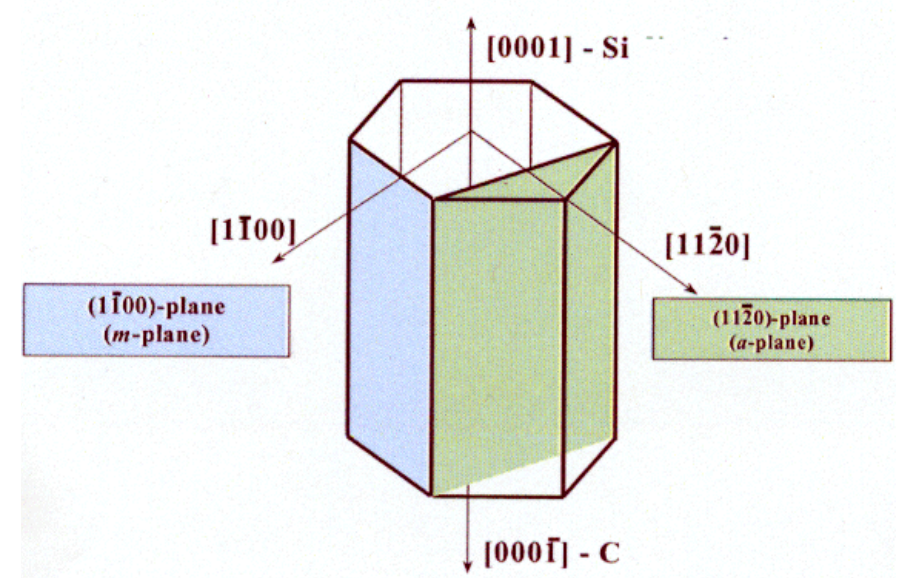

Figure 8.29. The $\mathrm{m}$ and a-planes are shown for the $6 \mathrm{H}$-SiC crystal. The c-plane is the plane perpendicular to the [0001] crystal direction.

$6 \mathrm{H}-\mathrm{SiC}$ also has different carrier mobility depending on whether the direction of current flow is parallel, or perpendicular to the c-axis. The electrodes for the first and second generation $6 \mathrm{H}-\mathrm{SiC}$ switches are on opposing a-plane faces of the substrate and photocurrent excited in the switch flows perpendicular to the c-axis. The electrodes for the third generation $6 \mathrm{H}$-SiC switches are on opposing m-plane faces of the substrate and photocurrent excited in the switch also flows perpendicular to the c-axis. The first and second generation $6 \mathrm{H}-\mathrm{SiC}$ switches have two optical facets on the c-plane of the substrate and two facets on the m-plane of the substrate. The third generation $6 \mathrm{H}-\mathrm{SiC}$ switches have two optical facets on the c-plane and two facets on the a-plane. An optical pulse having S, or P, polarity that is applied to the c-plane facets of the first, second, or third generation switches is always perpendicular to the c-axis, so there is no difference for 
substrates cut from the a, or m-plane. An optical pulse with S (P) polarity applied to either an aplane, or m-plane, facet is parallel (perpendicular) to the c-axis of the crystal. As a result, there is no operational difference in a photoconductive switch constructed with substrates cut from the aplane, or m-plane, of a $6 \mathrm{H}-\mathrm{SiC}$ crystal.

The quality of the third generation substrates is very good. Figure 8.30 is a microphotograph at a magnification of $5 \mathrm{X}$ of a 2.5 by $2.0 \mathrm{~mm}$ corner portion of the $\mathrm{m} 3$ substrate. The top edge of the $\mathrm{m} 3$ substrate is a polished c-facet and the right edge is a ground a-facet. The corner portion of the $\mathrm{m} 3$ substrate is free of micropipes. Figure 8.31 is a microphotograph of the same corner of the $\mathrm{m} 3$ substrate at a magnification of $10 \mathrm{X}$. No micropipes were found in the three third generation substrates. The polished and ground facets have some chips and some inclusions were found in the bulk of the substrates. The overall quality of the third generation substrates appears to be as good as the first generation $6 \mathrm{H}-\mathrm{SiC}$ substrates and superior to the second generation substrates. It should be noted that the maximum vanadium density for the third generation $6 \mathrm{H}-\mathrm{SiC}$ substrates is $3.0 \mathrm{e} 17 \mathrm{~cm}^{-3}$.

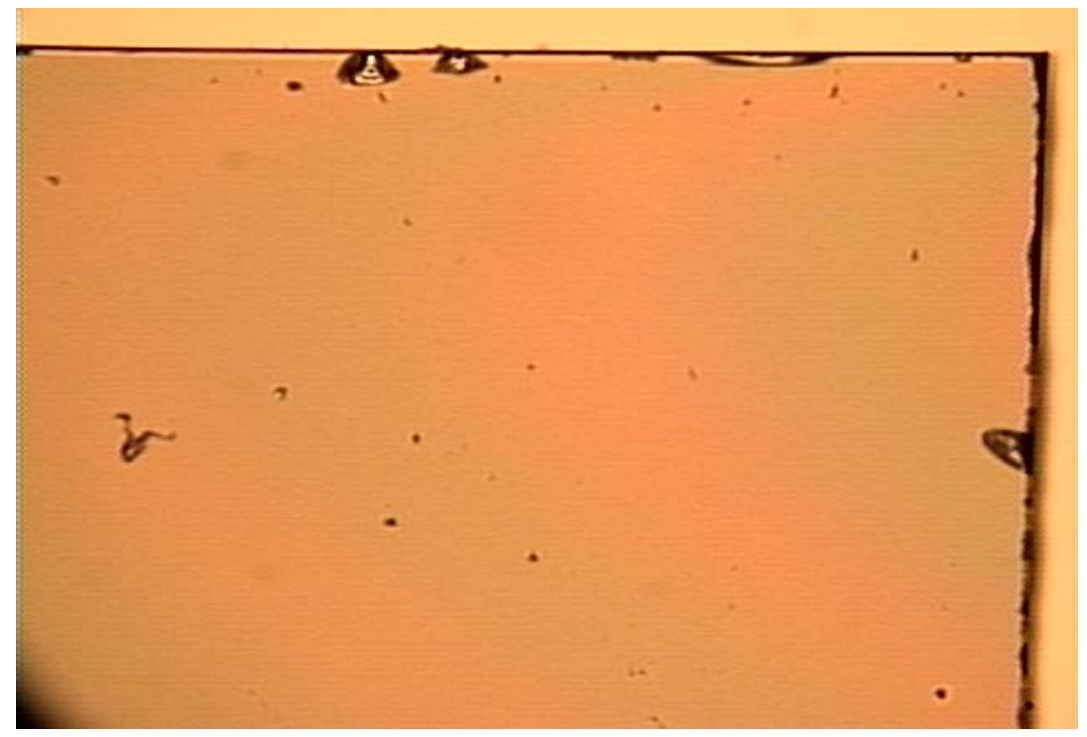

Figure 8.30. Microphotograph of a corner section of the third generation substrate $\mathrm{m} 3$ at a magnification of $5 X$. 


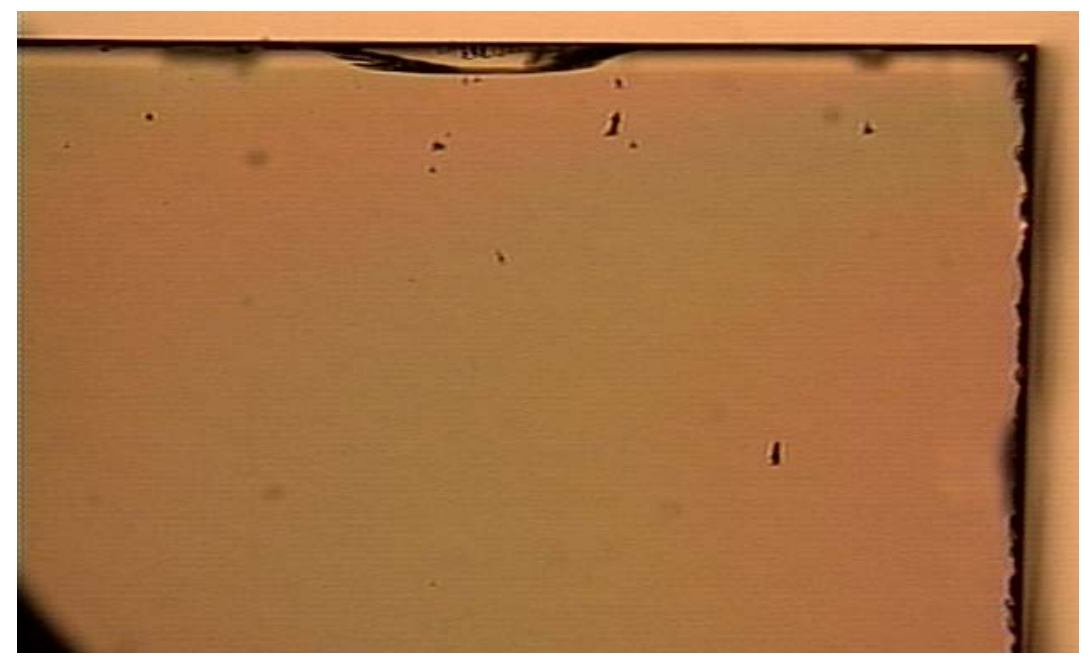

Figure 8.31. Microphotograph of a corner of the $\mathrm{m} 3$ substrate at a magnification of $10 \mathrm{X}$.

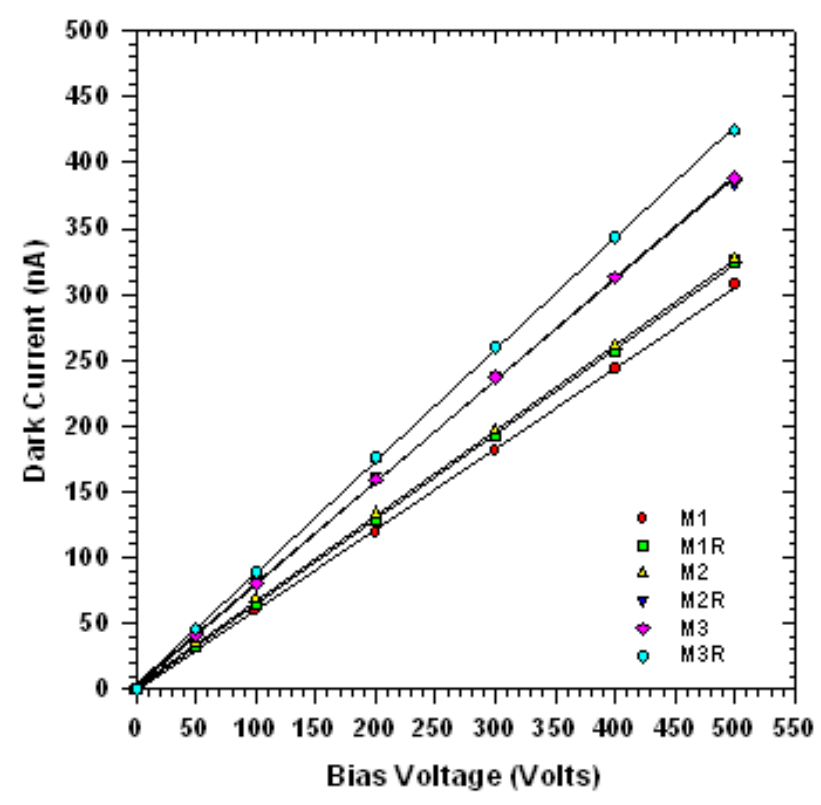

Figure 8.32. Dark current and reversed dark current plotted as a function of bias voltage for the $\mathrm{m} 1, \mathrm{~m} 2$, and $\mathrm{m} 3$ third generation switches.

Three photoconductive switch assemblies were constructed with substrates from the third generation $6 \mathrm{H}-\mathrm{SiC}$ crystal. The third generation switches are designated $\mathrm{m} 1, \mathrm{~m} 2$, and $\mathrm{m} 3$. The dark currents for the m1, m2, and m3 switches are shown in Figure 8.32. Also shown in Figure 8.32 is the dark current for the third generation switches after the polarity of the switches was 
reversed. These plots are designated M1R, M2R and M3R on the plots. Normally, the initial switch dark current measurement was performed in the morning, the polarity of the switch was reversed and the second dark current measurement was performed in the afternoon. The room temperature was $\sim 5-10{ }^{\circ} \mathrm{F}$ warmer in the afternoon compared to the morning, resulting in slightly higher dark currents for the reversed polarity measurements. The third generation switch dark current is a linear function of charge voltage for both the normal and reversed polarity measurements. The dark current measurement for the three, third generation switches is very consistent. The values of the normal (initial measurement) dark resistances are 1.652e9, 1.538e9 and $1.279 \mathrm{e} 9$ ohms for the $\mathrm{m} 1, \mathrm{~m} 2$, and $\mathrm{m} 3$ switches, respectively. The average value of the third generation switch dark resistances is $\sim 60 \%$ of the average value of the dark resistance for the second generation switches (A, B, C, and D). The third generation switches are slightly thicker $(1.022 \mathrm{~mm})$ than the second generation switches $(0.958 \mathrm{~mm})$, but they are longer and wider. The differences in the second and third generation switch cross section and thickness cancel one another out and the third generation dark resistivity is also $~ 60 \%$ of that for the second generation switches. The lower dark resistivity of the third generation switches is a result of a the third generation material substrates having a lower net compensation ratio (V/(N-B), lower vanadium density and higher net nitrogen density. The third generation switches have a higher density of free electrons from the nitrogen donors and fewer vanadium acceptors levels to capture them which results in a higher density of free electrons in the substrate material in thermal equilibrium at room temperature. 


\subsection{1) Third Generation Material Switch Test Results at 532 nm}

The third generation $6 \mathrm{H}$-SiC switches $\mathrm{m} 1, \mathrm{~m} 2$, and $\mathrm{m} 3$ were tested as photoconductive switches using the modified low voltage test board. The third generation switches were tested at a charge voltage of 267 volts with a load resistance of 50.8 ohms. Figure 8.33 shows an overlay of three optical pulses and the resulting switch photocurrents for the m2 switch for an optical pulse energy of $1 \mathrm{~mJ}$ at $532 \mathrm{~nm}$. The peak optical power for the optical pulses in Figure 8.33 is $0.1181 \mathrm{MW}$. The peak current through switch $\mathrm{m} 2$ is 3.54 amps. The peak optical power leads the peak photocurrent by 1.55 ns and the optical and photocurrent pulse widths are 8 and 15 ns FWHM, respectively.

The optical power and m2 switch photocurrent waveforms for an applied optical energy of $20 \mathrm{~mJ}$ is shown in Figure 8.34. The peak optical power and photocurrent in Figure 8.34 are 2.2171 MW and 5.2 Amps, respectively. Figure 8.34, like the results for the second generation switches at the higher applied optical powers, gives the impression that the switch

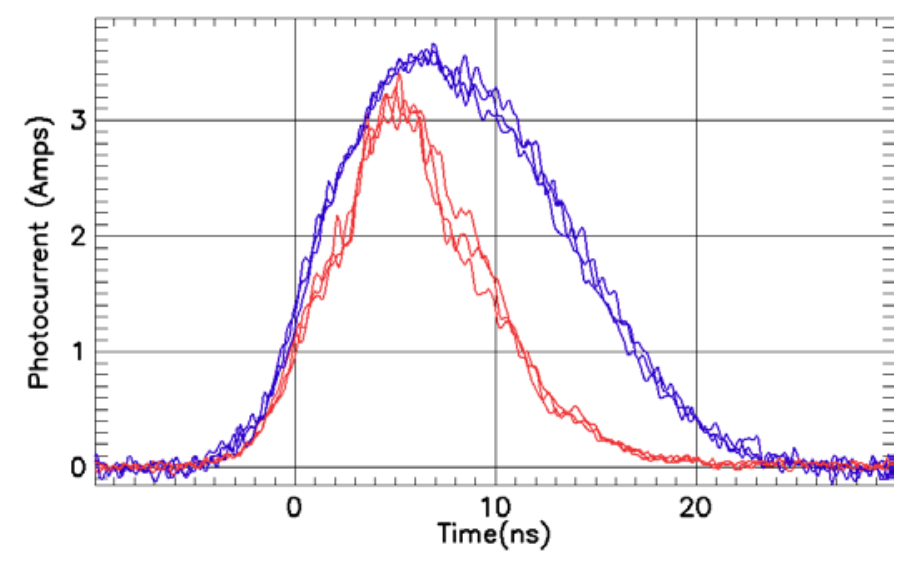

Figure 8.33. Overlay of optical power (red) and $\mathrm{m} 2$ switch photocurrent (blue) waveforms plotted versus time.

photocurrent leads the optical pulse. This is the result of the switch - circuit interaction described in Chapter 7 and the higher efficiency of this material. The peak optical power of Figure 8.34 is a factor of 18.8 time that of the peak optical power in Figure 8.33. As a result, an optical power of 
0.1181 MW (which corresponds to the peak power of Figure 8.33) is achieved very early in the foot of the optical pulse of Figure 8.34 and generates a significant photocurrent.

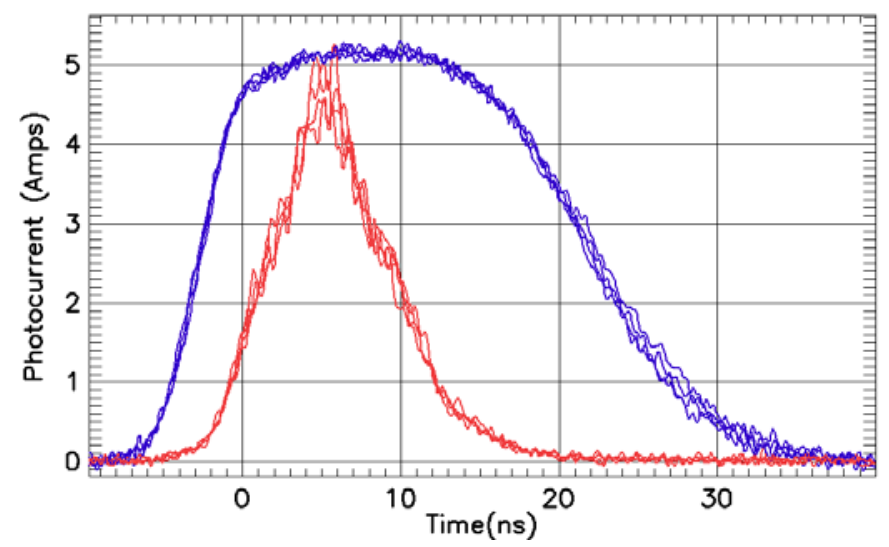

Figure 8.34. Overlay of optical (red) and switch $\mathrm{m} 2$ photocurent (blue) waveforms plotted as a function of time.

The optical pulse and switch m2 current have pulse widths of 8 and 26 ns FWHM, respectively. The m2 switch current pulse width of Figure 8.34 is now significantly wider than the switch current shown in Figure 8.33. This is also a result of the peak optical power of Figure 8.34 being much higher than that of Figure 8.33. The optical power of Figure 8.34 reaches the point where the resistance of switch $\mathrm{m} 2$ is much less than the $50.8 \mathrm{ohm}$ load resistance fairly early in its rise. This results in a wider photocurrent pulse with a flatter peak. The peak optical power leads the peak m2 switch current by 2.0 ns in Figure 8.34. This has increased from $1.55 \mathrm{~ns}$ in Figure 8.33.

The performance of the m1, m2, and m3 switches are summarized in Figure 8.35 and 8.36. These Figures show plots of the minimum switch resistance and maximum conductance for the $\mathrm{m} 1, \mathrm{~m} 2$ and $\mathrm{m} 3$ switches as functions of the peak applied power at $532 \mathrm{~nm}$. The third generation switch minimum resistance is $\sim 25$ ohms at a peak optical power of $0.118 \mathrm{MW}$ and decreases to $~ 0.8$ ohms for a peak applied optical power of $2.2 \mathrm{MW}$ at $532 \mathrm{~nm}$. There is very 


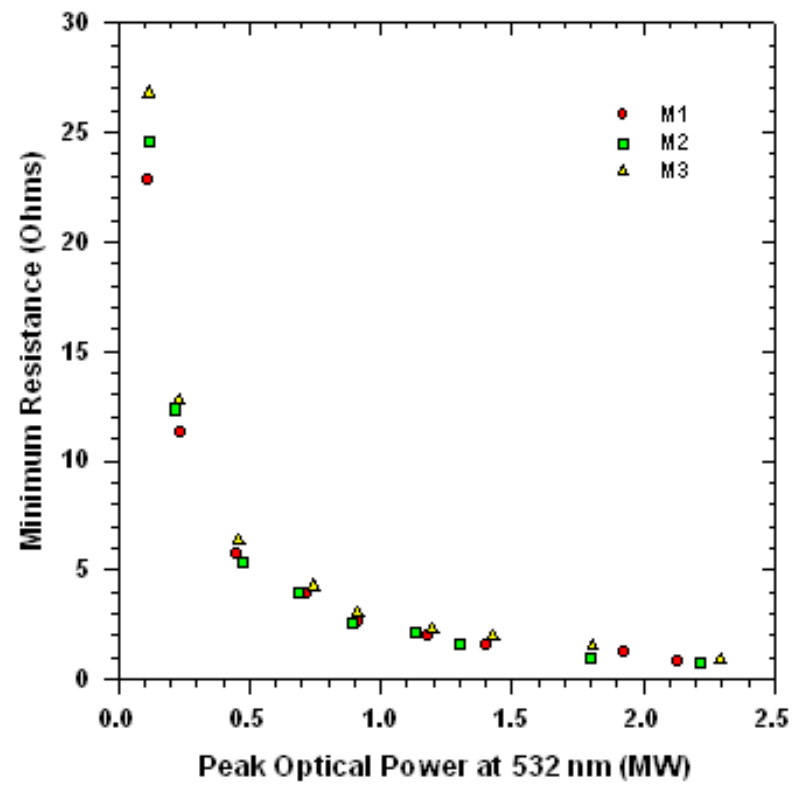

Figure 8.35. Minimum switch resistance of the third generation switches $m 1, m 2$, and $m 3$ plotted as a function of the peak applied optical power at $532 \mathrm{~nm}$

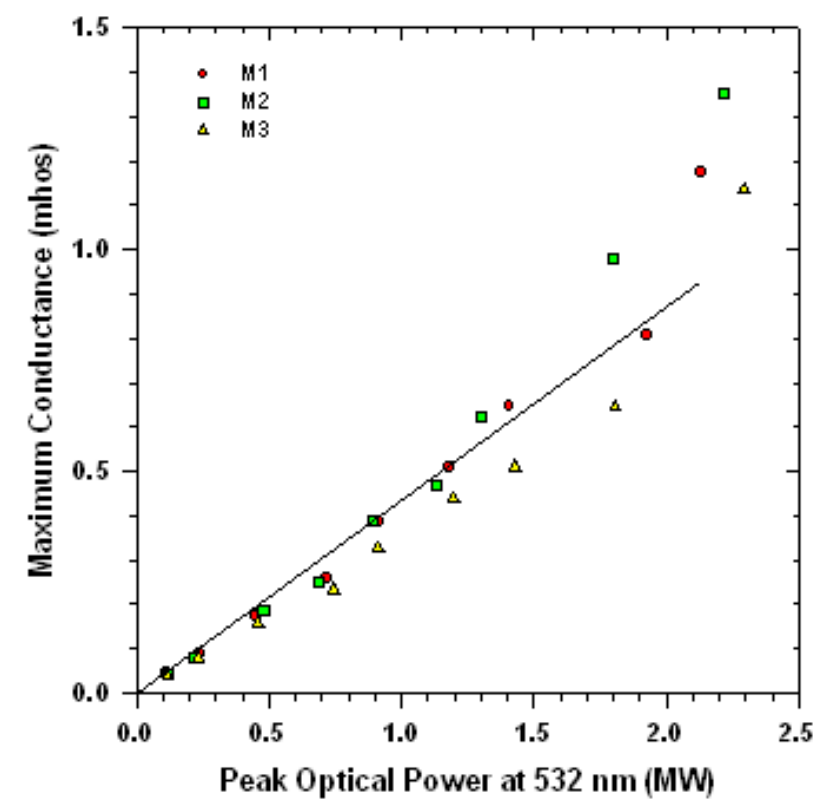

Figure 8.36. Maximum conductance of the third generation switches $m 1, m 2$, and $m 3$ plotted as a function of peak applied optical power at $532 \mathrm{~nm}$.

little difference in the performance of the $\mathrm{m} 1, \mathrm{~m} 2$, and $\mathrm{m} 3$ switches. All the third generation $6 \mathrm{H}$ $\mathrm{SiC}$ switches have a lower on resistance for a given peak optical power at $532 \mathrm{~nm}$ than either of 
the second generation switches, A and B. Figure 8.36 is a plot of the maximum switch conductance of $\mathrm{m} 1, \mathrm{~m} 2$, and $\mathrm{m} 3$ as a function of peak applied optical power at $532 \mathrm{~nm}$. The maximum conductance appears to be a linear function of peak optical power for peak optical powers below 1.3 MW. There is a fair amount of scatter in the maximum conductance data at peak optical powers above 1.3 MW. The minimum switch on resistance falls below 2.0 ohms for peak optical powers greater than 1.3 MW. The accuracy of the calculation to obtain minimum resistance decreases as the minimum resistance gets to very low values. This occurs because we are calculating the voltage division between the load resistance and switch resistance at the peak value of switch current. The switch voltage is taken to be the difference between the test circuit charge voltage and the load voltage. However, the load voltage is approximately equal to the charge voltage at applied optical powers that reduce the switch on resistance to very low values. As a result, small errors in the charge and load voltage measurements can produce excess scatter in the maximum conductance and minimum resistance values. It is our experience that the measurements and calculations are accurate and reliable for minimum resistances down to the 2 - 4 ohm level. In addition, two-photon absorption begins to make a significant contribution to the switch current at optical intensities above $20 \mathrm{MW} / \mathrm{cm}^{2}$ at $532 \mathrm{~nm}$ excitation wavelength.

Figure 8.37 is a comparison of the maximum conductivity for the second (red) and third (blue) generation switches as a function of peak power density at $532 \mathrm{~nm}$. The third generation $6 \mathrm{H}-\mathrm{SiC}$ switches have a higher maximum conductivity than either of the second generation switches for corresponding values of peak power density at $532 \mathrm{~nm}$. The m3 switch, the 'worst' of the third generation switches, has maximum conductivities that are $1.5-1.6$ times higher than the best (switch A) second generation switch. We believe that this is a direct result of the third 


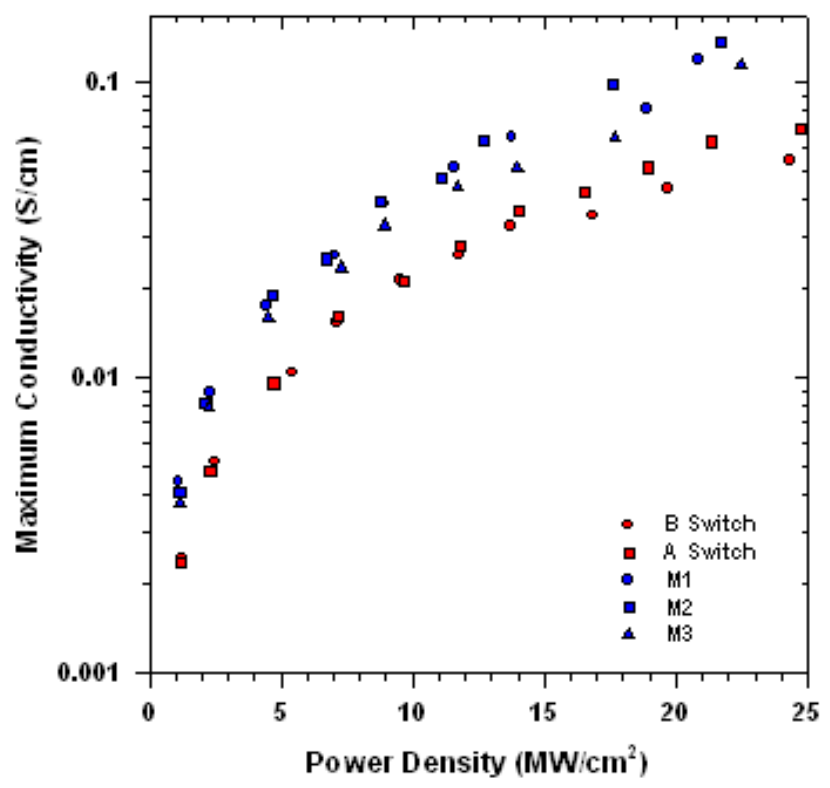

Figure 8.37. Maximum conductivity of the second (red) and third (blue) generation $6 \mathrm{H}-\mathrm{SiC}$ switches plotted versus peak applied optical power density at $532 \mathrm{~nm}$.

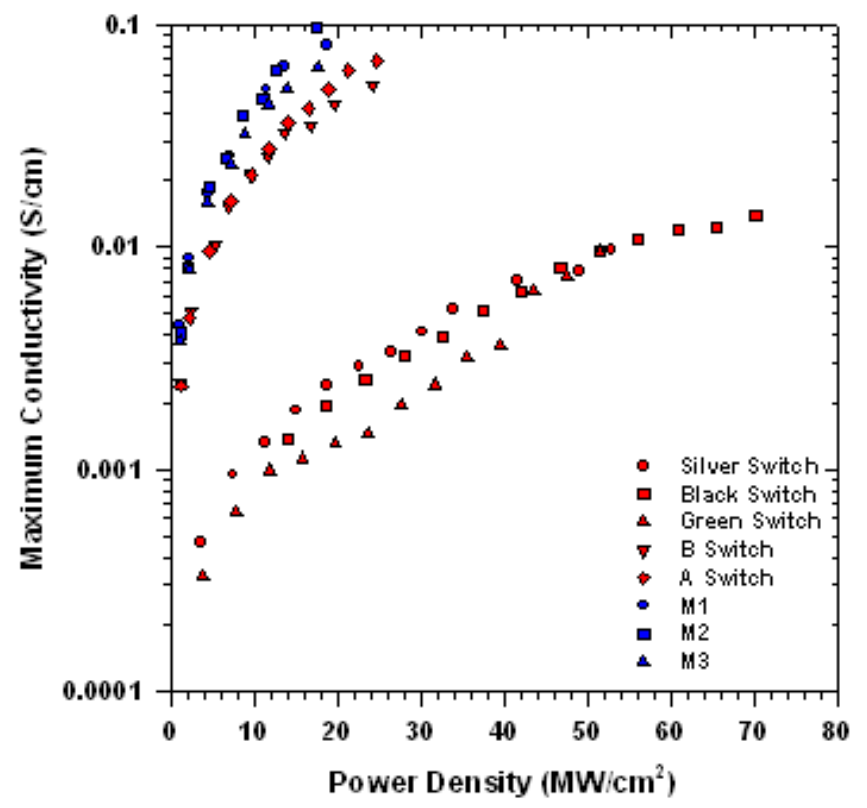

Figure 8.38. Maximum conductivity of first, second and third generation (blue) $6 \mathrm{H}-\mathrm{SiC}$ switches plotted as a function of peak applied optical power density at $532 \mathbf{n m}$.

generation $6 \mathrm{H}-\mathrm{SiC}$ material having a lower (higher) average density of vanadium (nitrogen) that leads to a higher total number of vanadium acceptor levels filled with electrons in the third 
generation material compared to the second generation material. The switching performance of the first, second and third generation $6 \mathrm{H}-\mathrm{SiC}$ switches at the $532 \mathrm{~nm}$ wavelength are compared in Figure 8.38. The poorest performing third generation switch (m3) has a maximum conductivity that is a factor of 25 - 30 times higher than the best performing first generation switch (silver switch) at common values of peak applied power densities at $532 \mathrm{~nm}$. If we assume equal carrier mobility and recombination times in the first and third generation materials, the third generation material has a quantum efficiency that is, at a minimum, $20-45$ times higher than the first generation material.

\section{6) Conclusions}

The testing of three sets of $6 \mathrm{H}-\mathrm{SiC}$ substrates with substantially different vanadium, nitrogen, and boron densities have shown significant differences in their extrinsic photoconductive switching properties at 1064 and $532 \mathrm{~nm}$. Extrinsic photoconductive switching performance has been greatly improved in $6 \mathrm{H}-\mathrm{SiC}$ by increasing the net donor density $(\mathrm{N}-\mathrm{B})$ while maintaining semi-insulating properties with vanadium compensation. Quantum efficiencies - switch efficiencies of the 6H-SiC extrinsic switch were increased by a factor of 20

- 50 at $532 \mathrm{~nm}$ excitation. The improvement in quantum efficiency was accomplished by increasing the $\mathrm{N}$ and $\mathrm{V}$ densities while reducing the $\mathrm{B}$ density compared to initial $6 \mathrm{H}-\mathrm{SiC}$ test substrates. These results establish the capability to design and optimize the performance of extrinsic $6 \mathrm{H}-\mathrm{SiC}$ photoconductive switches by controlling the doping profiles of nitrogen, vanadium and boron. In addition, no micropipes were detected in the third generation $6 \mathrm{H}-\mathrm{SiC}$ substrates. The third generation $6 \mathrm{H}-\mathrm{SiC}$ substrates offer low on resistance and high maximum conductivity with virtually no micropipes. The third generation $6 \mathrm{H}-\mathrm{SiC}$ substrates appear to be 
device grade substrates that can used to construct high power, extrinsic, photoconductive switches.

The purpose of improving $6 \mathrm{H}-\mathrm{SiC}$ switch performance was to construct a switch that could be used for compact accelerator applications. The switch performance goals stated in Chapter 1 are a minimum on resistance of $1 \mathrm{ohm}$, operating voltage of $15-20 \mathrm{kV}$ and peak currents of greater than 1 kilo-amp. So far, we have only described low voltage testing of the third generation $6 \mathrm{H}-\mathrm{SiC}$ switch substrates. We will describe high voltage, high current tests of the third generation material in the following chapter.

\section{7) References}

8.1) J. R. Jenny, et al, "Electrical and optical investigation of the position of the vanadium related defects in the $4 \mathrm{H}$ and $6 \mathrm{H} \mathrm{SiC}$ bandgaps”, Materials Research Society Symposium - Proceedings, Vol. 423, pp. 507 - 512, 1996.

8.2) G. Tamulaitis, et al., "Carrier lifetime in conductive and vanadium doped 6H-SiC substrates”, Applied Physics Letters, Vol. 84, No. 3, pp. 335 - 337, 2004

8.3) R. H. Bube, Photoelectronic Properties of Semiconductors, pp. 9 - 10, Cambridge University Press, 1992.

8.4) V. Gavryushin, "On the possibility of the direct study of local electron - phonon interaction in semiconductors”, JETP Letters, Vol. 78, No. 5, pp. 309 - 313, 2003.

8.5) V. Gavryushin, et al, "Characterization of intrinsic and impurity deep levels in ZnSe and ZnO crystals by nonlinear spectroscopy”, Journal of Crystal Growth, Vol. 138, pp. 924 933, 1994. 


\section{Chapter 9: High Voltage, High Power Testing of the Third Generation, 6H- SiC, Extrinsic Photoconductive Switch}

In the previous chapters, we have demonstrated the feasibility of $6 \mathrm{H}-\mathrm{SiC}$ and $2 \mathrm{H}-\mathrm{GaN}$ extrinsic photoconductive switches, identified a method of improving the quantum efficiency of vanadium compensated $6 \mathrm{H}-\mathrm{SiC}$, and demonstrated improved quantum and switching efficiency of a vanadium compensated $6 \mathrm{H}-\mathrm{SiC}$, extrinsic photoconductive switch. The subject of this chapter is to report on the performance of a high voltage, vanadium compensated, improved quantum efficiency, $6 \mathrm{H}-\mathrm{SiC}$, extrinsic photoconductive switch in a high power switching application. Switch design is based on results of chapters 5, 6, 7 and 8. The substrate has improved nitrogen and vanadium dopant levels and the boron and aluminum levels are minimized (third generation $6 \mathrm{H}-\mathrm{SiC}$ material). The nominal size of the substrates is $1 \mathrm{~cm}$ by 1 $\mathrm{cm}$ to allow for sufficient optical penetration and $1 \mathrm{~mm}$ thick so operation at $\geq 16 \mathrm{kV}$ can be achieved.

The two substrates that were used to construct the switches were cut from the $6 \mathrm{H}-\mathrm{SiC}$ crystals m-plane. The m-6 (m-9) substrate is 1.02 (1.01) mm thick, 10.35 (10.31) mm in length along the a-plane and 9.77 (9.94) $\mathrm{mm}$ long along the c-plane. The 9.77 (9.94) mm long facets (cplane facets) were polished to a planar optical finish and the 10.35 (10.31) mm facets (a-plane facets) were ground to a planar finish. The substrates were cleaned and metalized at LLNL. The cleaning process, for the vanadium compensated $6 \mathrm{H}-\mathrm{SiC}$ substrates, is listed below.

1) 3, 10 minute baths performed in an ultrasonic cleaner using acetone, methanol and isopropyl alcohol with $\mathrm{N}_{2}$ blow dry between solvent baths.

2) Wet etch for 5 minutes in a 3:1 $\left(\mathrm{H}_{2} \mathrm{SO}_{4}: \mathrm{H}_{2} \mathrm{O}_{2}\right)$ Piranha etch solution at room temperature, DI $\mathrm{H}_{2} \mathrm{O}$ rinse for 5 minutes, $\mathrm{N}_{2}$ blow dry. 
3) Wet etch in 49\% $\mathrm{HF}$ :DI $\mathrm{H}_{2} \mathrm{O}$ solution for 5 minutes, $\mathrm{DI} \mathrm{H}_{2} \mathrm{O}$ rinse for 5 minutes, $\mathrm{N}_{2}$ blow dry

The clean and etched substrates are placed in the stainless steel mask assembly shown in Figure 9.1. The stainless steel mask assembly consists of the three pieces shown in Figure 9.1, the pocket plate (A), coined plate (B) and top plate (C). The substrates are loaded into the $\sim 1$
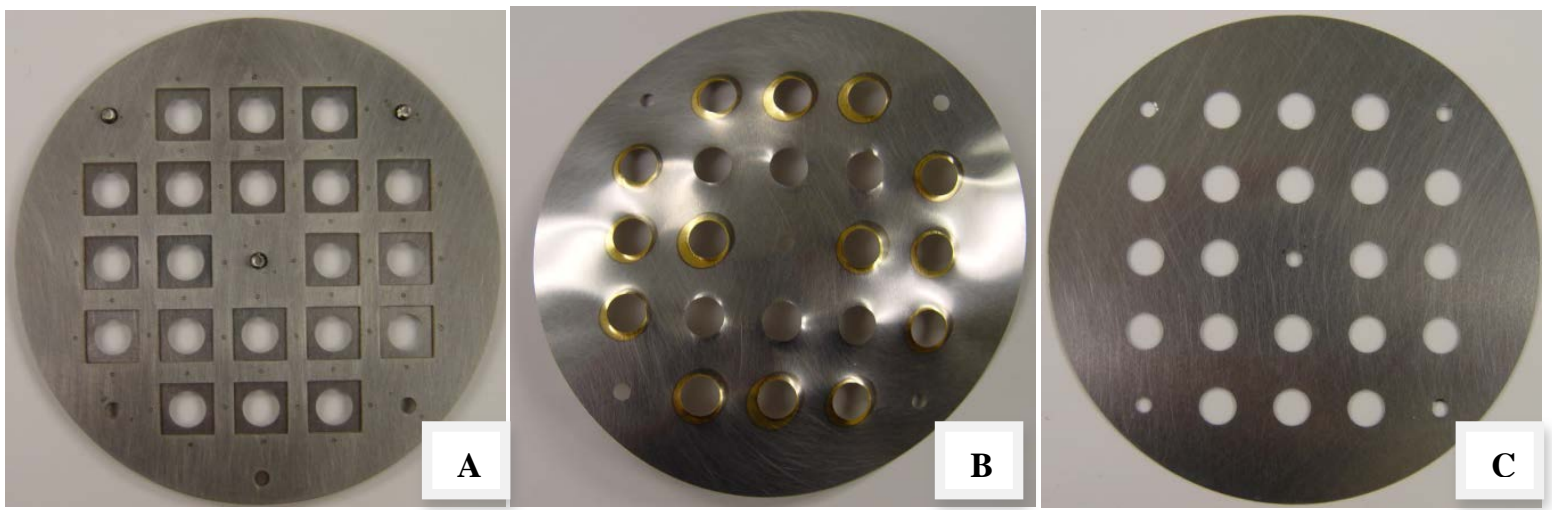

Figure 9.1. The stainless steel mask assembly components

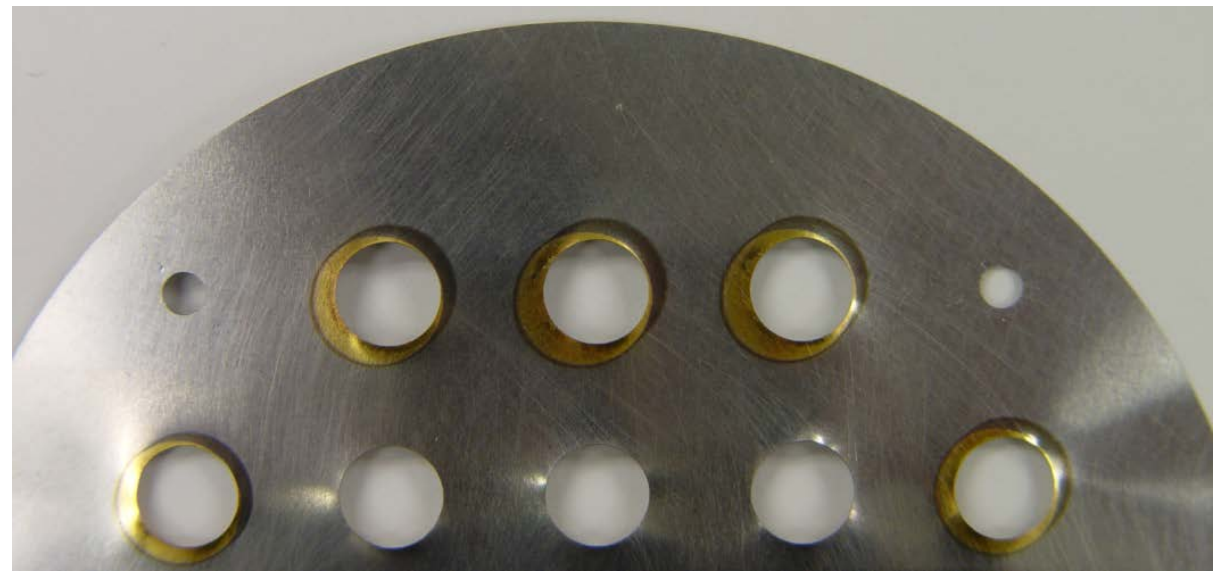

Figure 9.2. Turned in edges of the coined plate used to pin $6 \mathrm{H}-\mathrm{SiC}$ substrates in mask.

mm deep pockets of the pocket plate, are held in place by the coined plate and the coined plate is covered by top plate. The top and pocket plates have $7.5 \mathrm{~mm}$ diameter circular holes that define the electrode metallization on the top and bottom of the $6 \mathrm{H}-\mathrm{SiC}$ substrates. The coined plate also has $7.5 \mathrm{~mm}$ diameter holes that have had the edges of the circular holes turned in (coined) by 
by pressing a ball bearing into them. This turns the edges of the holes inward as shown in Figure 9.2. The turned in edges of the holes in the coined plate pins the $6 \mathrm{H}-\mathrm{SiC}$ substrates against the bottom of the pockets in the pocket plate when the mask assembly is screwed together, preventing the substrates from moving in the mask assembly. The stainless steel mask assembly is three inches in diameter and is mounted in a planetary arrangement that revolves around the metal vapor source of the electron beam evaporation system. The initial metal layer of $100 \mathrm{~nm}$ of nickel is deposited in $7.5 \mathrm{~mm}$ diameter, circular patterns centered on opposing sides of the substrate. The substrates are removed from the stainless steel mask and the nickel electrode patterns are annealed at $1000{ }^{\circ} \mathrm{C}$ for two minutes to form a nickel silicide layer at the substrate surface. The substrate is etched in a room temperature sulfuric acid bath for 30 minutes to remove excess nickel. The sulfuric acid bath is followed by a DI $\mathrm{H}_{2} \mathrm{O}$ bath (5 min.) and $\mathrm{N}_{2}$ blow dry. The substrates are replaced into the stainless steel mask and re-installed in the electron beam evaporation system for depositions of $7.5 \mathrm{~mm}$, circular patterns of $100 \mathrm{~nm}$ of titanium, $200 \mathrm{~nm}$ of platinum and $500 \mathrm{~nm}$ of gold centered on both sides of the substrate. The metalized substrates are switch assemblies that are ready for testing. The first test that is usually performed on the $6 \mathrm{H}-$ $\mathrm{SiC}$ switch assemblies is dark current measurements.

The dark resistance for the metalized m-6 and m-9 substrates is shown in Figure 9.3. The m-6 (m-9) substrate has a dark resistance and resistivity of 1.852e9 (1.418e9) and 1.836e10 (1.439e10) ohm $-\mathrm{cm}$. These results are in good agreement with the dark resistances measured for the $\mathrm{m} 1, \mathrm{~m} 2$, and $\mathrm{m} 3$ substrates (see Figure 8.13 ) that ranged from $1.279 \mathrm{e} 9$ to $1.652 \mathrm{e} 9$ ohms. This is expected since the m-plane substrates share the same doping profile. 


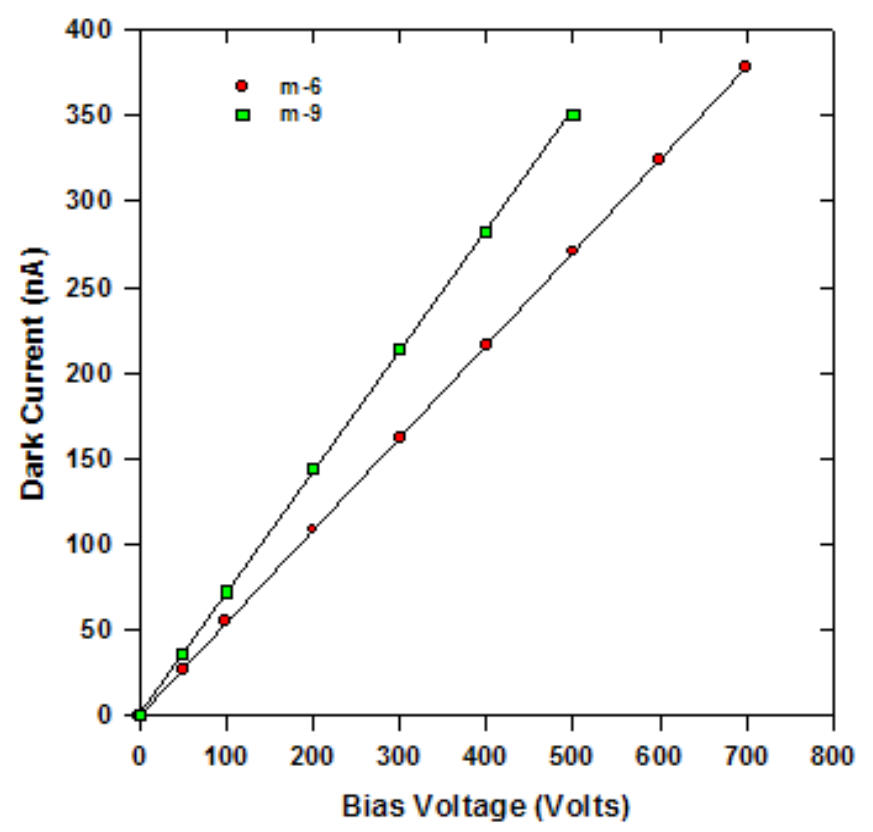

Figure 9.3. Metallized substrates $\mathrm{m}-6$ and $\mathrm{m}-9$ dark current plotted as a function of bias voltage. The dark resistance and resistivity of the $\mathrm{m}-6(\mathrm{~m}-9)$ substrate are $1.852 \mathrm{e} 9(1.418 \mathrm{e} 9)$ ohms and 1.836e10 (1.439e10) ohm $-\mathrm{cm}$.

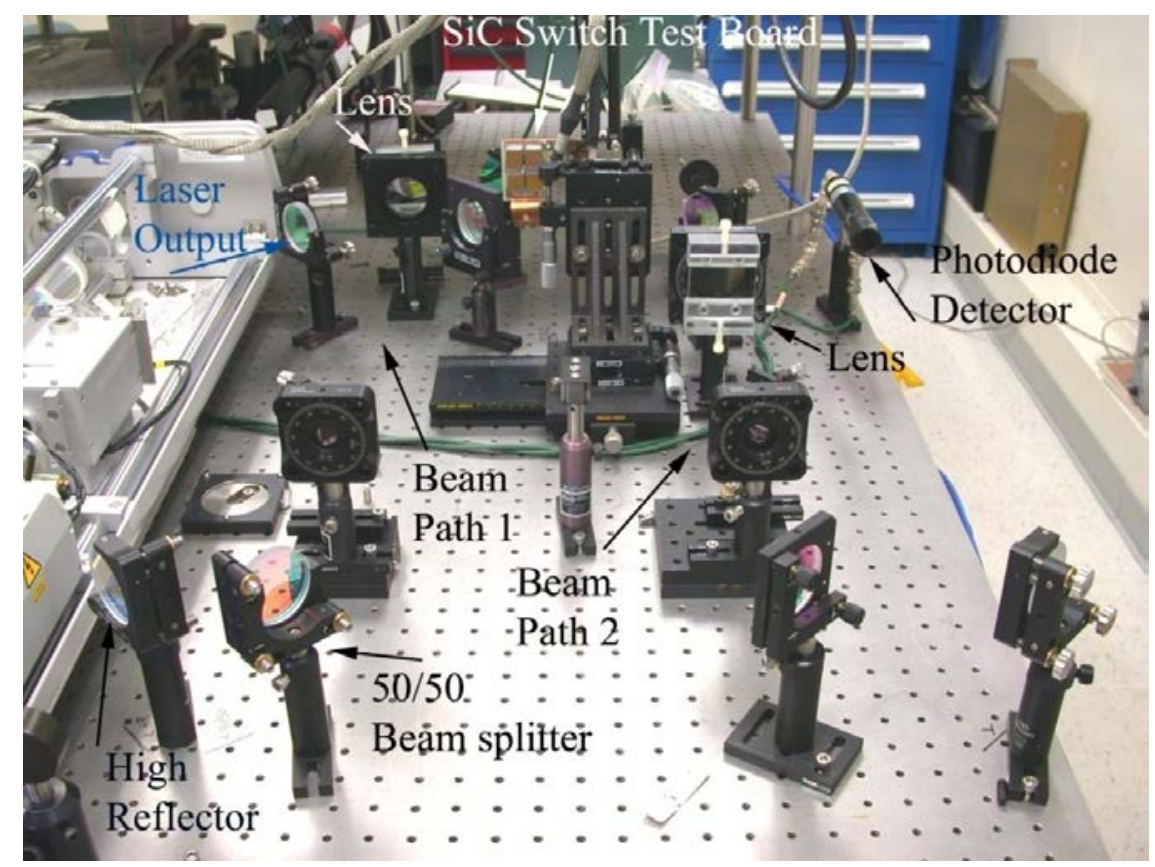

Figure 9.4. Optical layout for the application of optical pulses at opposing facets on the $m-6$ switch. 
The photoconductive switches constructed with the m-6 and m-9 substrates were tested in the modified low voltage test circuit (Figures 8.15 - 8.17). Optical pulses of equal intensity were applied across the central $9 \mathrm{~mm}$ wide portion of opposing facets along the a-plane $(10.35 \mathrm{~mm}$ long substrate edge). The optical pulses slightly overfilled the $1.02 \mathrm{~mm}$ thick facet. The optical system layout for applying optical pulse at two opposing switch facets is shown in Figure 9.4. The low voltage photoconductive switch tests were performed at a charge voltage of 270 volts for an optical energy (peak power) range of $1 \mathrm{~mJ}(0.0975 \mathrm{MW})$ per facet to $20 \mathrm{~mJ}$ (2.013 MW) per facet at $532 \mathrm{~nm}$. Figure 9.5 is an overlay of five switch photocurrent and optical pulse waveforms for a peak optical power of 2.013 MW applied at two opposing facets. The optical pulse width is 8.8 ns FWHM. The calculated value of the minimum switch resistance from the data of Figure 9.5 is 660 milliohms.

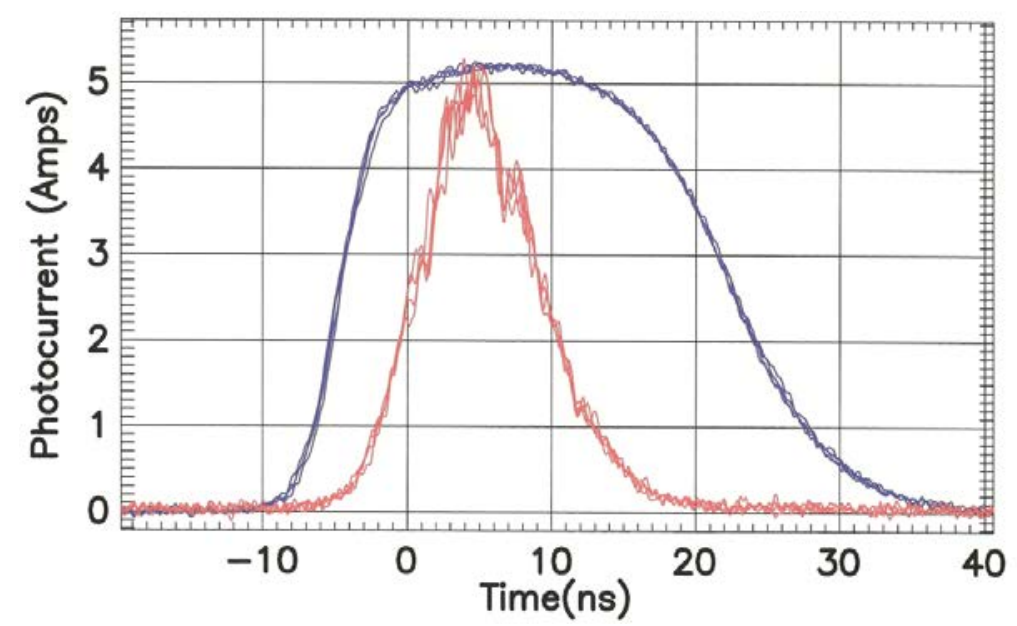

Figure 9.5. Plots of photocurrent and optical intensity waveforms for $m-6$ switch for operation at charge voltage of 270 volts and peak optical intensity of $4.026 \mathrm{MW}$ at $532 \mathrm{~nm}$ (corresponds to $20 \mathrm{~mJ}$ at opposing ground facets). 


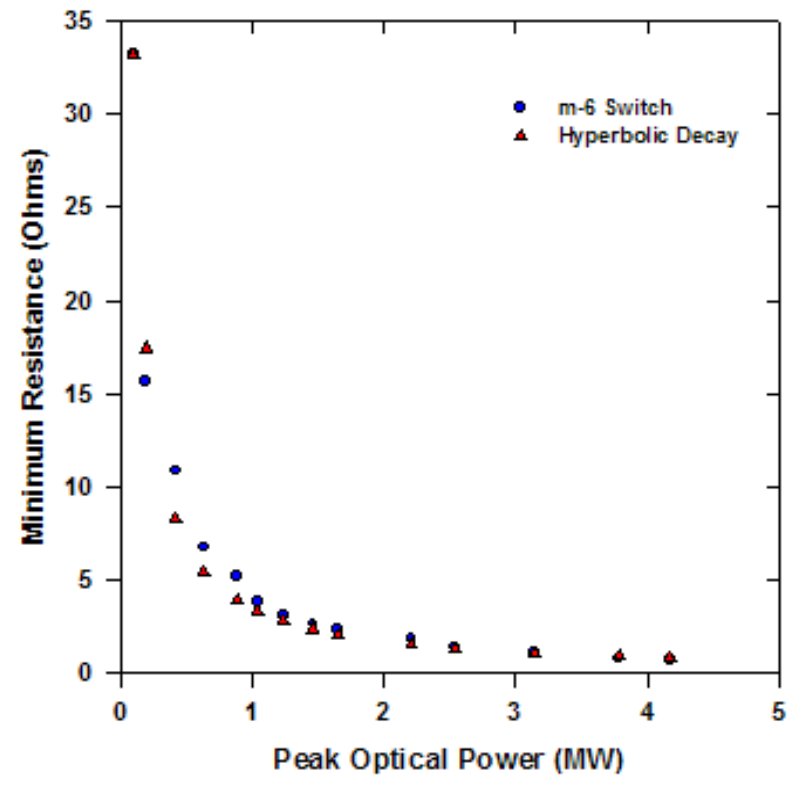

Figure 9.6. Minimum resistance of the $\mathrm{m}-6$ switch plotted as a function of total applied peak optical power (sum of peak powers applied to two opposing facets).

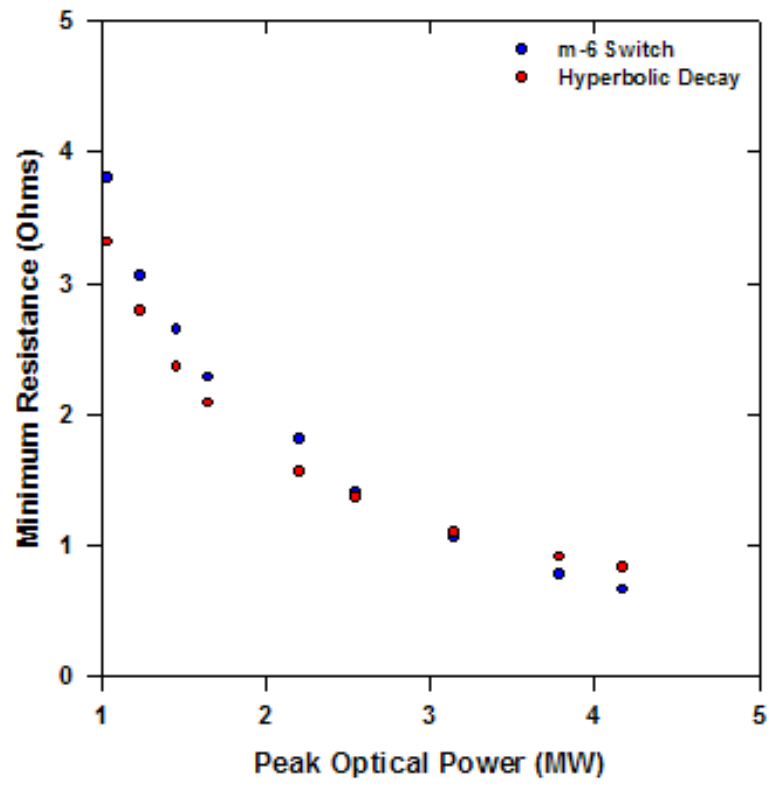

Figure 9.7. Expanded view of the of the m-6 minimum resistance data plotted in Figure 9.6. The minimum on resistance becomes sub-ohmic between 3 and $4 \mathrm{MW}$ of total applied peak optical power.

The minimum switch resistance of the m-6 switch plotted versus total applied optical power is shown in Figures 9.6 and 9.7. Figure 9.7 is an expanded view of the data in Figure 9.6. 
The minimum resistance of the m-6 switch becomes less than an ohm at peak optical powers greater than $3 \mathrm{MW}$ at $532 \mathrm{~nm}$.

The maximum switch conductance for the m-6 switch is plotted as a function of total peak optical power in Figure 8.8. A peak maximum conductance of 1.51 mhos is obtained at a peak applied optical power of 4.17 MW. The maximum conductance plot starts to become supralinear at peak applied optical powers greater than $3 \mathrm{MW}$ at $532 \mathrm{~nm}$, the point where the calculated resistance falls below $1 \mathrm{ohm}$. There are two mechanisms that contribute to the switch conductance becoming supralinear. First, two-photon absorption begins to make a measurable contribution to carrier generation at high optical intensities $(\beta=3.2 \mathrm{~cm} / \mathrm{GW})$. The 3 MW peak optical power corresponds to a peak optical intensity of $15 \mathrm{MW} / \mathrm{cm}^{2}$. Carrier generation by twophoton absorption is proportional to the square of the optical intensity and will cause the switch

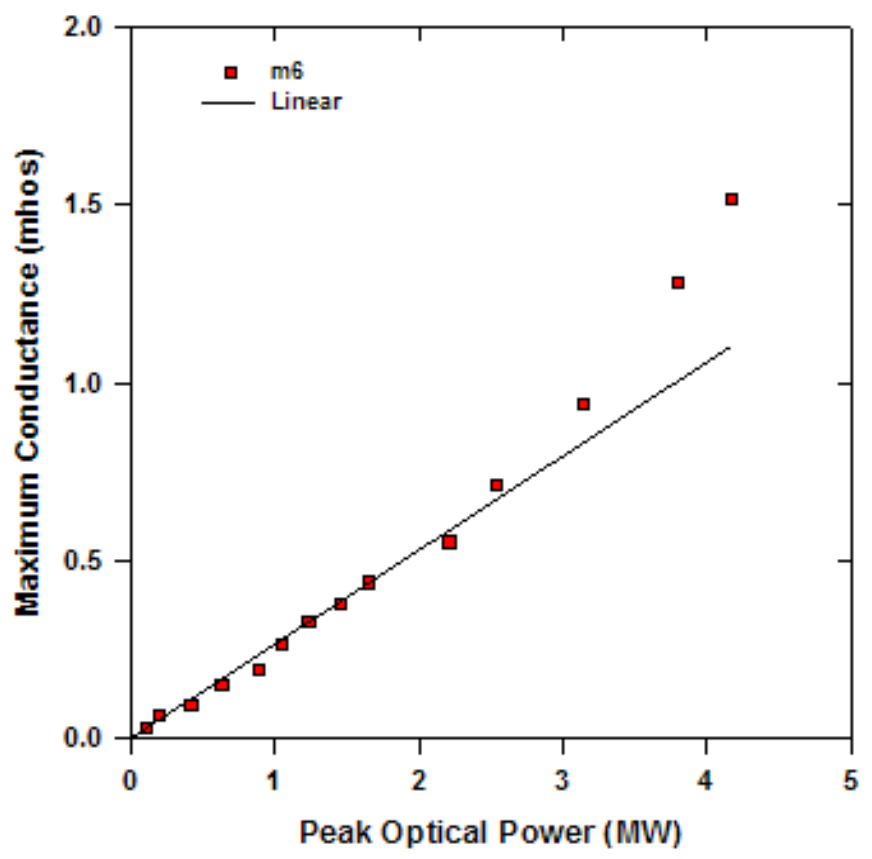

Figure 9.8. The maximum conductance of the $m-6$ switch plotted as a function of peak applied optical power at $532 \mathrm{~nm}$. The maximum conductance becomes superlinear at peak powers greater than $3 \mathrm{MW}$. 
conductance to have a supralinear component. Second, the calculated switch resistance results become somewhat unreliable at resistances below 2 - 3 ohms. This occurs since we are subtracting the load voltage from the charge voltage, which are very close in value at high optical intensities. The voltage probes used for the load and charge voltage measurements both have an error of 2 percent. The precise value of the calculated switch resistance cannot be accurately obtained when the switch resistance falls below a value of a few percent of the load resistance (50.8 ohms), or, 2 - 3 ohms.

\section{1) High Voltage Tests}

The high voltage test board of Figures 5.11 and 5.12 was modified to achieve operation at charge voltages of $16 \mathrm{kV}$. This was accomplished by replacing the $56 \mathrm{nF}, 4 \mathrm{kV}$, ceramic chip capacitors of the high voltage board with $3.3 \mathrm{nF}, 10 \mathrm{kV}$ ceramic chip capacitors. The modified board is shown in Figures 9.9 - 9.11. Figure 9.9 is the top view of the modified board showing six sets of two series connected $3.3 \mathrm{nF}, 10 \mathrm{kV}$ capacitors, connected in parallel for a total board storage capacitance of $9.8 \mathrm{nF}$ at $20 \mathrm{kV}$. The copper sheet conductors connect the high voltage side of the storage capacitors to the anode of the m-6 switch assembly. The load resistors on the modified high voltage board were also replaced with non-inductive, ceramic resistors with repetitive high voltage pulse rating of $8 \mathrm{kV}$. Figure 8.10 shows the load resistors mounted on the bottom side of the board. The load resistor is constructed from ten parallel sets of two 51 ohm resistors connected in series to produce an effective load resistance of 10.2 ohms. The copper pad located in the center of the bottom of the board makes connection to the cathode of the m- 6 switch assembly. The m-6 switch mounted on the board is shown in the side view of Figure 9.11. The modified high voltage test board is submerged in a Flourinert FC-77 bath to prevent surface tracking around the edge of the switch. The board storage capacitance is pulse charged to full 


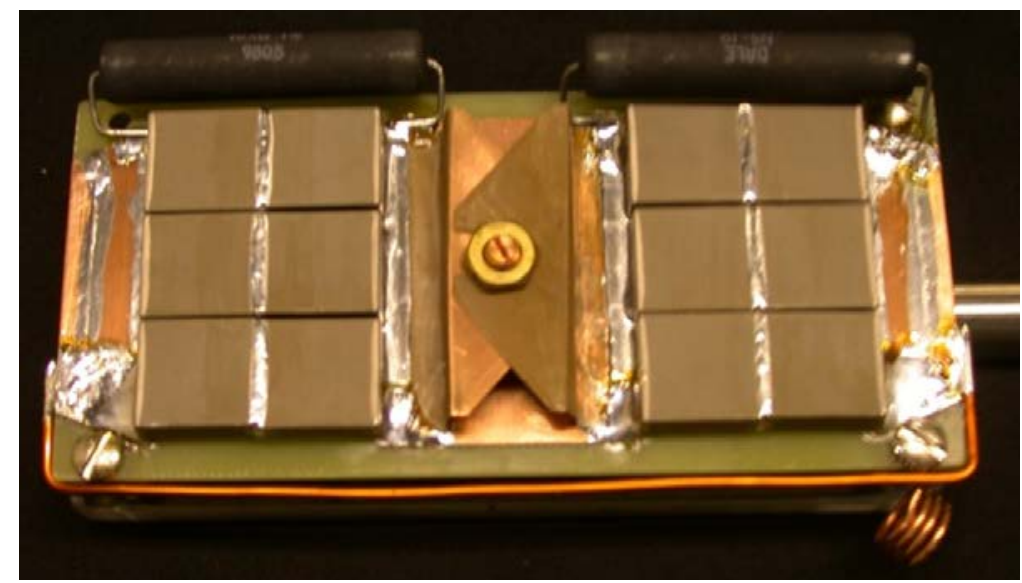

Figure 9.9. Top of the modified high voltage board showing storage capacitor bank and copper sheet connections to the anode of the m-6 switch assembly.

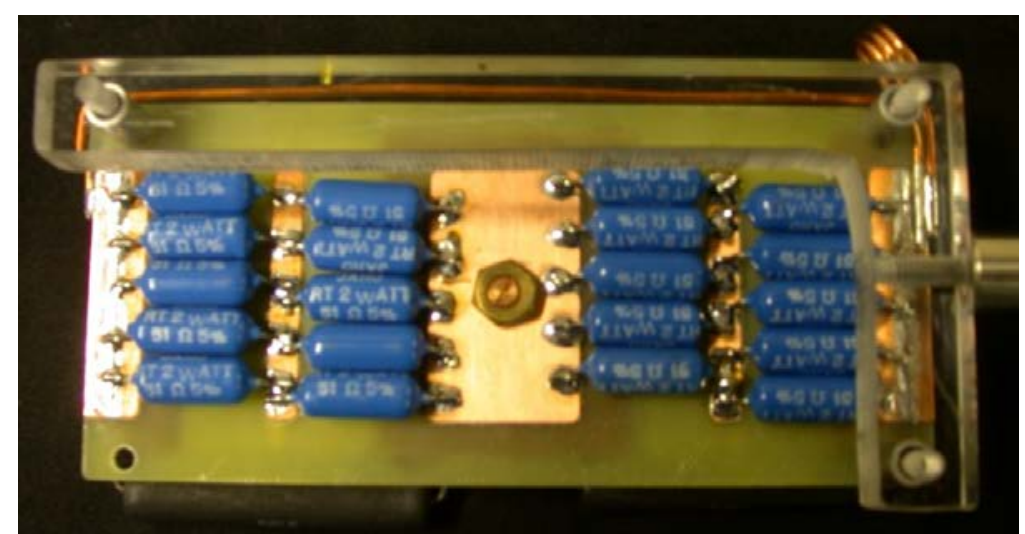

Figure 9.10. Bottom side of modified high voltage board showing load resistor assembly

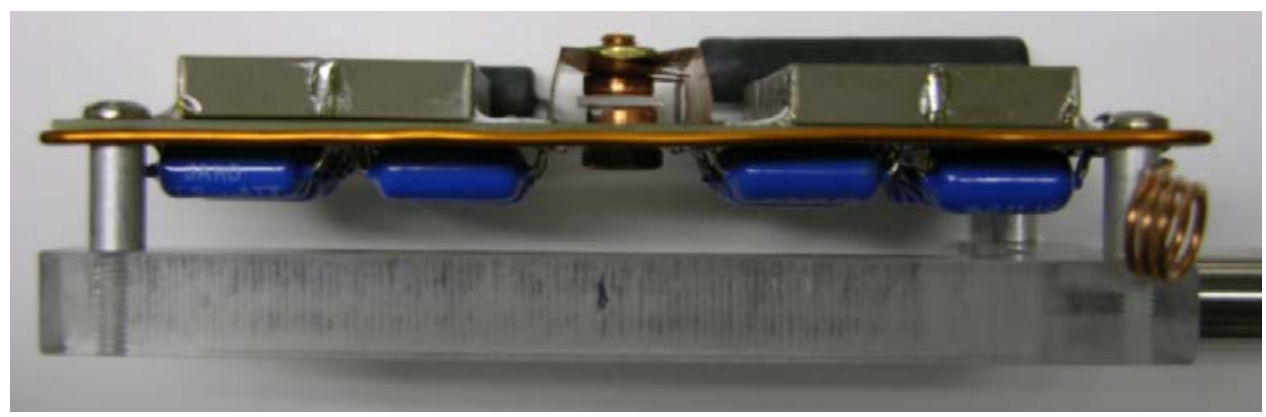

Figure 9.11. Side view of modified high voltage board shows capacitor bank (top), load resistor (bottom) and m-6 switch mounted on board. Optical pulse is applied to the facet shown in figure and to opposite facet of m-6 switch.

voltage in $1 \mu \mathrm{s}$. High voltage and current tests were performed on the m- 6 switch assembly up to a charge voltage of $17 \mathrm{kV}$. The charge voltage on the high voltage test board storage capacitor 
for a peak charge voltage of $16.5 \mathrm{kV}$ is shown in Figure 9.12. The voltage unit in Figure 9.12 is 5 $\mathrm{kV} /$ div. The switch is optically triggered at the peak of the charge voltage which is $16.5 \mathrm{kV}$. The charge voltage is measured with a Tektronix P6015 high voltage probe. This probe has a 4 ns rise time and a bandwidth $<50 \mathrm{MHz}$, so the charge voltage signal after the $6 \mathrm{H}-\mathrm{SiC}$ is triggered is somewhat suspect. There is a slight temporal offset between the charge voltage and

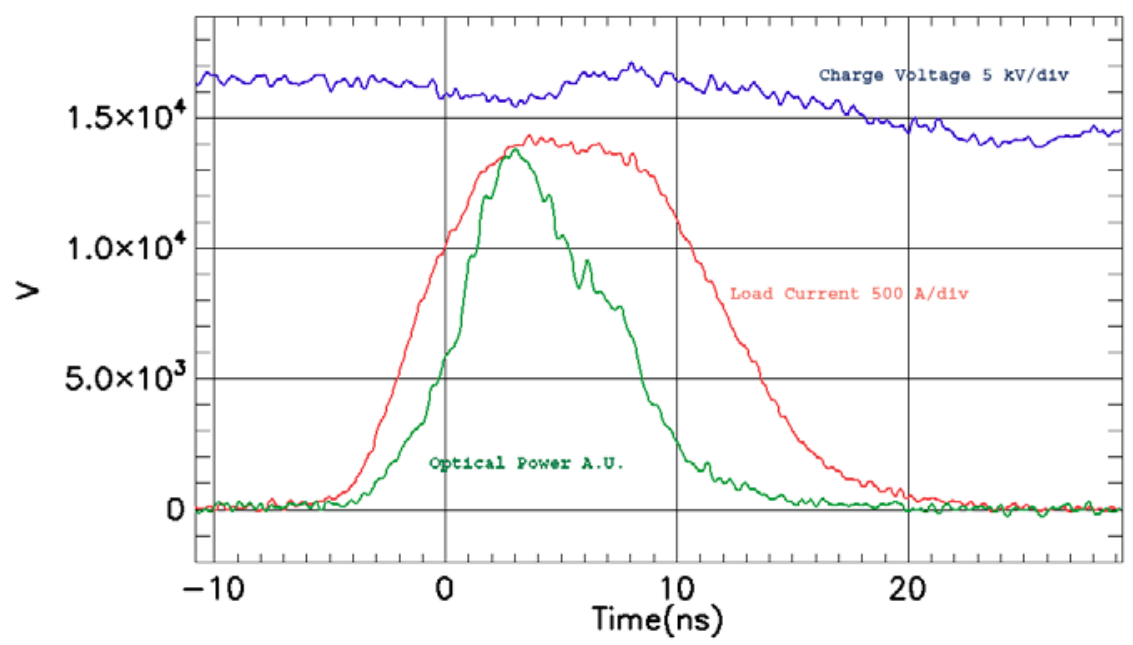

Figure 9.12. Charge voltage waveform at the time the m-6 photoconductive switch is fired. The units of the $y$-axis are $5 \mathrm{kV} / \mathrm{div}$. The charge voltage was measured with a Tektronix P6015 high voltage probe. There is a slight time shift between the voltage and current/optical pulses due to different cable delays.

photocurrent/optical pulses due to different cable delays. The photocurrent and optical pulse associated with the charge voltage of Figure 9.12 are also shown. The peak current is 1400 amperes for a total peak optical power of 4.9 MW (2.45 MW applied to two facets). Figure 9.13 is an overlay of five photocurrent and optical power waveforms for a peak charge voltage of 16.5 $\mathrm{kV}$ and a total peak applied optical power of $4.9 \mathrm{MW}$ at $532 \mathrm{~nm}$. It is difficult to calculate the minimum switch resistance because there is significant voltage droop on the charge capacitors during current flow through the photoconductive switch. This is due to an increase in charge 
being removed from the storage capacitors on the modified high voltage board as a result of higher operating voltages and a load resistance that has been reduced to one fifth the resistance

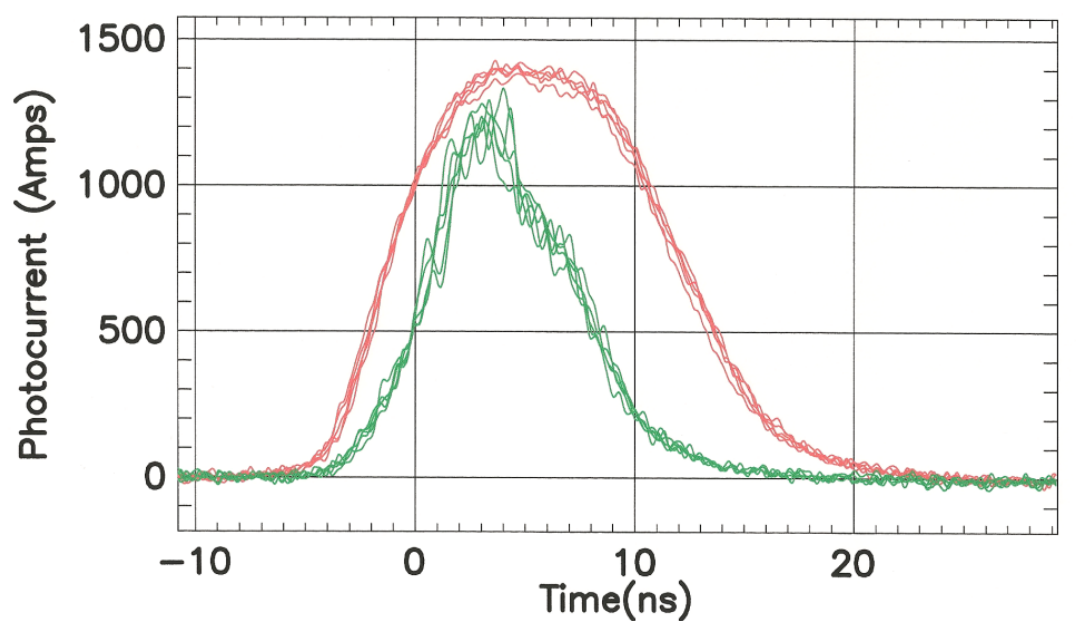

Figure 9.13. Photocurrent and optical pulse plotted as functions of time for a charge voltage of $16.5 \mathrm{kV}$ and total applied optical energy of $4.9 \mathrm{MW}$ at $532 \mathrm{~nm}$.

(10.2 ohms compared to 50.2 ohms) of the original high voltage board. The problem is compounded since the storage capacitor of the modified high voltage board was constructed using higher voltage, but lower capacitance, ceramic capacitors. This resulted in a storage capacitor of $9.8 \mathrm{nF}$ for the modified high voltage board compared to $112 \mathrm{nF}$ for the original high voltage board.

An estimate of the efficiency of the $6 \mathrm{H}-\mathrm{SiC}$ photoconductive switch can be obtained by comparing the energy delivered to the $10.2 \mathrm{ohm}$ load and the energy lost in the storage capacitor. The charge voltage on the modified high voltage board just before and after switch conduction is shown in Figure 9.14 for a peak charge voltage of $16.54 \mathrm{kV}$. The voltage droop in the capacitor voltage appears as step change in the charge voltage occurring just at the zero time mark in Figure 9.14. The duration of photoconductive switch current pulse is $~ 30$ ns as shown in Figure 9.13. The initial capacitor voltage at the time the $6 \mathrm{H}-\mathrm{SiC}$ photoconductive switch is fired is 16.54 $\mathrm{kV}$. The final capacitor voltage, thirty nanoseconds later, is $15 \mathrm{kV}$, obtained by averaging the oscillations on the capacitor voltage signal. 


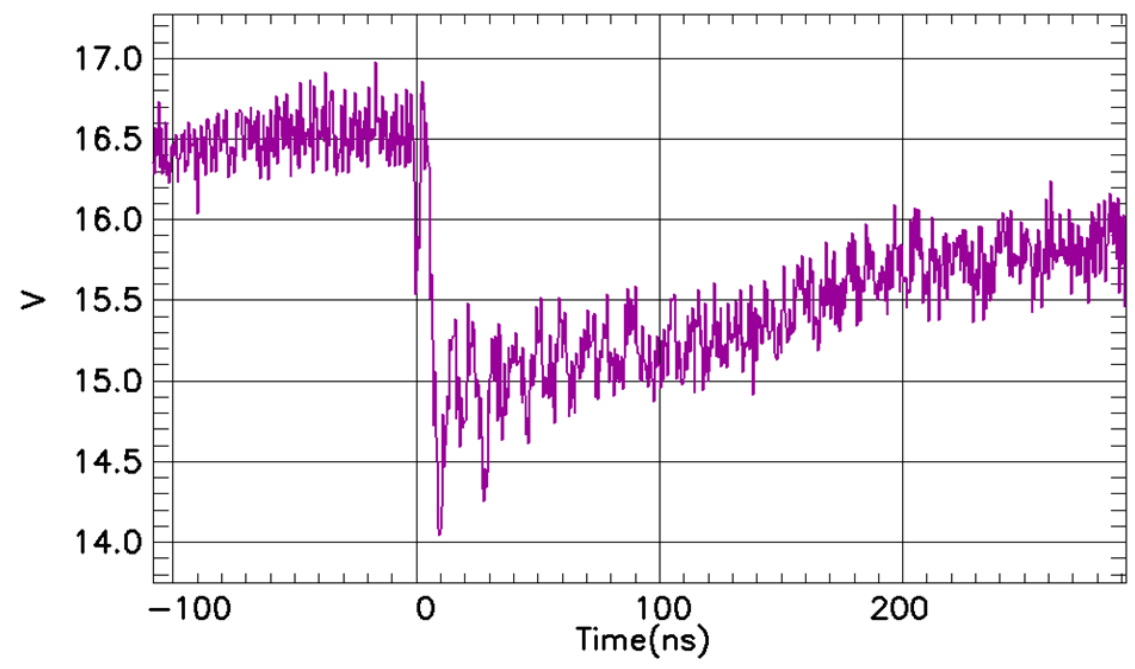

Figure 9.14. Charge voltage (storage capacitor voltage) waveform plotted versus time for time interval just before and after $6 \mathrm{H}$-SiC photoconductive switch current conduction. The voltage scale is in $\mathbf{5 0 0}$ volts per division.

The switch efficiency can be estimated by comparing the energy lost from the storage capacitor to the energy transmitted to the load resistance. The energy lost from the storage capacitor is expressed in equation (9.1). Where $\mathrm{V}_{\mathrm{i}}$ and $\mathrm{V}_{\mathrm{f}}$ are the

$$
E_{l o s t}=C \frac{\left(V_{i}^{2}-V_{f}^{2}\right)}{2}
$$

storage capacitor voltages at the beginning and end of the photoconductive switch pulse, respectively. Substituting the values listed above for $V_{i}$ and $V_{f}$ listed above we obtain a value of $0.238 \mathrm{~J}$ for $\mathrm{E}_{\text {lost }}$.

The energy dissipated in the 10.2 ohm resistive load can be calculated using equation (9.2).

$$
E_{\text {load }}=\int_{t_{i}}^{t_{f}} I_{\text {load }}^{2}(t) R_{\text {load }} d t
$$


Where $t_{i}$ and $t_{f}$ are the times that mark the beginning and end of the load current $\left(\mathrm{I}_{\text {load }}\right)$ and $\mathrm{R}_{\text {load }}$ is the $10.2 \mathrm{ohm}$ resistive load. The current waveform corresponding to the $16.54 \mathrm{kV}$ charge pulse was numerically integrated and $E_{\text {load }}$ was calculated to be $0.21 \mathrm{~J}$. The switch efficiency is the ratio of $\mathrm{E}_{\text {load }}$ and $\mathrm{E}_{\text {lost}}$, or in this case, $0.882(88.2 \%)$. The missing $11.8 \%$ of $\mathrm{E}_{\text {lost }}$ is assumed to be dissipated in the $6 \mathrm{H}-\mathrm{SiC}$ photoconductive switch. This is a reasonable switch efficiency considering the rate of rise and fall of the optical trigger pulse (see Figure 9.13). The applied optical power is less than $3 \mathrm{MW}$ for the first 5.5 ns and the last 10 ns of the pulse. The applied optical power was greater than $3 \mathrm{MW}$ for 6 ns of the pulse duration. The peak optical power of 3 MW was the point when the minimum resistance of the switch used in this test (m-6) reached 1 ohm (see Figure 8.7). As a result, the switch spends a fair amount of time with a resistance greater $\geq 1 \mathrm{ohm}$ and a shorter period with a resistance $<1 \mathrm{ohm}$, ending up with an efficiency of 88.2 \%. We can estimate the minimum switch resistance by numerically integrating the load current with respect to time up to the point that the load current reaches its maximum. This integral is the charge removed from the storage capacitor. The storage capacitor voltage droop at the time the load current reaches its maximum is calculated using equation (9.3).

$$
\Delta Q=\int_{0}^{\Delta t} I_{\text {load }}(t) d t=C \Delta V_{c}
$$

Where $\mathrm{C}$ is the value of the storage capacitor on the modified high voltage board (9.8 $\mathrm{nF})$ and $\Delta \mathrm{V}_{\mathrm{c}}$ is the droop of the capacitor voltage representing the decrease from its starting value (16.54 $\mathrm{kV}$ ). The numerical integration of the load current for a peak charge voltage of $16.54 \mathrm{kV}$ yielded a value of $7.841 \mu \mathrm{C}$ for $\Delta \mathrm{Q}$. A value of 800.2 Volts is obtained for $\Delta \mathrm{V}_{\mathrm{c}}$ by dividing $\Delta \mathrm{Q}$ by the value of the storage capacitor $(9.8 \mathrm{nF})$. The capacitor voltage at the point in time that the load current reaches its peak value is $15.74 \mathrm{kV}$ that is obtained by subtracting the droop (800.2 Volts) 
from the starting voltage $(16.54 \mathrm{kV})$. The circuit resistance of 11.23 ohms is obtained by dividing $15.74 \mathrm{kV}$ by the peak current 1401 Amps. The minimum switch resistance of 1.03 ohms is obtained by subtracting the load resistance of 10.2 ohms from the minimum circuit resistance of 11.23 ohms.

The photoconductive switch fabricated from the m6 substrate accumulated $\sim 200$ pulses at charge voltages ranging from 15 to $17 \mathrm{kV}$ and 300 to 400 pulses with currents in excess of 1 kA without any discernable damage, or degradation in performance. The extrinsic photoconductive switches fabricated with the third generation vanadium and nitrogen doped 6H$\mathrm{SiC}$ material has proven to be a high voltage, high power, compact switch capable of operation at high electric field gradient. The remaining development of this switch lies in electrode design and switch packaging to extend its operating range to $50 \mathrm{kV}$ and higher.

\section{2) Conclusions}

We started the development of vanadium compensated, 6H-SiC extrinsic photoconductive switches with switch performance goals of $\geq 15 \mathrm{kV}$ operating voltage, $\geq 1.0 \mathrm{kA}$ pulsed current and a minimum switch on resistance of $1 \mathrm{ohm}$. We have achieved all these goals with the third generation, vanadium compensated, $6 \mathrm{H}-\mathrm{SiC}$ material. The operating voltage, peak current, and minimum on resistance goal were all met simultaneously. The operating voltage and peak pulsed current goals were exceeded without special geometries to relieve peak switch electric fields, or reduce switch current density. The goal of a $1 \mathrm{ohm}$ minimum on resistance was achieved for $1 \mathrm{~mm}$ thick, $1 \mathrm{~cm}^{2}$ substrates by improving the quantum and switching efficiency through judicious control of the vanadium, nitrogen and boron impurity densities in the $6 \mathrm{H}-\mathrm{SiC}$ 
substrates. The changes in impurity doping profiles led to a factor of 50 improvement in switch performance of the third generation $6 \mathrm{H}-\mathrm{SiC}$ material compared to the initial material.

The third generation $6 \mathrm{H}-\mathrm{SiC}$ material has been verified as a material that can be used to construct high voltage, high power, compact, extrinsic photoconductive switches ready for application in compact accelerators. However, this is only the first step. Switch reliability, scaling to higher operating voltages, and improved methods of coupling the optical trigger pulse to the switch facets are subjects that are important for the development of a commercial extrinsic photoconductive switch. The next chapter will contain some recommendations for further development of the vanadium compensated, $6 \mathrm{H}-\mathrm{SiC}$, extrinsic photoconductive switch. 


\section{Chapter 10: Conclusions and Recommendations}

\section{1) Conclusions}

The successful development of a vanadium compensated, $6 \mathrm{H}-\mathrm{SiC}$ extrinsic photoconductive switch for use as a closing switch for compact accelerator applications was realized by improvements made to the vanadium, nitrogen and boron impurity densities. The changes made to the impurity densities were based on the physical intuition outlined in Chapter 6 and the rate equation models of Chapter 7. The final impurity 'recipe' calls for vanadium, nitrogen and boron densities of $2.5 \mathrm{e} 17 \mathrm{~cm}^{-3}, 1.25 \mathrm{e} 17 \mathrm{~cm}^{-3}$ and $\leq 1 \mathrm{e} 16 \mathrm{~cm}^{-3}$, respectively. This recipe was originally developed to maximize the quantum efficiency of the vanadium compensated $6 \mathrm{H}-\mathrm{SiC}$, while maintaining a thermally stable semi-insulating material. The rate equation models described in Chapter 7 indicate that, besides increasing the quantum efficiency, the impurity recipe should be expected to also increase the carrier recombination time.

The third generation vanadium compensated $6 \mathrm{H}-\mathrm{SiC}$ has average impurity densities close to the recipe values. Extrinsic photoconductive switches constructed from the third generation vanadium compensated, $6 \mathrm{H}-\mathrm{SiC}, 1 \mathrm{~mm}$ thick, $1 \mathrm{~cm}^{2}$, substrates have achieved high power operation at $16 \mathrm{kV}$ with pulsed currents exceeding 1400 Amperes and a minimum on resistance of $1 \mathrm{ohm}$. These results are in excess of the switching goals outlined in Chapter 1 . The extrinsic photoconductive switch performance of the third generation $6 \mathrm{H}-\mathrm{SiC}$ material was improved by a factor of 50 for excitation at the $532 \mathrm{~nm}$ wavelength compared to the initial $6 \mathrm{H}$ $\mathrm{SiC}$ material. Switches based on this material have been incorporated into a prototype compact proton medical accelerator being developed by the Compact Particle Acceleration Corporation (CPAC) [10.1] 
The vanadium compensated, $6 \mathrm{H}-\mathrm{SiC}$, extrinsic photoconductive switch operates differently when excited by 1064, or $532 \mathrm{~nm}$, wavelength light. The 6H-SiC extrinsic photoconductive switch is a unipolar device when excited with $1064 \mathrm{~nm}$ light. The carriers are electrons excited from filled vanadium acceptor levels and other electron traps located within $1.17 \mathrm{eV}$ of the conduction band. The switch is bipolar at $532 \mathrm{~nm}$ since the carriers consist of holes, as well as electrons. The holes are primarily generated by the excitation of valence band electrons into empty trap/acceptor levels and by two-photon absorption. Carrier generation by two-photon absorption becomes more important at high applied optical intensity at $532 \mathrm{~nm}$ and contributes to the supralinear behavior of switch conductance as a function of optical power.

We believe that the vanadium compensated $6 \mathrm{H}-\mathrm{SiC}$ material is trap dominated at low nitrogen to vanadium ratios. The trap dominated vanadium compensated $6 \mathrm{H}-\mathrm{SiC}$ exhibits low quantum efficiency when excited with 1064 and $532 \mathrm{~nm}$ light and has a carrier recombination time of $\sim 150-300$ ps. The vanadium compensated $6 \mathrm{H}-\mathrm{SiC}$ transitions to an impurity dominated material as the ratio of nitrogen to vanadium is increased to 0.5 . The increased nitrogen doping produces a material with much higher quantum efficiency and carrier recombination time of 0.9 to $1.0 \mathrm{~ns}$.

The iron compensated $2 \mathrm{H}-\mathrm{GaN}$ did not perform well as an extrinsic photoconductive switch. The density of carriers generated at $1064 \mathrm{~nm}$ was, low indicating that there were very few electrons trapped in the iron acceptor level located at $0.5-0.6 \mathrm{eV}$ below the conduction band. Carrier generation at $532 \mathrm{~nm}$ was dominated by two photon absorption resulting in the switch conductance increasing as the square of applied optical intensity. A minimum switch resistance of 0.8 ohms was calculated for the $400 \mu \mathrm{m}$ thick, 1.2 by $1.2 \mathrm{~cm}, 2 \mathrm{H}-\mathrm{GaN}$ switch for an 
applied optical intensity of $41.25 \mathrm{MW} / \mathrm{cm}^{2}$. An optical intensity of $\sim 70 \mathrm{MW} / \mathrm{cm}^{2}$ at $532 \mathrm{~nm}$ would be required to achieve a $0.8 \mathrm{ohm}$ on resistance for a $1 \mathrm{~mm}$ thick, $1 \mathrm{~cm}^{2}, 2 \mathrm{H}-\mathrm{GaN}$ switch.

\section{2) Recommendations}

Future work on the development of vanadium compensated, 6H-SiC, extrinsic photoconductive switches should be focused on increasing switch reliability, operating voltage and power, along with improvements to how light is applied to the switch facets. There are also two basic material parameters that need to be measured for the third generation, vanadium compensated, $6 \mathrm{H}-\mathrm{SiC}$. My primary recommendation is to measure the recombination time, and electron and hole mobilities for the third generation, vanadium compensated, $6 \mathrm{H}-\mathrm{SiC}$ material. The carrier mobility and recombination time are important material parameters for any photoconductive switch. The recombination time determines the ultimate bandwidth of the photoconductive switch. The mobility multiplied by the recombination time is the 'lifetime mobility' product which is the measure of a photoconductor's sensitivity to optical excitation [10.2]. The mobility can be measured using the Temperature Dependent Hall Effect (TDH) method [10.3, 10.4]. Recombination times less than 1 ns can be measured by optical methods $[10.5,10.6]$, while recombination times greater than 1 ns can be measured using microwave techniques $[10.7,10.8]$.

The extrinsic photoconductive switches constructed from the third generation, vanadium compensated, $6 \mathrm{H}-\mathrm{SiC}$ material have demonstrated high power performance at a charge voltage of $16 \mathrm{kV}$ and peak current of 1400 Amps. High power testing consisted of hundreds of pulses. However, extrinsic photoconductive switches used in a commercial compact accelerator will have to operate for hundreds of million to billions of pulses without failure. This makes switch 
reliability equally as important as the demonstration of high power switch performance reported in this work. The vanadium compensated, $6 \mathrm{H}-\mathrm{SiC}$, extrinsic photoconductive switches should undergo an extensive series of switching tests to determine switch life as a function of peak current, average current and operating voltage.

The extrinsic, vanadium compensated, $6 \mathrm{H}-\mathrm{SiC}$ photoconductive switches have a limited maximum operating voltage when constructed as described in this dissertation. Alternate geometries will have to be developed to enable switch operation at higher electrical fields and operating voltages. The Rogowski, Bruce, and Chang [10.9 - 10.11] electrode configurations will allow higher switch operating voltages and electric fields. A Rogowski profile machined into the opposing faces of a substrate is shown in Figure 10.1(a) [10.12]. The metalized Rogowski profiles guarantee that the maximum electric field is located at the center of the substrate. The normalized electric field for the Rogowski profiled electrodes of Figure 10 (a) is plotted as a function of distance from point a to point b along the electrode surface in Figure 10 (b). The electric field is normalized to the value of electric field at the electrode center. This approach is difficult to implement since it requires machining of the $6 \mathrm{H}-\mathrm{SiC}$ material. In addition, the machining of the Rogowski profile shown in Figure 10.1 (a) reduces the thickness of the substrate to half its original value on the switch centerline. As a result, you need to start with a substrate that has twice the thickness required for the switch operating voltage. 


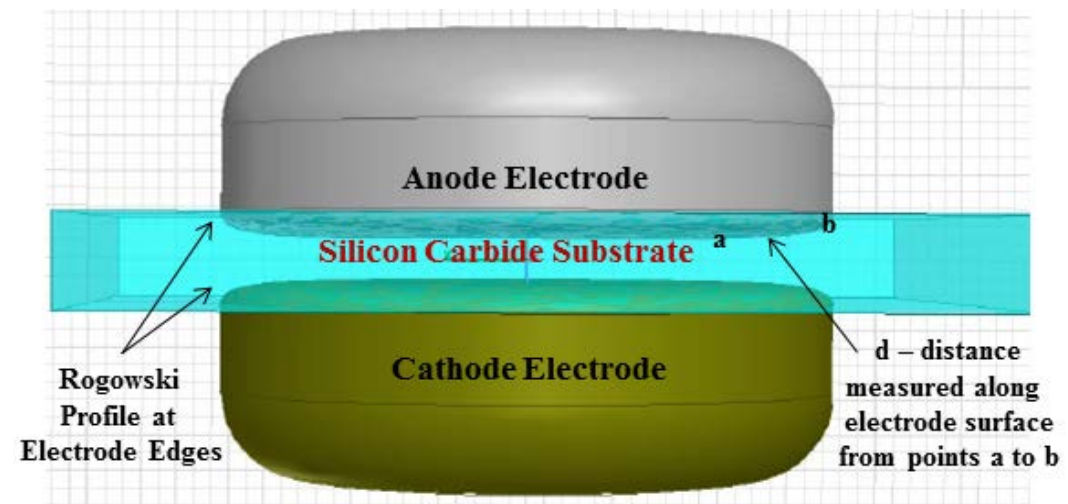

(a)

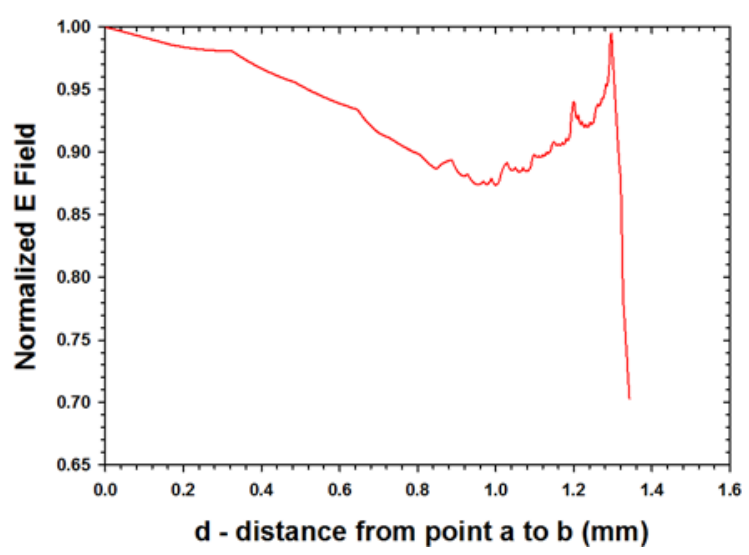

(b)

Figure 10.1 (a). Rogowski profile machined into opposing faces of a switch substrate and (b)calculated normalized electric field at anode surface from point a to point $b$.

A similar approach to achieving higher operating voltage is to encapsulate the $6 \mathrm{H}-\mathrm{SiC}$ substrate in a dielectric material with a dielectric constant equal to the dielectric constant of $6 \mathrm{H}-$ SiC ( 10.0) [10.13]. The Rogowski, Bruce, Chang, or any other profile can be machined, or cast, into the assembly. In this case the encapsulating dielectric is cast, or machined, to achieve the desired electrode profile, so machining the $6 \mathrm{H}-\mathrm{SiC}$ is avoided. This approach is depicted in Figure 10.2. A $1 \mathrm{~cm}$ by $1 \mathrm{~cm}, 1 \mathrm{~mm}$ thick, $6 \mathrm{H}-\mathrm{SiC}$ substrate is encapsulated in a transparent epoxy. The mold used to cast this assembly includes a Rogowski profile centered on the $6 \mathrm{H}-\mathrm{SiC}$ 
substrate. Future work should build on these first attempts at increasing switch operating voltage and electric field.
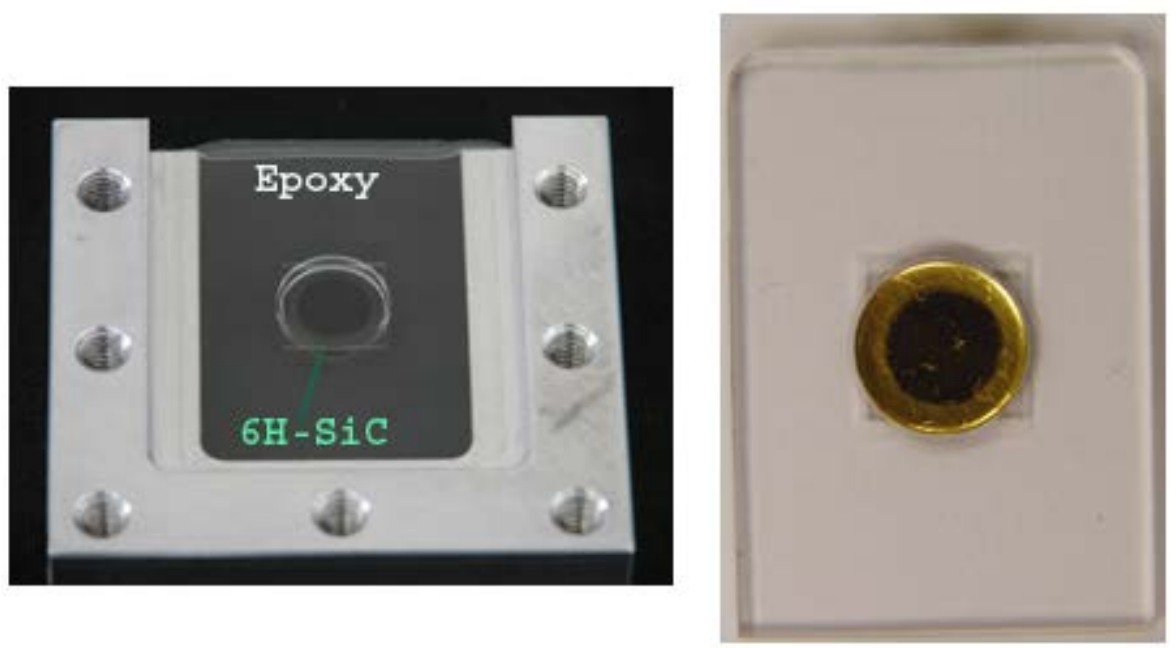

Figure 10.2. 6H-SiC substrate is potted in a transparent epoxy. Rogowski profile is cast into transparent epoxy. Metalized contact with Rogowski profile is shown on right.

The potted assembly shown in Figure 10.2 enables higher switch operating voltage, but prevents light from being directly applied to a switch facet. The optical intensity required to achieve low switch on resistance far exceeds the damage threshold of the encapsulating epoxy. As a result, an alternate method must be used to apply the optical trigger pulse to the switch facets for a potted switch design.

In general, a design where the light is fiber coupled to the switch facet should be the ultimate goal, since direct transmission of light to the switch facet is not always practical. Fibers, or waveguides, can be used to route light to the switch facets. The fibers and waveguides can be bonded to the switch facet and become incorporated into the potted switch design. The main technical requirement is that the light is applied to the switch facet uniformly. This may require a 
combination of both fibers and waveguides to achieve a uniform optical pulse at the switch facet as shown in Figure 10.3. The number, diameter and position of fibers at the waveguide facet will need to be balanced against the length of the waveguide (L) to assure that the optical pulse is uniform at the $6 \mathrm{H}-\mathrm{SiC}$ switch facet.

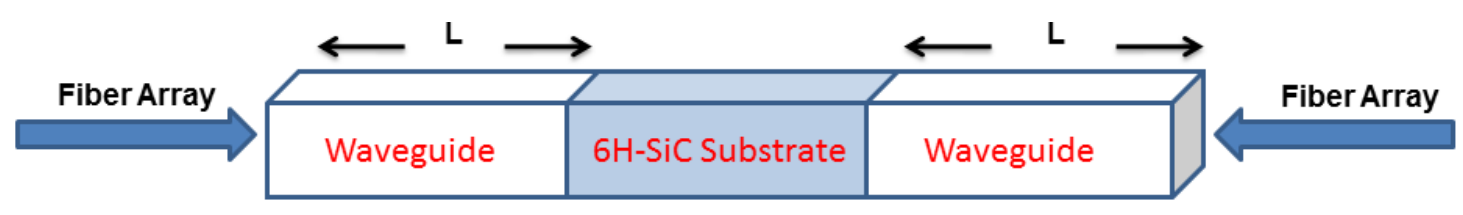

\section{Figure 10.3. Block diagram of fiber - waveguide system used to apply a uniform light pulse at the $6 \mathrm{H}-\mathrm{SiC}$ switch facet.}

There is also further material development that will enable switch development. The availability of micropipe-free, vanadium compensated, $6 \mathrm{H}-\mathrm{SiC}$ will allow for switch substrates cut from the material c plane. This would assure uniform doping of vanadium, nitrogen and boron across the switch substrate. There is a great deal of development needed to achieve reliable, high power, compact, $6 \mathrm{H}-\mathrm{SiC}$, extrinsic photoconductive switches. This dissertation represents only the beginning.

The $2 \mathrm{H}-\mathrm{GaN}$ material also performed well (poorly) as a photoconductive switch at the $532 \mathrm{~nm}(1064 \mathrm{~nm})$ wavelength. The $2 \mathrm{H}-\mathrm{GaN}$ switch performance at $1064 \mathrm{~nm}$ indicated that the bulk of the electrons from the shallow oxygen donors were trapped at levels deeper than the iron acceptor level. The 2H-GaN switch conductance at the $532 \mathrm{~nm}$ wavelength was dominated by electron hole pairs generated by two-photon absorption. This results in an interesting situation for the semi-insulating $2 \mathrm{H}-\mathrm{GaN}$ material. It seems clear that an extrinsic, $2 \mathrm{H}-\mathrm{GaN}$ 
photoconductive switch can be obtained by achieving the proper densities of the deep iron acceptors and shallow donors. The shallow donor can be either oxygen, or silicon. The development of a switch of this type is analogous to the $6 \mathrm{H}-\mathrm{SiC}$ extrinsic switch based on the vanadium and nitrogen dopants. This development should be pursued.

A photoconductive switch was constructed using semi-insulating, $2 \mathrm{H}-\mathrm{GaN}$ material that demonstrated sub-ohm switch on resistance based on carrier generation by two-photon absorption at the $532 \mathrm{~nm}$ wavelength. An eight hundred milliohm minimum switch on resistance was measured for a $400 \mu \mathrm{m}$ thick switch using trap a dominated $2 \mathrm{H}-\mathrm{GaN}$ material. Carrier generation by two-photon absorption has an advantage over linear extrinsic carrier generation. Carrier generation based on two-photon absorption does not require any carriers trapped at extrinsic levels in the switch material. In fact, any optical absorption by carriers trapped at extrinsic levels in the switch material would represent a loss for this type of photoconductive switch, since that would subtract from the photons available for the nonlinear two-photon absorption process. This makes photoconductive switches based on two-photon absorption in semi-insulating, $2 \mathrm{H}-\mathrm{GaN}$ ideally suited to construct from trap dominated materials with fast recovery. $2 \mathrm{H}-\mathrm{GaN}$ photoconductive switches based on two-photon absorption merit further study and development.

\section{3) References}

10.1) Compact Particle Acceleration Corporation, www.cpac.pro

10.2) R. H. Bube, Photoelectronic Properties of Semiconductors, pp. 18 - 20, Cambridge University Press, 1992 
10.3) W. C. Mitchel, et al, "Electrical properties of unintentionally doped semi-insulating and conducting 6H-SiC", Journal of Applied Physics, Vol. 100, No. 1, Paper Number 043706, 2006

10.4) Z - Q. Fang, et al, "Deep centers in semi-insulating Fe-doped native GaN structures grown by hydride vapour phase epitaxy", Physica Status Solidi (C), Current Topics in Solid State Physics, Vol. 5, No. 6, pp. 1508 - 1511, 2008

10.5) G. Tamulaitus, et al, "Carrier lifetime in conductive and vanadium doped 6H-SiC substrates”, Applied Physics Letters, Vol. 84, No. 3, pp. 335 - 337, 2004

10.6) V. Grivickas, et al, "Excess carrier lifetime of bulk n-type 3C-SiC, Applied Physics Letters, Vol. 95, No. 24, 2009

10.7) R. J. Kumar, et al, "Microwave photoconductivity decay characterization of high purity 4H-SiC substrates”, Journal of Applied Physics, Vol. 102, No.1, 2007

10.8) M. Kato, et al, "Excess carrier lifetime mapping for bulk SiC wafers by microwave photoconductivity decay method and its relationship with structural defect distribution", Materials Science Forum, Vol. 457 - 460, No. 1, pp. 505 - 508, 2004

10.9) J. D. Cobine, Gaseous Conductors - Theory and Engineering Applications, pp. 177 180, Dover, 1958

10.10) F. M. Bruce, "Calibration of uniform field spark gaps for high volume measurement at power frequencies", Journal of the Institute of Electrical Engineers, Vol. 94, pp. 138 149, 1947

10.11) T. J. Chang, "Improved uniform field electrode profiles for TEA lasers and high voltage applications”, Review of Scientific Instruments, Vol. 44, pp. 405 - 407, 1973

10.12) S. A. Hawkins and J. S. Sullivan, "Optically initiated silicon carbide high voltage switch with contoured-profile electrode interfaces", United States Patent US 8258632 B1

10.13) J. S. Sullivan, et al, "High-voltage photo-switch package module having encapsulation with profiled metallized concavities”, United States Patent US 20130056842 A1 